In cooperation with the SWEETWATER AUTHORITY

Water- and Air-Quality Monitoring of the Sweetwater Reservoir Watershed, San Diego County, CaliforniaPhase One Results, Continued, 1999-2001

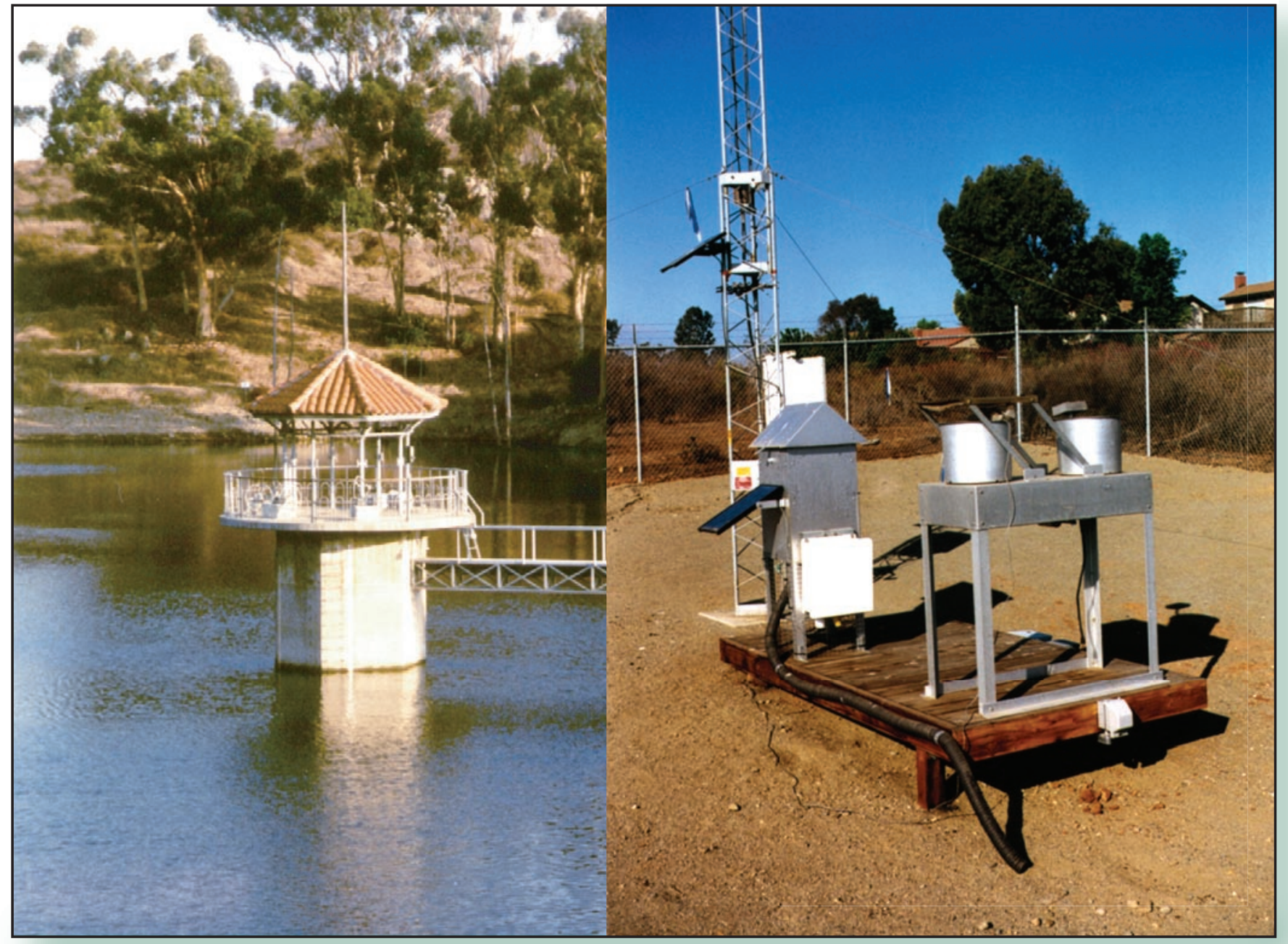

Data Series 233

U.S. DEPARTMENT OF THE INTERIOR U.S. GEOLOGICAL SURVEY 
Cover: Photographs supplied by Michael S. Majewski, U.S. Geological Survey. 


\section{Water- and Air-Quality Monitoring of the Sweetwater Reservoir Watershed, San Diego County, California-Phase One Results, Continued, 1999-2001}

By Gregory 0. Mendez, William T. Foreman, Jagdeep S. Sidhu, and Michael S. Majewski

In cooperation with the Sweetwater Authority

Data Series 233 


\section{U.S. Department of the Interior \\ Dirk Kempthorne, Secretary \\ U.S. Geological Survey \\ Mark D. Myers, Director}

U.S. Geological Survey, Reston, Virginia: 2007

For product and ordering information:

World Wide Web: http://www.usgs.gov/pubprod

Telephone: 1-888-ASK-USGS

For more information on the USGS--the Federal source for science about the Earth, its natural and living resources, natural hazards, and the environment:

World Wide Web: http://www.usgs.gov

Telephone: 1-888-ASK-USGS

Any use of trade, product, or firm names is for descriptive purposes only and does not imply endorsement by the U.S. Government.

Although this report is in the public domain, permission must be secured from the individual copyright owners to reproduce any copyrighted materials contained within this report.

Suggested citation:

Mendez, G.0., Foreman, W.T., Sidhu, J.S., and Majewski, M.S., 2007, Water- and air-quality monitoring of the Sweetwater Reservoir watershed, San Diego County, California-Phase one results, continued, 1999-2001: U.S. Geological Survey Data Series 233, 270 p. 


\section{Contents}

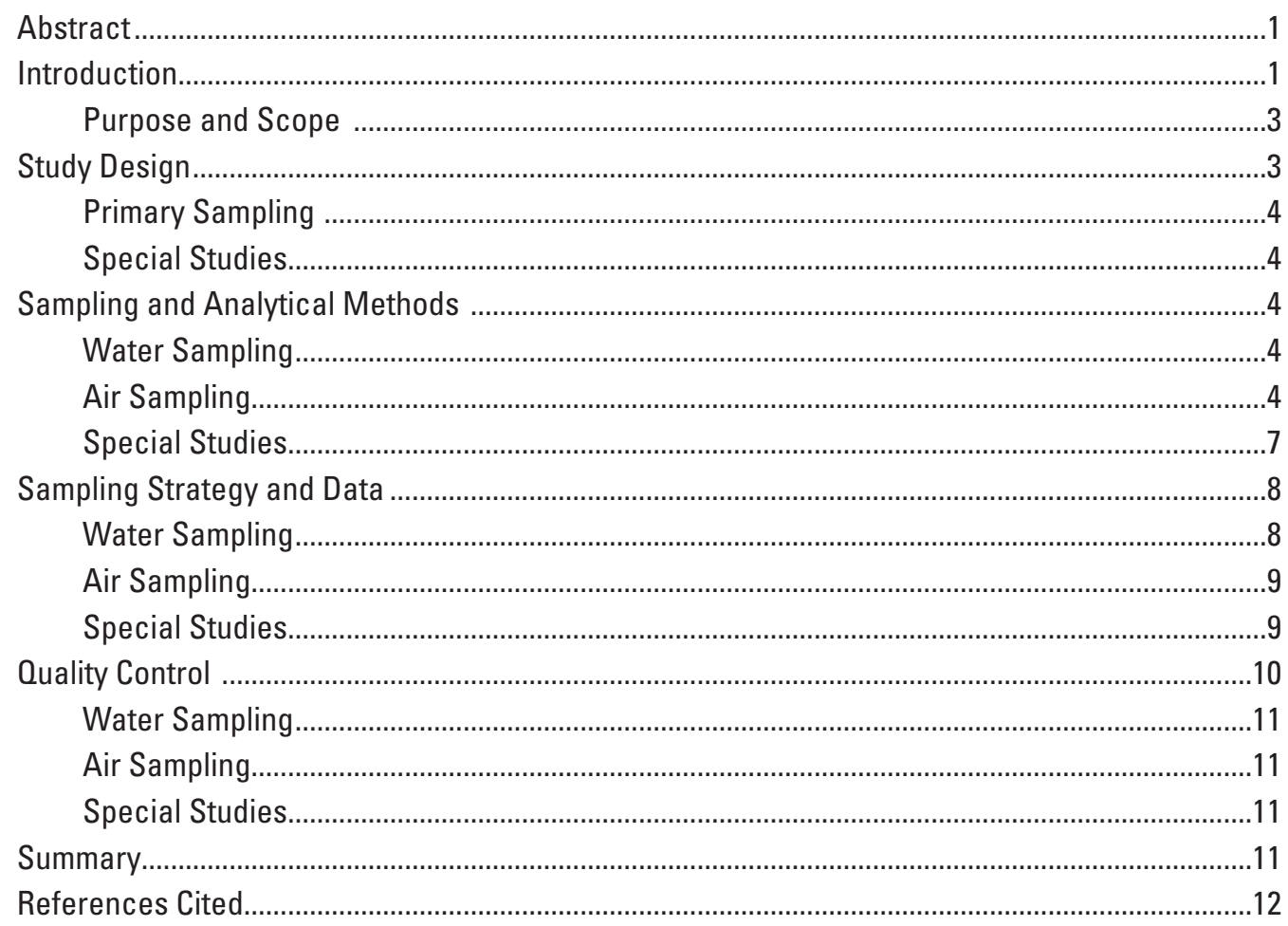

\section{Figures}

Figure 1. Map showing location of the study area, Sweetwater River watershed, the Sweetwater and Loveland Reservoirs, the Reynolds Desalination Plant, and selected wells.

Figure 2. Map showing Sweetwater Reservoir sampling sites and the most probable alignment of SR 125, San Diego County, California $\ldots \ldots \ldots \ldots \ldots \ldots \ldots \ldots \ldots \ldots$

Figure 3. Map showing Loveland Reservoir sampling sites, San Diego County, California ..... 6 


\section{Tables}

Table 1. Site identification numbers, site names, state well numbers, and other

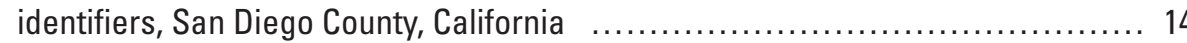

Table 2. Water-quality depth-profile field data for Sweetwater and Loveland Reservoirs sampling sites and for Sweetwater River above Sweetwater Reservoir, San Diego County, California

Table 3. Analytical results for selected volatile organic compounds in whole water using USGS National Water Quality Laboratory Schedule 2020 in Sweetwater and Loveland Reservoirs, San Diego County, California 25

Table 4. Analytical results for pesticide compounds in filtered water using U.S. Geological Survey National Water Quality Laboratory Schedule 2001 for Sweetwater and Loveland Reservoirs, San Diego County, California

Table 5A. Analytical results for volatile organic compounds with low breakthrough volumes in air for the Sweetwater Reservoir air sampling site, San Diego County, California

Table 5B. Analytical results for volatile organic compounds with high breakthrough volumes in air from the Sweetwater Reservoir air sampling site, San Diego County, California

Table 6. Analytical results for polycyclic aromatic hydrocarbon compounds in air for the Sweetwater Reservoir air sampling site, San Diego County, California

Table 7A. Pesticide compounds analyzed in air from modified U.S. Geological Survey National Water Quality Laboratory schedules for the Sweetwater Reservoir air sampling site, San Diego County, California

Table 7B. Analytical results for pesticide concentrations in air using modified U.S. Geological Survey National Water Quality Laboratory Schedule 2001 for the Sweetwater Reservoir air sampling site, San Diego County, California

Table 7C. Analytical results for pesticide concentrations in air using modified U.S. Geological Survey National Water Quality Laboratory Schedule 2002 for the Sweetwater Reservoir air sampling site, San Diego County, California ...... 134

Table 8. Analytical results for first special study—pesticide compounds in filtered water using U.S. Geological Survey National Water Quality Laboratory Schedule 2002 - in Sweetwater River and Sweetwater Reservoir, San Diego County, California

Table 9A. Analytical results for second special study — pesticides in semipermeable membrane device (SPMD) samples—for the Sweetwater Reservoir, San Diego County, California

Table 9B. Analytical results for second special study-polycyclic aromatic hydrocarbons and other semivolatile organic compounds in semipermeable membrane device (SPMD) samples_-for the Sweetwater Reservoir, San Diego County, California ... 147

Table 10. Analytical results for third special study—dissolved copper-at the Perdue Treatment Plant, the Reynolds Desalination Facility, and Sweetwater Wells, San Diego County, California

Table 11. Analytical results of fourth special study—volatile organic compounds in whole water-using U.S. Geological Survey National Water Quality Laboratory Schedule 2020 for selected National City wells, San Diego County, California ...... 151

Table 12. Analytical results for fifth special study-dissolved trace metals in filtered water-for the Sweetwater Reservoir, San Diego County, California

Table 13. Analytical results for sixth (last) special study-selected wastewater compounds in whole water-for the Sweetwater and Loveland Reservoirs, San Diego County, California 
Table 14. Quality-control analytical results for volatile organic compound (VOC) concentrations using U.S. Geological Survey National Water Quality Laboratory Schedule 2020 for water samples from Sweetwater and Loveland Reservoirs, San Diego County, California

Table 15. Quality-control analytical results for pesticide concentrations using U.S. Geological Survey National Water Quality Laboratory Schedule 2001 for filtered water samples from Perdue Treatment Plant, San Diego County, California ....

Table 16A. Quality-control analytical cartridge travel blank results for volatile organic compounds with low breakthrough volumes for the Sweetwater Reservoir air sampling site, San Diego County, California

Table 16B. Quality-control analytical cartridge spike results for volatile organic compounds with low breakthrough volumes for the Sweetwater Reservoir air sampling site, San Diego County, California

Table 16C. Quality-control analytical cartridge lot blank results for volatile organic compounds with low breakthrough volumes for the Sweet-water Reservoir air sampling site, San Diego County, California

Table 16D. Quality-control analytical laboratory blank results for volatile organic compounds with low breakthrough volumes for the Sweetwater Reservoir air sampling site, San Diego County, California

Table 17A. Quality-control analytical cartridge travel blank results for volatile organic compounds (VOC) with high breakthrough volumes for the Sweetwater Reservoir air sampling site, San Diego County, California

Table 17B. Quality-control analytical results for cartridge spike for volatile organic compounds (VOC) with high breakthrough volumes for the Sweetwater Reservoir air sampling site, San Diego County, California

Table 17C. Quality-control analytical cartridge lot blank results for volatile organic compounds (VOC) with high breakthrough volumes for the Sweetwater Reservoir air sampling site, San Diego County, California ....

Table 17D. Quality-control analytical laboratory blank results for volatile organic compounds (VOC) with high breakthrough volumes for the Sweetwater Reservoir air sampling site, San Diego County, California

Table 18A. Quality-control laboratory reagent blank results for polycyclic aromatic hydrocarbon compound concentrations for the Sweetwater Reservoir air sampling site, San Diego County, California

Table 18B. Quality-control laboratory reagent spike results for polycyclic aromatic hydrocarbon compound concentrations for the Sweetwater Reservoir air sampling site, San Diego County, California

Table 19A. Quality-control laboratory reagent blank results for pesticide concentrations using modified U.S. Geological Survey National Water Quality Laboratory Schedule 2001 for the Sweetwater Reservoir air sampling site, San Diego County, California

Table 19B. Quality-control laboratory spike results for pesticide concentrations using modified U.S. Geological Survey National Water Quality Laboratory Schedule 2001 for the Sweetwater Reservoir air sampling site, San Diego County, California

Table 20A. Quality-control laboratory reagent blank results for pesticide concentrations using modified U.S. Geological Survey National Water Quality Laboratory Schedule 2002 for the Sweetwater Reservoir air sampling site, San Diego County, California 
Table 20B. Quality-control laboratory reagent spike results for pesticide concentrations in air using modified U.S. Geological Survey National Water Quality Laboratory Schedule 2002 for the Sweetwater Reservoir air sampling site, San Diego County, Californ

Table 21. Quality-control analytical results for surrogates added to the semipermeable membrane device in the Sweetwater Reservoir, San Diego County, California.....

Table 22. Quality-control laboratory reagent blank and reagent spike results for selected wastewater compounds in whole water at the U.S. Geological Survey National Water Quality Laboratory, Colorado

\section{Conversion Factors}

\begin{tabular}{lcl}
\hline \multicolumn{1}{c}{ Multiply } & By & \multicolumn{1}{c}{ To obtain } \\
\hline acre-foot (acre-ft) & 1,233 & cubic meter $\left(\mathrm{m}^{3}\right)$ \\
acre-foot $(\mathrm{acre}-\mathrm{ft})$ & 0.001233 & cubic hectometer $\left(\mathrm{hm}^{3}\right)$ \\
centimeter $(\mathrm{cm})$ & 0.3937 & inch (in.) \\
gram $(\mathrm{g})$ & 0.03527 & ounce, avoirdupois (oz) \\
hectometer, cubic $\left(\mathrm{hm}^{3}\right)$ & 810.7 & acre-foot (acre-ft) \\
kilogram $(\mathrm{kg})$ & 2.205 & pound, avoirudupois (lb) \\
kilometer $(\mathrm{km})$ & 0.6214 & mile (mi) \\
liter $(\mathrm{L})$ & 33.82 & ounce, fluid (oz) \\
meter $(\mathrm{m})$ & 3.281 & foot $(\mathrm{ft})$ \\
millimeter $(\mathrm{mm})$ & 0.03937 & inch (in.) \\
\hline
\end{tabular}

\section{Notes}

Temperature in degrees Celsius $\left({ }^{\circ} \mathrm{C}\right)$ may be converted to degrees Fahrenheit $\left({ }^{\circ} \mathrm{F}\right)$ as follows:

$$
{ }^{\circ} \mathrm{F}=\left(1.8 \times{ }^{\circ} \mathrm{C}\right)+32
$$

Temperature in degrees Fahrenheit $\left({ }^{\circ} \mathrm{F}\right)$ may be converted to degrees Celsius $\left({ }^{\circ} \mathrm{C}\right)$ as follows:

$$
{ }^{\circ} \mathrm{C}=\left({ }^{\circ} \mathrm{F}-32\right) / 1.8
$$

Vertical coordinate information is referenced to the North American Vertical Datum of 1988 (NAVD 88).

Horizontal coordinate information is referenced to the North American Datum of 1983 (NAD 83).

Altitude, as used in this report, refers to distance above the vertical datum.

Specific conductance is given in microsiemens per centimeter at 25 degrees Celsius $\left(\mu \mathrm{S} / \mathrm{cm}\right.$ at $\left.25^{\circ} \mathrm{C}\right)$.

Concentrations of chemical constituents in water are given either in milligrams per liter (mg/L) or micrograms per liter $(\mu \mathrm{g} / \mathrm{L})$. 


\section{Abbreviations, Acronyms, and Initialisms}

(Note: Clarification or additional information is provided in parentheses)

acre-ft, acre-foot

$\mathrm{cm}$, centimeter

g, gram

$\mathrm{gm}^{-3}$, gram per cubic meter

$\mathrm{km}$, kilometer

$\mathrm{km}^{2}$, square kilometer

$\mathrm{L}$, liter

$\mathrm{m}$, meter

$\mathrm{m}^{3}$, cubic meter

$\mathrm{mg}$, milligram

min, minute

$\mathrm{mm}$, millimeter

$\mu \mathrm{g}$, microgram

$\mu \mathrm{S} / \mathrm{cm}$, microsiemens per centimeter

CARB, California Air Resources Board

GC/MS, gas chromatography/mass spectrometry

GFF, glass fiber filter

LFDD, low-flow diversion dam

LLR, Loveland Reservoir

MCL, maximum contaminant level

MDL, method detection limit

MRDP, Methods Research and Development Program

NAWQA, National Water Quality Assessment (USGS)

NPDES, National Pollutant Discharge Elimination System

NWQL, National Water Quality Laboratory (USGS)

PAH, polynuclear aromatic hydrocarbons

PCB, polychlorinated biphenyls

PUF, polyurethane foam

SDF, San Diego Formation

SPMD, semipermeable membrane device

$\mathrm{SR}$, state route

SWR, Sweetwater Reservoir

URDS, Urban Runoff Diversion System

USGS, U.S. Geological Survey

VOC, volatile organic compound 


\section{Well-Numbering System}

Wells are identified and numbered according to their location in the rectangular system for the subdivision of public lands. Identification consists of the township number, north or south; the range number, east or west; and the section number. Each section is divided into sixteen 40-acre tracts lettered consecutively (except I and $\mathrm{O})$, beginning with "A" in the northeast corner of the section and progressing in a sinusoidal manner to "R" in the southeast corner. Within the 40-acre tract, wells are sequentially numbered in the order they are inventoried. The final letter refers to the base line and meridian. In California, there are three base lines and meridians; Humboldt (H), Mount Diablo (M), and San Bernardino (S). All wells in the study area are referenced to the San Bernardino base line and meridian (S). Well numbers consist of 15 characters and follow the format 017S002W-028R001. In this report, well numbers are abbreviated and written 17S/2W-28R1. Wells in the same township and range are referred to only by their section designation, 28R1. The following diagram shows how the number for well $17 \mathrm{~S} / 2 \mathrm{~W}-28 \mathrm{R} 1$ is derived.

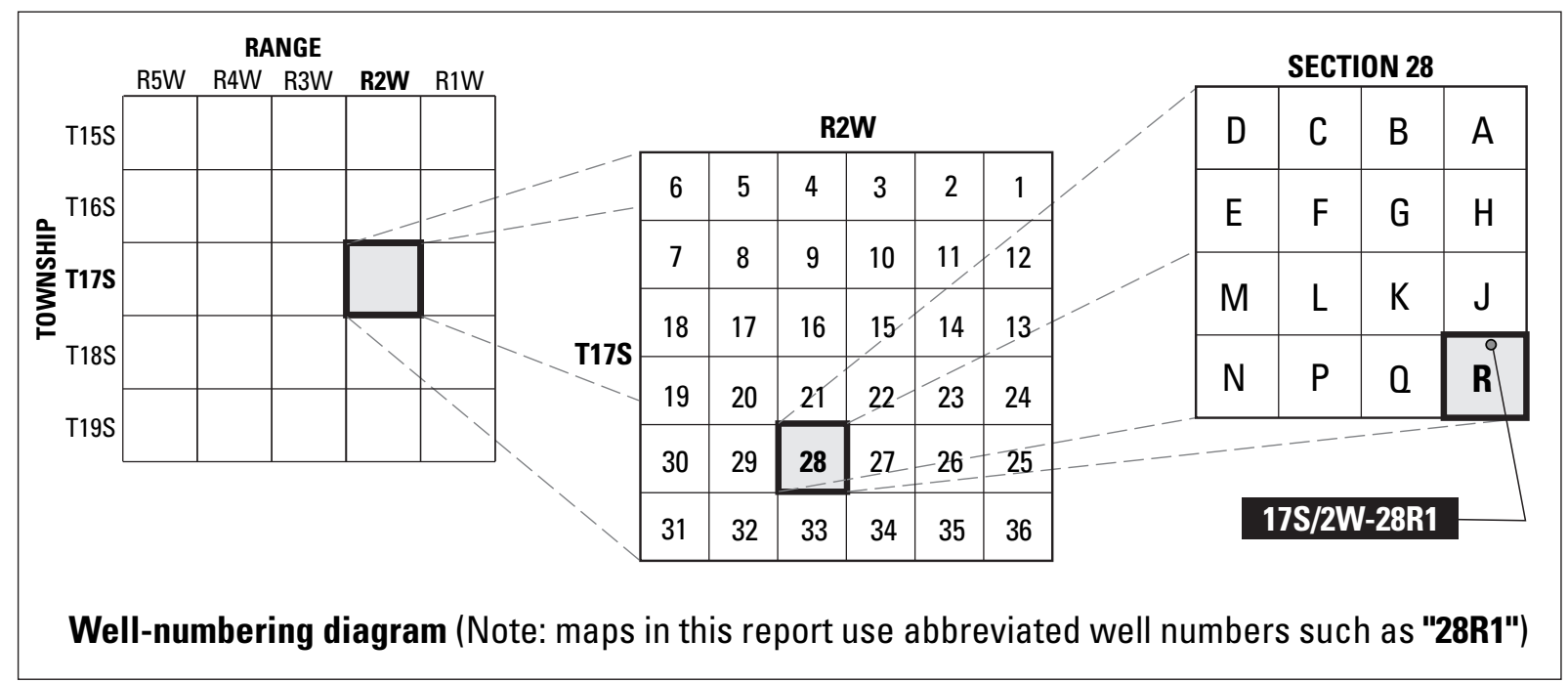




\title{
Water- and Air-Quality Monitoring of the Sweetwater Reservoir Watershed, San Diego County, California-Phase One Results, Continued, 1999-2001
}

\author{
By Gregory 0. Mendez, William T. Foreman, Jagdeep S. Sidhu, and Michael S. Majewski
}

\section{Abstract}

In 1998, the U.S. Geological Survey, in cooperation with the Sweetwater Authority, began a study to assess the overall health of the Sweetwater watershed with respect to chemical contamination. The study included regular sampling of air and water at Sweetwater Reservoir for chemical contaminants, including volatile organic compounds, polycyclic aromatic hydrocarbons, pesticides, and major and trace elements. Background water samples were collected at Loveland Reservoir for volatile organic compounds and pesticides.

The purpose of this study was to monitor changes in contaminant composition and concentration in the air and water resulting from the construction and operation of State Route 125 near Sweetwater Reservoir. To accomplish this, the study was divided into two phases. Phase One sampling was designed to establish baseline conditions for target compounds in terms of detection frequency and concentration in air and water. Phase Two sampling is planned to continue at the established monitoring sites during and after construction of State Route 125 to assess the chemical impact this roadway alignment project may have on the water quality in the reservoir. In addition to the ongoing data collection, several special studies were initiated to assess the occurrence of specific chemicals of concern, such as low-use pesticides, trace metals, and wastewater compounds.

This report describes the study design, and the sampling and analytical methods, and presents the results for the second and third years of the study (October 1999 to September 2001). Data collected during the first year of sampling (October 1998 to September 1999) were published in 2002.

\section{Introduction}

The Sweetwater Authority (hereinafter referred to as the "Authority"), under the guidance of its Board of Directors, operates a public drinking-water supply system for over 175,000 residential and commercial customers in Chula Vista, National City, and Bonita, California. The Sweetwater Reservoir (SWR) (fig. 1), which has a storage capacity of $34.6 \mathrm{hm}^{3}$, is located about $15 \mathrm{~km}$ southeast of San Diego, California. The Authority also stores water at Loveland Reservoir (LLR) (fig. 1), which has a storage capacity of $31.3 \mathrm{hm}^{3}$ and is located about $30 \mathrm{~km}$ east of SWR near Alpine, California. In addition to the two reservoirs, the Authority operates two deep wells in National City and several alluvial wells near the Sweetwater River in Chula Vista. The Reynolds Desalination Facility, formerly known as the Demineralization Facility, in Chula Vista, treats brackish ground water from the wells along the Sweetwater River. Approximately 70 percent of the water the Authority provides comes from local supplies that include the Sweetwater River and ground water. The remaining water is imported from the Colorado River and northern California sources through pipelines and aqueducts. Both local reservoir and imported waters are treated at the Robert A. Perdue treatment plant located at the SWR.

The Sweetwater River watershed covers $466 \mathrm{~km}^{2}$ (fig. 1). Although much of the land is undeveloped, the watershed includes the Sycuan and Viejas Indian Reservations, part of the Cleveland National Forest, agricultural land, rural residential acreage, urban and suburban residential development, mining and industrial land use, commercial recreation, and commercial business development. The watershed currently includes three 18-hole golf courses. During the data-collection period described (water years 2000 and 2001), no recreational activities were allowed at the SWR. Shore fishing is allowed at Loveland Reservoir in a restricted area at the east end of the reservoir. The Authority maintains a motorized boat at each reservoir for routine water-quality sampling and regular shore patrols. 


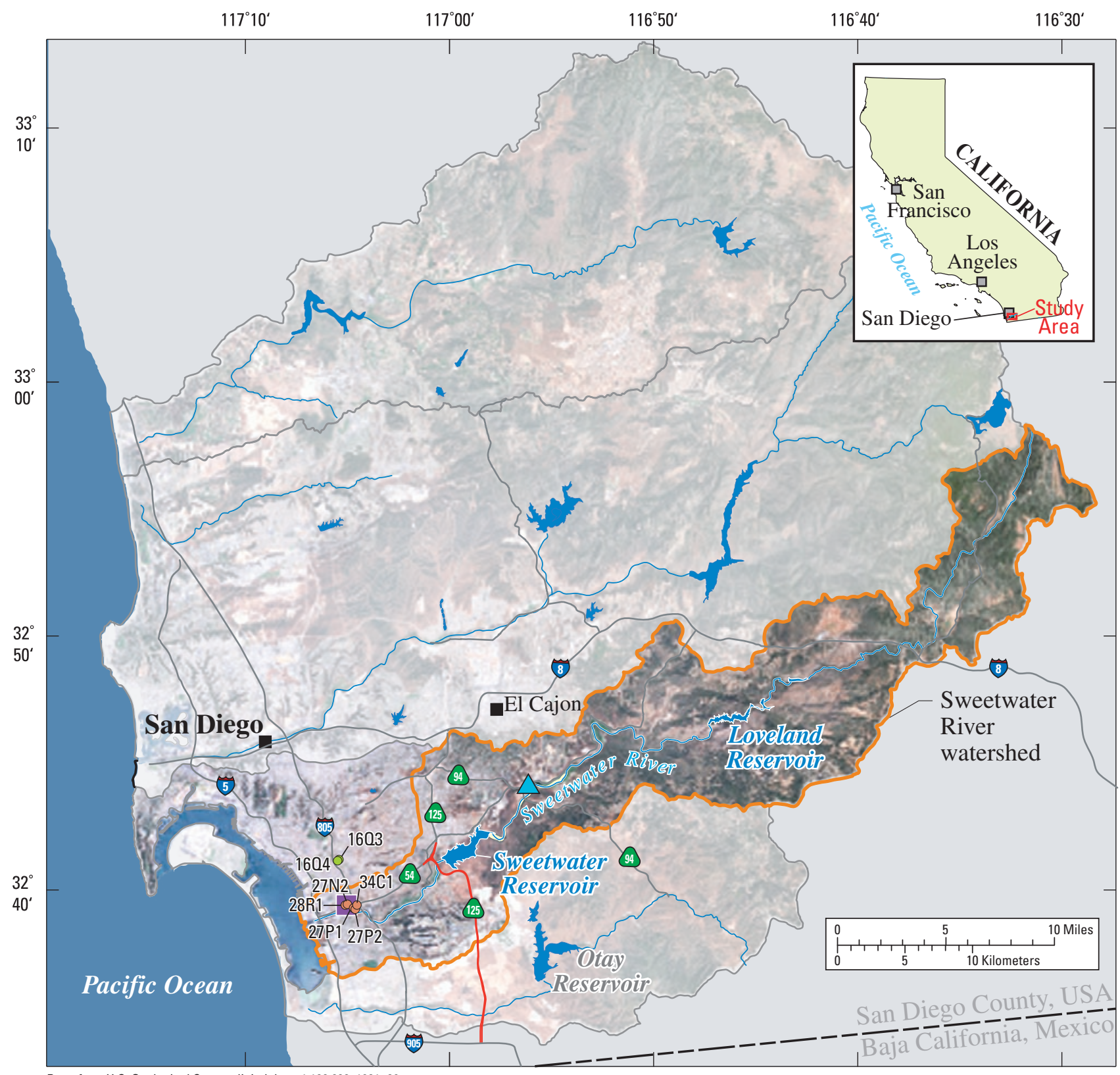

Base from U.S. Geological Survey digital data, 1:100,000, 1981-89;

Universal Transverse Mercator Projection (NGVD 29), Zone 11.

\section{EXPLANATION}

- Alluvial wells $\quad$ Reynolds Desalination Facility

- National City wells $\triangle$ Sweetwater River at Steele Canyon bridge

Figure 1. Location of the study area, Sweetwater River watershed, the Sweetwater and Loveland Reservoirs, the Reynolds Desalination Plant, and selected wells. 
The impact of local inputs of anthropogenic compounds, such as organic chemicals and pesticides, on the watershed and reservoir water quality is largely unknown. The Authority is concerned about the impact that increasing growth and development in the Sweetwater River watershed will have on the quality of their drinking-water supply and has initiated a variety of efforts to protect the watershed. These efforts include source assessments, total-organic-carbon assessments, watershed stakeholders' outreach program to identify issues, and the construction and operation of an urban-runoff diversion system. These programs help the Authority evaluate and manage the overall environmental health of the watershed by monitoring changes that can degrade the quality of the water supply and necessitate additional water treatment as the population increases and land use intensifies. Responding to these concerns, in 1998, the Authority initiated a monitoring study in cooperation with the U.S. Geological Survey (USGS).

In addition to the increasing urbanization pressures within the watershed, another Authority concern and the primary reason for this study, is the construction and operation of State Route (SR) 125. In 1984, the San Diego Association of Governments added SR 125 to the Regional Transportation Plan as part of San Diego's future highway system. The SR 125 project (fig. 1) consists of approximately $18 \mathrm{~km}$ of roadway construction and alignment that extends from SR 54 (northern terminus) to Interstate 905 (southern terminus). The project plans call for the initial construction of a four-lane toll way that may be expanded to include additional lanes for dedicated transit purposes such as high occupancy vehicles or light-rail (California Department of Transportation, 2001). More than 200,000 vehicles per day, including more than 10 percent of heavy diesel trucks from both the United States and Mexico, are expected to travel SR 125. The alignment will be elevated about $30 \mathrm{~m}$ above land surface at its highest point, and the most likely construction scenario will bring SR 125 within about $150 \mathrm{~m}$ of the reservoir at its nearest point. Construction of SR 125 was scheduled to begin in 2005 and is expected to be completed in 2007. Because the SWR is downwind of all proposed alignments, the Authority became concerned that toxic vehicle emissions and pesticides used on the roadside, as well as paved-road dusts, might enter the reservoir by atmospheric deposition in concentrations that could affect public health and have an impact on the cost of treating the drinking-water supply.

In 1996, the Authority commissioned a study (Ogden Environmental and Energy Services, 1997) to model the atmospheric depositional loading to the SWR. The model included a variety of toxic compounds from vehicular fuel combustion emissions and any attendant health risks associated with all SR 125 alignment scenarios. The predicted concentrations of selected contaminants were compared with the standards set by the California Safe Drinking Water Act (California Environmental Protection Agency, 1986) and with California and federal maximum contaminant levels (MCL). The model results indicated that drinking-water guidance levels for one or more contaminants would be exceeded in all three alternative freeway construction options. This study was repeated reaching similar conclusions (Byard and Giroux, 1999). Both the Authority and U.S. Environmental Protection Agency Region IX concluded that the findings in the Ogden and the Byard and Giroux reports warranted the implementation of a monitoring program to characterize the impact that atmospheric deposition of vehicular emissions from the operation of SR 125 may have on the quality of the drinking water stored in the SWR.

\section{Purpose and Scope}

The purpose of this report is to describe the data that were collected from October 1999 to September 2001 (water years 2000 and 2001), prior to the start of construction of SR 125. This report is the second in a series that describes the monitoring activities and presents the data that will be used to assess the potential impact on water quality in the Sweetwater Reservoir resulting from land-use changes and development in the watershed. Data collected during the first year of sampling (October 1998 to September 1999) were published in Majewski and others (2002). A full interpretation and assessment of the data will be completed at the end of the monitoring study.

The scope of the USGS study is to compare analytical results of samples from three environmental media-air, water, and bed sediment — to determine whether any measured changes in reservoir water quality are the result of atmospheric deposition from the construction and operation of SR 125. Because new chemical contaminants of concern are identified regularly, this project has worked closely with the Methods Research and Development Program (MRDP) group at USGS National Water Quality Laboratory (NWQL) to help bring online new analytical methods for chemicals of concern, such as endocrine disrupting compounds, pharmaceuticals, wastewater indicator compounds, and the ever increasing number of pesticides being used. The MRDP at NWQL continually develops new analytical methods for the various classes of emerging chemical contaminants.

\section{Study Design}

The study consists of two phases: Phase One sampling started in October 1998 and will continue until construction starts for SR 125, when Phase Two sampling will begin. The objectives of Phase One are to determine the occurrence and concentration of selected organic and inorganic compounds in water, air, and bed sediments and to establish baseline conditions for the target analytes before construction of SR 125 begins. The objectives of Phase Two are to continue monitoring for the same chemical constituents, to compare the analytical results with those from Phase One, and to assess the impact of SR 125 on water quality in the SWR. Two types of studies were done: a study using primary samples to determine a common set of compounds and special studies to assess the occurrence of specific chemicals of concern. 


\section{Primary Sampling}

Each of the environmental media (water, air, and sediment) in the Sweetwater watershed is being sampled and analyzed for those compounds expected to accumulate in them. The media and compounds that will be sampled throughout the study, the core (primary) data, include the following:

- Surface water-volatile organic compounds (VOC) and pesticides;

- Air-VOCs, polycyclic aromatic hydrocarbons (PAH), and pesticides;

- Bed sediments-PAHs, total polychlorinated biphenyls (PCB), pesticides, and major and trace metals.

Originally, Phase One incorporated seven water-sampling sites at SWR (sites 1-7, fig. 2), and two at LLR (ig. 3). Regularly scheduled water sampling began at these sites during the first year of the study (water year 1999). Starting with the second year, only three of the seven sites at SWR and one site at LLR were used for sampling.

Phase Two sampling will start when construction of SR 125 begins. Water and air monitoring will continue at the established sites during and after the construction of SR 125. Data from the California Air Resources Board (CARB) Air Toxics monitoring site at Chula Vista will be used to assess the number and concentration of airborne anthropogenic compounds originating from areas upwind of the SR 125 alignment. Phase Two monitoring also will be used to assess the continuing inputs of anthropogenic compounds resulting from the land-use practices in the watershed by comparing compound detections and frequency with data collected in Phase One.

The air-sampling site is located adjacent to SWR, downwind of the proposed SR 125 routes and upwind of SWR along a transect of the predominant wind direction (site denoted by a star symbol on fig. 2 ). Air samples were not collected at LLR because its foothill location $(30 \mathrm{~km}$ east of SWR) is considered sufficiently downwind of SR 125 to be minimally affected by any airborne contaminants originating from this highway. The air-sampling site is wired for AC power (120 volts), equipped with telephone modem access, and secured from the general public by a 2-m high chain-link fence.

Surficial bed sediments were not sampled during the time frame covered by this report, but were sampled at the beginning of Phase One, as previously reported by Majewski and others (2002), at each reservoir water-sampling site and at the urban-runoff diversion system ponds. Also, at the beginning of Phase One, a one-time coring of the SWR bed sediments was completed (Majewski and others, 2002). The bed sediments will be resampled at the beginning of Phase Two and again several months before the completion of the study.

\section{Special Studies}

During the course of this project, several special studies have been initiated to help the Authority better understand the effects that different operational modes have on water quality. These special studies, which are of limited scope and only conducted at a targeted subset of sampling locations, are expected to provide up-to-date water-quality information on chemicals of current concern. Six special studies were conducted during the 2-year time frame covered by this report (1999-2001). Two of these special studies involved analysis of ground water.

\section{Sampling and Analytical Methods}

\section{Water Sampling}

Reservoir water samples for all analyses were collected using a 1.2-L, Teflon Kemmerer sampler (Wildco, Saginaw, MI). Procedures for collecting and processing water samples for dissolved chemical determinations are based on protocols used by the NAWQA Program (Shelton, 1994, 1997). Samples from the low-flow diversion dam (LFDD, fig. 2) were obtained as grab samples collected by submerging the sample bottle, removing the cap, filling the bottle, and recapping the bottle while still submerged. The finished (treated) drinking-water samples and imported raw-water samples were collected from spigots located at the distribution points. The water lines were flushed for 5 minutes before the sample bottles were filled.

Each primary water sample was analyzed for VOCs and pesticides at USGS National Water Quality Laboratory. The VOC analysis determined 87 compounds by purge and trap capillary-column gas chromatography/mass spectrometry $(\mathrm{GC} /$ MS) with full-scan ion monitoring (Connor and others, 1998). Water samples for pesticide analyses were filtered through 0.7-micrometer (nominal pore diameter) glass-fiber filters and were analyzed for pesticides using laboratory schedule 2001 at USGS NWQL. Schedule 2001 determines 47 pesticides and pesticide transformation products by $\mathrm{C}-18$ solid-phase extraction (SPE) and capillary-column GC/MS with selected-ion monitoring (GC/MS-SIM) (Zaugg and others, 1995).

\section{Air Sampling}

During the time frame of the sample collection and analyses that were performed for this report, there were no published USGS methods for the techniques used in this monitoring program to measure pesticides and PAHs, and as such, these techniques are considered "research methods." Analytical results that were obtained using these research methods have not been entered into the USGS database, but rather are presented in this report along with a full description of the methods that were used to obtain the data. 


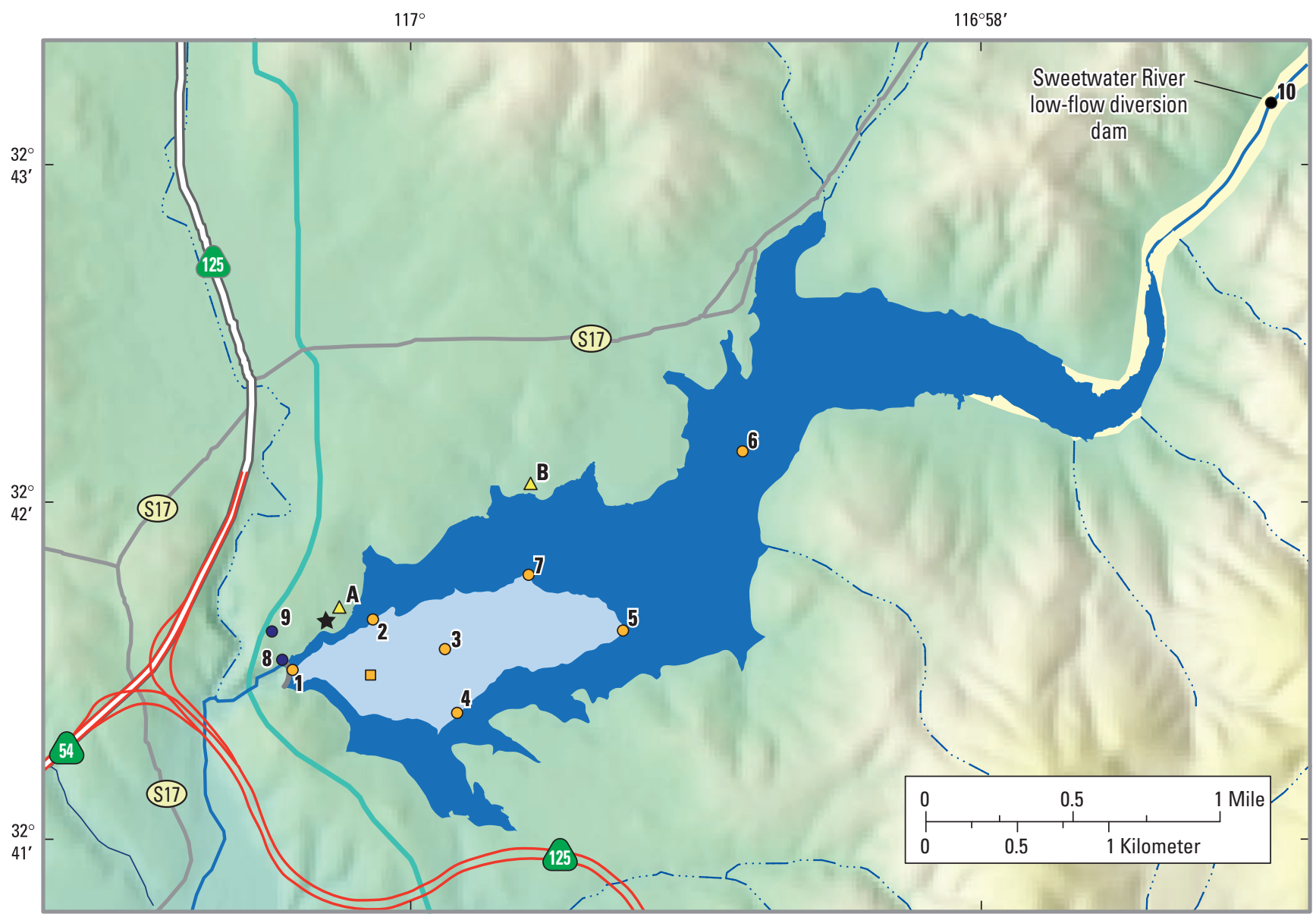

Base from U.S. Geological Survey digital data, 1:100,000, 1981-89; Universal Transverse Mercator Projection (NGVD 29), Zone 11.

\section{EXPLANATION}

Sweetwater Reservoirmaximum pool boundary

Sweetwater Reservoirminimum pool boundary

$=$ SR-125 alignment (under construction)

$=$ Highway

- Road

- Sweetwater River

-.. Intermittent stream

- Pipeline
○ Water sample and surficial bed sediment (Sweetwater Reservoir) -

1 Near pump tower

2 Near Vista del Lago

3 Center of minimum pool

4 Near recreation area

5 Minimum pool boundary east

6 East end reservoir fill boundary

7 Near Gum Tree Cove pond
- Water sample only-

8 Perdue Treatment Plant finished water

9 Perdue Treatment Plant imported raw water

10 Sweetwater River at low-flow diversion dam

Figure 2. Sweetwater Reservoir sampling sites and the most probable alignment of SR 125, San Diego County, California.

In conjunction with the USGS, the low-volume air sampling and analytical methods for VOCs were developed by, and all samples were analyzed by, the research group of Dr. James Pankow at the Oregon Graduate Institute of the Oregon Health and Science University. The air samples for VOC analyses were collected by adsorption onto cartridges and analyzed by thermal-desorption GC/MS procedures as detailed in Pankow and others (1998). Using these procedures, ambient, gas-phase, atmospheric VOC concentrations were monitored using two programmable low-volume air sampling pumps
(224-PCXR8, SKC INC., Eight Four, Pennsylvania). One type of sampler was used to pull a 1.5-L sample of air through a glass cartridge containing $50 \mathrm{mg}$ of Carbotrap B in series with $280 \mathrm{mg}$ of Carboxen 1000 (Supelco, Bellafonte, Pennsylvania) for the analysis of seven VOCs with the highest volatilities (lowest breakthrough volumes), including several chlorofluorocarbons. A second type of sampler was used to pull a 5-L sample of air through a glass cartridge containing $180 \mathrm{mg}$ of Carbotrap B in series with 70 mg of Carboxen 1000 (Supelco, Bellafonte, Pennsylvania) for the analysis of the remaining 


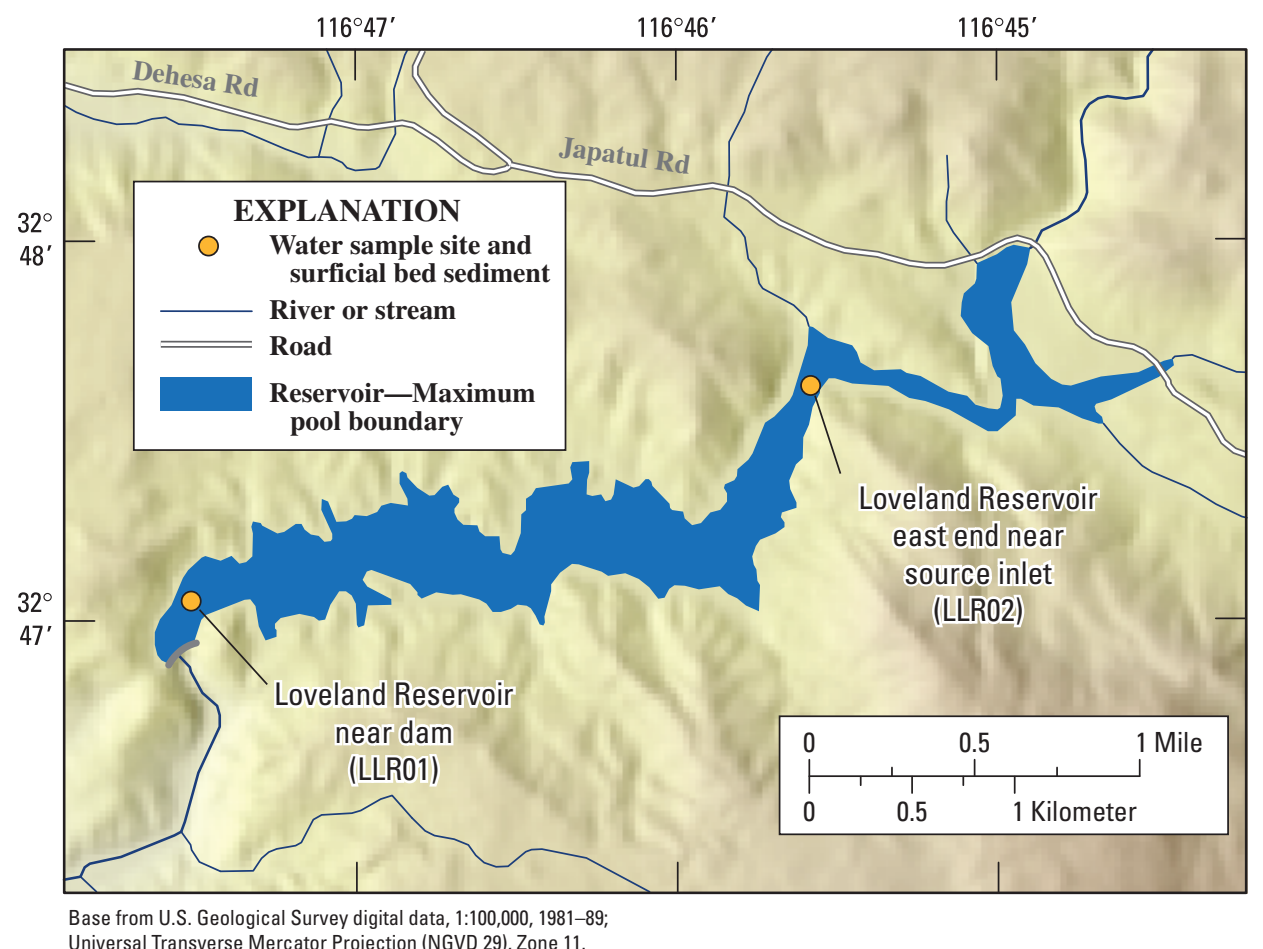

Figure 3. Loveland Reservoir sampling sites, San Diego County, California.

79 VOCs with lower volatilities (higher breakthrough volumes). Each sample was a 24-hour composite collected every 12th day. The timing of the air-sample collection for VOCs was coordinated with the CARB Air Toxics Program. Samples were analyzed for $86 \mathrm{VOCs}$, which ranged in volatility from dichlorofluoromethane (CFC-12), which had the highest volatility, to 1,2,3-trichlorobenzene, which had the lowest volatility (Pankow and others, 1998).

The research methods used in this monitoring program for sampling and analyzing semivolatile organic compounds (PAHs and pesticides) in air are comparable to those described by Foreman and others (2000) for pesticides, and to USEPA methods TO-4A for pesticides (U.S. Environmental Protection Agency, 1999a) and TO-13A for PAHs (U.S. Environmental Protection Agency, 1999b). These air methods were developed to complement the pesticides in water methods (Zaugg and others, 1995; Sandstrom and others, 2001) and PAH/alkylPAH in sediment method (Olson and others, 2004) applied to the SWR monitoring program.

The high-volume air samples for the analysis of PAHs or pesticides were collected by drawing air through a $90-\mathrm{mm}$ diameter glass-fiber filter (GFF; type A/E, Pall Corp., East Hills, NY) followed by a cartridge containing two polyurethane foam (PUF) plugs. The GFF collects atmospheric particles from which the operationally defined particle-phase concentration of each analyte is determined. The PUF plugs collect the operationally defined gas-phase concentration of each analyte. Prior to use, the GFFs were cleaned by baking at 450 degrees Celsius $\left({ }^{\circ} \mathrm{C}\right)$, desiccated for at least 2 hours until cool, weighed to the nearest 0.2 milligram (mg) on a balance, wrapped in baked aluminum foil, and stored in resealable polyethylene bags. At the SWR air-sampling site, a GFF was removed from the foil with clean (methanolrinsed) stainless steel forceps and placed in a perfluoralkoxy fluoropolymer (Teflon-PFA) filter holder (series 90, Savillex Corp., Minnetonka, MN) that was modified in two ways. The outer closure piece of the filter holder was cut open to provide an 80-mm diameter opening that would improve air flow and expose most of the GFF surface for atmospheric particle collection. The holder's inner closure piece was machined to include a $3.8-\mathrm{cm}$ female National Pipe Thread (NPT) taper to allow direct connection to the $3.8-\mathrm{cm}$ male NPT threaded connection on the inlet of the PUF cartridge.

PUF plugs for the cartridges were $5 \mathrm{~cm}$ in diameter by $7.6 \mathrm{~cm}$ long and were prepared from open-cell foam having an average density of $0.043 \mathrm{gm}^{-3}$ and containing no polybrominated diphenyl ether flame retardants (Netherland Rubber Company, Cincinnati, OH). PUF plugs were cleaned by rinsing with tap water and then sequentially extracted for at least 12 hours each with acetone, 30-percent ethyl acetate in hexane, and dichloromethane in a Soxhlet apparatus. Residual solvent was squeezed from the PUF plugs using a stainless-steel potato masher, and the plugs were then dried in a vacuum oven at $40^{\circ} \mathrm{C}$ for at least 48 hours before being stored in sealed 500-mL wide-mouth jars with Teflon-lined lids. At SWR, primary (top) and secondary (bottom) PUF plugs were positioned in series inside a $24.2-\mathrm{cm}$ long by $3.5-\mathrm{cm}$ internal diameter Teflon-PFA cartridge (Savillex Corp., Minnetonka, MN) with the bottom PUF plug held in place against a TeflonPFA screen. PUF plugs were carefully inserted into the PUF 
cartridge using clean stainless steel forceps to ensure that the PUF plugs were well fitted to the cartridge wall with no creases that would allow air to migrate around the plug instead of passing through the foam.

The PUF cartridge was then connected to the GFF filter holder, and the GFF-PUF sampling train was positioned inside a high-volume sampler enclosure (Graesby-GMW, Village of Cleves, Ohio) comparable to that described in USEPA method TO-4A (U.S. Environmental Protection Agency, 1999a). The outlet of the PUF cartridge was connected via $0.95-\mathrm{cm}$ outer diameter Teflon tubing to a high-volume blower motor. Air samples were collected by pulling ambient air through the GFF-PUF sampling train at flow rates of 23 to $55 \mathrm{~L} / \mathrm{min}$ for seven days, providing sample volumes ranging from 234 to $585 \mathrm{~m}^{3}$ (cubic meter) for samples described in this report. Sampling times were controlled by a timer, and sample volume was calculated by multiplying the sample collection time by the air flow rate determined using a calibrated flow meter. Following sample collection, the GFF was removed from the holder using clean forceps, returned to the aluminum foil, folded in half (particle-laden side inward), and sealed in the foil and bag. PUF plugs were returned to the jars using forceps, and the jars were labeled to identify the top or bottom PUF and were tightly sealed with the lids. GFF and PUF were stored at $4^{\circ} \mathrm{C}$ (maximum) prior to overnight shipment on ice to USGS NWQL.

At NWQL, air sample components were stored at $-5^{\circ} \mathrm{C}$ (maximum) until analysis. GFFs were desiccated for 24 hours and weighed to $\pm 0.2 \mathrm{mg}$ to determine particle weight. This weight was divided by the sample's air volume to determine the total suspended particle (TSP) concentration. Each GFF was placed in a 500-mL flat bottom flask, and each PUF plug was placed in a Soxhlet apparatus. The GFF and PUF plugs were fortified with surrogate compounds (see Quality Control section later in report) and extracted with 100-mL (GFF) or 300-mL (PUF) of 30-percent ethyl acetate in hexane for at least 12 hours. Top and bottom PUF plugs were extracted and analyzed separately during this reporting period to determine PUF collection efficiency for gas-phase analytes. Extracts were dried with sodium sulfate and reduced in volume to $1 \mathrm{~mL}$ using Kuderna-Danish distillation and nitrogen gas evaporation. Each extract was then transferred to a 0.5-g octadecylsilyl (C-18) solid-phase extraction (SPE) column (Isolute 221-

0050-BS, Biotage AB, Charlottesville, VA) positioned over a 1-g Florisil-PR column (Isolute 712-0100-C). Analytes were eluted from these columns with $6 \mathrm{~mL}$ of ethyl acetate, which was reduced in volume to about $0.3 \mathrm{~mL}$ by nitrogen gas evaporation. Extracts were transferred with $0.15-\mathrm{mL}$ ethyl acetate rinse to 2-mL GC vials containing $500 \mathrm{ng}$ of 1,4-dichlorobenzene and five perdeuterated polycyclic aromatic hydrocarbons (naphthalene-d8, acenaphthene-d10, phenanthrene-d10, chrysene-d12, and perylene-d12) in ethyl acetate as internal injection standards.
The extracts were then analyzed by three different GC/MS-SIM methods. Pesticides and degradates comparable to those determined by NWQL water schedule 2001 were measured using the operational conditions described by Zaugg and others (1995) and Lindley and others (1996). A subset of parent pesticides and degradates comparable to those determined by NWQL water method 2002 were measured using the GC/MS conditions given by Sandstrom and others (2001). Finally, PAHs and alkyl-PAHs were analyzed using GC/MS conditions comparable to that described by Olson and others (2004) for a subset of those compounds determined by that sediment method except that the mass spectrometer was operated in the selected-ion monitoring mode to provide lower detection levels. Compound concentrations determined from these three methods are reported in nanograms per cubic meter of air $\left(\mathrm{ng} / \mathrm{m}^{3}\right)$.

\section{Special Studies}

In addition to the primary sampling of water and air to provide a core dataset for the understanding of potential water-quality impacts on the SWR, six "special studies" were conducted during the 1999-2000 time frame covered by this report. These special studies were conducted to obtain waterquality information about specific processes and chemicals of current concern. Two of these special studies (the first and the sixth) were conducted to investigate the occurrence of additional organic chemical contaminants in water using two new research methods that were under development at the NWQL. The other four special studies (the second through the fifth) used traditional methods to supplement the data from the primary sampling.

The first special study collected water samples for determination of moderate-use pesticides and degradates using procedures that subsequently became official USGS schedule 2002 (Sandstrom and others, 2001). Water samples collected by this method were filtered through $0.7-\mu \mathrm{m}$ nominal pore size GFFs into baked 1-L sample bottles, capped, and then shipped on ice to the USGS NWQL. Analyses were performed using the C-18 SPE and GC/MS techniques described by Sandstrom and others (2001). The second special study investigated the dissolved-phase concentrations of organochlorine compounds, PAHs, and other semivolatile compounds in the SWR by use of semi-permeable membrane devices (SPMD) developed by the USGS (Huckins and others, 1990).

The third special study was an investigation of dissolved copper, which was analyzed using the same analytical methods as were used for the other water samples collected for the primary sampling, as described by Shelton (1994 and 1997). The fourth special study was an investigation of VOCs in two drinking-water production wells located north of the Reynolds desalination plant (fig. 1). The fifth special study analyzed dissolved trace metals, which were analyzed by inductivelycoupled plasma/mass spectrometry using methods described in Faires (1993). 
The sixth special study collected whole-water samples for the determination of wastewater indicator compounds. Samples for wastewater compounds were extracted with dichloromethane in a continuous liquid-liquid extractor. Extracts were reduced in volume to $0.5 \mathrm{~mL}$ by micro-Kuderna-Danish distillation and nitrogen evaporation. Perdeuterated PAH internal standards were added to the extracts and analyzed by GC/MS-SIM (samples collected in December 2000) or GC/ MS operated in full scan mode (samples from March 2001). The GC/MS full scan analysis is comparable to that described by Zaugg and others (2002). All water samples for organic analyses were shipped on ice to the laboratories for analysis.

\section{Sampling Strategy and Data}

Some data tables show more than one laboratory reporting level (LRL) for a compound or element in different samples. This occurs, in part, because reporting levels are updated annually, as necessary, as a component of the NWQL's yearly assessment of long-term method detection levels and LRLs (Childress and others, 1999). Reporting levels also sometimes vary based on differences in sample volumes or the presence of interferences. Some concentrations are reported as estimated (E). In this case, the compound was determined to be present in the sample on the basis of mass spectral information, but the concentration is less certain because the determined concentration is below the lowest calibration standard or the LRL, whichever is greater, or because of recognized method limitations for certain compounds.

\section{Water Sampling}

Water samples were collected at three sites in SWR (sites 1, 3, and 6, fig 2), two sites at the Perdue Treatment Plant (sites 8 and 9, fig.2), one site on the Sweetwater River above SWR (site 10, fig.2), and one site at LLR (LLR01, fig. 3). The results for water samples are entered into the USGS National Water Information System database. All sites within SWR, with the exception of site 6 , were within the reservoir's minimum pool boundary to ensure that water would be available for sampling throughout the year. Site 6 was located in the eastern third of the reservoir in very shallow water (fig. 2). As the water level fell, the water depth at this site decreased and the bed sediments became exposed. When this happened, the sampling site was moved to a location where the water depth was about $1 \mathrm{~m}$ deep. Sampling sites at both SWR and LLR were marked with stationary buoys anchored to the bottom of the reservoir.

Three water-sampling sites (sites 8, 9, and 10, fig. 2) were established outside the reservoir boundaries. Site 8 monitors the quality of the finished (treated) water as it leaves the treatment plant for distribution to customers. Site 9 monitors the quality of the imported raw water before it enters the treatment plant or resevoir. Site 10, Sweetwater River at the Low-Flow
Diversion Dam (LFDD), monitors the quality of the watershed drainage water entering SWR. During low flows, the water from LFDD is diverted into the Urban Runoff Diversion System (URDS) ponds. Local urban runoff and the first flush (initial flow after a dry period, when pollutants might occur in higher concentrations) in the Sweetwater River are diverted into the URDS ponds to prevent unwanted water from entering the SWR. All site identification numbers, sampling site names, and other identifiers are listed in table 1.

In most cases, imported water is pumped by pipeline directly into the treatment plant. Occasionally, this water is pumped directly into SWR to augment the local supply. When imported water is pumped directly into the reservoir, it significantly increases the water level with volume increases on the order of tens of thousands of acre-feet.

Baseline water sampling at both SWR and LLR began in September 1998 and continued at 2-month intervals through September 1999 . This bimonthly sampling allowed monitoring of various operational modes of the reservoirs, such as replenishment or withdrawal events that significantly change the water level in the reservoirs. Baseline water sampling also showed the spatial variability in chemical occurrence and concentration in each reservoir. Beginning in October 1999, the sampling frequency was reduced to once every third month (quarterly). The number of sampling sites at SWR was reduced from seven to three, and the two sampling sites at LLR were reduced to one. The reduction in the number of sampling sites was done to focus the sampling efforts on "indicator" sites-those sites that are believed to provide the most relevant information without compromising the scientific integrity of the project. These sites are

- SWR01-located near the dam and the intake for reservoir water to the treatment plant; to monitor the quality of water entering the treatment plant.

- SWR03-located near the center of the minimum pool; to monitor the quality of water at the center of the reservoir.

- SWR06-located near the east end of the reservoir; to monitor the quality of water entering the reservoir from the Sweetwater River.

- LLR01-located near the dam; to monitor the quality of the reserve storage water.

Before any reservoir water was sampled, depth profiles of dissolved oxygen, $\mathrm{pH}$, specific conductance, and temperature were measured at 1-m intervals from the surface to the reservoir bottom at each sampling location using a multiparameter water-quality monitor (table 2). At LLR, the depth-profile measurements below $10 \mathrm{~m}$ were taken every $2 \mathrm{~m}$ because the depth of LLR is much greater than the depth of SWR. If the temperature profile indicated a thermocline, two sets of water samples were collected at the site: one at midepilimnion and one at midhypolimnion. The epilimnion can be defined as the layer in a lake extending from the surface to a depth where photosynthesis no longer occurs. The hypolimnion can be defined as the poorly illuminated lower region of a stratified lake where denser, colder water currents are minimal. The 
temperature of the hypolimnion is nearly uniform and oxygen is depleted. This stratum of water is characterized by decay rather than by the production of organic matter. If no thermocline was evident, only one sample set was collected at a point midway between the water surface and the reservoir bottom.

The concentrations of VOC analytes detected in water ranged from $0.01 \mu \mathrm{g} / \mathrm{L}$ to $66.6 \mu \mathrm{g} / \mathrm{L}$, and the results are given in table 3 . The concentrations of pesticide analytes detected in filtered water ranged from $0.002 \mu \mathrm{g} / \mathrm{L}$ to $0.02 \mu \mathrm{g} / \mathrm{L}$, and the results are given in table 4 . Any additional analyses that were added to the sampling regime were performed on samples collected from selected sites. That is, these analyses were done to show occurrence and possible source of new analytes of interest, not necessarily their distribution within the watershed.

\section{Air Sampling}

The purpose of the air data collected at the air sample site (fig. 2) is to establish the occurrence, temporal patterns, and ambient levels of selected airborne organic compounds (VOCs, PAHs, and pesticides). This site was installed downwind of the proposed SR 125 routes and upwind of SWR (fig. 2), along a transect of the predominant wind direction. The site includes a fully instrumented meteorological station that records hourly averages of wind speed and direction, ambient air temperature and relative humidity at two heights, rainfall, barometric pressure, and atmospheric stability. Establishment of this air sampling station followed the guidelines outlined by the National Atmospheric Deposition Program (Bigelow, 1984), with the help of the South Coast Air Pollution Control Board (William Brick, oral commun., 1999).

The first VOC air sample was collected on March 23, 1999. Each sample was a 24-hour composite collected every 12th day. The timing of the VOC sample collection was coordinated with the CARB Air Toxics Program. The LRL for these analytes ranged from 0.02 to 0.06 part per billion by volume. The results for the VOC air samples collected during the time frame of this report are given in table 5A for the 7 compounds with low breakthrough volumes. Table 5B gives the results for the remaining 79 VOCs.

The first PAH and pesticide air sample was collected during the week of May 11, 1999. Each sample was a 24-hour/7-day weekly composite collected every third week. Air samples were not collected at LLR because its foothill location (30 km east of SWR) is considered sufficiently downwind of SR 125 to be minimally impacted by any airborne contaminants originating from it. Analytical results for PAHs in air samples collected during the time frame of this report are given in table 6. PAHs with molecular weights less than 178 Daltons are not reported because of incomplete collection on PUF at the sampled volumes (You and Bidleman, 1984). Compounds analyzed for pesticides in air samples using a modification of NWQL water Schedules 2001 and 2002 are given in table 7A. Results for these pesticides analyzed using a modification of NWQL water sample Schedules 2001 and
2002 are given in tables $7 \mathrm{~B}$ and $7 \mathrm{C}$, respectively. Not all of the compounds that were determined by the corresponding water methods are reported for the air samples because certain analytes were known to have incomplete collection on the PUF plugs based on a previous study (Foreman and others, 2000) or inadequate analytical performance in the air method.

\section{Special Studies}

The first special study, which began in January 2000, focused on moderate-use pesticides in filtered water analyzed using NWQL Schedule 2002 (table 8). Between January 27 and February 12, 2000, water was transferred $\left(11.2 \mathrm{hm}^{3}\right)$ from LLR to SWR via the Sweetwater River. Because there are several golf courses along the Sweetwater River between LLR and SWR, this water transfer was an opportunity to examine whether pesticides used on the golf courses would accumulate in the river channel during extended dry periods and be carried downstream with the initial flow of the river after a dry period (first flush). This first-flush sampling occurred on the 29th and 30th of January 2000 at two sites along the Sweetwater River. One sample was collected at the Steele Canyon Bridge at Cottonwood Golf Course site (fig. 1) as the released water was passing through the area. Four additional samples were collected at the LFDD (site 10, fig. 2) at approximately 12hour intervals. One sample was collected in early January at the LFDD for comparison with the first-flush data. In addition, several samples from SWR (sites 1 and 3, fig. 2), the LFDD, and the imported raw water (site 9, fig. 2) were taken in June and September 2000 and analyzed using this method (table 8).

The second special study investigated concentrations of dissolved-phase organochlorine compounds (legacy persistent organic pollutants), PAHs, and other semivolatile organic compounds using semipermeable membrane devices (SPMD). The organochlorine compounds and PAHs, especially, have low water solubility and are of concern with regard to human health. This study was conducted during a period of extended dry weather when the water level in SWR was low. Three SPMDs were deployed in January 2001 near the pump tower (site 1, fig. 2); one was implaced for one month and two for two months. The results are presented in tables 9A and 9B.

SPMDs are flat polyethylene plastic tubes filled with triolein, a lipid-like material. The polyethylene membrane of the SPMD allows dissolved (bioavailable) contaminants to pass through the membrane while excluding water. The triolein inside the SPMD is similar in characteristics to a highly purified fish fat. The contaminants dissolve in the triolein just as they do in the fats of a fish. The SPMD was placed on a rack, which was inserted into a protective stainless steel container that was then submerged in the water at a depth of $1 \mathrm{~m}$. SPMDs collect contaminants in the water that are often hard to detect through normal chemical sampling procedures because they are present at such low dissolved-phase concentrations. SPMDs measure the bioavailable form of a chemical that an organism can absorb and incorporate. Very low concentrations 
of certain contaminants (such as PAHs, organochlorine pesticides, and polychlorinated biphenyls) may still be important in the environment because of their ability to bioconcentrate in animals (by dietary uptake or uptake through fish gills with subsequent accumulation in the animal's fat). The concentrations of these chemicals in rivers can change daily or even hourly, but their concentrations in reservoirs are expected to be more constant. The SPMD allows the calculation of an average concentration of each contaminant per kilogram of SPMD during the period that the sampling device is in the water.

The third special study analyzed additional water samples from various sources for copper concentration in April and July 2001 (table 10). In April, two water samples were taken at the Perdue Treatment Plant_-finished and imported waters; and one taken at the Reynolds Desalination Facility-discharge water. The Reynolds Desalination Facility uses reverseosmosis treatment to remove dissolved salts and microscopic particles that could be found in alluvial ground water. The facility, completed in 1999, can produce four million gallons of drinking water a day. The sample collected at the Desalination Facility was from the plant discharge, which is referred to as "sample point 45 " because it is the compliance point for the facility's National Pollutant Discharge Elimination System (NPDES) permit. The sample was collected just before the discharge went into a concrete channel that flows to the Sweetwater River. This sample was a composite sample taken over a 21-hour period.

In July, ten water samples were collected for dissolved copper determination (table 10): one at the Perdue Treatment Plant (finished water) (site 8, fig. 2), four at the Reynolds Desalination Facility (fig. 1), and five at selected San Diego Formation wells (SDF1 through 5, table 1, fig. 1). The Desalination Facility samples were collected from the inflow, feed, effluent, and discharge. The inflow sample point is raw water coming into the plant from ground-water wells. The feed sample point is the inflow water filtered through a cartridge with sulfuric acid with a scale inhibitor added. The effluent sample point is water that goes into the drinking-water system. The discharge sample point is water that goes back to the Sweetwater River. The five ground-water wells that were sampled supply water that feeds into the plant. Depending on the needs of the plant, all or a combination of these San Diego Formation wells make up their inflow.

The fourth special study analyzed VOCs in water from two National City wells that the SWA uses to supplement their drinking-water supply. These wells were sampled so that SWA would have a better understanding of measured VOCs in their supplemental drinking water. The results from sampling these two wells in April 2000 are listed in table 11.

The fifth special study analyzed dissolved trace metals in reservoir water samples collected in March 2001 (table 12). A variety of trace metals occur naturally, but are also emitted from auto exhaust, tire wear, and road dust. These analyses were added to help understand the role of atmospheric deposition of trace metals originating from the SR125 alignment and their affect on water quality.
The last special study analyzed contaminants typically associated with wastewater effluent in reservoir whole-water samples (sites 1, 8, 9, fig. 2, and LLR01, fig. 3) collected in December 2000 and March 2001 (table 13). Some compounds have a second LRL because the laboratory quality control required that the level be raised to decrease the possibility of false positives. The laboratory reporting levels for some compounds were higher in the March 2001 samples.

\section{Quality Control}

Three types of quality-control samples were used in this study: blanks, spikes, and replicates. Blanks and spikes are used to estimate result bias, and replicates are used to estimate result variability. Additionally, surrogate compounds were added to VOC, PAH, and pesticide samples at the laboratory to monitor sample-specific performance of the analytical method.

Blanks are intended to be free of the analytes of interest and can be prepared in the laboratory or field. Blank samples are analyzed to test for bias that could result from contamination of environmental samples by the analytes of interest or by interfering compounds during any stage of sample collection, processing, or analysis. For this study, blanks for water samples were collected by processing USGS laboratory certified reagent water, known to be free of the analytes of interest, through all parts of the sample-collection process. Field blanks were collected at an environmental sampling location by passing the blank water through all field-cleaned sampling equipment. Field blanks were used to demonstrate that all stages of sample collection and processing did not introduce measurable contamination. Equipment blanks were collected annually to monitor for potential contamination due to sample collection and processing equipment. Source-solution blanks were collected to verify that the laboratory certified reagent water was free of the analytes of interest. Travel (trip) blanks were used to identify contamination that could occur during sample transport and analysis rather than as a result of sample collection and processing. Lot blanks were analyzed at the laboratory to test the cleanliness of the cartridges. Laboratory blanks (water and air methods) were used to demonstrate that the laboratory sample processing steps did not introduce measurable contamination.

Spiked samples are prepared by fortifying reagent water, an environmental water sample, or the air sampling media, with a known concentration of selected analytes. For this study, spiked samples were used to measure bias in analyte recovery. Matrix spikes also were used to test the effects of various sampled matrices on the recovery of specific compounds, including matrix interference or matrix-induced analyte degradation. One field water sample was spiked, in replicate, for pesticides during this study period.

Replicate water samples were collected and processed identically to the environmental sample and used to measure the variability during sample processing and analysis. 


\section{Water Sampling}

One field quality-control sample was collected during every sampling campaign except during September 2001. The type of quality-control sample collected and the sampling location were chosen randomly. The blank and replicate sample quality-control data for VOCs in water samples are given in table 14. The field-matrix spike and spike replicate quality control data for pesticides in water samples are given in table 15 .

The quality of analytical results was monitored by adding surrogate compounds to each sample before it was processed for analysis. These surrogate compounds were added at the NWQL to monitor sample preparation and analysis. For water samples, 1,2-dichloroethane-d4, toluene-d8, and 1,4-bromofluorobenzene were added to each VOC sample, and diazinon-d10 and $\alpha-\mathrm{HCH}-\mathrm{d} 6$ were added to each pesticide sample. Surrogate results, in percent recovery, for each type of water sample is given in tables 3 and $\underline{4}$, respectively.

\section{Air Sampling}

Each VOC air sample collected has an associated cartridge travel blank, cartridge (reagent) spike, cartridge lot blank, and laboratory blank that were analyzed along with the environmental sample. The quality-control data for chlorofluorocarbons and other compounds with low breakthrough volumes are given in tables 16A, $\underline{16 \mathrm{~B}}, \underline{16 \mathrm{C}}$, and $\underline{16 \mathrm{D}}$, respectively. The quality-control data for the remaining 80 VOCs are given in tables 17A, 17B, 17C, and 17D, respectively.

Air samples for semivolatile organic compounds comprise three components (a GFF and a top and a bottom PUF plug) that were individually extracted. These extracts were analyzed by three distinct GC/MS procedures at the NWQL for PAHs and pesticides. Each air sample component was fortified with surrogate compounds at the NWQL to monitor sample preparation and analysis. Recovery data for surrogate compounds nitrobenzene-d5, 2-fluorobiphenyl, and terphenyl-d14 used to monitor performance for the PAH analysis are given in table 6 . Recovery data for surrogate compounds diazinon-d 10 and $\alpha-\mathrm{HCH}-\mathrm{d} 6$ used to monitor performance for the pesticides analyses by modified Schedules 2001 and 2002 are given in tables 7B and $\underline{7 \mathrm{C}}$, respectively.

The laboratory reagent blank and laboratory reagent spike results for PAHs are given in tables $18 \mathrm{~A}$ and $18 \mathrm{~B}$, respectively. Corresponding laboratory blank and laboratory spike results for air sample analysis are in tables 19A and $\underline{19 \mathrm{~B}}$ using modified schedule 2001-type pesticides, and in tables 20A and 20B for modified schedule 2002-type pesticides.

\section{Special Studies}

The laboratory blank quality-control data associated with the second special study with the SPMD samples are given in table 21 . The laboratory spike and laboratory blank qual- ity-control data for the sixth (last) special study of wastewater compounds are given in table 22 .

\section{Summary}

The primary purpose of this study was to monitor changes in contaminant composition and concentration in the air and water during the construction and operation of a major thoroughfare being built upwind of the Sweetwater Reservoir. To accomplish this, the study was divided into two phases. Phase One sampling was designed to establish baseline conditions for target compounds (primary sampling) in terms of detection frequency and concentration in air and water. Phase Two sampling is planned to continue during and after construction of the roadway to assess the chemical impact this alignment project may have on the water quality in the reservoir. In addition to the ongoing data collection, six special studies were initiated during the reporting period of Phase One to assess the occurrence of specific chemicals of concern, such as moderate-use pesticides and degradates, trace metals, and wastewater compounds in water samples.

Many compounds were analyzed to provide a better understanding of the effects urbanization may have on a drinking-water reservoir. Before any reservoir water was sampled, depth profiles were made of temperature, $\mathrm{pH}$, dissolved oxygen, and specific conductance which were measured at each reservoir sampling location (table 2). Analytical results for the primary water sampling are given in tables 3 and 4 . Analytical results for the primary air sampling are given in tables $\underline{5}-\underline{7}$. Data for the six special studies are given in tables 8-13. Quality-control data for water samples are given in tables 14 and 15. Quality assurance data for air sampling are given in tables 16-20. Quality-control data for two special studies are given in tables 21 and 22 . 


\section{References Cited}

Bigelow, D.S., 1984, Instruction manual: NADP/NTN site selection and installation: Fort Collins, Colorado, Colorado State University, Natural Resource Ecology Laboratory, variously paged [available from National Atmospheric Deposition Program Office, Illinois State Water Survey, 2004 Griffith Drive, Champaign, IL 61820].

Byard, J.L., 1999, Impact of SR125 vehicle emissions on the Sweetwater Reservoir-executive summary report: Chula Vista, Calif., Sweetwater Authority, 32 p.

California Department of Transportation, 2001, State Route 125 (From I-905 to State Route 54): District 11 Fact Sheet, accessed December 14, 2001, at http://www.dot.ca.gov/ dist11/facts/125south.pdf.

http://www.dot.ca.gov/dist11/facts/125south.pdf

California Environmental Protection Agency, 1986, California Safe Drinking Water and Toxic Enforcement Act of 1986 (Proposition 65): Health and Safety Code, chap. 6.6, Sections 25249.5 through 25249.13 .

Connor, B.F., Rose, D.L., Noriega, M.C., Murtagh, L.K., and Abney, S.R., 1998, Methods of analysis by the U.S. Geological Survey National Water Quality Laboratory-Determination of 86 volatile organic compounds in water gas chromatography/mass spectrometry, including detections less than reporting limits: U.S. Geological Survey OpenFile Report 97-829, 78 p.

Faires, L.M., 1993, Methods of analysis by the U.S. Geological Survey National Water Quality Laboratory-Determination of metals in water by inductively coupled plasma-mass spectrometry: U.S. Geological Survey Open-File Report 92-634, 28 p.

Foreman, W.T., Connor, B.F., Furlong, E.T., Vaught, D.G., and Merten, L.M., 1995, Methods of analysis by the U.S. Geological Survey National Water Quality Laboratory-Determination of organochlorine pesticides and polychlorinated biphenyls in bottom sediment by dual capillary-column gas chromatography with electron-capture detection: U.S. Geological Survey Open-File Report 95-140, 78 p.

Foreman, W.T., Goolsby, D.A., Majewski, M.S., Wiebe, F.W., and Battaglin, W.A., 1997, Acetochlor and other herbicides in air and rain in the Midwestern United States [abs.], in Bridging the global environment: Technology, communication, and education: Society of Environmental Toxicology and Chemistry Annual Meeting, 18th, San Francisco, Calif., November 16-20, 1997, Abstract Book no. 229, p. 45.
Foreman, W.T., Majewski, M.S., Goolsby, D.A., Wiebe, F.W., and Coupe, R.H., 2000, Pesticides in the atmosphere of the Mississippi River Valley, Part II-Air: Science of the Total Environment, v. 248, no. 2, 3, p. 213-226.

Huckins, J.N., Tubergen, M.W., and Manuweera, G.K., 1990, Semipermeable membrane devices containing model lipid: a new approach to monitoring the availability of lipophilic contaminants and estimating their bioconcentration potential: Chemosphere v. 20, p. 533-552.

Lindley, C.E., Stewart, J.T., and Sandstrom, M.W., 1996, Determination of low concentrations of acetochlor in water by automated solid-phase extraction and gas chromatography with mass selective detection: Journal of the Association of Official Analytical Chemists International, v. 79, no. 4, p. 962-966.

Majewski, M.S., Foreman, W.T., Goolsby, D.A., and Nakagaki, Naomi, 1998, Airborne pesticide residues along the Mississippi River: Environmental Science and Technology, v. 32, no. 23 , p. 3689-3698.

Majewski, M.S., Sishu, J.S., and Mendez, G.O., 2002, Waterquality monitoring of Sweetwater and Loveland Reservoirs, San Diego County, California_-Phase One results, 19981999: U.S. Geological Survey Open-File Report 02-186, $134 \mathrm{p}$.

Ogden Environmental and Energy Services, 1997, SR-125 South Route alternatives: potential air emissions impact on Sweetwater Reservoir: San Diego, Calif. [available from Sweetwater Authority, 505 Garrett Avenue, Chula Vista, CA 95912].

Olson, M.C., Iverson, J.L., Furlong, E.T., and Schroeder, M.P., 2004, Methods of analysis by the U.S. Geological Survey National Water Quality Laboratory--Determination of polycyclic aromatic hydrocarbon compounds in sediment by gas chromatography/mass spectrometry: U.S. Geological Survey Water-Resources Investigations Report 03-4318, 45 p., accessed March 2003 at http://nwql.usgs.gov/Public/ pubs/WRIR03-4318/WRIR03-4318.pdf.

Pankow, J.F., Luo, Wentai, Isabelle, L.M., Bender, D.A., and Baker, R.J., 1998, Determination of a wide range of volatile organic compounds in ambient air using multisorbent adsorption/ thermal desorption and gas chromatography/ mass spectrometry: Analytical Chemistry, v. 70, no. 24, p. 5213-5221.

Sandstrom, M.W., Stroppel, M.E., Foreman, W.T., and Schroeder, M.P., 2001, Methods of analysis by the U.S. Geological Survey National Water Quality Laboratory-Determination of moderate-use pesticides and selected degradates in water by $\mathrm{C}-18$ solid-phase extraction and gas chromatography/mass spectrometry: U.S. Geological Survey WaterResources Investigations Report 01-4098, 70 p. 
Shelton, L.R., 1994, Field guide for collecting and processing stream-water samples for the National Water-Quality Assessment Program: U.S. Geological Survey Open-File Report 94-455, 42 p.

Shelton, L.R., 1997, Field guide for collecting samples for analysis of volatile organic compounds in stream water for the National Water-Quality Assessment Program: U.S. Geological Survey Open-File Report 97-401, 14 p.

U.S. Environmental Protection Agency, 1999a, Compendium of methods for the determination of toxic organic compounds in ambient air (2nd ed), Compendium method TO-4A-Determination of pesticides and polychlorinated biphenyls in ambient air using high volume polyurethane foam (PUF) sampling followed by gas chromatography/ multi-detector detection (GC/MD): Center for Environmental Research Information, Office of Research and Development EPA/625/R-96/010b, 49 p., accessed May 2006 at http://www.epa.gov:80/ttnamti1/files/ambient/airtox/to4ar2r.pdf.
U.S. Environmental Protection Agency, 1999b, Compendium of methods for the determination of toxic organic compounds in ambient air (2nd ed), Compendium method TO-13A-Determination of polycyclic aromatic hydrocarbons (PAHs) in ambient air using gas chromatography/mass spectrometry (GC/MS): Center for Environmental Research Information, Office of Research and Development EPA/625/ R-96/010b, 78 p., accessed May 2006 at http://www.epa. gov/ttn/amtic/files/ambient/airtox/to-13arr.pdf.

You, F., and Bidleman, T.F., 1984, Influence of volatility on the collection of polycyclic aromatic hydrocarbon vapors with polyurethane foam: Environmental Science and Technology, v. 18, p. 330-333.

Zaugg, S.D., Sandstrom, M.W., Smith, S.G., and Fehlberg, K.M., 1995, Methods of analysis by the U.S. Geological Survey National Water Quality Laboratory-Determination of pesticides in water by $\mathrm{C}-18$ solid-phase extraction and capillary-column gas chromatography/mass spectrometry with selected-ion monitoring: U.S. Geological Survey Open-File Report 95-181, 49 p. 


\section{Water- and Air-Quality Monitoring, Sweetwater Reservoir Watershed, San Diego County, California, 1999-2001}

Table 1. Site identification numbers, site names, state well numbers, and other identifiers, San Diego County, California.

[See figure 2 for most site locations. QA, quality assurance; QC, quality control]

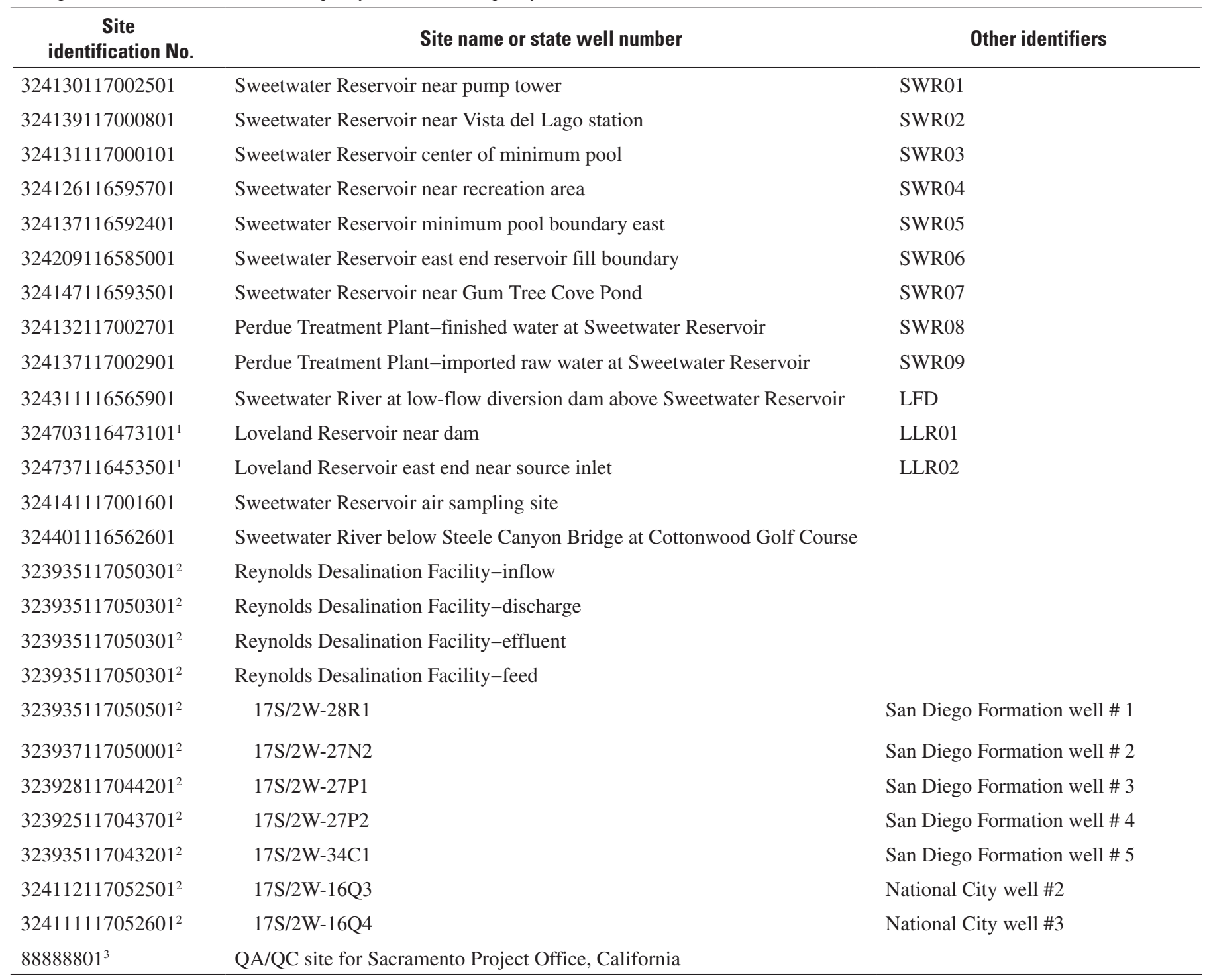

${ }^{1}$ Loveland Reservoir sites located on figure 3.

${ }^{2}$ Well sites and desalination facility located on figure 1.

${ }^{3}$ Quality control identification number. 
Table 2. Water-quality depth-profile field data for Sweetwater and Loveland Reservoir sampling sites and for Sweetwater River above Sweetwater Reservoir, San Diego County, California.

[Time is denoted in 24-hour scale; the five digit number in parentheses below the compound name, the parameter code, is used in the U.S. Geological Survey's computerized data system (National Water Information System) to uniquely identify a specific constituent or property; m, meter; mg/L, milligram per liter; $\mu \mathrm{S} / \mathrm{cm}$, microsiemens per centimeter; ${ }^{\circ} \mathrm{C}$, degree Celsius; ND, no data]

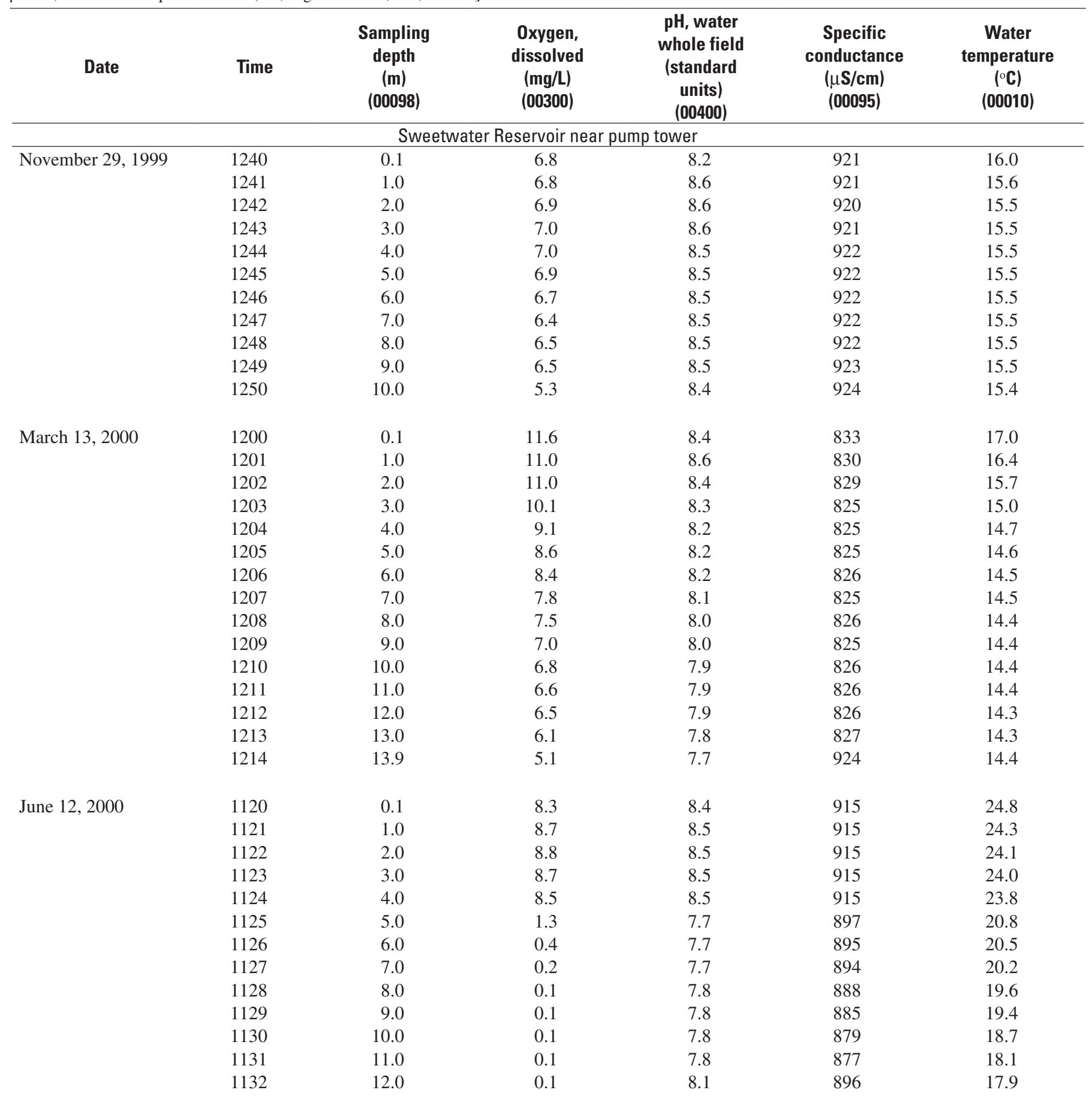


Table 2. Water-quality depth-profile field data for Sweetwater and Loveland Reservoir sampling sites and for Sweetwater River above Sweetwater Reservoir, San Diego County, California-Continued.

[Time is denoted in 24-hour scale; the five digit number in parentheses below the compound name, the parameter code, is used in the U.S. Geological Survey's computerized data system (National Water Information System) to uniquely identify a specific constituent or property; m, meter; mg/L, milligram per liter; $\mu \mathrm{S} / \mathrm{cm}$, microsiemens per centimeter; ${ }^{\circ} \mathrm{C}$, degree Celsius; ND, no data]

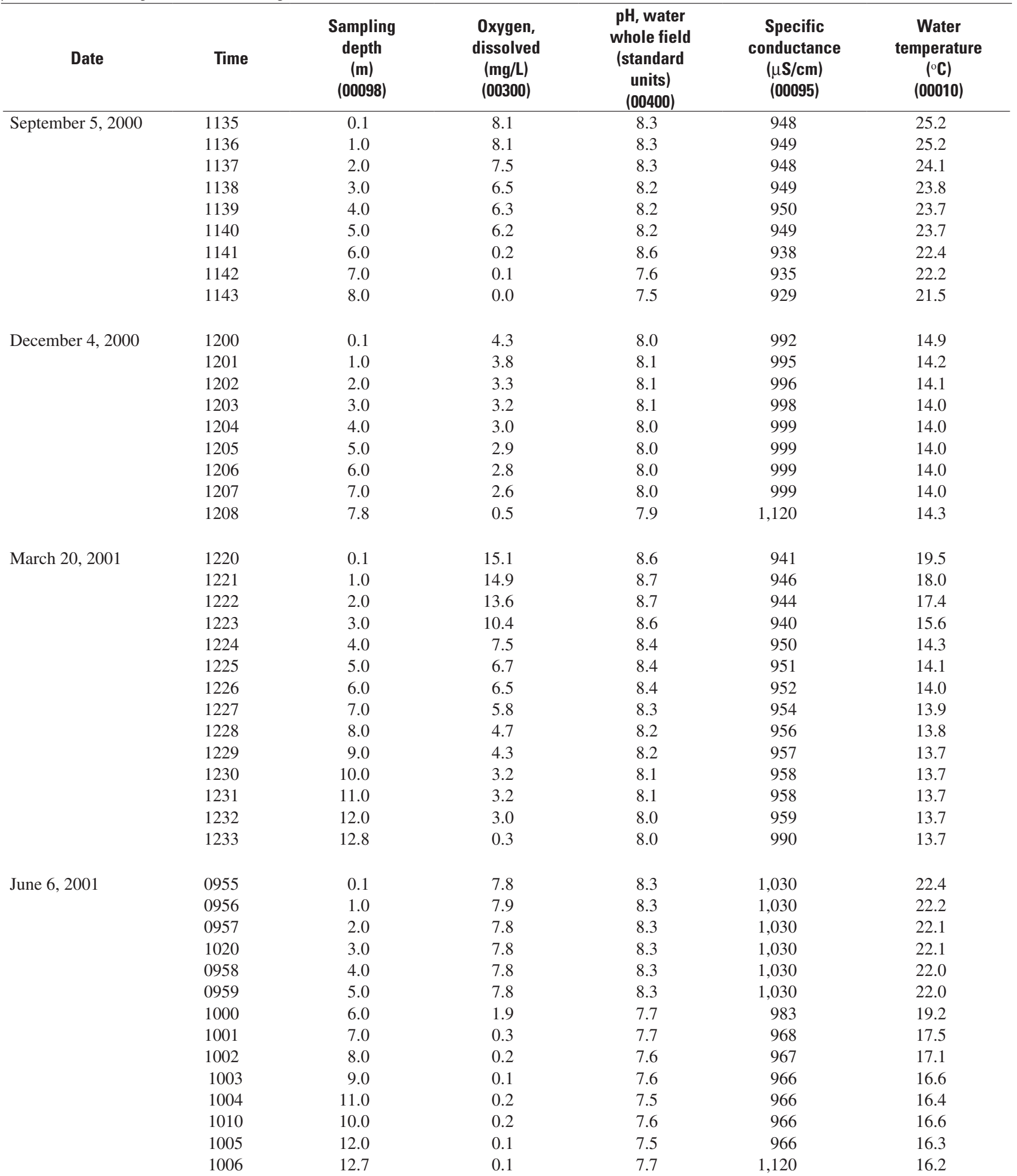


Table 2. Water-quality depth-profile field data for Sweetwater and Loveland Reservoir sampling sites and for Sweetwater River above Sweetwater Reservoir, San Diego County, California-Continued.

[Time is denoted in 24-hour scale; the five digit number in parentheses below the compound name, the parameter code, is used in the U.S. Geological Survey's computerized data system (National Water Information System) to uniquely identify a specific constituent or property; m, meter; mg/L, milligram per liter; $\mu \mathrm{S} / \mathrm{cm}$, microsiemens per centimeter; ${ }^{\circ} \mathrm{C}$, degree Celsius; ND, no data]

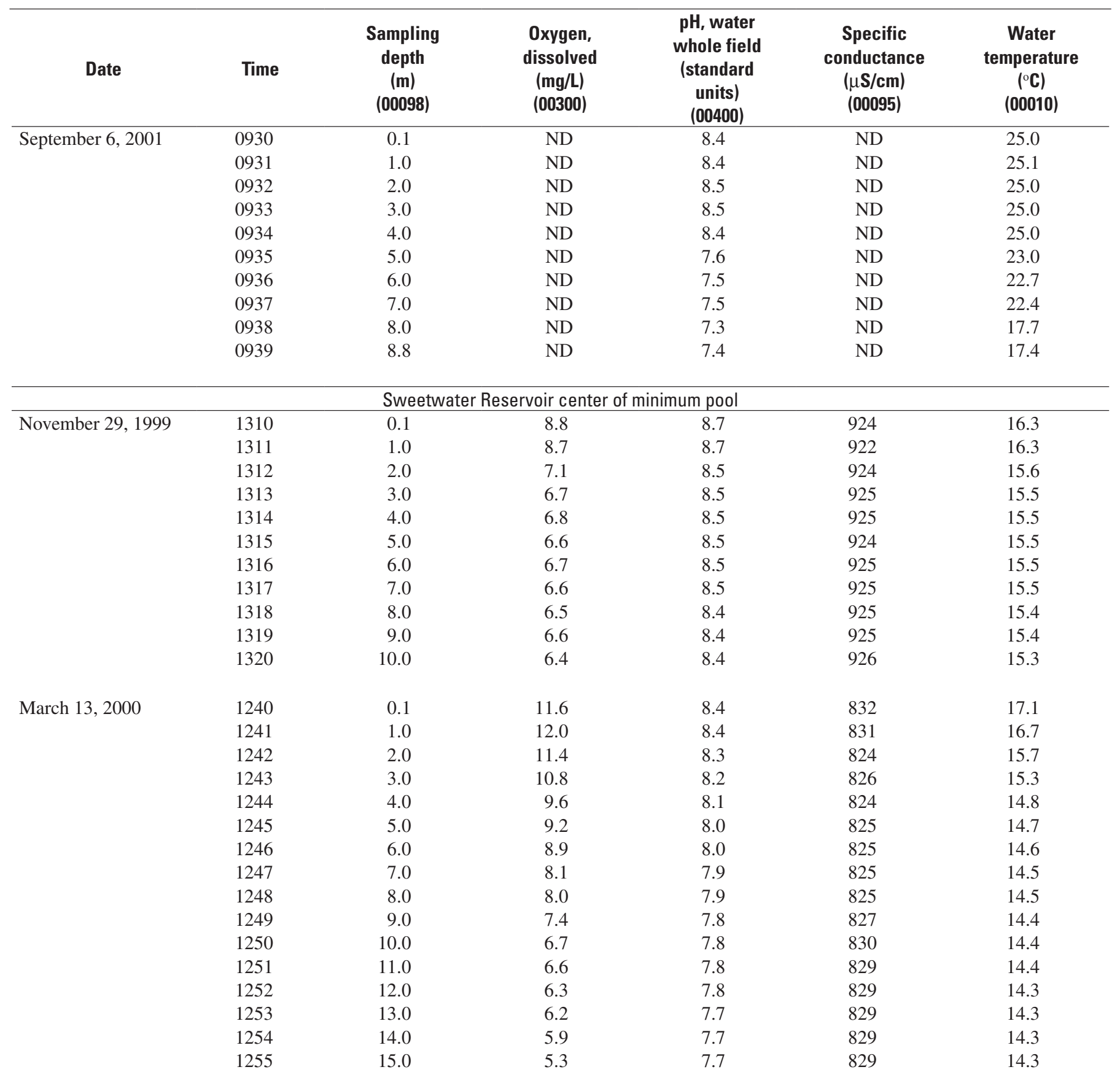


Table 2. Water-quality depth-profile field data for Sweetwater and Loveland Reservoir sampling sites and for Sweetwater River above Sweetwater Reservoir, San Diego County, California-Continued.

[Time is denoted in 24-hour scale; the five digit number in parentheses below the compound name, the parameter code, is used in the U.S. Geological Survey's computerized data system (National Water Information System) to uniquely identify a specific constituent or property; m, meter; mg/L, milligram per liter; $\underline{\mu \mathrm{S} / \mathrm{cm} \text {, microsiemens per centimeter; }{ }^{\circ} \mathrm{C} \text {, degree Celsius; ND, no data] }}$

\begin{tabular}{|c|c|c|c|c|c|c|}
\hline Date & Time & $\begin{array}{c}\text { Sampling } \\
\text { depth } \\
(\mathrm{m}) \\
(00098)\end{array}$ & $\begin{array}{c}\text { Oxygen, } \\
\text { dissolved } \\
(\mathrm{mg} / \mathrm{L}) \\
(00300)\end{array}$ & $\begin{array}{c}\mathrm{pH} \text {, water } \\
\text { whole field } \\
\text { (standard } \\
\text { units) } \\
(00400)\end{array}$ & $\begin{array}{c}\text { Specific } \\
\text { conductance } \\
(\mu S / \mathrm{cm}) \\
(00095)\end{array}$ & $\begin{array}{c}\text { Water } \\
\text { temperature } \\
\left({ }^{\circ} \mathrm{C}\right) \\
(00010)\end{array}$ \\
\hline \multirow[t]{8}{*}{ June 12, 2000} & 1220 & 0.1 & 8.3 & 8.5 & 917 & 24.9 \\
\hline & 1221 & 1.0 & 8.4 & 8.5 & 916 & 24.9 \\
\hline & 1222 & 2.0 & 8.5 & 8.5 & 916 & 24.5 \\
\hline & 1225 & 5.0 & 1.2 & 7.8 & 905 & 21.6 \\
\hline & 1226 & 6.0 & 0.7 & 7.8 & 900 & 21.2 \\
\hline & 1227 & 7.0 & 0.6 & 7.8 & 892 & 20.2 \\
\hline & 1228 & 8.0 & 0.4 & 7.8 & 889 & 19.8 \\
\hline & 1229 & 9.0 & 0.3 & 7.8 & 879 & 18.7 \\
\hline \multirow[t]{10}{*}{ September 5, 2000} & 1225 & 0.1 & 8.3 & 8.6 & 946 & 25.4 \\
\hline & 1226 & 1.0 & 8.2 & 8.6 & 947 & 24.8 \\
\hline & 1227 & 2.0 & 6.8 & 8.6 & 947 & 24.0 \\
\hline & 1228 & 3.0 & 6.3 & 8.5 & 947 & 23.9 \\
\hline & 1229 & 4.0 & 6.1 & 8.5 & 948 & 23.8 \\
\hline & 1230 & 5.0 & 6.1 & 8.5 & 949 & 23.7 \\
\hline & 1231 & 6.0 & 0.2 & 8.0 & 942 & 23.0 \\
\hline & 1232 & 7.0 & 0.1 & 7.8 & 934 & 22.1 \\
\hline & 1233 & 8.0 & 0.0 & 7.7 & 921 & 20.2 \\
\hline & 1234 & 9.0 & 0.0 & 7.7 & 920 & 19.5 \\
\hline \multirow{4}{*}{ December 4, 2000} & 1236 & 6.0 & 3.1 & 8.2 & 997 & 14.0 \\
\hline & 1237 & 7.0 & 2.7 & 8.1 & 999 & 14.0 \\
\hline & 1238 & 8.0 & 2.2 & 8.1 & 998 & 14.0 \\
\hline & 1239 & 8.3 & 0.5 & 8.2 & 1,000 & 14.0 \\
\hline \multirow[t]{10}{*}{ March 20, 2001} & 1314 & 4.0 & 8.4 & 8.6 & 944 & 14.7 \\
\hline & 1315 & 5.0 & 7.8 & 8.5 & 948 & 14.3 \\
\hline & 1316 & 6.0 & 7.1 & 8.4 & 951 & 14.2 \\
\hline & 1317 & 7.0 & 6.4 & 8.4 & 952 & 14.1 \\
\hline & 1318 & 8.0 & 5.3 & 8.3 & 954 & 13.9 \\
\hline & 1319 & 9.0 & 5.1 & 8.3 & 955 & 13.8 \\
\hline & 1320 & 10.0 & 5.1 & 8.3 & 955 & 13.8 \\
\hline & 1321 & 11.0 & 4.2 & 8.2 & 956 & 13.7 \\
\hline & 1322 & 12.0 & 3.6 & 8.2 & 957 & 13.7 \\
\hline & 1323 & 13.0 & 2.9 & 8.2 & 958 & 13.7 \\
\hline
\end{tabular}


Table 2. Water-quality depth-profile field data for Sweetwater and Loveland Reservoir sampling sites and for Sweetwater River above Sweetwater Reservoir, San Diego County, California—Continued.

[Time is denoted in 24-hour scale; the five digit number in parentheses below the compound name, the parameter code, is used in the U.S. Geological Survey's computerized data system (National Water Information System) to uniquely identify a specific constituent or property; m, meter; mg/L, milligram per liter; $\mu \mathrm{S} / \mathrm{cm}$, microsiemens per centimeter; ${ }^{\circ} \mathrm{C}$, degree Celsius; ND, no data]

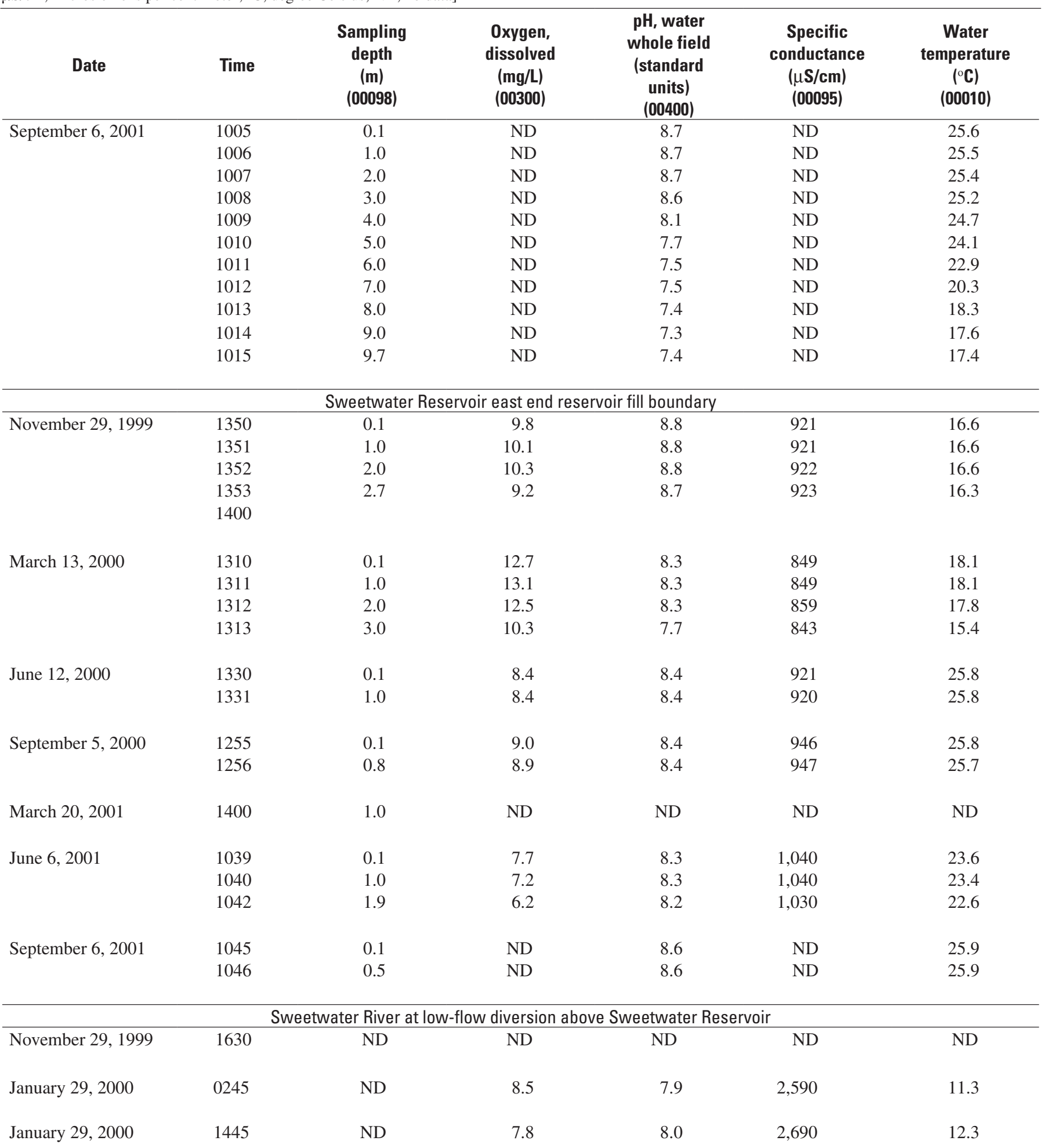


Table 2. Water-quality depth-profile field data for Sweetwater and Loveland Reservoir sampling sites and for Sweetwater River above Sweetwater Reservoir, San Diego County, California-Continued.

[Time is denoted in 24-hour scale; the five digit number in parentheses below the compound name, the parameter code, is used in the U.S. Geological Survey's computerized data system (National Water Information System) to uniquely identify a specific constituent or property; m, meter; mg/L, milligram per liter; $\mu \mathrm{S} / \mathrm{cm}$, microsiemens per centimeter; ${ }^{\circ} \mathrm{C}$, degree Celsius; ND, no data]

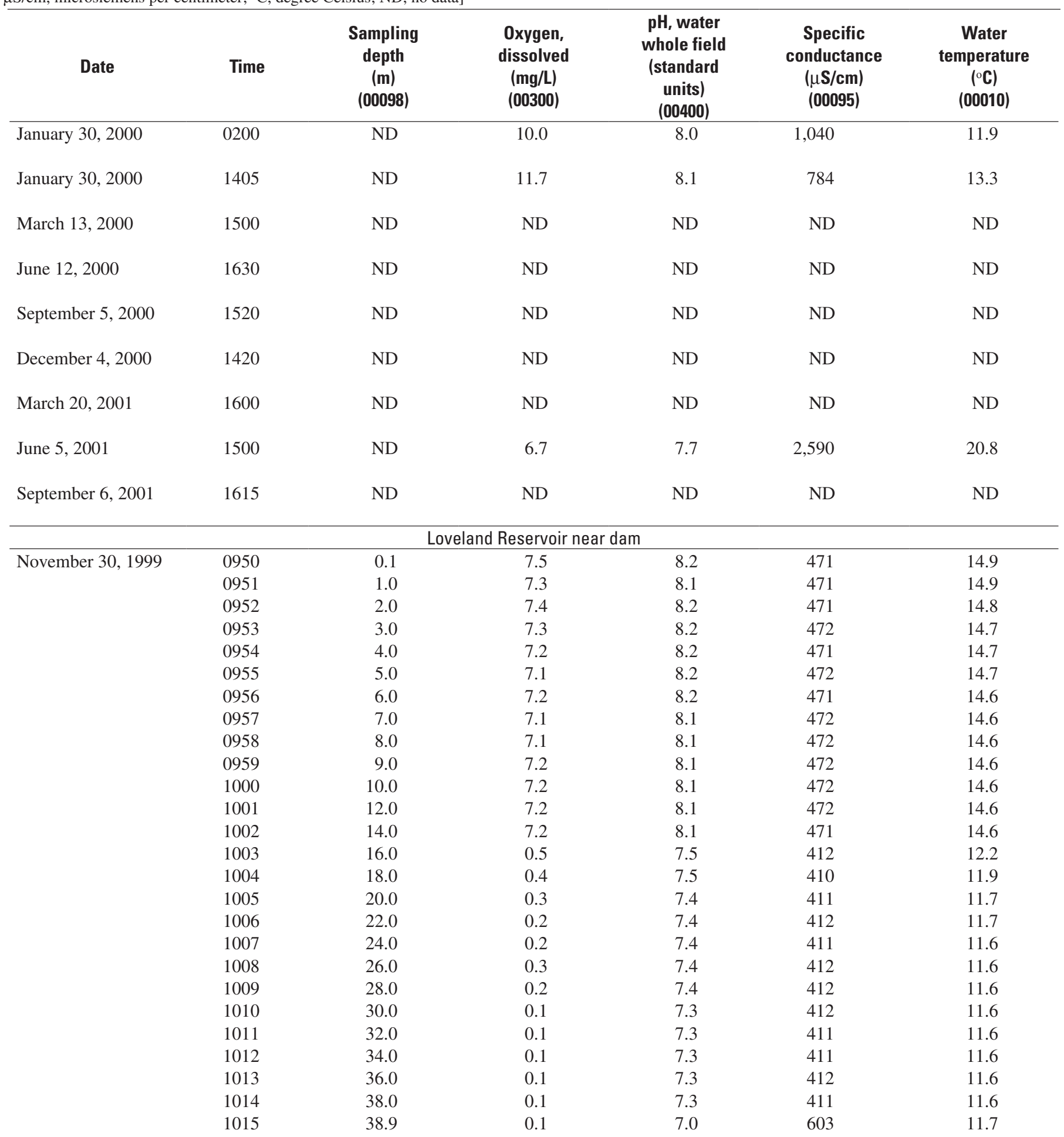


Table 2. Water-quality depth-profile field data for Sweetwater and Loveland Reservoir sampling sites and for Sweetwater River above Sweetwater Reservoir, San Diego County, California-Continued.

[Time is denoted in 24-hour scale; the five digit number in parentheses below the compound name, the parameter code, is used in the U.S. Geological Survey's computerized data system (National Water Information System) to uniquely identify a specific constituent or property; m, meter; $\mathrm{mg} / \mathrm{L}$, milligram per liter; $\mu \mathrm{S} / \mathrm{cm}$, microsiemens per centimeter; ${ }^{\circ} \mathrm{C}$, degree Celsius; ND, no data]

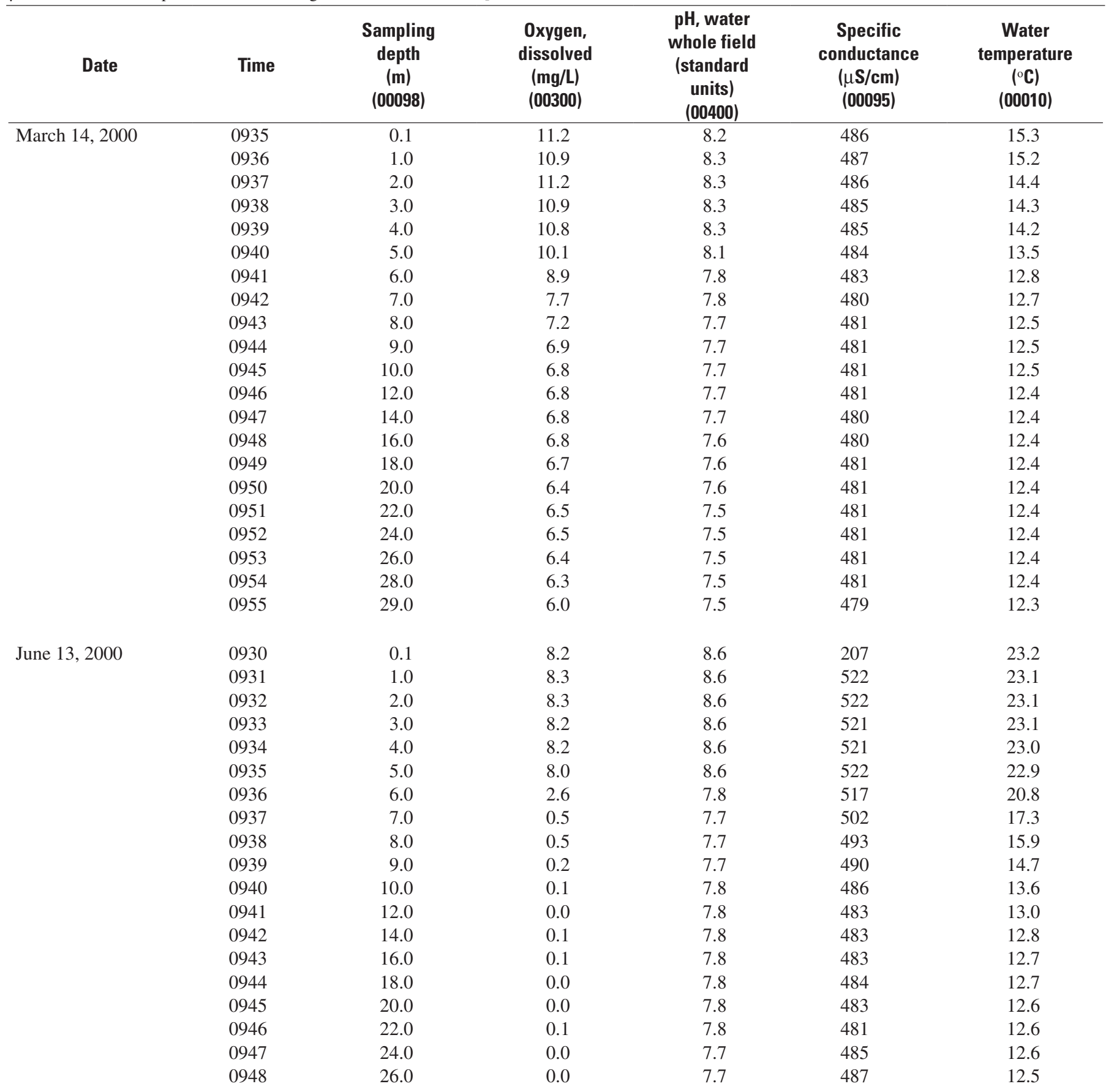


Table 2. Water-quality depth-profile field data for Sweetwater and Loveland Reservoir sampling sites and for Sweetwater River above Sweetwater Reservoir, San Diego County, California-Continued.

[Time is denoted in 24-hour scale; the five digit number in parentheses below the compound name, the parameter code, is used in the U.S. Geological Survey's computerized data system (National Water Information System) to uniquely identify a specific constituent or property; m, meter; mg/L, milligram per liter; $\mu \mathrm{S} / \mathrm{cm}$, microsiemens per centimeter; ${ }^{\circ} \mathrm{C}$, degree Celsius; ND, no data]

\begin{tabular}{|c|c|c|c|c|c|c|}
\hline Date & Time & $\begin{array}{c}\text { Sampling } \\
\text { depth } \\
\text { (m) } \\
(00098)\end{array}$ & $\begin{array}{c}\text { Oxygen, } \\
\text { dissolved } \\
(\mathrm{mg} / \mathrm{L}) \\
(00300)\end{array}$ & $\begin{array}{c}\text { pH, water } \\
\text { whole field } \\
\text { (standard } \\
\text { units) } \\
(00400) \\
\end{array}$ & $\begin{array}{c}\text { Specific } \\
\text { conductance } \\
(\mu \mathrm{S} / \mathrm{cm}) \\
(00095)\end{array}$ & $\begin{array}{c}\text { Water } \\
\text { temperature } \\
\left({ }^{\circ} \mathrm{C}\right) \\
(00010)\end{array}$ \\
\hline \multirow[t]{14}{*}{ September 6, 2000} & 0925 & 0.1 & 7.5 & 8.3 & 522 & 23.3 \\
\hline & 0926 & 1.0 & 7.4 & 8.3 & 523 & 23.2 \\
\hline & 0927 & 2.0 & 7.3 & 8.3 & 523 & 23.2 \\
\hline & 0930 & 5.0 & 7.1 & 8.3 & 523 & 23.1 \\
\hline & 0931 & 6.0 & 7.2 & 8.3 & 523 & 23.1 \\
\hline & 0932 & 7.0 & 7.0 & 8.3 & 523 & 23.0 \\
\hline & 0933 & 8.0 & 1.4 & 7.5 & 507 & 19.9 \\
\hline & 0934 & 9.0 & 0.1 & 7.5 & 500 & 16.5 \\
\hline & 0939 & 18.0 & 0.0 & 7.7 & 489 & 12.8 \\
\hline & 0940 & 20.0 & 0.1 & 7.7 & 489 & 12.7 \\
\hline & 0941 & 22.0 & 0.1 & 7.7 & 488 & 12.7 \\
\hline & 0942 & 24.0 & 0.1 & 7.7 & 491 & 12.6 \\
\hline & 0943 & 26.0 & 0.0 & 7.6 & 492 & 12.6 \\
\hline & 0944 & 27.0 & 0.0 & 7.6 & 527 & 12.6 \\
\hline \multirow[t]{13}{*}{ December 5, 2000} & 0950 & 0.1 & 6.6 & 8.0 & 513 & 13.4 \\
\hline & 0951 & 1.0 & 6.5 & 8.1 & 515 & 13.2 \\
\hline & 0952 & 2.0 & 6.4 & 8.1 & 515 & 13.1 \\
\hline & 0953 & 3.0 & 6.2 & 8.1 & 515 & 13.0 \\
\hline & 1002 & 14.0 & 5.4 & 7.9 & 516 & 12.7 \\
\hline & 1003 & 16.0 & 5.4 & 7.9 & 516 & 12.6 \\
\hline & 1004 & 18.0 & 5.4 & 7.9 & 515 & 12.6 \\
\hline & 1005 & 20.0 & 5.3 & 7.9 & 517 & 12.6 \\
\hline & 1006 & 22.0 & 5.3 & 7.9 & 515 & 12.6 \\
\hline & 1007 & 24.0 & 5.4 & 7.8 & 515 & 12.6 \\
\hline & 1008 & 26.0 & 5.4 & 7.8 & 515 & 12.6 \\
\hline & 1009 & 28.0 & 5.2 & 7.8 & 516 & 12.6 \\
\hline & 1010 & 28.2 & 1.1 & 7.5 & 549 & 12.6 \\
\hline
\end{tabular}


Table 2. Water-quality depth-profile field data for Sweetwater and Loveland Reservoir sampling sites and for Sweetwater River above Sweetwater Reservoir, San Diego County, California-Continued.

[Time is denoted in 24-hour scale; the five digit number in parentheses below the compound name, the parameter code, is used in the U.S. Geological Survey's computerized data system (National Water Information System) to uniquely identify a specific constituent or property; m, meter; mg/L, milligram per liter; $\mu \mathrm{S} / \mathrm{cm}$, microsiemens per centimeter; ${ }^{\circ} \mathrm{C}$, degree Celsius; ND, no data]

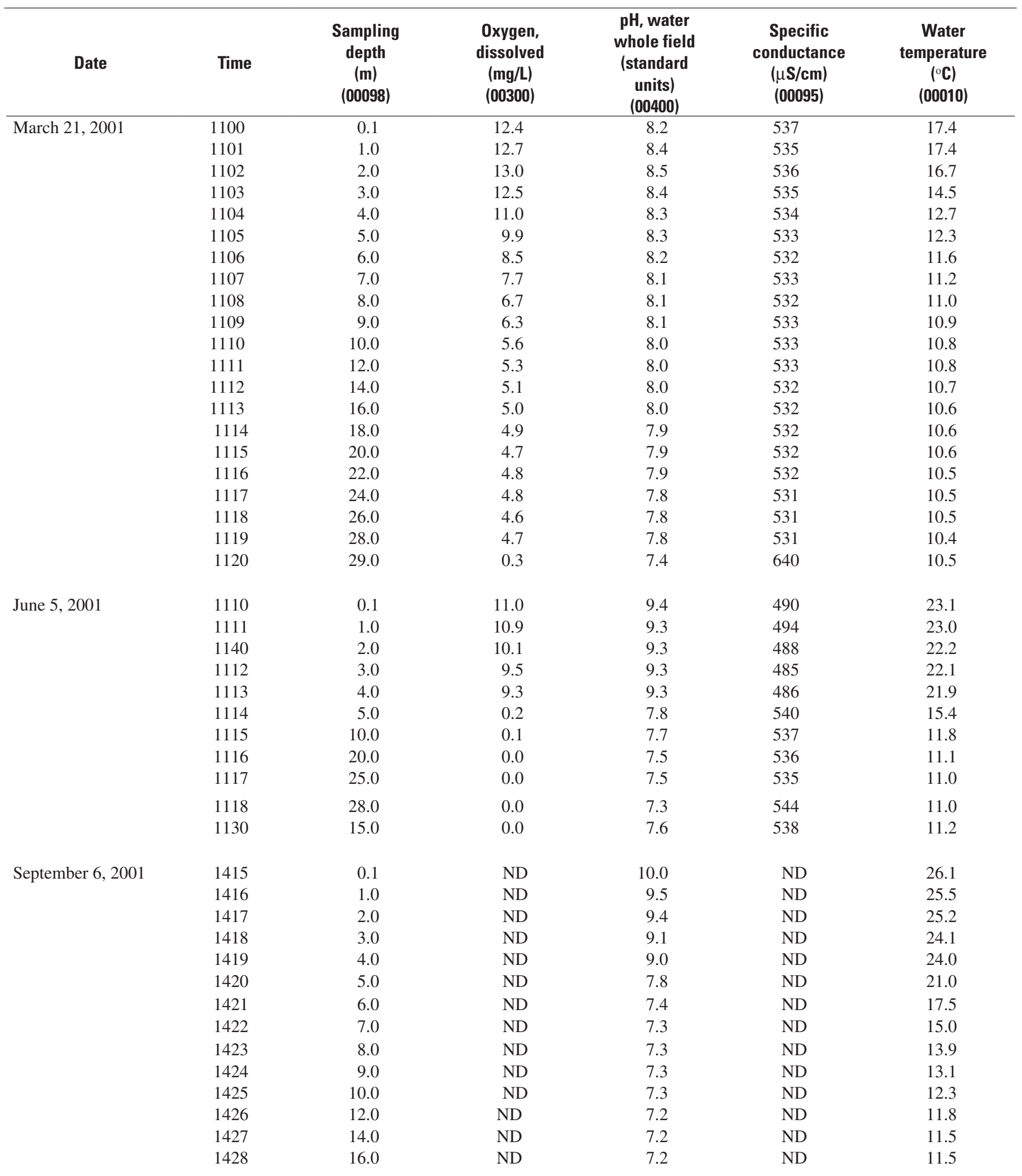


Table 2. Water-quality depth-profile field data for Sweetwater and Loveland Reservoir sampling sites and for Sweetwater River above Sweetwater Reservoir, San Diego County, California-Continued.

[Time is denoted in 24-hour scale; the five digit number in parentheses below the compound name, the parameter code, is used in the U.S. Geological Survey's computerized data system (National Water Information System) to uniquely identify a specific constituent or property; m, meter; mg/L, milligram per liter; $\mu \mathrm{S} / \mathrm{cm}$, microsiemens per centimeter; ${ }^{\circ} \mathrm{C}$, degree Celsius; ND, no data]

\begin{tabular}{|c|c|c|c|c|c|c|}
\hline Date & Time & $\begin{array}{c}\text { Sampling } \\
\text { depth } \\
\text { (m) } \\
(00098)\end{array}$ & $\begin{array}{c}\text { Oxygen, } \\
\text { dissolved } \\
(\mathrm{mg} / \mathrm{L}) \\
(00300)\end{array}$ & $\begin{array}{c}\text { pH, water } \\
\text { whole field } \\
\text { (standard } \\
\text { units) } \\
(00400)\end{array}$ & $\begin{array}{c}\text { Specific } \\
\text { conductance } \\
(\mu \mathrm{S} / \mathrm{cm}) \\
(00095)\end{array}$ & $\begin{array}{c}\text { Water } \\
\text { temperature } \\
\left({ }^{\circ} \mathrm{C}\right) \\
(00010)\end{array}$ \\
\hline & 1429 & 18.0 & ND & 7.2 & ND & 11.4 \\
\hline & 1430 & 20.0 & ND & 7.2 & ND & 11.4 \\
\hline & 1431 & 22.0 & ND & 7.2 & ND & 11.3 \\
\hline & 1434 & 28.0 & ND & 7.2 & ND & 11.3 \\
\hline & 1435 & 29.0 & ND & 7.1 & ND & 11.3 \\
\hline \multicolumn{7}{|c|}{ Sweetwater River below Steele Canyon Bridge at Cottonwood Golf Course } \\
\hline January 29,2000 & 1000 & ND & ND & 8.4 & 2,750 & 11.2 \\
\hline
\end{tabular}




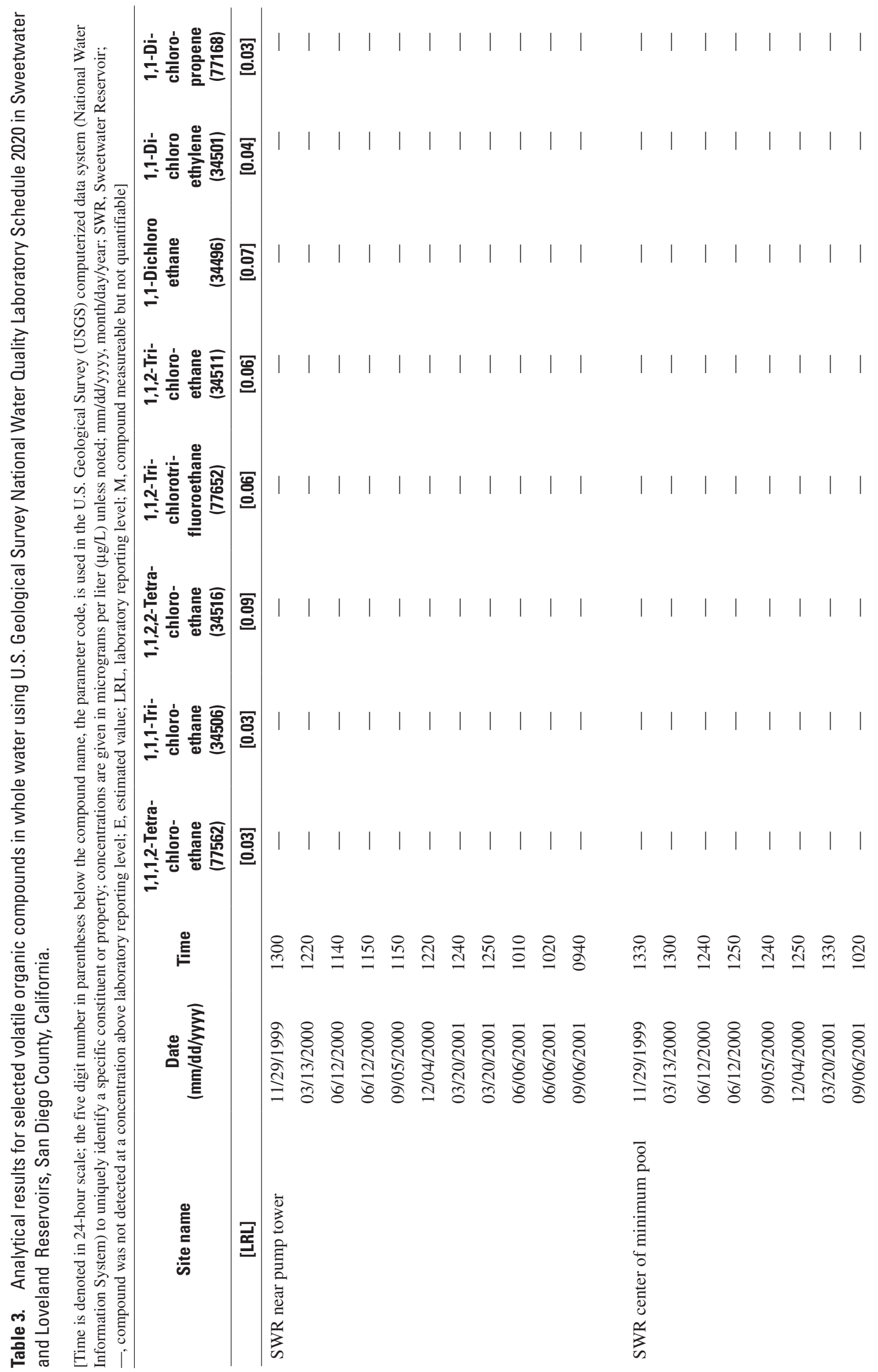




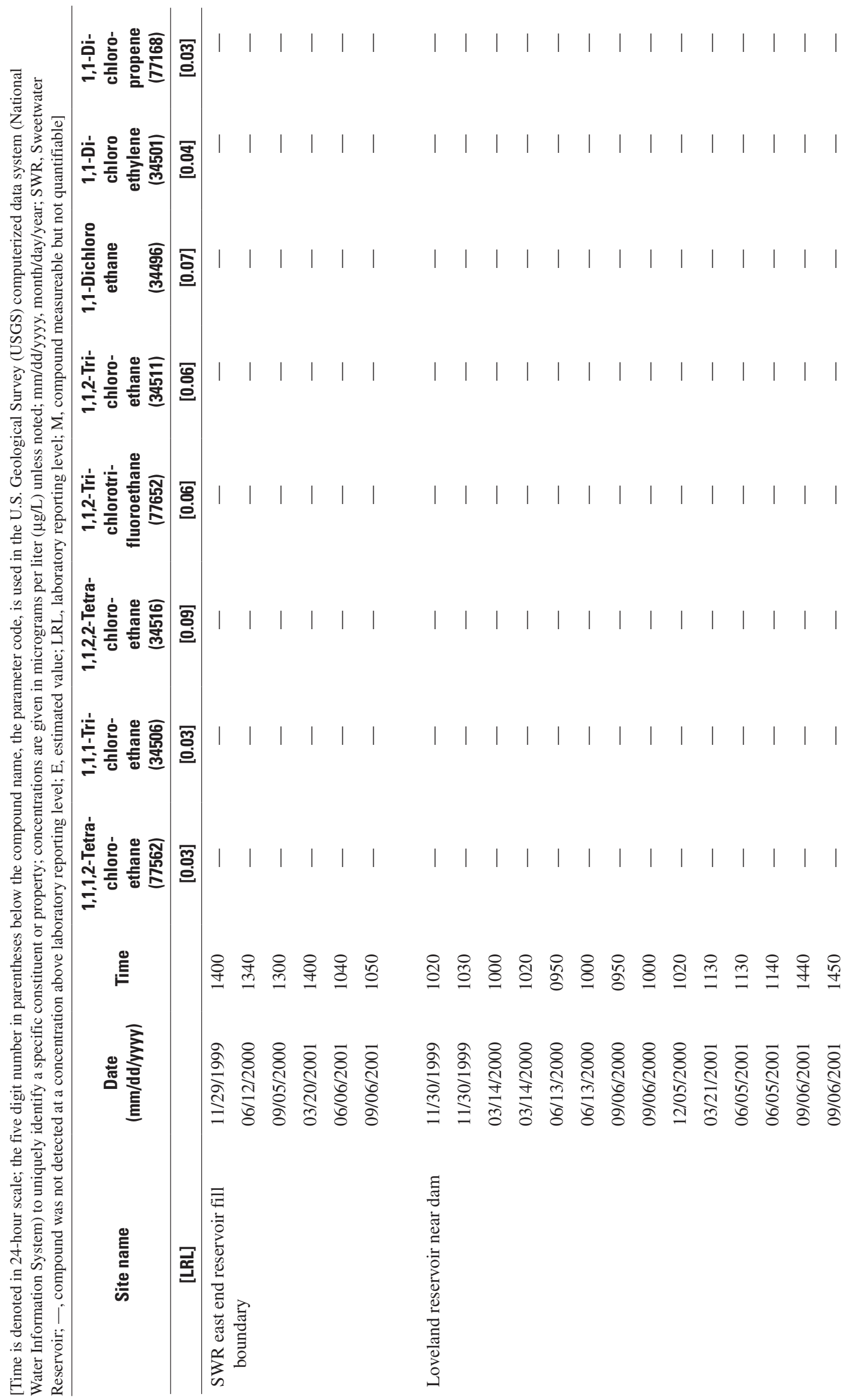




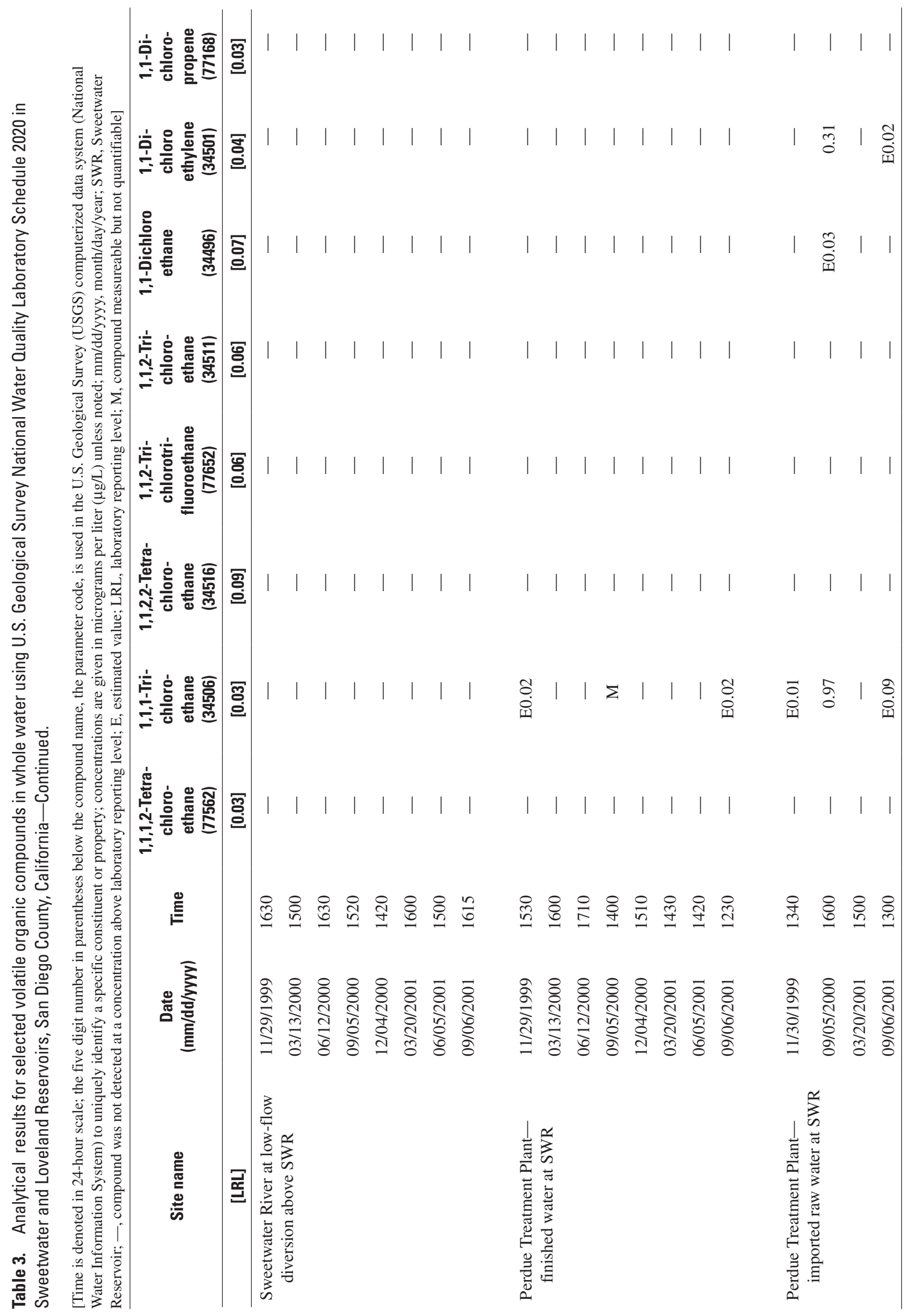




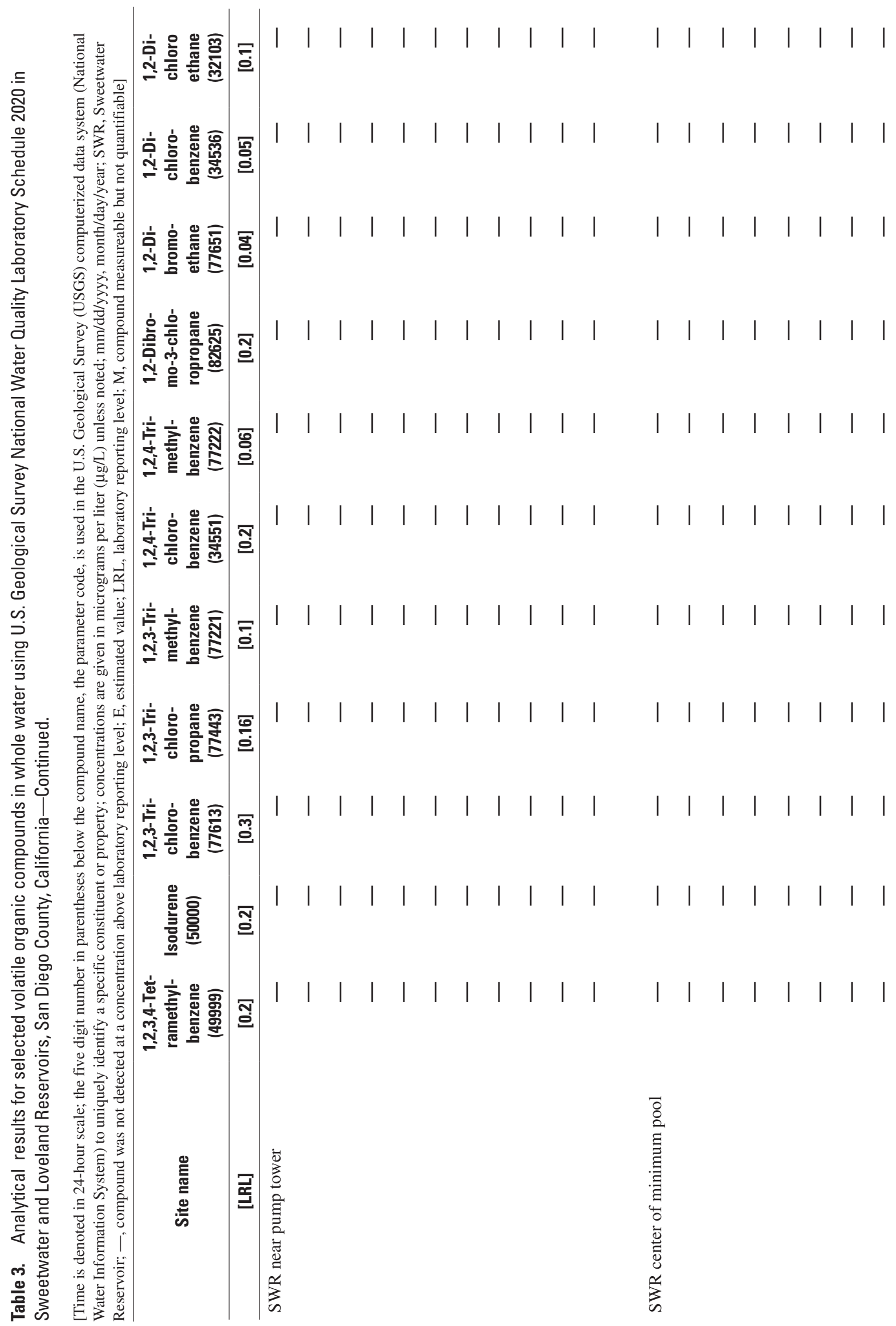




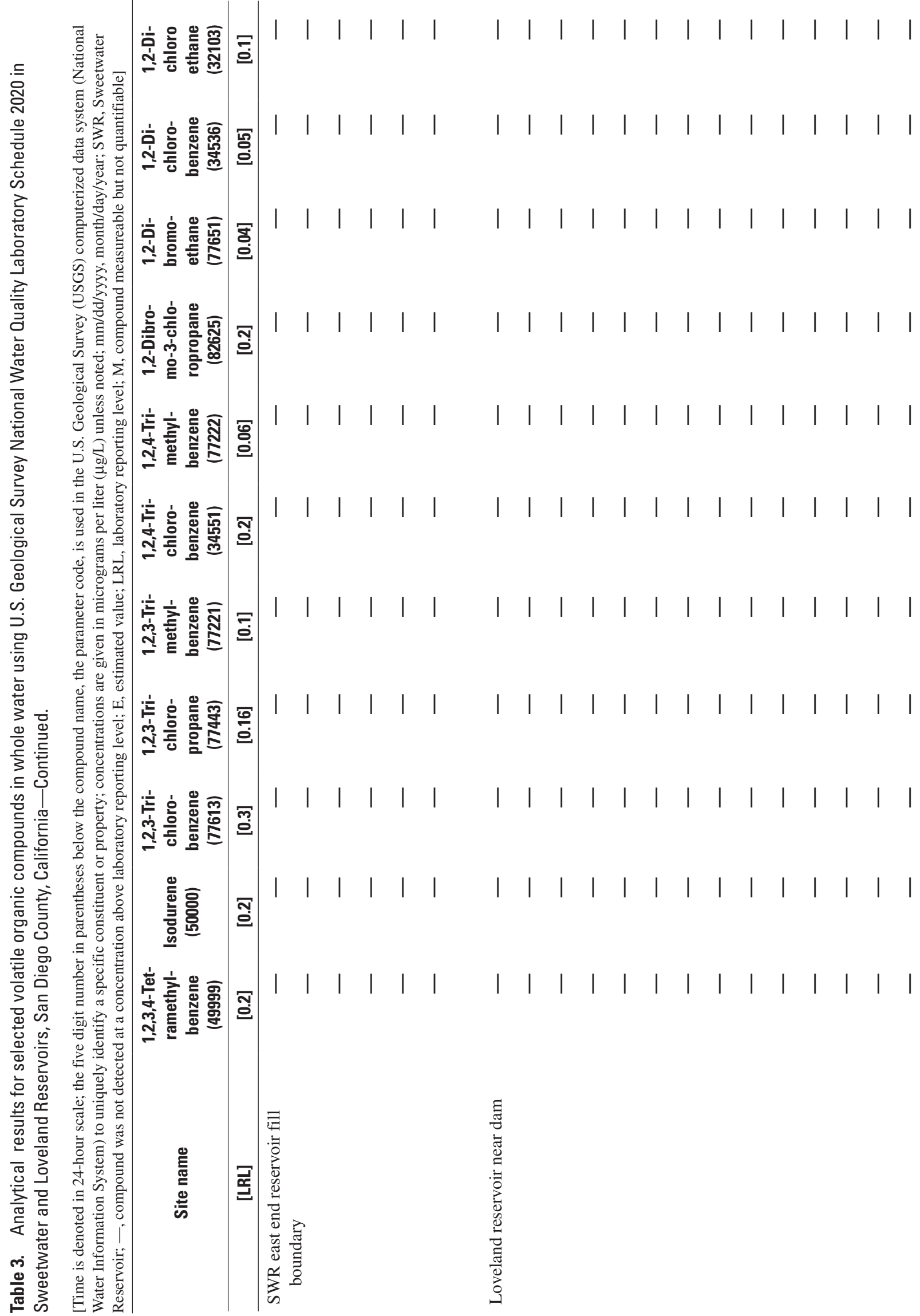




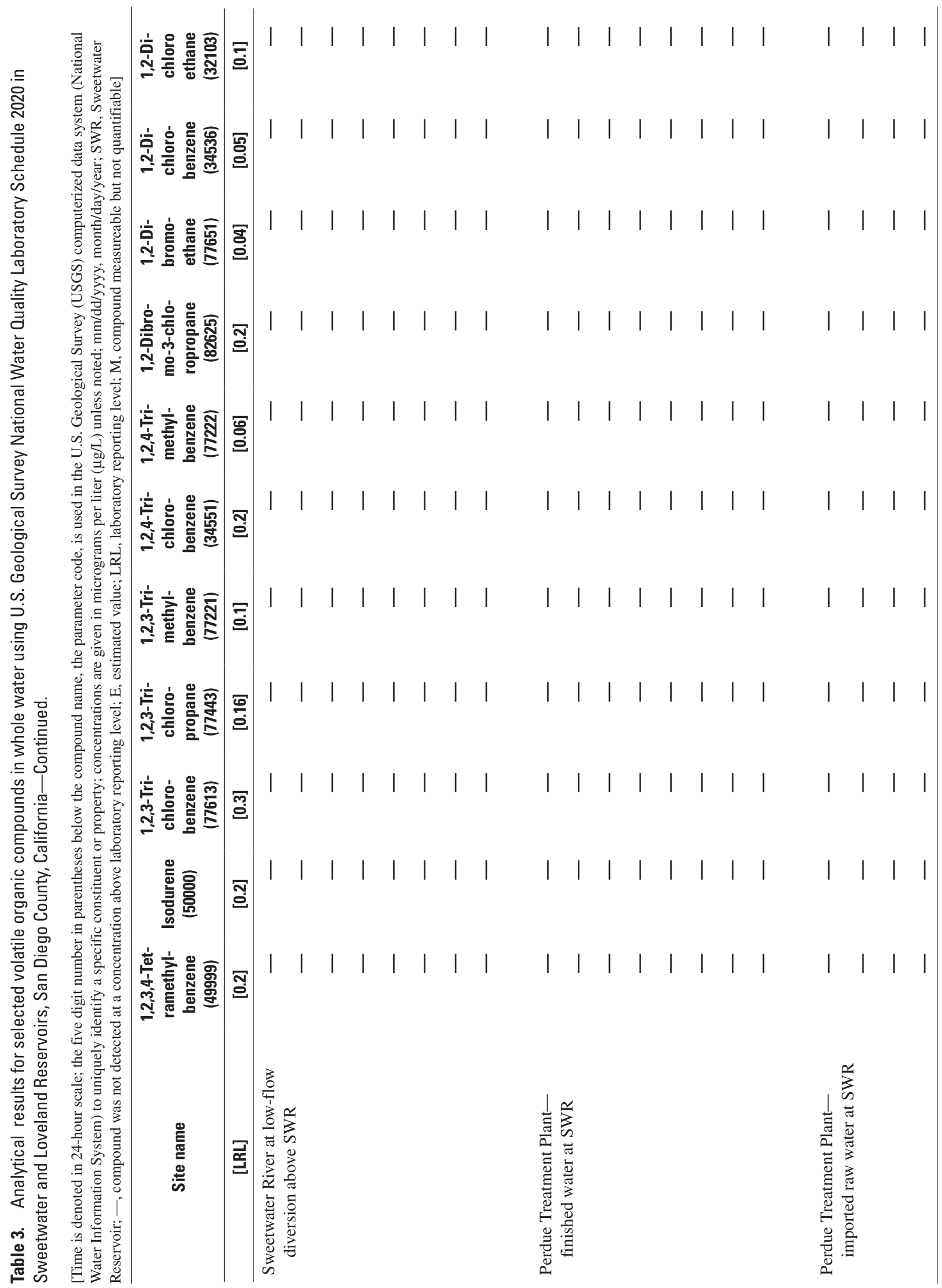




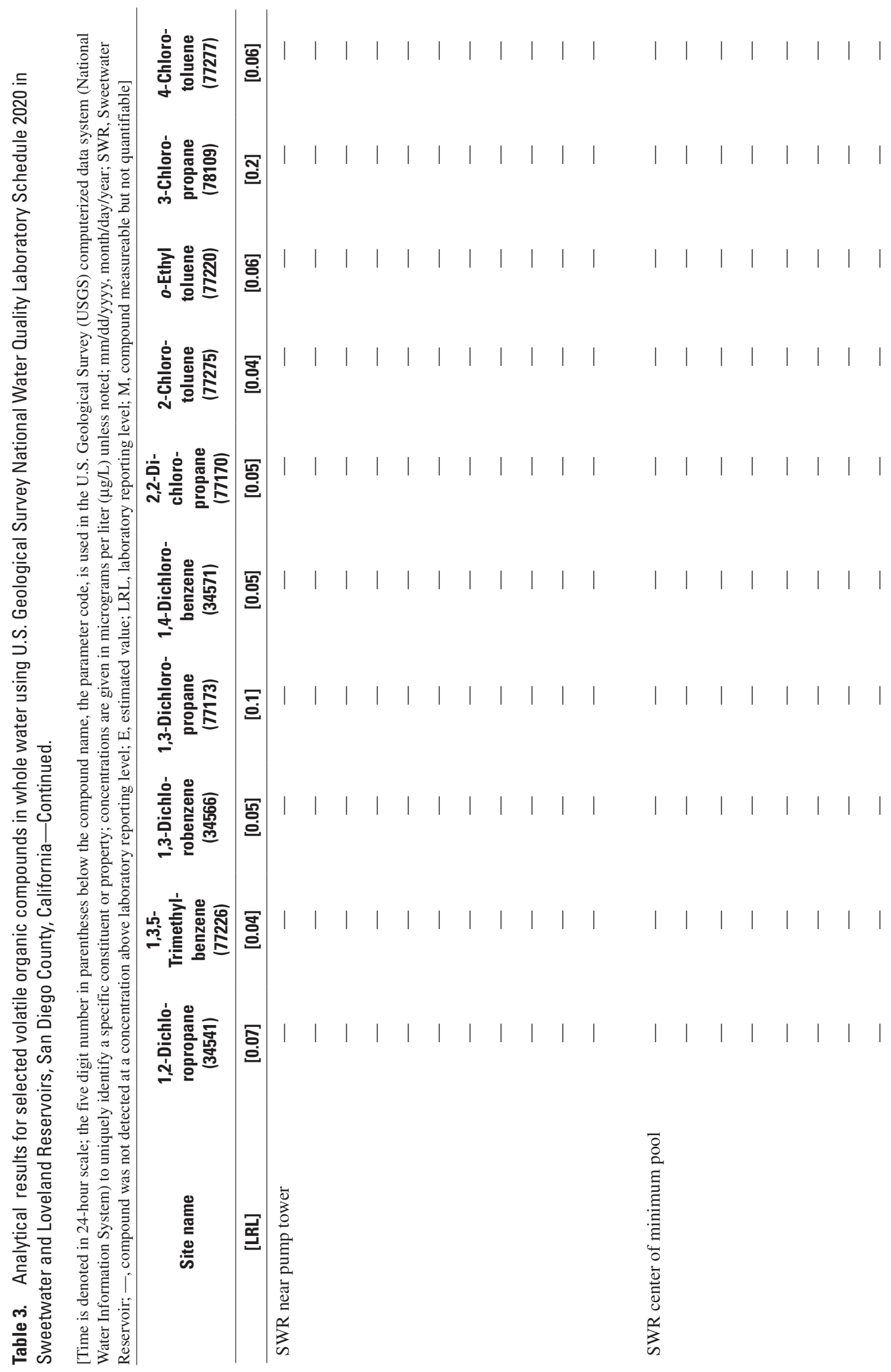




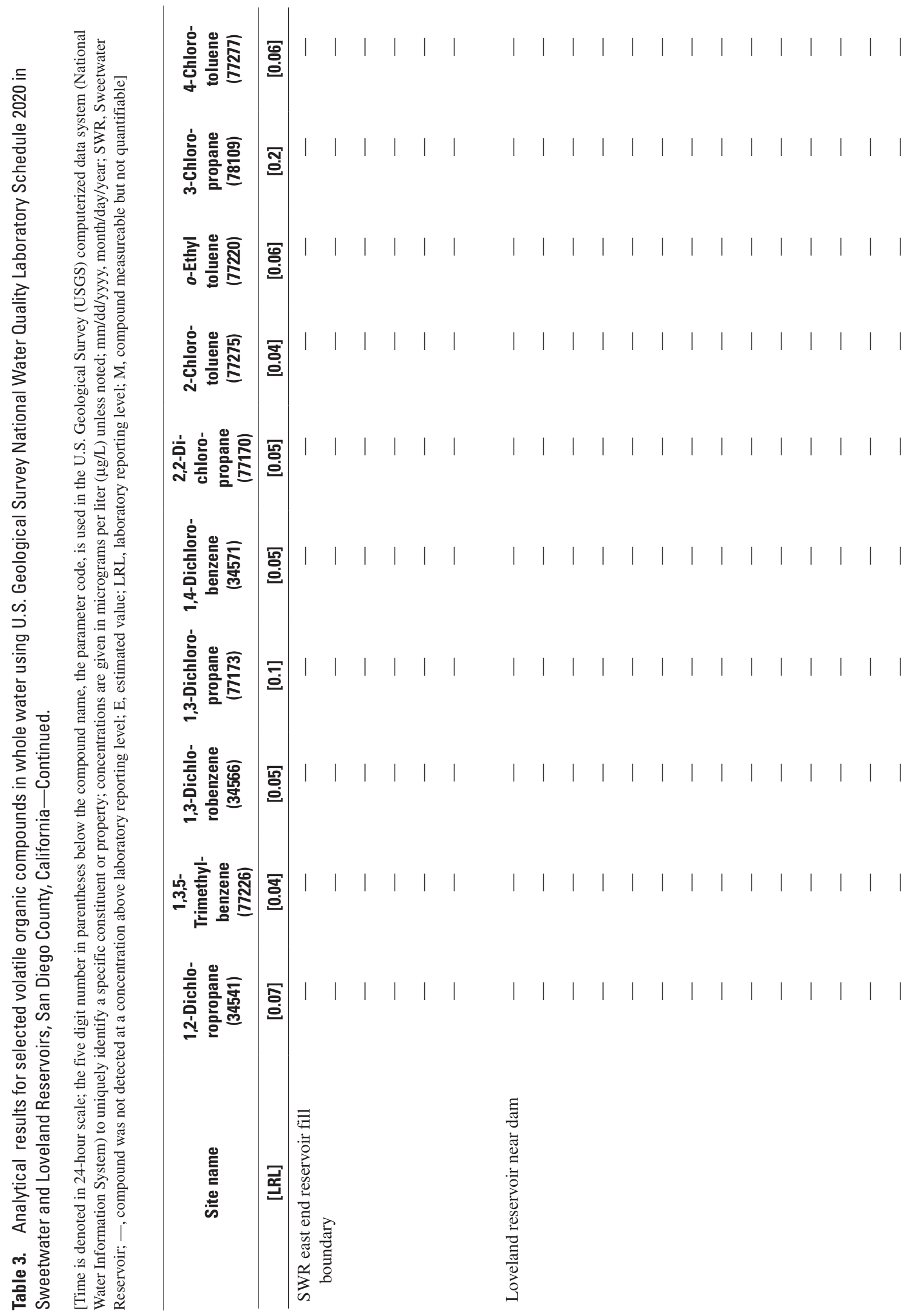




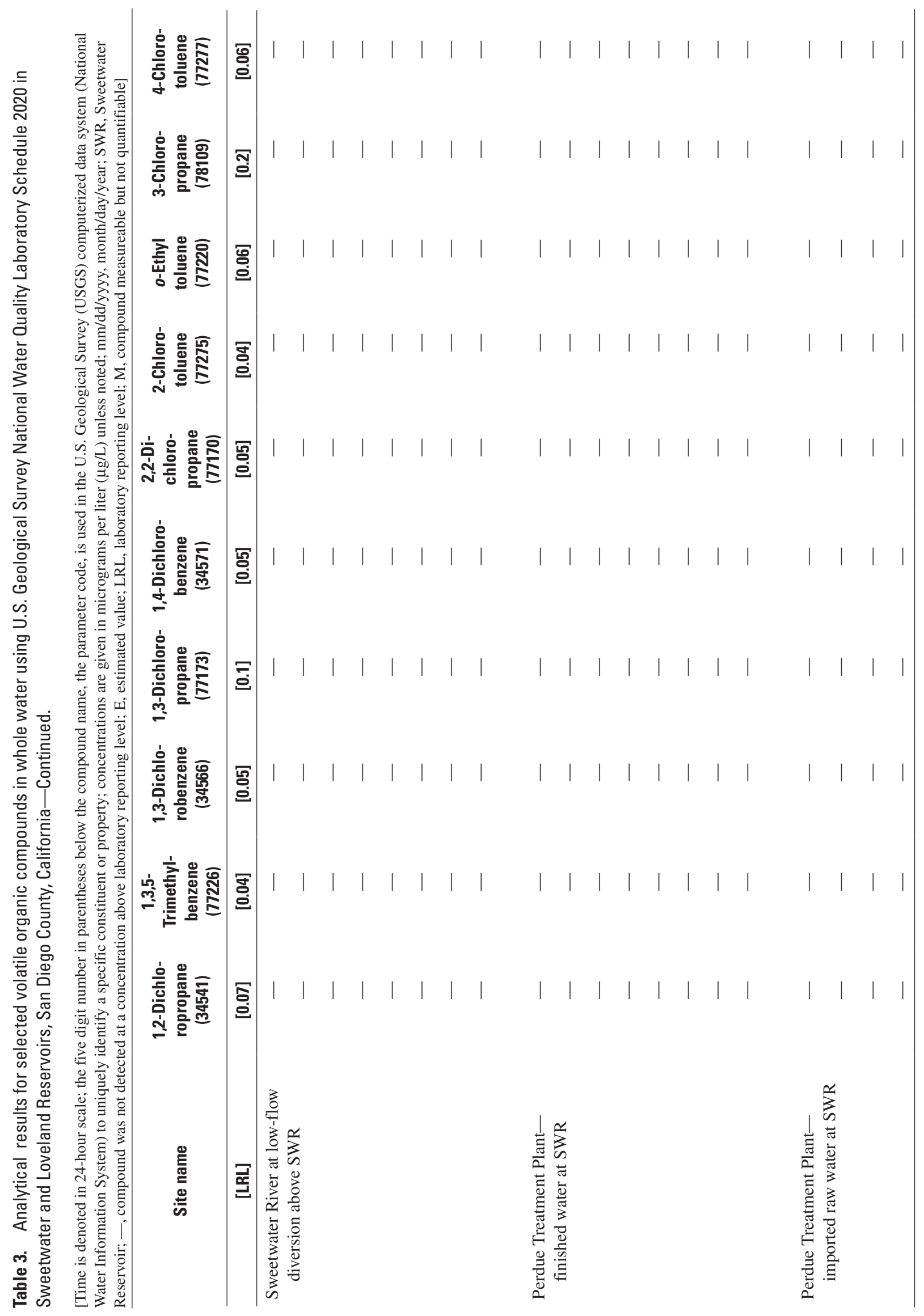




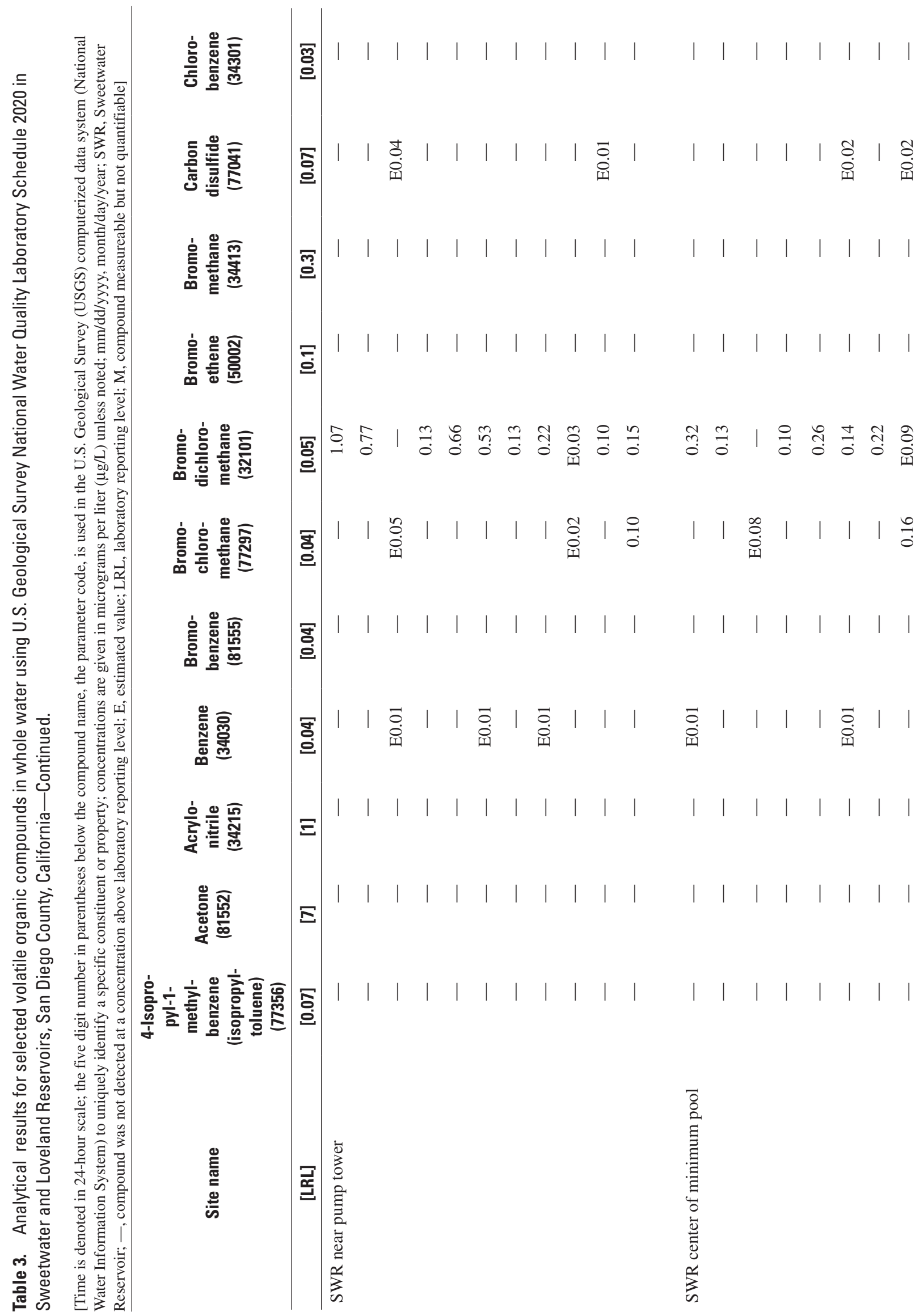




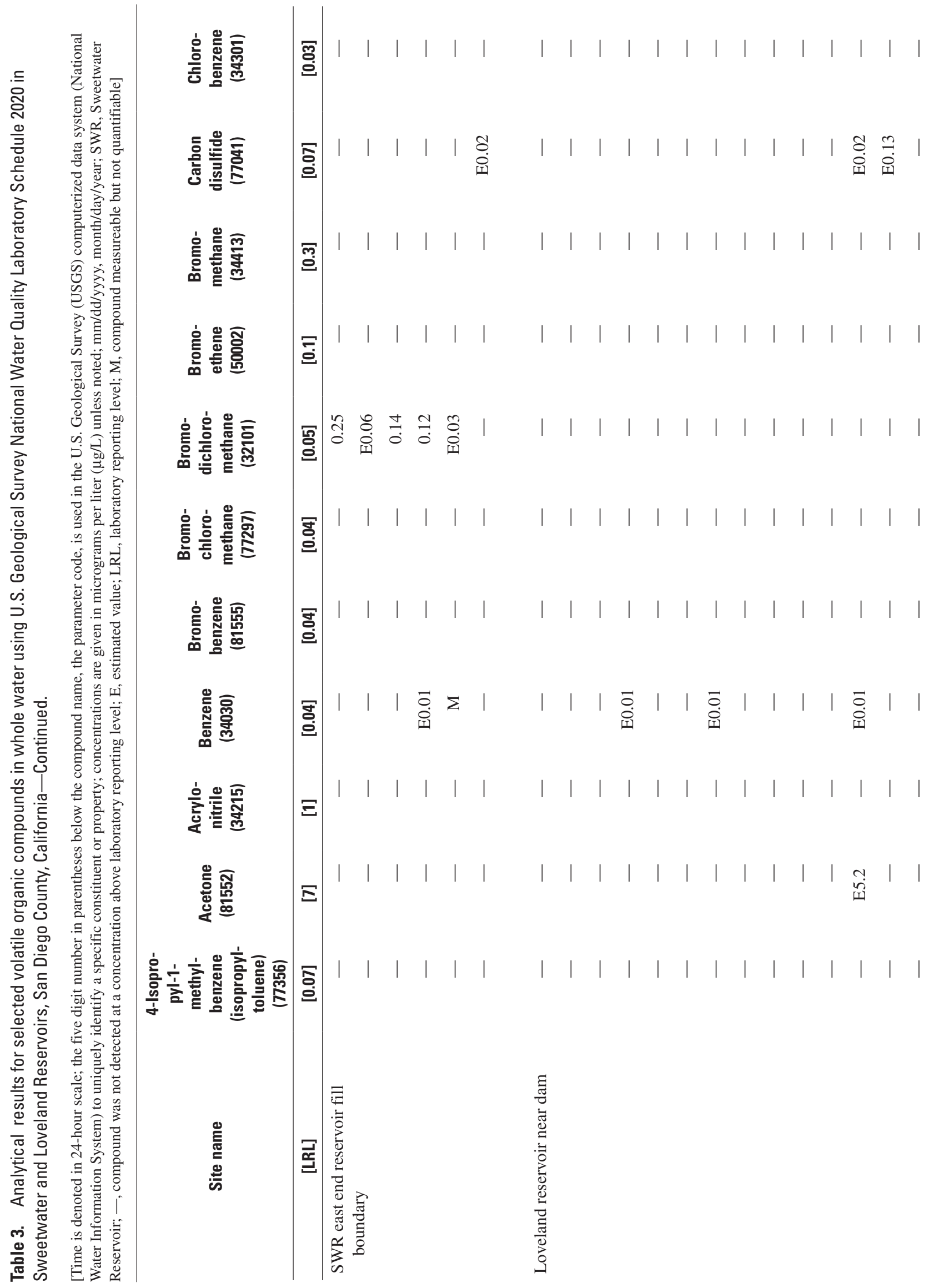




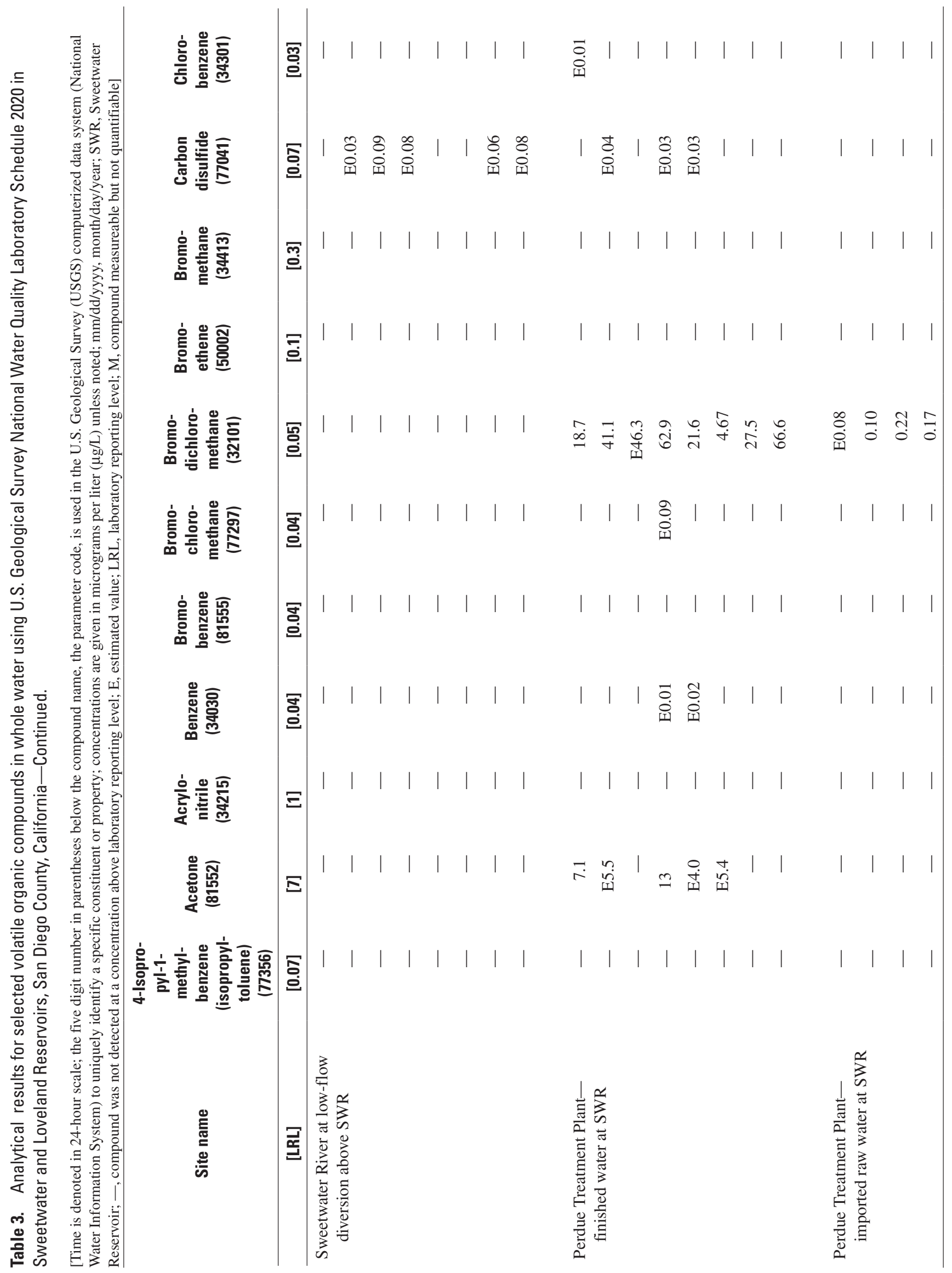




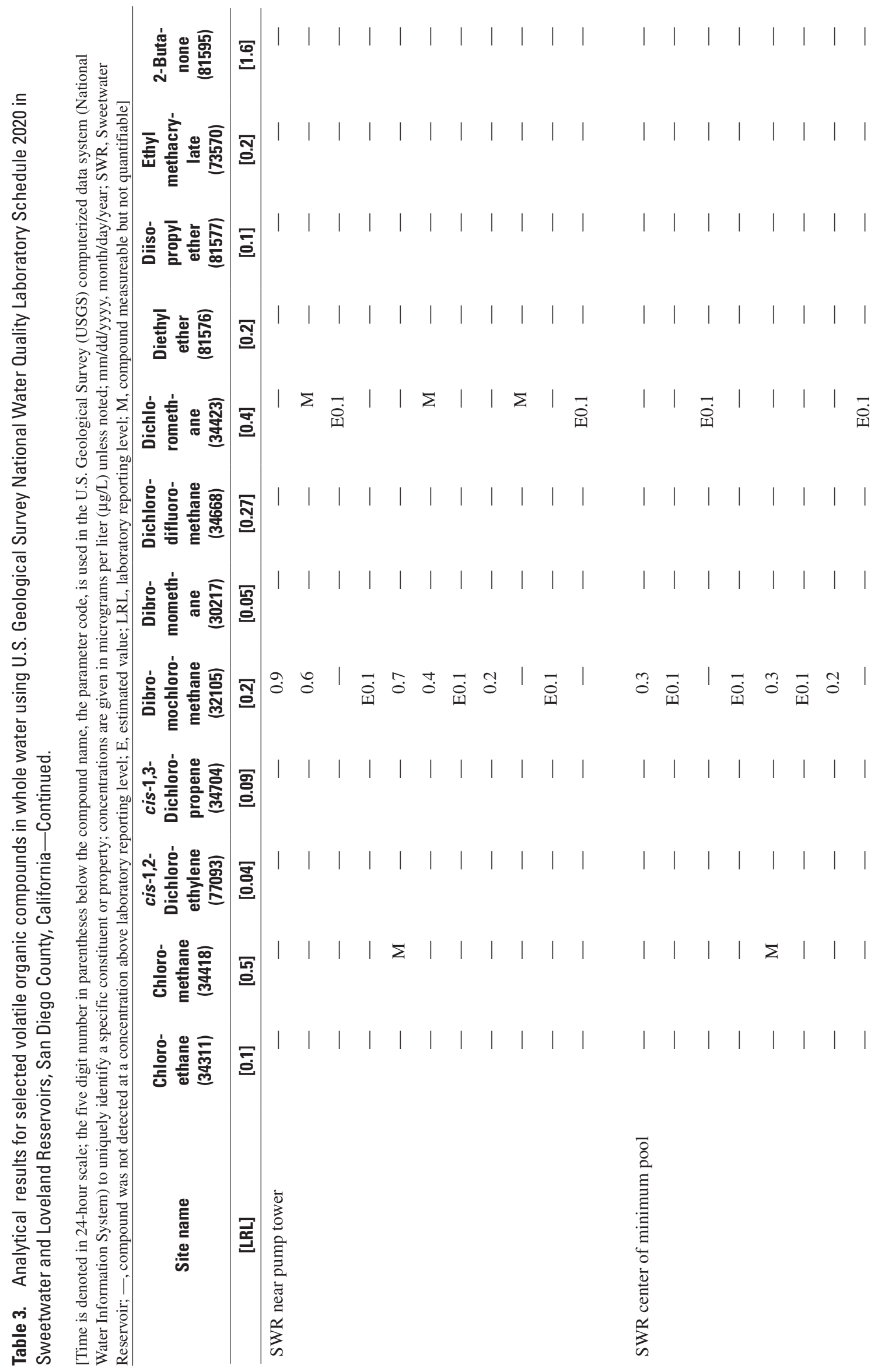




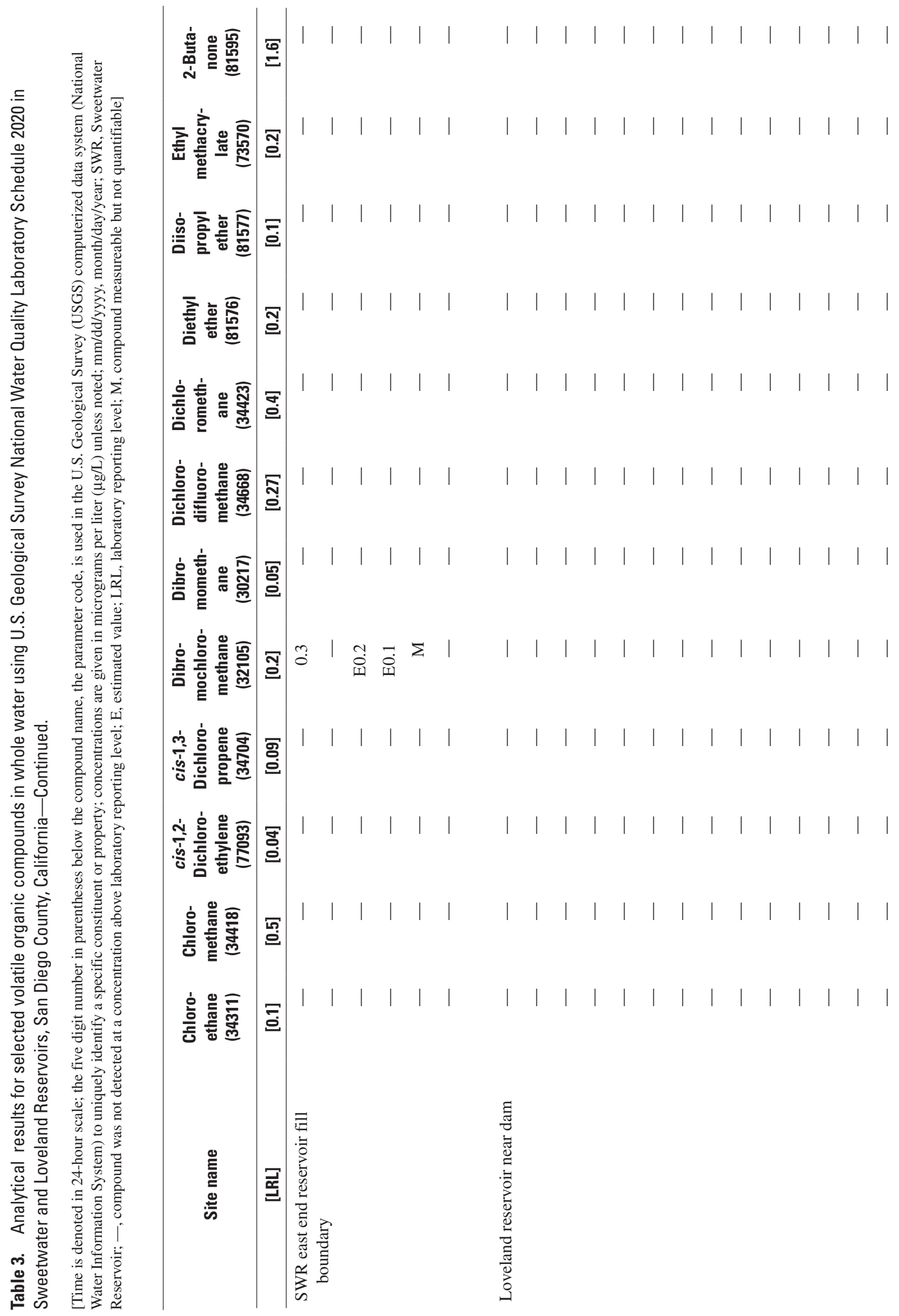




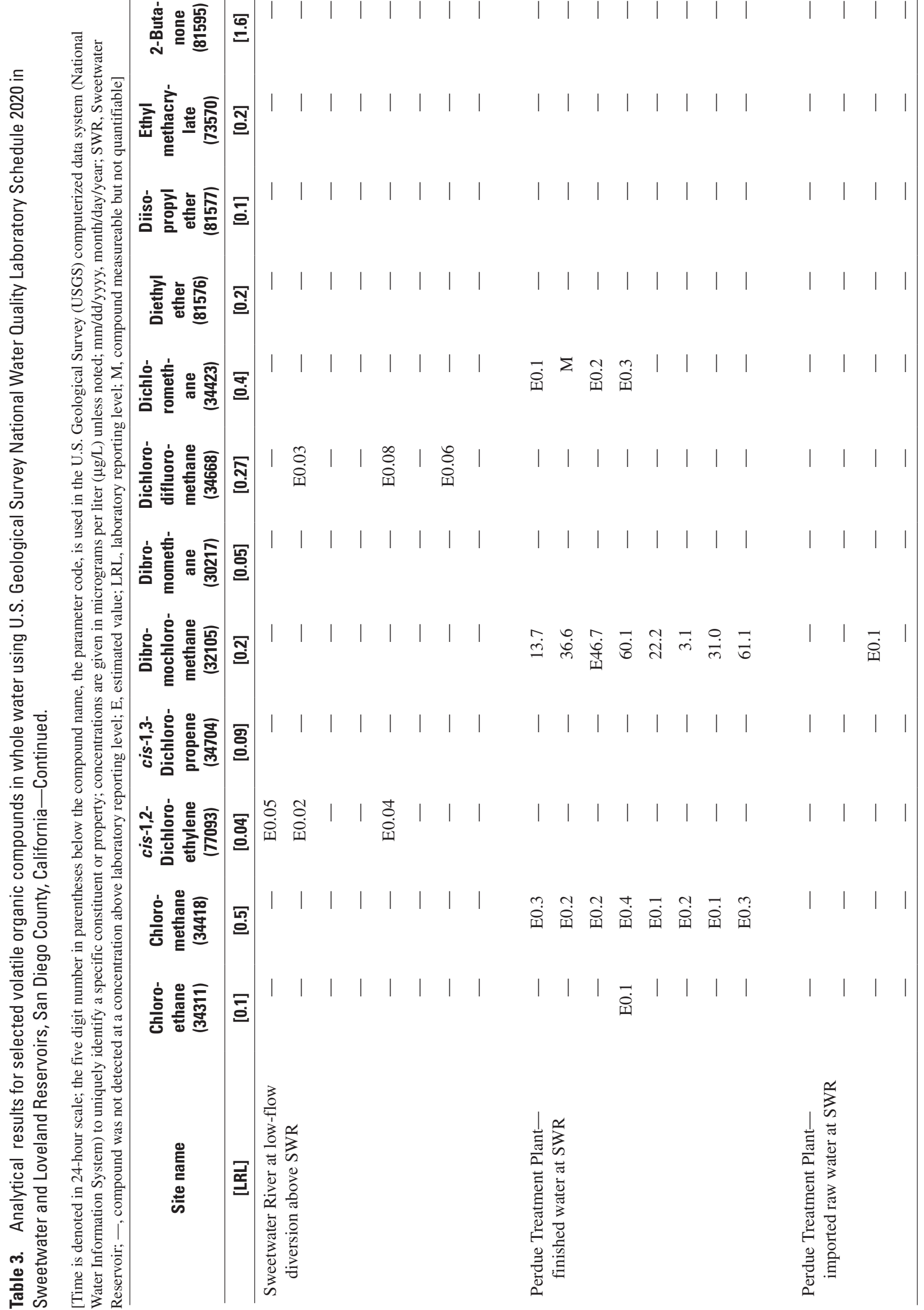




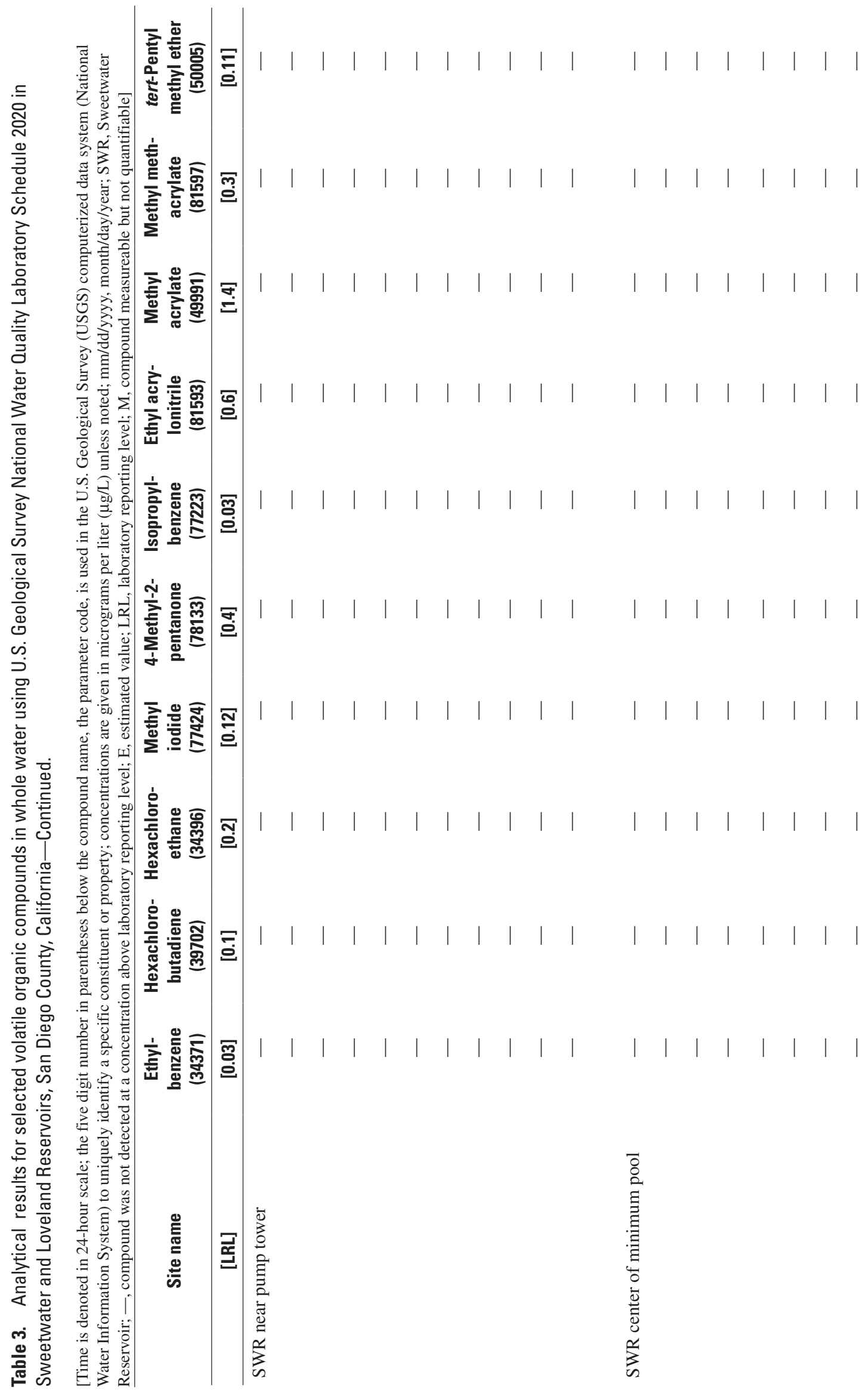




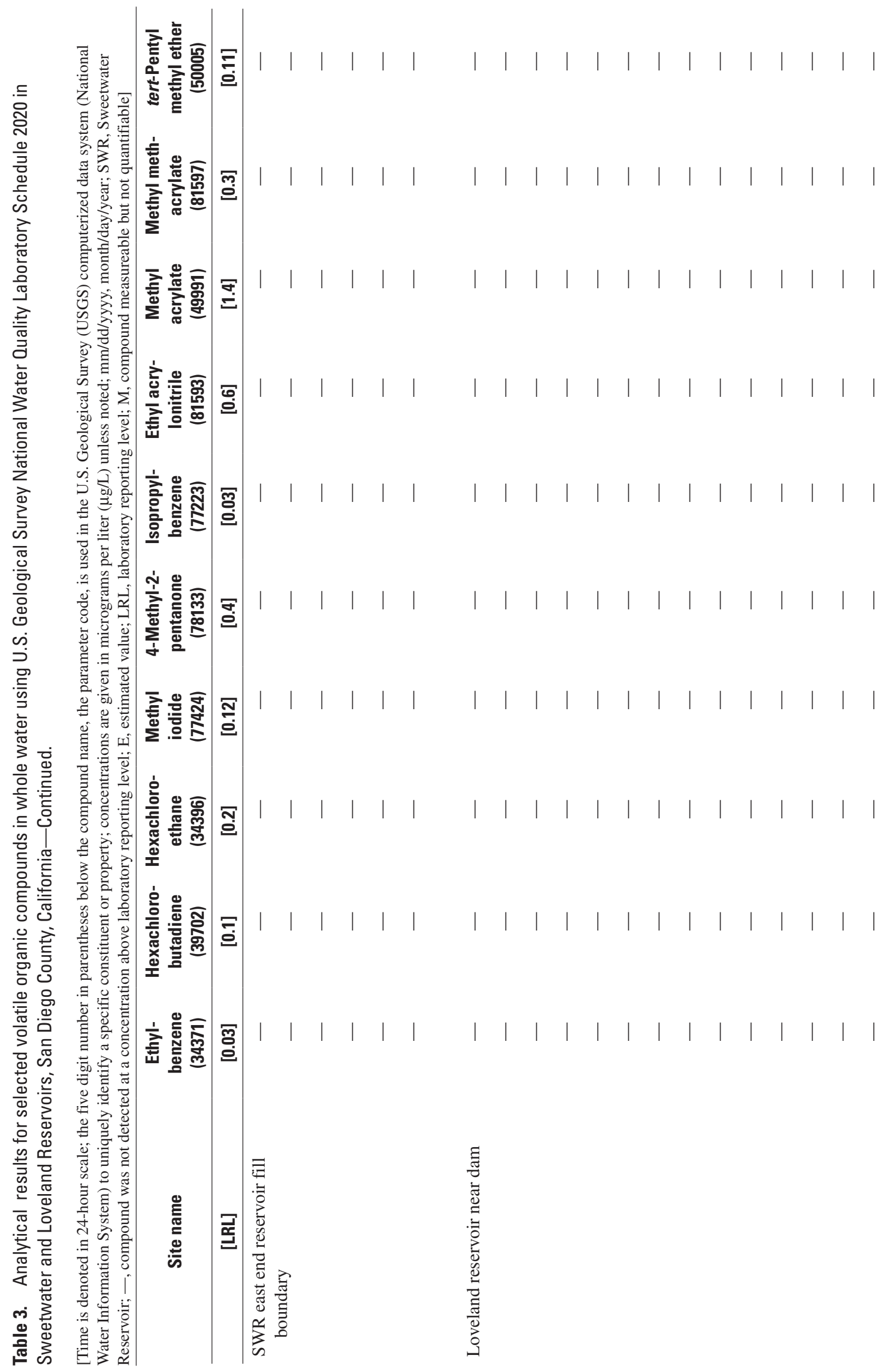




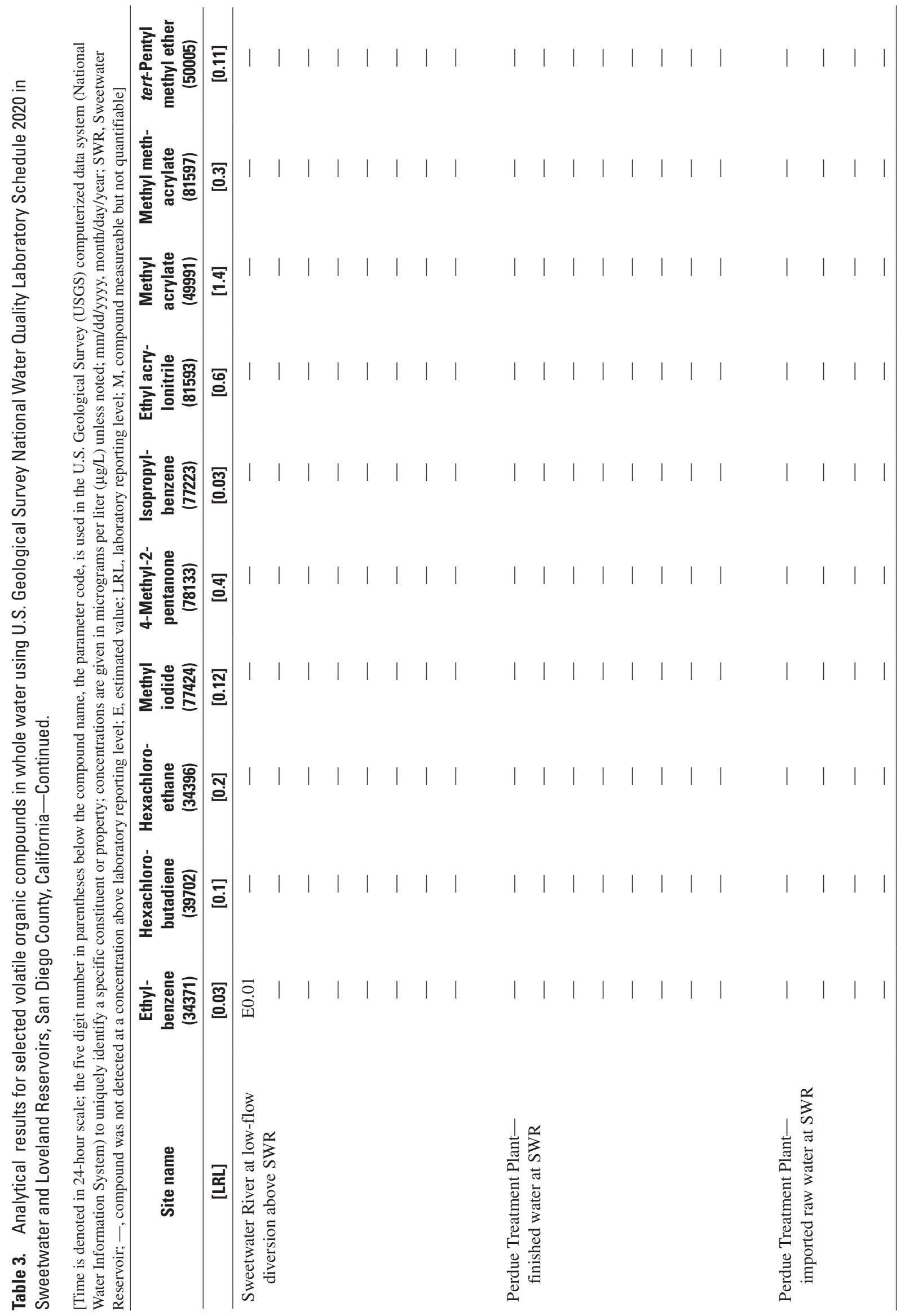




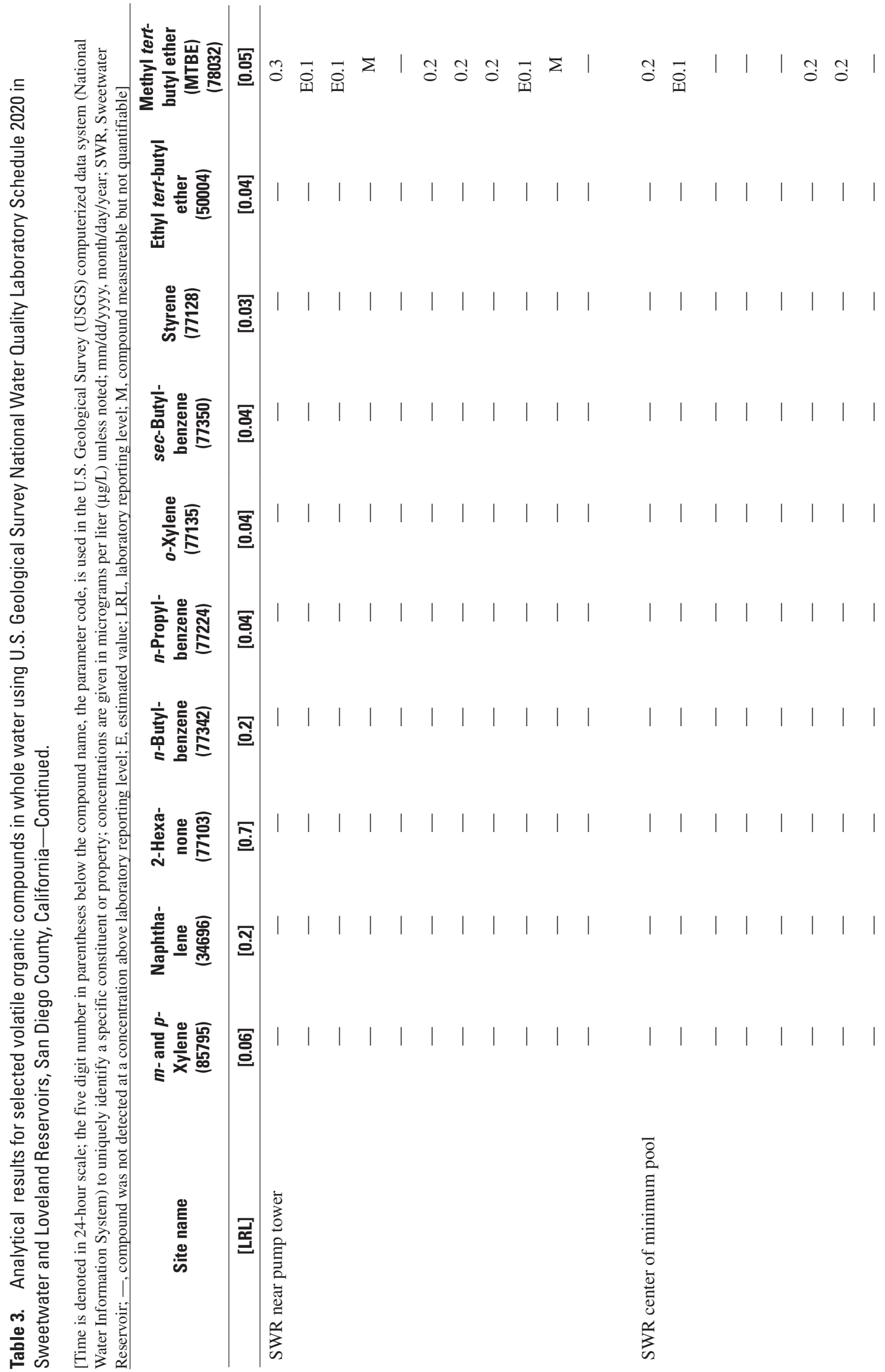




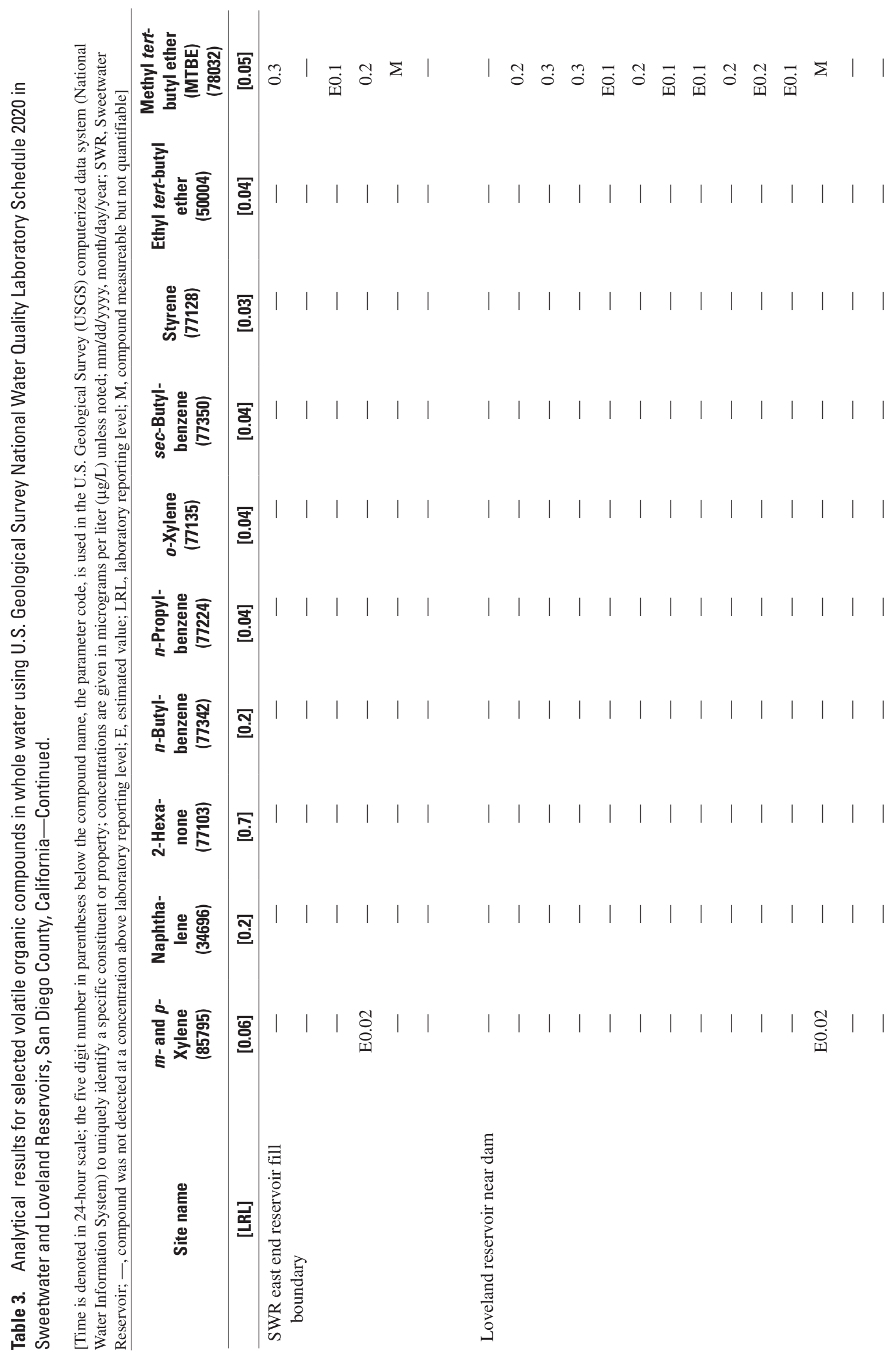




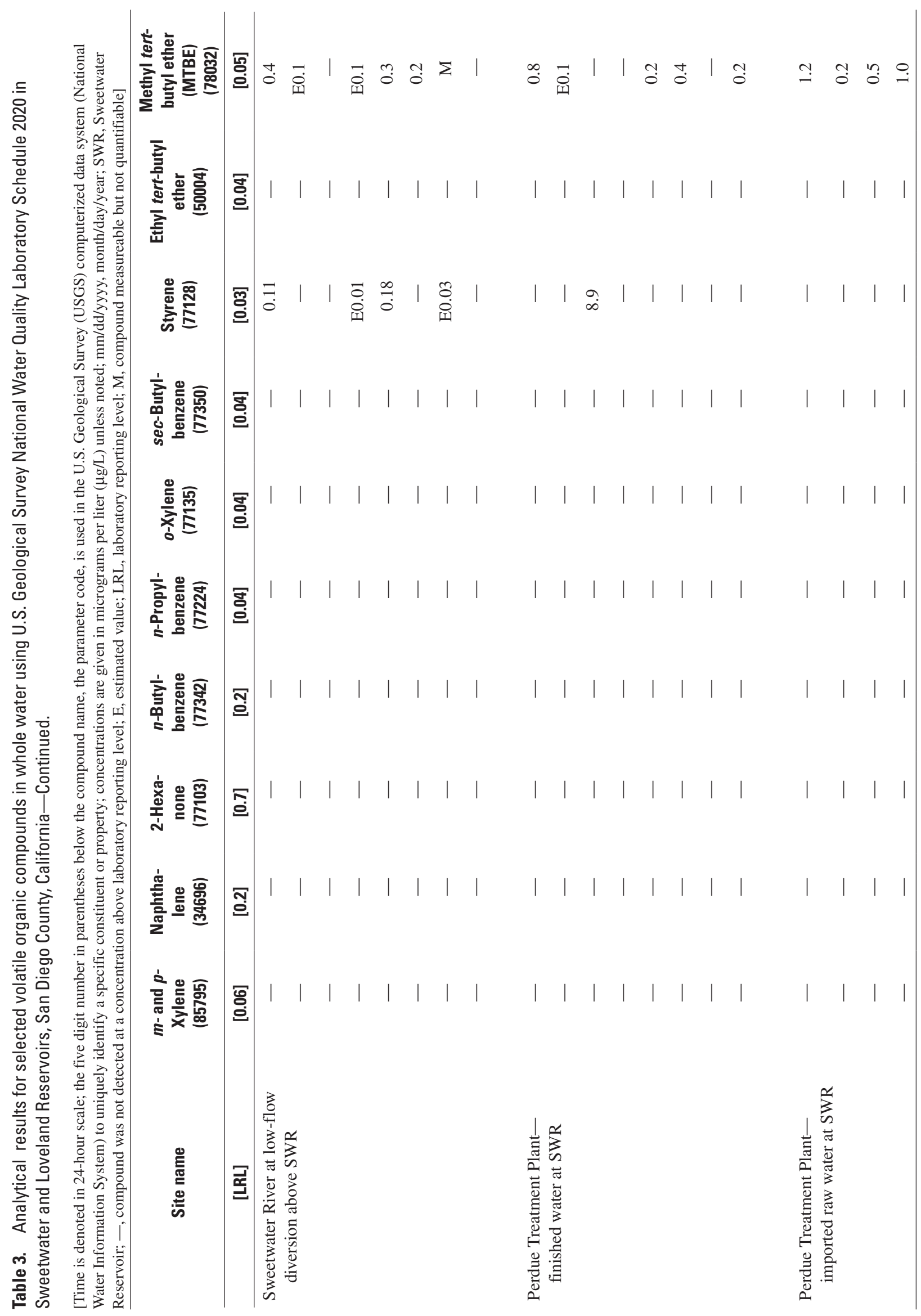




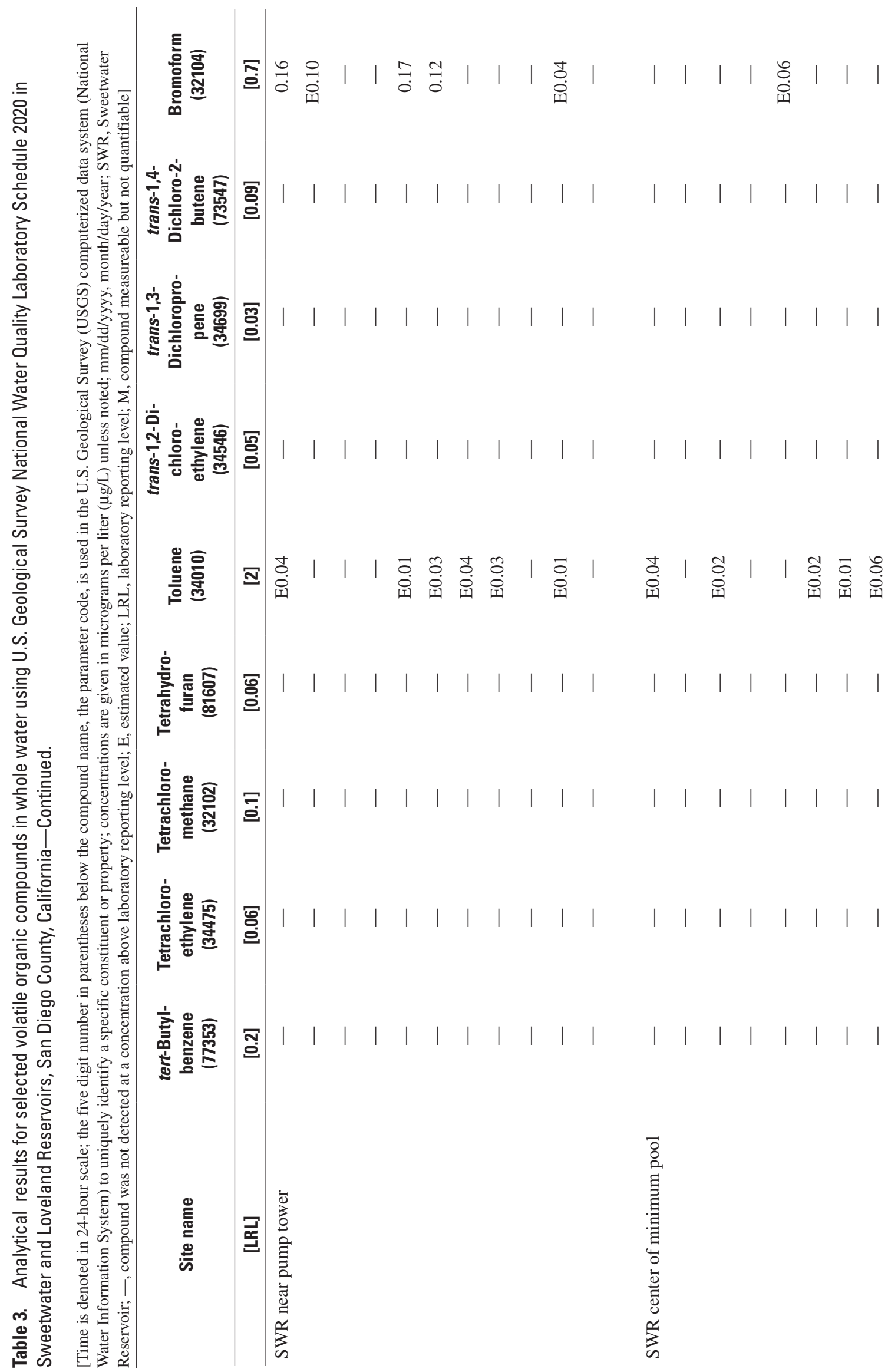




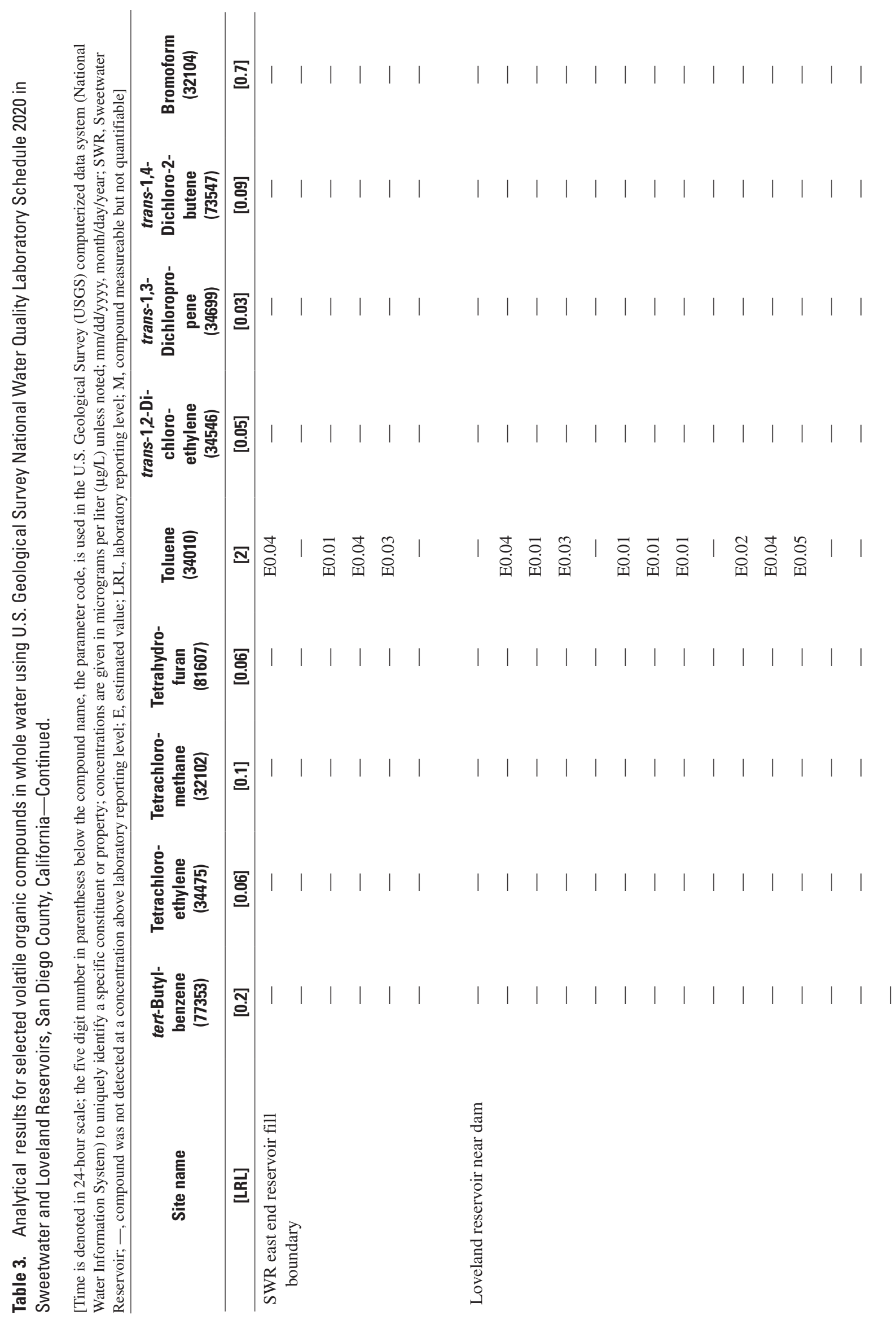




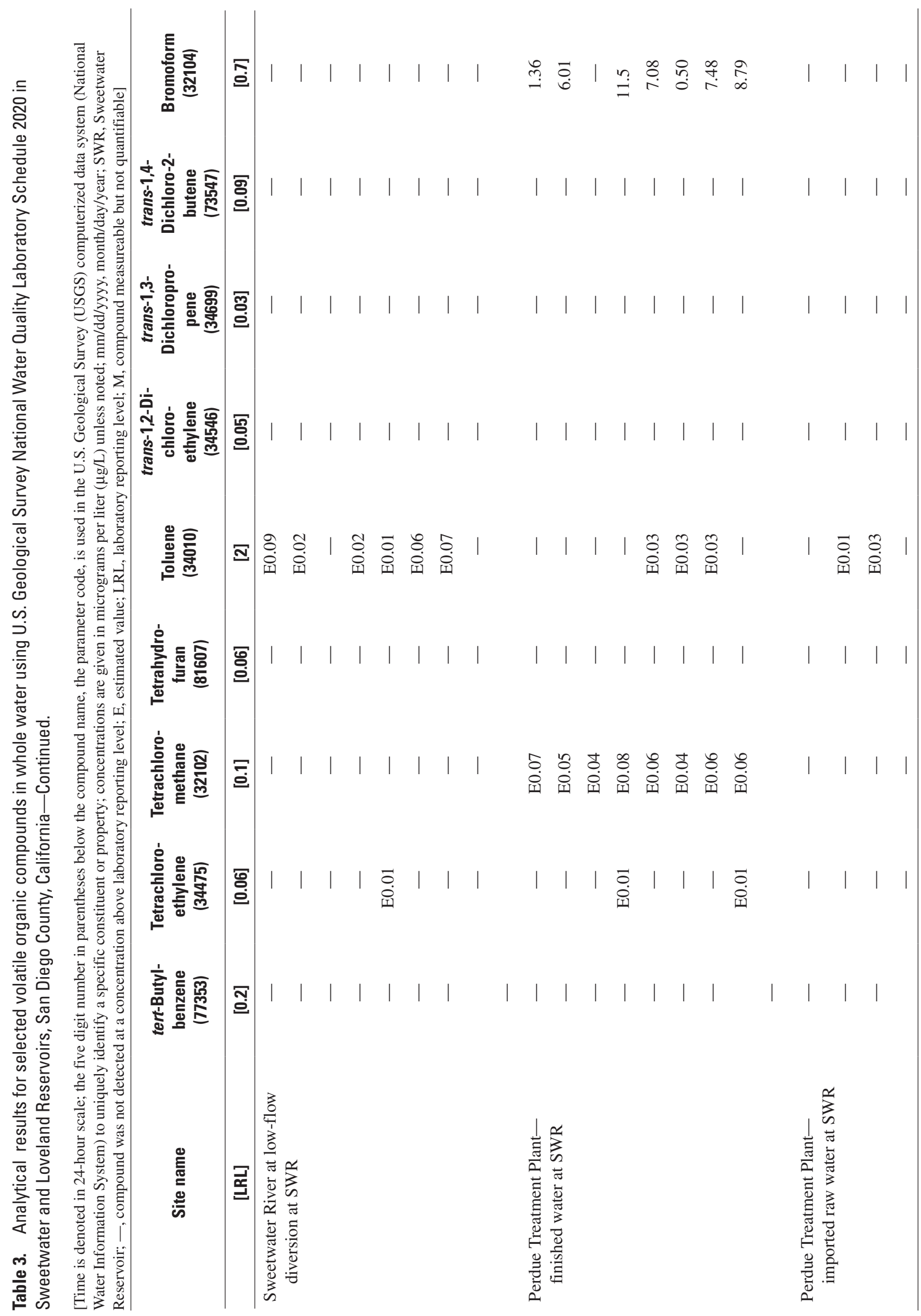




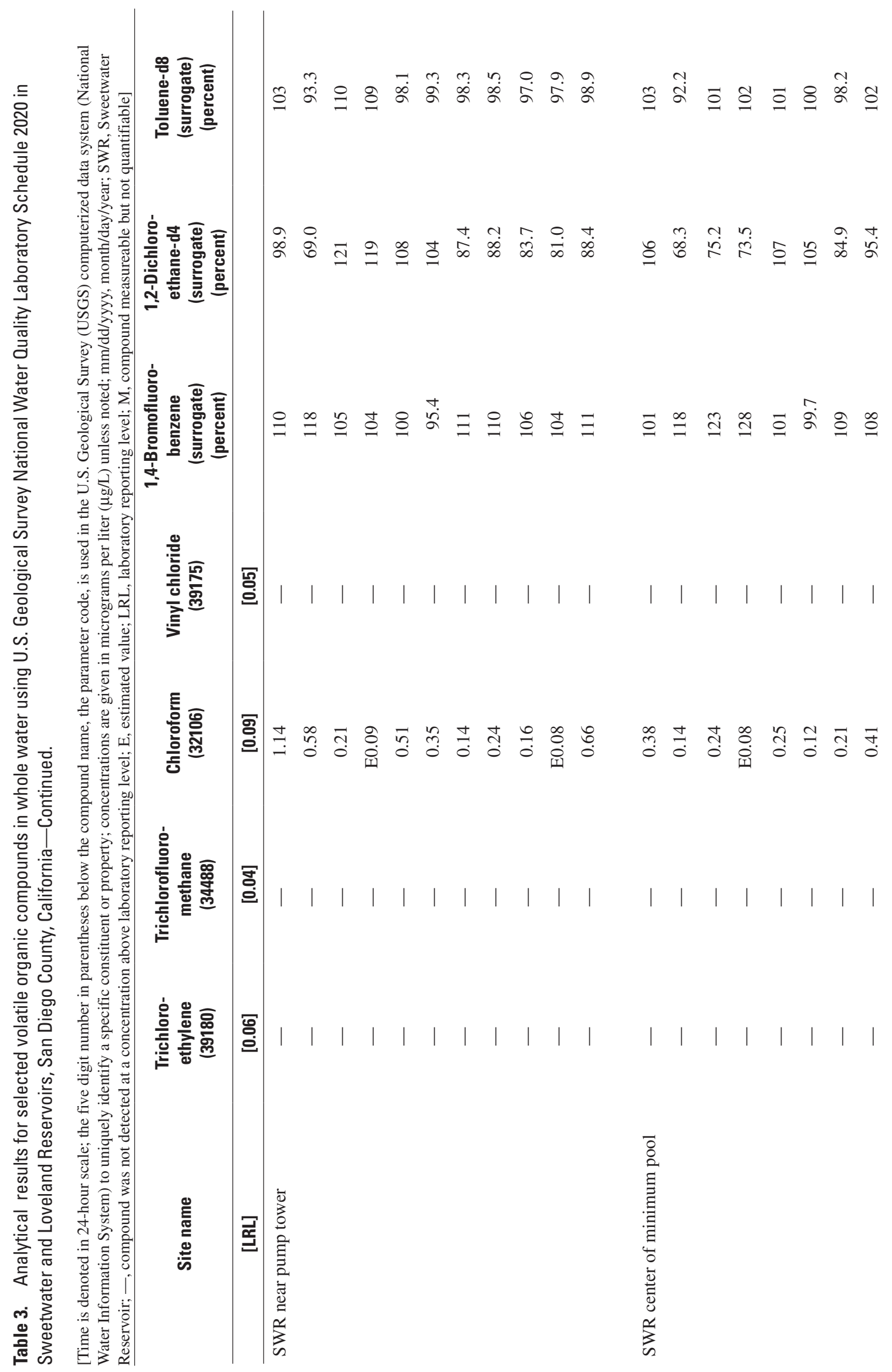




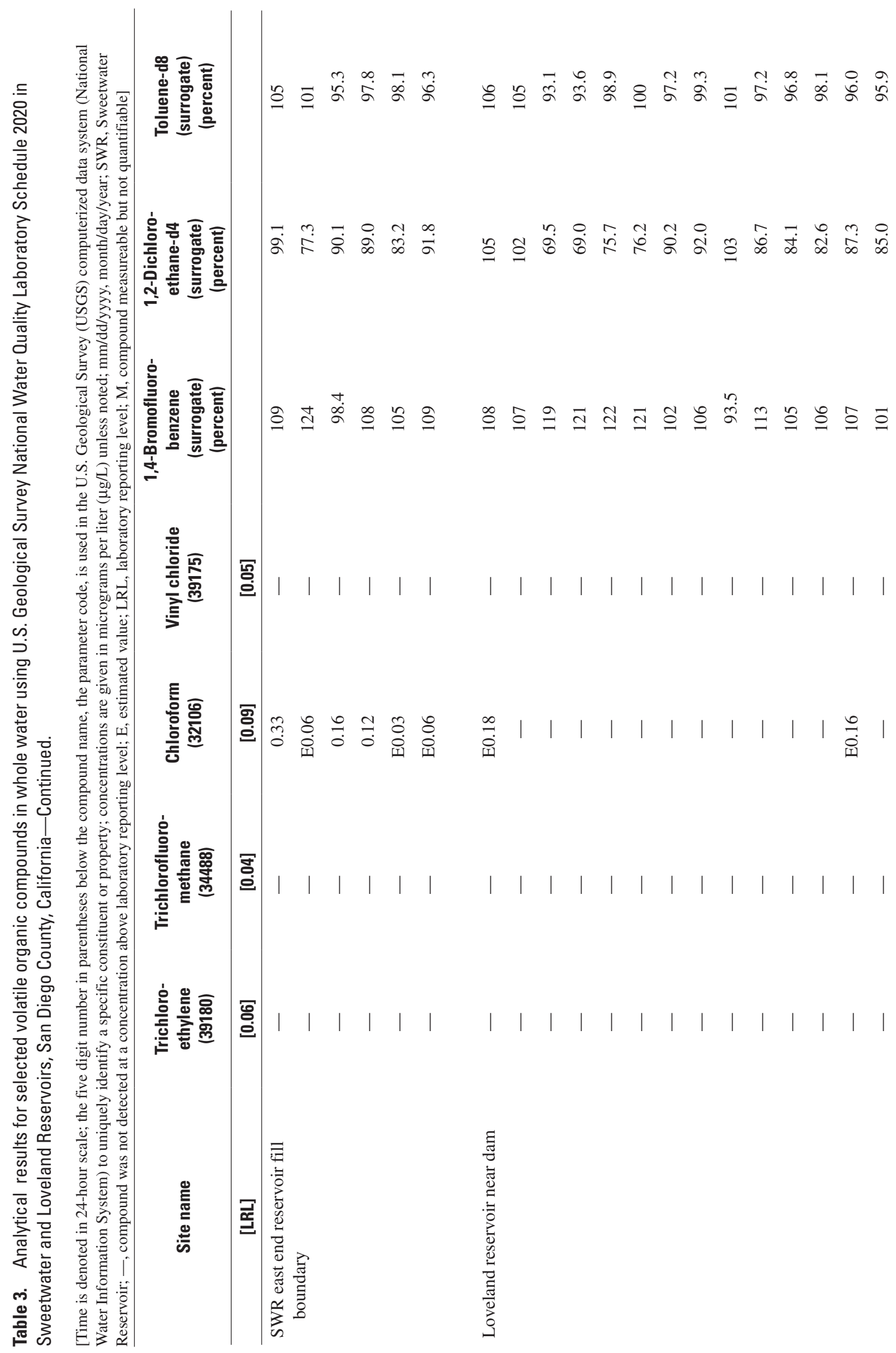




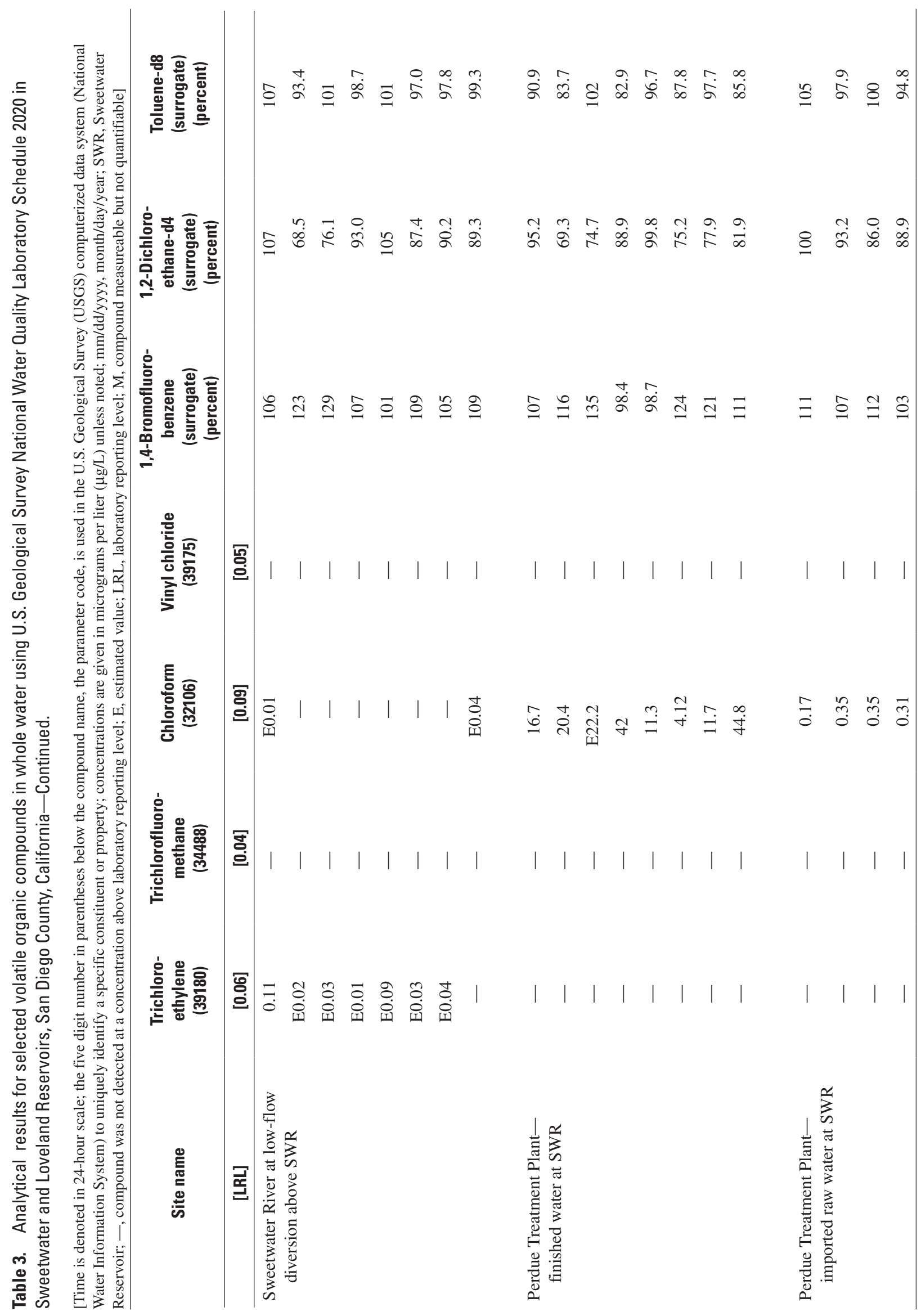




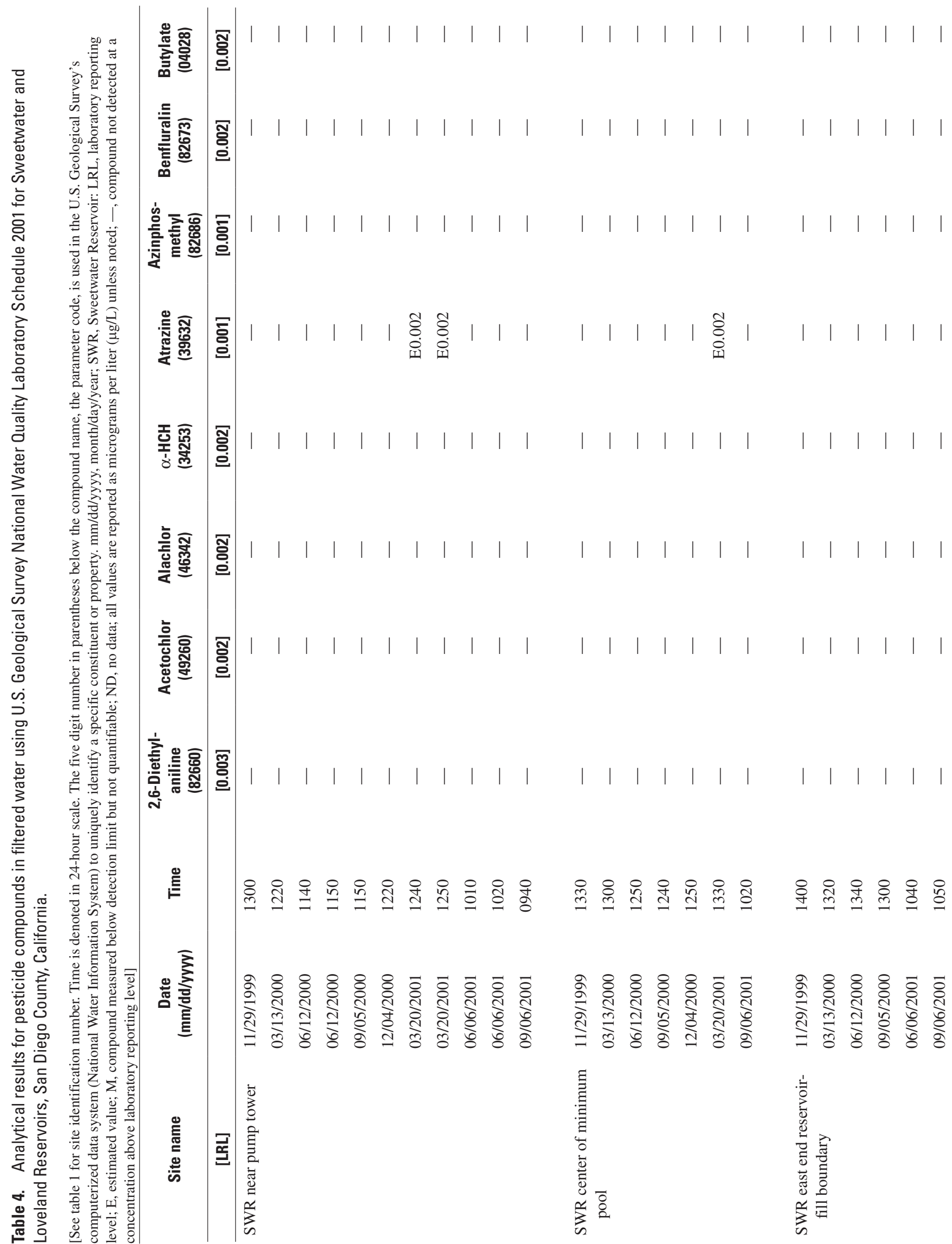




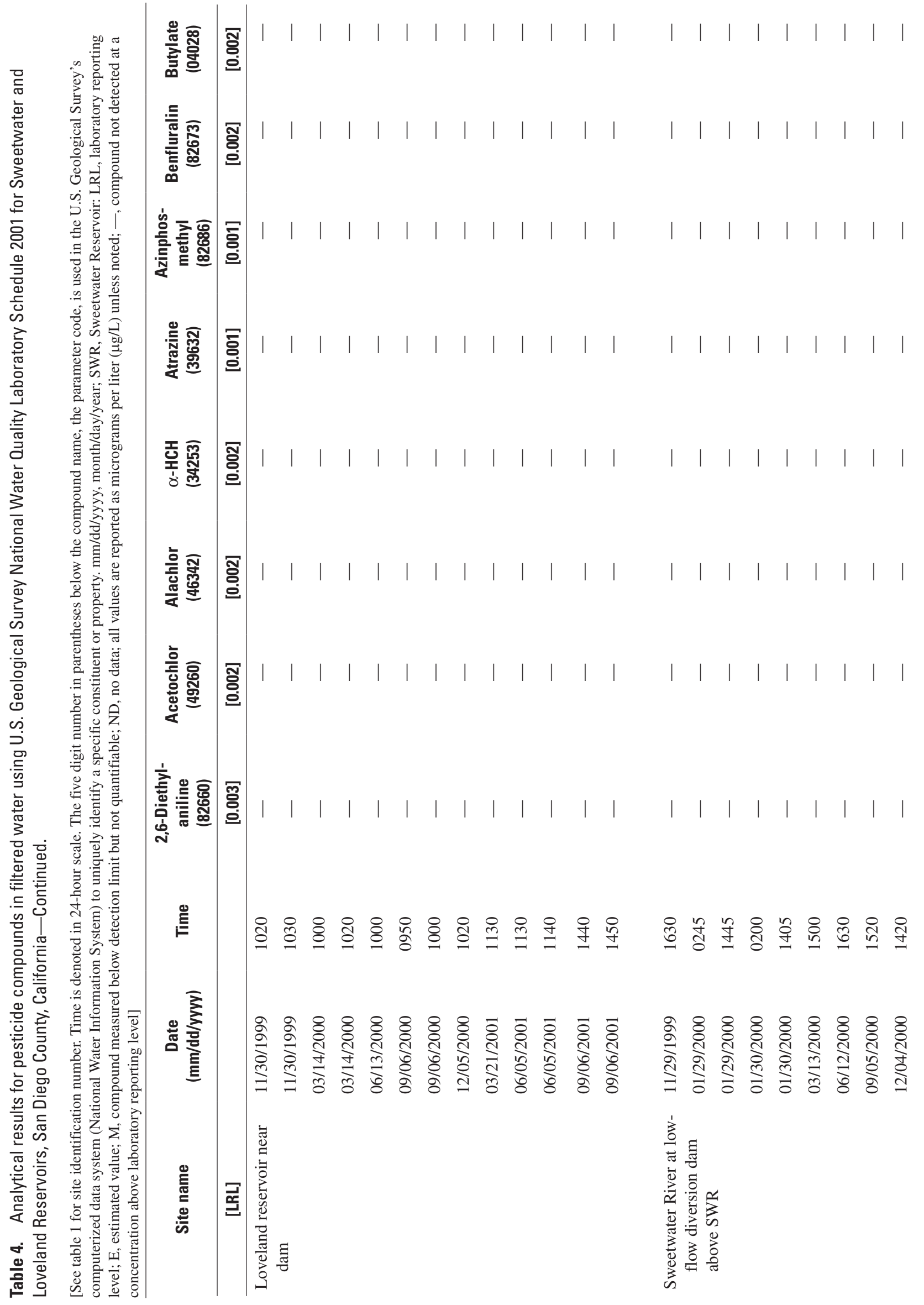




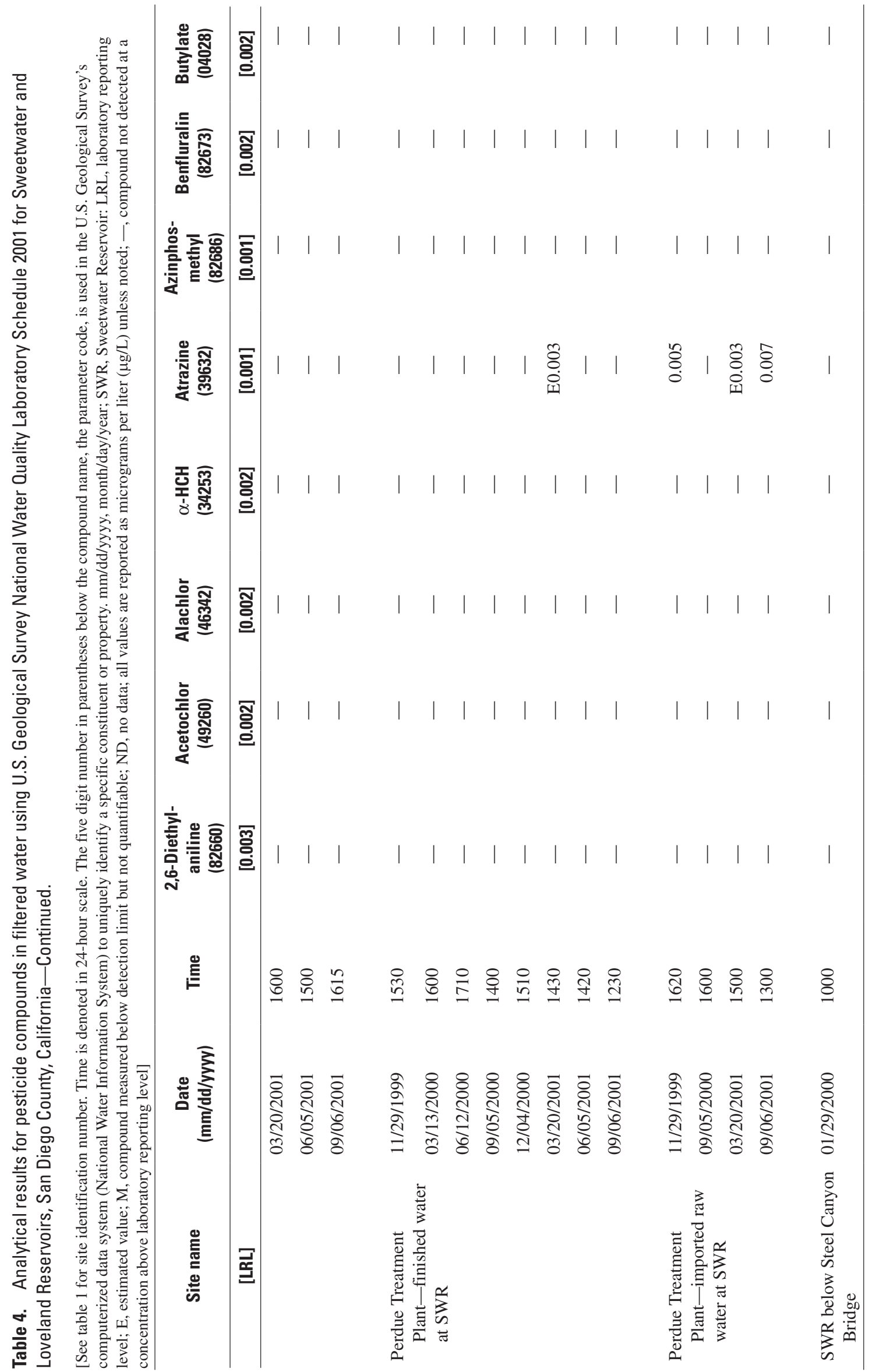




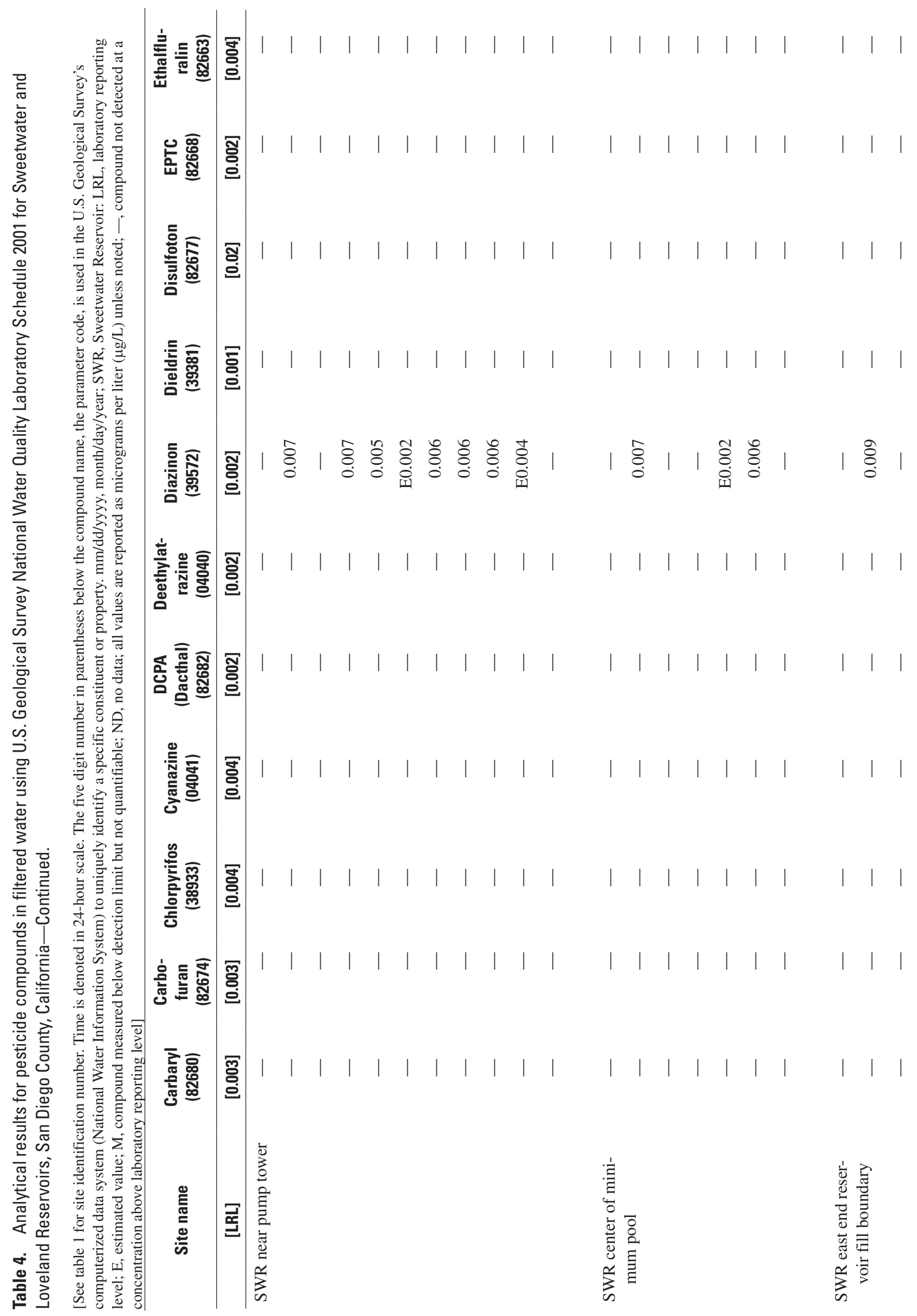




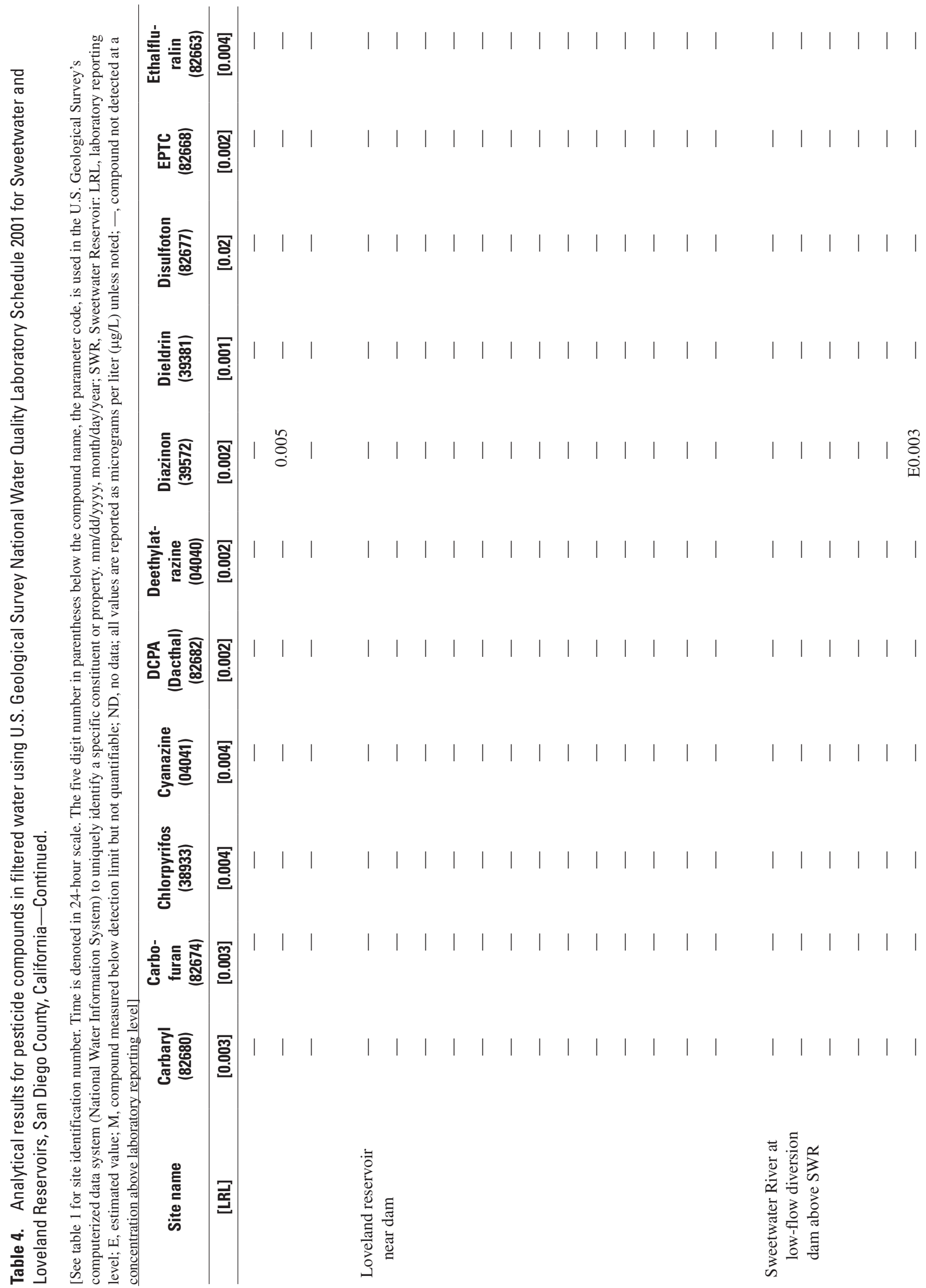


Table $4 \quad 57$

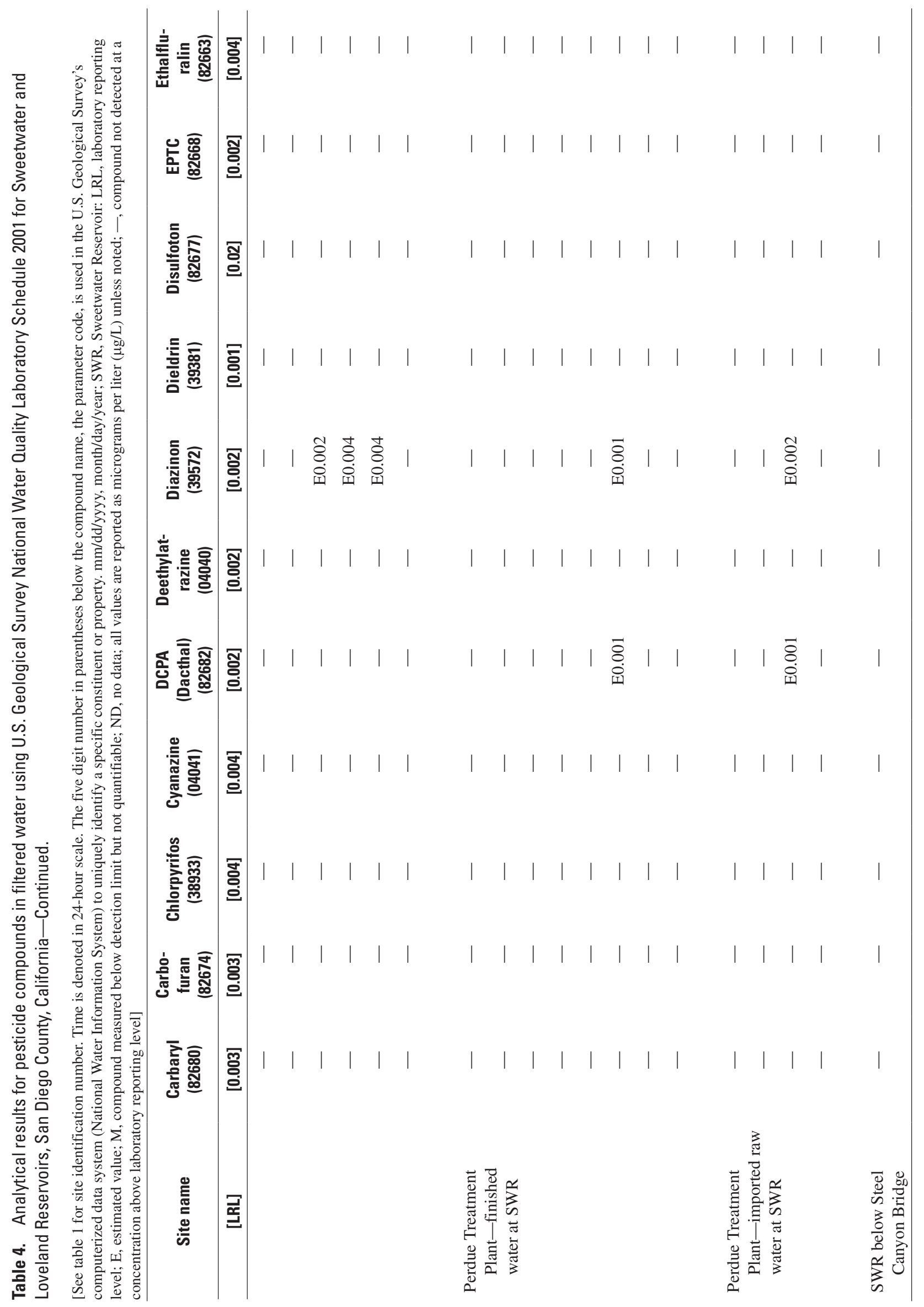




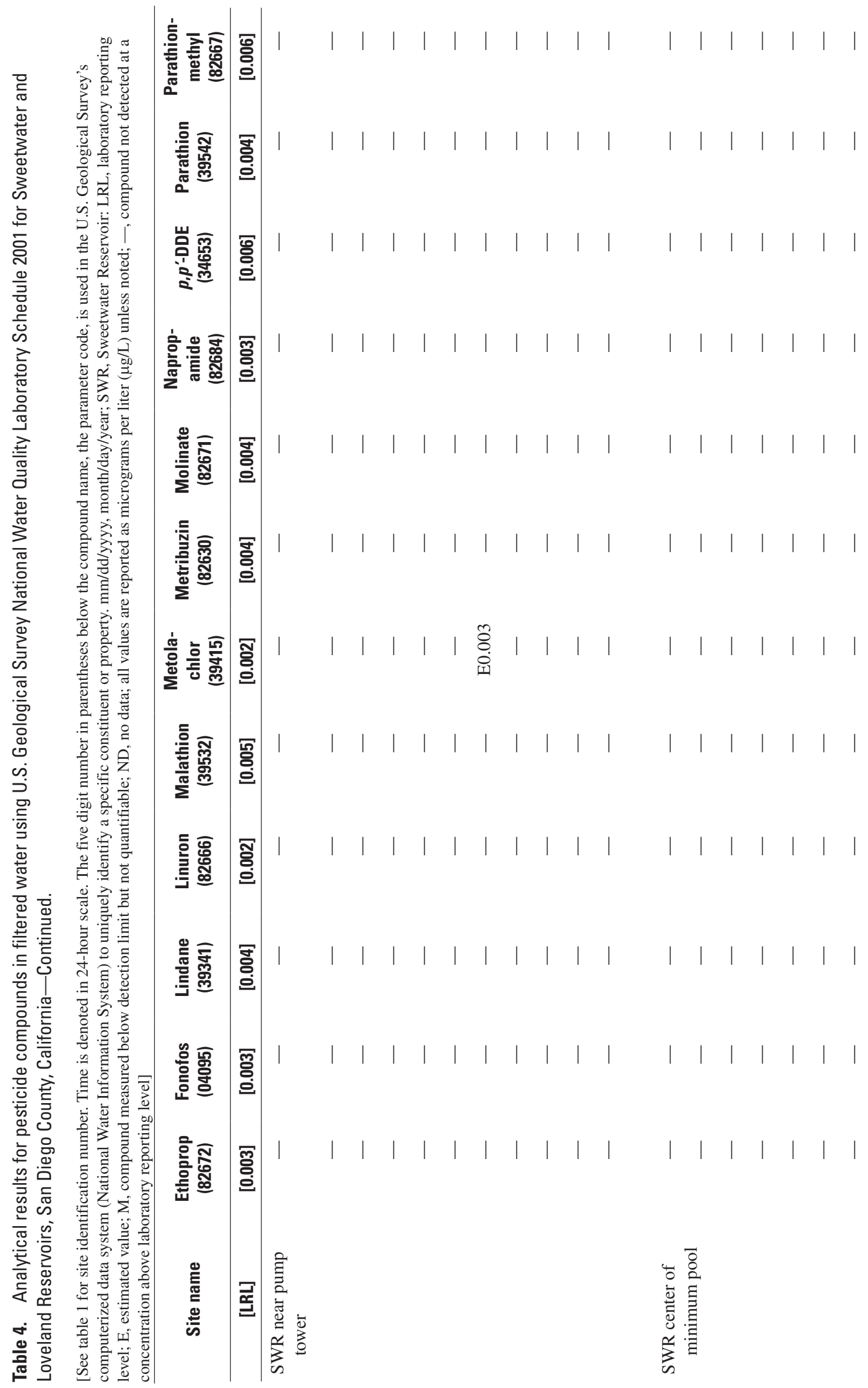




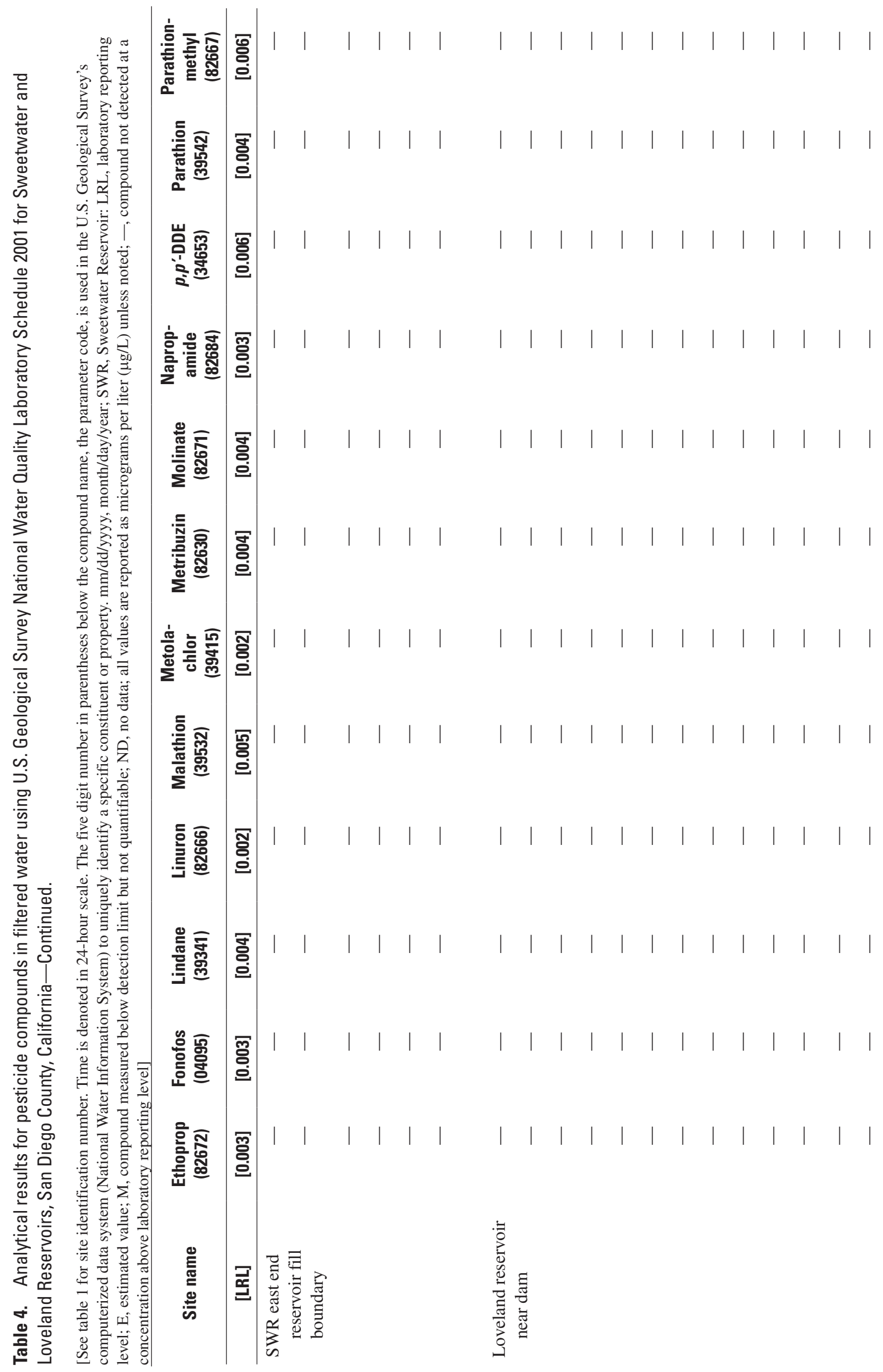




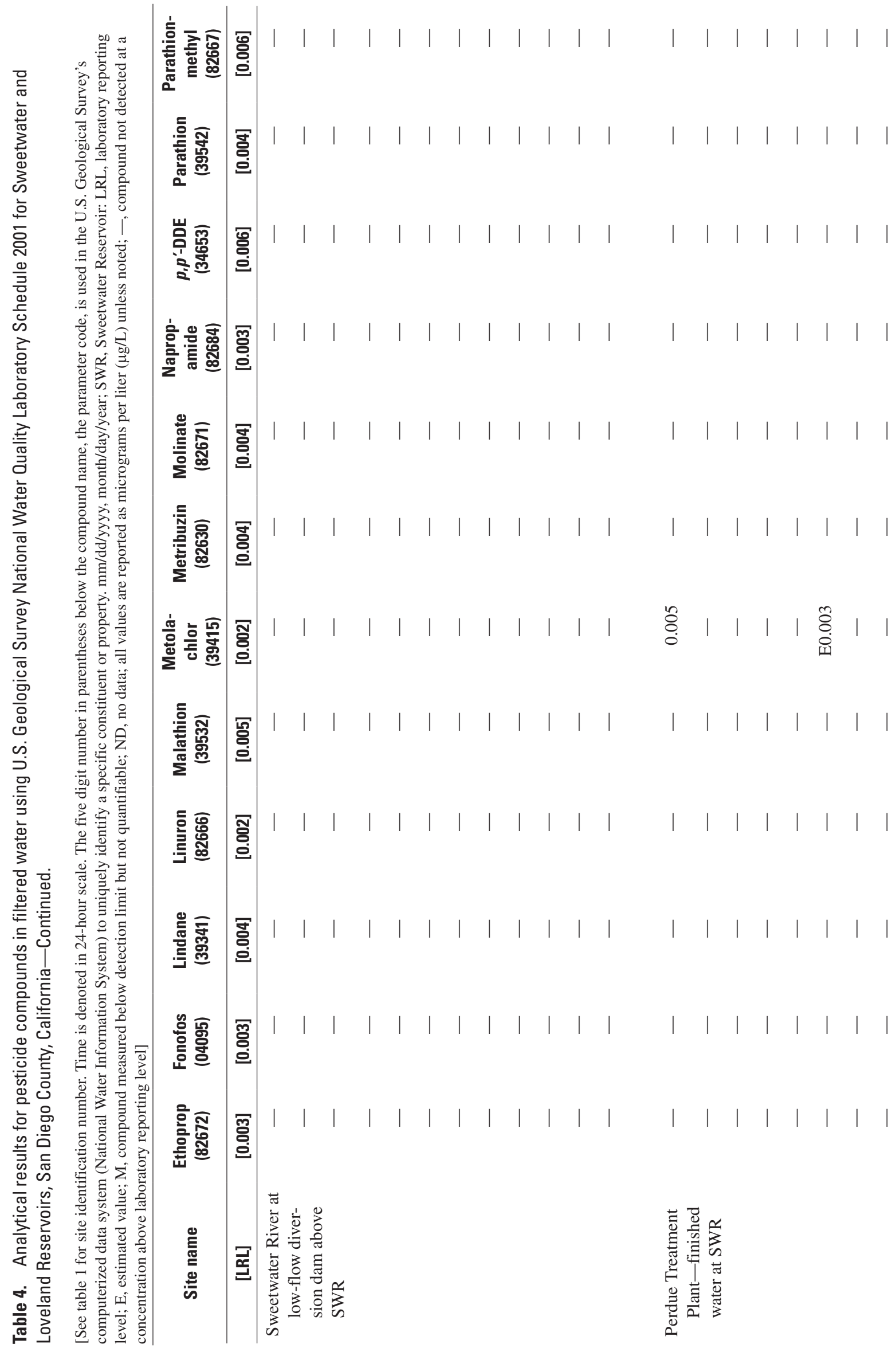


Table $4 \quad 61$

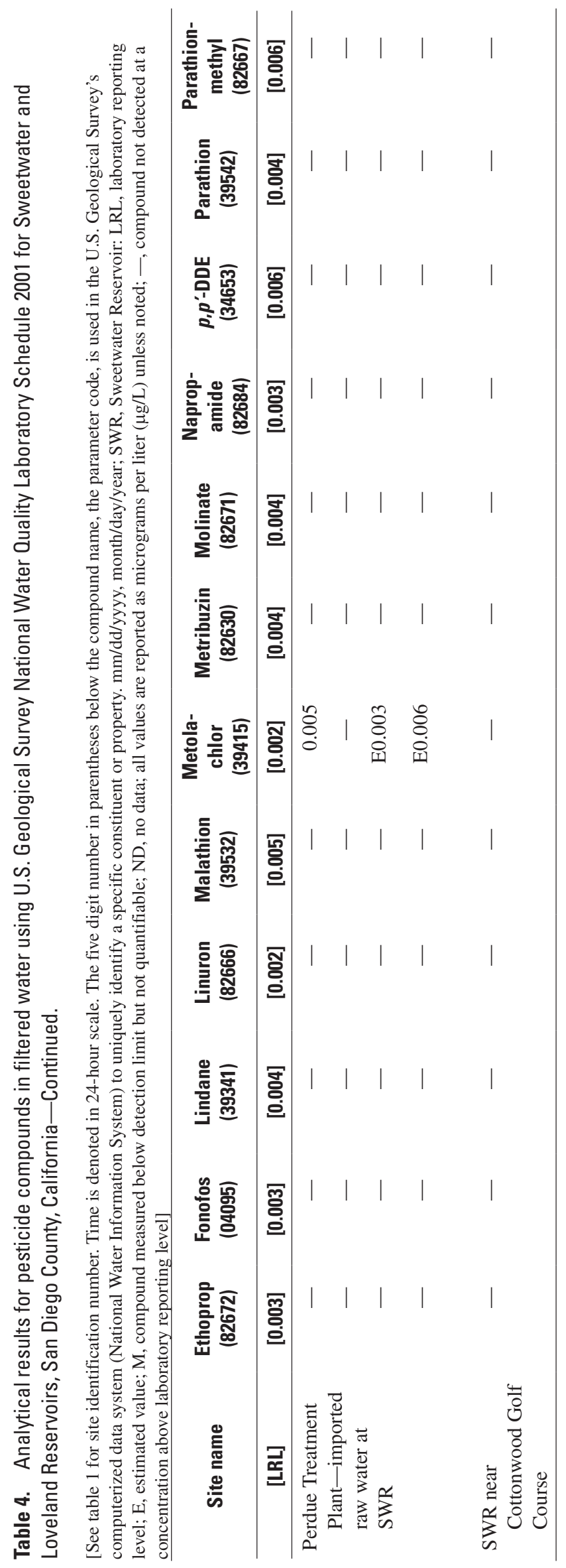




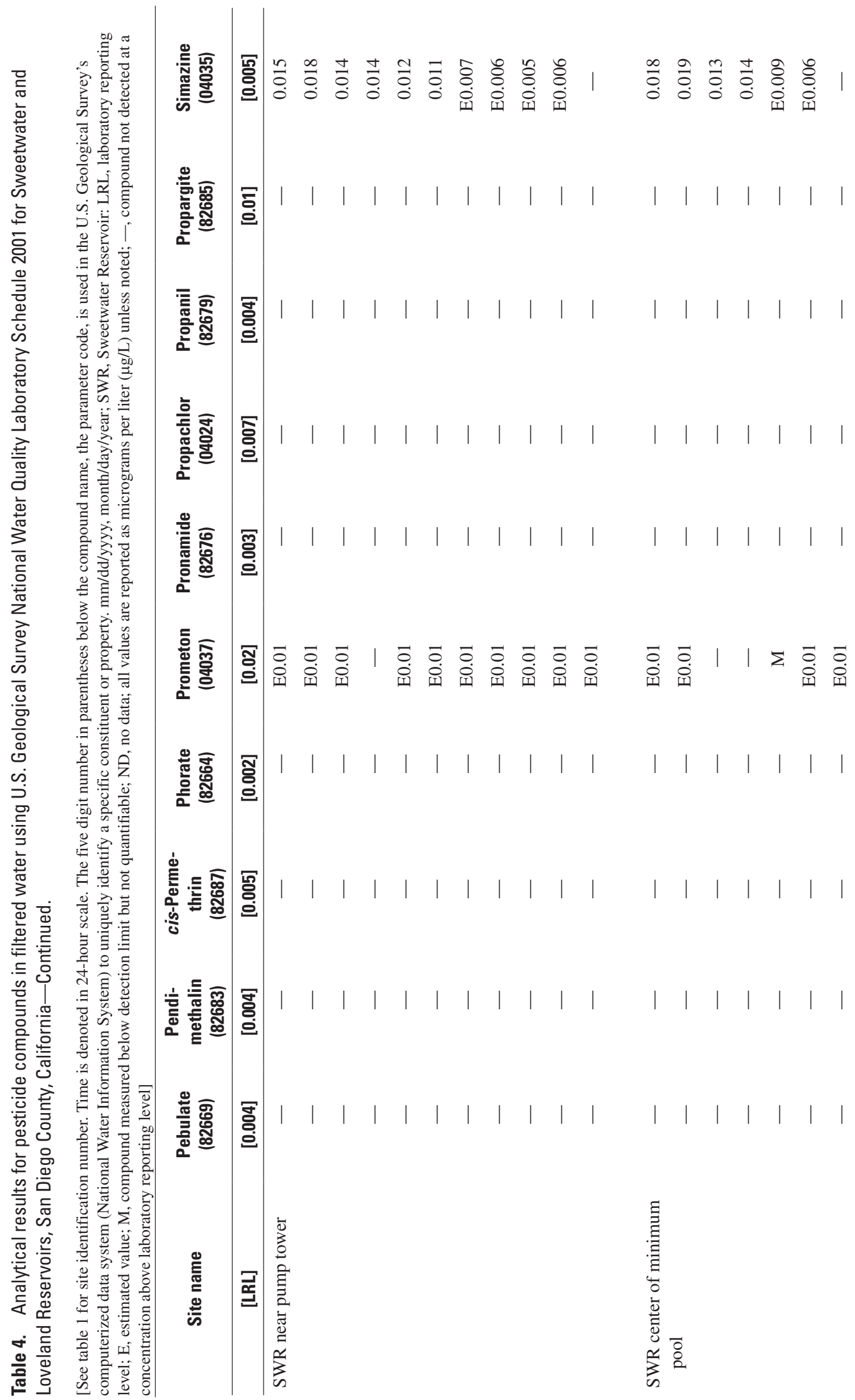


Table $4 \quad 63$

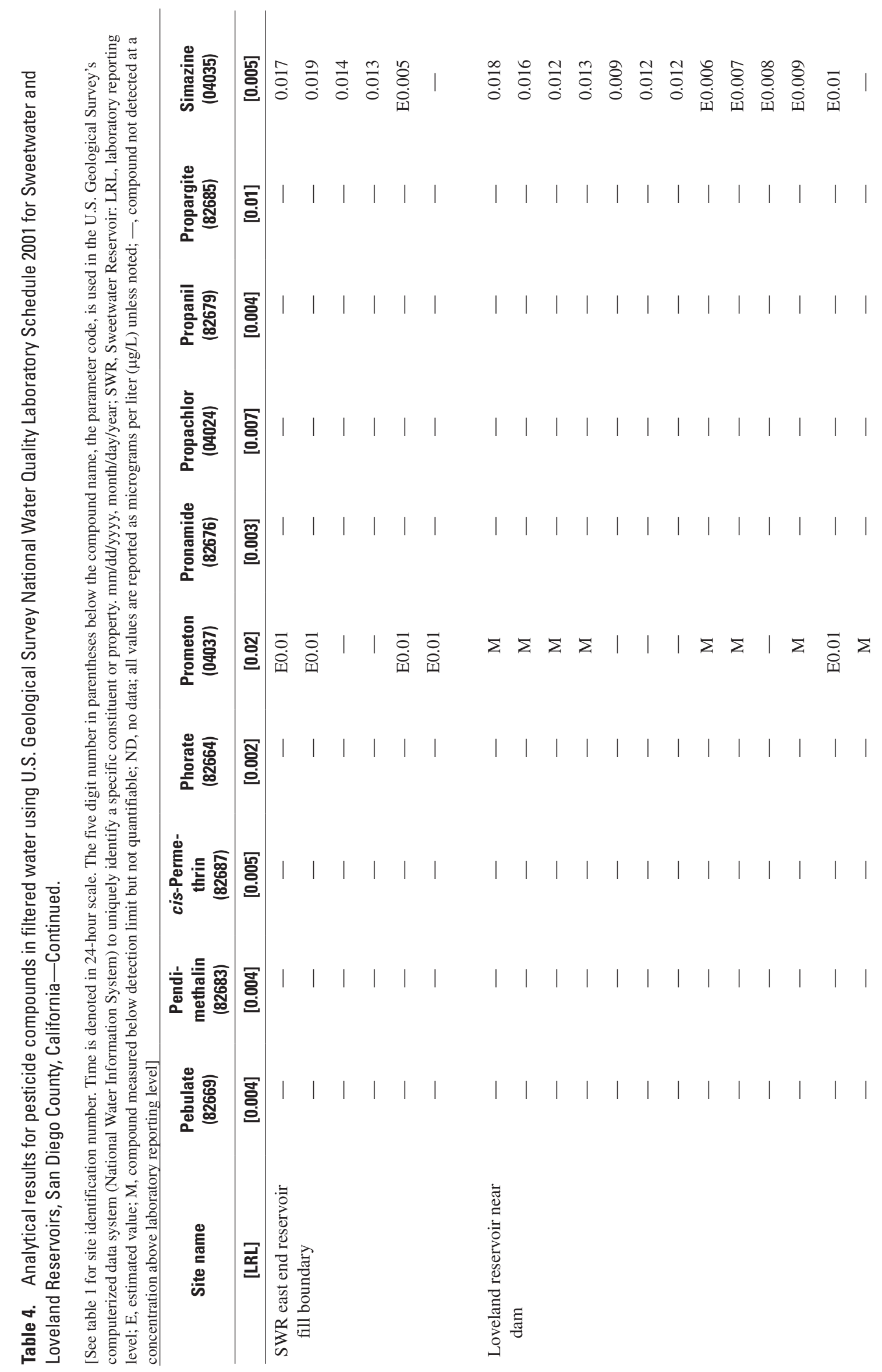




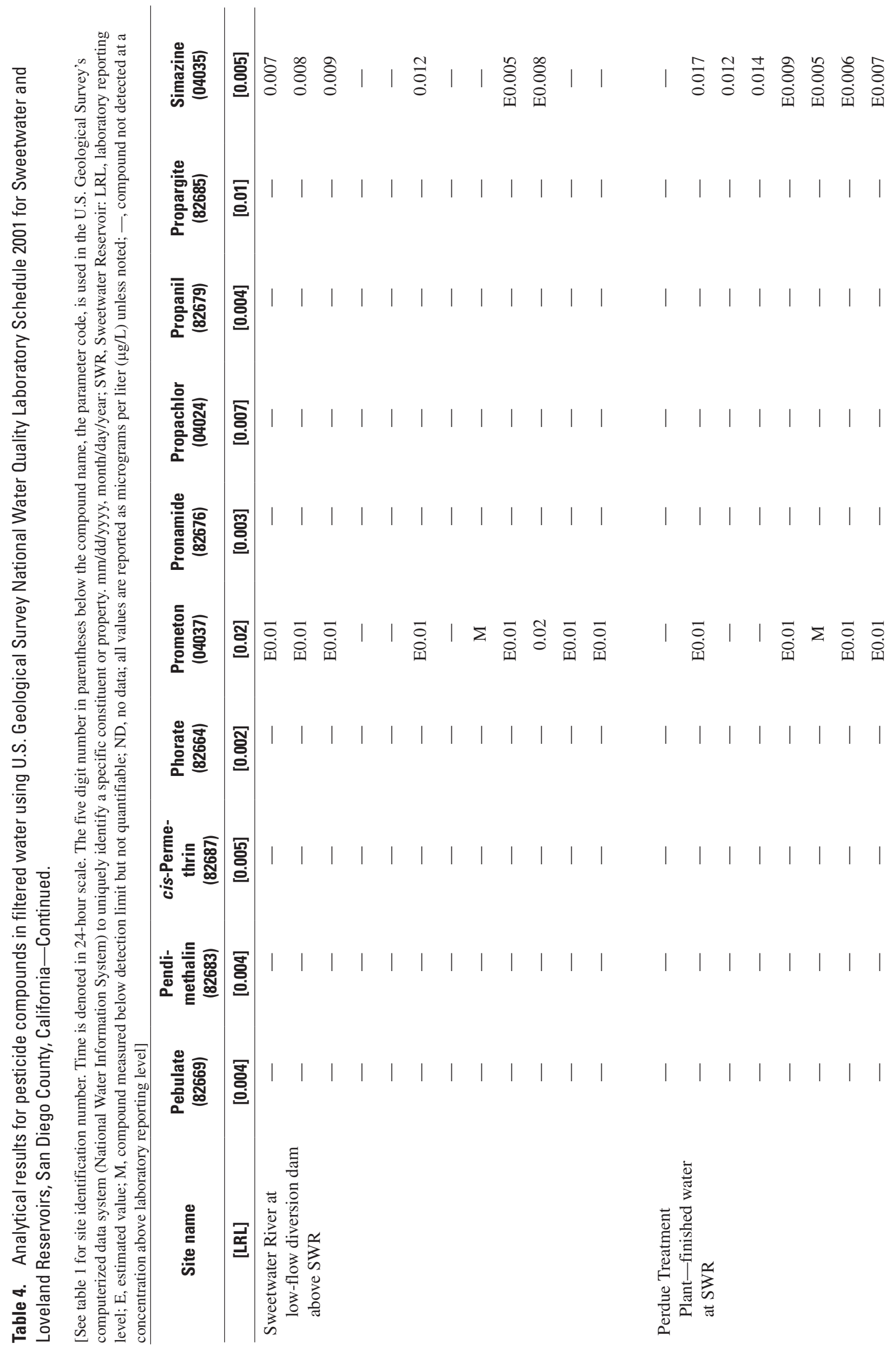


Table $4 \quad 65$

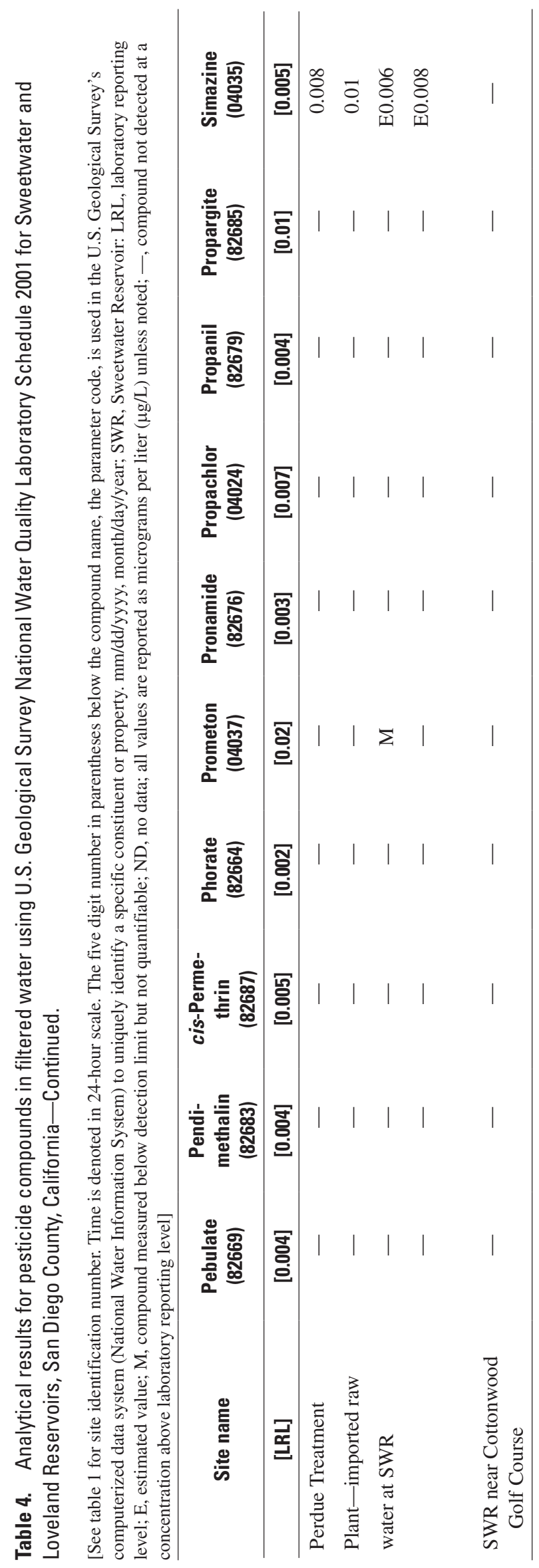




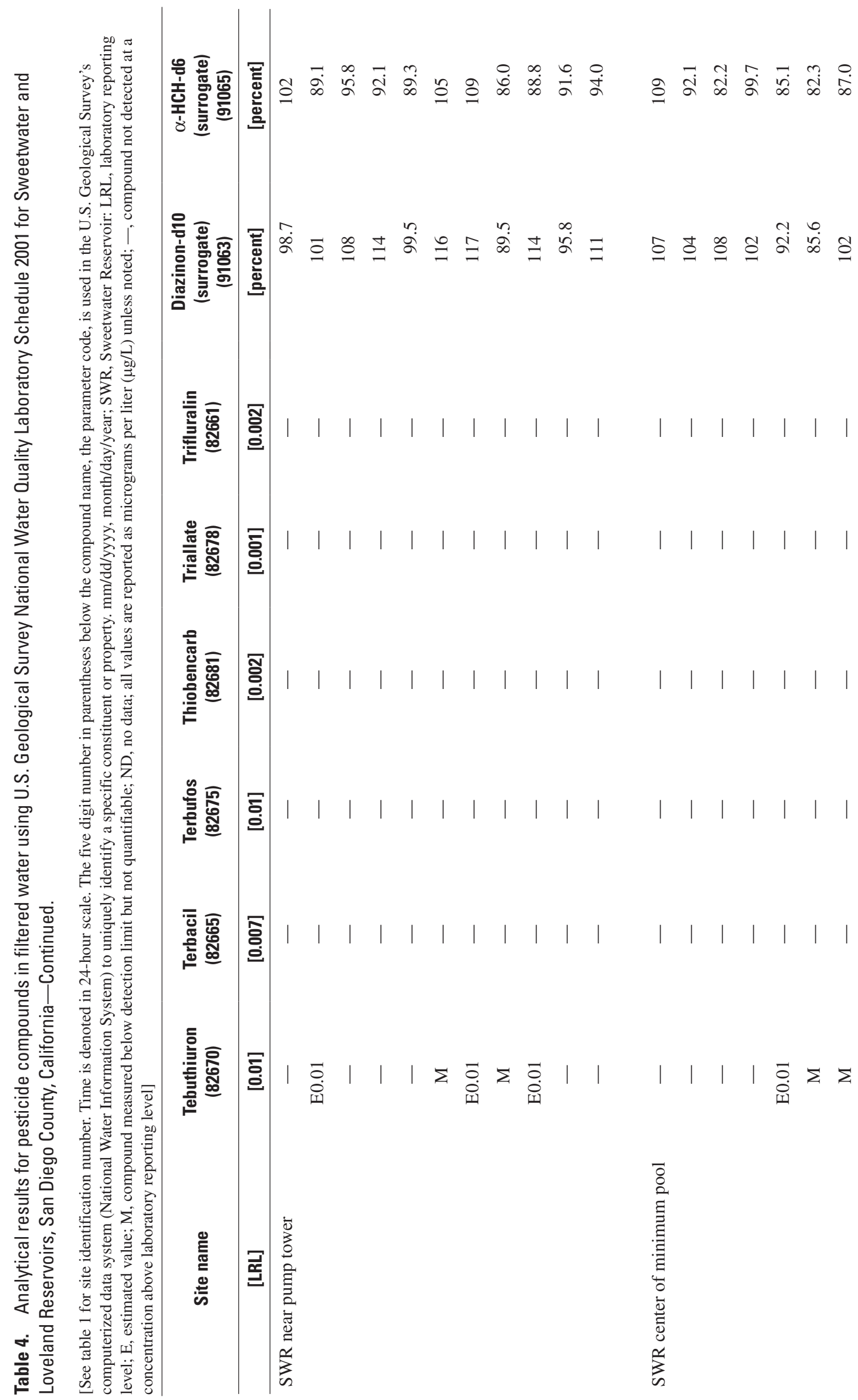


Table $4 \quad 67$

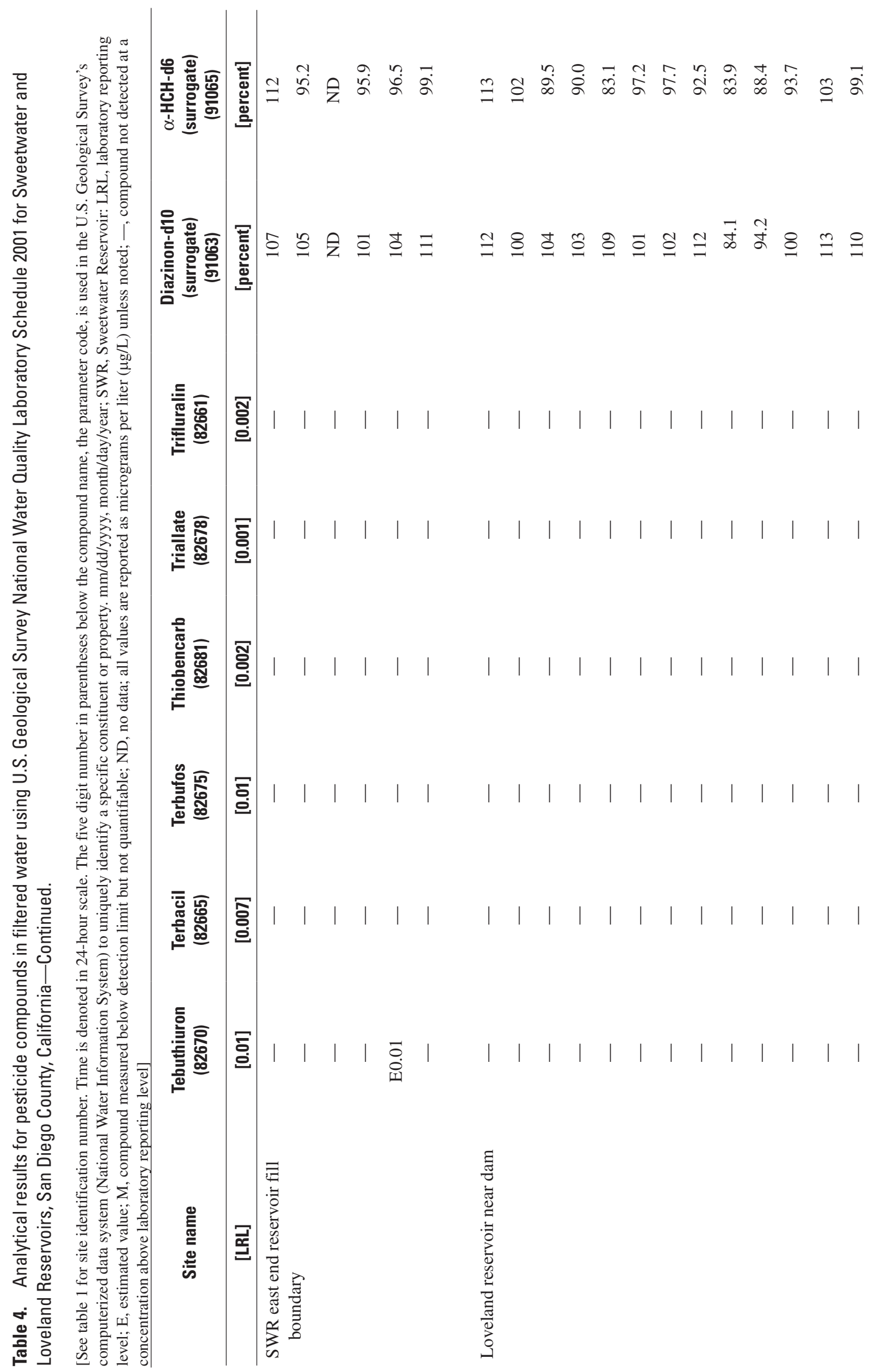




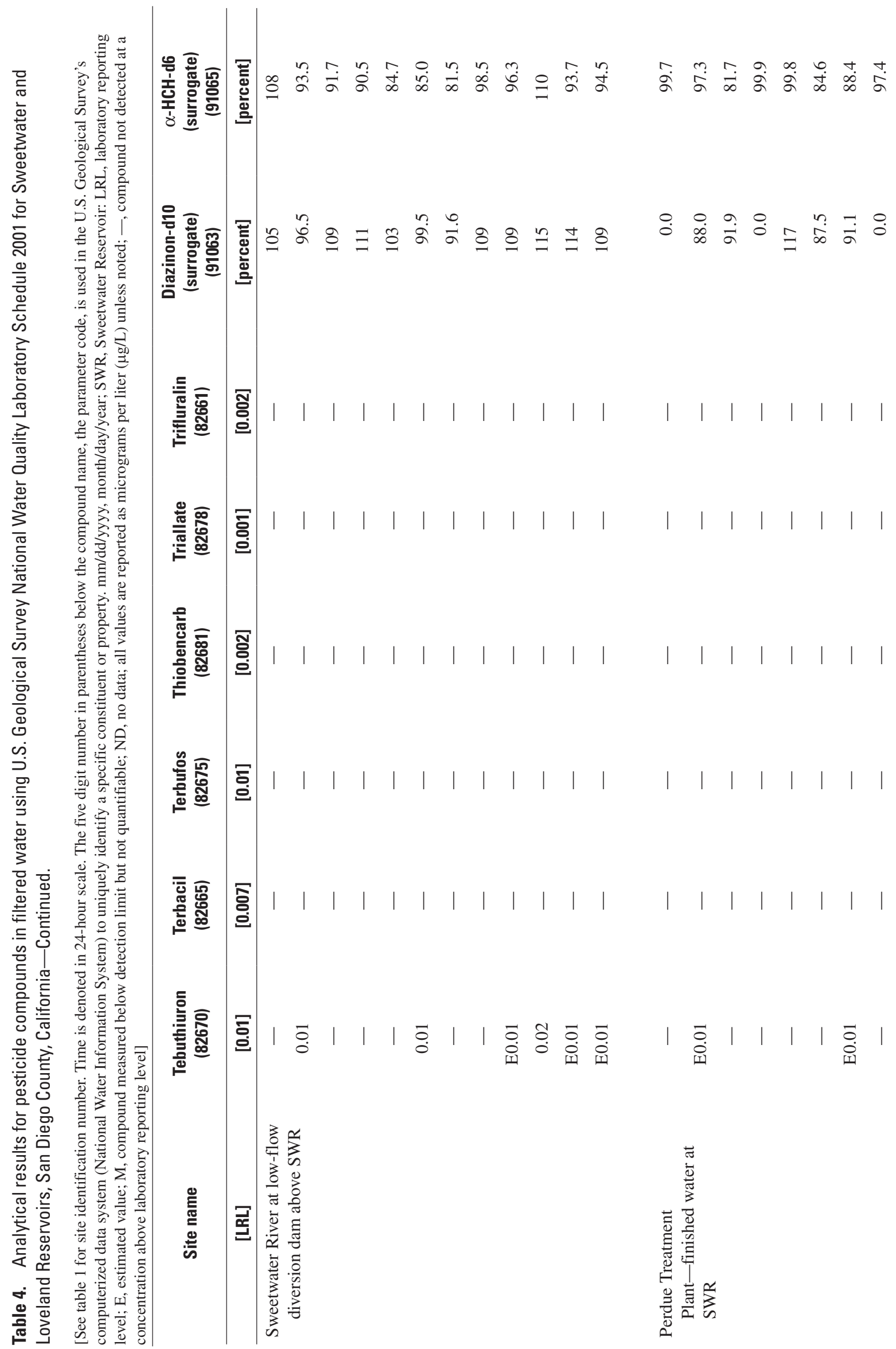




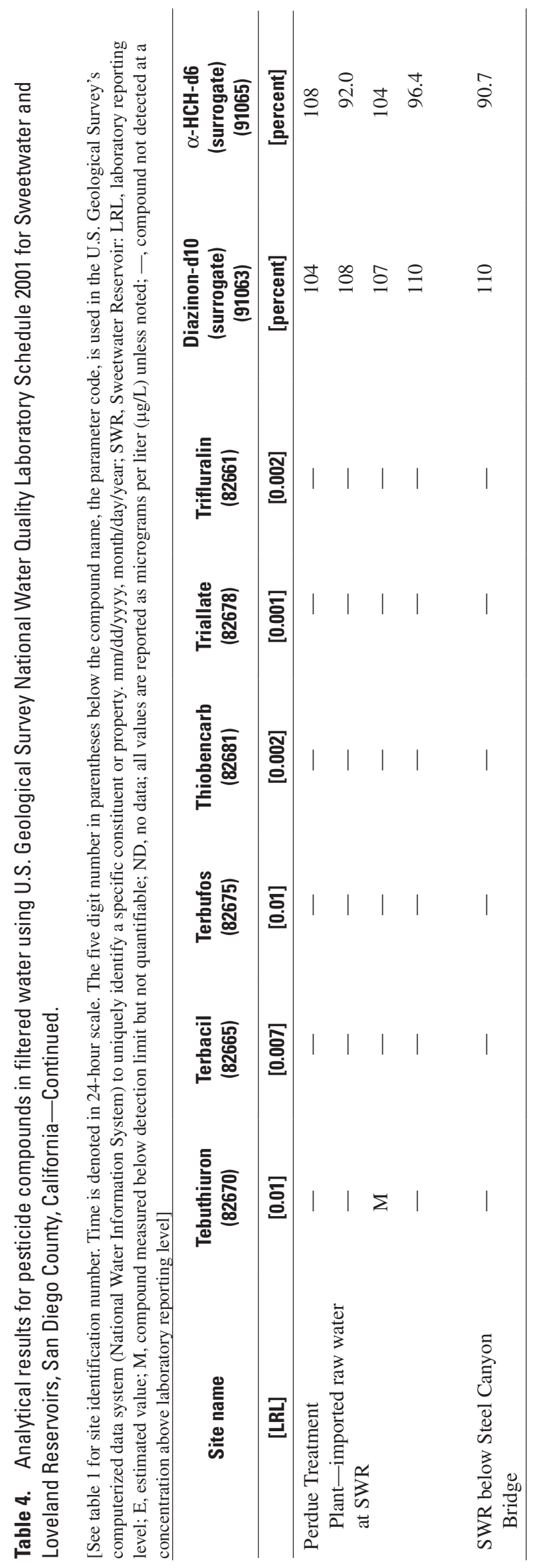


Table 5A. Analytical results for volatile organic compounds with low breakthrough volumes in air for the Sweetwater Reservoir air sampling site, San Diego County, California.

[[The site identification number is 324141117001601. Concentrations are given in parts per billion by volume (ppbv). Data for travel blanks and environmental samples collected on 10/27/2000 were not used because samples were contaminated during transport. E, estimated value; mm/dd/yyyy, month/day/year; NA, not analyzed; - , compound not detected at a concentration above laboratory reporting level]

\begin{tabular}{|c|c|c|c|c|c|c|c|}
\hline $\begin{array}{c}\text { Date } \\
\text { (mm/dd/yyyy) }\end{array}$ & $\begin{array}{l}\text { Dichloro- } \\
\text { difluoro- } \\
\text { methane } \\
\text { (CFC-12) }\end{array}$ & $\begin{array}{l}\text { Chloro- } \\
\text { methane }\end{array}$ & $\begin{array}{c}\text { Chloro- } \\
\text { ethene } \\
\text { (vinyl } \\
\text { chloride) }\end{array}$ & $\begin{array}{l}\text { Bromo- } \\
\text { methane }\end{array}$ & $\begin{array}{l}\text { Chloro- } \\
\text { ethane }\end{array}$ & $\begin{array}{c}\text { Bromo- } \\
\text { ethene } \\
\text { (vinyl } \\
\text { bromide) }\end{array}$ & $\begin{array}{c}\text { 1,1,2,-Trichloro- } \\
\text { 1,2,2-trifluoro- } \\
\text { ethane } \\
\text { (CFC-113) }\end{array}$ \\
\hline 10/02/1999 & 0.664 & - & - & - & - & - & E0.062 \\
\hline $10 / 13 / 1999$ & 0.638 & - & - & - & - & - & E0.074 \\
\hline $10 / 26 / 1999$ & 0.763 & - & - & - & - & - & E0.079 \\
\hline 11/07/1999 & 0.575 & - & - & - & - & - & E0.075 \\
\hline $11 / 19 / 1999$ & 0.642 & E0.084 & - & - & - & - & E0.066 \\
\hline $12 / 13 / 1999$ & 0.419 & - & - & - & - & - & E0.054 \\
\hline $12 / 13 / 1999$ & 0.407 & - & - & - & - & - & E0.048 \\
\hline $12 / 25 / 1999$ & 0.670 & 0.448 & - & - & - & - & E0.076 \\
\hline 01/06/2000 & E0.709 & - & - & 0.126 & - & - & E0.079 \\
\hline $01 / 18 / 2000$ & 1.56 & E0.237 & - & - & - & - & 0.210 \\
\hline $01 / 30 / 2000$ & 0.546 & - & - & - & - & - & - \\
\hline $02 / 11 / 2000$ & E0.614 & 0.777 & - & 2.49 & - & - & E0.069 \\
\hline 03/18/2000 & 0.379 & - & - & - & - & - & E0.050 \\
\hline $04 / 11 / 2000$ & 0.726 & - & - & - & - & - & E0.148 \\
\hline $04 / 23 / 2000$ & 0.802 & - & - & - & - & - & E0.103 \\
\hline $05 / 05 / 2000$ & 0.789 & - & - & - & - & - & E0.097 \\
\hline $05 / 17 / 2000$ & 0.709 & - & - & - & - & - & E0.085 \\
\hline $05 / 29 / 2000$ & 0.493 & - & - & - & - & - & E0.062 \\
\hline $06 / 10 / 2000$ & 0.714 & E0.211 & - & - & - & - & E0.077 \\
\hline $06 / 29 / 2000$ & 0.268 & - & - & - & - & - & E0.065 \\
\hline 07/11/2000 & 0.629 & - & - & - & - & - & E0.074 \\
\hline $07 / 23 / 2000$ & 0.667 & E0.291 & - & - & - & - & E0.078 \\
\hline 08/04/2000 & 0.505 & 0.603 & - & - & 3.65 & - & E0.004 \\
\hline 08/16/2000 & 0.952 & - & - & - & - & - & E0.106 \\
\hline $08 / 28 / 2000$ & NA & NA & NA & NA & NA & NA & NA \\
\hline 09/09/2000 & 0.769 & - & - & - & - & - & E0.084 \\
\hline 09/21/2000 & 1.27 & - & - & - & - & - & E0.127 \\
\hline $10 / 03 / 2000$ & 1.40 & - & - & - & - & - & E0.169 \\
\hline $11 / 08 / 2000$ & 1.24 & - & - & - & - & - & E0.191 \\
\hline $11 / 20 / 2000$ & 1.14 & - & - & - & - & - & E0.128 \\
\hline $12 / 02 / 2000$ & 0.800 & E0.237 & - & - & - & - & E0.096 \\
\hline $12 / 14 / 2000$ & 1.11 & - & - & - & - & - & E0.137 \\
\hline $12 / 26 / 2000$ & 1.30 & - & 0.58 & - & - & - & 0.198 \\
\hline 01/07/2001 & NA & NA & NA & NA & NA & NA & NA \\
\hline
\end{tabular}


Table 5A. Analytical results for volatile organic compounds with low breakthrough volumes in air for the Sweetwater Reservoir air sampling site, San Diego County, California—Continued.

[[The site identification number is 324141117001601. Concentrations are given in parts per billion by volume (ppbv). Data for travel blanks and environmental samples collected on 10/27/2000 were not used because samples were contaminated during transport. E, estimated value; mm/dd/yyyy, month/day/year; NA, not analyzed; _ , compound not detected at a concentration above laboratory reporting level]

\begin{tabular}{|c|c|c|c|c|c|c|c|}
\hline $\begin{array}{c}\text { Date } \\
\text { (mm/dd/yyyy) }\end{array}$ & $\begin{array}{l}\text { Dichloro- } \\
\text { difluoro- } \\
\text { methane } \\
\text { (CFC-12) }\end{array}$ & $\begin{array}{l}\text { Chloro- } \\
\text { methane }\end{array}$ & $\begin{array}{l}\text { Chloro- } \\
\text { ethene } \\
\text { (vinyl } \\
\text { chloride) }\end{array}$ & $\begin{array}{l}\text { Bromo- } \\
\text { methane }\end{array}$ & $\begin{array}{l}\text { Chloro- } \\
\text { ethane }\end{array}$ & $\begin{array}{l}\text { Bromo- } \\
\text { ethene } \\
\text { (vinyl } \\
\text { bromide) }\end{array}$ & $\begin{array}{c}\text { 1,1,2,-Trichloro- } \\
\text { 1,2,2-trifluoro- } \\
\text { ethane } \\
\text { (CFC-113) }\end{array}$ \\
\hline $01 / 19 / 2001$ & NA & NA & NA & NA & NA & NA & NA \\
\hline $01 / 31 / 2001$ & 1.04 & - & 1.87 & - & - & - & E0.141 \\
\hline $02 / 12 / 2001$ & 0.601 & - & - & - & - & - & E0.100 \\
\hline $02 / 24 / 2001$ & 0.661 & - & - & - & - & - & E0.095 \\
\hline $02 / 24 / 2001$ & 0.460 & - & - & - & - & - & E0.079 \\
\hline 03/08/2001 & 0.304 & - & - & - & - & - & E0.059 \\
\hline 03/08/2001 & 0.477 & - & - & - & - & - & E0.113 \\
\hline 03/20/2001 & 0.987 & - & - & - & - & - & E0.140 \\
\hline 03/20/2001 & 0.931 & - & - & - & - & - & E0.139 \\
\hline 04/01/2001 & 0.939 & - & - & - & - & - & E0.119 \\
\hline $04 / 13 / 2001$ & 1.22 & - & - & - & - & - & E0.175 \\
\hline $04 / 25 / 2001$ & 0.895 & - & - & - & - & - & E0.122 \\
\hline 05/07/2001 & 1.13 & - & - & - & - & - & E0.139 \\
\hline 05/19/2001 & 0.962 & - & - & - & - & - & E0.172 \\
\hline $05 / 31 / 2001$ & 1.14 & - & - & - & - & - & 0.209 \\
\hline $05 / 31 / 2001$ & 1.12 & - & - & - & - & - & E0.181 \\
\hline $06 / 12 / 2001$ & 0.770 & - & - & - & - & - & E0.150 \\
\hline $06 / 24 / 2001$ & 0.617 & - & - & - & - & - & E0.167 \\
\hline $07 / 05 / 2001$ & 1.22 & - & - & - & - & - & 0.198 \\
\hline 07/05/2001 & 1.75 & - & - & - & - & - & 0.201 \\
\hline 07/16/2001 & 0.913 & - & - & - & - & - & E0.113 \\
\hline 08/08/2001 & - & - & - & - & - & - & - \\
\hline $08 / 23 / 2001$ & NA & NA & NA & NA & NA & NA & NA \\
\hline 09/16/2001 & 0.087 & - & - & - & - & - & E0.013 \\
\hline 09/28/2001 & 1.09 & - & - & - & - & - & E0.147 \\
\hline
\end{tabular}


Table 5B. Analytical results for volatile organic compounds with high breakthrough volumes in air from the Sweetwater Reservoir air sampling site, San Diego County, California.

[The site identification number is 324141117001601. Concentrations are given in parts per billion by volume (ppbv). Data for travel blanks and environmental samples collected on 10/27/2000 were not used because samples were contaminated during transport. E, estimated value; mm/dd/yyyy, month/day/year; NA, not analyzed; —, compound not detected at a concentration above laboratory reporting level]

\begin{tabular}{|c|c|c|c|c|c|c|c|c|}
\hline $\begin{array}{c}\text { Date } \\
(\mathrm{mm} / \mathrm{dd} / \mathrm{yyyy})\end{array}$ & $\begin{array}{l}\text { Dibromo- } \\
\text { methane }\end{array}$ & $\begin{array}{l}\text { Bromo- } \\
\text { dichloro- } \\
\text { methane }\end{array}$ & $\begin{array}{c}\text { Carbon } \\
\text { tetra- } \\
\text { chloride }\end{array}$ & $\begin{array}{l}\text { 1,2-Dichloro- } \\
\text { ethane }\end{array}$ & $\begin{array}{c}\text { Bromoform } \\
\text { (Tribromo- } \\
\text { methane) }\end{array}$ & $\begin{array}{c}\text { Dibromo- } \\
\text { chloro- } \\
\text { methane }\end{array}$ & $\begin{array}{l}\text { Chloroform } \\
\text { (Trichloro- } \\
\text { methane) }\end{array}$ & Toluene \\
\hline 10/02/1999 & - & E0.010 & E0.083 & E0.003 & - & E0.002 & E0.025 & 2.04 \\
\hline 10/13/1999 & - & E0.015 & E0.099 & E0.005 & - & - & E0.035 & 2.12 \\
\hline 10/26/1999 & - & - & E0.092 & E0.004 & - & - & E0.008 & 0.887 \\
\hline $12 / 13 / 1999$ & - & - & E0.085 & E0.006 & - & - & E0.009 & 1.28 \\
\hline 12/13/1999 & - & - & E0.064 & E0.005 & - & - & E0.018 & 1.11 \\
\hline $12 / 25 / 1999$ & - & - & E0.080 & E0.006 & - & - & E0.022 & 1.97 \\
\hline 01/06/2000 & - & - & E0.030 & E0.003 & - & - & E0.005 & 0.584 \\
\hline 03/18/2000 & - & - & E0.015 & - & - & - & - & 0.219 \\
\hline $04 / 11 / 2000$ & - & - & E0.113 & E0.004 & - & - & E0.002 & 0.353 \\
\hline $04 / 23 / 2000$ & - & - & E0.128 & E0.008 & - & - & E0.011 & 0.596 \\
\hline 05/05/2000 & - & E0.003 & E0.105 & - & - & - & E0.016 & 0.600 \\
\hline 05/17/2000 & - & E0.003 & E0.093 & E0.004 & - & - & E0.008 & 0.480 \\
\hline 05/29/2000 & - & E0.008 & E0.130 & E0.004 & - & E0.004 & E0.038 & 0.423 \\
\hline 06/10/2000 & - & E0.004 & E0.140 & - & - & E0.002 & E0.026 & 0.309 \\
\hline 06/29/2000 & - & E0.025 & E0.110 & - & E0.005 & E0.021 & E0.022 & 0.737 \\
\hline 09/09/2000 & - & E0.008 & E0.201 & E0.006 & E0.004 & E0.006 & E0.033 & 0.805 \\
\hline $09 / 21 / 2000$ & - & - & E0.197 & E0.009 & - & - & - & 2.73 \\
\hline $10 / 03 / 2000$ & - & E0.014 & 0.351 & E0.028 & E0.010 & E0.005 & E0.064 & 2.44 \\
\hline $11 / 08 / 2000$ & - & - & 0.297 & E0.026 & E0.007 & E0.009 & 0.120 & 3.60 \\
\hline $11 / 20 / 2000$ & - & - & 0.401 & E0.047 & - & - & 0.105 & 3.85 \\
\hline $12 / 02 / 2000$ & - & - & E0.216 & E0.019 & - & - & E0.050 & 4.62 \\
\hline $12 / 14 / 2000$ & - & - & 0.412 & E0.034 & - & - & 0.086 & 4.75 \\
\hline $12 / 26 / 2000$ & NA & NA & NA & NA & NA & NA & NA & NA \\
\hline 01/07/2001 & - & - & E0.229 & E0.016 & - & - & E0.051 & 4.55 \\
\hline 01/19/2001 & - & - & E0.160 & E0.010 & - & - & E0.014 & 0.396 \\
\hline
\end{tabular}


Table 5B. Analytical results for volatile organic compounds with high breakthrough volumes in air from the Sweetwater Reservoir air sampling site, San Diego County, California-Continued.

[The site identification number is 324141117001601. Concentrations are given in parts per billion by volume (ppbv). Data for travel blanks and environmental samples collected on 10/27/2000 were not used because samples were contaminated during transport. E, estimated value; mm/dd/yyyy, month/day/year; NA, not analyzed; - , compound not detected at a concentration above laboratory reporting level]

\begin{tabular}{|c|c|c|c|c|c|c|c|c|}
\hline $\begin{array}{c}\text { Date } \\
\text { (mm/dd/yyyy) }\end{array}$ & $\begin{array}{l}\text { Dibromo- } \\
\text { methane }\end{array}$ & $\begin{array}{l}\text { Bromo- } \\
\text { dichloro- } \\
\text { methane }\end{array}$ & $\begin{array}{c}\text { Carbon } \\
\text { tetra- } \\
\text { chloride }\end{array}$ & $\begin{array}{l}\text { 1,2-Dichloro- } \\
\text { ethane }\end{array}$ & $\begin{array}{l}\text { Bromoform } \\
\text { (Tribromo- } \\
\text { methane) }\end{array}$ & $\begin{array}{l}\text { Dibromo- } \\
\text { chloro- } \\
\text { methane }\end{array}$ & $\begin{array}{l}\text { Chloroform } \\
\text { (Trichloro- } \\
\text { methane) }\end{array}$ & Toluene \\
\hline $01 / 31 / 2001$ & - & - & E0.134 & E0.010 & - & - & - & 6.53 \\
\hline $02 / 12 / 2001$ & NA & NA & NA & NA & NA & NA & NA & NA \\
\hline $02 / 24 / 2001$ & - & - & E0.107 & - & - & - & E0.010 & 0.547 \\
\hline 03/08/2001 & NA & NA & NA & NA & NA & NA & NA & NA \\
\hline 03/20/2001 & - & - & - & E0.002 & - & - & - & E0.035 \\
\hline $04 / 01 / 2001$ & - & - & E0.132 & E0.012 & E0.001 & - & E0.026 & 1.75 \\
\hline $04 / 13 / 2001$ & - & - & - & - & - & - & - & 0.104 \\
\hline 05/19/2001 & - & - & E0.002 & - & - & - & - & - \\
\hline $05 / 31 / 2001$ & - & - & - & - & - & - & - & - \\
\hline $05 / 31 / 2001$ & - & - & - & - & - & - & - & - \\
\hline $06 / 12 / 2001$ & NA & $\mathrm{NA}$ & NA & NA & NA & NA & NA & NA \\
\hline $06 / 24 / 2001$ & - & - & - & - & - & - & - & - \\
\hline $07 / 05 / 2001$ & - & - & - & - & - & - & - & - \\
\hline $07 / 05 / 2001$ & - & - & E0.033 & - & - & - & - & 0.152 \\
\hline $07 / 16 / 2001$ & - & - & - & - & - & - & - & - \\
\hline
\end{tabular}


Table 5B. Analytical results for volatile organic compounds with high breakthrough volumes in air from the Sweetwater Reservoir air sampling site, San Diego County, California-Continued.

[The site identification number is 324141117001601 . Concentrations are given in parts per billion by volume (ppbv). Data for travel blanks and environmental samples collected on 10/27/2000 were not used because samples were contaminated during transport. E, estimated value; mm/dd/yyyy, month/day/year; NA, not analyzed; - , compound not detected at a concentration above laboratory reporting level]

\begin{tabular}{|c|c|c|c|c|c|c|c|c|c|}
\hline $\begin{array}{c}\text { Date } \\
\text { (mm/dd/yyyy) }\end{array}$ & Benzene & $\begin{array}{l}\text { 2-Propene- } \\
\text { nitrile } \\
\text { (Acrylonitrile) }\end{array}$ & $\begin{array}{l}\text { Chloro- } \\
\text { benzene }\end{array}$ & $\begin{array}{c}\text { Ethyl- } \\
\text { benzene }\end{array}$ & $\begin{array}{l}\text { Hexa- } \\
\text { chloro- } \\
\text { ethane }\end{array}$ & $\begin{array}{l}\text { Dichloro- } \\
\text { methane } \\
\text { (Methylene } \\
\text { chloride) }\end{array}$ & $\begin{array}{l}\text { Tetra- } \\
\text { chloro- } \\
\text { ethene } \\
\text { (PCE) }\end{array}$ & $\begin{array}{l}\text { 1,1-Di- } \\
\text { chloro- } \\
\text { ethane }\end{array}$ & $\begin{array}{l}\text { 1,1-Di- } \\
\text { chloro- } \\
\text { ethene }\end{array}$ \\
\hline 10/02/1999 & 0.609 & - & - & 0.274 & - & 0.147 & E0.059 & - & - \\
\hline 10/13/1999 & 0.888 & - & E0.001 & 0.323 & - & 0.136 & E0.055 & - & - \\
\hline 10/26/1999 & 0.328 & - & - & 0.130 & - & E0.026 & E0.021 & - & - \\
\hline 11/07/1999 & 0.475 & - & - & 0.177 & - & E0.022 & E0.039 & - & - \\
\hline 11/19/1999 & 0.539 & - & E0.001 & 0.164 & - & 0.136 & E0.035 & - & - \\
\hline 12/13/1999 & 0.463 & - & - & 0.170 & - & E0.072 & E0.033 & - & - \\
\hline 12/13/1999 & 0.720 & - & E0.005 & 0.174 & - & 0.143 & E0.017 & - & - \\
\hline $12 / 25 / 1999$ & 0.877 & - & E0.004 & 0.298 & - & 0.117 & E0.056 & - & - \\
\hline 01/06/2000 & 0.410 & - & E0.007 & E0.059 & - & 0.241 & E0.025 & - & - \\
\hline 01/18/2000 & - & - & E0.005 & E0.010 & - & - & E0.008 & - & - \\
\hline 01/30/2000 & 0.206 & - & - & E0.067 & - & - & E0.010 & - & - \\
\hline $02 / 11 / 2000$ & 0.201 & - & E0.001 & E0.060 & - & E0.015 & E0.016 & - & - \\
\hline $03 / 18 / 2000$ & E0.064 & - & - & E0.020 & - & - & E0.008 & - & - \\
\hline $04 / 11 / 2000$ & 0.161 & - & E0.001 & E0.033 & - & E0.031 & E0.009 & - & - \\
\hline $04 / 23 / 2000$ & 0.257 & - & E0.001 & 0.093 & - & E0.075 & E0.021 & - & - \\
\hline 05/05/2000 & 0.286 & - & E0.001 & 0.095 & - & E0.052 & 0.151 & - & - \\
\hline $05 / 17 / 2000$ & 0.169 & - & E0.001 & E0.064 & - & E0.037 & - & - & - \\
\hline 05/29/2000 & 0.126 & - & E0.003 & E0.063 & - & 0.112 & E0.021 & - & - \\
\hline 06/10/2000 & 0.152 & - & E0.002 & E0.038 & - & 0.180 & E0.018 & - & - \\
\hline 06/29/2000 & 0.334 & - & E0.009 & 0.089 & - & E0.074 & E0.035 & - & - \\
\hline $07 / 11 / 2000$ & 0.388 & - & E0.014 & 0.123 & - & 0.164 & E0.033 & - & - \\
\hline 07/23/2000 & 0.393 & - & E0.007 & 0.267 & - & 0.152 & E0.030 & - & - \\
\hline 08/04/2000 & E0.094 & - & E0.002 & E0.058 & - & E0.047 & - & - & - \\
\hline 08/16/2000 & 0.293 & - & E0.002 & 0.111 & - & E0.061 & E0.029 & - & - \\
\hline $08 / 28 / 2000$ & 0.121 & - & E0.004 & E0.046 & - & E0.063 & - & - & - \\
\hline 09/09/2000 & 0.228 & - & E0.003 & 0.081 & - & E0.084 & E0.032 & - & - \\
\hline $09 / 21 / 2000$ & 0.670 & - & E0.004 & 0.295 & - & E0.054 & 0.056 & - & - \\
\hline $10 / 03 / 2000$ & 0.842 & - & E0.005 & 0.349 & - & 0.172 & 0.079 & - & - \\
\hline $11 / 08 / 2000$ & 1.87 & - & E0.011 & 0.677 & - & 0.700 & 0.232 & - & - \\
\hline $11 / 20 / 2000$ & 1.65 & - & E0.002 & 0.948 & - & 0.289 & 0.103 & - & - \\
\hline $12 / 02 / 2000$ & 1.75 & - & E0.005 & 0.561 & - & 0.344 & 0.107 & - & - \\
\hline $12 / 14 / 2000$ & 2.07 & - & E0.011 & 0.477 & - & 0.255 & 0.143 & - & - \\
\hline $12 / 26 / 2000$ & NA & NA & NA & NA & NA & NA & NA & NA & NA \\
\hline 01/07/2001 & 1.58 & - & E0.003 & 0.659 & - & 0.402 & 0.105 & - & - \\
\hline 01/19/2001 & 0.330 & - & - & E0.068 & - & E0.031 & - & - & - \\
\hline $01 / 31 / 2001$ & 0.405 & - & E0.011 & 0.156 & - & 0.130 & E0.017 & - & - \\
\hline $02 / 12 / 2001$ & NA & NA & NA & NA & NA & NA & NA & NA & NA \\
\hline
\end{tabular}


Table 5B. Analytical results for volatile organic compounds with high breakthrough volumes in air from the Sweetwater Reservoir air sampling site, San Diego County, California-Continued.

[The site identification number is 324141117001601 . Concentrations are given in parts per billion by volume (ppbv). Data for travel blanks and environmental samples collected on 10/27/2000 were not used because samples were contaminated during transport. E, estimated value; mm/dd/yyyy, month/day/year; NA, not analyzed; - , compound not detected at a concentration above laboratory reporting level]

\begin{tabular}{lccccccccc}
\hline $\begin{array}{c}\text { Date } \\
\text { (mm/dd/yyy) }\end{array}$ & Benzene & $\begin{array}{c}\text { 2-Propene- } \\
\text { nitrile } \\
\text { (Acrylonitrile) }\end{array}$ & $\begin{array}{c}\text { Chloro- } \\
\text { benzene }\end{array}$ & $\begin{array}{c}\text { Ethyl- } \\
\text { benzene }\end{array}$ & $\begin{array}{c}\text { Hexa- } \\
\text { chloro- } \\
\text { ethane }\end{array}$ & $\begin{array}{c}\text { Dichloro- } \\
\text { methane } \\
\text { (Methylene } \\
\text { chloride) }\end{array}$ & $\begin{array}{c}\text { Tetra- } \\
\text { chloro- } \\
\text { ethene } \\
\text { (PCE) }\end{array}$ & $\begin{array}{c}\text { 1,1-Di- } \\
\text { chloro- } \\
\text { ethane }\end{array}$ & $\begin{array}{c}\text { 1,1-Di- } \\
\text { chloro- } \\
\text { ethene }\end{array}$ \\
\hline $02 / 24 / 2001$ & 0.402 & - & - & 0.133 & - & E0.068 & E0.014 & - & - \\
$02 / 24 / 2001$ & 0.652 & - & E0.002 & 0.162 & - & 0.162 & E0.019 & - & - \\
$03 / 08 / 2001$ & NA & NA & NA & NA & NA & NA & NA & NA & NA \\
$03 / 08 / 2001$ & NA & NA & NA & NA & NA & NA & NA & NA & NA \\
$03 / 20 / 2001$ & 0.167 & - & E0.016 & E0.016 & - & E0.047 & - & - & - \\
$04 / 01 / 2001$ & 0.552 & - & E0.003 & 0.216 & - & E0.047 & E0.050 & - & - \\
$04 / 13 / 2001$ & 0.652 & - & E0.028 & E0.007 & - & 0.267 & - & - & 0.496 \\
$04 / 13 / 2001$ & 0.431 & - & E0.002 & 0.188 & - & 0.110 & 0.070 & - & - \\
$04 / 25 / 2001$ & E0.092 & - & E0.001 & E0.048 & - & E0.041 & E0.019 & - & - \\
$05 / 07 / 2001$ & 0.220 & - & - & E0.073 & - & E0.080 & 0.122 & - & - \\
$05 / 19 / 2001$ & - & - & E0.002 & E0.008 & - & 0.123 & - & - & - \\
$05 / 31 / 2001$ & - & - & - & E0.006 & - & - & - & - & - \\
$05 / 31 / 2001$ & - & - & E0.003 & - & - & - & - & - & 0.351 \\
$06 / 12 / 2001$ & NA & NA & NA & NA & NA & NA & NA & NA & NA \\
$06 / 24 / 2001$ & 0.689 & - & E0.028 & E0.007 & - & 0.427 & - & - & - \\
$07 / 05 / 2001$ & 0.265 & - & E0.012 & E0.014 & - & E0.045 & - & - & - \\
$07 / 05 / 2001$ & - & - & - & E0.020 & - & - & E0.007 & - & - \\
$07 / 16 / 2001$ & 0.228 & - & - & - & - & - & - & - & - \\
$08 / 08 / 2001$ & - & - & - & - & - & - & - & - & - \\
$08 / 23 / 2001$ & 0.289 & - & - & 0.113 & - & 0.126 & E0.038 & - & - \\
$09 / 16 / 2001$ & 0.318 & - & E0.007 & E0.049 & - & 0.274 & E0.016 & - & - \\
$09 / 28 / 2001$ & 0.339 & - & E0.011 & - & - & 0.309 & - & - & 0.318 \\
\hline
\end{tabular}


Table 5B. Analytical results for volatile organic compounds with high breakthrough volumes in air from the Sweetwater Reservoir air sampling site, San Diego County, California-Continued.

[The site identification number is 324141117001601. Concentrations are given in parts per billion by volume (ppbv). Data for travel blanks and environmental samples collected on 10/27/2000 were not used because samples were contaminated during transport. E, estimated value; mm/dd/yyyy, month/day/year; NA, not analyzed; - , compound not detected at a concentration above laboratory reporting level]

\begin{tabular}{|c|c|c|c|c|c|c|c|c|}
\hline $\begin{array}{c}\text { Date } \\
\text { (mm/dd/yyyy) }\end{array}$ & $\begin{array}{c}1,1,1- \\
\text { Trichloro- } \\
\text { ethane }\end{array}$ & $\begin{array}{c}1,1,2- \\
\text { Trichloro- } \\
\text { ethane }\end{array}$ & $\begin{array}{c}1,1,2,2- \\
\text { Tetrachloro- } \\
\text { ethane }\end{array}$ & $\begin{array}{c}1,2- \\
\text { Dichloro- } \\
\text { benzene }\end{array}$ & $\begin{array}{l}\text { 1,2-Dichloro- } \\
\text { propane }\end{array}$ & $\begin{array}{l}\text { trans-1,2- } \\
\text { Dichloro- } \\
\text { ethene }\end{array}$ & $\begin{array}{c}1,2,4- \\
\text { Trichloro- } \\
\text { benzene }\end{array}$ & $\begin{array}{l}\text { 1,3-Dichloro- } \\
\text { benzene }\end{array}$ \\
\hline 10/02/1999 & 0.094 & - & - & - & - & - & - & - \\
\hline 10/13/1999 & 0.085 & - & - & - & - & - & - & - \\
\hline 10/26/1999 & E0.060 & - & - & - & - & - & - & - \\
\hline 11/07/1999 & E0.070 & - & - & - & - & - & - & - \\
\hline 11/19/1999 & 0.102 & - & - & - & - & - & - & - \\
\hline $12 / 13 / 1999$ & 0.099 & - & - & - & - & - & - & - \\
\hline 12/13/1999 & E0.067 & - & - & - & - & - & - & - \\
\hline $12 / 25 / 1999$ & 0.082 & - & - & - & - & - & - & - \\
\hline 01/06/2000 & E0.024 & - & - & - & - & - & - & - \\
\hline $01 / 18 / 2000$ & - & - & - & - & - & - & - & - \\
\hline 01/30/2000 & E0.044 & - & - & - & - & - & - & - \\
\hline $02 / 11 / 2000$ & E0.014 & - & - & - & - & - & - & - \\
\hline 03/18/2000 & E0.011 & - & - & - & - & - & - & - \\
\hline $04 / 11 / 2000$ & E0.057 & - & - & - & - & - & - & - \\
\hline $04 / 23 / 2000$ & E0.061 & - & - & - & - & - & - & - \\
\hline 05/05/2000 & E0.051 & - & - & - & - & - & - & E0.025 \\
\hline 05/17/2000 & E0.050 & - & - & - & - & - & - & E0.011 \\
\hline 05/29/2000 & E0.062 & - & - & - & - & - & - & - \\
\hline 06/10/2000 & E0.058 & - & - & - & - & - & - & - \\
\hline 06/29/2000 & E0.056 & - & - & - & - & - & - & - \\
\hline $07 / 11 / 2000$ & E0.051 & - & - & - & - & - & - & - \\
\hline 07/23/2000 & E0.054 & - & E0.004 & - & - & - & - & - \\
\hline 08/04/2000 & E0.041 & - & - & - & - & - & - & - \\
\hline 08/16/2000 & E0.067 & - & - & - & - & - & - & - \\
\hline $08 / 28 / 2000$ & E0.067 & - & - & - & - & - & - & - \\
\hline 09/09/2000 & 0.086 & - & - & - & - & - & - & - \\
\hline $09 / 21 / 2000$ & 0.121 & - & - & - & - & - & - & - \\
\hline $10 / 03 / 2000$ & 0.203 & - & - & - & - & - & - & - \\
\hline $11 / 08 / 2000$ & 0.180 & - & - & - & - & - & - & - \\
\hline $11 / 20 / 2000$ & 0.169 & - & - & - & - & - & - & - \\
\hline $12 / 02 / 2000$ & 0.117 & - & - & - & - & - & - & - \\
\hline $12 / 14 / 2000$ & 0.200 & - & - & - & - & - & - & - \\
\hline $12 / 26 / 2000$ & NA & NA & NA & NA & NA & NA & NA & NA \\
\hline 01/07/2001 & 0.115 & - & - & - & - & - & - & - \\
\hline 01/19/2001 & E0.049 & - & - & - & - & - & - & - \\
\hline $01 / 31 / 2001$ & E0.051 & - & - & - & - & - & - & - \\
\hline $02 / 12 / 2001$ & NA & NA & NA & NA & NA & NA & NA & NA \\
\hline $02 / 24 / 2001$ & E0.034 & - & - & - & - & - & - & - \\
\hline
\end{tabular}


Table 5B. Analytical results for volatile organic compounds with high breakthrough volumes in air from the Sweetwater Reservoir air sampling site, San Diego County, California-Continued.

[The site identification number is 324141117001601. Concentrations are given in parts per billion by volume (ppbv). Data for travel blanks and environmental samples collected on 10/27/2000 were not used because samples were contaminated during transport. E, estimated value; mm/dd/yyyy, month/day/year; NA, not analyzed; - , compound not detected at a concentration above laboratory reporting level]

\begin{tabular}{|c|c|c|c|c|c|c|c|c|}
\hline $\begin{array}{c}\text { Date } \\
\text { (mm/dd/yyyy) }\end{array}$ & $\begin{array}{c}1,1,1- \\
\text { Trichloro- } \\
\text { ethane }\end{array}$ & $\begin{array}{c}1,1,2- \\
\text { Trichloro- } \\
\text { ethane }\end{array}$ & $\begin{array}{c}1,1,2,2- \\
\text { Tetrachloro- } \\
\text { ethane }\end{array}$ & $\begin{array}{c}1,2- \\
\text { Dichloro- } \\
\text { benzene }\end{array}$ & $\begin{array}{c}\text { 1,2-Dichloro- } \\
\text { propane }\end{array}$ & $\begin{array}{c}\text { trans-1,2- } \\
\text { Dichloro- } \\
\text { ethene }\end{array}$ & $\begin{array}{c}1,2,4- \\
\text { Trichloro- } \\
\text { benzene }\end{array}$ & $\begin{array}{l}\text { 1,3-Dichloro- } \\
\text { benzene }\end{array}$ \\
\hline $02 / 24 / 2001$ & E0.044 & - & - & - & - & - & - & - \\
\hline 03/08/2001 & NA & NA & NA & $\mathrm{NA}$ & NA & NA & NA & NA \\
\hline 03/08/2001 & NA & NA & NA & NA & NA & NA & NA & NA \\
\hline 03/20/2001 & - & - & - & - & - & - & - & - \\
\hline $04 / 01 / 2001$ & E0.065 & - & - & - & - & - & - & - \\
\hline $04 / 13 / 2001$ & - & - & - & - & - & - & - & - \\
\hline 04/13/2001 & E0.066 & - & - & - & - & - & - & - \\
\hline $04 / 25 / 2001$ & E0.051 & - & - & - & - & - & - & - \\
\hline 05/07/2001 & 0.084 & - & - & - & - & - & - & - \\
\hline 05/19/2001 & E0.001 & - & - & - & - & - & - & - \\
\hline $05 / 31 / 2001$ & E0.001 & - & - & - & - & - & - & - \\
\hline $05 / 31 / 2001$ & - & - & - & - & - & - & - & - \\
\hline $06 / 12 / 2001$ & NA & NA & NA & NA & NA & NA & NA & NA \\
\hline $06 / 24 / 2001$ & - & - & - & - & - & - & - & - \\
\hline 07/05/2001 & - & - & - & - & - & - & - & - \\
\hline $07 / 05 / 2001$ & E0.018 & - & - & - & - & - & - & - \\
\hline $07 / 16 / 2001$ & - & - & - & - & - & - & - & - \\
\hline 08/08/2001 & - & - & - & - & - & - & - & - \\
\hline 08/23/2001 & 0.086 & - & - & E0.025 & - & - & - & - \\
\hline 09/16/2001 & E0.014 & - & - & - & - & - & - & - \\
\hline 09/28/2001 & - & - & - & - & - & - & - & - \\
\hline
\end{tabular}


Table 5B. Analytical results for volatile organic compounds with high breakthrough volumes in air from the Sweetwater Reservoir air sampling site, San Diego County, California-Continued.

[The site identification number is 324141117001601 . Concentrations are given in parts per billion by volume (ppbv). Data for travel blanks and environmental samples collected on 10/27/2000 were not used because samples were contaminated during transport. E, estimated value; mm/dd/yyyy, month/day/year; NA, not analyzed; - , compound not detected at a concentration above laboratory reporting level]

\begin{tabular}{|c|c|c|c|c|c|c|c|c|}
\hline $\begin{array}{c}\text { Date } \\
\text { (mm/dd/yyyy) }\end{array}$ & $\begin{array}{c}\text { 1,1,1- } \\
\text { Trichloro- } \\
\text { ethane }\end{array}$ & $\begin{array}{c}\text { 1,1,2- } \\
\text { Trichloro- } \\
\text { ethane }\end{array}$ & $\begin{array}{c}\text { 1,1,2,2- } \\
\text { Tetrachloro- } \\
\text { ethane }\end{array}$ & $\begin{array}{c}\text { 1,2- } \\
\text { Dichloro- } \\
\text { benzene }\end{array}$ & $\begin{array}{l}\text { 1,2-Dichloro- } \\
\text { propane }\end{array}$ & $\begin{array}{c}\text { trans-1,2- } \\
\text { Dichloro- } \\
\text { ethene }\end{array}$ & $\begin{array}{c}\text { 1,2,4- } \\
\text { Trichloro- } \\
\text { benzene }\end{array}$ & $\begin{array}{l}\text { 1,3-Dichloro- } \\
\text { benzene }\end{array}$ \\
\hline $10 / 02 / 1999$ & 0.094 & - & - & - & - & - & - & - \\
\hline $10 / 13 / 1999$ & 0.085 & - & - & - & - & - & - & - \\
\hline $10 / 26 / 1999$ & E0.060 & - & - & - & - & - & - & - \\
\hline $12 / 13 / 1999$ & 0.099 & - & - & - & - & - & - & - \\
\hline $12 / 13 / 1999$ & E0.067 & - & - & - & - & - & - & - \\
\hline $12 / 25 / 1999$ & 0.082 & - & - & - & - & - & - & - \\
\hline 01/06/2000 & E0.024 & - & - & - & - & - & - & - \\
\hline $02 / 11 / 2000$ & E0.014 & - & - & - & - & - & - & - \\
\hline 03/18/2000 & E0.011 & - & - & - & - & - & - & - \\
\hline $04 / 11 / 2000$ & E0.057 & - & - & - & - & - & - & - \\
\hline $04 / 23 / 2000$ & E0.061 & - & - & - & - & - & - & - \\
\hline $05 / 05 / 2000$ & E0.051 & - & - & - & - & - & - & E0.025 \\
\hline $05 / 17 / 2000$ & E0.050 & - & - & - & - & - & - & E0.011 \\
\hline $05 / 29 / 2000$ & E0.062 & - & - & - & - & - & - & - \\
\hline $06 / 10 / 2000$ & E0.058 & - & - & - & - & - & - & - \\
\hline $06 / 29 / 2000$ & E0.056 & - & - & - & - & - & - & - \\
\hline 09/09/2000 & 0.086 & - & - & - & - & - & - & - \\
\hline $09 / 21 / 2000$ & 0.121 & - & - & - & - & - & - & - \\
\hline $10 / 03 / 2000$ & 0.203 & - & - & - & - & - & - & - \\
\hline $11 / 08 / 2000$ & 0.180 & - & - & - & - & - & - & - \\
\hline $11 / 20 / 2000$ & 0.169 & - & - & - & - & - & - & - \\
\hline $12 / 02 / 2000$ & 0.117 & - & - & - & - & - & - & - \\
\hline $12 / 14 / 2000$ & 0.200 & - & - & - & - & - & - & - \\
\hline $12 / 26 / 2000$ & NA & NA & NA & NA & NA & NA & NA & NA \\
\hline 01/07/2001 & 0.115 & - & - & - & - & - & - & - \\
\hline $01 / 19 / 2001$ & E0.049 & - & - & - & - & - & - & - \\
\hline $01 / 31 / 2001$ & E0.051 & - & - & - & - & - & - & - \\
\hline $02 / 12 / 2001$ & NA & NA & NA & NA & NA & NA & NA & NA \\
\hline $02 / 24 / 2001$ & E0.034 & - & - & - & - & - & - & - \\
\hline
\end{tabular}


Table 5B. Analytical results for volatile organic compounds with high breakthrough volumes in air from the Sweetwater Reservoir air sampling site, San Diego County, California-Continued.

[The site identification number is 324141117001601. Concentrations are given in parts per billion by volume (ppbv). Data for travel blanks and environmental samples collected on 10/27/2000 were not used because samples were contaminated during transport. E, estimated value; mm/dd/yyyy, month/day/year; NA, not analyzed; - , compound not detected at a concentration above laboratory reporting level]

\begin{tabular}{|c|c|c|c|c|c|c|c|c|}
\hline $\begin{array}{c}\text { Date } \\
\text { (mm/dd/yyyy) }\end{array}$ & $\begin{array}{c}1,1,1- \\
\text { Trichloro- } \\
\text { ethane }\end{array}$ & $\begin{array}{c}1,1,2- \\
\text { Trichloro- } \\
\text { ethane }\end{array}$ & $\begin{array}{c}1,1,2,2- \\
\text { Tetrachloro- } \\
\text { ethane }\end{array}$ & $\begin{array}{c}1,2- \\
\text { Dichloro- } \\
\text { benzene }\end{array}$ & $\begin{array}{c}\text { 1,2-Dichloro- } \\
\text { propane }\end{array}$ & $\begin{array}{c}\text { trans-1,2- } \\
\text { Dichloro- } \\
\text { ethene }\end{array}$ & $\begin{array}{c}1,2,4- \\
\text { Trichloro- } \\
\text { benzene }\end{array}$ & $\begin{array}{l}\text { 1,3-Dichloro- } \\
\text { benzene }\end{array}$ \\
\hline $02 / 24 / 2001$ & E0.044 & - & - & - & - & - & - & - \\
\hline 03/08/2001 & NA & NA & NA & NA & NA & NA & NA & NA \\
\hline 03/08/2001 & NA & NA & NA & NA & NA & NA & NA & NA \\
\hline 03/20/2001 & - & - & - & - & - & - & - & - \\
\hline $04 / 01 / 2001$ & E0.065 & - & - & - & - & - & - & - \\
\hline $04 / 13 / 2001$ & - & - & - & - & - & - & - & - \\
\hline $04 / 13 / 2001$ & E0.066 & - & - & - & - & - & - & - \\
\hline $04 / 25 / 2001$ & E0.051 & - & - & - & - & - & - & - \\
\hline 05/07/2001 & 0.084 & - & - & - & - & - & - & - \\
\hline 05/19/2001 & E0.001 & - & - & - & - & - & - & - \\
\hline $05 / 31 / 2001$ & E0.001 & - & - & - & - & - & - & - \\
\hline $05 / 31 / 2001$ & - & - & - & - & - & - & - & - \\
\hline $06 / 12 / 2001$ & NA & NA & NA & NA & NA & NA & NA & NA \\
\hline $06 / 24 / 2001$ & - & - & - & - & - & - & - & - \\
\hline $07 / 05 / 2001$ & - & - & - & - & - & - & - & - \\
\hline $07 / 05 / 2001$ & E0.018 & - & - & - & - & - & - & - \\
\hline $07 / 16 / 2001$ & - & - & - & - & - & - & - & - \\
\hline 08/08/2001 & - & - & - & - & - & - & - & - \\
\hline $08 / 23 / 2001$ & 0.086 & - & - & E0.025 & - & - & - & - \\
\hline 09/16/2001 & E0.014 & - & - & - & - & - & - & - \\
\hline 09/28/2001 & - & - & - & - & - & - & - & - \\
\hline
\end{tabular}


Table 5B. Analytical results for volatile organic compounds with high breakthrough volumes in air from the Sweetwater Reservoir air sampling site, San Diego County, California-Continued.

[The site identification number is 324141117001601 . Concentrations are given in parts per billion by volume (ppbv). Data for travel blanks and environmental samples collected on 10/27/2000 were not used because samples were contaminated during transport. E, estimated value; mm/dd/yyyy, month/day/year; NA, not analyzed; - , compound not detected at a concentration above laboratory reporting level]

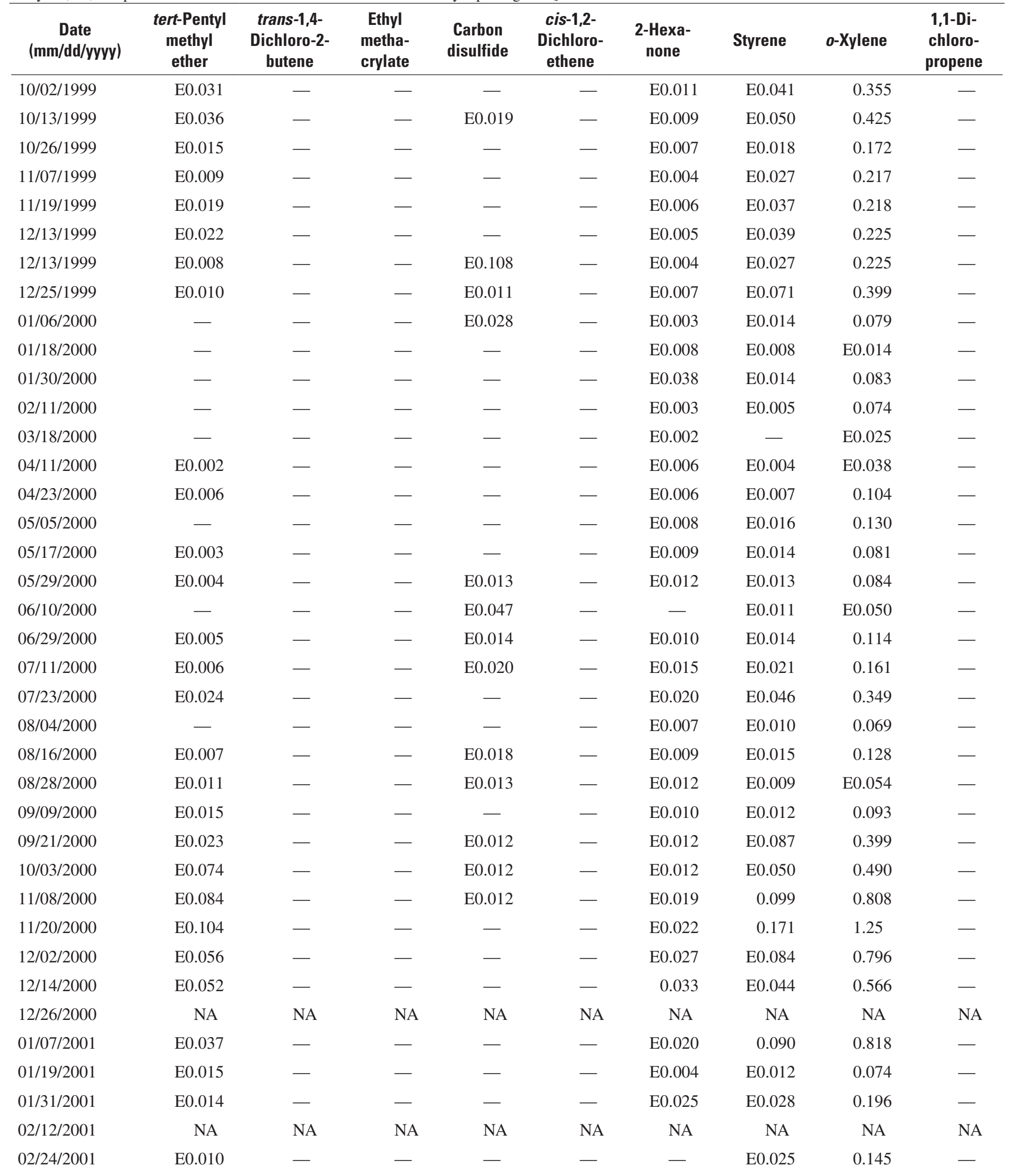


Table 5B. Analytical results for volatile organic compounds with high breakthrough volumes in air from the Sweetwater Reservoir air sampling site, San Diego County, California-Continued.

[The site identification number is 324141117001601. Concentrations are given in parts per billion by volume (ppbv). Data for travel blanks and environmental samples collected on 10/27/2000 were not used because samples were contaminated during transport. E, estimated value; mm/dd/yyyy, month/day/year; NA, not analyzed; - , compound not detected at a concentration above laboratory reporting level]

\begin{tabular}{cccccccccc}
\hline $\begin{array}{c}\text { Date } \\
\text { (mm/dd/yyyy) }\end{array}$ & $\begin{array}{c}\text { tert-Pentyl } \\
\text { methyl } \\
\text { ether }\end{array}$ & $\begin{array}{c}\text { trans-1,4- } \\
\text { Dichloro-2- } \\
\text { buten }\end{array}$ & $\begin{array}{c}\text { Ethyl } \\
\text { metha- } \\
\text { crylate }\end{array}$ & $\begin{array}{c}\text { Carbon } \\
\text { disulfide }\end{array}$ & $\begin{array}{c}\text { cis-1,2- } \\
\text { Dichloro- } \\
\text { ethene }\end{array}$ & $\begin{array}{c}\text { 2-Hexa- } \\
\text { none }\end{array}$ & Styrene & $\begin{array}{c}\text { o-Xylene } \\
\text { 1,1-Di- } \\
\text { chloro- } \\
\text { propene }\end{array}$ \\
\hline $02 / 24 / 2001$ & E0.011 & - & - & E0.026 & - & - & E0.034 & 0.175 & - \\
$03 / 08 / 2001$ & NA & NA & NA & NA & NA & NA & NA & NA & NA \\
$03 / 08 / 2001$ & NA & NA & NA & NA & NA & NA & NA & NA & NA \\
$03 / 20 / 2001$ & - & - & - & E0.046 & - & E0.034 & 0.022 & E0.023 & - \\
$04 / 01 / 2001$ & E0.008 & - & - & E0.019 & - & E0.014 & E0.037 & 0.261 & - \\
$04 / 13 / 2001$ & - & - & - & 0.274 & - & E0.021 & E0.022 & E0.014 & - \\
$04 / 13 / 2001$ & E0.008 & - & - & - & - & E0.016 & E0.037 & 0.229 & - \\
$04 / 25 / 2001$ & - & - & - & - & - & E0.007 & E0.005 & E0.055 & - \\
$05 / 07 / 2001$ & - & - & - & E0.038 & - & - & - & 0.086 & - \\
$05 / 19 / 2001$ & - & - & - & E0.089 & - & - & - & - & - \\
$05 / 31 / 2001$ & - & - & - & E0.030 & - & - & - & - & - \\
$05 / 31 / 2001$ & - & - & - & E0.048 & - & - & - & - & - \\
$06 / 12 / 2001$ & NA & NA & NA & NA & NA & NA & NA & NA & NA \\
$06 / 24 / 2001$ & - & - & - & E0.054 & - & E0.081 & - & - & - \\
$07 / 05 / 2001$ & - & - & - & 0.172 & - & 0.163 & E0.035 & E0.037 & - \\
$07 / 05 / 2001$ & - & - & - & - & - & E0.006 & - & E0.020 & - \\
$07 / 16 / 2001$ & - & - & - & - & - & - & - & - & - \\
$08 / 08 / 2001$ & - & - & - & - & - & - & - & - & - \\
$08 / 23 / 2001$ & - & - & - & E0.012 & - & E0.015 & E0.028 & 0.197 & - \\
$09 / 16 / 2001$ & - & - & - & 0.064 & - & E0.007 & - & E0.056 & - \\
$09 / 28 / 2001$ & - & - & - & 0.527 & - & - & - & - & - \\
\hline
\end{tabular}


Table 5B. Analytical results for volatile organic compounds with high breakthrough volumes in air from the Sweetwater Reservoir air sampling site, San Diego County, California-Continued.

[The site identification number is 324141117001601. Concentrations are given in parts per billion by volume (ppbv). Data for travel blanks and environmental samples collected on 10/27/2000 were not used because samples were contaminated during transport. E, estimated value; mm/dd/yyyy, month/day/year; NA, not analyzed; - compound not detected at a concentration above laboratory reporting level]

\begin{tabular}{|c|c|c|c|c|c|c|c|c|}
\hline $\begin{array}{c}\text { Date } \\
\text { (mm/dd/yyyy) }\end{array}$ & $\begin{array}{l}\text { 2,2-Dichloro- } \\
\text { propane }\end{array}$ & $\begin{array}{l}\text { 1,3-Dichloro- } \\
\text { propane }\end{array}$ & $\begin{array}{c}o \text {-Ethyl } \\
\text { toluene }\end{array}$ & $\begin{array}{c}1,2,3- \\
\text { Trimethyl- } \\
\text { benzene }\end{array}$ & $\begin{array}{c}\text { 1,2,4- } \\
\text { Trimethyl- } \\
\text { benzene }\end{array}$ & $\begin{array}{c}\text { Isopropyl- } \\
\text { benzene } \\
\text { (Cumene) }\end{array}$ & $\begin{array}{l}n \text {-Propyl- } \\
\text { benzene }\end{array}$ & $\begin{array}{c}\text { 1,3,5- } \\
\text { Trimethyl- } \\
\text { benzene }\end{array}$ \\
\hline $10 / 02 / 1999$ & - & - & E0.075 & E0.065 & 0.308 & E0.013 & E0.054 & 0.090 \\
\hline $10 / 13 / 1999$ & - & - & 0.093 & 0.089 & 0.407 & E0.017 & E0.068 & 0.114 \\
\hline $10 / 26 / 1999$ & - & - & E0.035 & E0.031 & 0.151 & E0.006 & E0.025 & E0.043 \\
\hline $12 / 13 / 1999$ & - & - & E0.045 & E0.041 & 0.199 & E0.008 & E0.034 & E0.055 \\
\hline $12 / 13 / 1999$ & - & - & E0.040 & E0.043 & 0.183 & E0.007 & E0.030 & E0.052 \\
\hline $12 / 25 / 1999$ & - & - & 0.081 & 0.075 & 0.357 & E0.013 & E0.056 & 0.102 \\
\hline $01 / 06 / 2000$ & - & - & E0.021 & E0.016 & 0.094 & E0.004 & E0.015 & E0.026 \\
\hline $03 / 18 / 2000$ & - & - & E0.005 & - & E0.021 & E0.001 & E0.004 & E0.006 \\
\hline $04 / 11 / 2000$ & - & - & E0.006 & - & E0.024 & E0.001 & E0.005 & E0.006 \\
\hline $04 / 23 / 2000$ & - & - & E0.014 & E0.009 & E0.051 & E0.004 & E0.014 & E0.014 \\
\hline $05 / 05 / 2000$ & - & - & E0.033 & E0.026 & 0.144 & E0.006 & E0.026 & E0.035 \\
\hline $05 / 17 / 2000$ & - & - & E0.016 & E0.012 & E0.059 & E0.003 & E0.013 & E0.016 \\
\hline $05 / 29 / 2000$ & - & - & E0.022 & E0.012 & 0.094 & E0.005 & E0.016 & E0.020 \\
\hline $06 / 10 / 2000$ & - & - & E0.012 & E0.009 & E0.057 & E0.003 & E0.008 & E0.014 \\
\hline $06 / 29 / 2000$ & - & - & E0.022 & E0.016 & 0.082 & E0.005 & E0.019 & E0.023 \\
\hline $09 / 09 / 2000$ & - & - & E0.023 & E0.017 & 0.098 & E0.005 & E0.019 & E0.024 \\
\hline $09 / 21 / 2000$ & - & - & 0.092 & 0.080 & 0.388 & E0.013 & E0.067 & 0.099 \\
\hline $10 / 03 / 2000$ & - & - & 0.091 & E0.075 & 0.418 & E0.014 & E0.067 & 0.112 \\
\hline $11 / 08 / 2000$ & - & - & 0.248 & 0.222 & 1.08 & E0.037 & 0.181 & 0.259 \\
\hline $11 / 20 / 2000$ & - & - & 0.323 & 0.334 & 1.63 & E0.046 & 0.229 & 0.406 \\
\hline $12 / 02 / 2000$ & - & - & 0.159 & 0.146 & 0.699 & E0.029 & 0.117 & 0.207 \\
\hline $12 / 14 / 2000$ & - & - & 0.140 & 0.096 & 0.555 & E0.027 & 0.108 & 0.147 \\
\hline $12 / 26 / 2000$ & NA & NA & NA & NA & NA & NA & NA & NA \\
\hline $01 / 07 / 2001$ & - & - & 0.155 & 0.181 & 0.768 & E0.029 & 0.112 & 0.209 \\
\hline $01 / 19 / 2001$ & - & - & E0.010 & E0.006 & E0.046 & E0.032 & E0.011 & E0.012 \\
\hline $01 / 31 / 2001$ & - & - & E0.036 & E0.025 & 0.140 & E0.010 & E0.029 & E0.041 \\
\hline $02 / 12 / 2001$ & NA & NA & $\mathrm{NA}$ & NA & NA & NA & NA & NA \\
\hline $02 / 24 / 2001$ & - & - & E0.032 & E0.032 & 0.147 & E0.005 & E0.028 & E0.037 \\
\hline
\end{tabular}


Table 5B. Analytical results for volatile organic compounds with high breakthrough volumes in air from the Sweetwater Reservoir air sampling site, San Diego County, California-Continued.

[The site identification number is 324141117001601. Concentrations are given in parts per billion by volume (ppbv). Data for travel blanks and environmental samples collected on 10/27/2000 were not used because samples were contaminated during transport. E, estimated value; mm/dd/yyyy, month/day/year; NA, not analyzed; - , compound not detected at a concentration above laboratory reporting level]

\begin{tabular}{|c|c|c|c|c|c|c|c|c|}
\hline $\begin{array}{c}\text { Date } \\
\text { (mm/dd/yyyy) }\end{array}$ & $\begin{array}{l}\text { 2,2-Dichloro- } \\
\text { propane }\end{array}$ & $\begin{array}{l}\text { 1,3-Dichloro- } \\
\text { propane }\end{array}$ & $\begin{array}{l}o \text {-Ethyl } \\
\text { toluene }\end{array}$ & $\begin{array}{c}1,2,3- \\
\text { Trimethyl- } \\
\text { benzene }\end{array}$ & $\begin{array}{c}1,2,4- \\
\text { Trimethyl- } \\
\text { benzene }\end{array}$ & $\begin{array}{l}\text { Isopropyl- } \\
\text { benzene } \\
\text { (Cumene) }\end{array}$ & $\begin{array}{l}n \text {-Propyl- } \\
\text { benzene }\end{array}$ & $\begin{array}{c}\mathbf{1 , 3 , 5 -} \\
\text { Trimethyl- } \\
\text { benzene }\end{array}$ \\
\hline $02 / 24 / 2001$ & - & - & E0.037 & E0.039 & 0.183 & E0.007 & E0.036 & E0.046 \\
\hline 03/08/2001 & NA & NA & NA & NA & NA & NA & NA & NA \\
\hline 03/08/2001 & NA & NA & NA & NA & NA & NA & NA & NA \\
\hline 03/20/2001 & - & - & E0.005 & E0.004 & E0.018 & E0.003 & E0.005 & E0.006 \\
\hline 04/01/2001 & - & - & E0.055 & E0.040 & 0.210 & E0.010 & E0.044 & E0.060 \\
\hline $04 / 13 / 2001$ & - & - & E0.003 & E0.002 & E0.010 & E0.002 & E0.003 & E0.003 \\
\hline $04 / 13 / 2001$ & - & - & E0.055 & E0.037 & E0.192 & E0.011 & E0.045 & E0.049 \\
\hline $04 / 25 / 2001$ & - & - & E0.007 & - & E0.031 & E0.002 & E0.007 & E0.007 \\
\hline 05/07/2001 & - & - & E0.021 & E0.017 & E0.064 & E0.049 & E0.018 & E0.020 \\
\hline 05/19/2001 & - & - & E0.002 & E0.002 & E0.007 & E0.001 & E0.001 & E0.002 \\
\hline $05 / 31 / 2001$ & - & - & E0.002 & E0.003 & E0.009 & E0.002 & E0.002 & E0.003 \\
\hline $05 / 31 / 2001$ & - & - & E0.003 & - & - & - & - & E0.004 \\
\hline $06 / 12 / 2001$ & NA & NA & NA & NA & NA & NA & NA & NA \\
\hline $06 / 24 / 2001$ & - & - & - & - & E0.006 & - & E0.001 & - \\
\hline 07/05/2001 & - & - & E0.009 & E0.010 & E0.045 & E0.006 & E0.008 & E0.014 \\
\hline $07 / 05 / 2001$ & - & - & E0.005 & E0.004 & E0.015 & E0.001 & E0.005 & E0.005 \\
\hline 07/16/2001 & - & - & - & - & - & - & E0.001 & - \\
\hline 08/08/2001 & - & - & - & - & - & - & - & - \\
\hline 08/23/2001 & - & - & E0.059 & E0.049 & 0.246 & E0.008 & E0.038 & E0.064 \\
\hline 09/16/2001 & - & - & E0.014 & E0.011 & E0.050 & E0.003 & E0.011 & E0.013 \\
\hline 09/28/2001 & - & E 0.009 & - & E0.002 & E0.005 & - & - & E0.001 \\
\hline
\end{tabular}


Table 5B. Analytical results for volatile organic compounds with high breakthrough volumes in air from the Sweetwater Reservoir air sampling site, San Diego County, California-Continued.

[The site identification number is 324141117001601 . Concentrations are given in parts per billion by volume (ppbv). Data for travel blanks and environmental samples collected on 10/27/2000 were not used because samples were contaminated during transport. E, estimated value; mm/dd/yyyy, month/day/year; NA, not analyzed; _-, compound not detected at a concentration above laboratory reporting level]

\begin{tabular}{|c|c|c|c|c|c|c|c|c|c|}
\hline $\begin{array}{c}\text { Date } \\
\text { (mm/dd/yyyy) }\end{array}$ & $\begin{array}{c}\text { 2-Chloro- } \\
\text { toluene }\end{array}$ & $\begin{array}{l}\text { 1-Chloro-4- } \\
\text { methyl- } \\
\text { benzene }\end{array}$ & $\begin{array}{l}\text { Bromo- } \\
\text { chloro- } \\
\text { methane }\end{array}$ & $\begin{array}{l}n \text {-Butyl- } \\
\text { benzene }\end{array}$ & $\begin{array}{c}\text { sec- } \\
\text { Butyl- } \\
\text { benzene }\end{array}$ & $\begin{array}{c}\text { tert- } \\
\text { Butyl- } \\
\text { benzene }\end{array}$ & $\begin{array}{c}\text { 4-Iso } \\
\text { propyl-1- } \\
\text { methyl- } \\
\text { benzene }\end{array}$ & $\begin{array}{c}1,2,3- \\
\text { Trichloro- } \\
\text { propane }\end{array}$ & $\begin{array}{c}1,1,1,2- \\
\text { Tetrachlo- } \\
\text { roethane }\end{array}$ \\
\hline 10/02/1999 & - & - & - & E0.011 & E0.005 & - & E0.035 & - & - \\
\hline 10/13/1999 & - & - & - & E0.016 & E0.006 & - & E0.045 & - & - \\
\hline 10/26/1999 & - & - & - & E0.006 & E0.002 & - & E0.020 & - & - \\
\hline 11/07/1999 & - & - & - & E0.006 & E0.003 & - & E0.017 & - & - \\
\hline 11/19/1999 & - & - & - & E0.008 & E0.002 & - & E0.024 & - & - \\
\hline 12/13/1999 & - & - & - & E0.008 & E0.003 & - & E0.026 & - & - \\
\hline 12/13/1999 & E0.002 & - & - & E0.007 & E0.002 & - & E0.015 & - & - \\
\hline $12 / 25 / 1999$ & E0.002 & - & - & E0.015 & E0.004 & - & E0.029 & - & - \\
\hline 01/06/2000 & - & - & - & E0.003 & E0.001 & - & E0.011 & - & - \\
\hline 01/18/2000 & - & - & - & E0.001 & - & - & E0.001 & - & - \\
\hline 01/30/2000 & - & - & - & E0.002 & E0.044 & - & E0.033 & - & - \\
\hline $02 / 11 / 2000$ & - & - & - & E0.002 & E0.001 & - & E0.004 & - & - \\
\hline 03/18/2000 & - & - & - & E0.001 & - & - & E0.002 & - & - \\
\hline 04/11/2000 & - & - & - & E0.001 & - & - & E0.002 & - & - \\
\hline 04/23/2000 & - & - & - & E0.001 & E0.001 & - & E0.002 & - & - \\
\hline 05/05/2000 & - & - & - & E0.005 & E0.001 & - & E0.014 & - & - \\
\hline 05/17/2000 & - & - & - & E0.002 & E0.001 & - & E0.007 & - & - \\
\hline 05/29/2000 & - & - & - & E0.003 & E0.001 & - & E0.008 & - & - \\
\hline 06/10/2000 & - & - & - & E0.002 & E0.001 & - & E0.003 & - & - \\
\hline 06/29/2000 & - & - & - & E0.003 & E0.001 & - & E0.018 & - & - \\
\hline 07/11/2000 & - & - & - & E0.007 & E0.003 & - & E0.037 & - & - \\
\hline 07/23/2000 & - & - & - & E0.014 & E0.004 & - & 0.089 & - & - \\
\hline 08/04/2000 & - & - & - & E0.003 & E0.006 & - & E0.004 & - & - \\
\hline 08/16/2000 & - & - & - & E0.005 & E0.002 & - & E0.023 & - & - \\
\hline 08/28/2000 & - & - & - & E0.001 & E0.001 & - & E0.004 & - & - \\
\hline 09/09/2000 & - & - & - & E0.004 & E0.002 & - & E0.006 & - & - \\
\hline 09/21/2000 & - & - & - & E0.016 & E0.007 & - & E0.006 & - & - \\
\hline $10 / 03 / 2000$ & - & - & - & E0.012 & E0.006 & - & E0.024 & - & - \\
\hline $11 / 08 / 2000$ & - & - & - & E0.047 & E0.015 & - & 0.079 & - & - \\
\hline $11 / 20 / 2000$ & - & - & - & 0.071 & E0.024 & - & 0.082 & - & - \\
\hline $12 / 02 / 2000$ & - & - & - & E0.022 & E0.013 & - & 0.039 & - & - \\
\hline $12 / 14 / 2000$ & - & - & - & E0.016 & E0.011 & - & 0.030 & - & - \\
\hline $12 / 26 / 2000$ & NA & NA & NA & NA & NA & NA & NA & NA & NA \\
\hline 01/07/2001 & - & - & - & E0.041 & E0.012 & - & E0.041 & - & - \\
\hline 01/19/2001 & - & - & - & E0.001 & E0.002 & - & E0.001 & - & 0.010 \\
\hline 01/31/2001 & - & - & - & E0.002 & E0.002 & - & E0.013 & - & - \\
\hline $02 / 12 / 2001$ & NA & NA & NA & NA & NA & NA & NA & NA & NA \\
\hline
\end{tabular}


Table 5B. Analytical results for volatile organic compounds with high breakthrough volumes in air from the Sweetwater Reservoir air sampling site, San Diego County, California-Continued.

[The site identification number is 324141117001601. Concentrations are given in parts per billion by volume (ppbv). Data for travel blanks and environmental samples collected on 10/27/2000 were not used because samples were contaminated during transport. E, estimated value; mm/dd/yyyy, month/day/year; NA, not analyzed; - , compound not detected at a concentration above laboratory reporting level]

\begin{tabular}{|c|c|c|c|c|c|c|c|c|c|}
\hline $\begin{array}{c}\text { Date } \\
\text { (mm/dd/yyyy) }\end{array}$ & $\begin{array}{c}\text { 2-Chloro- } \\
\text { toluene }\end{array}$ & $\begin{array}{c}\text { 1-Chloro-4- } \\
\text { methyl- } \\
\text { benzene }\end{array}$ & $\begin{array}{l}\text { Bromo- } \\
\text { chloro- } \\
\text { methane }\end{array}$ & $\begin{array}{l}n \text {-Butyl- } \\
\text { benzene }\end{array}$ & $\begin{array}{c}\text { sec- } \\
\text { Butyl- } \\
\text { benzene }\end{array}$ & $\begin{array}{c}\text { tert- } \\
\text { Butyl- } \\
\text { benzene }\end{array}$ & $\begin{array}{c}\text { 4-Iso } \\
\text { propyl-1- } \\
\text { methyl- } \\
\text { benzene }\end{array}$ & $\begin{array}{c}1,2,3- \\
\text { Trichloro- } \\
\text { propane }\end{array}$ & $\begin{array}{c}1,1,1,2- \\
\text { Tetrachlo- } \\
\text { roethane }\end{array}$ \\
\hline $02 / 24 / 2001$ & - & - & - & E0.008 & E0.003 & - & E0.022 & - & - \\
\hline $02 / 24 / 2001$ & - & - & - & E0.009 & E0.002 & - & E0.030 & - & - \\
\hline 03/08/2001 & NA & NA & NA & $\mathrm{NA}$ & NA & NA & NA & NA & NA \\
\hline 03/08/2001 & NA & NA & NA & $\mathrm{NA}$ & NA & NA & NA & NA & NA \\
\hline $03 / 20 / 2001$ & - & - & - & E0.004 & - & - & E0.002 & - & - \\
\hline $04 / 01 / 2001$ & - & - & - & E0.007 & E0.004 & - & E0.015 & - & - \\
\hline $04 / 13 / 2001$ & - & - & - & E0.001 & - & - & E0.002 & - & - \\
\hline $04 / 13 / 2001$ & - & - & - & E0.009 & E0.004 & - & E0.021 & - & - \\
\hline $04 / 25 / 2001$ & - & - & - & E0.001 & - & - & E0.002 & - & - \\
\hline 05/07/2001 & - & - & - & E0.004 & - & - & E0.021 & - & - \\
\hline 05/19/2001 & - & - & - & E0.003 & - & - & E0.001 & - & - \\
\hline $05 / 31 / 2001$ & - & - & - & E0.004 & - & - & E0.002 & - & - \\
\hline 05/31/2001 & - & - & - & - & - & - & - & - & - \\
\hline $06 / 12 / 2001$ & NA & NA & NA & NA & NA & NA & NA & NA & NA \\
\hline $06 / 24 / 2001$ & - & - & - & E0.005 & - & - & - & - & - \\
\hline $07 / 05 / 2001$ & - & - & - & E0.012 & - & - & E0.007 & - & - \\
\hline $07 / 05 / 2001$ & - & - & - & - & - & - & E0.002 & - & - \\
\hline 07/16/2001 & - & - & - & - & - & - & - & - & - \\
\hline 08/08/2001 & - & - & - & - & - & - & - & - & - \\
\hline $08 / 23 / 2001$ & - & - & - & - & - & - & E0.017 & - & - \\
\hline 09/16/2001 & - & - & - & E0.002 & E0.001 & - & E0.010 & - & - \\
\hline $09 / 28 / 2001$ & - & - & - & - & - & - & E0.001 & - & - \\
\hline
\end{tabular}


Table 5B. Analytical results for volatile organic compounds with high breakthrough volumes in air from the Sweetwater Reservoir air sampling site, San Diego County, California-Continued.

[The site identification number is 324141117001601 . Concentrations are given in parts per billion by volume (ppbv). Data for travel blanks and environmental samples collected on 10/27/2000 were not used because samples were contaminated during transport. E, estimated value; mm/dd/yyyy, month/day/year; NA, not analyzed; _ , compound not detected at a concentration above laboratory reporting level]

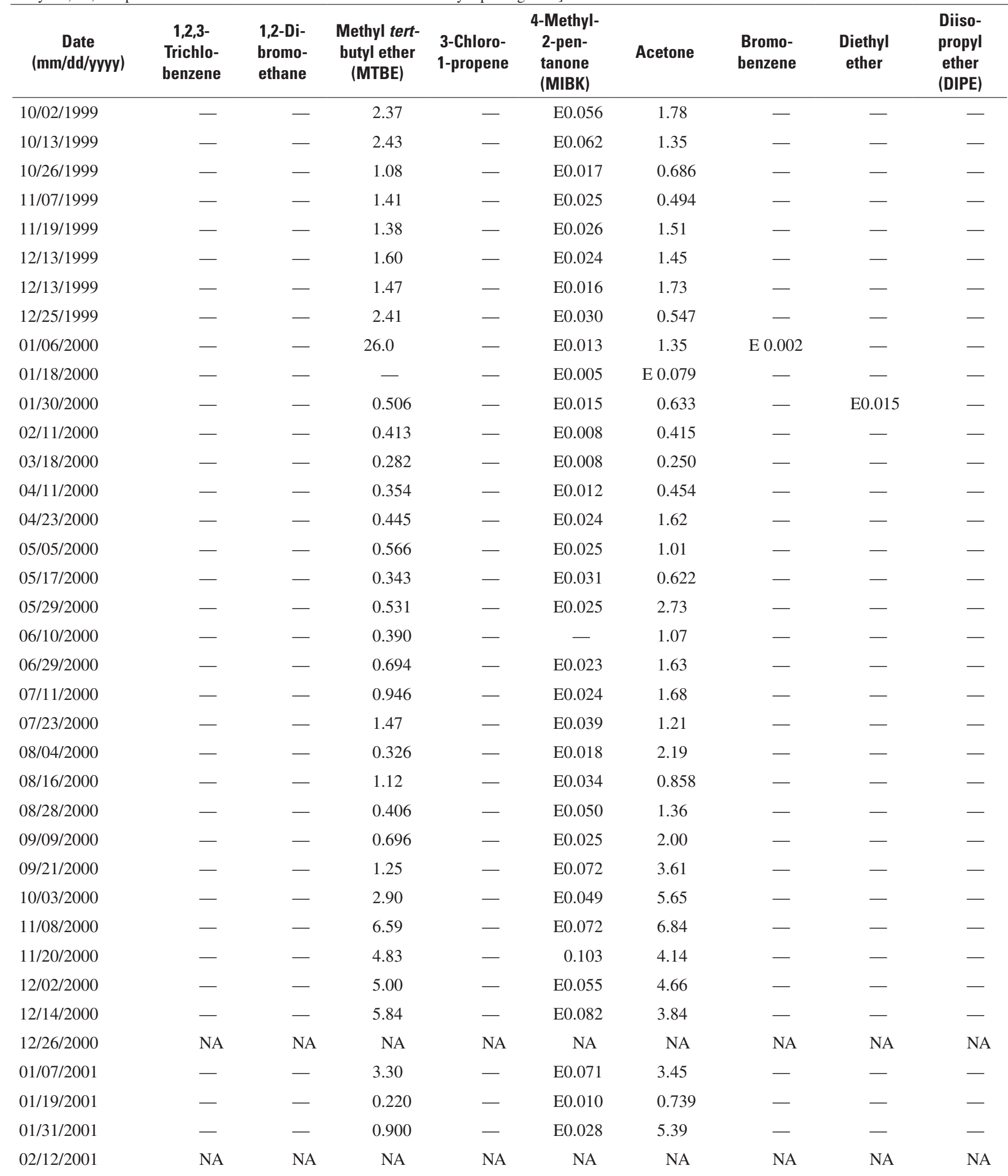


Table 5B. Analytical results for volatile organic compounds with high breakthrough volumes in air from the Sweetwater Reservoir air sampling site, San Diego County, California-Continued.

[The site identification number is 324141117001601 . Concentrations are given in parts per billion by volume (ppbv). Data for travel blanks and environmental samples collected on 10/27/2000 were not used because samples were contaminated during transport. E, estimated value; mm/dd/yyyy, month/day/year; NA, not analyzed; - , compound not detected at a concentration above laboratory reporting level]

\begin{tabular}{|c|c|c|c|c|c|c|c|c|c|}
\hline $\begin{array}{c}\text { Date } \\
\text { (mm/dd/yyyy) }\end{array}$ & $\begin{array}{c}1,2,3- \\
\text { Trichlo- } \\
\text { benzene }\end{array}$ & $\begin{array}{l}\text { 1,2-Di- } \\
\text { bromo- } \\
\text { ethane }\end{array}$ & $\begin{array}{l}\text { Methyl tert- } \\
\text { butyl ether } \\
\text { (MTBE) }\end{array}$ & $\begin{array}{l}\text { 3-Chloro- } \\
\text { 1-propene }\end{array}$ & $\begin{array}{l}\text { 4-Methyl- } \\
\text { 2-pen- } \\
\text { tanone } \\
\text { (MIBK) }\end{array}$ & Acetone & $\begin{array}{l}\text { Bromo- } \\
\text { benzene }\end{array}$ & $\begin{array}{l}\text { Diethyl } \\
\text { ether }\end{array}$ & $\begin{array}{l}\text { Diiso- } \\
\text { propyl } \\
\text { ether } \\
\text { (DIPE) }\end{array}$ \\
\hline $02 / 24 / 2001$ & - & - & 0.581 & - & - & 118 & - & - & E0.021 \\
\hline $02 / 24 / 2001$ & - & - & 0.768 & - & - & 142 & - & - & E0.016 \\
\hline 03/08/2001 & NA & NA & NA & NA & NA & NA & NA & NA & NA \\
\hline 03/08/2001 & NA & NA & NA & NA & NA & NA & NA & NA & NA \\
\hline 03/20/2001 & - & - & - & - & E0.008 & 2.07 & - & - & - \\
\hline 04/01/2001 & - & - & 1.43 & - & E0.035 & 1.94 & - & - & - \\
\hline $04 / 13 / 2001$ & - & - & - & - & E0.007 & 5.12 & - & - & - \\
\hline $04 / 13 / 2001$ & - & - & 1.35 & - & E0.052 & 4.06 & - & - & - \\
\hline $04 / 25 / 2001$ & - & - & 0.410 & - & E0.010 & 0.967 & - & - & - \\
\hline 05/07/2001 & - & - & 0.603 & - & - & 1.89 & - & - & - \\
\hline 05/19/2001 & - & - & - & - & - & - & - & - & - \\
\hline $05 / 31 / 2001$ & - & - & - & - & - & - & - & - & - \\
\hline $05 / 31 / 2001$ & - & - & - & - & - & 1.40 & - & - & - \\
\hline $06 / 12 / 2001$ & NA & NA & NA & NA & NA & NA & NA & NA & NA \\
\hline $06 / 24 / 2001$ & - & - & - & - & - & 1.68 & - & - & - \\
\hline $07 / 05 / 2001$ & - & - & - & - & - & - & - & - & - \\
\hline $07 / 05 / 2001$ & - & - & E0.112 & - & - & 1.20 & - & - & - \\
\hline $07 / 16 / 2001$ & - & - & - & - & - & - & - & - & - \\
\hline 08/08/2001 & - & - & - & - & - & - & - & - & - \\
\hline $08 / 23 / 2001$ & - & - & 1.28 & - & E0.037 & 2.86 & - & - & - \\
\hline $09 / 16 / 2001$ & - & - & 0.416 & - & E0.005 & 1.01 & - & - & - \\
\hline 09/28/2001 & - & - & - & - & - & - & - & - & - \\
\hline
\end{tabular}


Table 5B. Analytical results for volatile organic compounds with high breakthrough volumes in air from the Sweetwater Reservoir air sampling site, San Diego County, California-Continued.

[The site identification number is 324141117001601 . Concentrations are given in parts per billion by volume (ppbv). Data for travel blanks and environmental samples collected on 10/27/2000 were not used because samples were contaminated during transport. E, estimated value; mm/dd/yyyy, month/day/year; NA, not analyzed; - , compound not detected at a concentration above laboratory reporting level]

\begin{tabular}{|c|c|c|c|c|c|c|}
\hline $\begin{array}{c}\text { Date } \\
\text { (mm/dd/yyyv) }\end{array}$ & $\begin{array}{c}\text { Methyl } \\
\text { acrylonitrile }\end{array}$ & $\begin{array}{l}\text { 2-Butanone } \\
\text { (Methyl } \\
\text { ethyl ketone) }\end{array}$ & Methyl acrylate & Tetrahydrofuran & $\begin{array}{l}\text { 1,2-Dibromo- } \\
\text { 3-chloropropane } \\
\text { (DBCP) }\end{array}$ & $m$ - and $p$-Xylene \\
\hline $10 / 02 / 1999$ & - & 0.607 & - & E0.019 & - & 0.997 \\
\hline 10/13/1999 & - & 0.423 & - & E0.019 & - & 1.20 \\
\hline 10/26/1999 & - & 0.235 & - & E0.012 & - & 0.469 \\
\hline 11/07/1999 & - & 0.345 & - & E0.014 & - & 0.613 \\
\hline 11/19/1999 & E0.010 & 0.375 & - & E0.022 & - & 0.622 \\
\hline 12/13/1999 & E0.015 & 0.387 & - & E0.018 & - & 0.634 \\
\hline 12/13/1999 & - & 0.199 & - & E0.022 & - & 0.634 \\
\hline $12 / 25 / 1999$ & - & 0.230 & - & E0.030 & - & 1.12 \\
\hline 01/06/2000 & - & E0.126 & - & - & - & 0.229 \\
\hline 01/18/2000 & - & E0.039 & - & - & - & E0.036 \\
\hline 01/30/2000 & - & E0.012 & - & - & - & 0.232 \\
\hline $02 / 11 / 2000$ & - & E0.057 & - & - & - & 0.195 \\
\hline 03/18/2000 & - & E0.047 & - & - & - & E0.072 \\
\hline 04/11/2000 & - & 0.192 & - & - & - & 0.108 \\
\hline $04 / 23 / 2000$ & - & 0.324 & - & - & - & 0.276 \\
\hline 05/05/2000 & - & 0.243 & - & - & - & 0.333 \\
\hline 05/17/2000 & - & 0.267 & - & - & - & 0.212 \\
\hline 05/29/2000 & - & 0.198 & - & - & - & 0.239 \\
\hline 06/10/2000 & - & E0.112 & - & - & - & 0.143 \\
\hline 06/29/2000 & - & 0.201 & - & E0.013 & - & 0.303 \\
\hline 07/11/2000 & - & 0.313 & - & E0.017 & - & 0.429 \\
\hline 07/23/2000 & - & 0.389 & - & E0.005 & - & 1.07 \\
\hline 08/04/2000 & - & 0.306 & - & - & - & 0.209 \\
\hline 08/16/2000 & - & 0.313 & - & - & - & 0.387 \\
\hline 08/28/2000 & - & 0.219 & - & E0.013 & - & 0.179 \\
\hline 09/09/2000 & - & 0.457 & - & E0.006 & - & 0.282 \\
\hline 09/21/2000 & - & 0.555 & - & E0.011 & - & 1.03 \\
\hline $10 / 03 / 2000$ & - & 0.906 & - & E0.047 & - & 1.30 \\
\hline $11 / 08 / 2000$ & - & 1.59 & - & - & - & 2.10 \\
\hline $11 / 20 / 2000$ & - & 1.02 & - & E0.108 & - & 3.63 \\
\hline $12 / 02 / 2000$ & - & 0.411 & - & E0.070 & - & 2.38 \\
\hline $12 / 14 / 2000$ & - & 2.25 & - & - & - & 1.62 \\
\hline $12 / 26 / 2000$ & NA & NA & NA & NA & NA & NA \\
\hline 01/07/2001 & - & 0.340 & - & - & - & 2.58 \\
\hline 01/19/2001 & - & 0.220 & - & - & - & 0.212 \\
\hline $01 / 31 / 2001$ & - & 0.494 & - & - & - & 0.503 \\
\hline $02 / 12 / 2001$ & NA & NA & NA & NA & NA & NA \\
\hline $02 / 24 / 2001$ & - & 0.146 & - & - & - & 0.433 \\
\hline
\end{tabular}


Table 5B. Analytical results for volatile organic compounds with high breakthrough volumes in air from the Sweetwater Reservoir air sampling site, San Diego County, California-Continued.

[The site identification number is 324141117001601 . Concentrations are given in parts per billion by volume (ppbv). Data for travel blanks and environmental samples collected on 10/27/2000 were not used because samples were contaminated during transport. E, estimated value; mm/dd/yyyy, month/day/year; NA, not analyzed; - , compound not detected at a concentration above laboratory reporting level]

\begin{tabular}{|c|c|c|c|c|c|c|}
\hline $\begin{array}{c}\text { Date } \\
\text { (mm/dd/yyyy) }\end{array}$ & $\begin{array}{c}\text { Methyl } \\
\text { acrylonitrile }\end{array}$ & $\begin{array}{l}\text { 2-Butanone } \\
\text { (Methyl } \\
\text { ethyl ketone) }\end{array}$ & Methyl acrylate & Tetrahydrofuran & $\begin{array}{l}\text { 1,2-Dibromo- } \\
\text { 3-chloropropane } \\
\text { (DBCP) }\end{array}$ & $m$ - and $p$-Xylene \\
\hline $02 / 24 / 2001$ & - & 0.229 & - & - & - & 0.539 \\
\hline 03/08/2001 & NA & NA & NA & NA & NA & NA \\
\hline 03/08/2001 & NA & NA & NA & NA & NA & NA \\
\hline 03/20/2001 & E0.019 & 0.257 & - & - & - & E0.053 \\
\hline 04/01/2001 & - & 0.512 & - & - & - & 0.738 \\
\hline 04/13/2001 & - & E0.121 & - & - & - & E0.032 \\
\hline $04 / 13 / 2001$ & - & 0.647 & - & - & - & 0.652 \\
\hline $04 / 25 / 2001$ & - & E0.134 & - & - & - & 0.166 \\
\hline 05/07/2001 & - & 0.162 & - & - & - & 0.253 \\
\hline 05/19/2001 & - & - & - & - & - & E0.023 \\
\hline 05/31/2001 & - & - & - & - & - & - \\
\hline 05/31/2001 & - & - & - & - & - & - \\
\hline $06 / 12 / 2001$ & NA & NA & NA & NA & NA & NA \\
\hline $06 / 24 / 2001$ & - & E0.080 & - & - & - & - \\
\hline 07/05/2001 & - & - & - & - & - & E0.072 \\
\hline 07/05/2001 & - & E0.126 & - & - & - & E0.061 \\
\hline 07/16/2001 & - & - & - & - & - & - \\
\hline 08/08/2001 & - & - & - & - & - & - \\
\hline 08/23/2001 & - & - & - & - & - & 0.538 \\
\hline 09/16/2001 & - & E0.109 & - & - & - & 0.164 \\
\hline 09/28/2001 & - & - & - & - & - & - \\
\hline
\end{tabular}


Table 5B. Analytical results for volatile organic compounds with high breakthrough volumes in air from the Sweetwater Reservoir air sampling site, San Diego County, California-Continued.

[The site identification number is 324141117001601. Concentrations are given in parts per billion by volume (ppbv). Data for travel blanks and environmental samples collected on 10/27/2000 were not used because samples were contaminated during transport. E, estimated value; mm/dd/yyyy, month/day/year; NA, not analyzed; - , compound not detected at a concentration above laboratory reporting level]

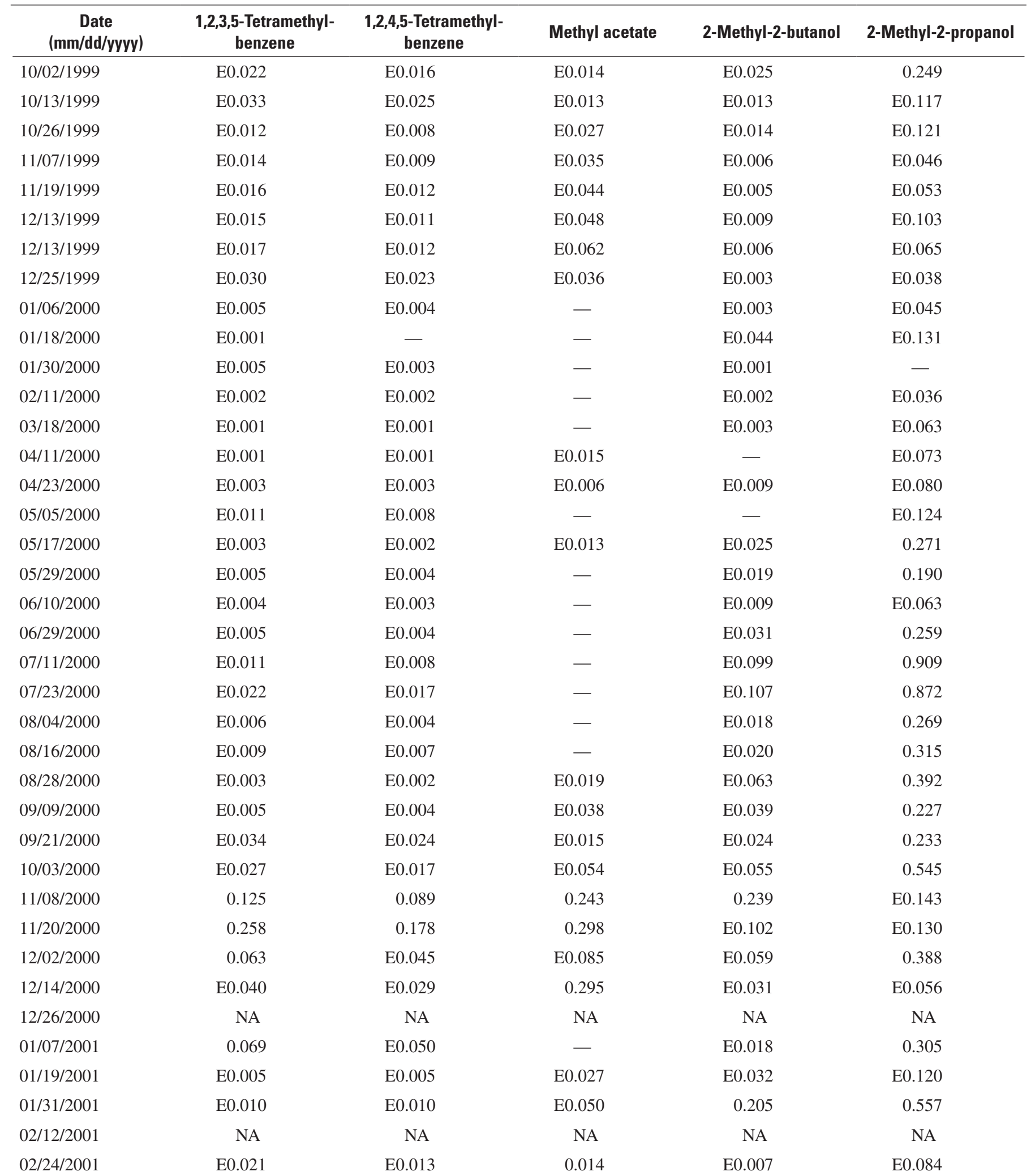


Table 5B. Analytical results for volatile organic compounds with high breakthrough volumes in air from the Sweetwater Reservoir air sampling site, San Diego County, California-Continued.

[The site identification number is 324141117001601. Concentrations are given in parts per billion by volume (ppbv). Data for travel blanks and environmental samples collected on 10/27/2000 were not used because samples were contaminated during transport. E, estimated value; mm/dd/yyyy, month/day/year; NA, not analyzed; - , compound not detected at a concentration above laboratory reporting level]

\begin{tabular}{|c|c|c|c|c|c|}
\hline $\begin{array}{c}\text { Date } \\
\text { (mm/dd/yyyy) }\end{array}$ & $\begin{array}{c}1,2,3,5-\text { Tetramethyl- } \\
\text { benzene }\end{array}$ & $\begin{array}{c}1,2,4,5-\text { Tetramethyl- } \\
\text { benzene }\end{array}$ & Methyl acetate & 2-Methyl-2-butanol & 2-Methyl-2-propanol \\
\hline $02 / 24 / 2001$ & E0.025 & E0.017 & 0.027 & E0.013 & E0.104 \\
\hline 03/08/2001 & NA & NA & NA & NA & NA \\
\hline 03/08/2001 & NA & NA & NA & NA & NA \\
\hline 03/20/2001 & E0.001 & E0.001 & - & E0.017 & 0.205 \\
\hline 04/01/2001 & E0.012 & E0.010 & - & E0.023 & 0.281 \\
\hline 04/13/2001 & E0.001 & E0.001 & - & E0.030 & 0.320 \\
\hline 04/13/2001 & E0.014 & E0.012 & - & E0.038 & 0.173 \\
\hline 04/25/2001 & E0.002 & E0.002 & - & - & E0.073 \\
\hline 05/07/2001 & E0.008 & E0.006 & - & E0.033 & 0.356 \\
\hline 05/19/2001 & - & - & - & E0.003 & E0.054 \\
\hline 05/31/2001 & - & - & - & E0.005 & - \\
\hline 05/31/2001 & - & - & - & E0.009 & E0.085 \\
\hline $06 / 12 / 2001$ & NA & NA & NA & NA & NA \\
\hline 06/24/2001 & - & - & - & E0.014 & 0.293 \\
\hline 07/05/2001 & - & - & - & E0.018 & 0.169 \\
\hline 07/05/2001 & E0.001 & E0.001 & - & E0.013 & E0.090 \\
\hline 07/16/2001 & - & - & - & - & - \\
\hline 08/08/2001 & - & - & - & - & - \\
\hline 08/23/2001 & E0.021 & E0.014 & - & E0.064 & E0.119 \\
\hline 09/16/2001 & E0.003 & E0.003 & - & E0.007 & E0.062 \\
\hline 09/28/2001 & - & - & - & - & - \\
\hline
\end{tabular}




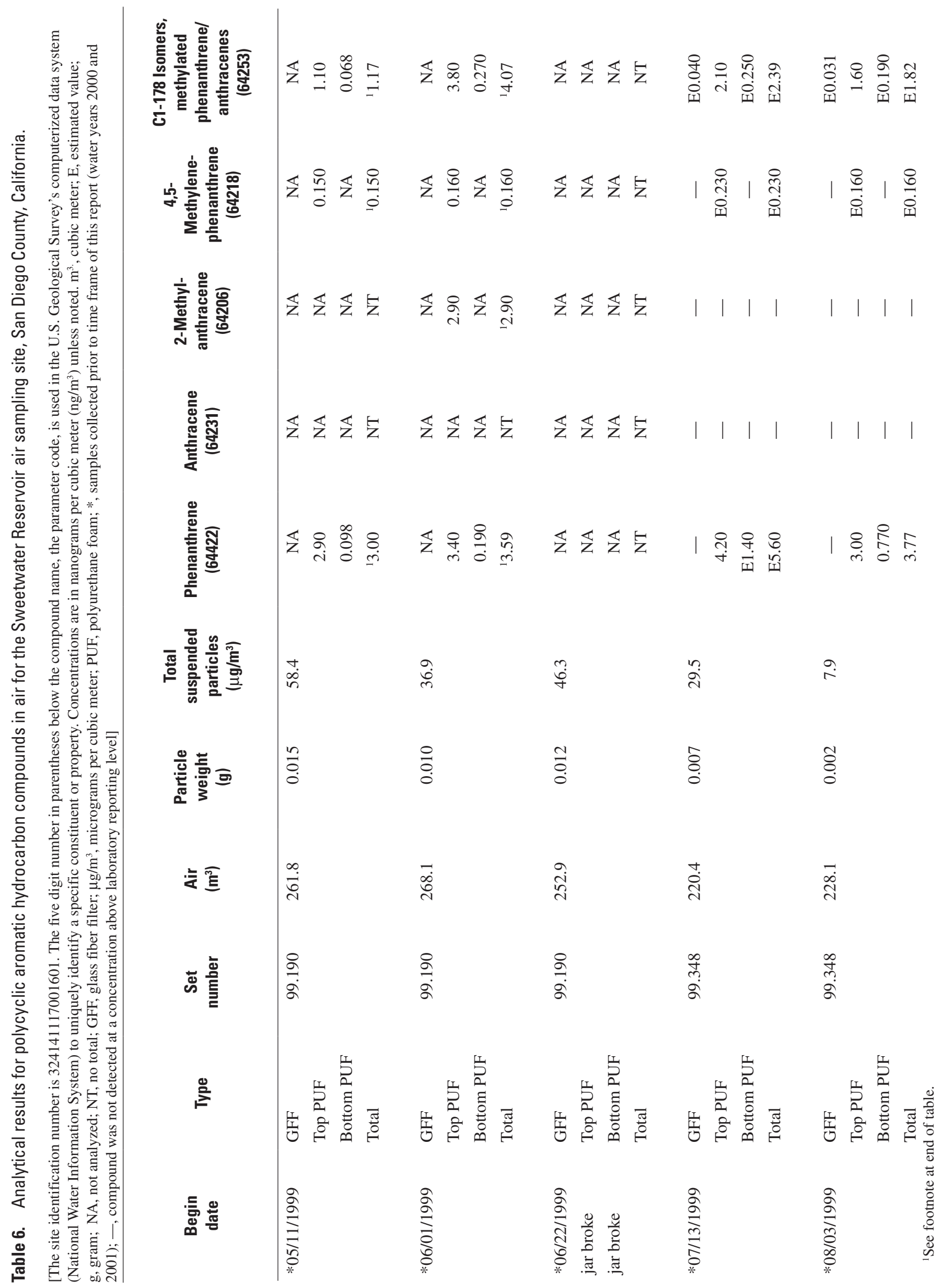




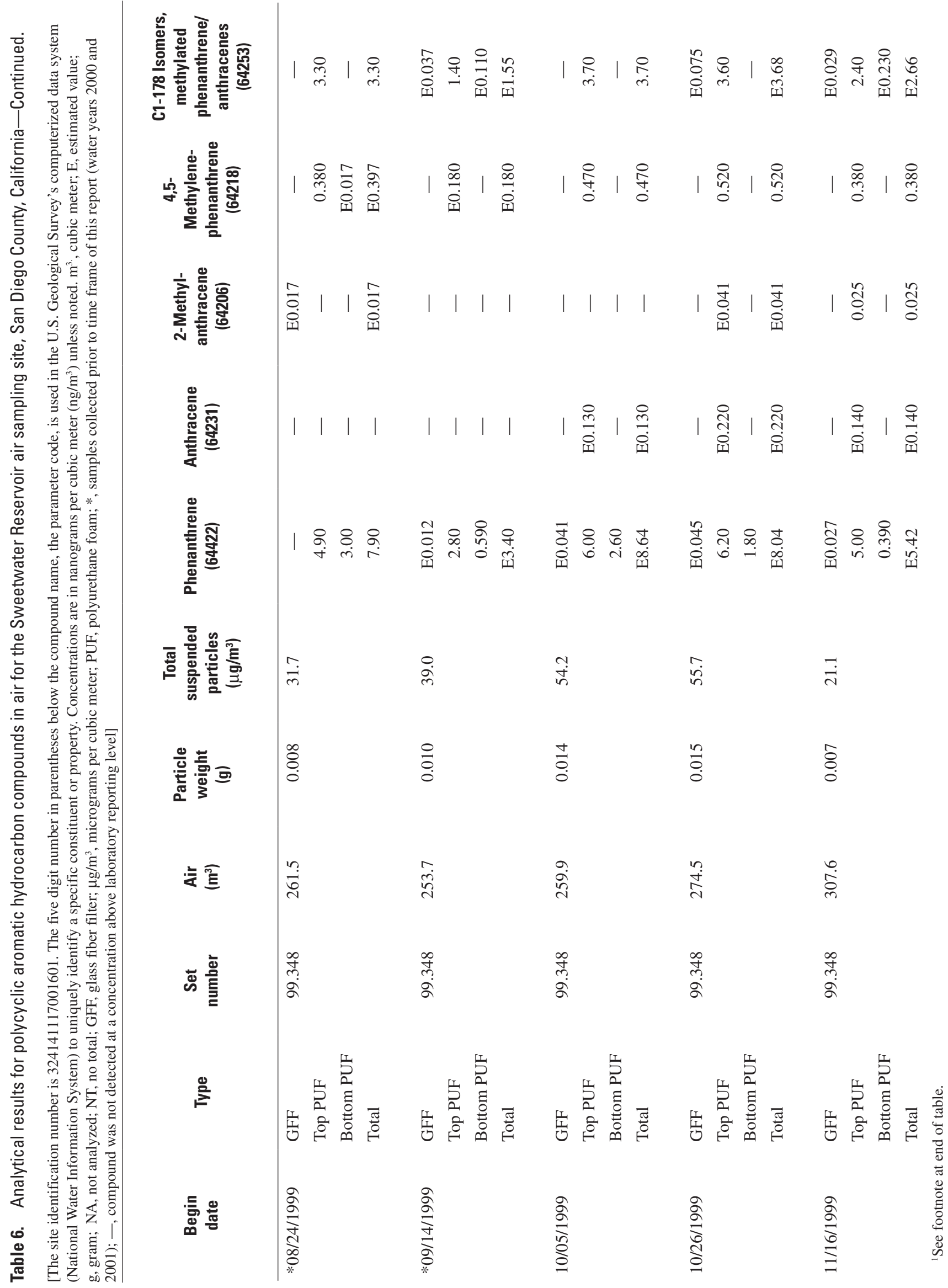




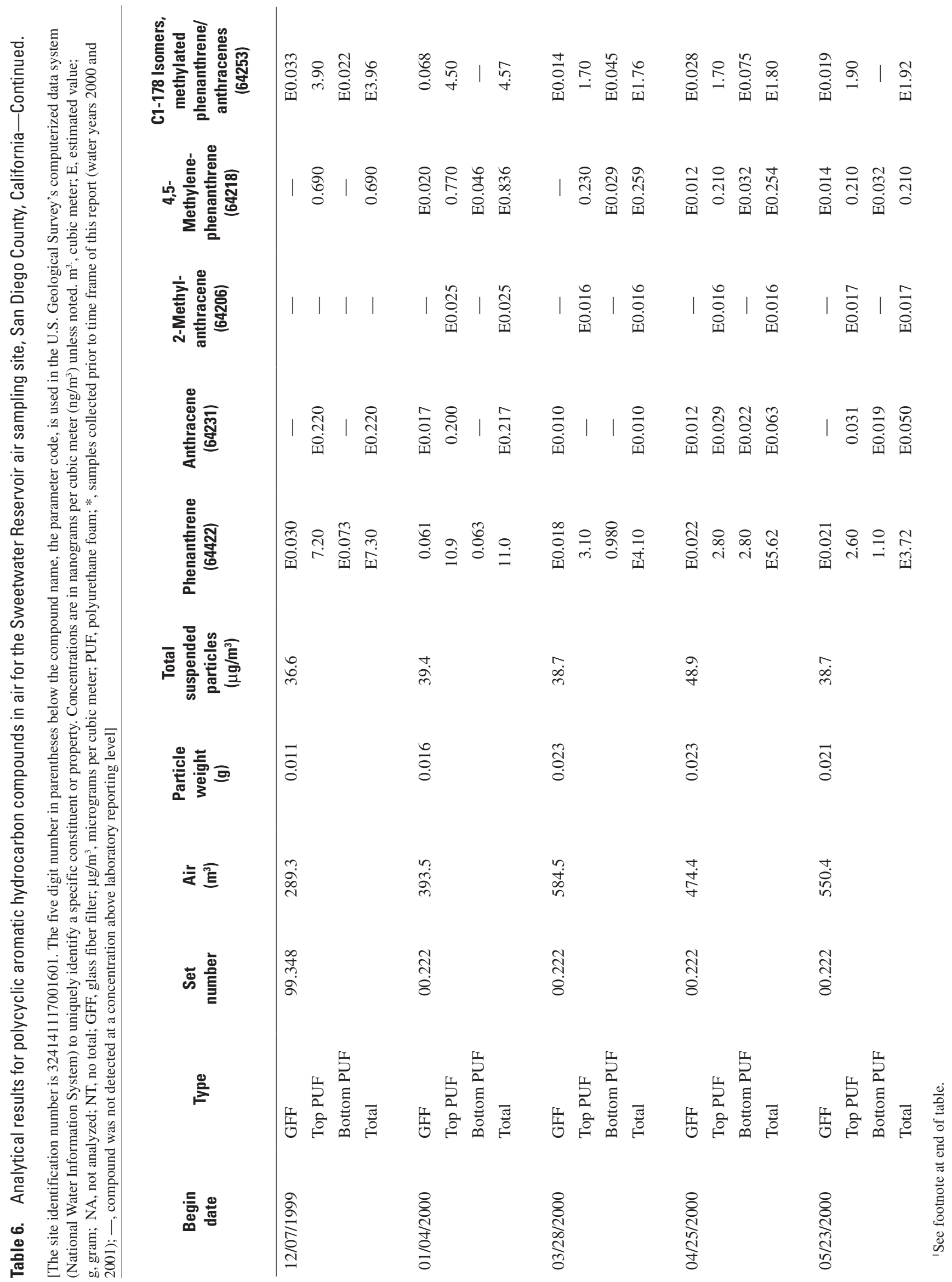




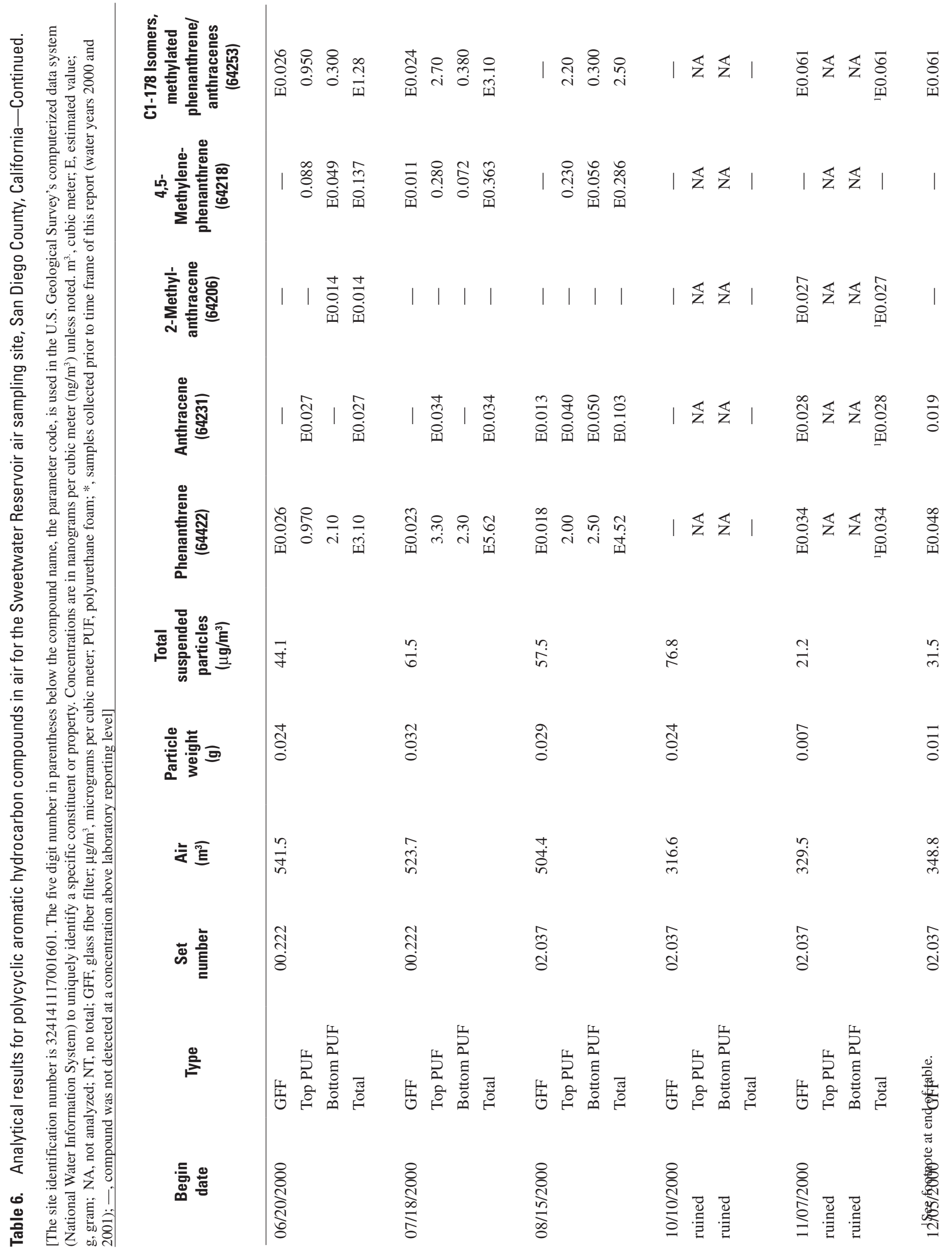




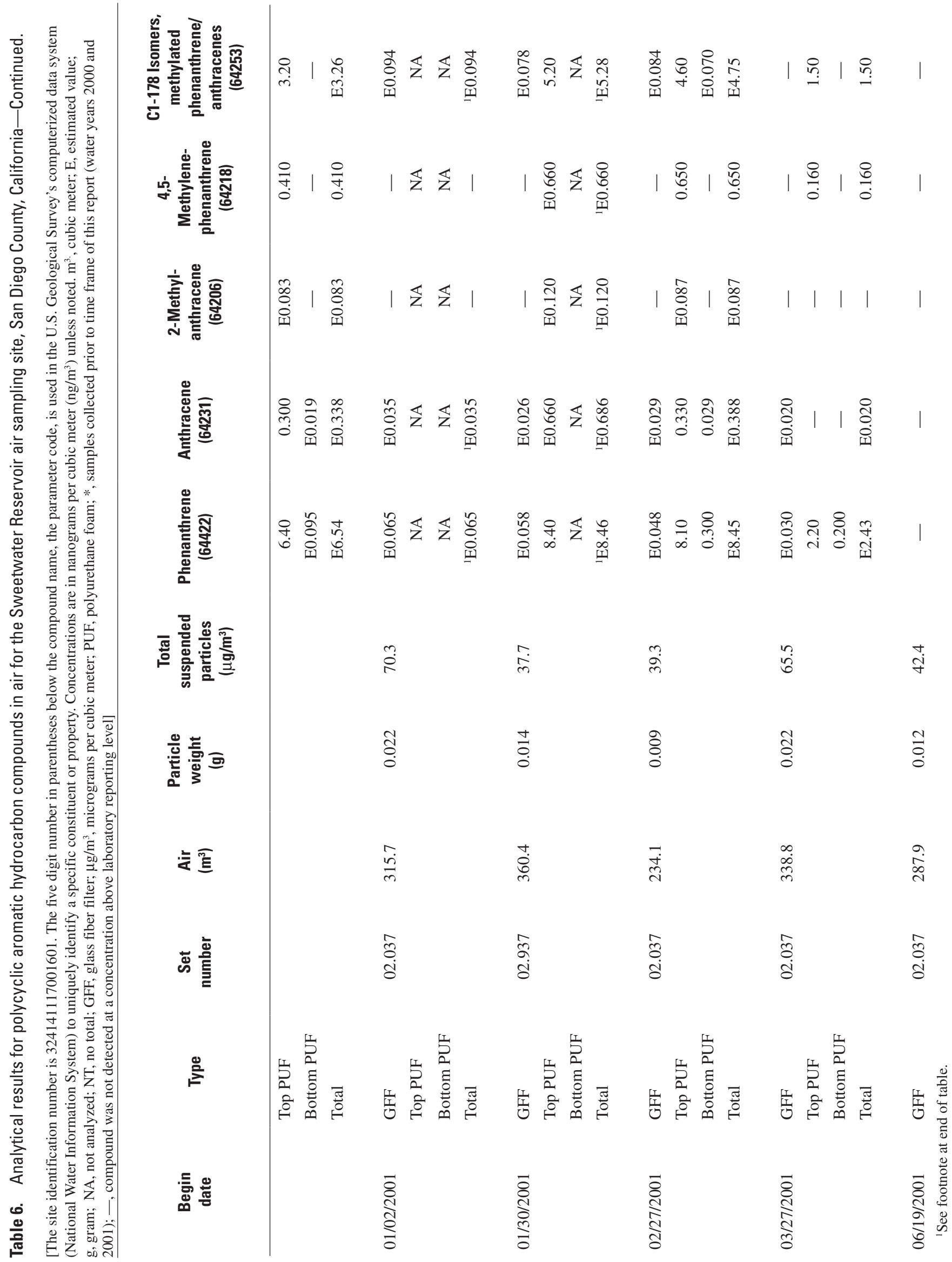




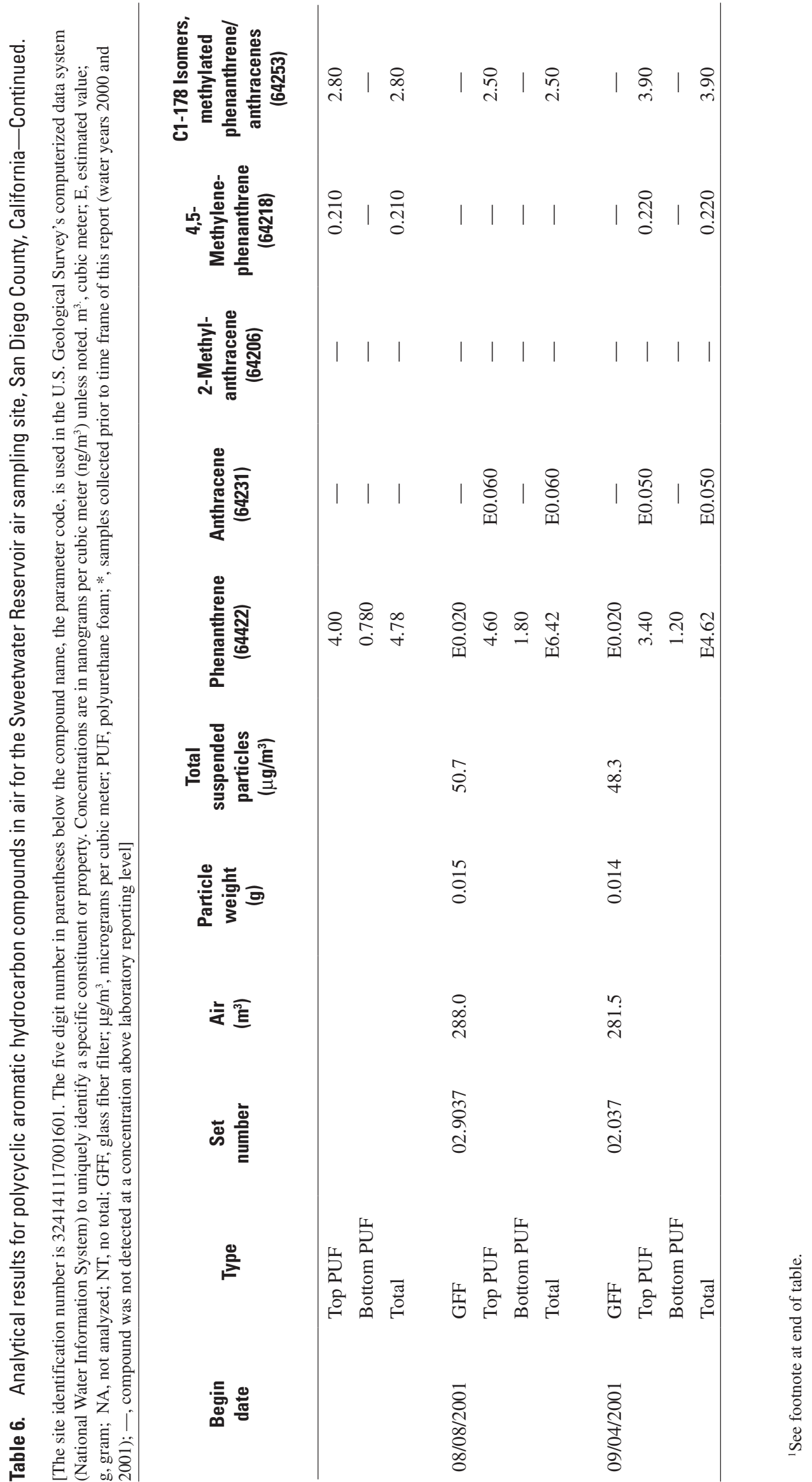




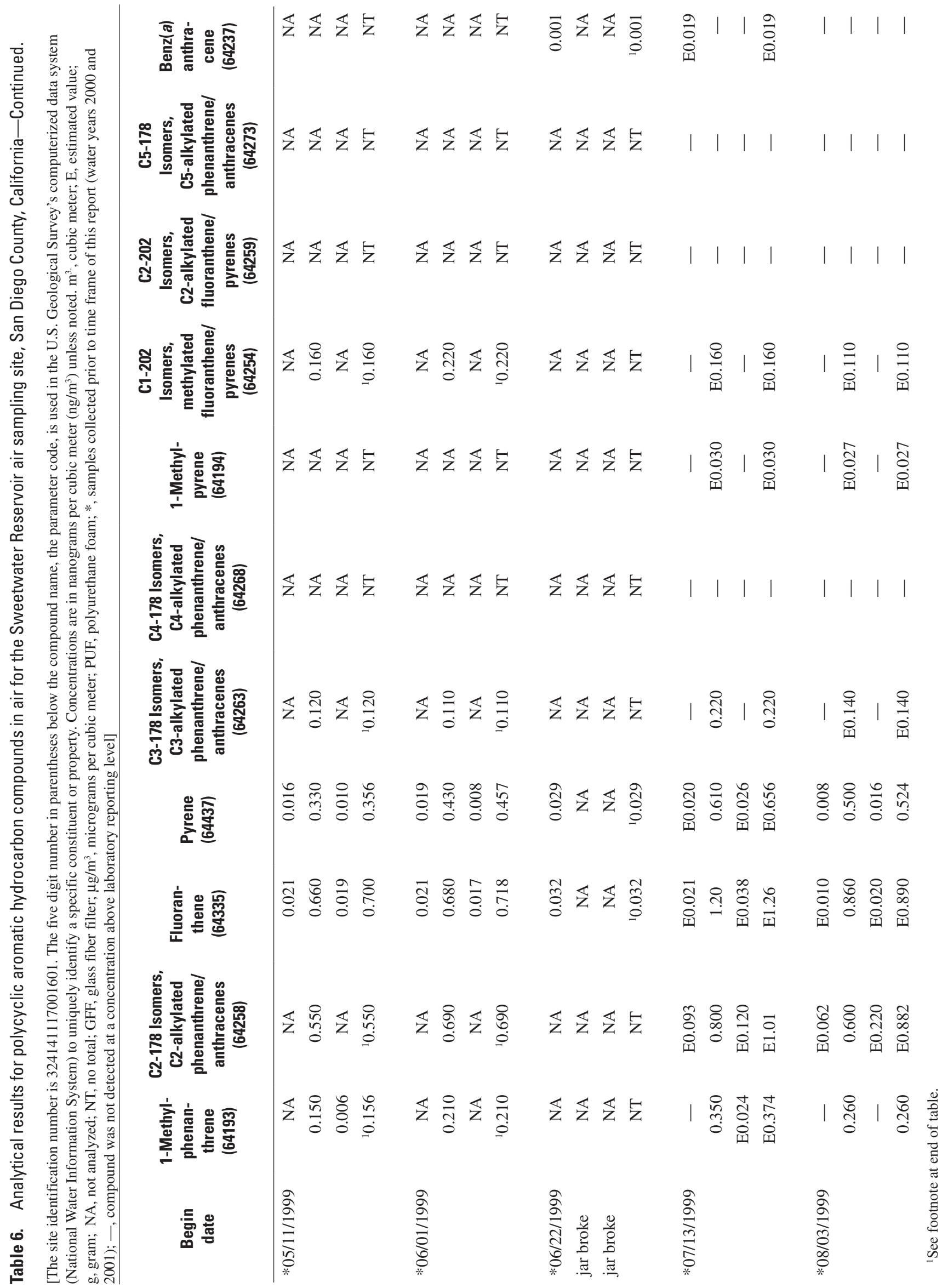




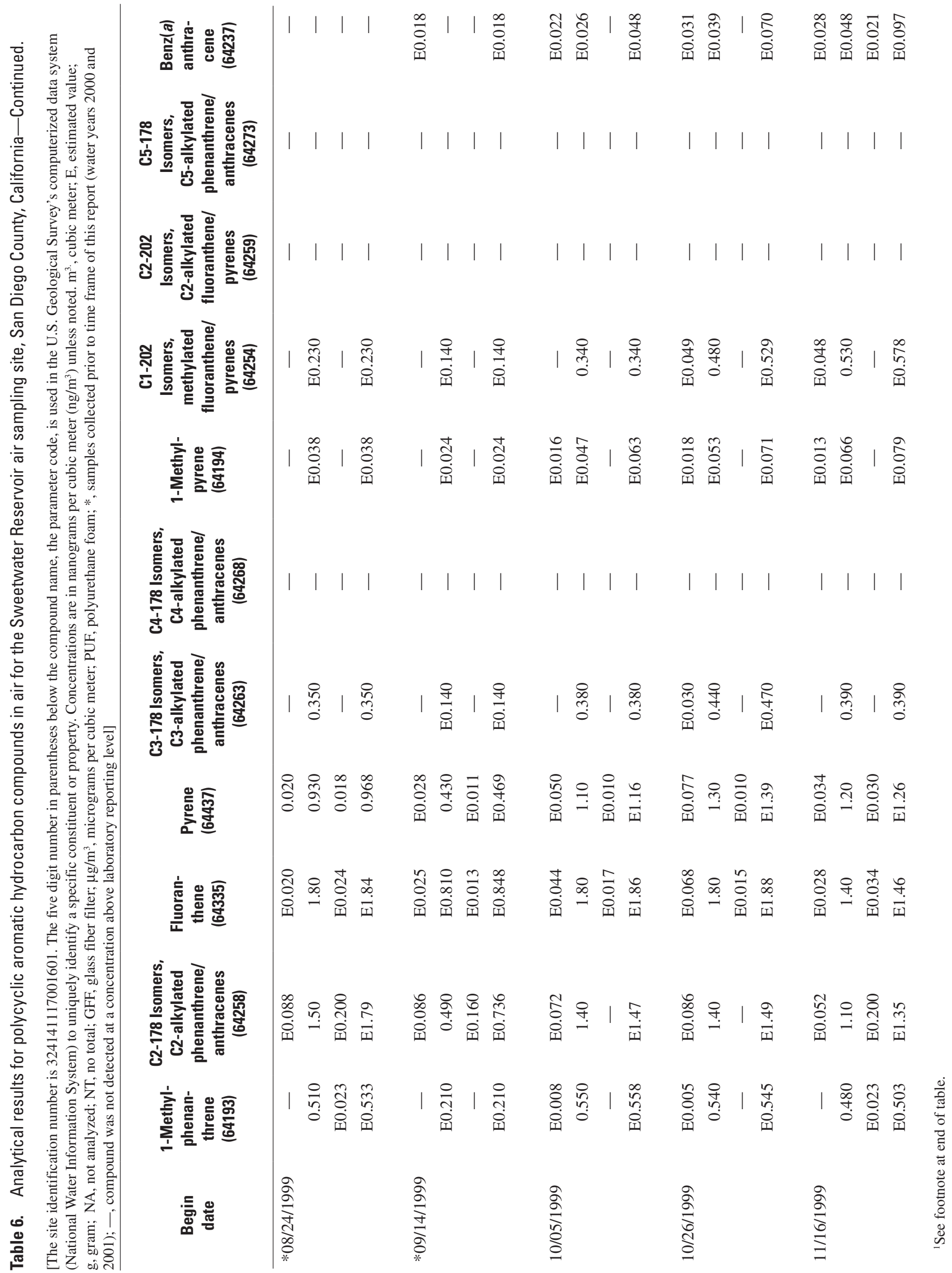




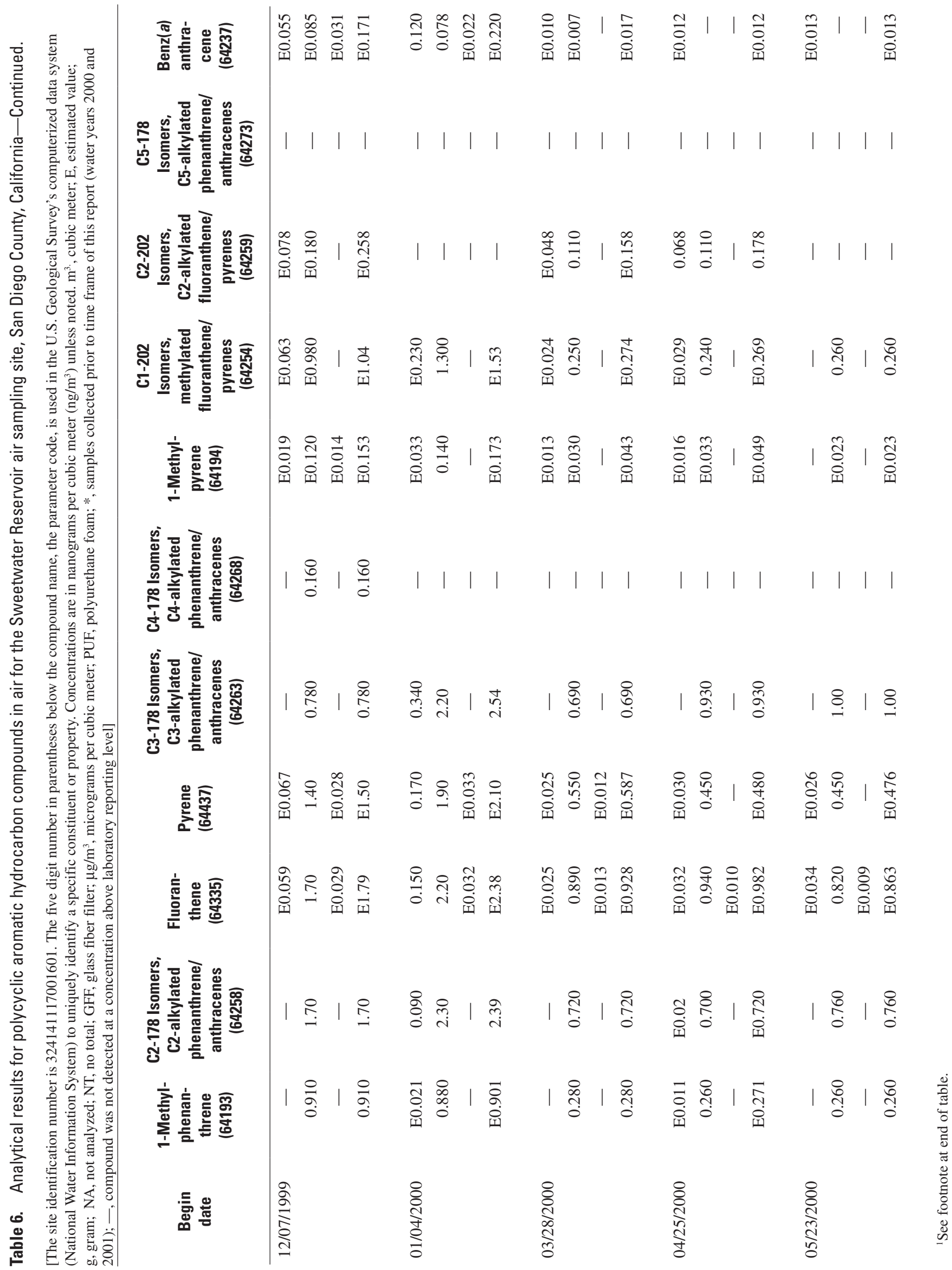




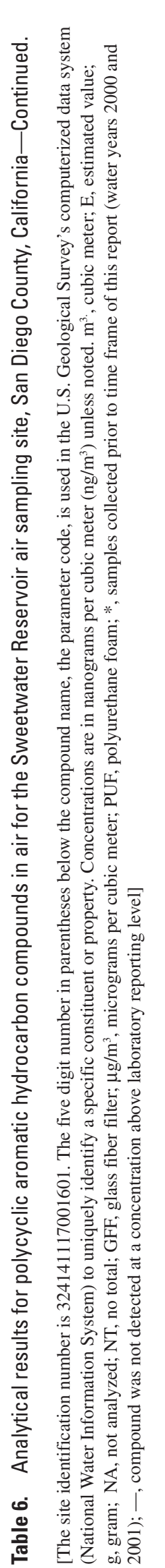

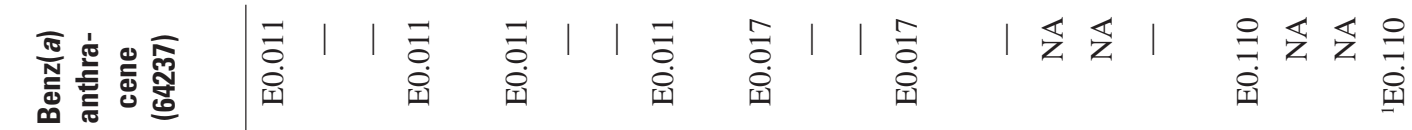

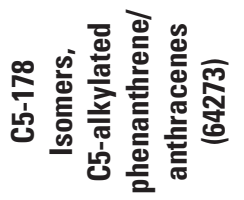

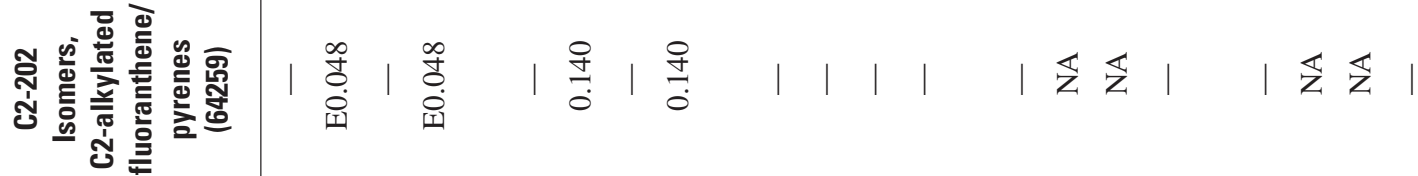

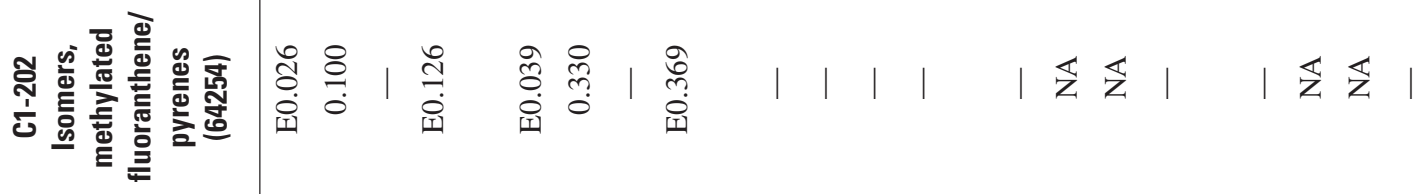

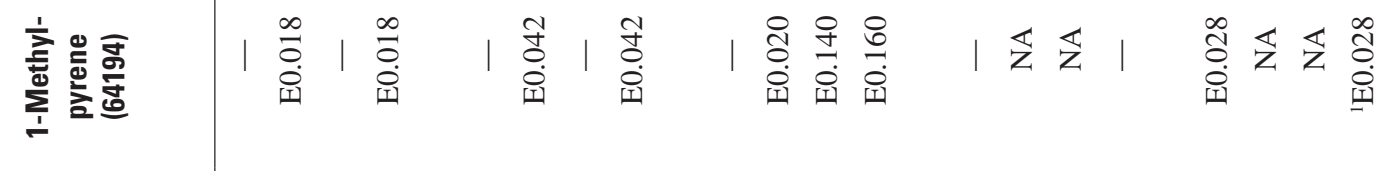

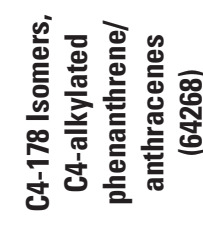

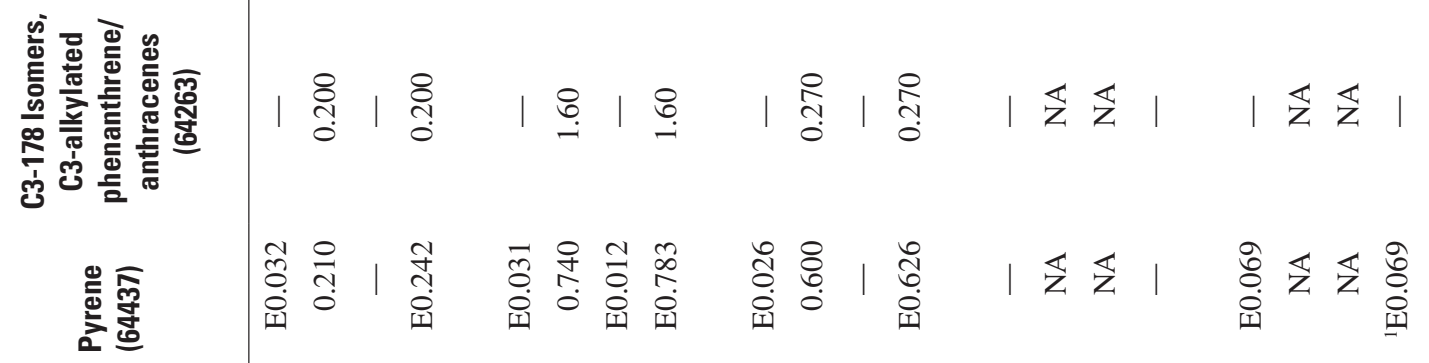

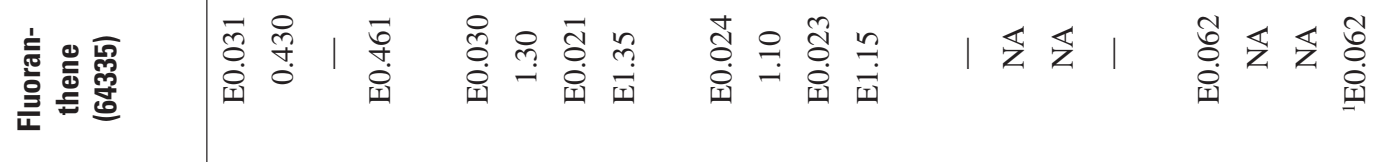

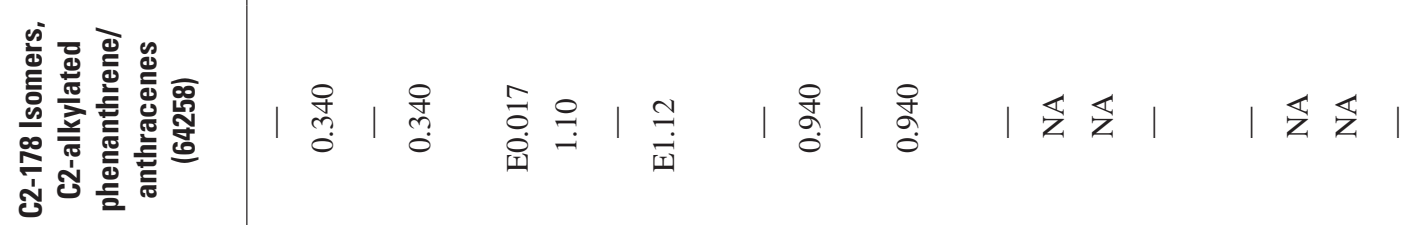

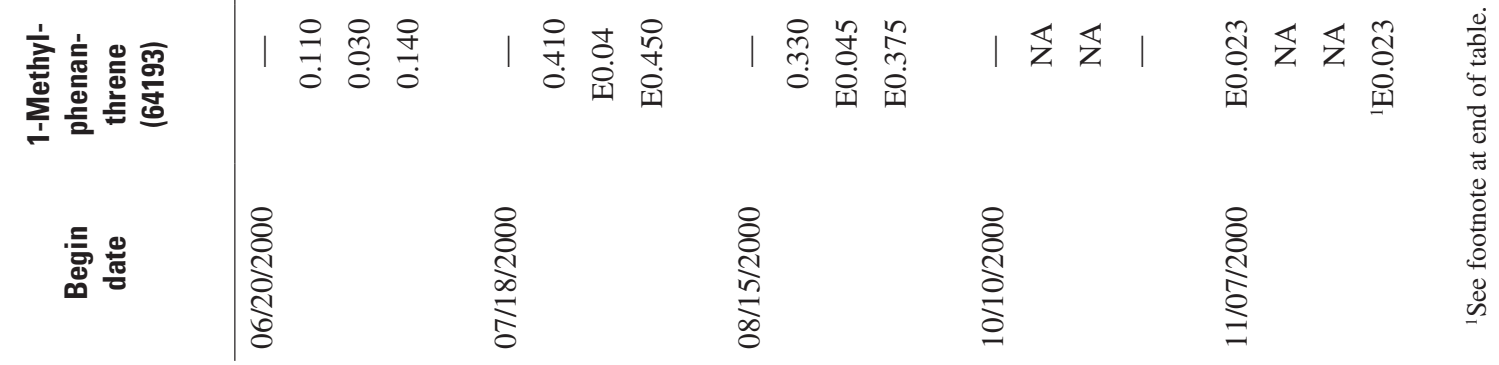




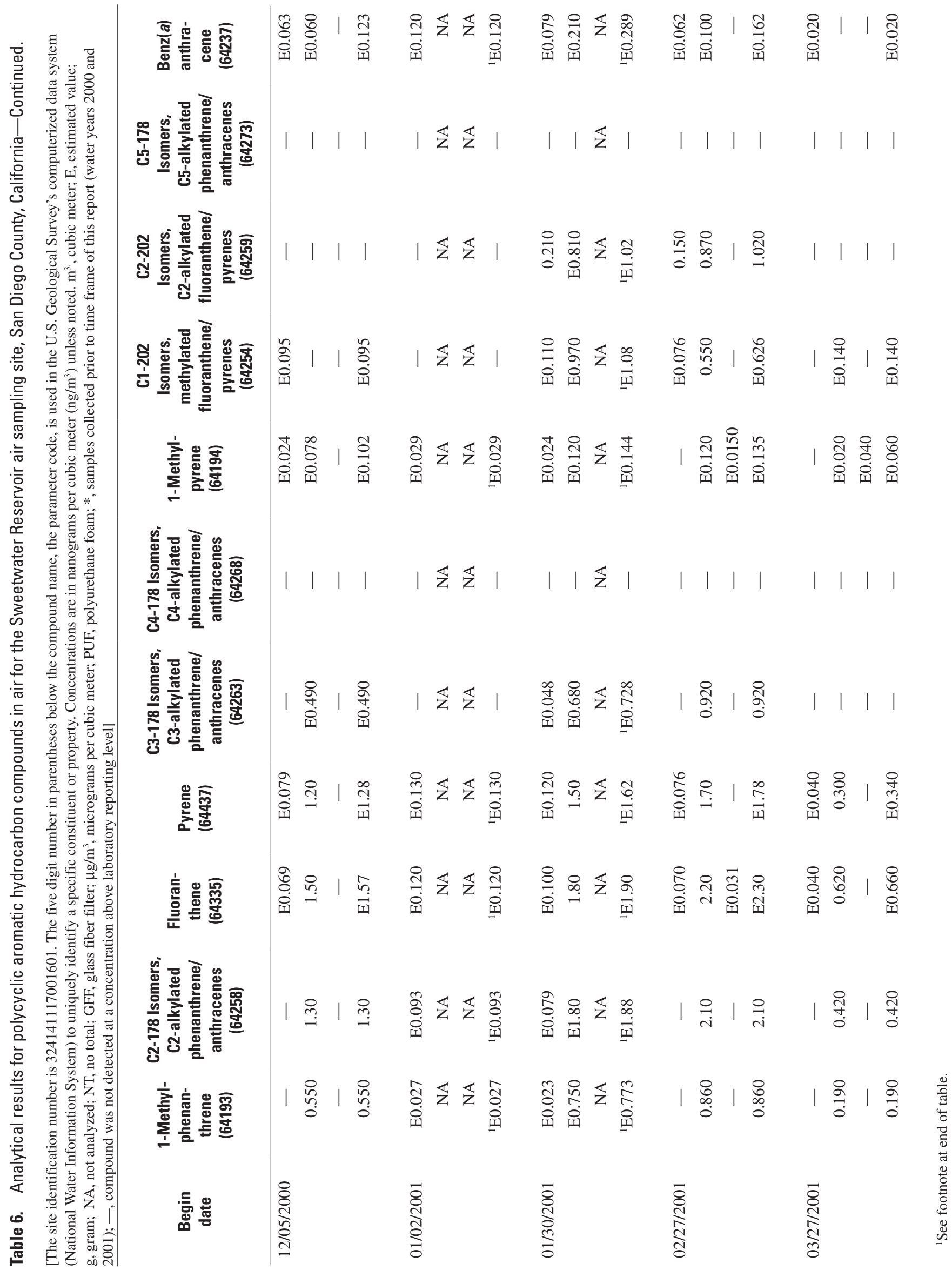




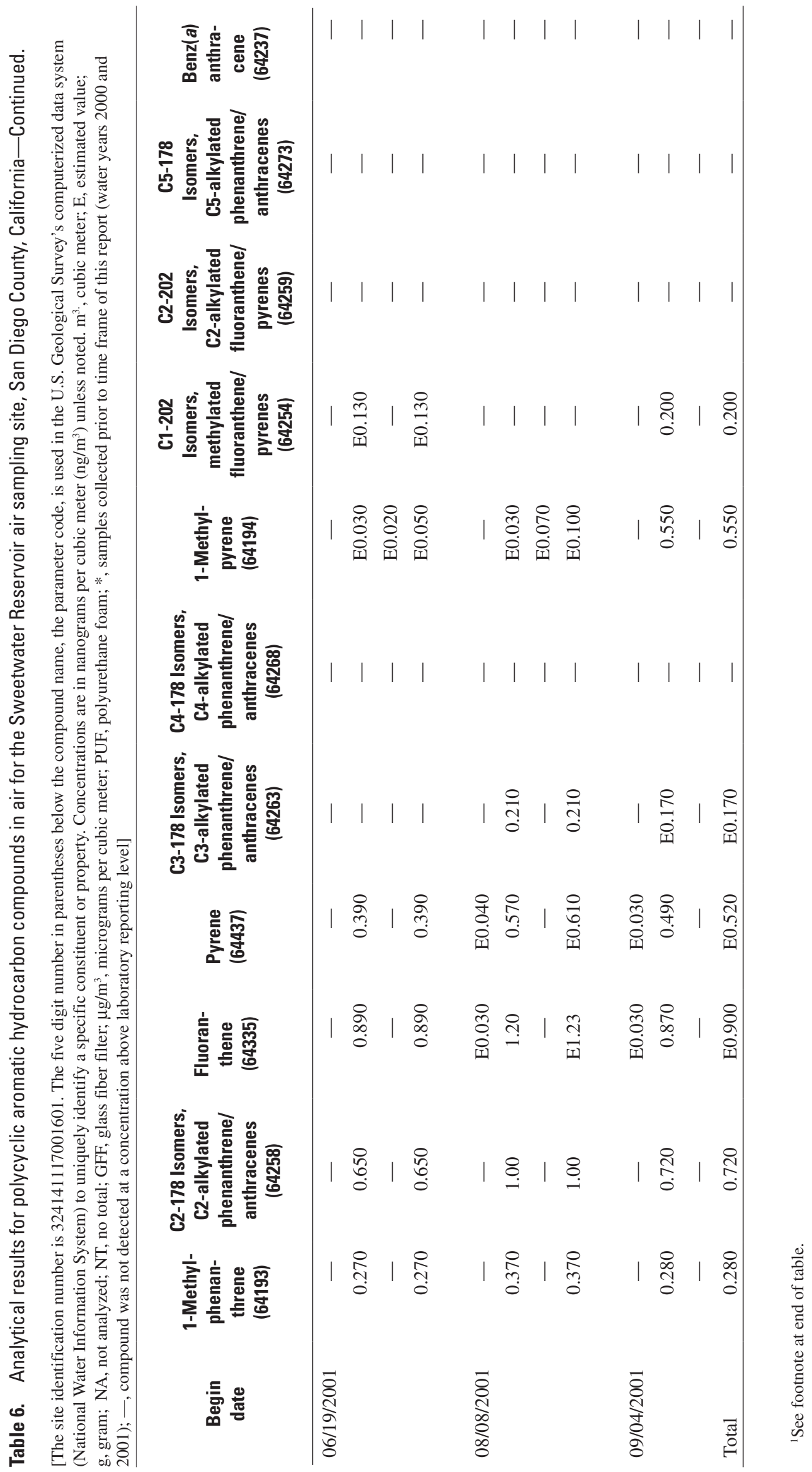




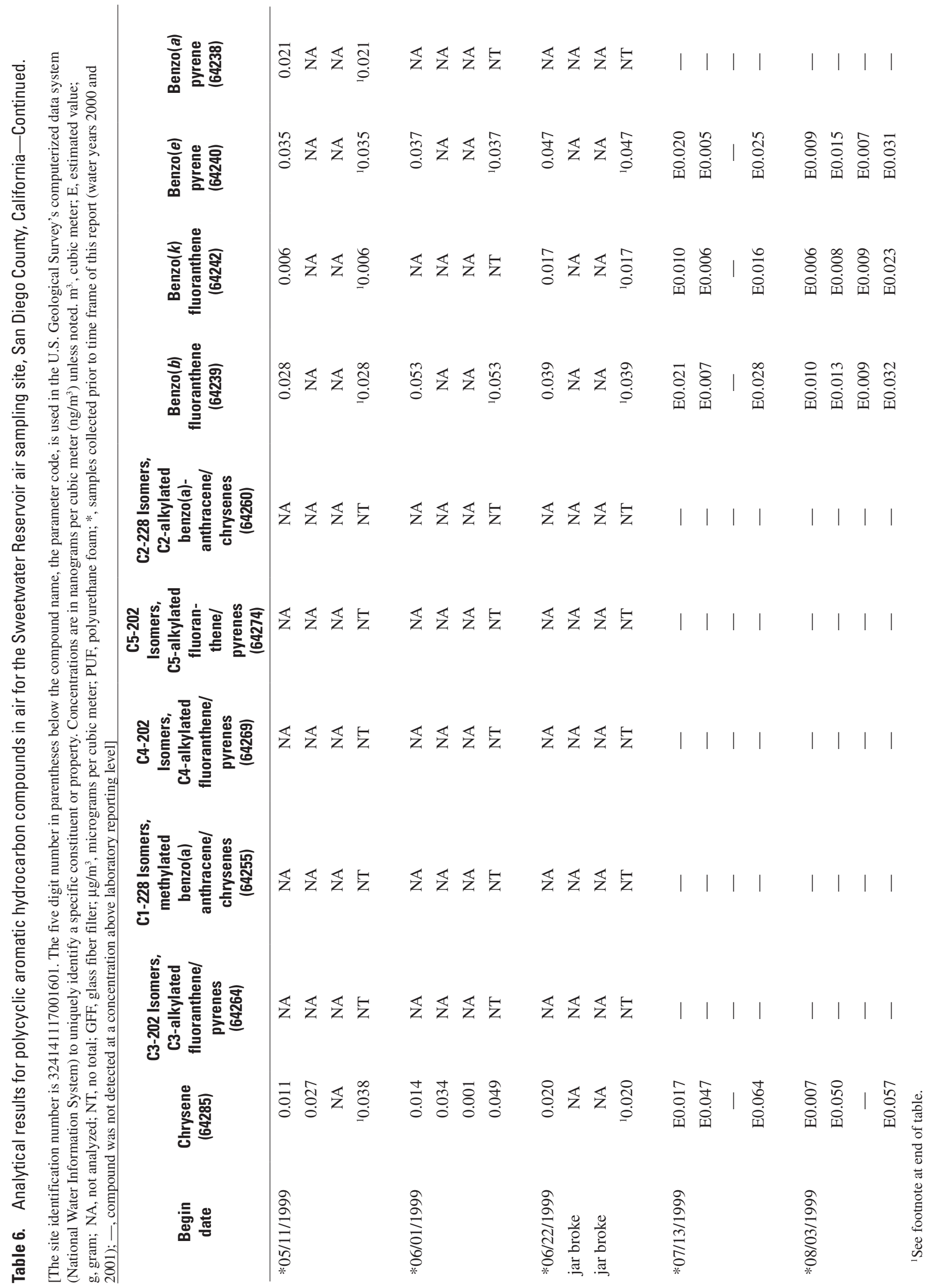


రं$$
\text { : }
$$$$
\text { . }
$$$$
\text { 政 }
$$$$
\text { . }
$$$$
\text { . }
$$

(9)

$$
\text { (2) }
$$

.

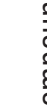

毫
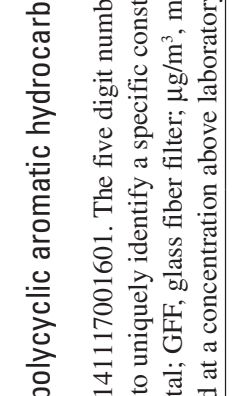

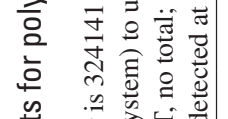
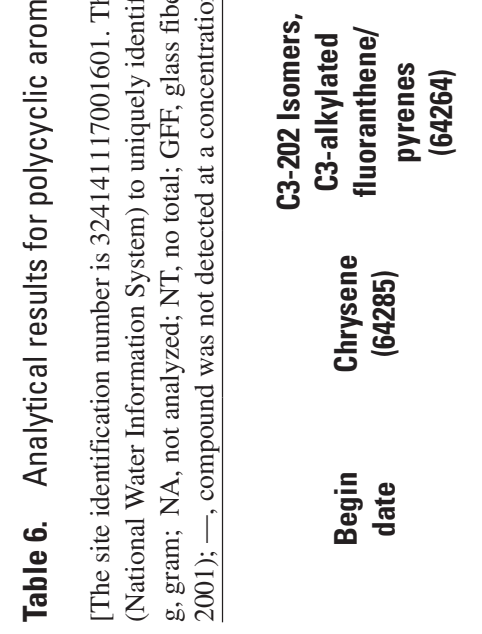

言警|

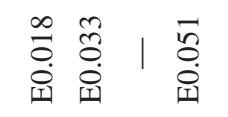

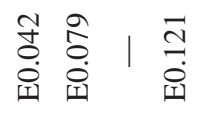

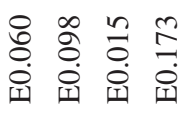

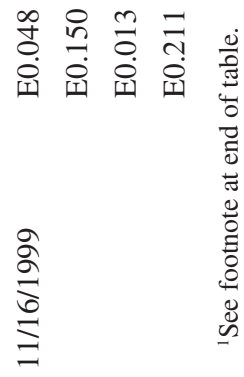




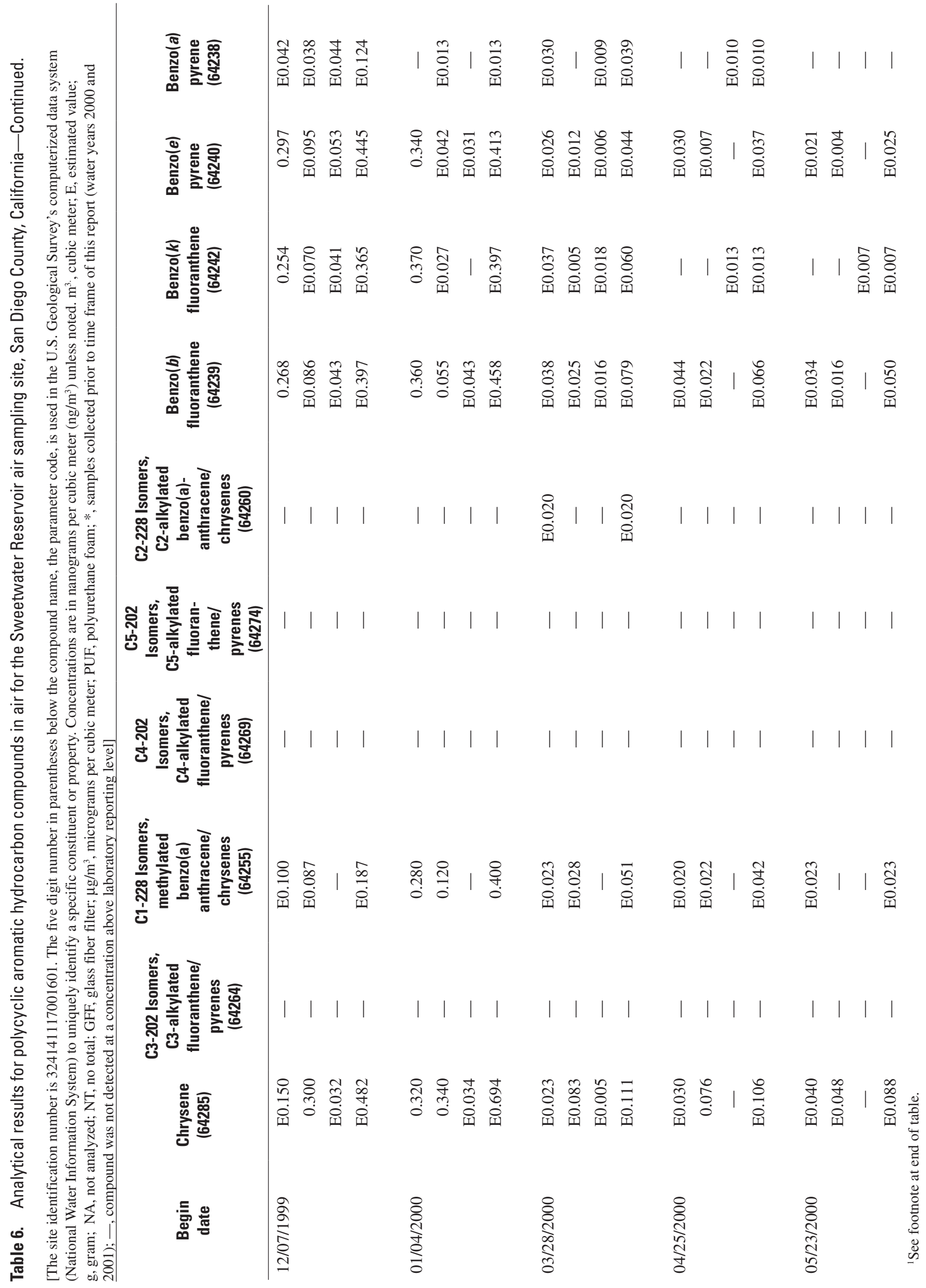




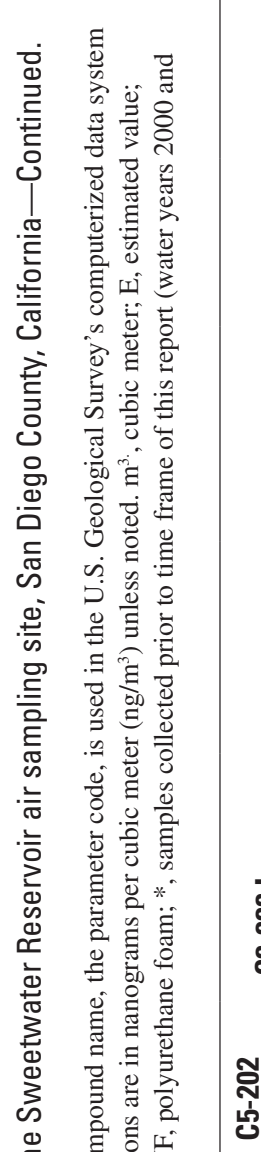

\begin{tabular}{|c|c|c|c|c|c|}
\hline 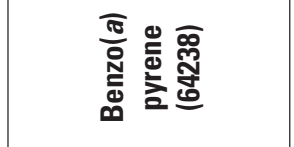 & 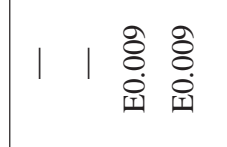 & 言| & 1111 & 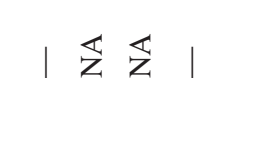 & 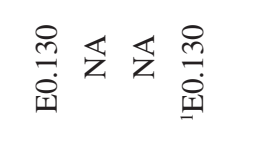 \\
\hline & 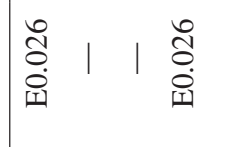 & 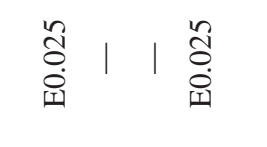 & 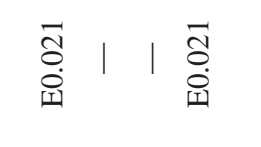 & $\left|\frac{1}{z} \frac{\pi}{z}\right|$ & 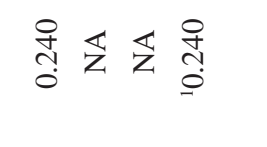 \\
\hline & | 1 | 1 兽 & 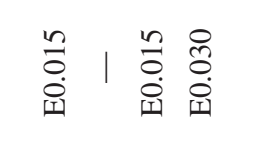 & 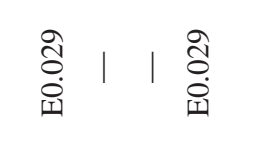 & 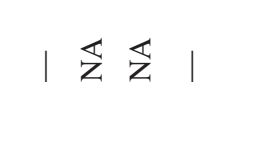 & 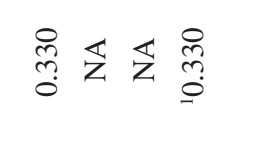 \\
\hline & | & 品总 | & 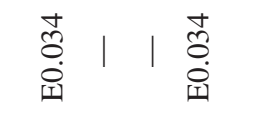 & $\left|\frac{\pi}{z}\right| \frac{\pi}{z} \mid$ & 总焉芸总 \\
\hline & $\mid \begin{array}{llll}1 & 1 & 1 & 1\end{array}$ & | | | | & 1111 & | $\left|\frac{1}{z} \frac{\pi}{z}\right|$ & | $\frac{\pi}{\mathrm{z}} \frac{\pi}{\mathrm{z}}$ \\
\hline 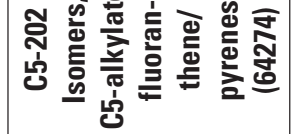 & 11 & I & 1111 & $\left|\frac{\pi}{z} \frac{\frac{\pi}{z}}{z}\right|$ & | $\frac{\tilde{z}}{z} \frac{\hat{z}}{z}$ \\
\hline 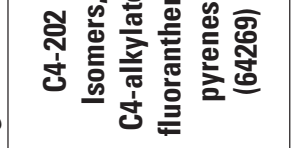 & $\begin{array}{lll}1 & 1 & 1\end{array}$ & & 1111 & $\left|\frac{1}{\frac{1}{z}}\right| \frac{\pi}{z} \mid$ & | $\frac{1}{\mathrm{z}} \frac{\hat{z}}{\mathrm{z}}$ \\
\hline & 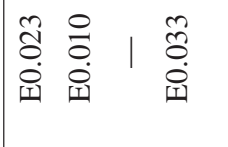 & 密言| & 1111 & $\left|\frac{1}{z} \frac{\frac{\pi}{厶}}{\mid}\right|$ & | $\frac{\tilde{z}}{\bar{z}}$ \\
\hline 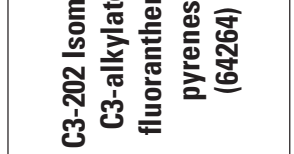 & 1111 & | | | | & 1111 & | $\left|\frac{\pi}{z} \frac{\pi}{z}\right|$ & | 考考 \\
\hline 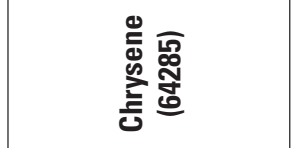 & 吾 & 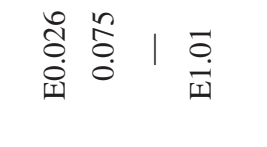 & 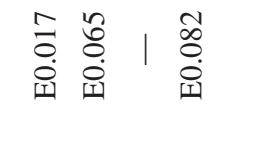 & 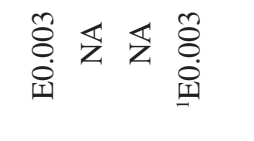 & 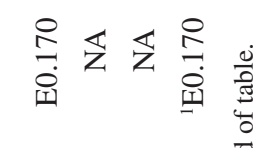 \\
\hline & हूँ̆ & 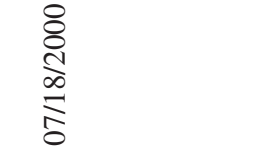 & 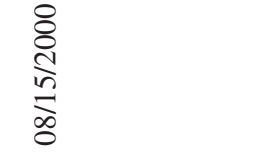 & 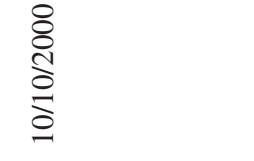 & 密 \\
\hline
\end{tabular}




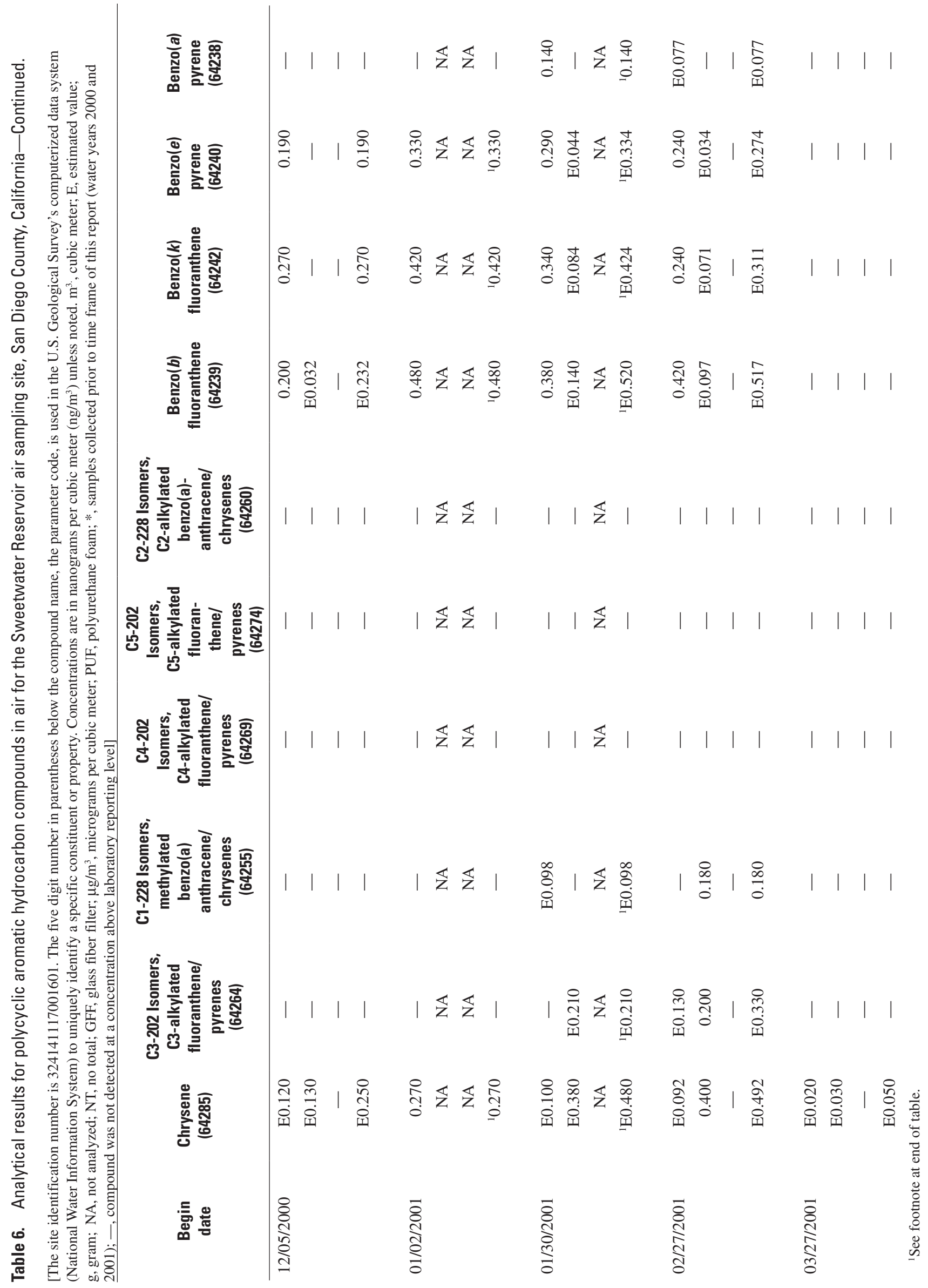




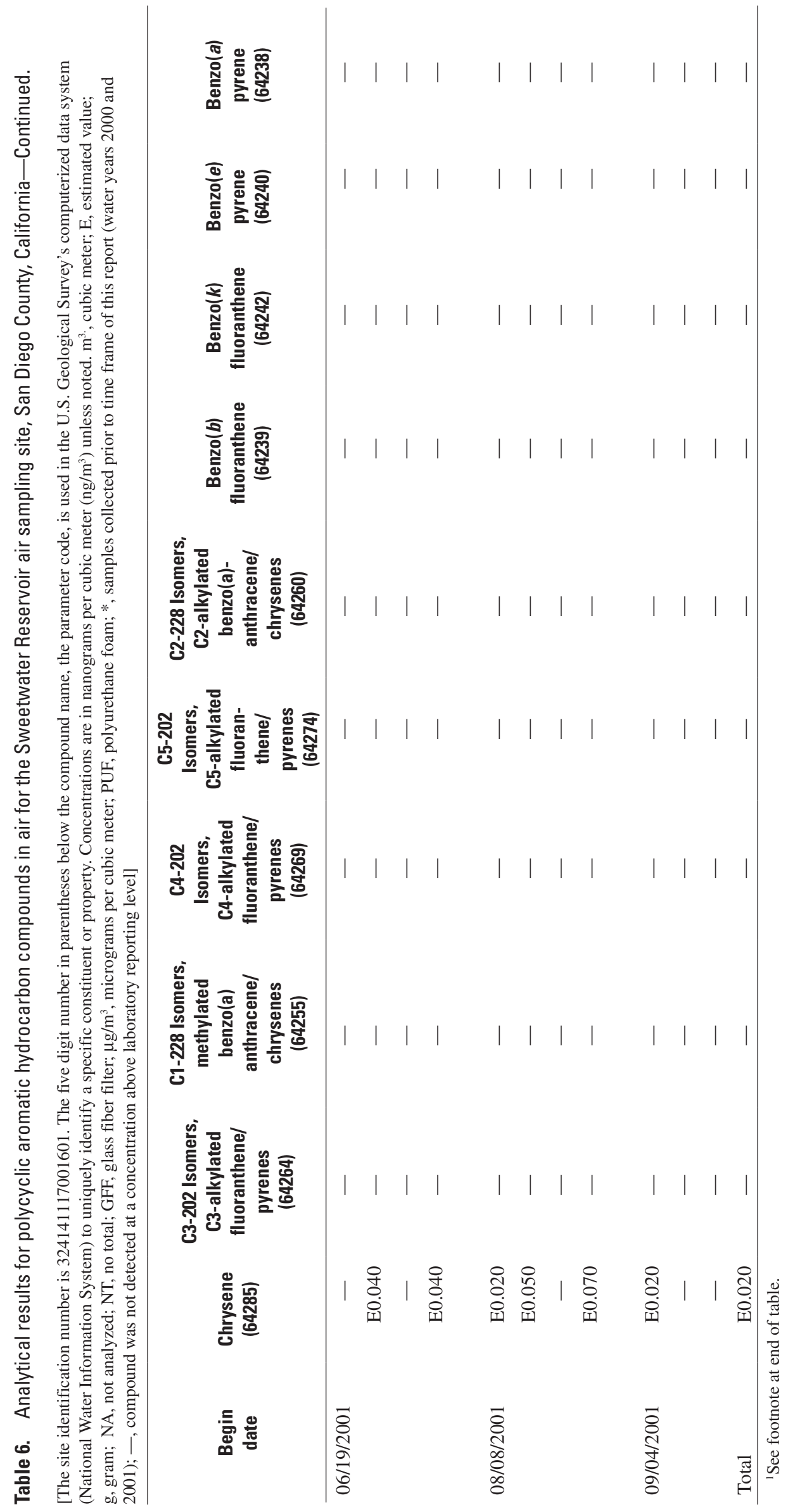




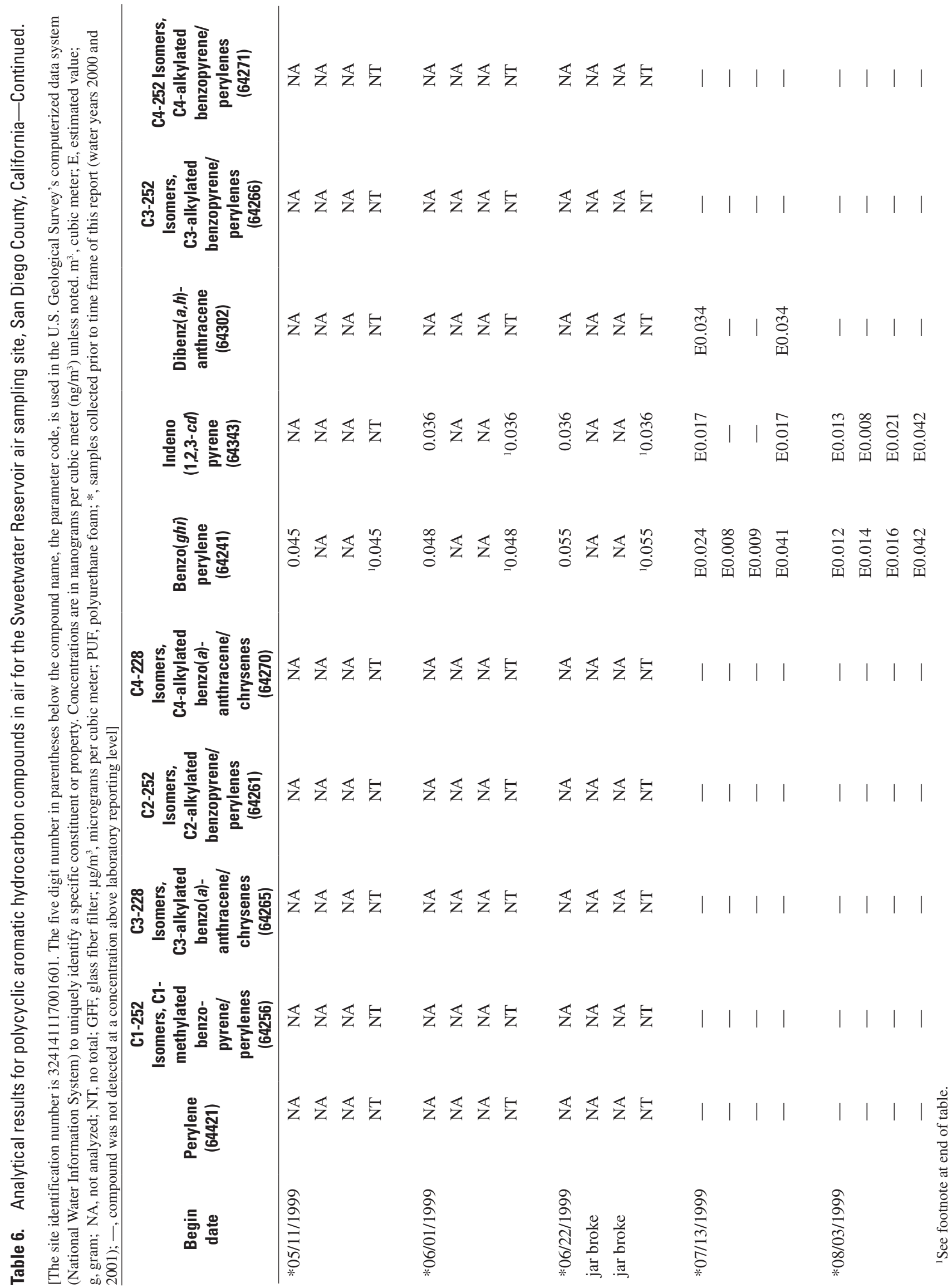




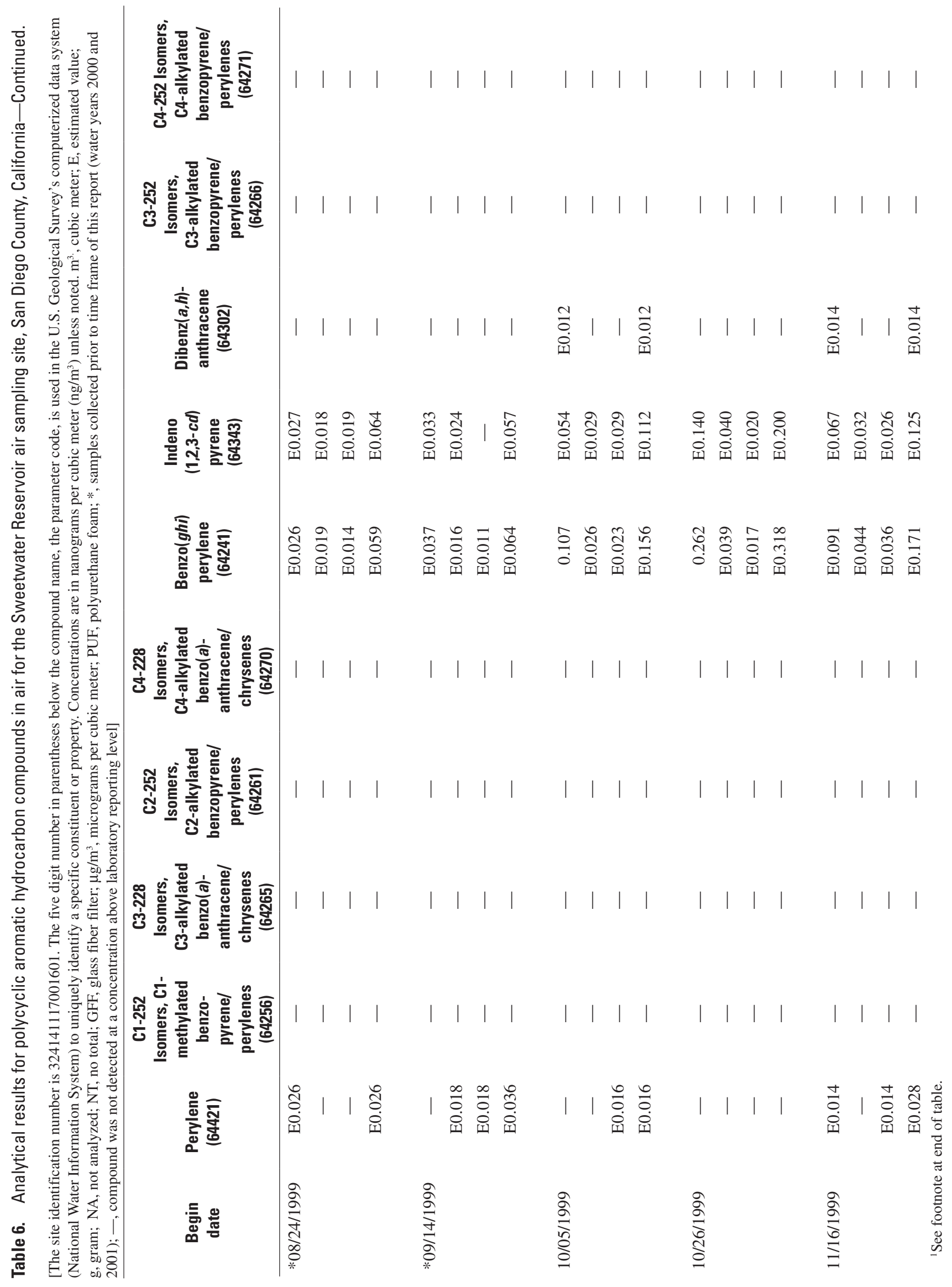




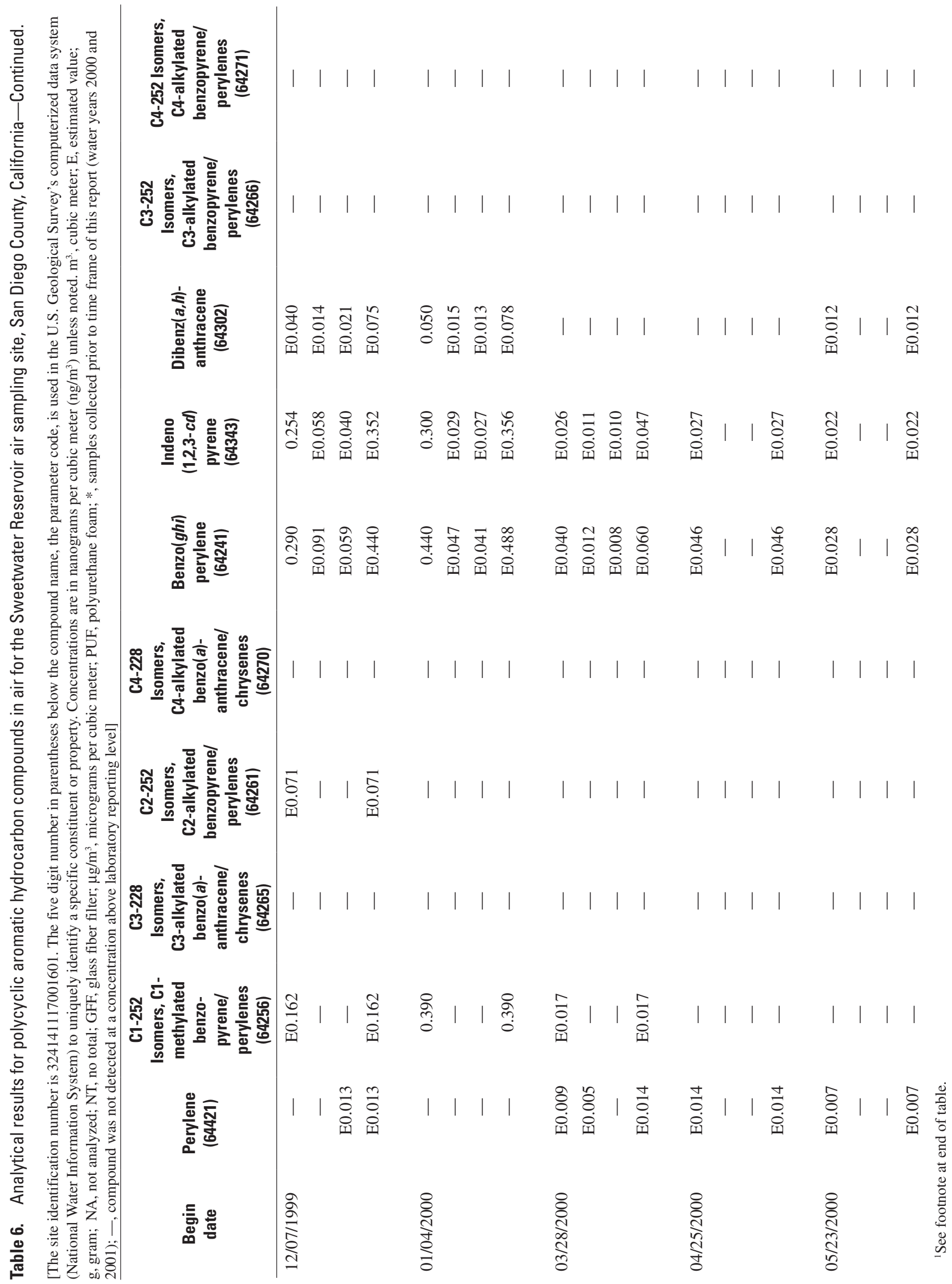




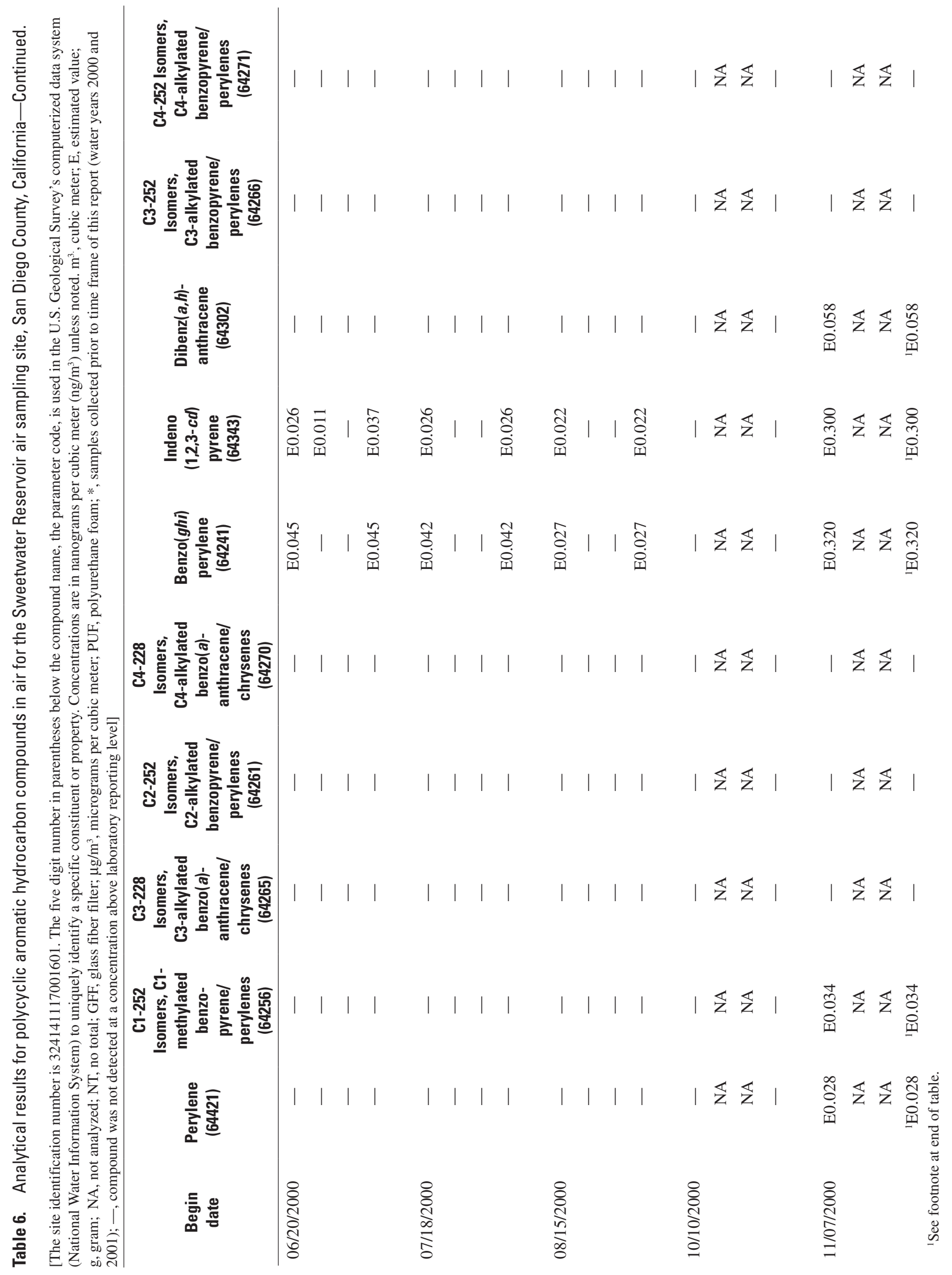




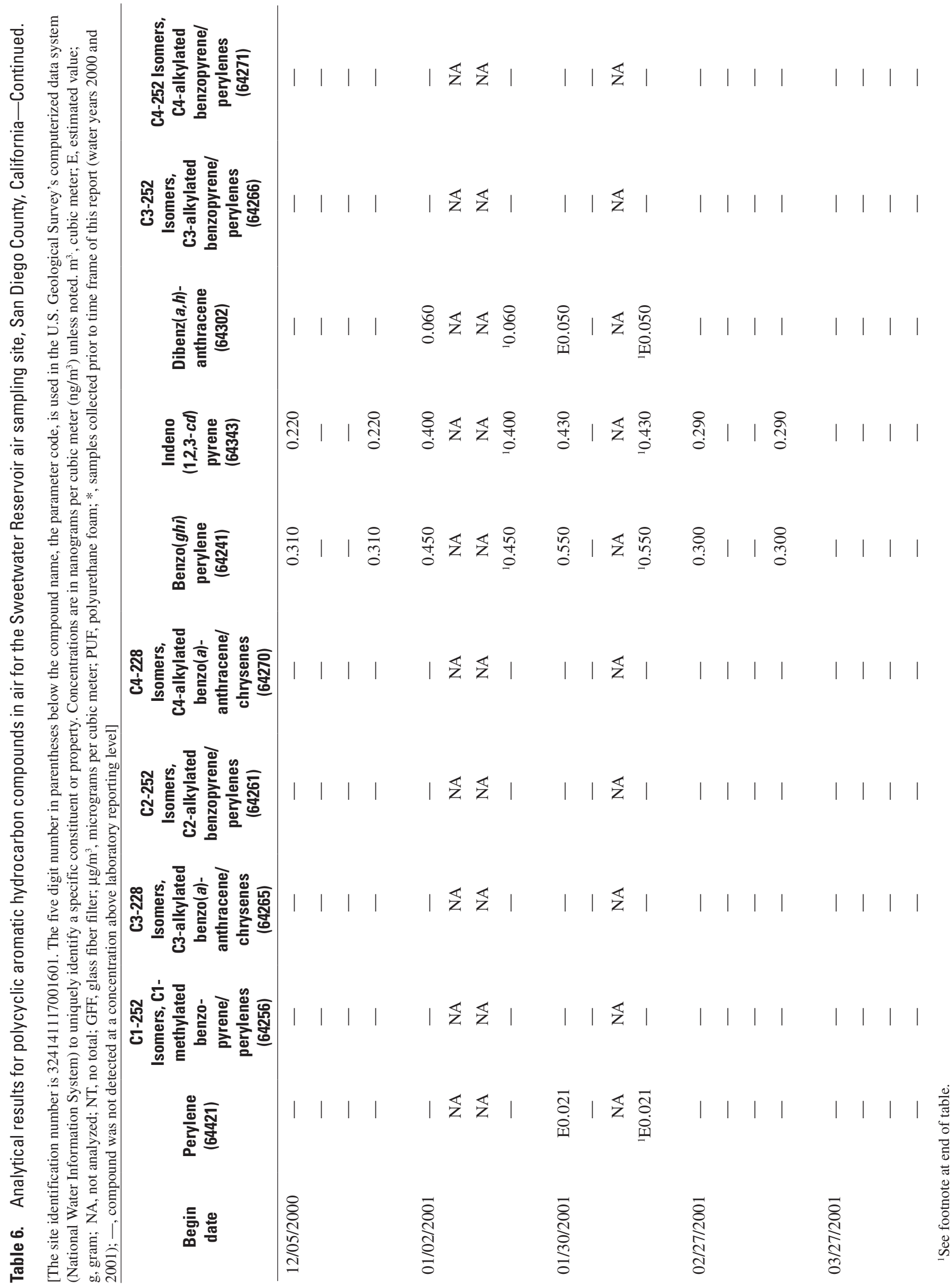




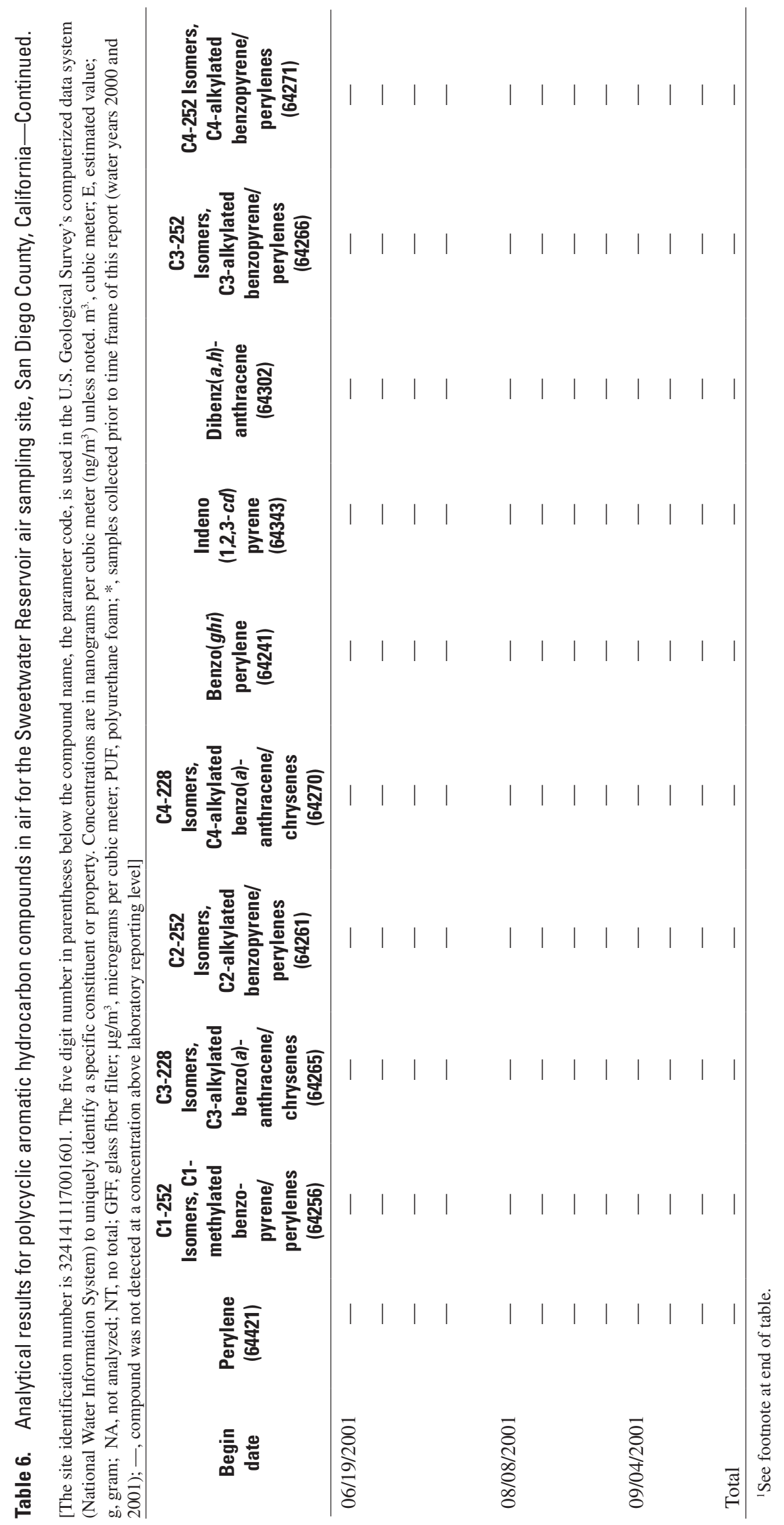




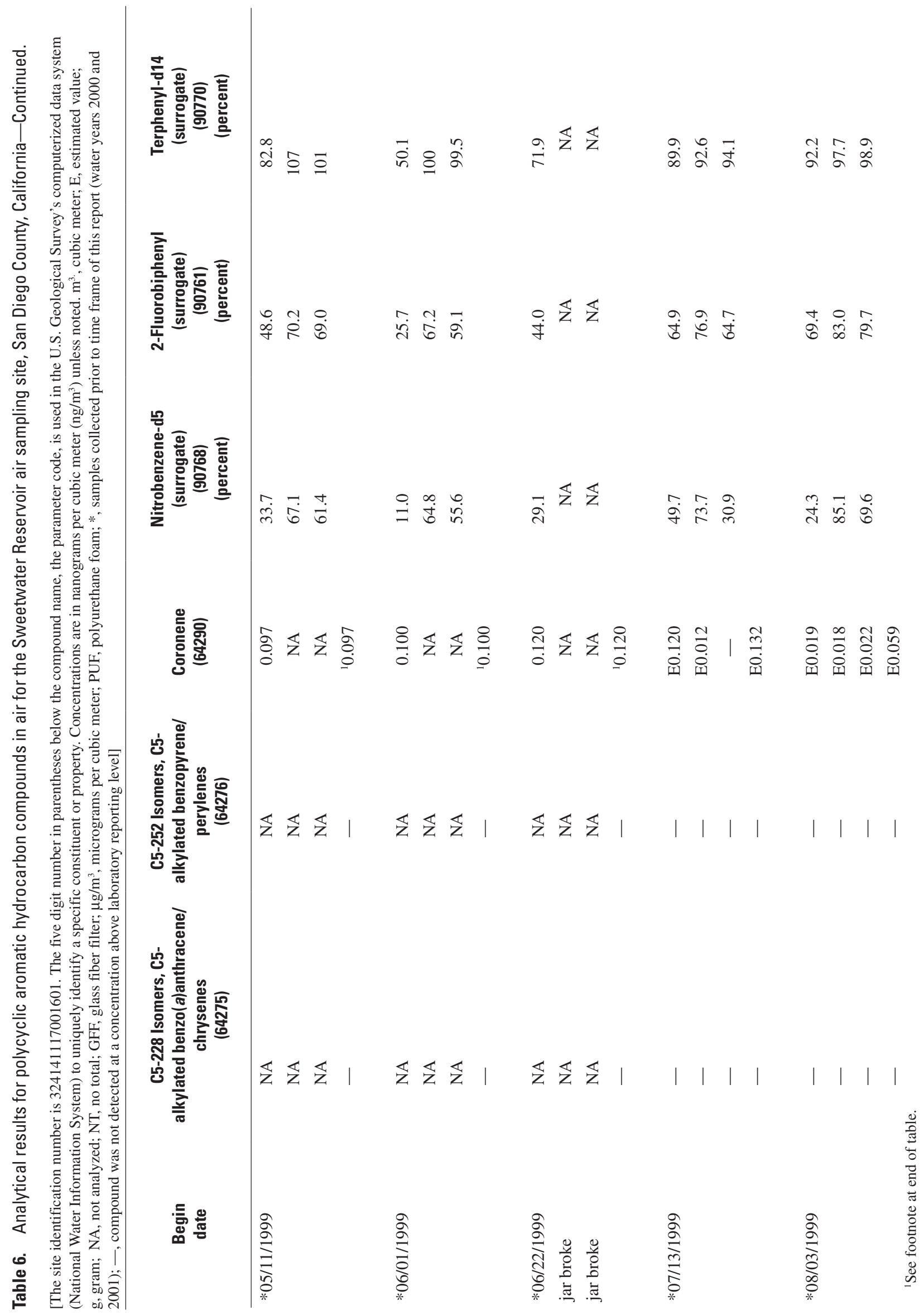




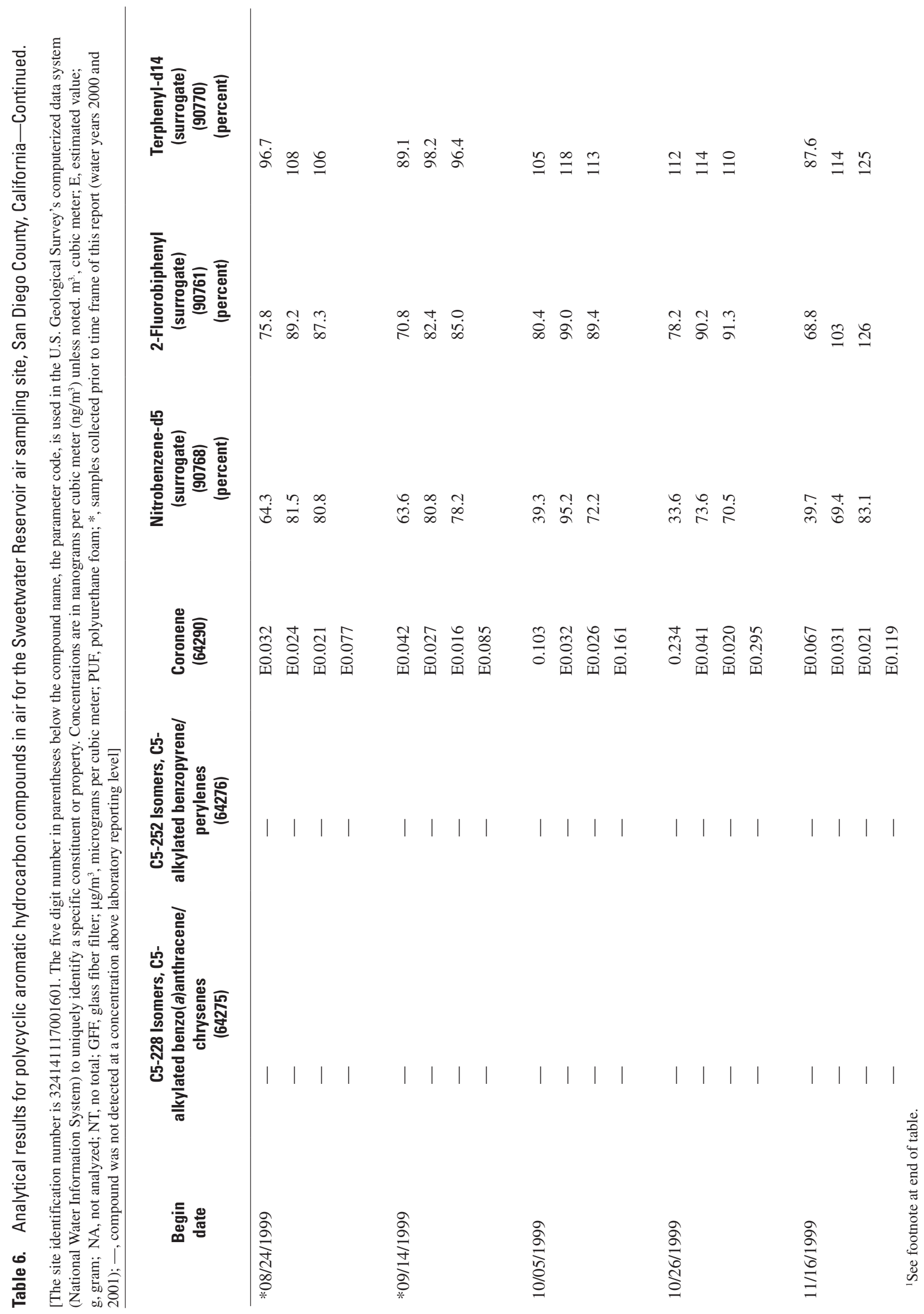




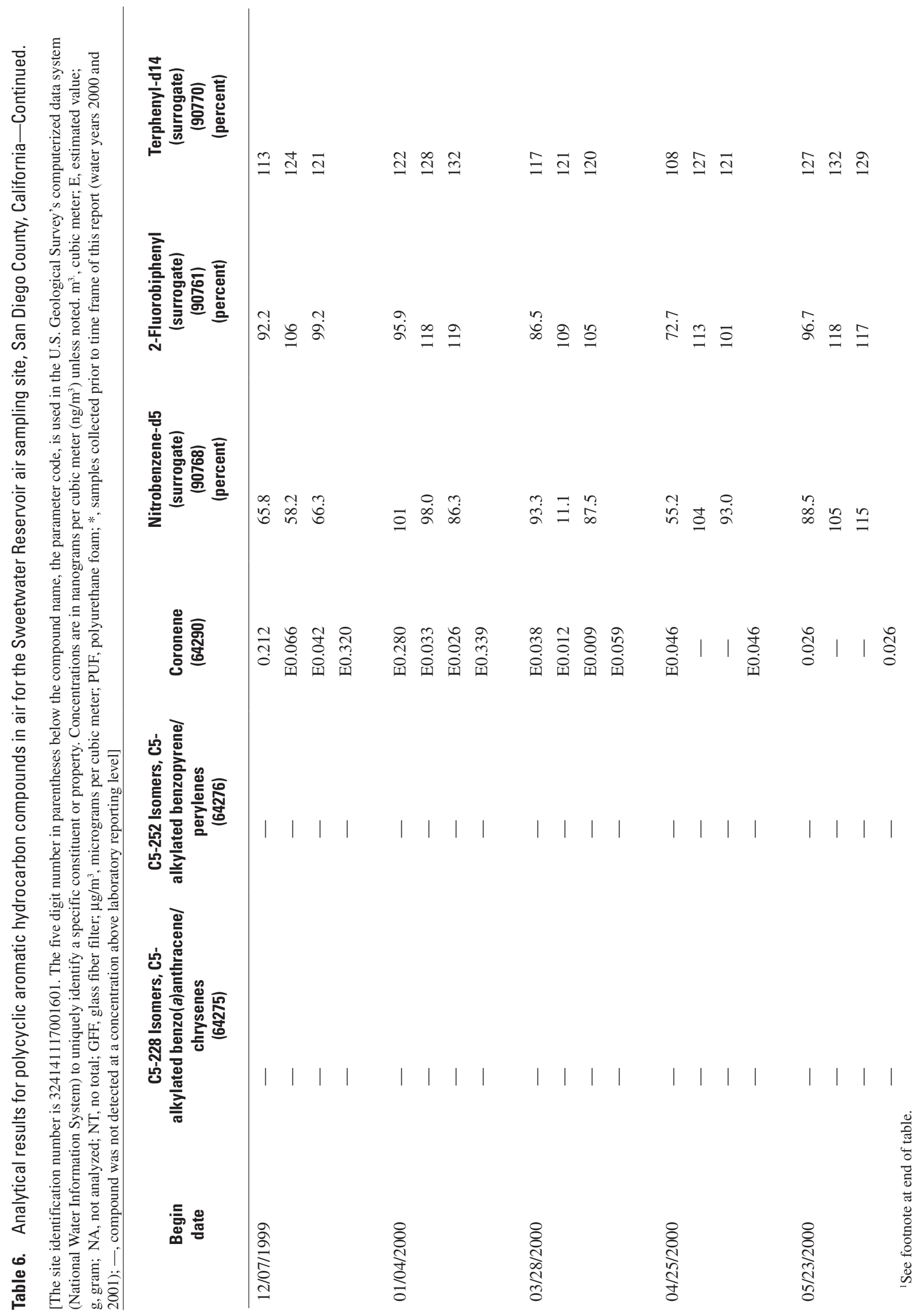




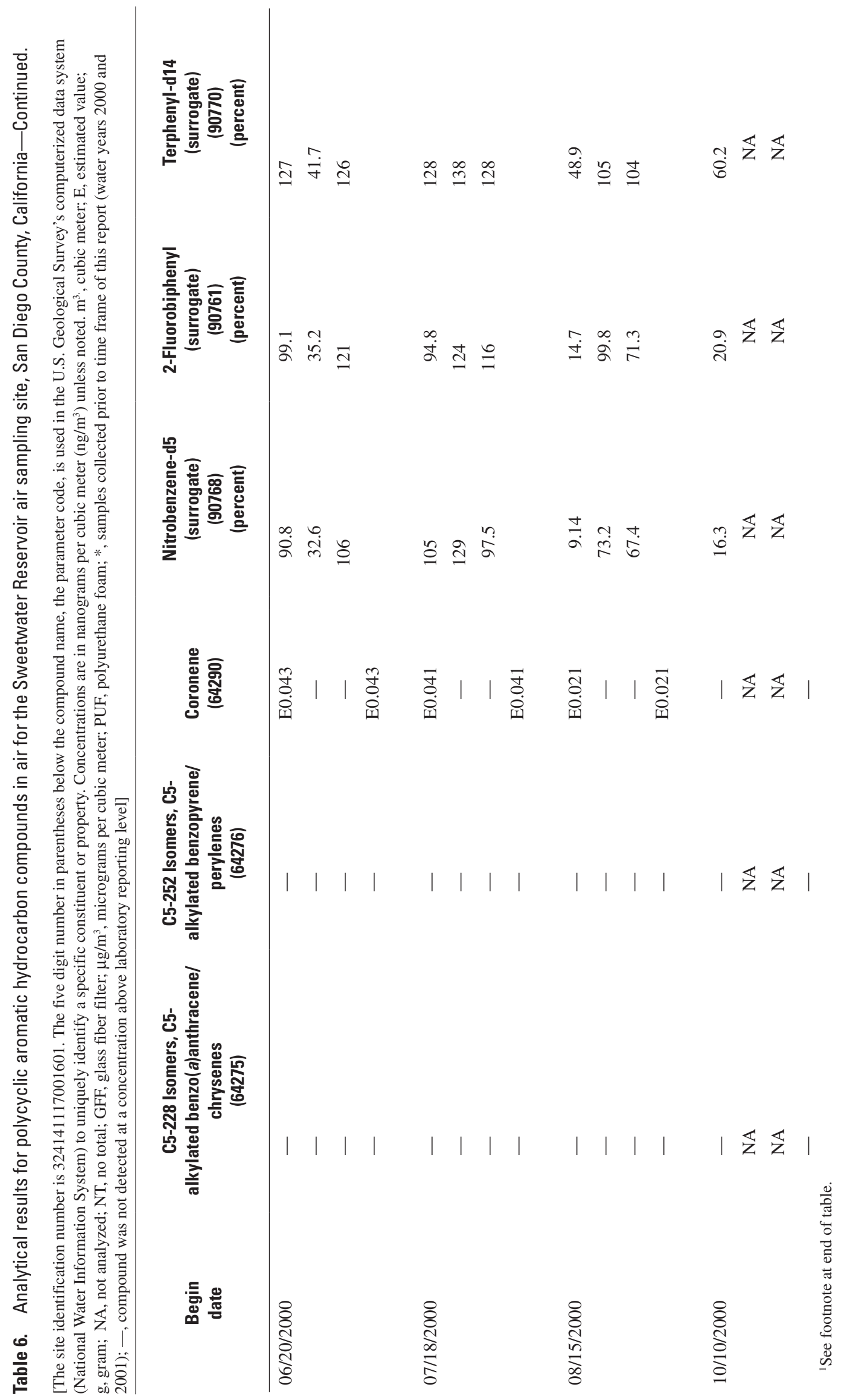




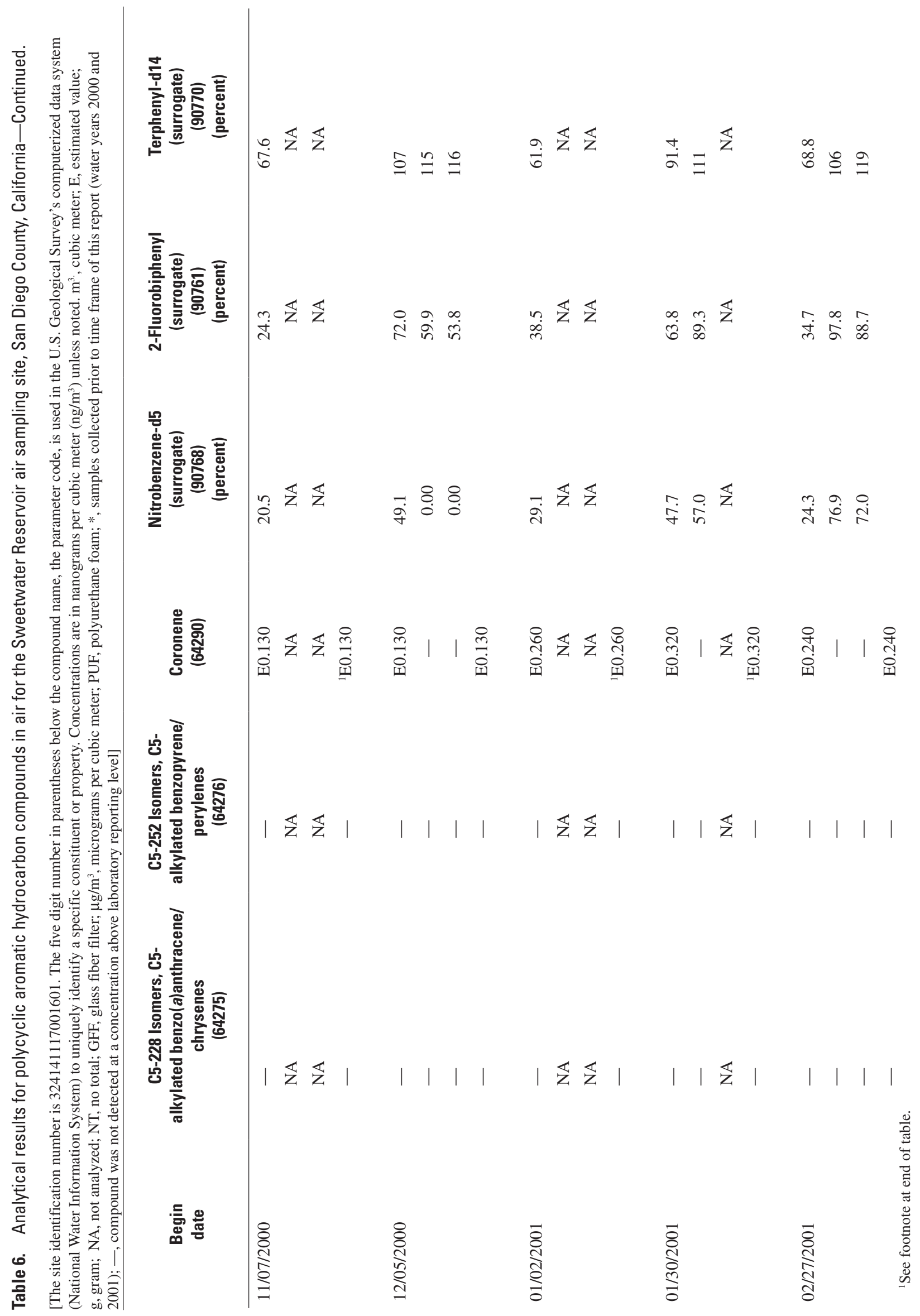




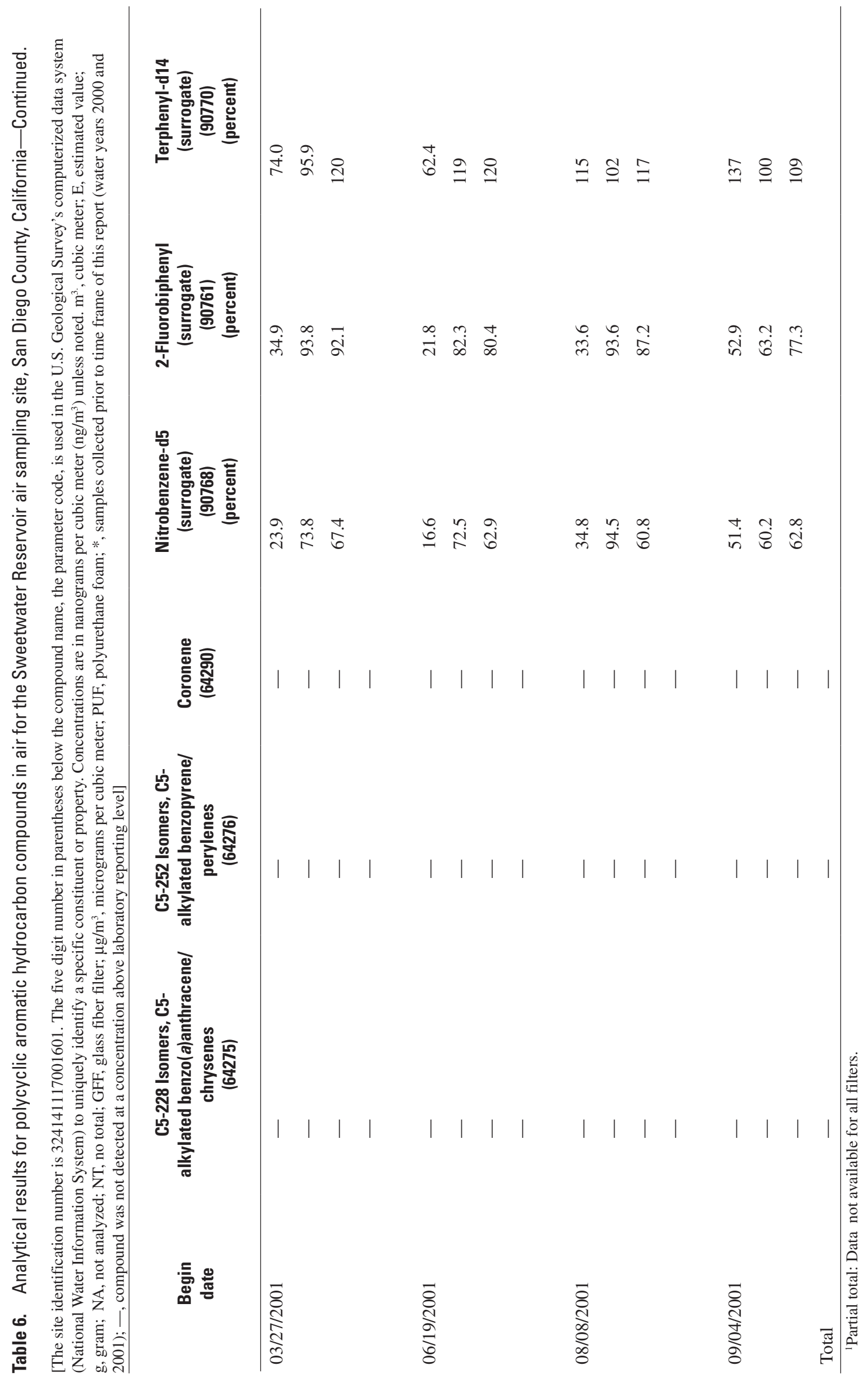


Table 7A. Pesticide compounds analyzed in air from modified U.S. Geological Survey National Water Quality Laboratory schedules for the Sweetwater Reservoir air sampling site, San Diego County, California.

[The site identification number is 324141117001601. Analytical results for pesticides, if detected, are shown on tables 7B,7C]

\begin{tabular}{|c|c|}
\hline \multicolumn{2}{|c|}{ Pesticide compound names } \\
\hline Modified schedule 2001 & Modified schedule 2002 \\
\hline (Detection results shown on table 7B) & (Detection results shown on table 7C) \\
\hline $\begin{array}{l}\text { 2-Chloro-4-isopropyl amino-6-amino-s- } \\
\text { triazine }\end{array}$ & 1,4-Naphthoquinone \\
\hline Acetochlor & 2-(4-tert-Butylphenoxy)-cyclohexanol \\
\hline Alachlor & 2,5-Dichloroaniline \\
\hline$\alpha-\mathrm{HCH}$ & 2-Amino- $N$-isopropylbenzamide \\
\hline Atrazine & 2-Chloro-2,6-diethylacetanilide \\
\hline Azinphos-methyl & 2-Ethyl-6-methylaniline \\
\hline Benfluralin & 3-Trifluoromethylaniline \\
\hline Carbaryl & 3,4-Dichloroaniline \\
\hline Carbofuran & 3,5-Dichloroaniline \\
\hline Chlorpyrifos & 4,4'-Dichlorobenzophenone \\
\hline Cyanazine & 4-Chloro-2-methylphenol \\
\hline DCPA (Dacthal) & 4-Chlorobenzylmethylsulfone \\
\hline Diazinon & $\alpha$-Endosulfan \\
\hline Dieldrin & $\beta$-Endosulfan \\
\hline Dimethoate & Azinphos-methyl oxygen analog \\
\hline Disulfoton & Bifenthrin \\
\hline$\alpha$-Endosulfan & cis-Propiconazole \\
\hline$\beta$-Endosulfan & Cycloate \\
\hline Endosulfan sulfate & Cyfluthrin \\
\hline Ethalfluralin & $\lambda$-Cyhalothrin \\
\hline Ethion & Cypermethrin \\
\hline Ethoprop & Dimethoate \\
\hline Fenthion sulfone & Disulfoton sulfone \\
\hline Fenthion sulfone oxygen analog & E-Dimethomorph \\
\hline Fonofos & Z-Dimethomorph \\
\hline Lindane & Endosulfan ether \\
\hline Linuron & Endosulfan sulfate \\
\hline Malathion & Ethion \\
\hline Methyl parathion & Ethion monoxon \\
\hline Metolachlor & Fenthion \\
\hline Metribuzin & Fenthion sulfoxide \\
\hline Molinate & Flumetralin \\
\hline Napropamide & Fonofos oxygen analog \\
\hline$p, p^{\prime}-\mathrm{DDE}$ & Iprodione \\
\hline Parathion & Isofenphos \\
\hline
\end{tabular}


Table 7A. Pesticide compounds analyzed in air from modified U.S. Geological Survey National Water Quality laboratory schedules for the Sweetwater Reservoir air sampling site, San Diego County, CaliforniaContinued.

[The site identification number is 324141117001601. Analytical results for pesticides, if detected, are shown on tables 7B,7C]

\begin{tabular}{|c|c|}
\hline \multicolumn{2}{|c|}{ Pesticide compound names } \\
\hline Modified schedule 2001 & Modified schedule 2002 \\
\hline (Detection results shown on table 7B) & (Detection results shown on table 7 C) \\
\hline Pendimethalin & Malaoxon \\
\hline cis-Permethrin & Methidathion \\
\hline Phorate & Methyl paraoxon \\
\hline Prometon & Myclobutanil \\
\hline Pronamide & $O$-Ethyl- $O$-methyl- $S$-propylphosphorothioate \\
\hline Propachlor & Oxyfluorfen \\
\hline Propanil & Paraoxon-ethyl \\
\hline Propargite & Profenofos \\
\hline Simazine & Prometryn \\
\hline Tebuthiuron & Propetamphos \\
\hline Terbufos & Sulfotepp \\
\hline Terbuthylazine & Tebupirimphos (Phostebupirim) \\
\hline Thiobencarb & Tebupirimphos oxygen analog \\
\hline Triallate & Tefluthrin \\
\hline \multirow[t]{2}{*}{ Trifluralin } & Temephos \\
\hline & Terbufos oxygen analog sulfone \\
\hline Diazinon-d10 (percent) & Terbuthylazine \\
\hline \multirow[t]{5}{*}{$\alpha-\mathrm{HCH}-\mathrm{d} 6$ (percent) } & Terbutos sulfone \\
\hline & trans-Propiconazole \\
\hline & Tribuphos \\
\hline & Diazinon-d10 (percent) \\
\hline & $\alpha$-HCH-d6 (percent) \\
\hline
\end{tabular}




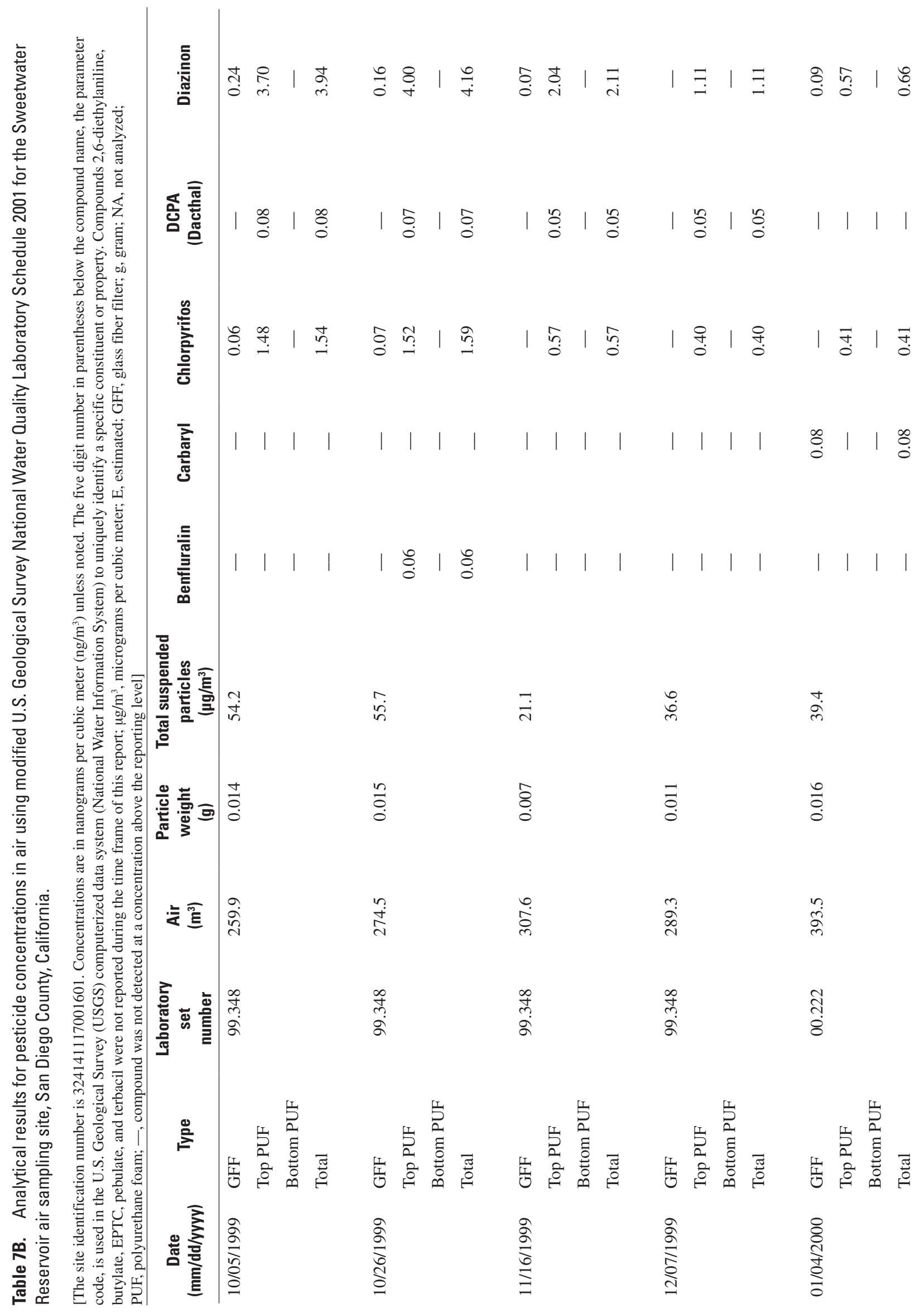




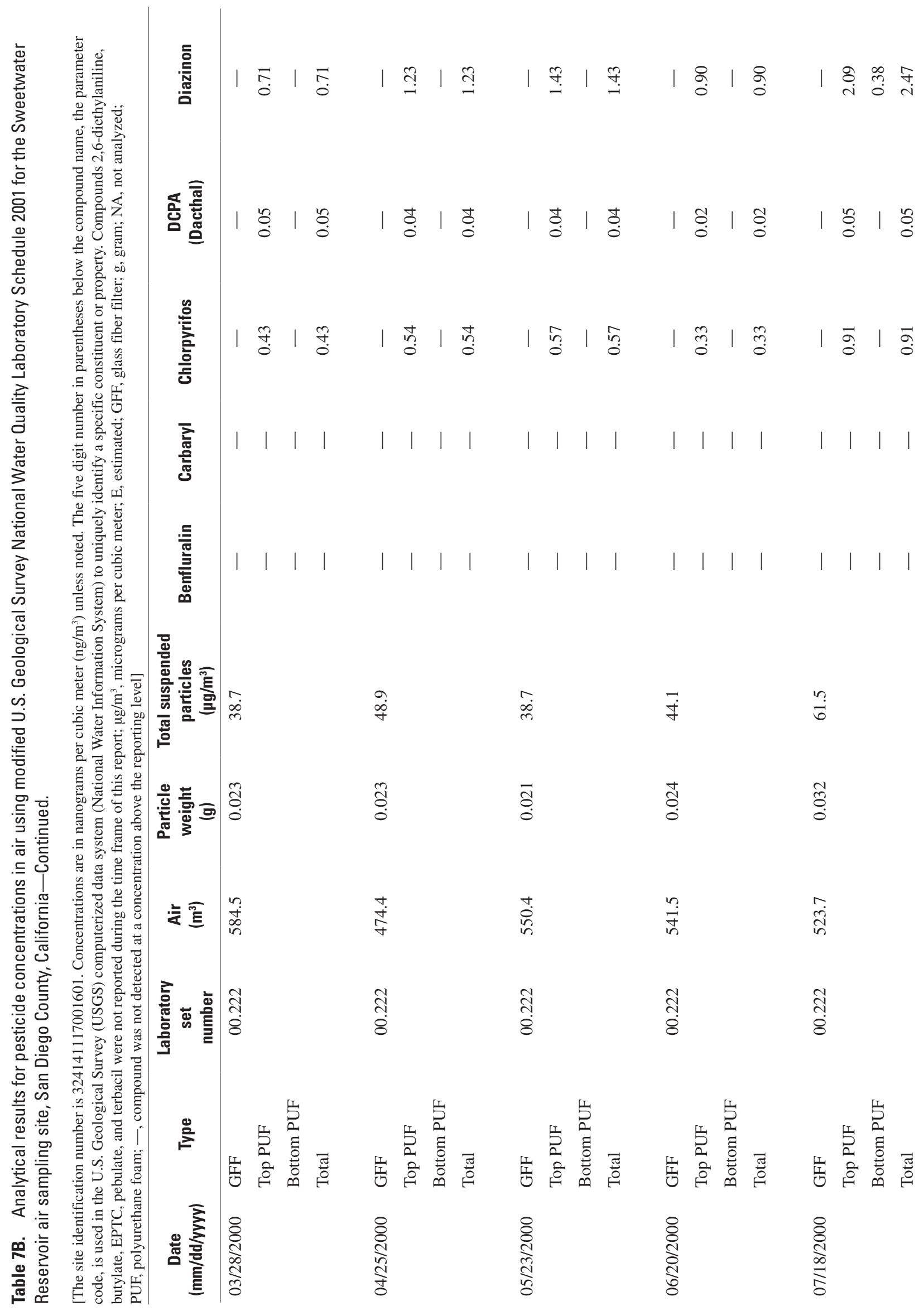




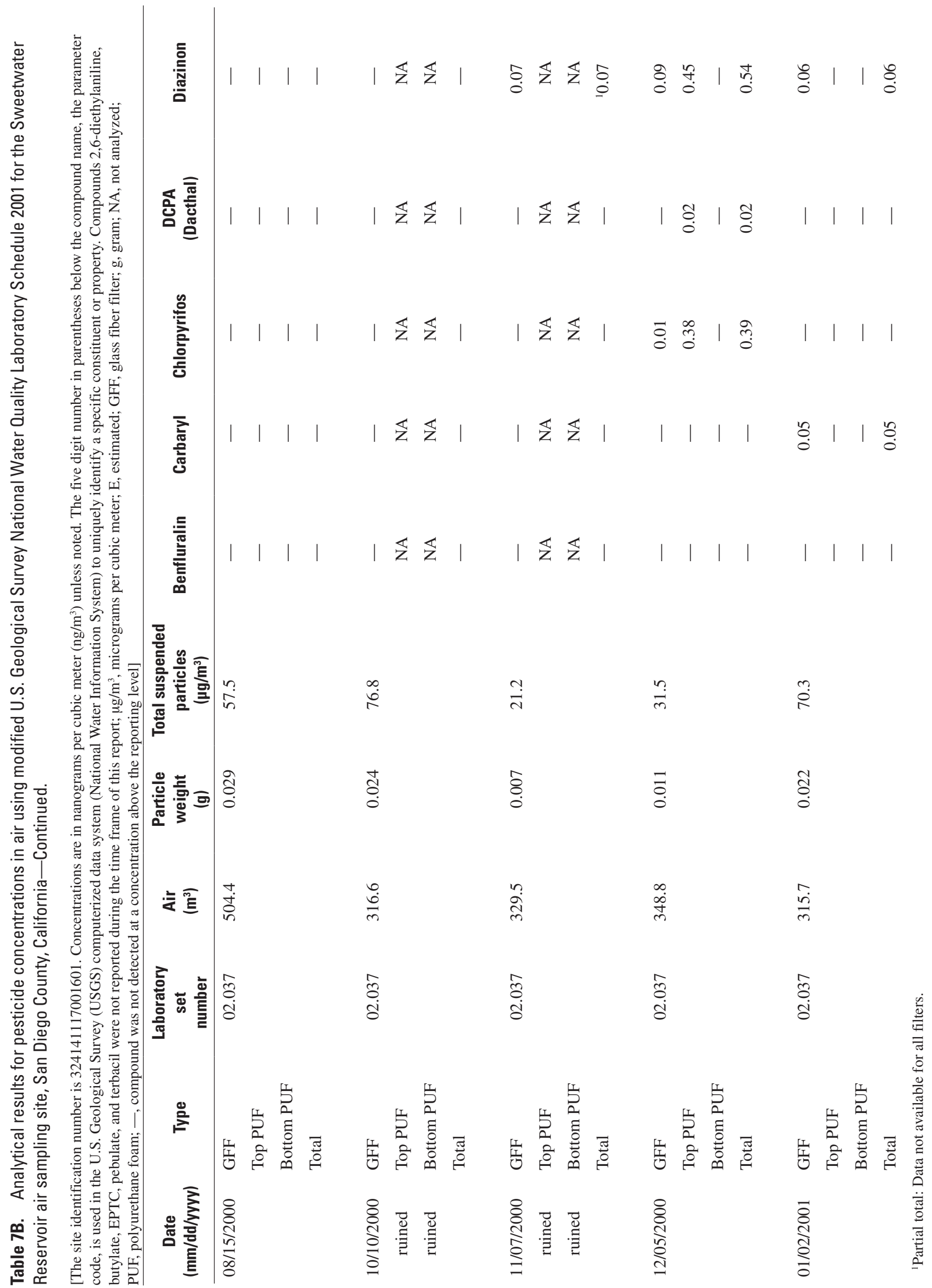




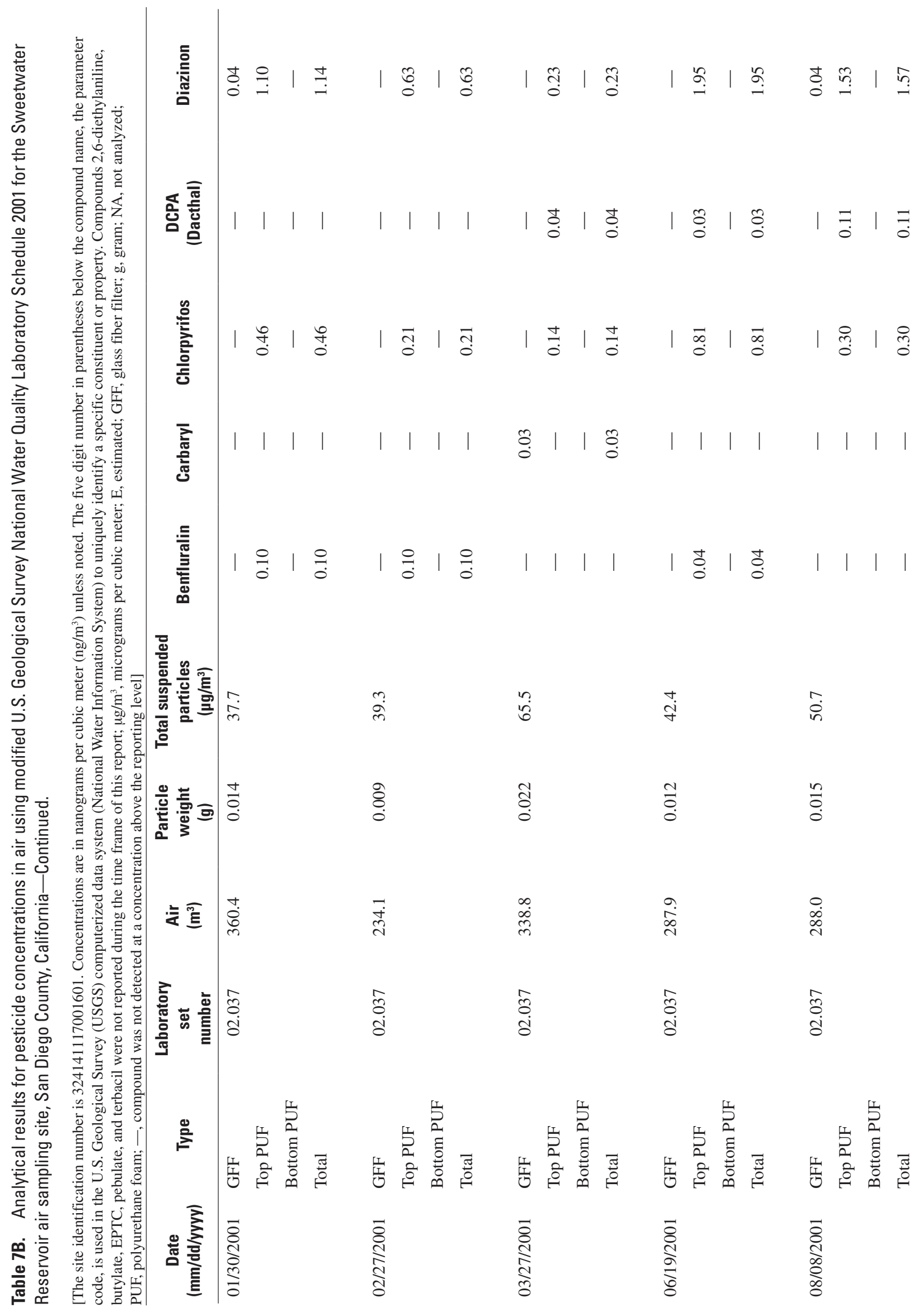




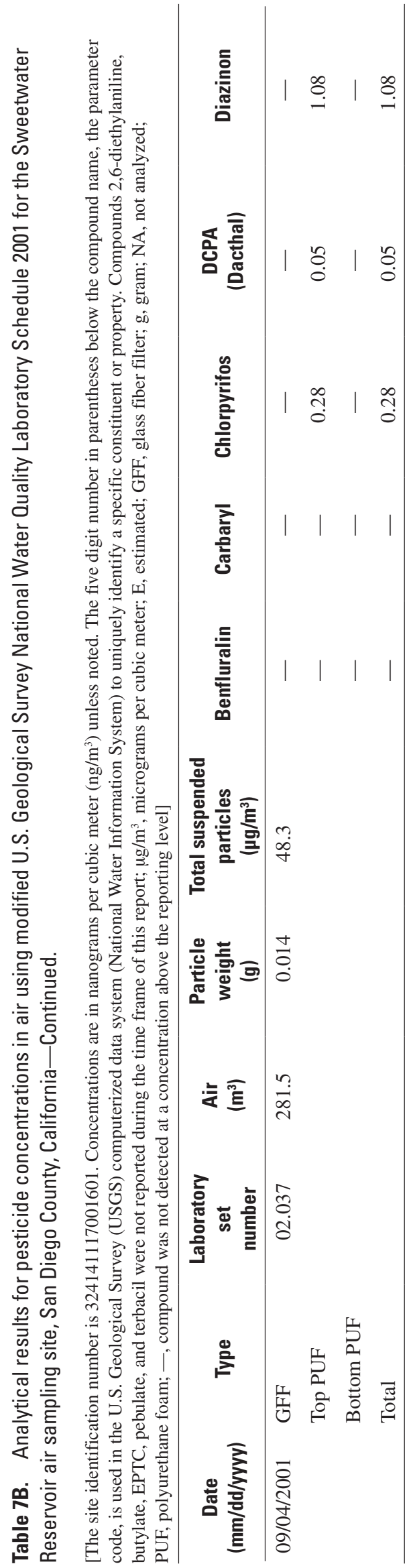




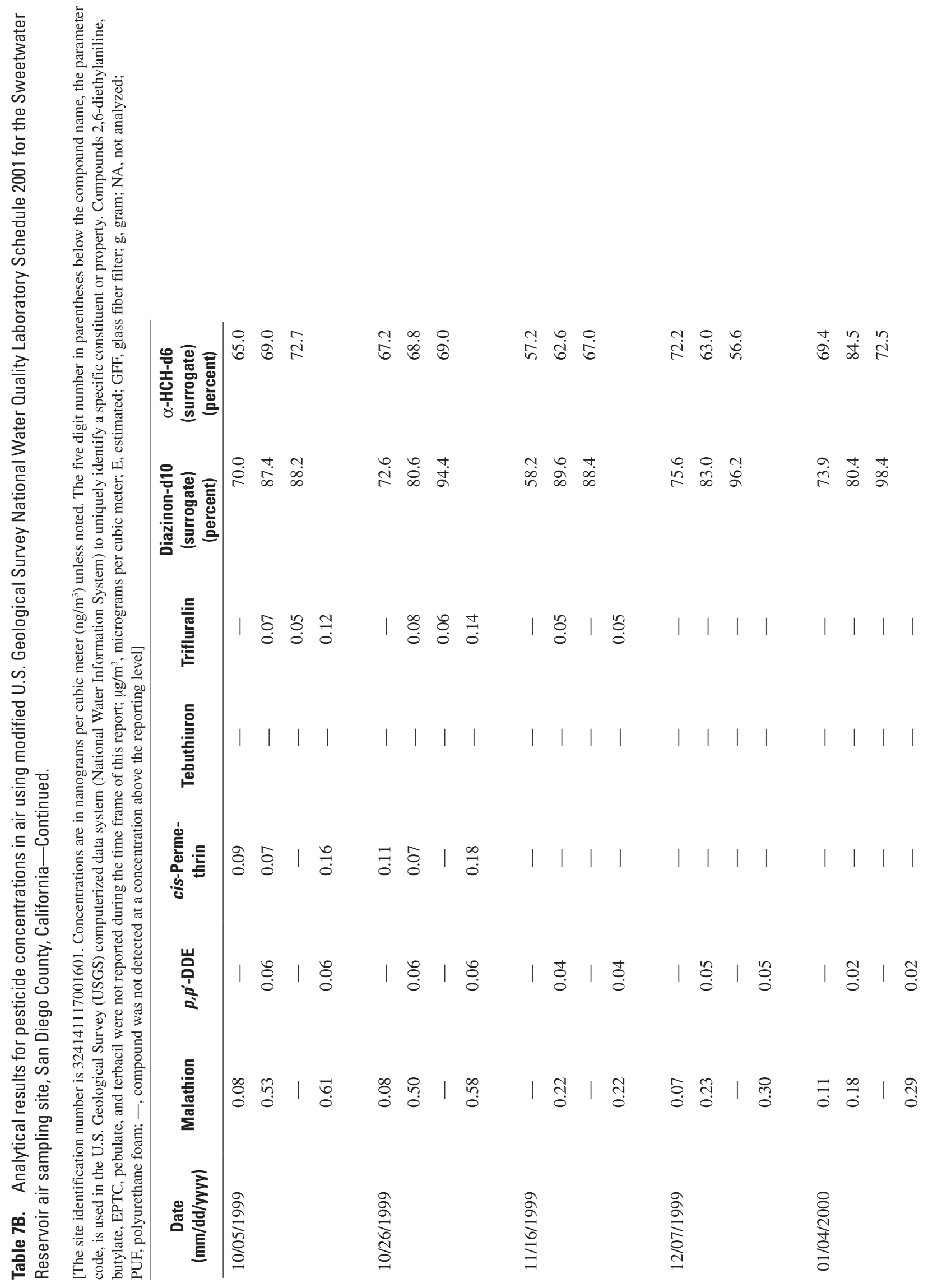




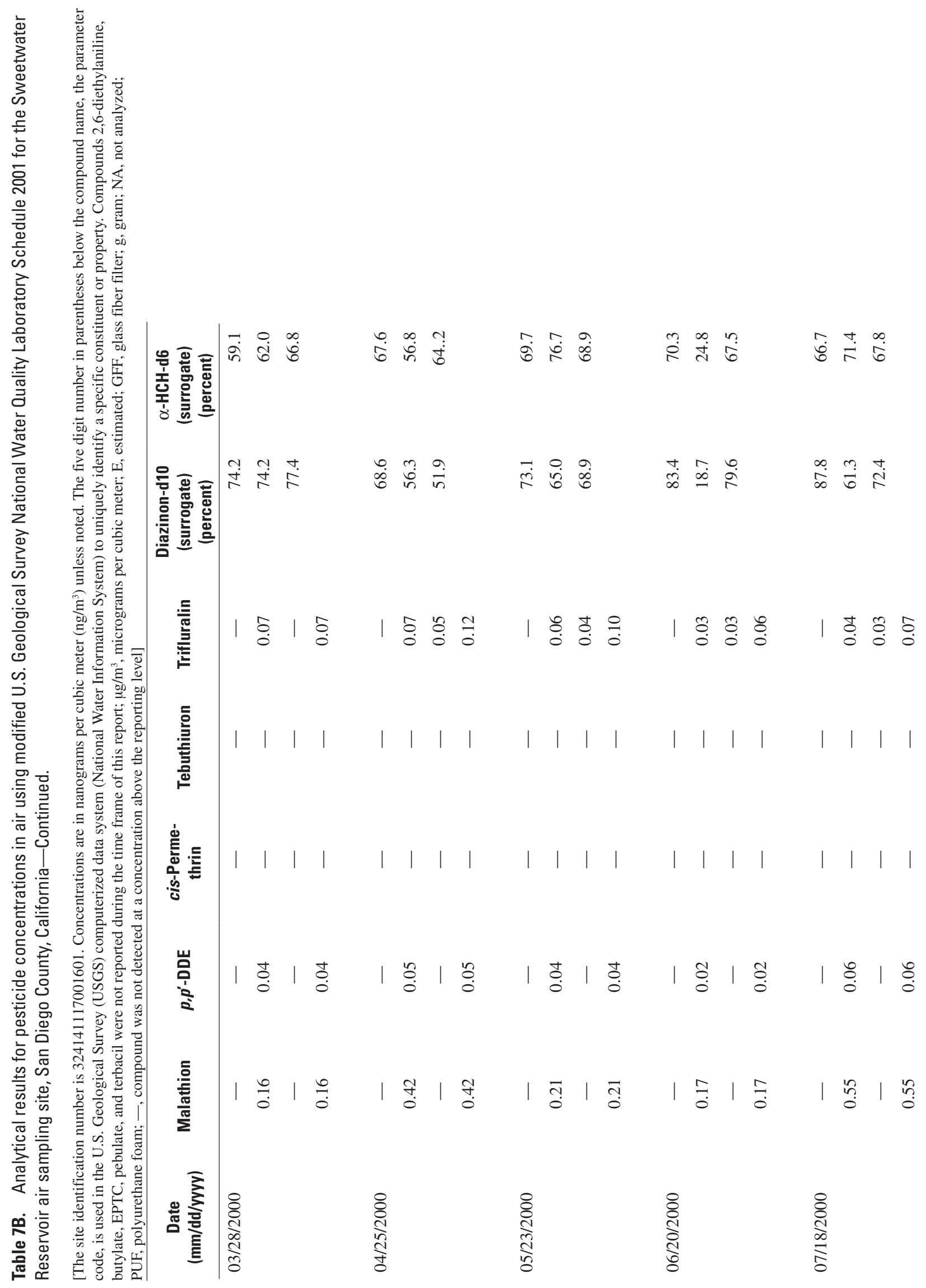




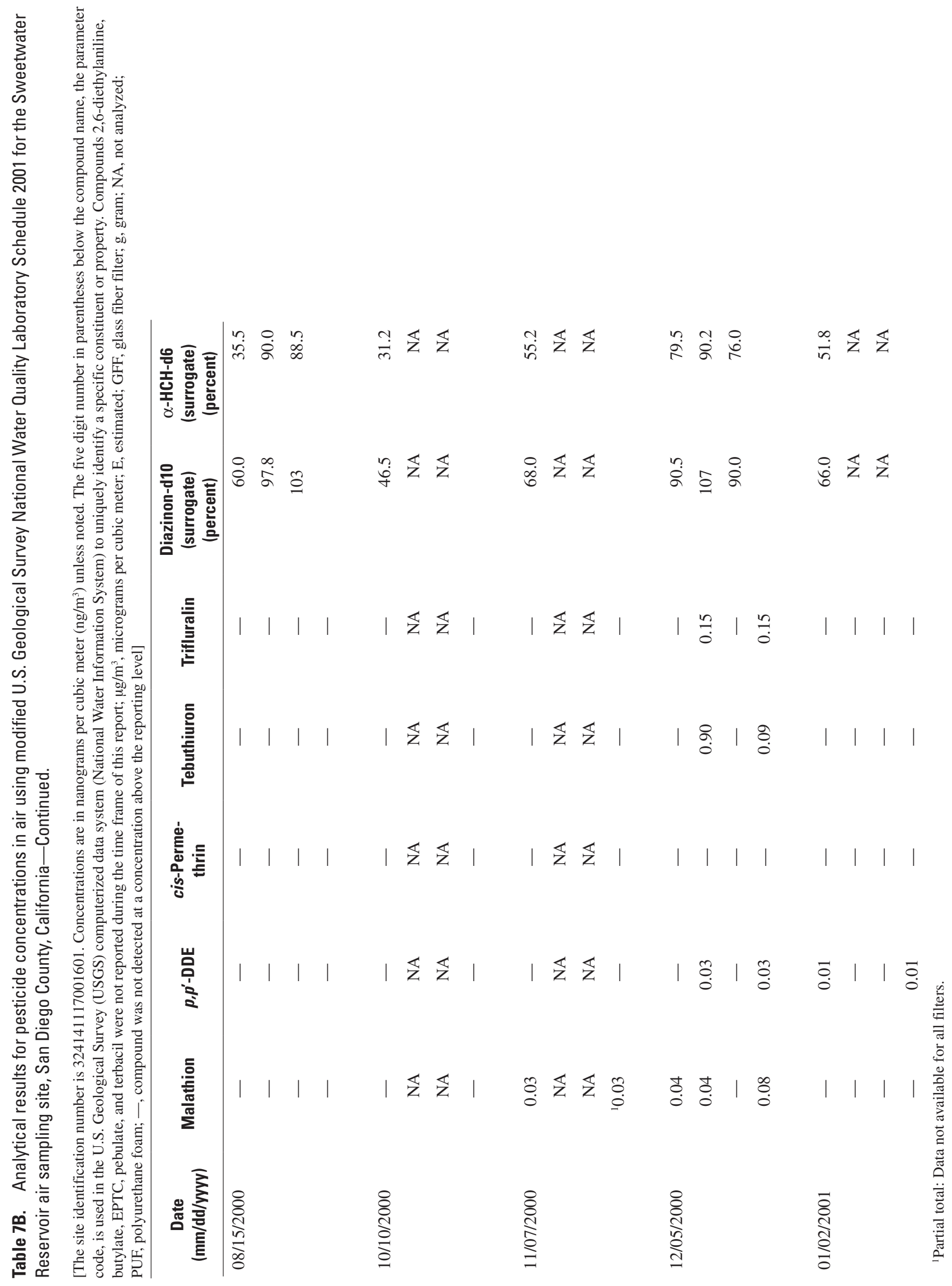




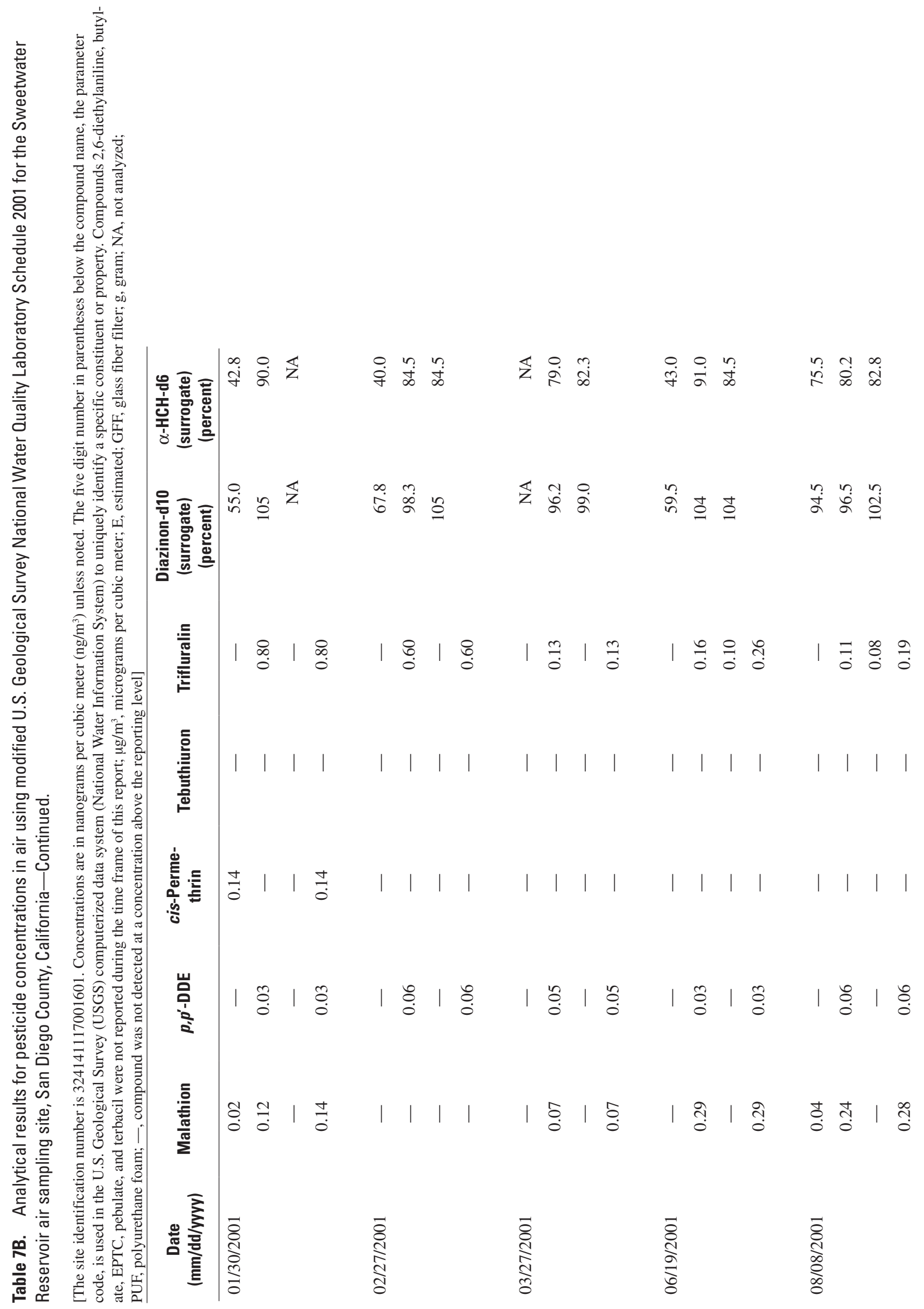




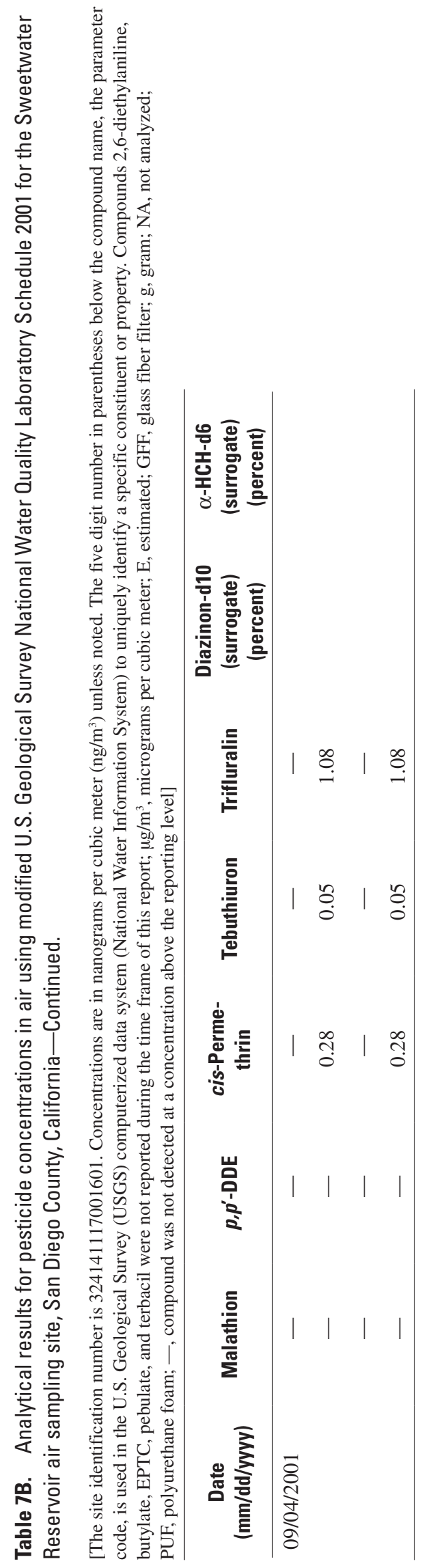




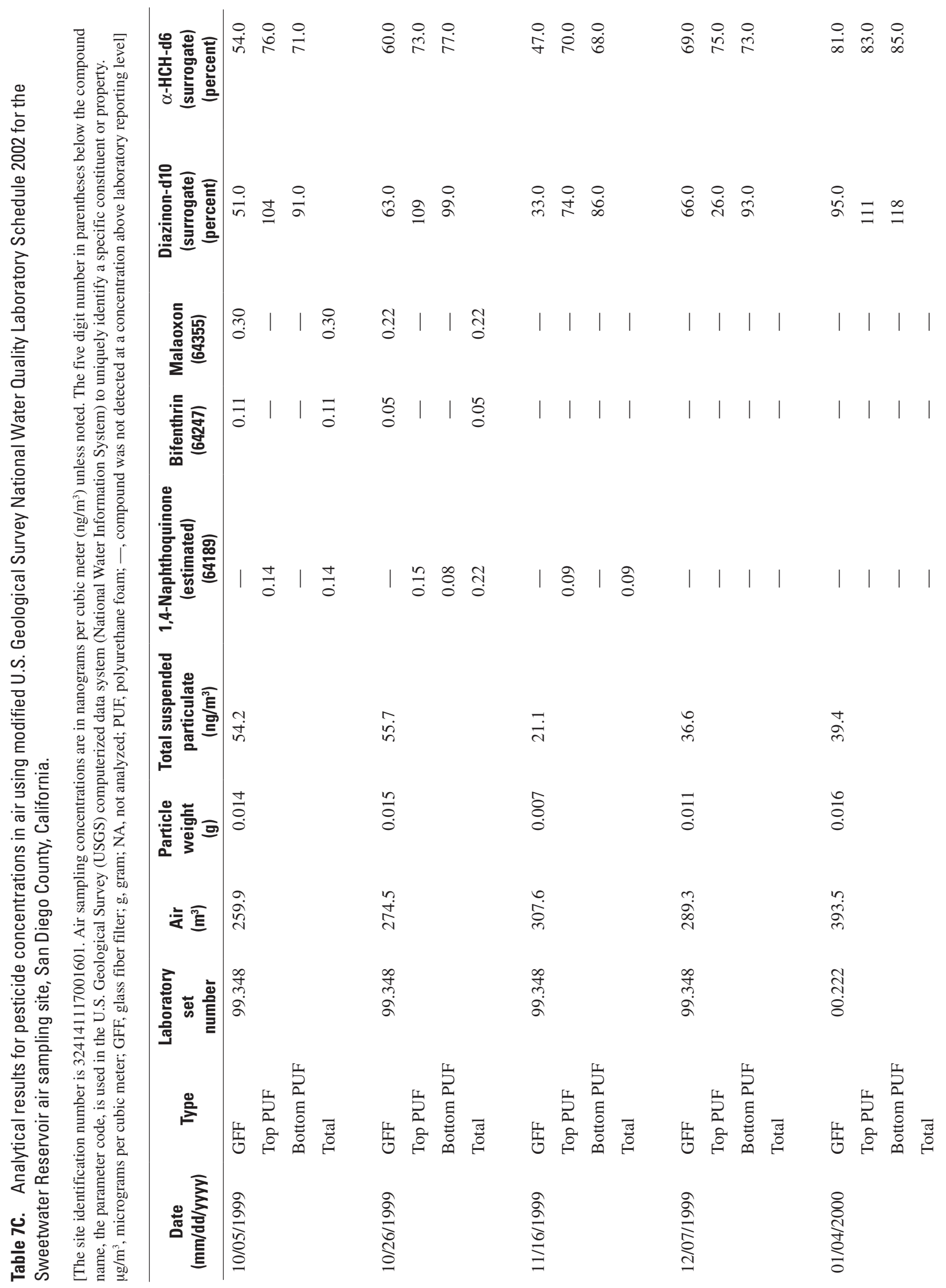




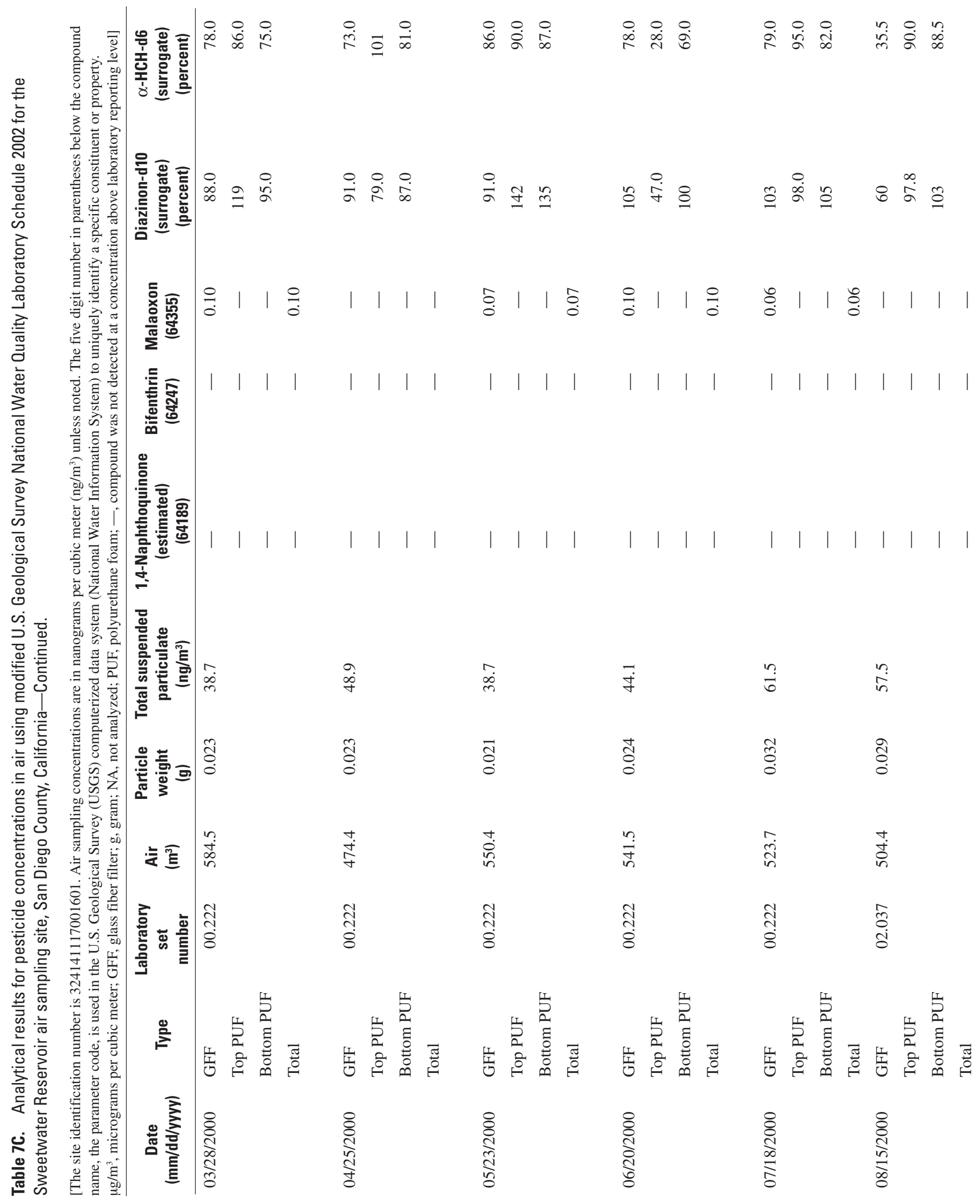




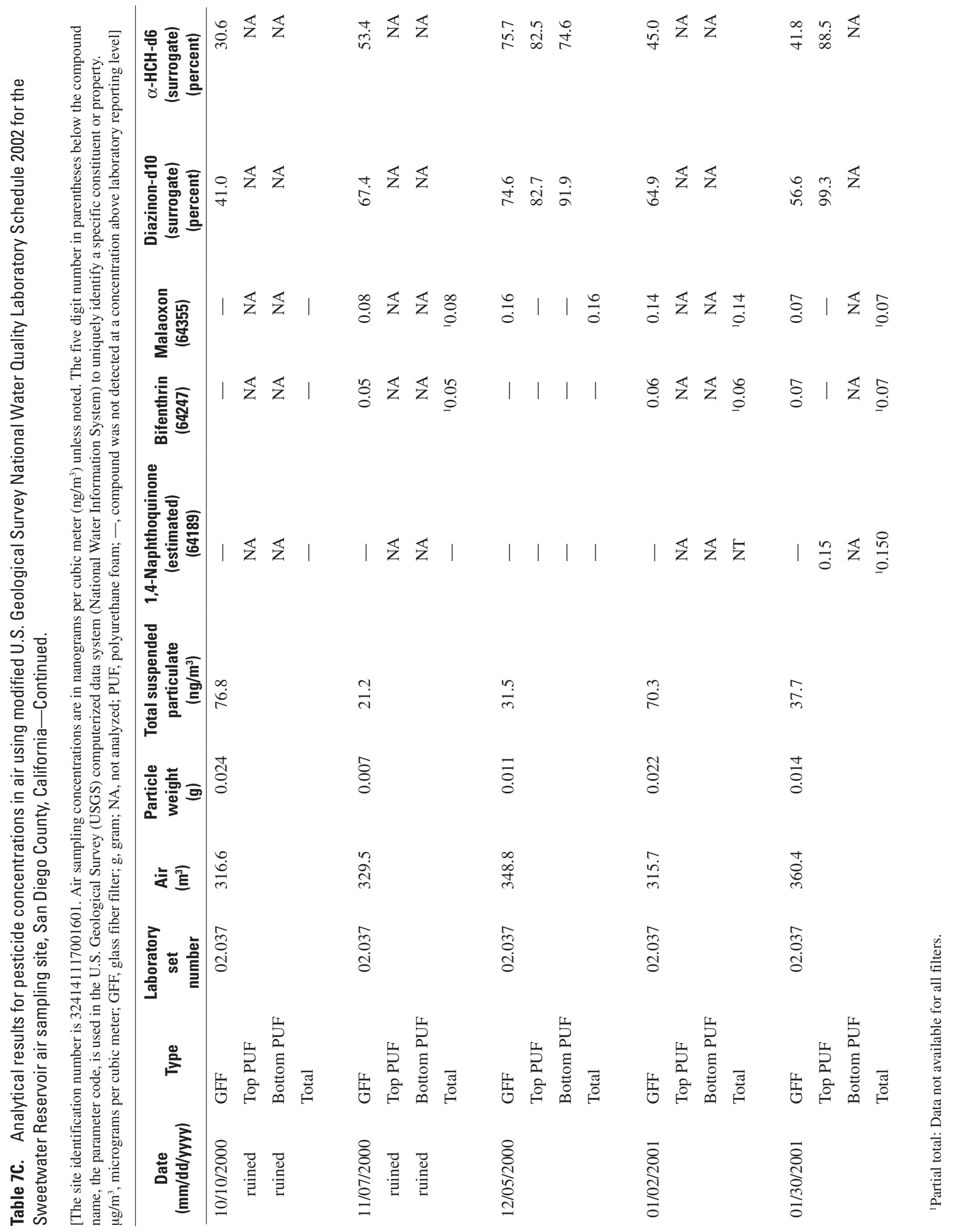




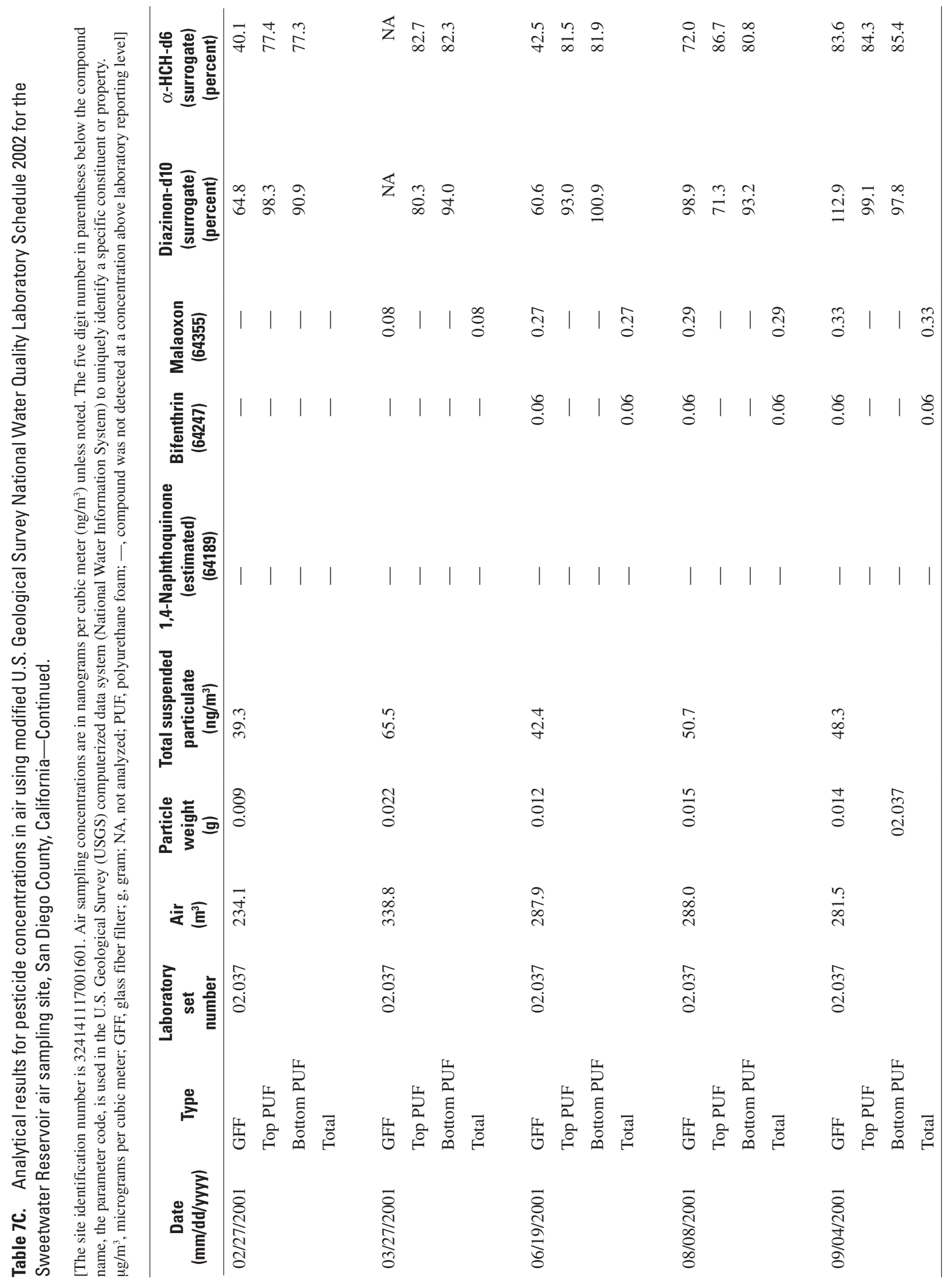




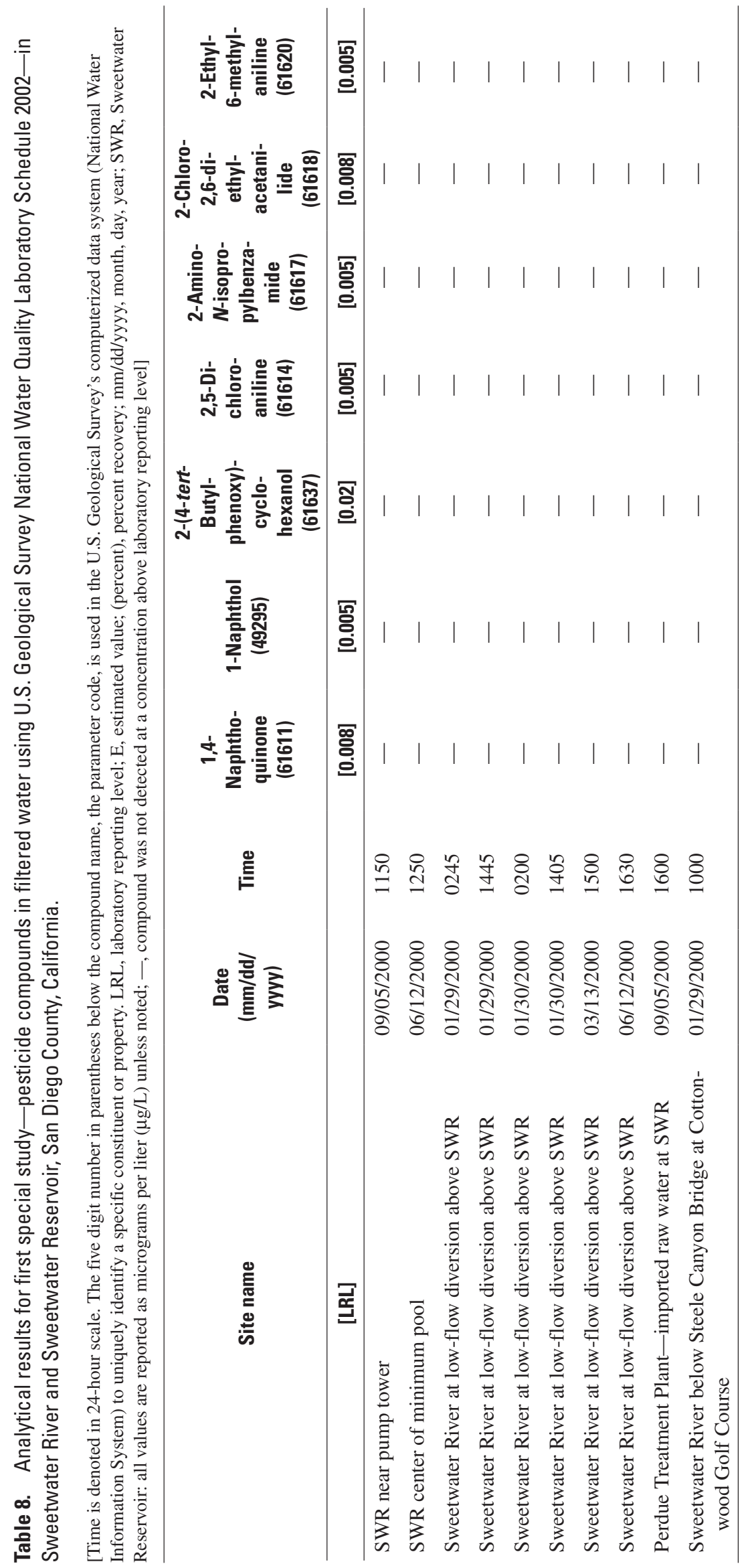




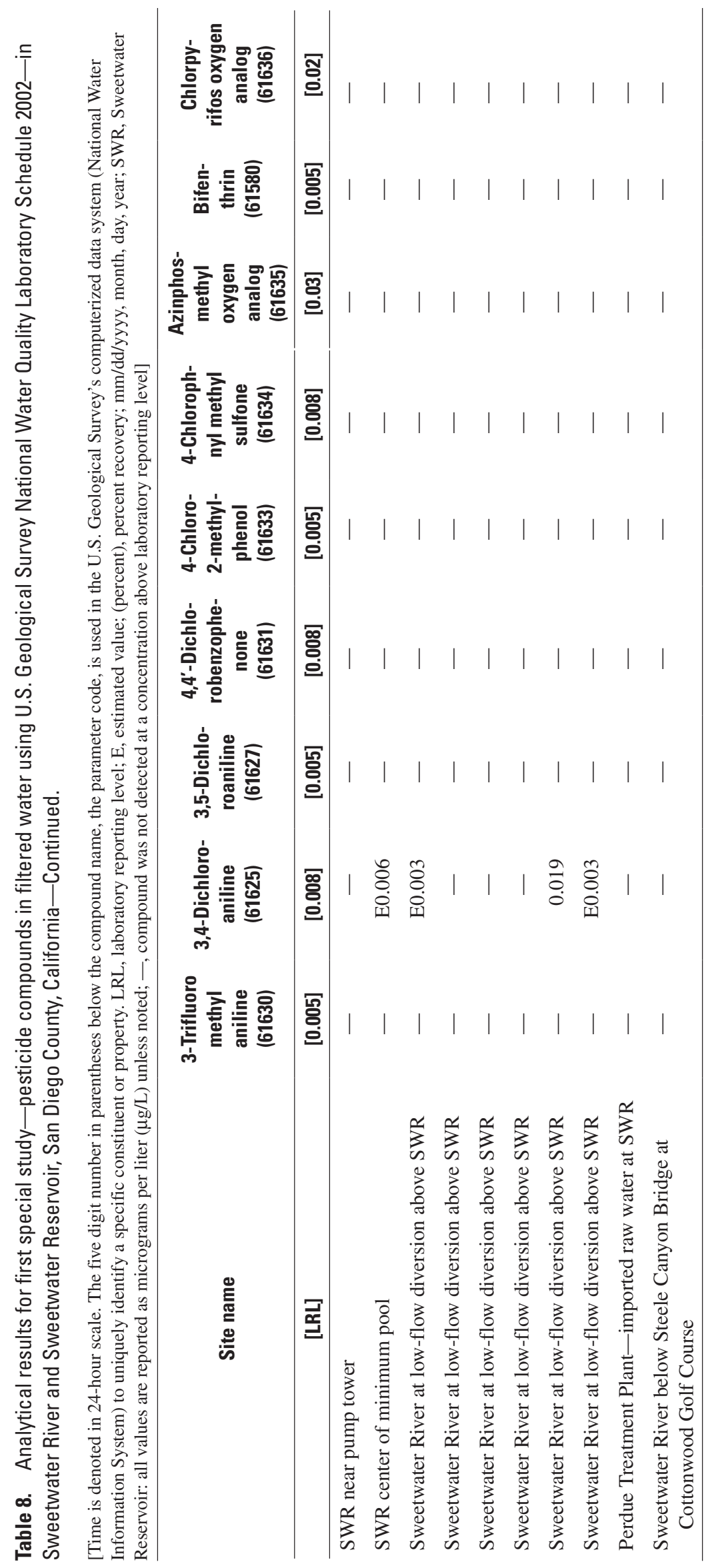




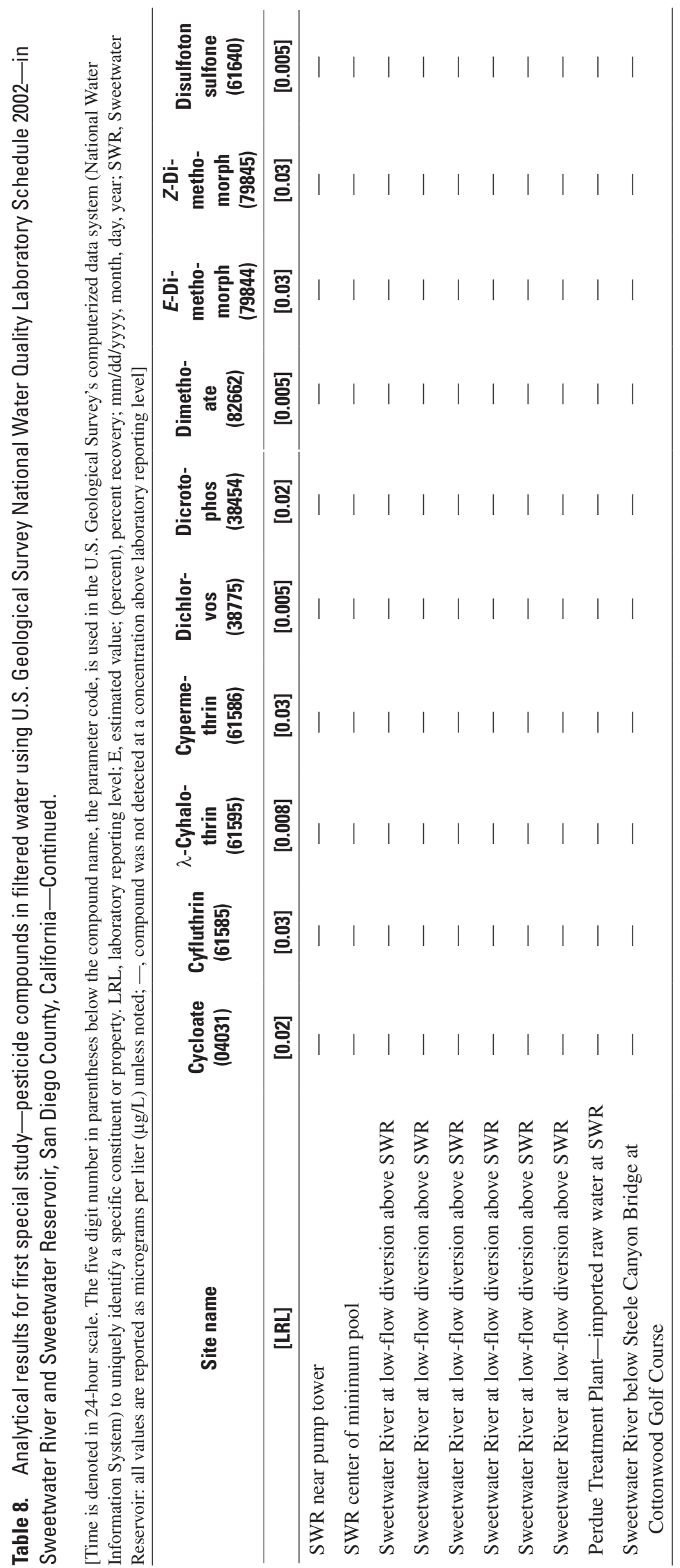




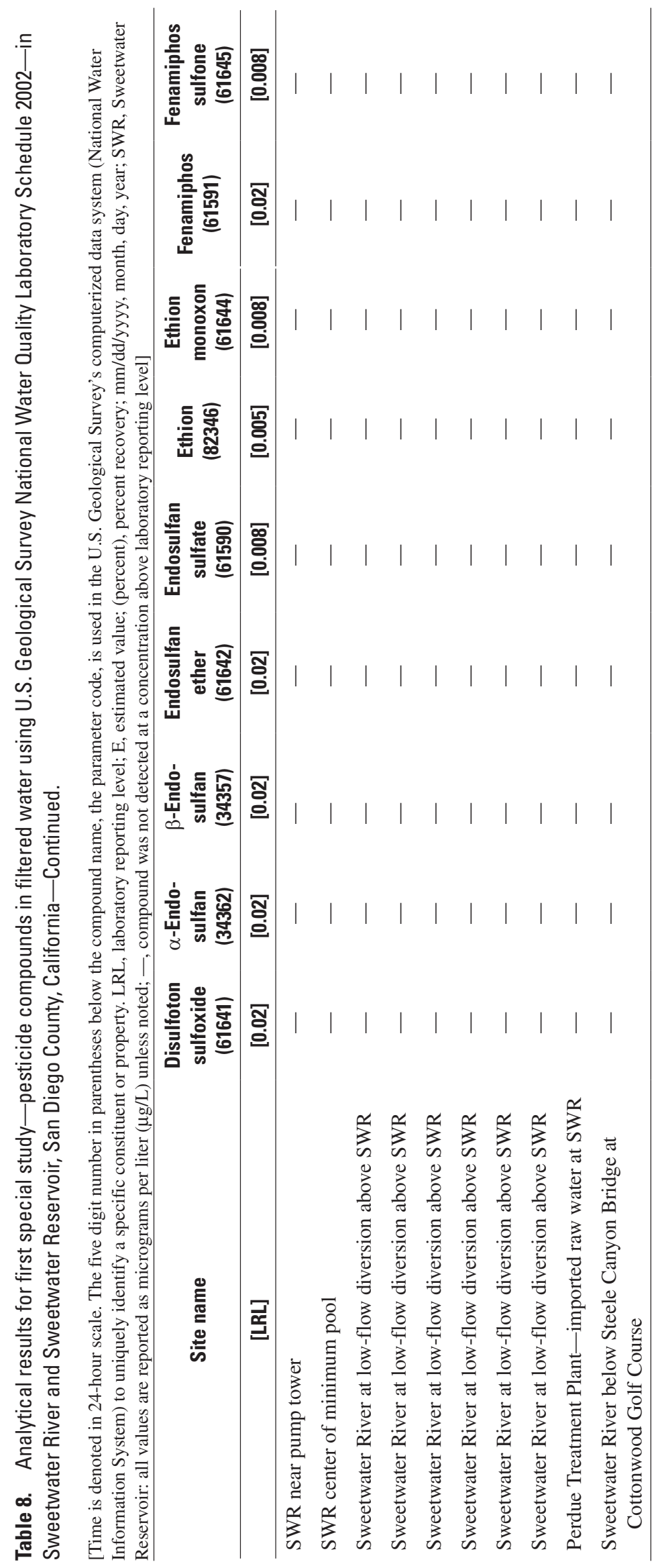




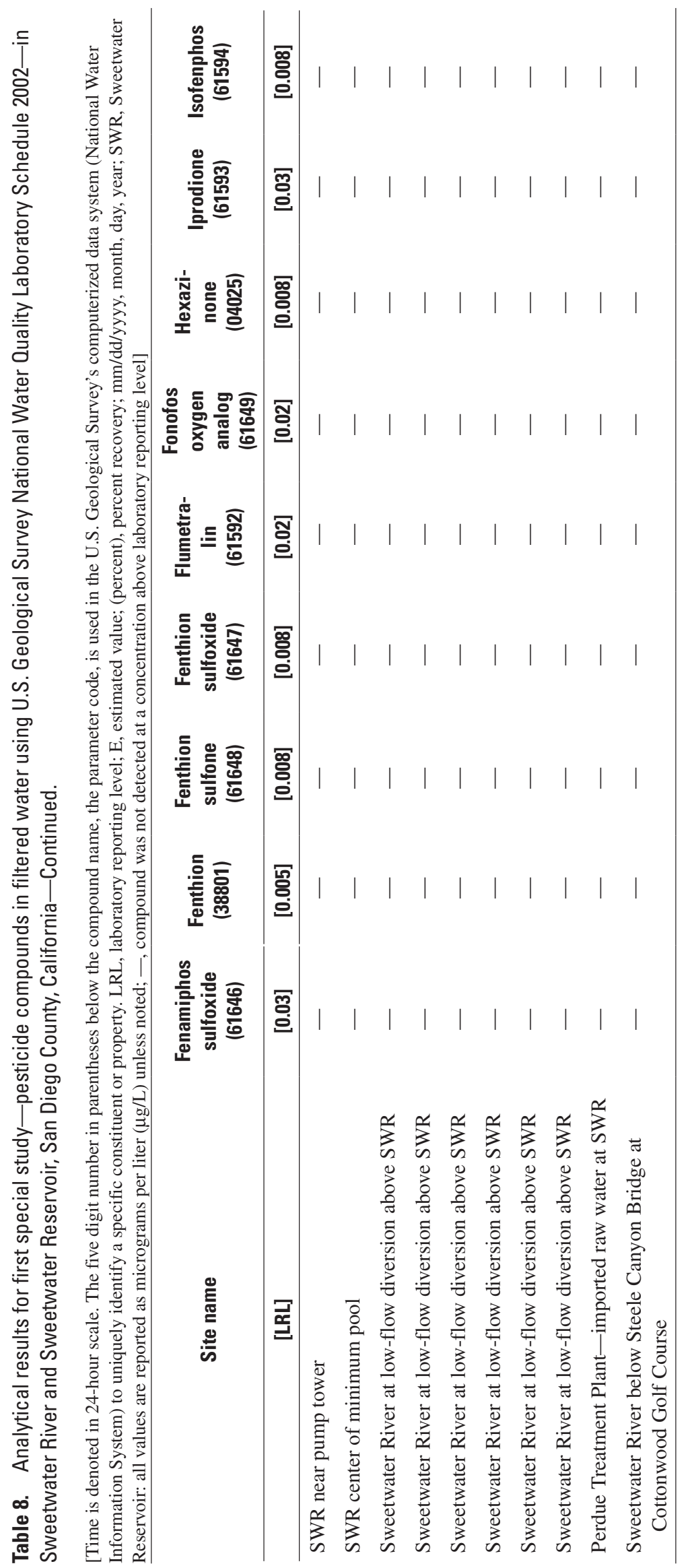




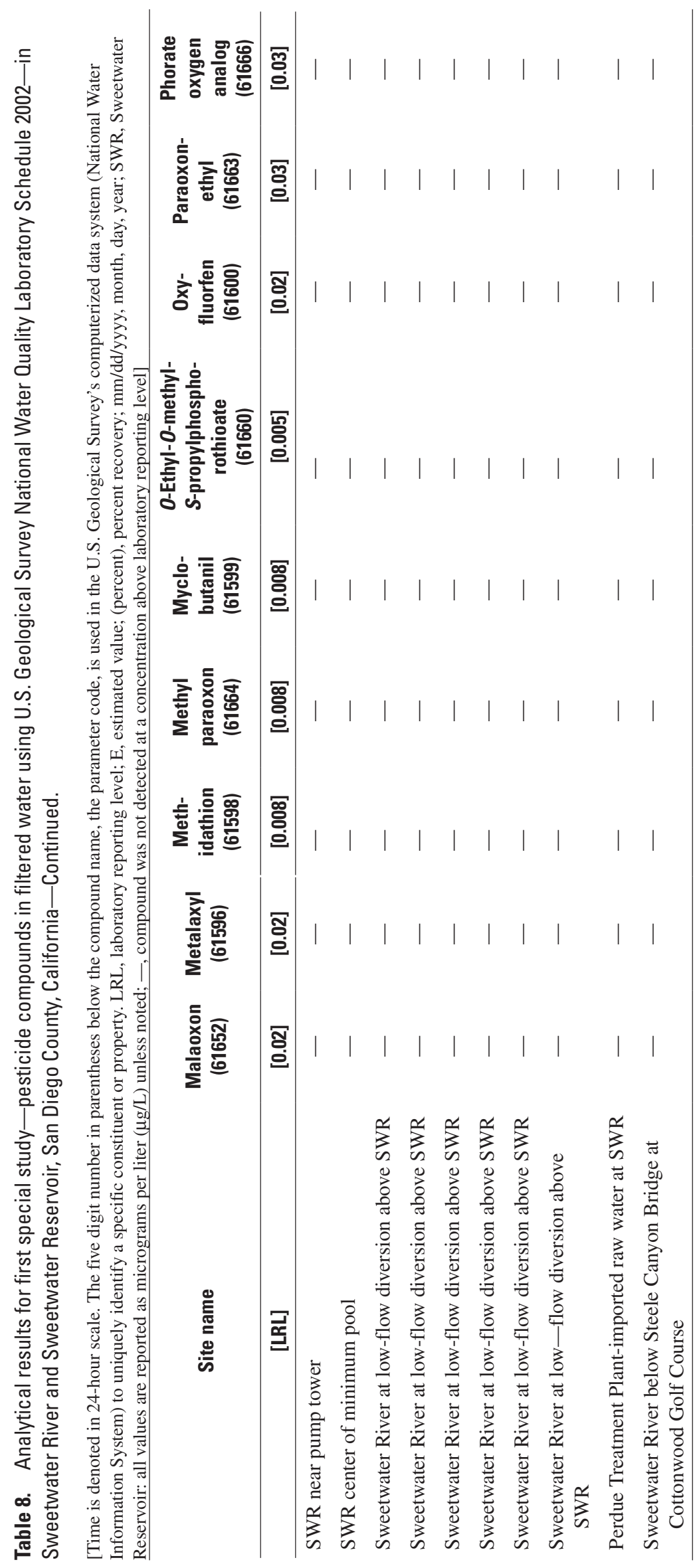




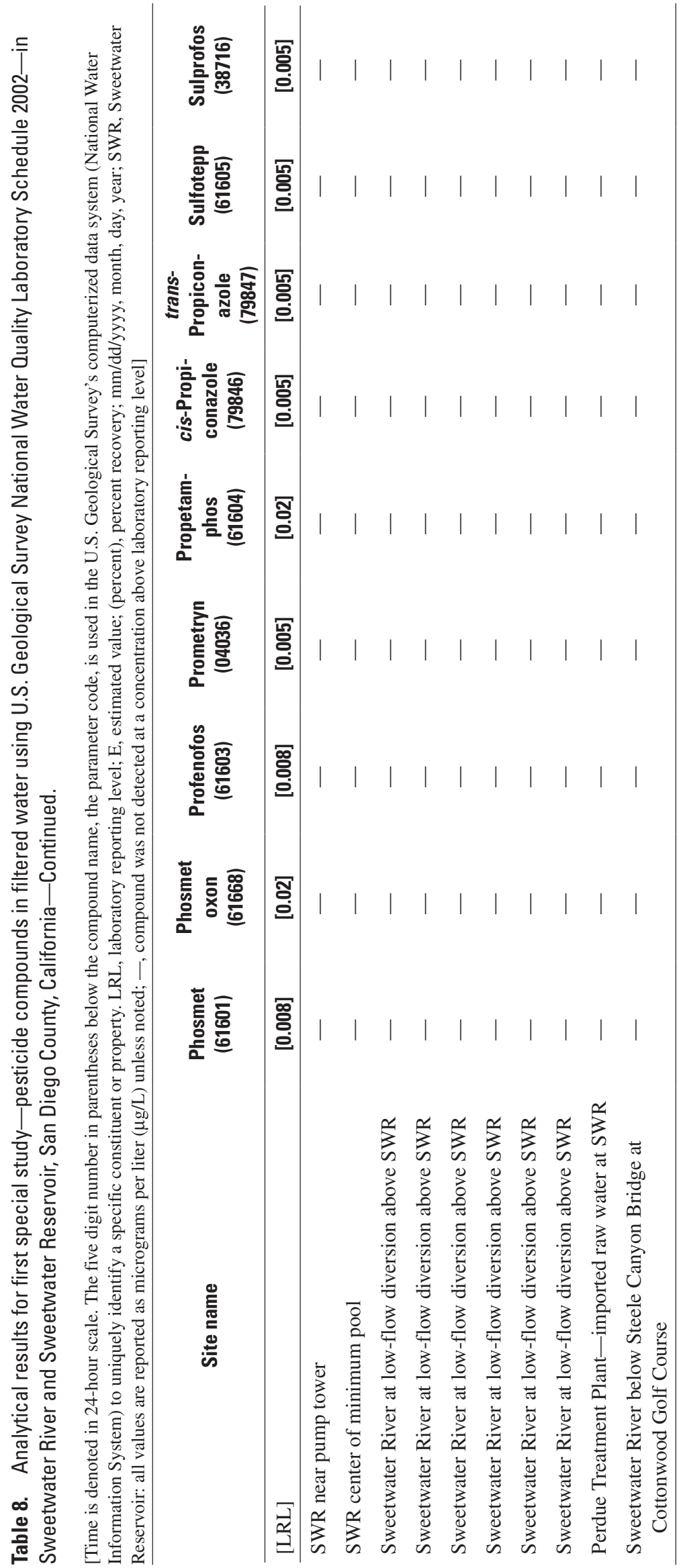




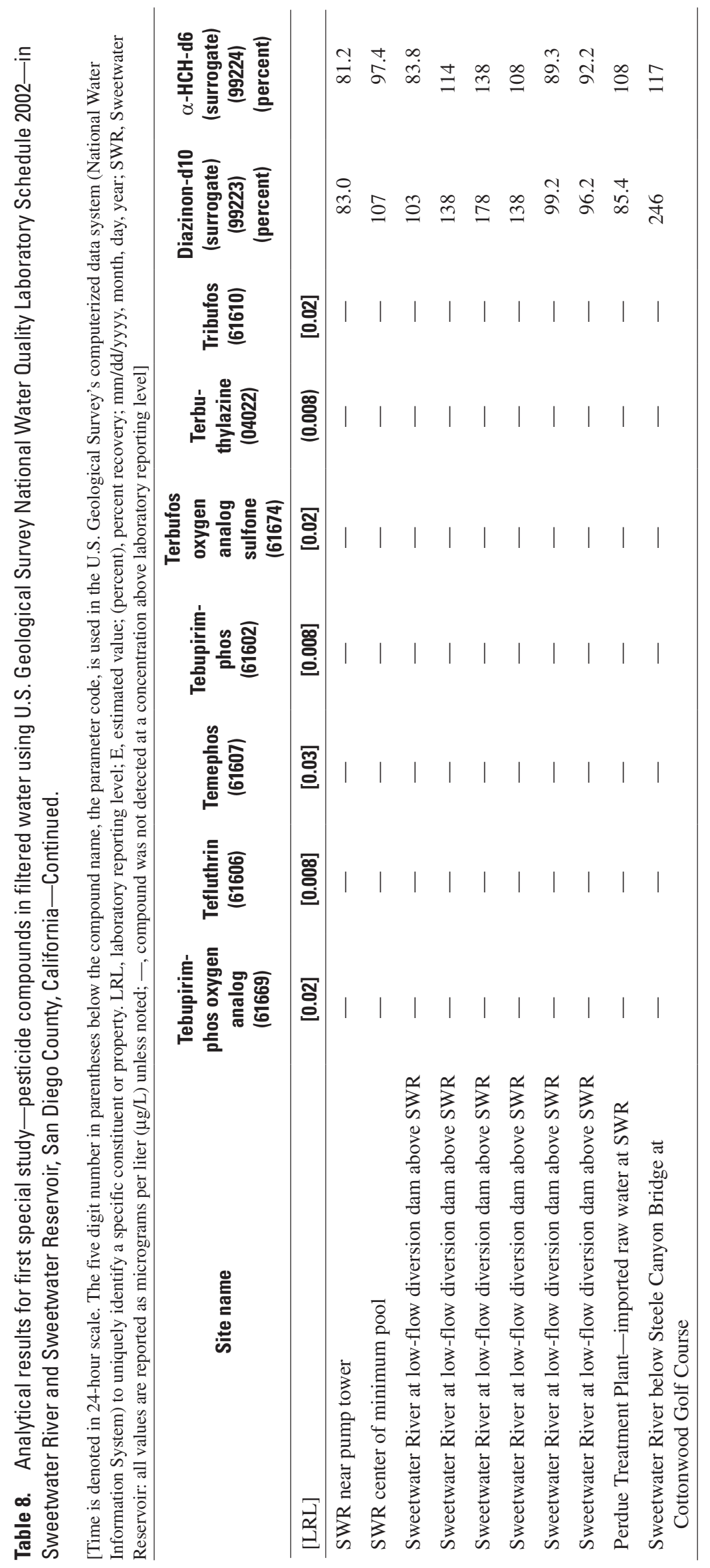


Table 9A. Analytical results for second special study_pesticides in semipermeable membrane device (SPMD) samples-for the Sweetwater Reservoir, San Diego County, California.

[Time is denoted in 24-hour scale. SWR, Sweetwater Reservoir; E, estimated value; kg, kilogram; $\mu \mathrm{g} / \mathrm{kg}$, microgram per kilogram; mm/dd/yyyy, month/day/ year; $<$, compound was not detected at a concentration above the laboratory reporting level]

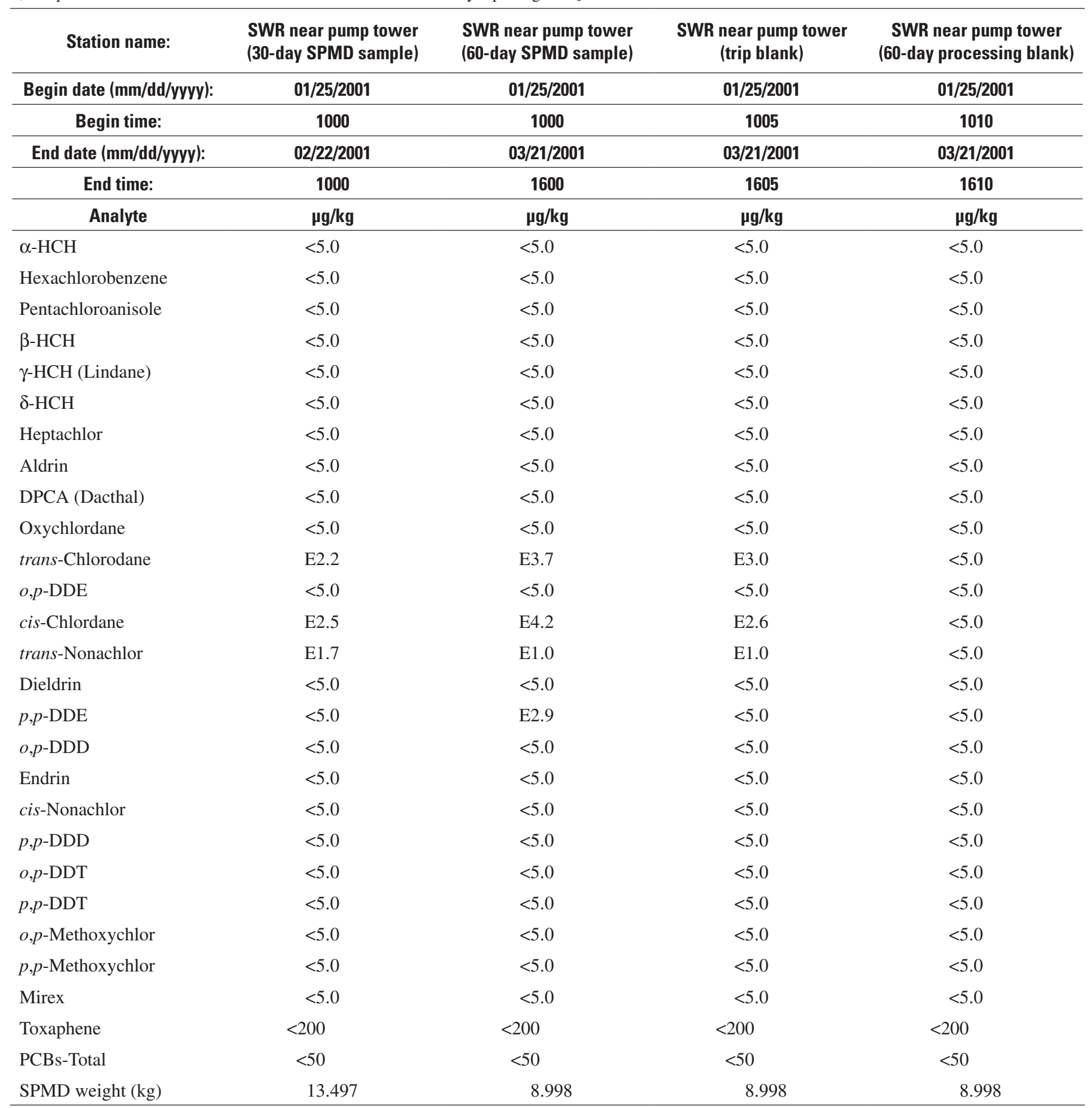


Table 9B. Analytical results for second special study—polycyclic aromatic hydrocarbons and other semivolatile organic compounds in semipermeable membrane device (SPMD) samples—-for the Sweetwater Reservoir, San Diego County, California.

[Time is denoted in 24-hour scale. E, estimated value; ID, identification; SWR, Sweetwater Reservoir; mm/dd/yyyy, month/day/year; $\mu \mathrm{g} / \mathrm{kg}$, microgram per kilogram; <, compound was not detected at a concentration above the laboratory reporting level]

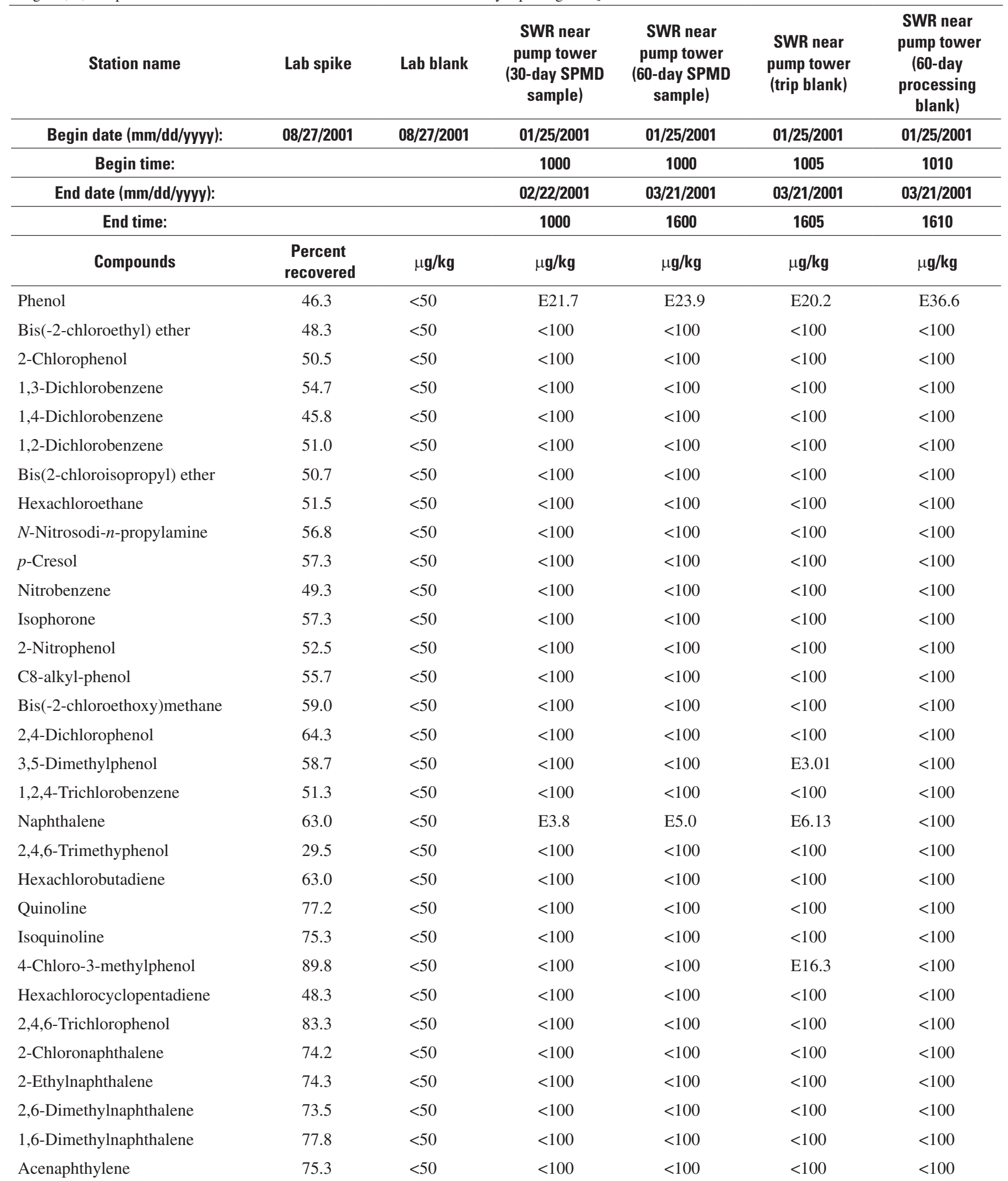


Table 9B. Analytical results for second special study—polycyclic aromatic hydrocarbons and other semivolatile organic compounds in semipermeable membrane device (SPMD) samples—for the Sweetwater Reservoir, San Diego County, California—Continued.

[Time is denoted in 24-hour scale. E, estimated value; ID, identification; SWR, Sweetwater Reservoir; mm/dd/yyyy, month/day/year; $\mu \mathrm{g} / \mathrm{kg}$, microgram per kilogram; <, compound was not detected at a concentration above the laboratory reporting level]

\begin{tabular}{|c|c|c|c|c|c|c|}
\hline Station name & Lab spike & Lab blank & $\begin{array}{c}\text { SWR near } \\
\text { pump tower } \\
\text { (30-day SPMD } \\
\text { sample) }\end{array}$ & $\begin{array}{c}\text { SWR near } \\
\text { pump tower } \\
\text { (60-day SPMD } \\
\text { sample) }\end{array}$ & $\begin{array}{l}\text { SWR near } \\
\text { pump tower } \\
\text { (trip blank) }\end{array}$ & $\begin{array}{l}\text { SWR near } \\
\text { pump tower } \\
\text { (60-day } \\
\text { processing } \\
\text { blank) }\end{array}$ \\
\hline Begin date (mm/dd/yyyy): & 08/27/2001 & 08/27/2001 & 01/25/2001 & $01 / 25 / 2001$ & $01 / 25 / 2001$ & $01 / 25 / 2001$ \\
\hline Begin time: & & & 1000 & 1000 & 1005 & 1010 \\
\hline End time: & & & 1000 & 1600 & 1605 & 1610 \\
\hline Compounds & $\begin{array}{l}\text { Percent } \\
\text { recovered }\end{array}$ & $\mu \mathbf{g} / \mathbf{k g}$ & $\mu \mathbf{g} / \mathbf{k g}$ & $\mu \mathbf{g} / \mathbf{k g}$ & $\mu \mathbf{g} / \mathbf{k g}$ & $\mu \mathbf{g} / \mathbf{k g}$ \\
\hline 1,2-Dimethylnaphthalene & 78.5 & $<50$ & $<100$ & $<100$ & $<100$ & $<100$ \\
\hline Dimethylphthalate & 87.3 & $<50$ & $<100$ & $<100$ & $<100$ & $<100$ \\
\hline 4-Nitrophenol & 86.5 & $<50$ & $<100$ & $<100$ & $<100$ & $<100$ \\
\hline 2,4-Dinitrotol & 84.7 & $<50$ & $<100$ & $<100$ & $<100$ & $<100$ \\
\hline 2,4-Dinitrophenol & 69.3 & $<50$ & $<100$ & $<100$ & $<100$ & $<100$ \\
\hline 2,3,6-Trimethylnaphthalene & 83.8 & $<50$ & $<100$ & $<100$ & $<100$ & $<100$ \\
\hline 9H-Fluorene & 83.2 & $<50$ & $<100$ & $<100$ & $<100$ & $<100$ \\
\hline 4-Chlorophenyl phenyl ether & 82.2 & $<50$ & $<100$ & $<100$ & $<100$ & $<100$ \\
\hline Diethylphthalate & 87.2 & $<50$ & $<100$ & $<100$ & $<100$ & $<100$ \\
\hline$N$-Nitrosodiphenylamine & 88.3 & $<50$ & $<100$ & $<100$ & $<100$ & $<100$ \\
\hline Azo-benzene & 84.3 & $<50$ & $<100$ & $<100$ & $<100$ & $<100$ \\
\hline Pentachlorophenol & 85.5 & $<50$ & $<100$ & $<100$ & $<100$ & $<100$ \\
\hline Pentachloronitrophenol & 88.7 & $<50$ & $<100$ & $<100$ & $<100$ & $<100$ \\
\hline Phenanthrene & 90.7 & $<50$ & E12.2 & E7.5 & $<100$ & $<100$ \\
\hline Anthracene & 88.0 & $<50$ & $<100$ & $<100$ & $<100$ & $<100$ \\
\hline Acridine & 88.7 & $<50$ & $<100$ & $<100$ & $<100$ & $<100$ \\
\hline Phenanthridine & 87.8 & $<50$ & $<100$ & $<100$ & $<100$ & $<100$ \\
\hline 9H-Carbazol & 88.5 & $<50$ & $<100$ & $<100$ & $<100$ & $<100$ \\
\hline 2-Methylanthracene & 87.0 & $<50$ & $<100$ & $<100$ & $<100$ & $<100$ \\
\hline $\operatorname{Benzo}(c)$ quinoline & 87.5 & $<50$ & $<100$ & $<100$ & $<100$ & $<100$ \\
\hline 4,5-Methylenephenanthrene & 86.7 & $<50$ & $<100$ & $<100$ & $<100$ & $<100$ \\
\hline 1-Methylphenanthrene & 86.3 & $<50$ & $<100$ & $<100$ & $<100$ & $<100$ \\
\hline Di- $n$-butyl phthalate & 87.8 & $<50$ & $\mathrm{E} 22.7$ & E10.2 & E15.1 & E3.1 \\
\hline Anthraquinone & 88.3 & $<50$ & $<100$ & $<100$ & $<100$ & $<100$ \\
\hline
\end{tabular}


Table 9B. Analytical results for second special study—polycyclic aromatic hydrocarbons and other semivolatile organic compounds in semipermeable membrane device (SPMD) samples—for the Sweetwater Reservoir, San Diego County, California—Continued.

[Time is denoted in 24-hour scale. E, estimated value; ID, identification; SWR, Sweetwater Reservoir; mm/dd/yyyy, month/day/year; $\mu \mathrm{g} / \mathrm{kg}$, microgram per kilogram; <, compound was not detected at a concentration above the laboratory reporting level]

\begin{tabular}{|c|c|c|c|c|c|c|}
\hline Station name & Lab spike & Lab blank & $\begin{array}{c}\text { SWR near } \\
\text { pump tower } \\
\text { (30-day SPMD } \\
\text { sample) }\end{array}$ & $\begin{array}{c}\text { SWR near } \\
\text { pump tower } \\
\text { (60-day SPMD } \\
\text { sample) }\end{array}$ & $\begin{array}{l}\text { SWR near } \\
\text { pump tower } \\
\text { (trip blank) }\end{array}$ & $\begin{array}{l}\text { SWR near } \\
\text { pump tower } \\
\text { (60-day } \\
\text { processing } \\
\text { blank) }\end{array}$ \\
\hline Begin date $(\mathrm{mm} / \mathrm{dd} / \mathrm{yyyy})$ : & 08/27/2001 & 08/27/2001 & 01/25/2001 & 01/25/2001 & $01 / 25 / 2001$ & 01/25/2001 \\
\hline Begin time: & & & 1000 & 1000 & 1005 & 1010 \\
\hline End date $(\mathrm{mm} / \mathrm{dd} / \mathrm{yyyy})$ : & & & $02 / 22 / 2001$ & $03 / 21 / 2001$ & 03/21/2001 & 03/21/2001 \\
\hline End time: & & & 1000 & 1600 & 1605 & 1610 \\
\hline Compounds & $\begin{array}{c}\text { Percent } \\
\text { recovered }\end{array}$ & $\mu \mathrm{g} / \mathrm{kg}$ & $\mu \mathrm{g} / \mathrm{kg}$ & $\mu \mathbf{g} / \mathbf{k g}$ & $\mu \mathbf{g} / \mathbf{k g}$ & $\mu \mathrm{g} / \mathbf{k g}$ \\
\hline Fluoranthene & 88.7 & $<50$ & E6.1 & E7.9 & $<100$ & $<100$ \\
\hline Pyrene & 88.3 & $<50$ & E3.4 & E2.4 & $<100$ & $<100$ \\
\hline 1-Methylpyrene & 88.0 & $<50$ & $<100$ & $<100$ & $<100$ & $<100$ \\
\hline Butylbenzyl-phthalate & 88.3 & $<50$ & $<100$ & $<100$ & $<100$ & $<100$ \\
\hline Benzo(a)anthracene & 91.5 & $<50$ & $<100$ & $<100$ & $<100$ & $<100$ \\
\hline Chrysene & 95.5 & $<50$ & $<100$ & $<100$ & $<100$ & $<100$ \\
\hline Bis(2-ethylhexyl)phthalate & 92.0 & $<50$ & E48.9 & E27.1 & E34.0 & E25.8 \\
\hline 2,2-Biquinoline & 93.3 & $<50$ & $<100$ & $<100$ & $<100$ & $<100$ \\
\hline Di-n-octyl phthalate & 94.2 & $<50$ & $<100$ & $<100$ & $<100$ & $<100$ \\
\hline $\operatorname{Benzo}(b)$ fluoranthene & 97.7 & $<50$ & $<100$ & $<100$ & $<100$ & $<100$ \\
\hline $\operatorname{Benzo}(k)$ fluoranthene & 92.0 & $<50$ & $<100$ & $<100$ & $<100$ & $<100$ \\
\hline Benzo $(a)$ pyrene & 95.7 & $<50$ & $<100$ & $<100$ & $<100$ & $<100$ \\
\hline Indeno(1,2,3-c)pyrene & 92.7 & $<50$ & $<100$ & $<100$ & $<100$ & $<100$ \\
\hline $\operatorname{Dibenz}(a, h)$ anthracene & 89.5 & $<50$ & $<100$ & $<100$ & $<100$ & $<100$ \\
\hline $\operatorname{Benzo}(g, h, i)$ perylene & 59.9 & $<50$ & $<100$ & $<100$ & $<100$ & $<100$ \\
\hline
\end{tabular}


Table 10. Analytical results for third special study—dissolved copper—at the Perdue Treatment Plant, the Reynolds Desalination Facility, and Sweetwater Wells, San Diego County, California.

[Time is denoted in 24-hour scale. The five digit number in parentheses below the compound name, the parameter code, is used in the U.S. Geological Survey's computerized data system (National Water Information System) to uniquely identify a specific constituent or property. E, estimated value; $\mu \mathrm{g} / \mathrm{L}$, microgram per liter; <, compound was not detected at a concentration above the laboratory reporting level]

\begin{tabular}{|c|c|c|c|}
\hline Station name & Date & Time & $\begin{array}{c}\text { Copper } \\
(\mu \mathrm{g} / \mathrm{L}) \\
(01040)\end{array}$ \\
\hline Perdue Treatment Plant-finished water at Sweetwater Reservoir & April 26, 2001 & 1110 & 2.8 \\
\hline Perdue Treatment Plant-finished water at Sweetwater Reservoir & July 24, 2001 & 1120 & 2.3 \\
\hline Perdue Treatment Plant-imported raw water at Sweetwater Reservoir & April 26, 2001 & 1130 & 4.1 \\
\hline Reynolds Desalination Facility-discharge & July 24, 2001 & 1010 & E1.1 \\
\hline Reynolds Desalination Facility-effluent & July 24, 2001 & 1020 & $<1$ \\
\hline Reynolds Desalination Facility-feed & July 24, 2001 & 1030 & 3.7 \\
\hline Reynolds Desalination Facility-inflow & July 24, 2001 & 1040 & $<1$ \\
\hline $17 \mathrm{~S} / 2 \mathrm{~W}-27 \mathrm{P} 1$ & July 24, 2001 & 0930 & 1.9 \\
\hline $17 \mathrm{~S} / 2 \mathrm{~W}-27 \mathrm{P} 2$ & July 24, 2001 & 0950 & $<1$ \\
\hline $17 \mathrm{~S} / 2 \mathrm{~W}-28 \mathrm{R} 1$ & July 24, 2001 & 1100 & 1.6 \\
\hline $17 \mathrm{~S} / 2 \mathrm{~W}-34 \mathrm{C} 1$ & July 24, 2001 & 1000 & $<1$ \\
\hline
\end{tabular}



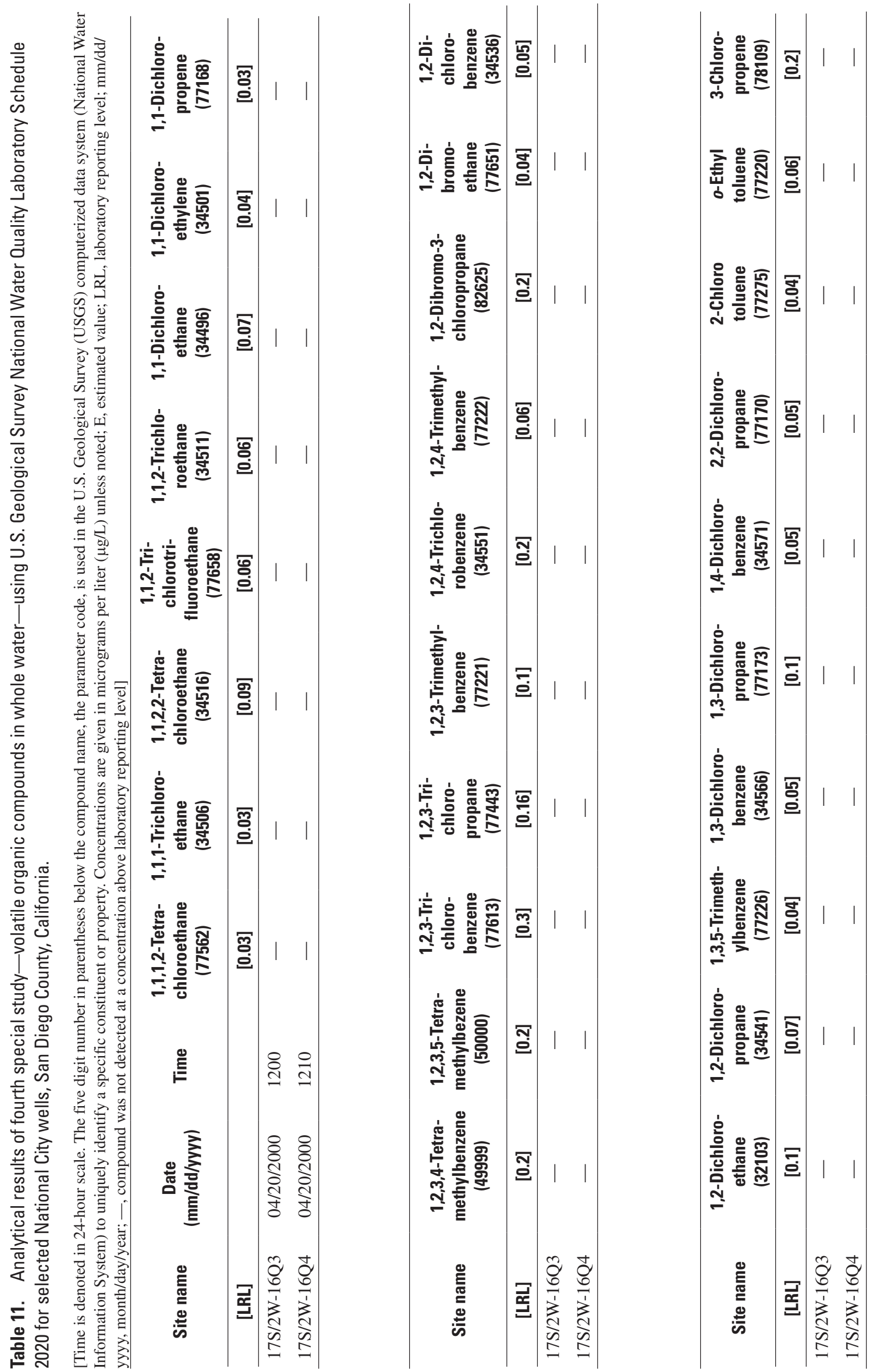

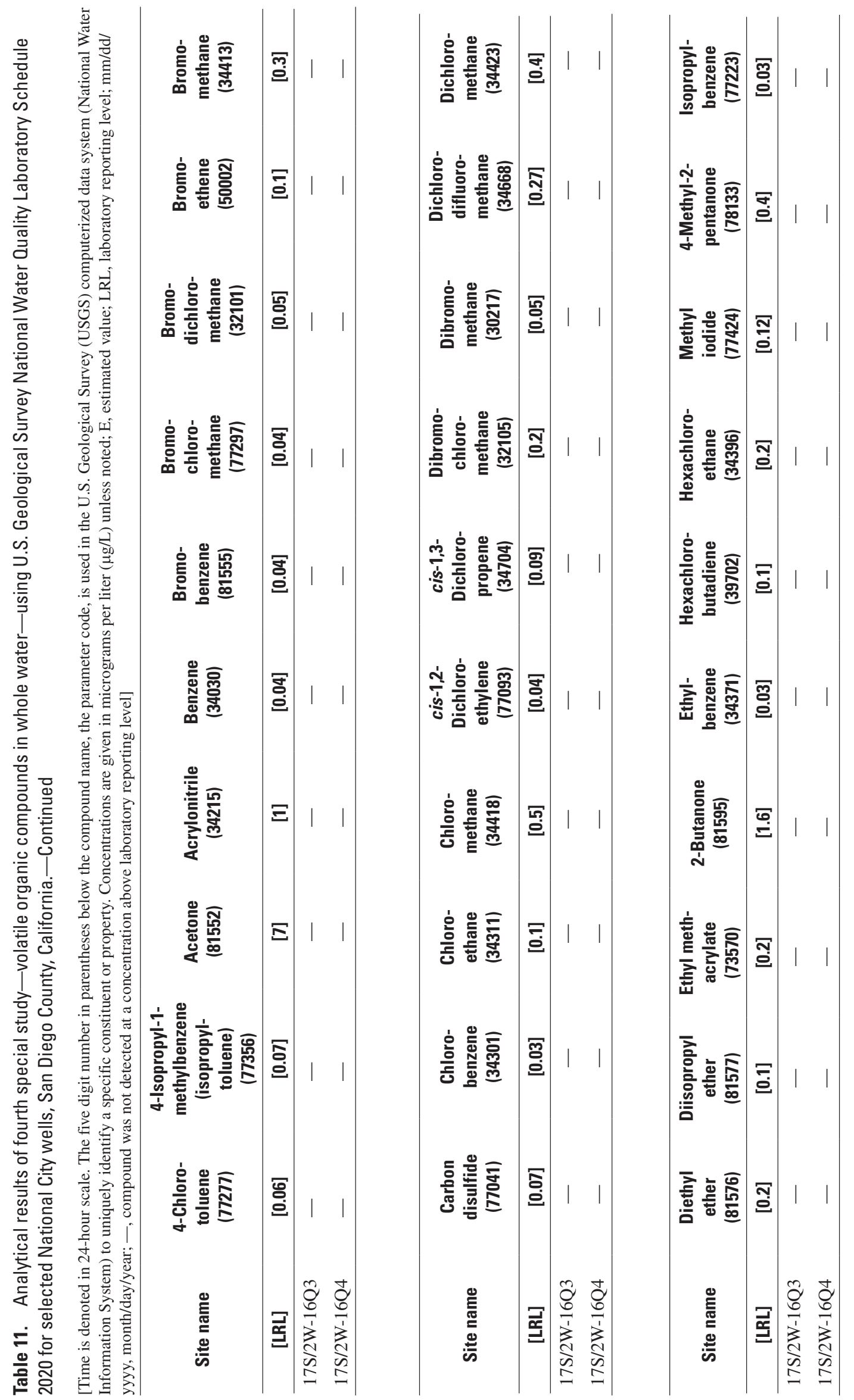

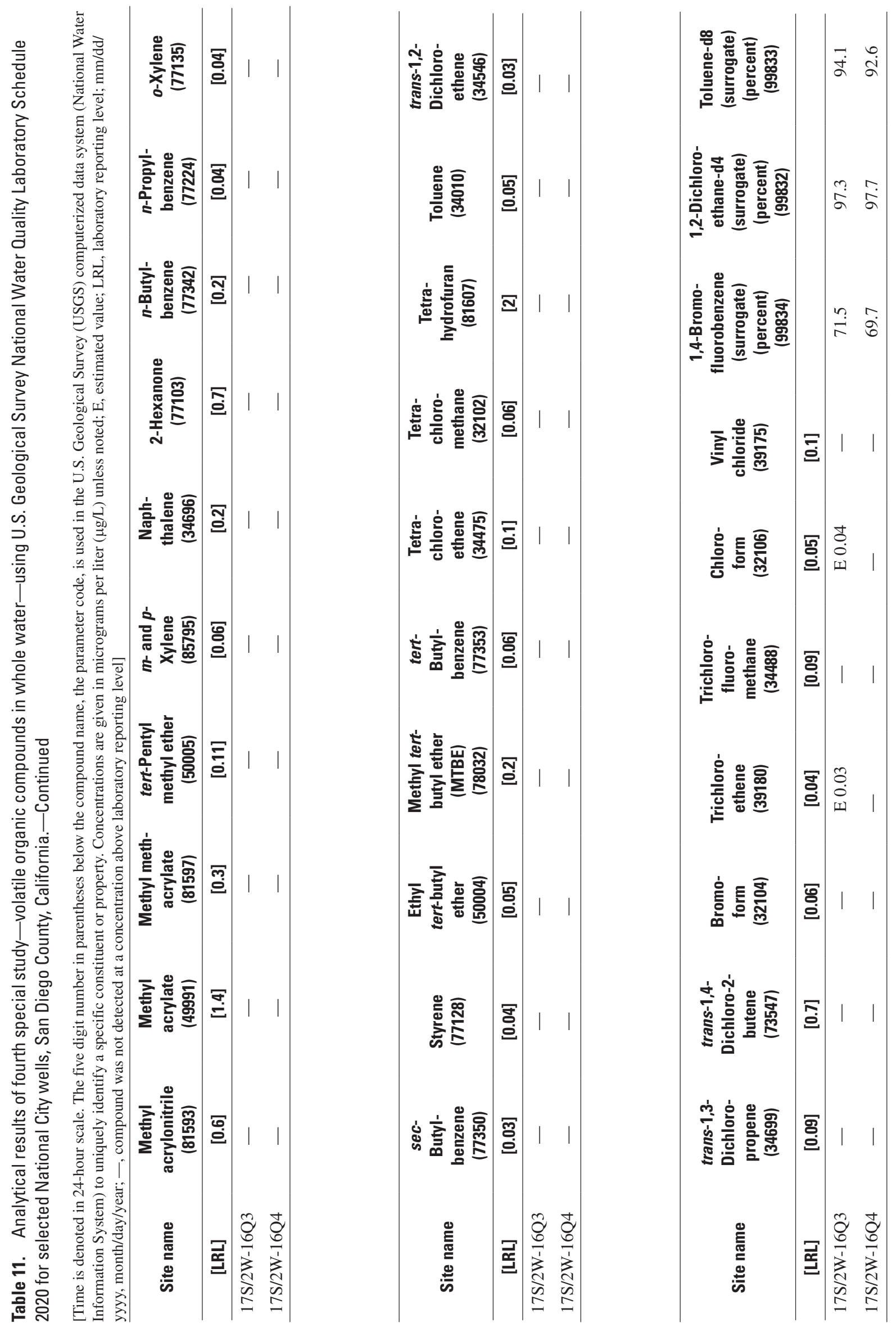


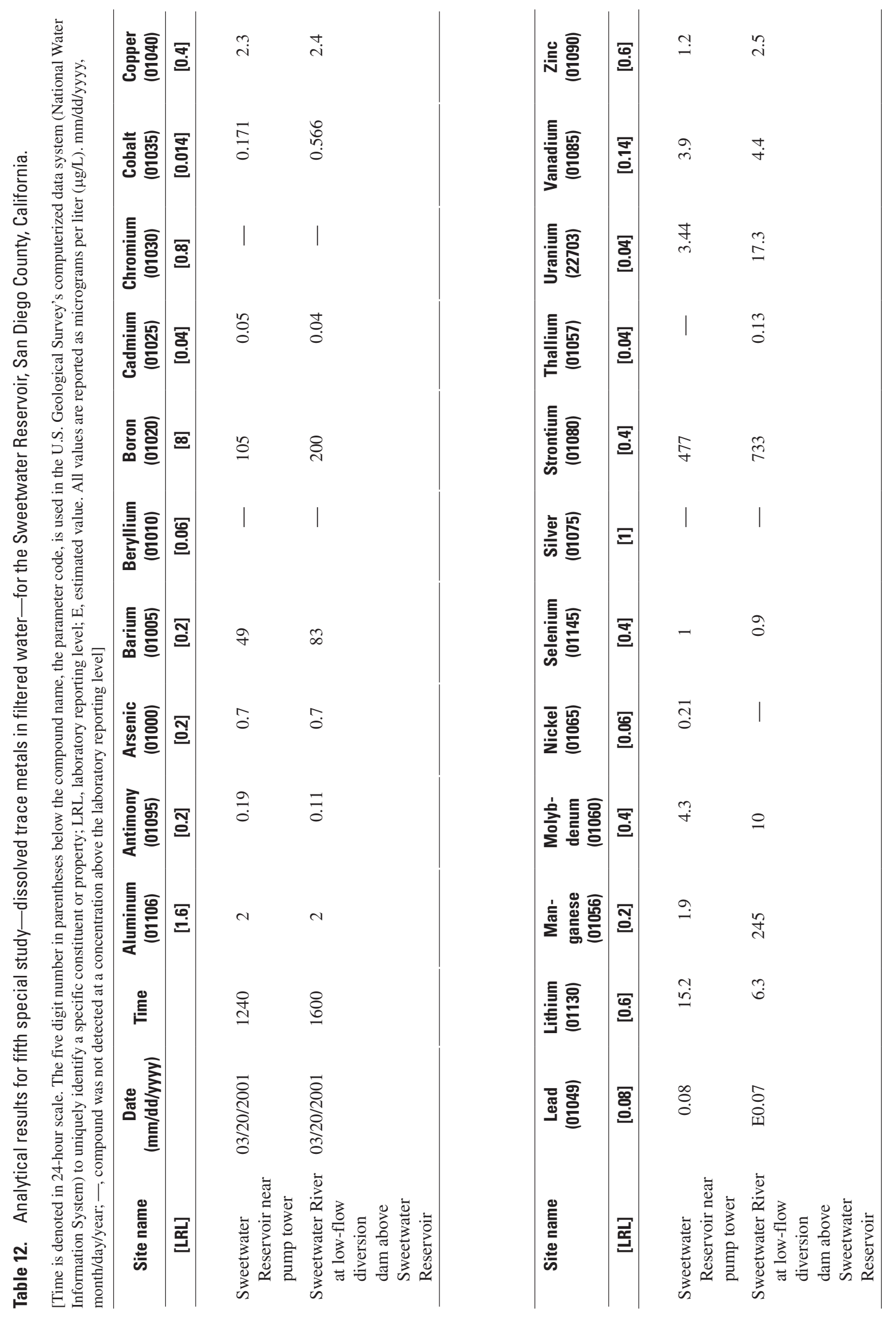



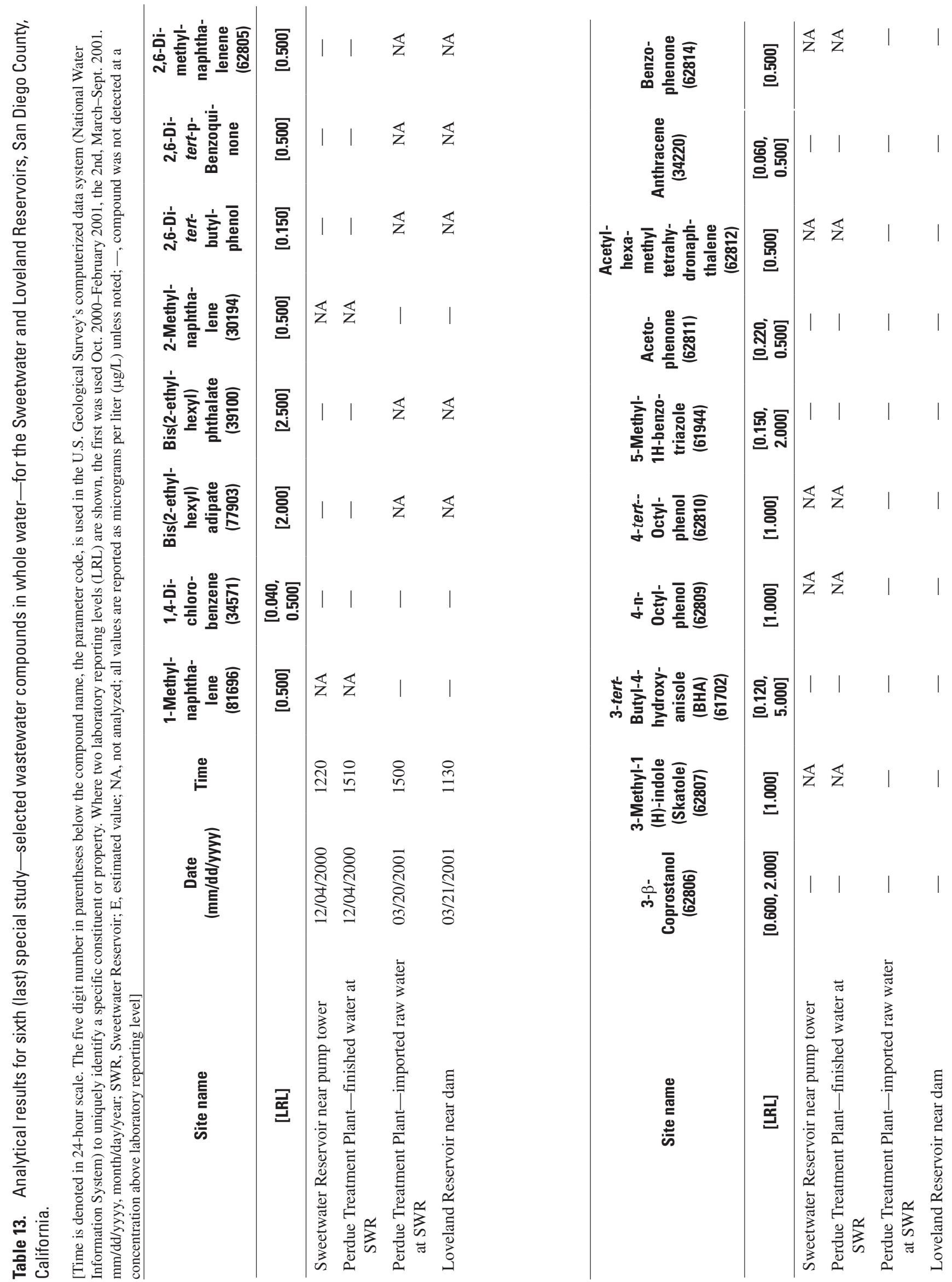

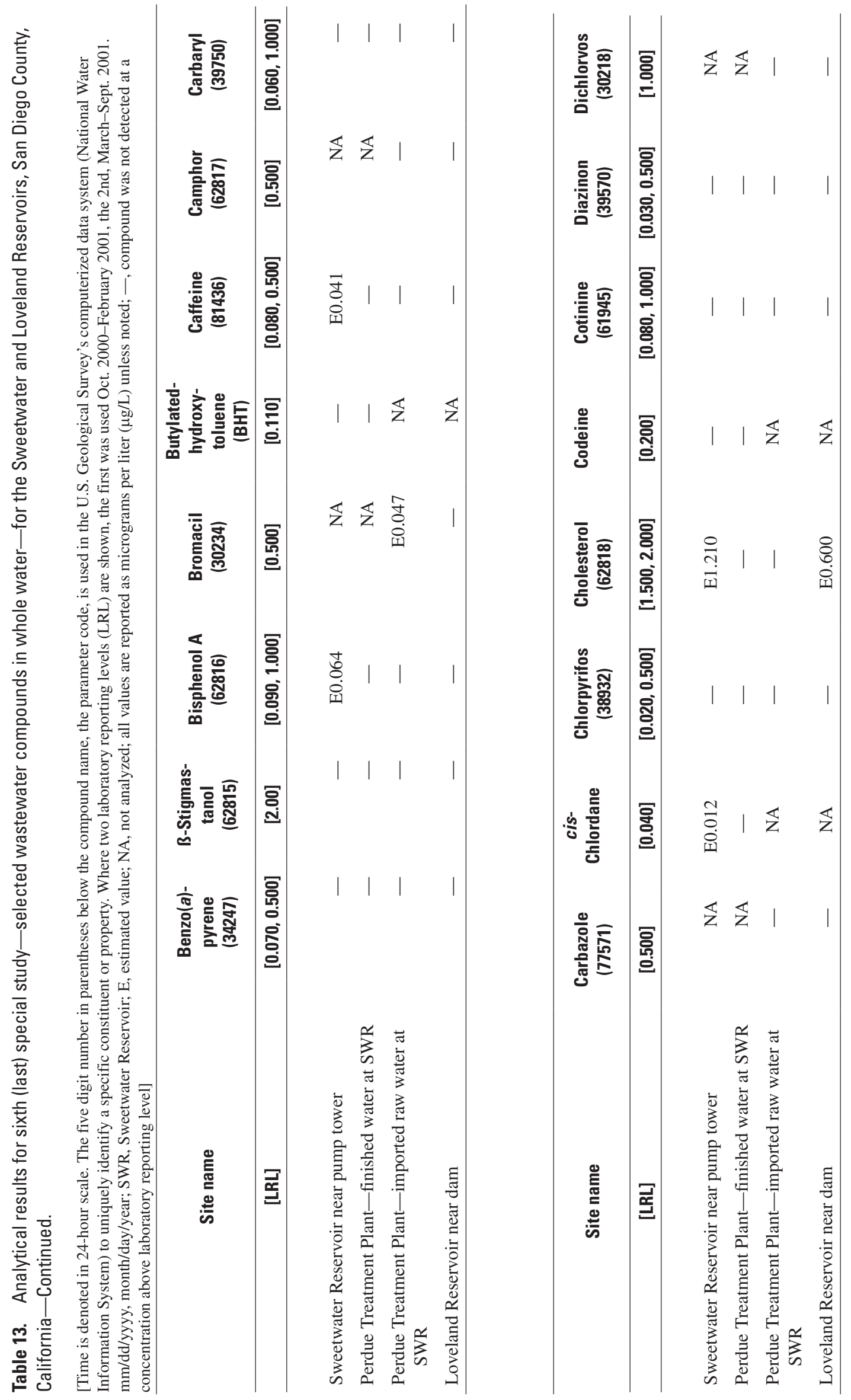

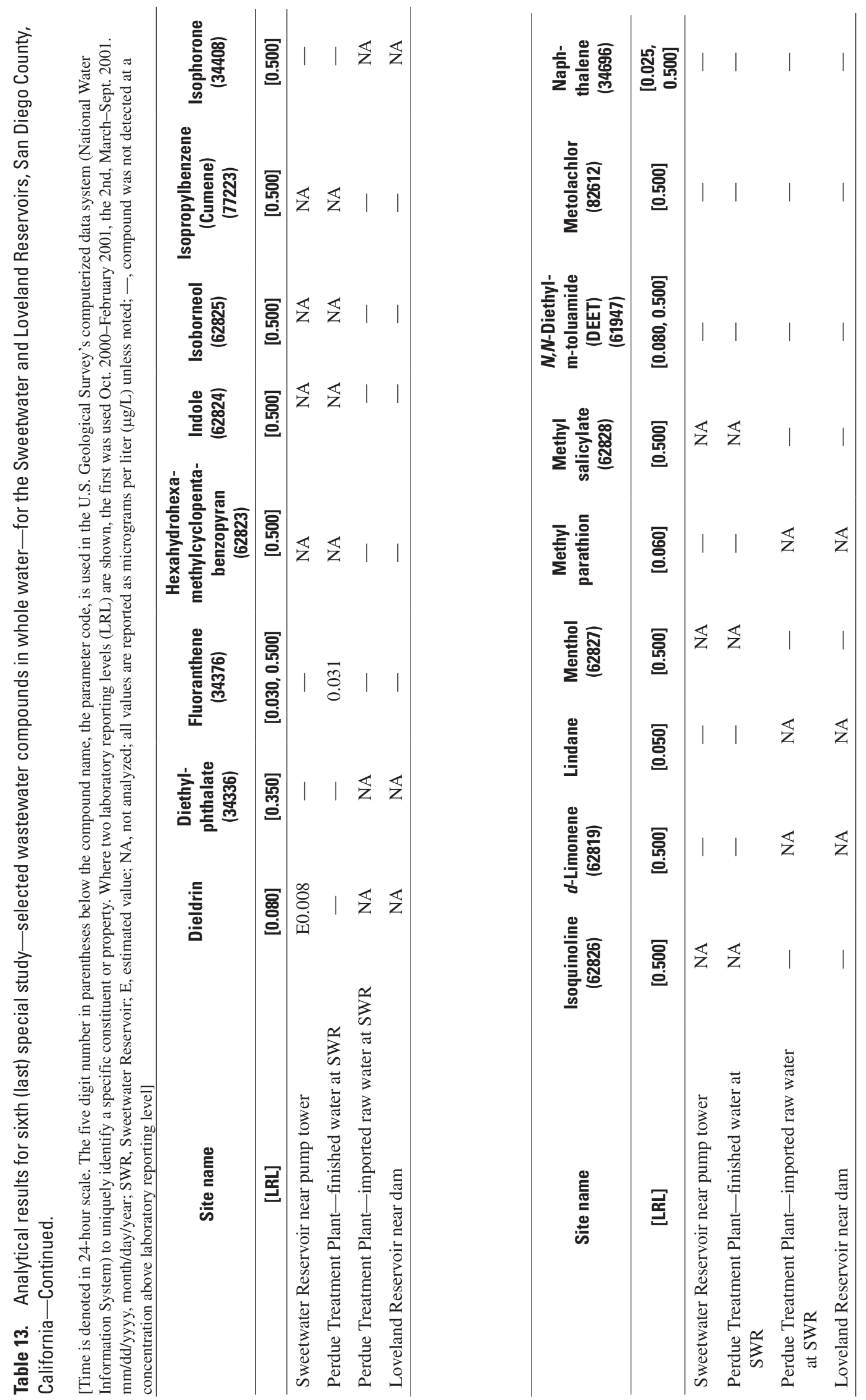

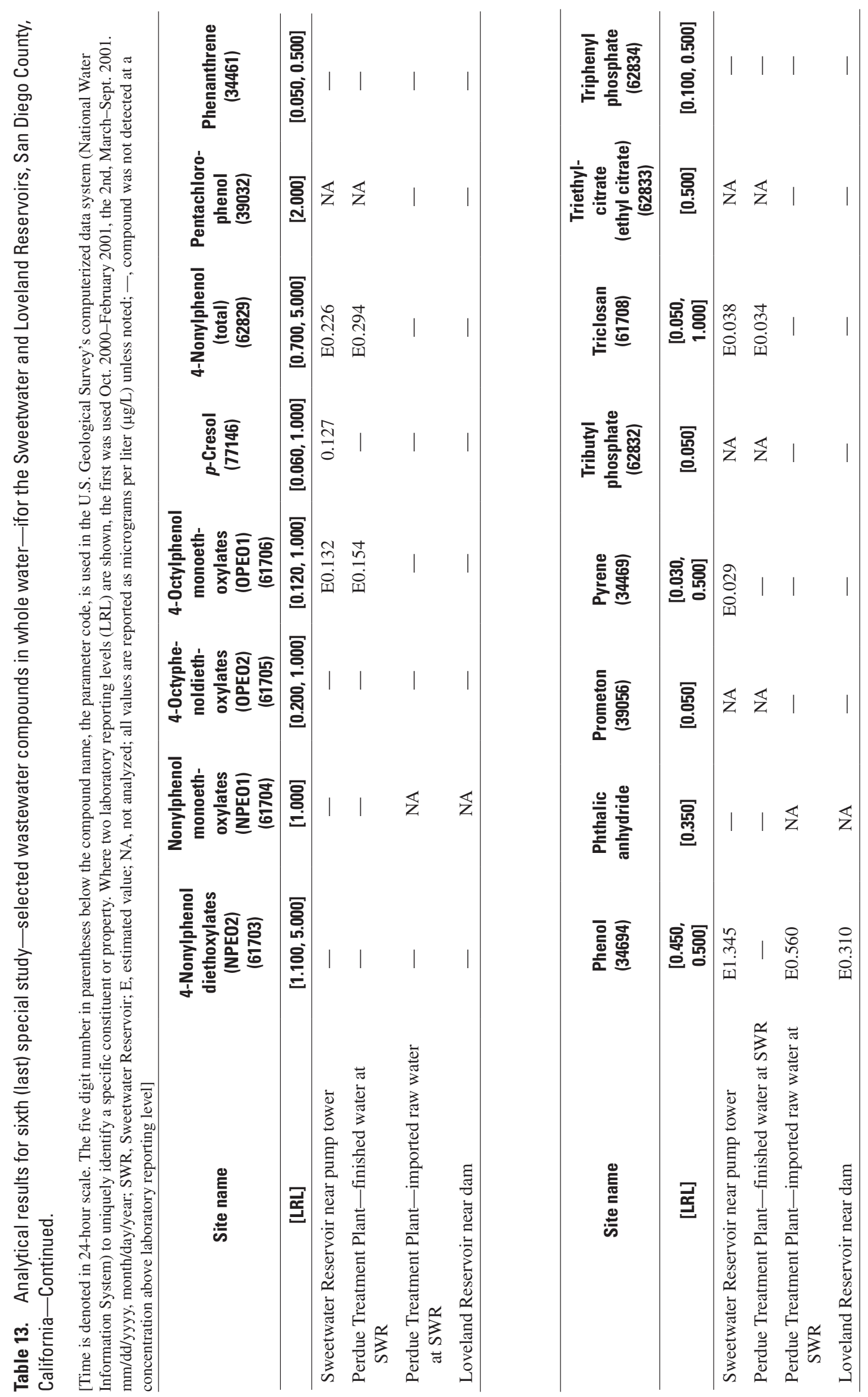


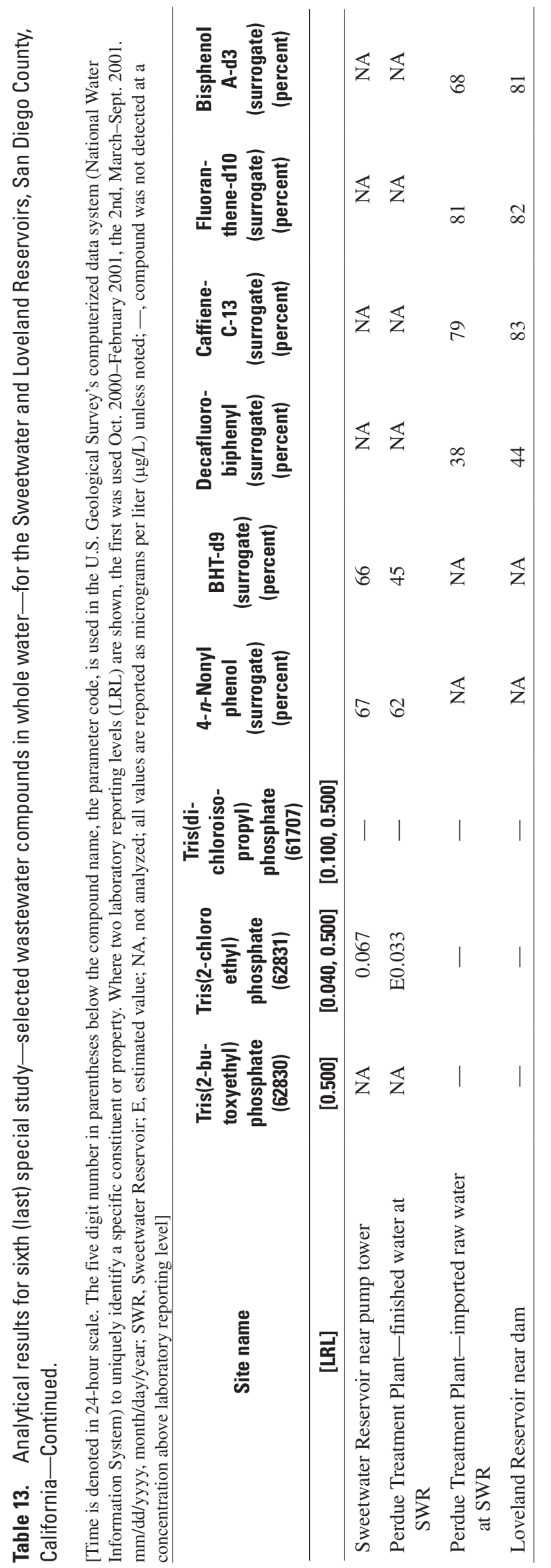




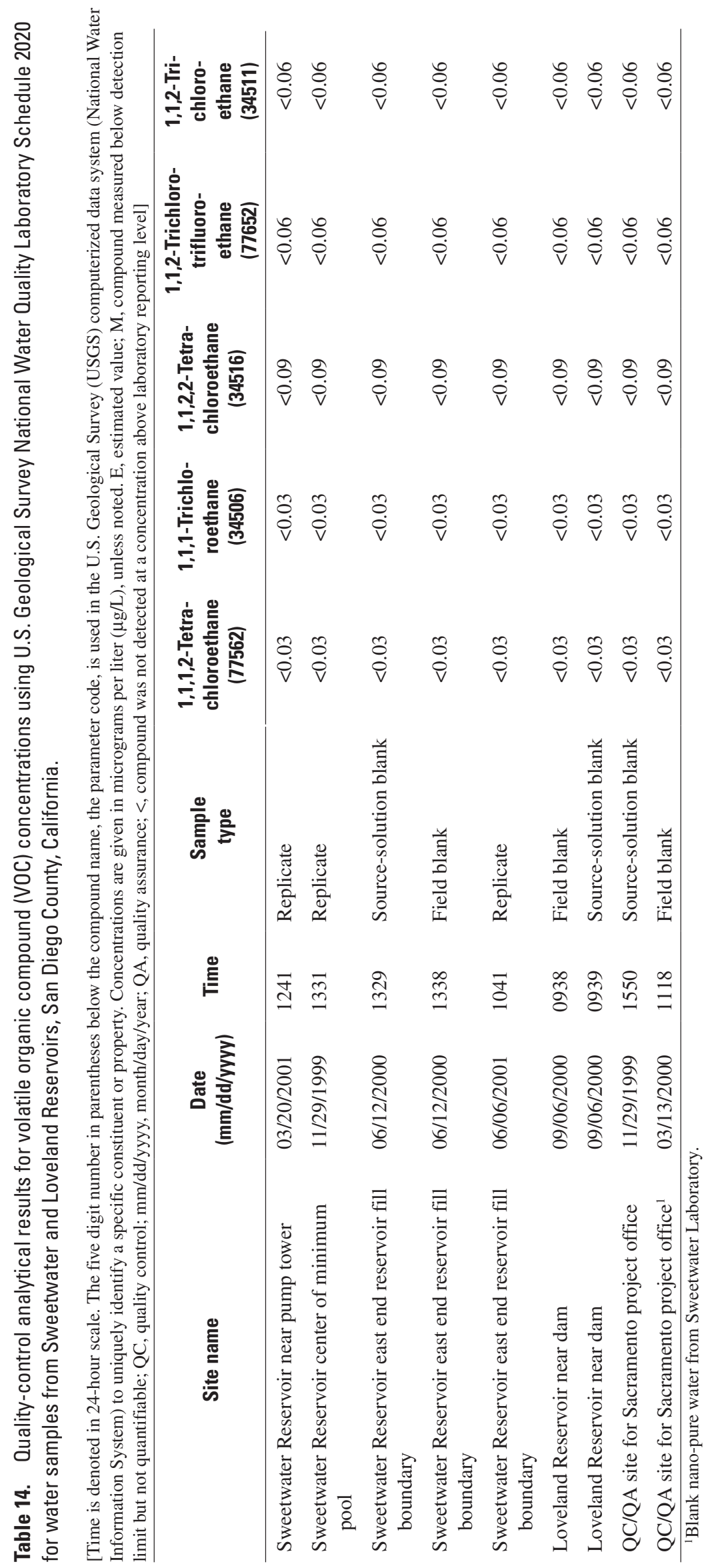




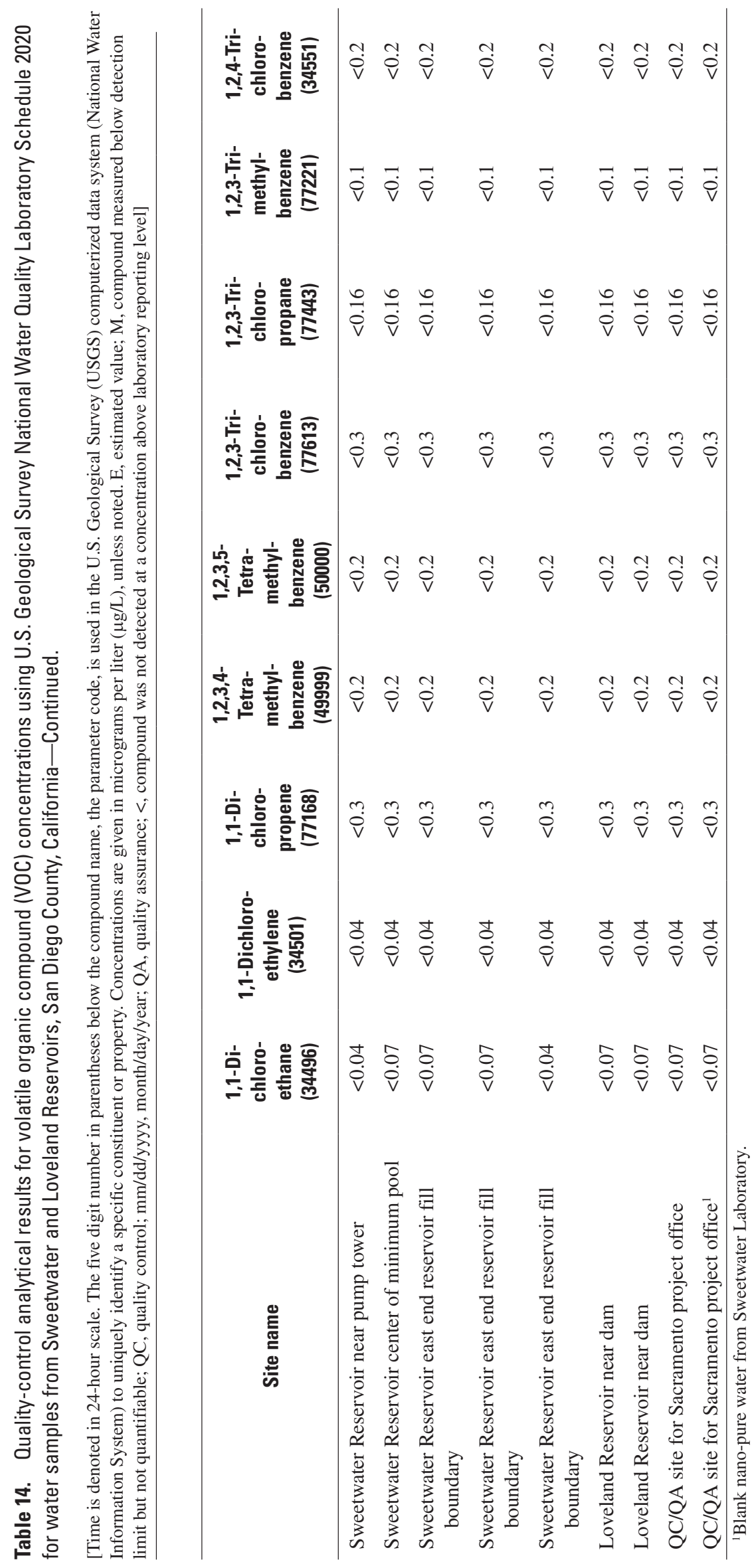




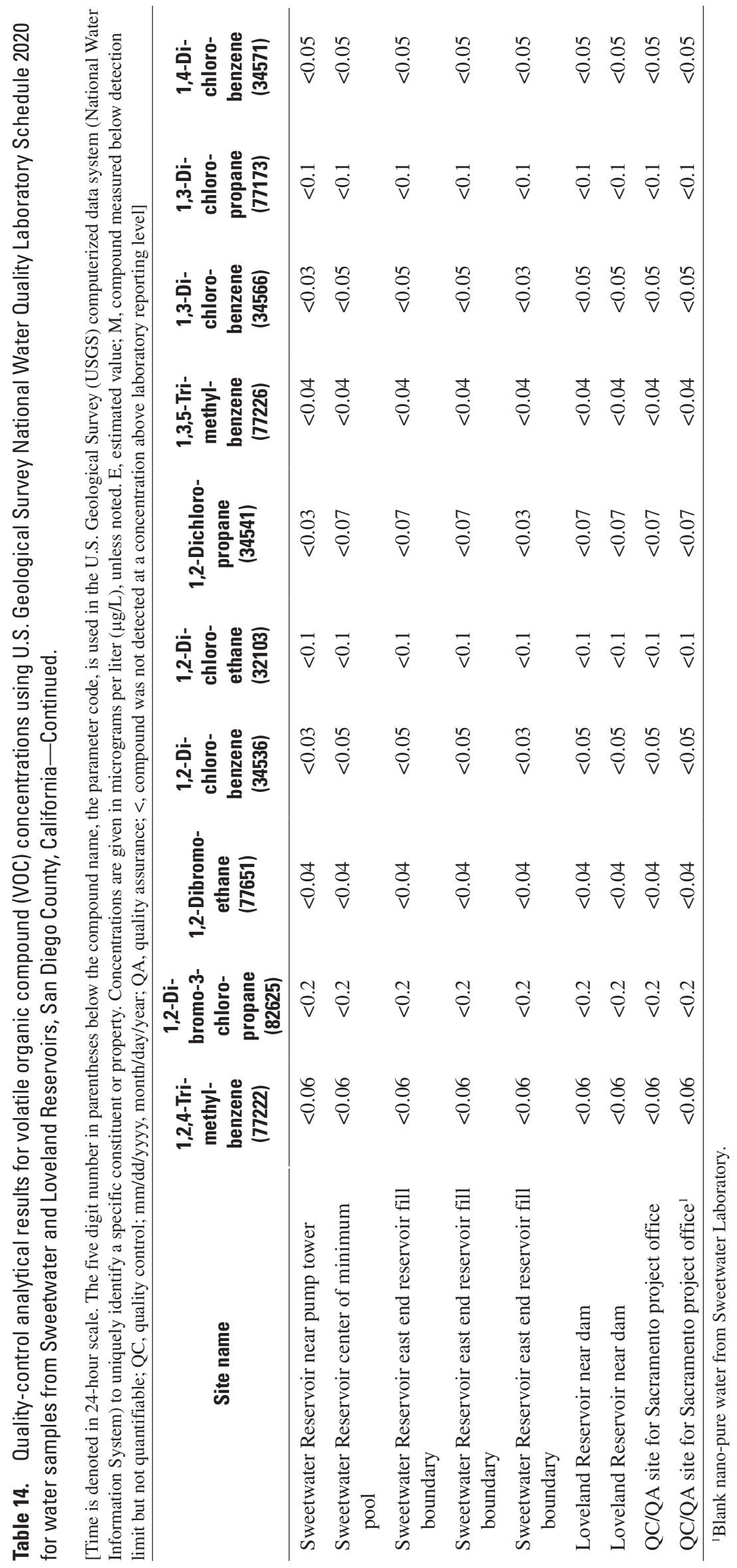




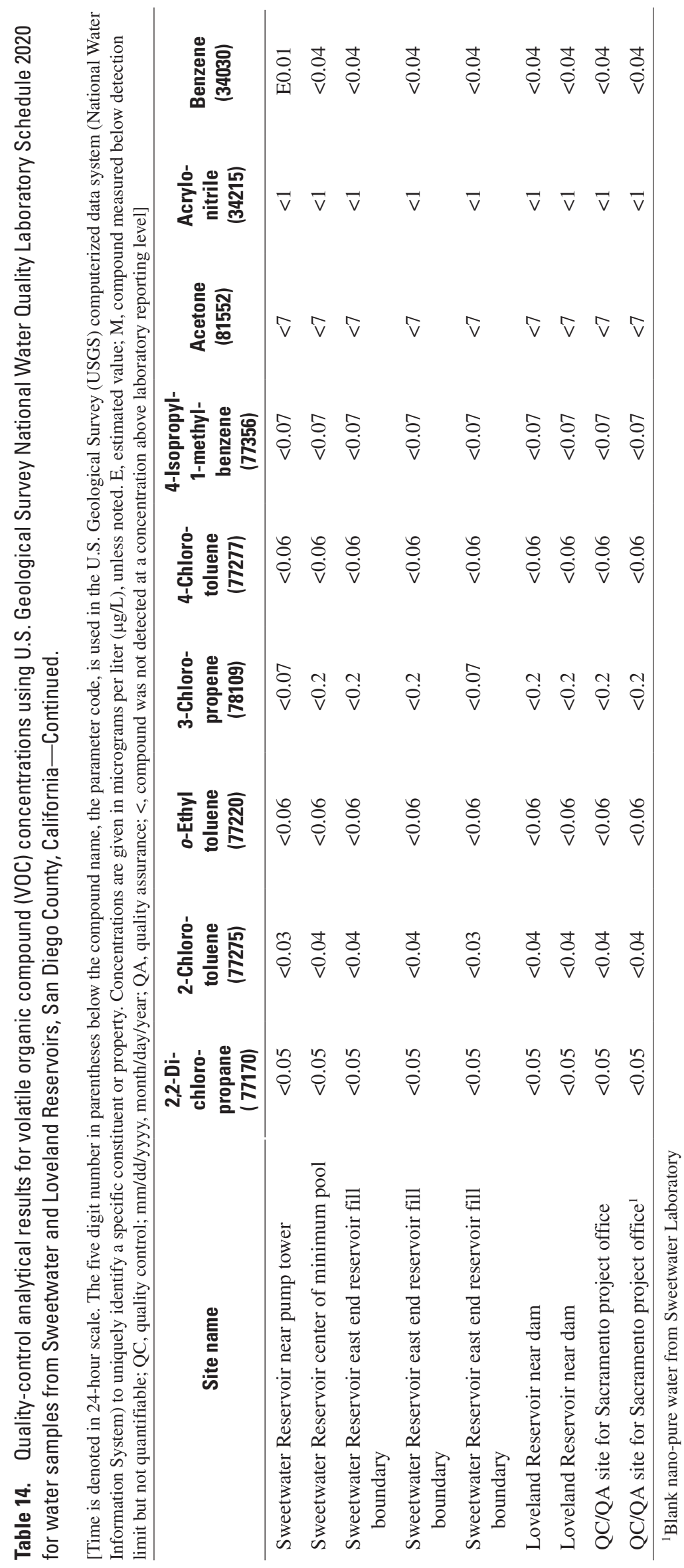




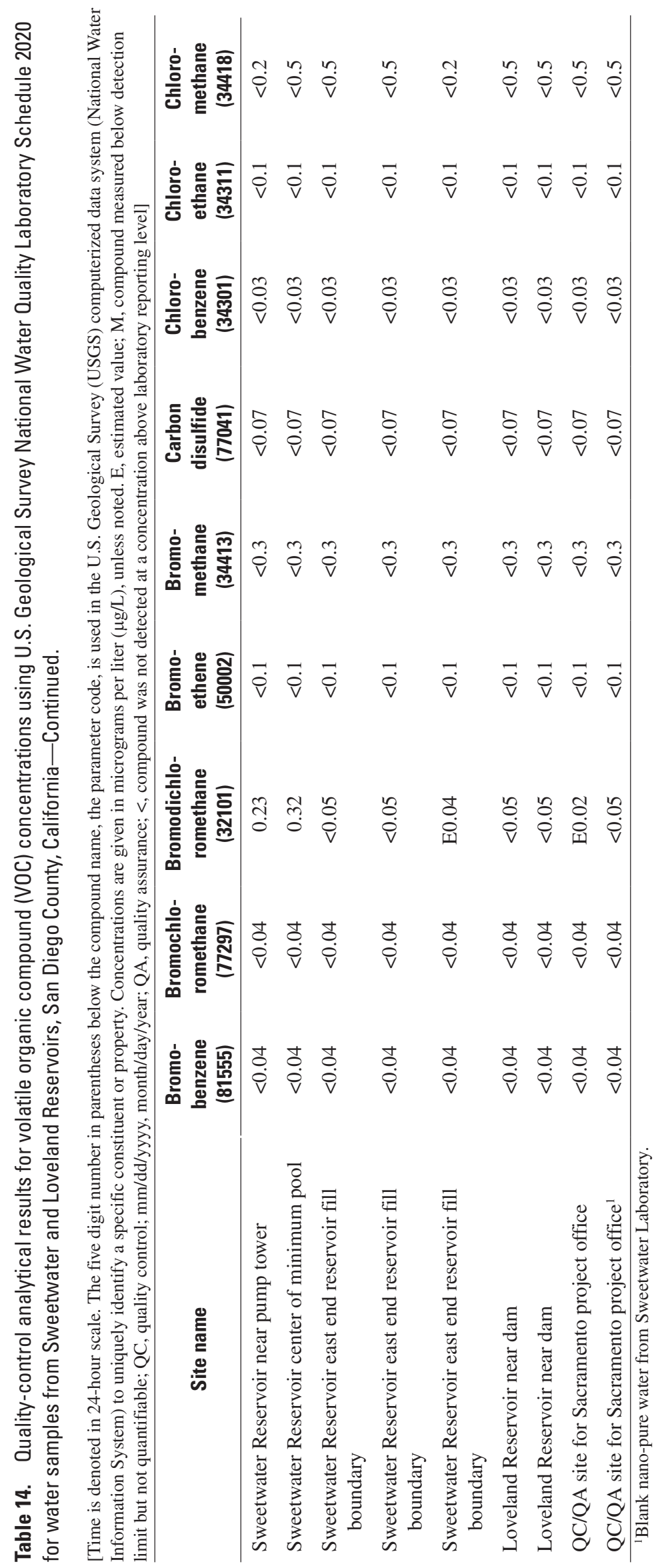




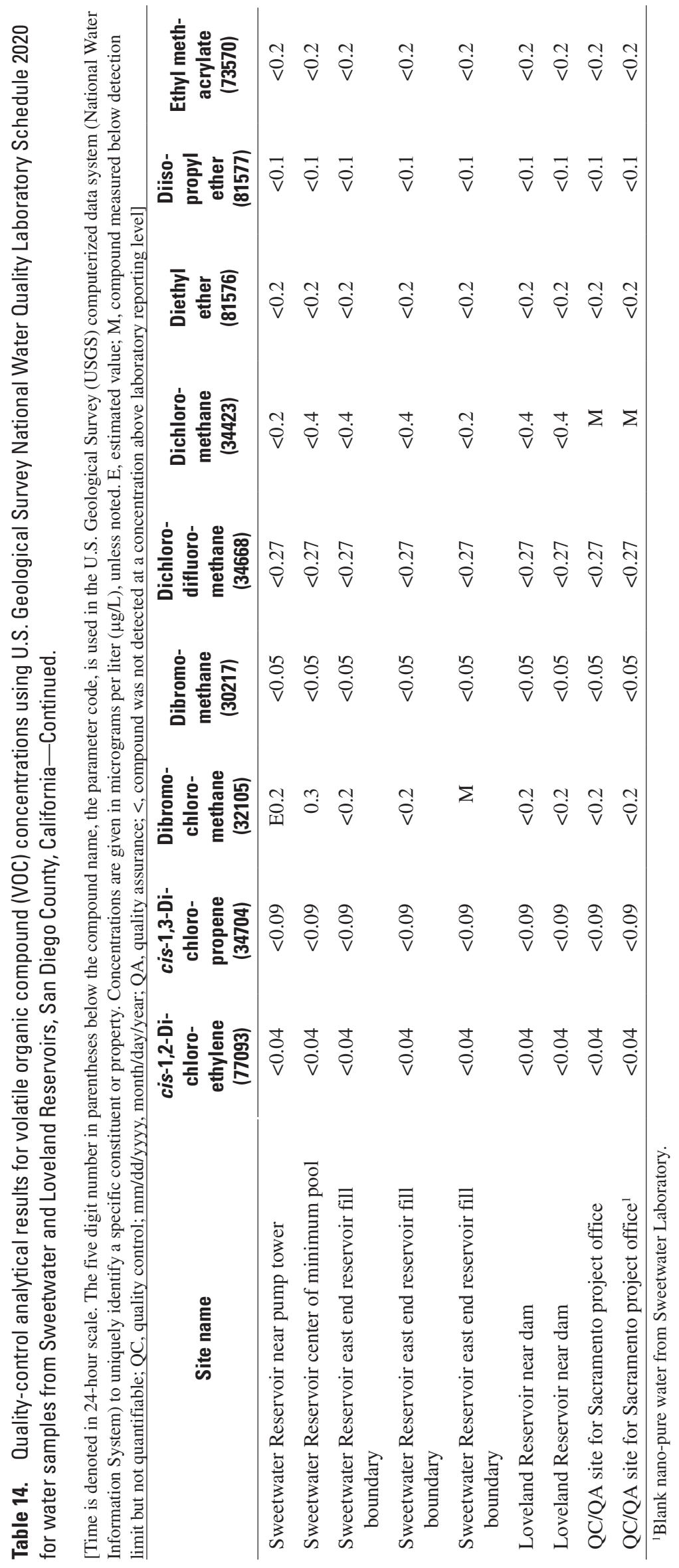




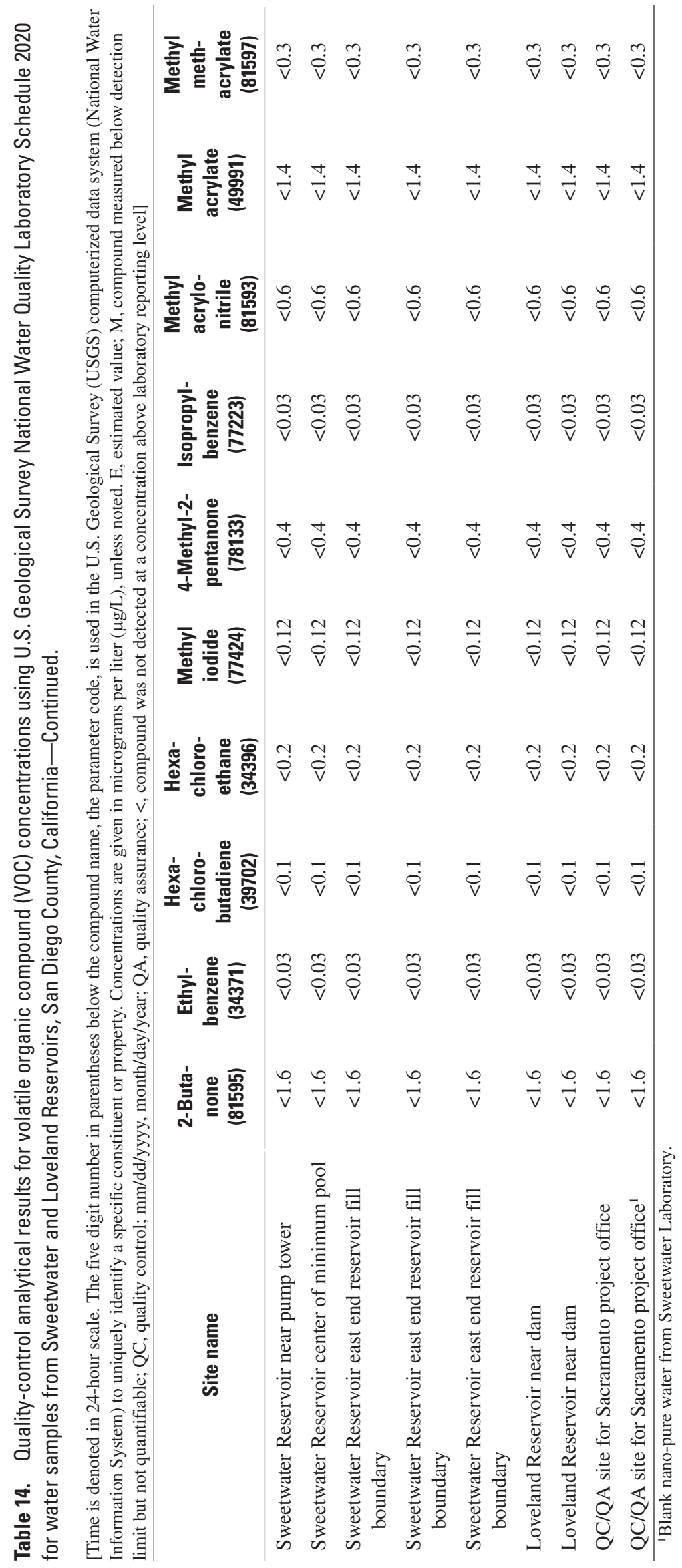




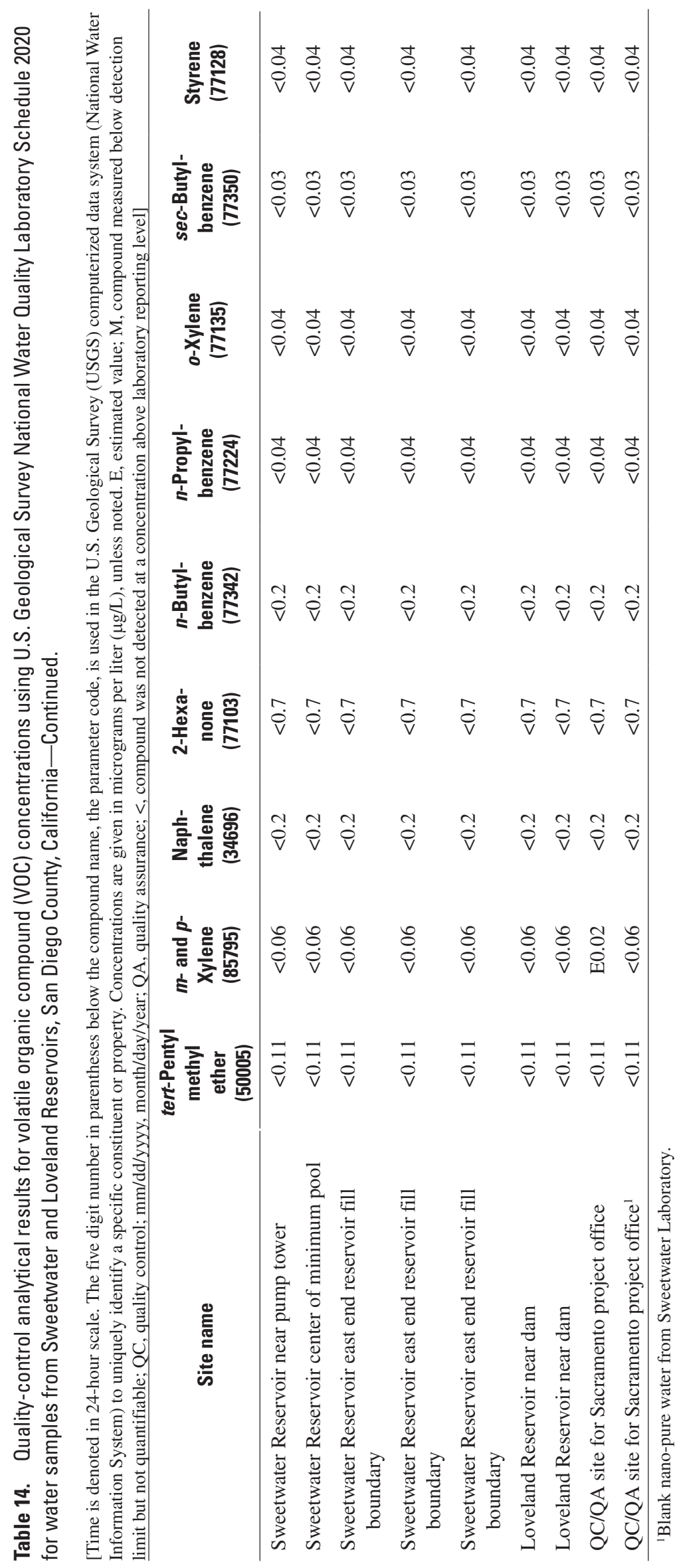




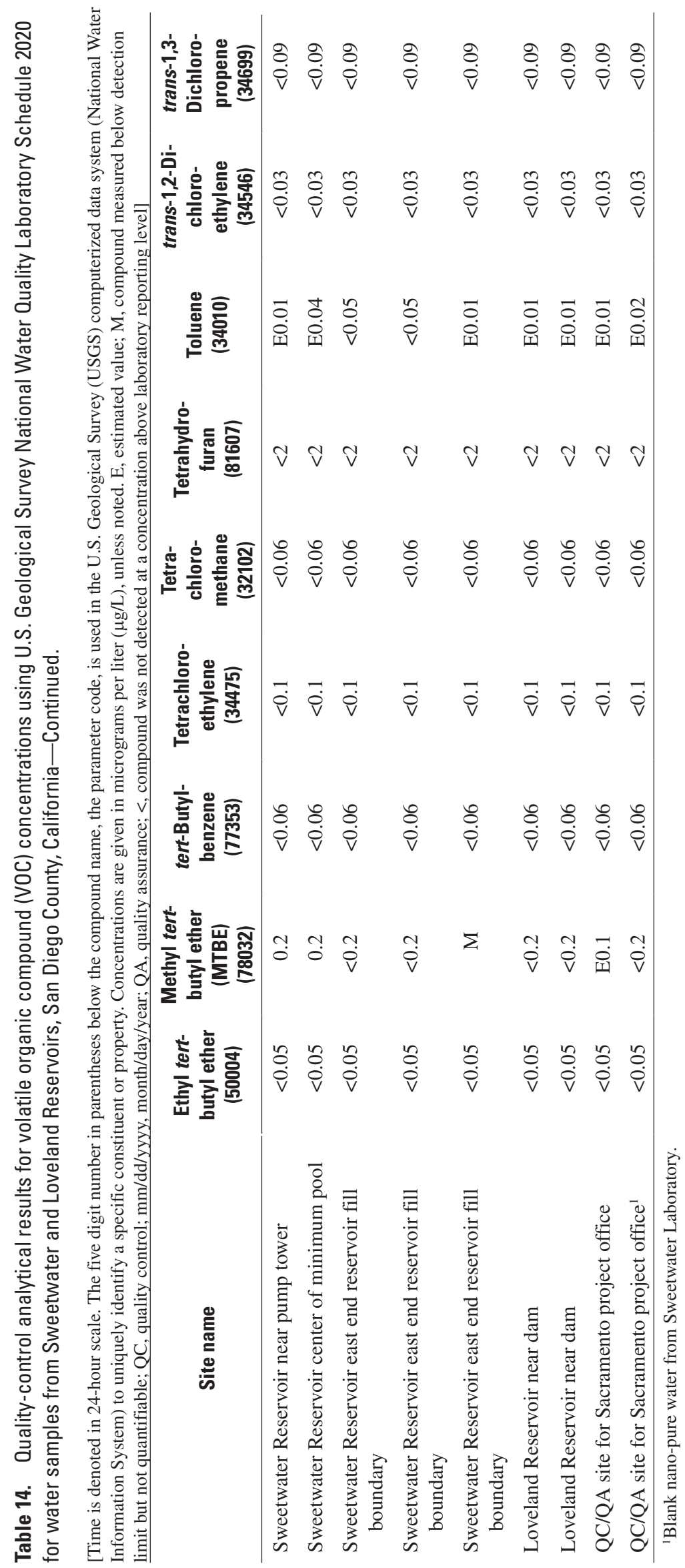




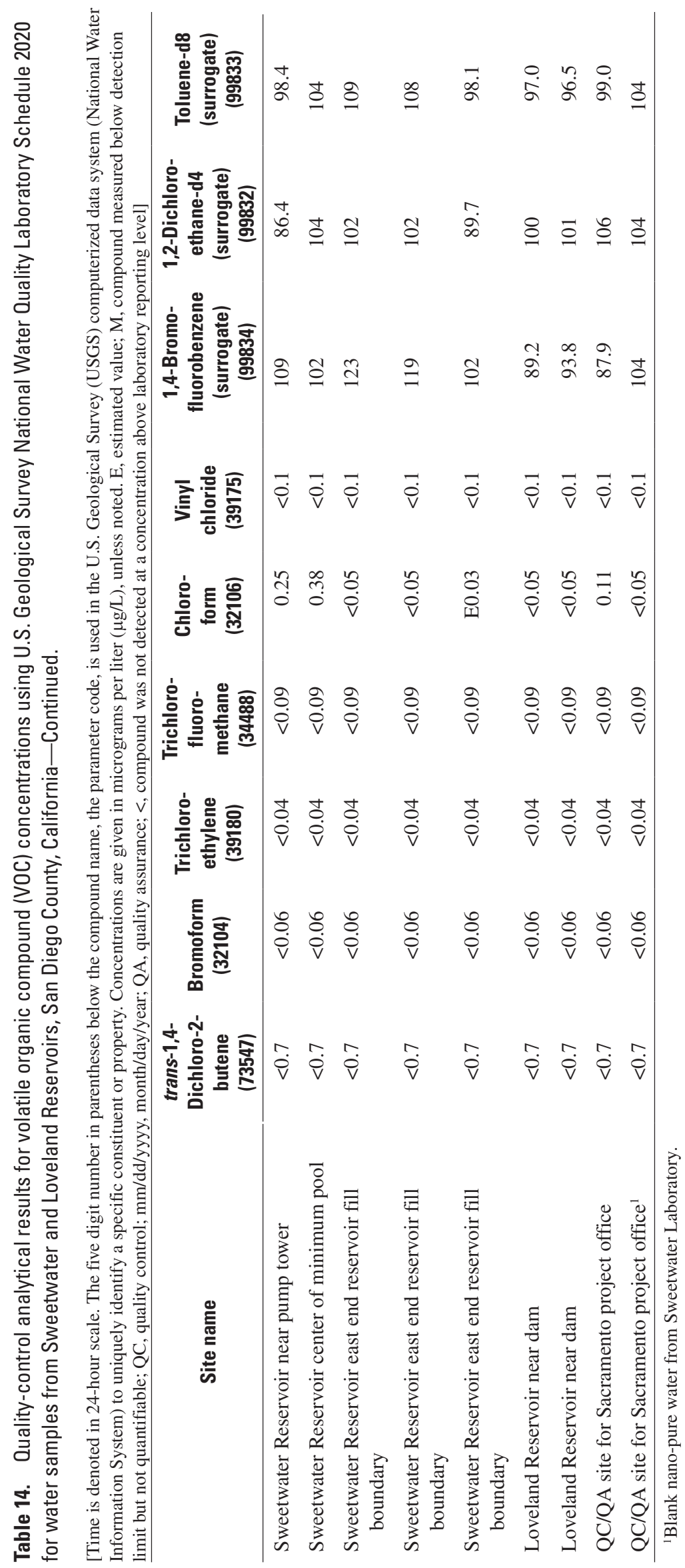




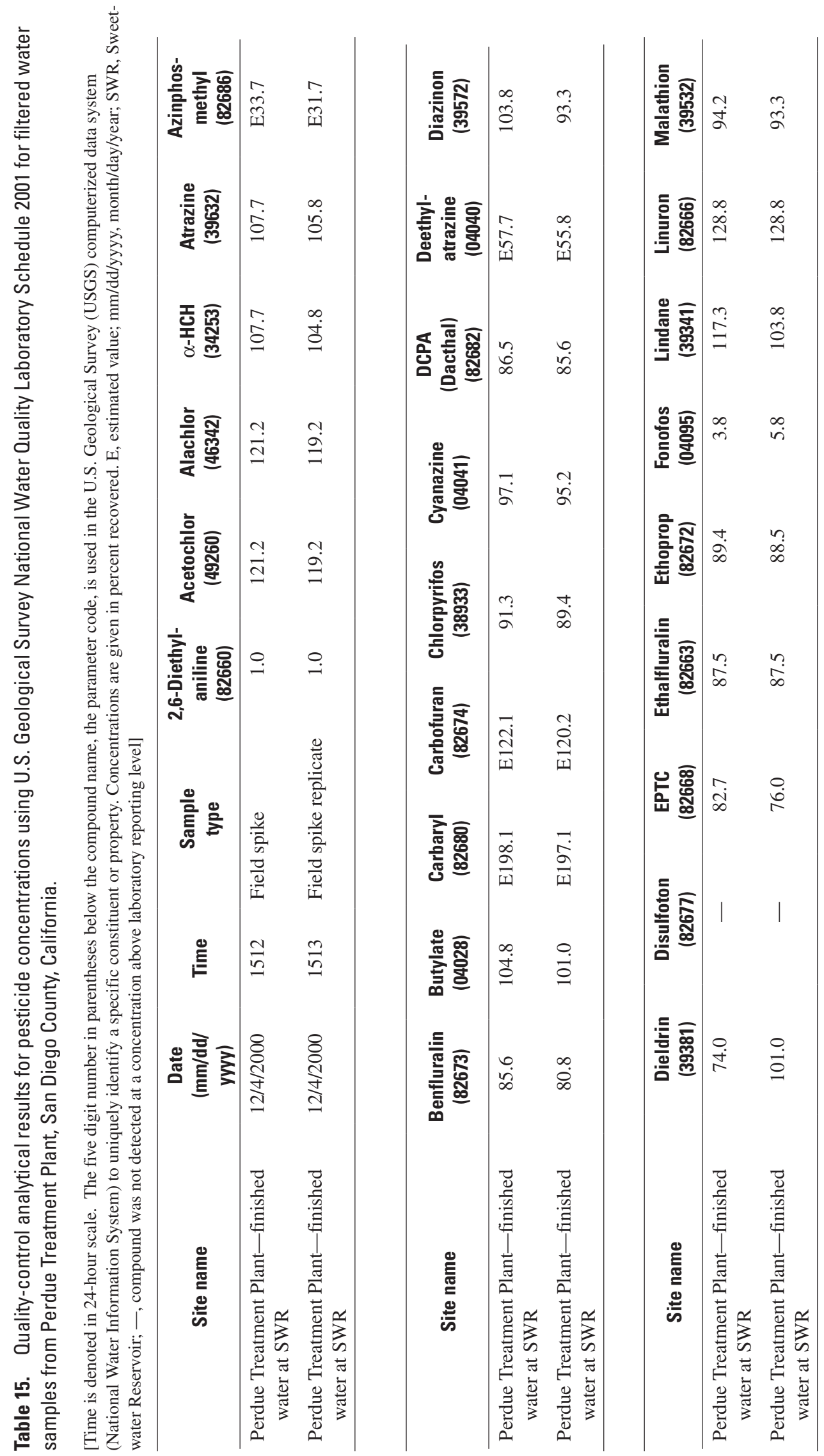




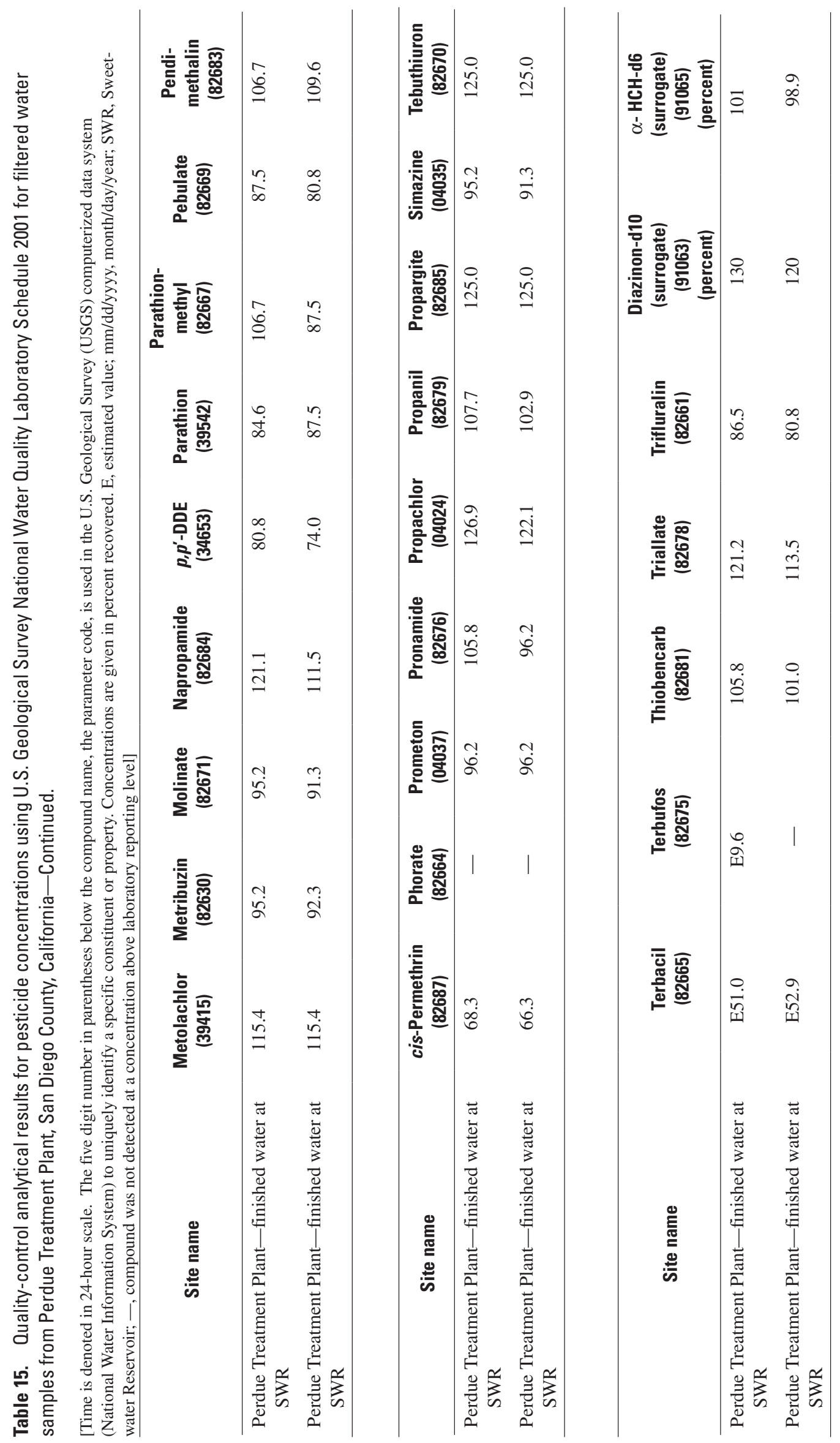




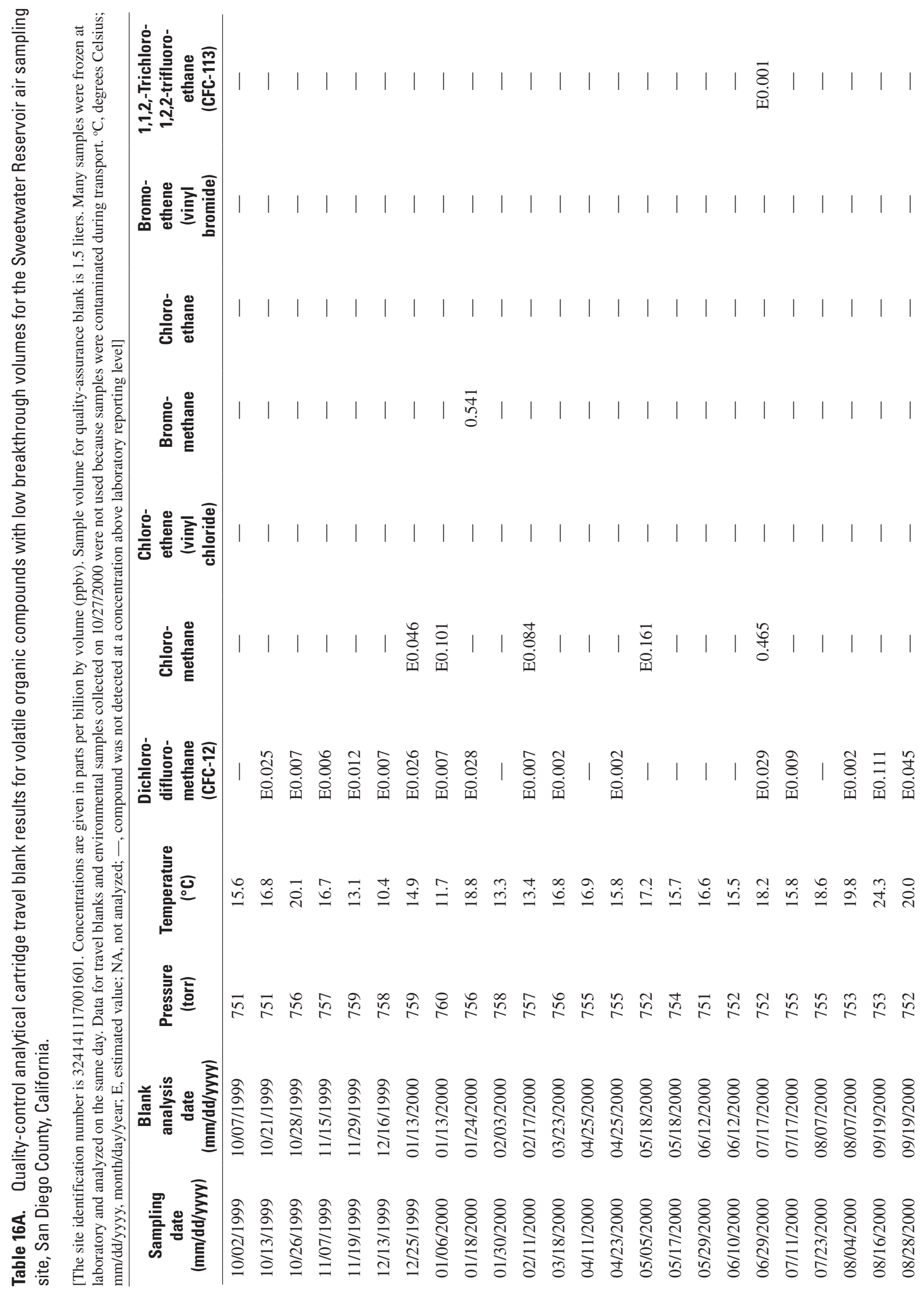




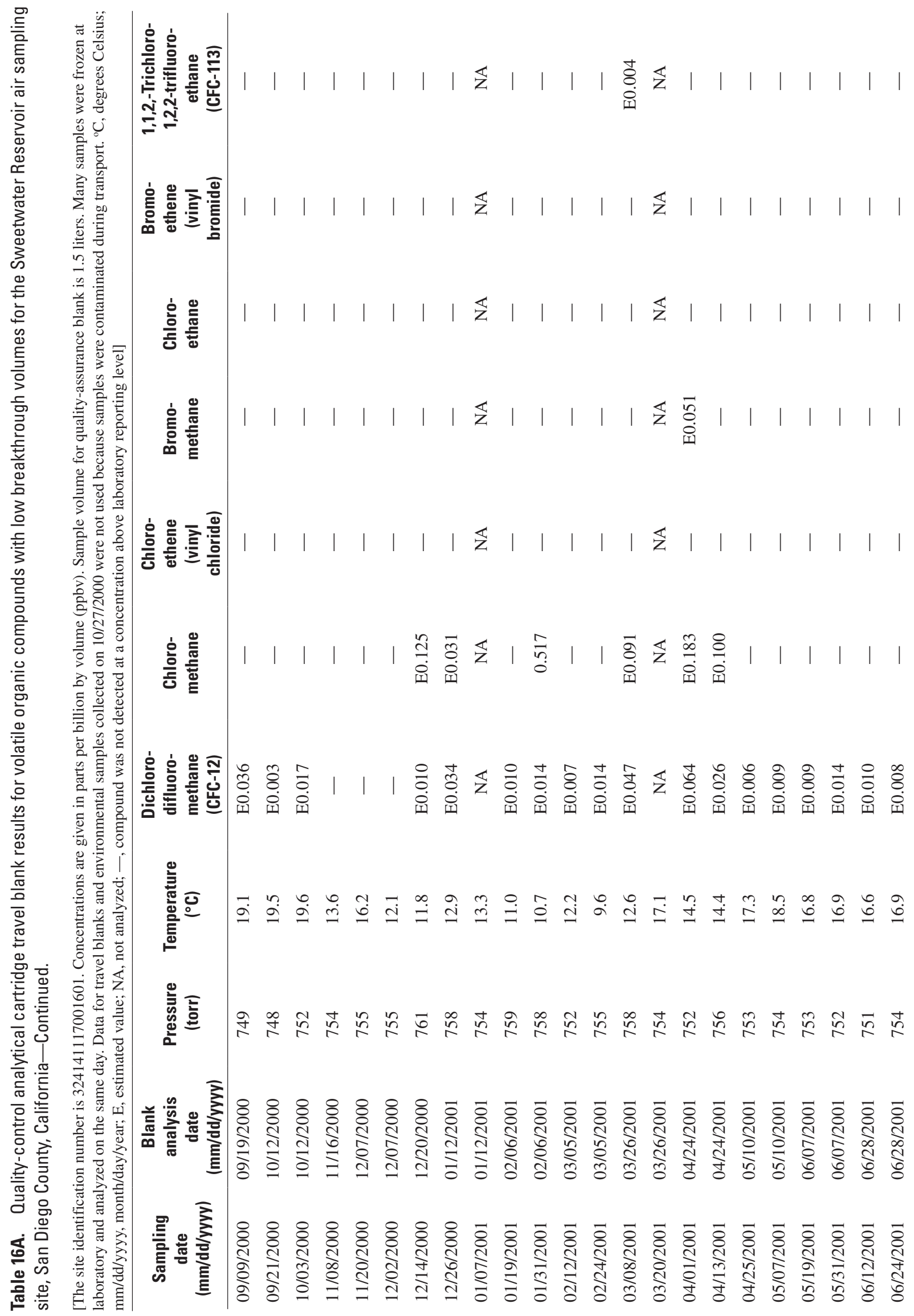




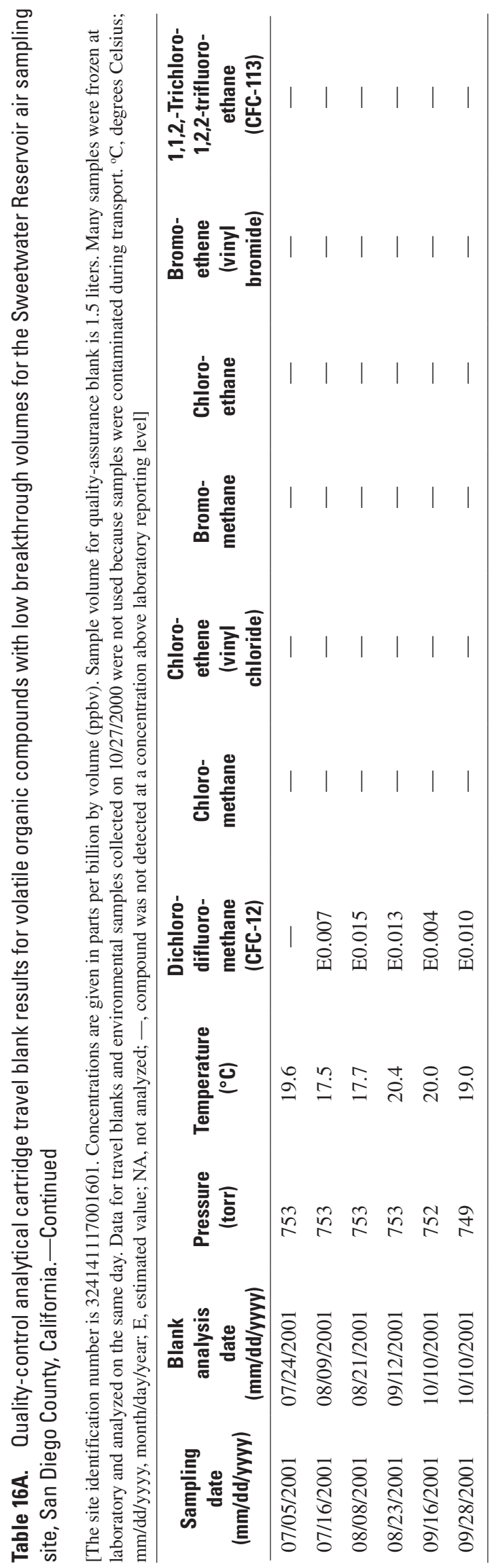




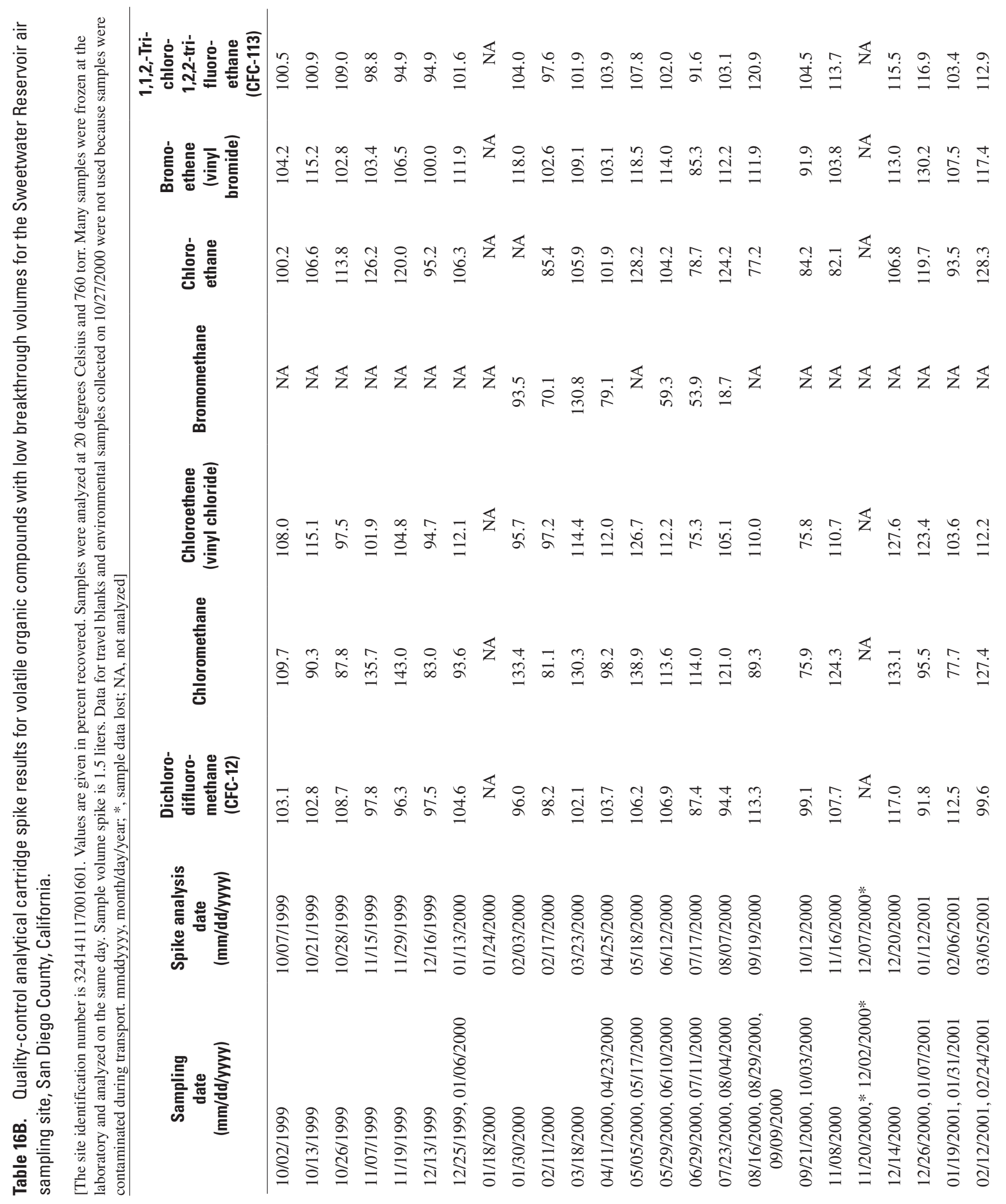




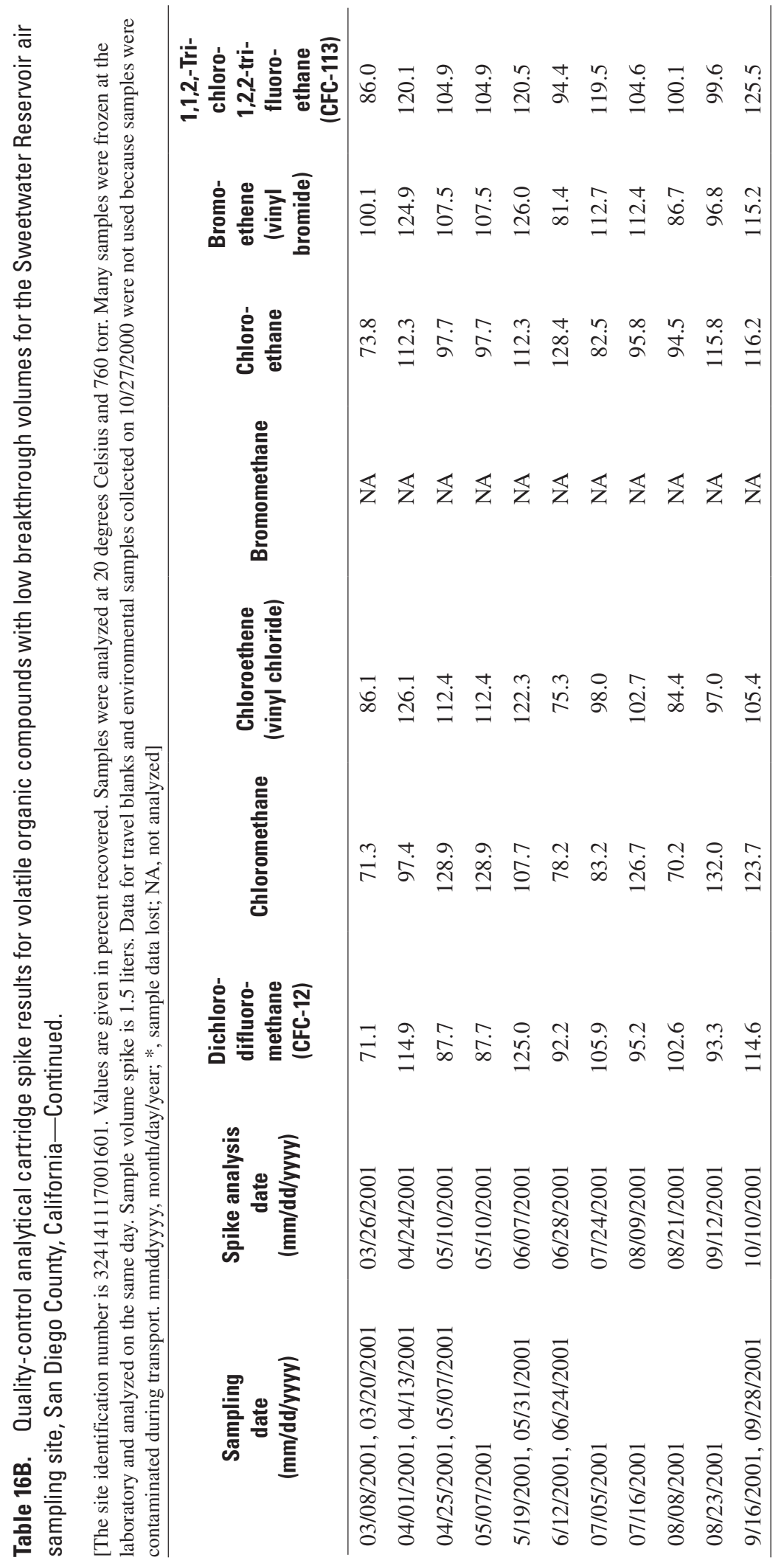


Table 16C. Quality-control analytical cartridge lot blank results for volatile organic compounds with low breakthrough volumes for the Sweetwater Reservoir air sampling site, San Diego County, California.

[The site identification number is 324141117001601 , Concentrations are given in parts per billion by volume (ppbv). Sample volume is 1.5 liters. Samples were analyzed at 20 degrees Celsius and 760 torr. Many samples were frozen at the laboratory and analyzed on the same day. Data for travel blanks and environmental samples collected on 10/27/2000 were not used because samples were contaminated during transport. mm/dd/yyyy, month/day/year; E, estimated value; NA, not analyzed; —, compound was not detected at concentration above laboratory reporting level]

\begin{tabular}{|c|c|c|c|c|c|c|c|c|}
\hline $\begin{array}{c}\text { Sampling } \\
\text { date } \\
\text { (mm/dd/yyyy) }\end{array}$ & $\begin{array}{c}\text { Blank } \\
\text { analysis } \\
\text { date } \\
\text { (mm/dd/ } \\
\text { yyyy) }\end{array}$ & $\begin{array}{l}\text { Dichloro- } \\
\text { difluoro- } \\
\text { methane } \\
\text { (CFC-12) }\end{array}$ & $\begin{array}{l}\text { Chloro- } \\
\text { methane }\end{array}$ & $\begin{array}{l}\text { Chloro- } \\
\text { ethene } \\
\text { (vinyl } \\
\text { chloride) }\end{array}$ & $\begin{array}{l}\text { Bromo- } \\
\text { methane }\end{array}$ & $\begin{array}{l}\text { Chloro- } \\
\text { ethane }\end{array}$ & $\begin{array}{c}\text { Bromo- } \\
\text { ethene } \\
\text { (vinyl bromide) }\end{array}$ & $\begin{array}{c}\text { 1,1,2,-Trichloro- } \\
\text { 1,2,2-trifluoro- } \\
\text { ethane } \\
\text { (CFC-113) }\end{array}$ \\
\hline 10/02/1999 & $10 / 07 / 1999$ & - & - & - & - & - & - & - \\
\hline 10/13/1999 & $10 / 21 / 1999$ & - & - & - & - & - & - & - \\
\hline 10/26/1999 & $10 / 28 / 1999$ & - & - & - & - & - & - & - \\
\hline 11/07/1999 & 11/15/1999 & - & - & - & - & - & - & - \\
\hline 11/19/1999 & $11 / 29 / 1999$ & - & - & - & - & - & - & - \\
\hline 12/13/1999 & $12 / 16 / 1999$ & - & - & - & - & - & - & - \\
\hline $12 / 25 / 1999$ & $01 / 13 / 2000$ & - & - & - & - & - & - & - \\
\hline 01/06/2000 & $01 / 13 / 2000$ & - & - & - & - & - & - & - \\
\hline 01/18/2000 & $01 / 24 / 2000$ & - & - & - & - & - & - & - \\
\hline 01/30/2000 & $02 / 03 / 2000$ & - & - & - & - & - & - & - \\
\hline $02 / 11 / 2000$ & $02 / 17 / 2000$ & - & - & - & - & - & - & - \\
\hline 03/18/2000 & $03 / 23 / 2000$ & - & - & - & - & - & - & - \\
\hline $04 / 11 / 2000$ & $04 / 25 / 2000$ & - & - & - & - & - & - & - \\
\hline $04 / 23 / 2000$ & $04 / 25 / 2000$ & - & - & - & - & - & - & - \\
\hline 05/05/2000 & $05 / 18 / 2000$ & - & E0.007 & - & - & - & - & - \\
\hline 05/17/2000 & $05 / 18 / 2000$ & - & - & - & - & - & - & - \\
\hline 05/29/2000 & $06 / 12 / 2000$ & - & - & - & - & - & - & - \\
\hline $06 / 10 / 2000$ & $06 / 12 / 2000$ & - & - & - & - & - & - & - \\
\hline 06/29/2000 & $07 / 17 / 2000$ & - & - & - & - & - & - & - \\
\hline 07/11/2000 & $07 / 17 / 2000$ & - & - & - & - & - & - & - \\
\hline 07/23/2000 & 08/07/2000 & E0.004 & - & - & - & - & - & - \\
\hline 08/04/2000 & $08 / 07 / 2000$ & E0.003 & - & - & - & - & - & - \\
\hline $08 / 16 / 2000$ & $09 / 19 / 2000$ & - & - & - & - & - & - & - \\
\hline 08/28/2000 & $09 / 19 / 2000$ & - & - & - & - & - & - & - \\
\hline 09/09/2000 & 09/19/2000 & E0.050 & - & - & - & - & - & - \\
\hline $09 / 21 / 2000$ & $10 / 12 / 2000$ & E0.004 & - & - & - & - & - & - \\
\hline $10 / 03 / 2000$ & $10 / 12 / 2000$ & - & - & - & - & - & - & - \\
\hline $11 / 08 / 2000$ & $11 / 16 / 2000$ & - & - & - & - & - & - & - \\
\hline $11 / 20 / 2000$ & $12 / 07 / 2000$ & - & - & - & - & - & - & - \\
\hline $12 / 02 / 2000$ & $12 / 07 / 2000$ & - & - & - & - & - & - & - \\
\hline $12 / 14 / 2000$ & $12 / 20 / 2000$ & - & - & - & - & - & - & - \\
\hline
\end{tabular}


Table 16C. Quality-control analytical cartridge lot blank results for volatile organic compounds with low breakthrough volumes for the Sweetwater Reservoir air sampling site, San Diego County, California—Continued.

[The site identification number is 324141117001601 , Concentrations are given in parts per billion by volume (ppbv). Sample volume is 1.5 liters. Samples were analyzed at 20 degrees Celsius and 760 torr. Many samples were frozen at the laboratory and analyzed on the same day. Data for travel blanks and environmental samples collected on 10/27/2000 were not used because samples were contaminated during transport. mm/dd/yyyy, month/day/year; E, estimated value; NA, not analyzed; - - compound was not detected at concentration above laboratory reporting level]

\begin{tabular}{ccccccccc}
\hline $\begin{array}{c}\text { Sampling } \\
\text { date } \\
\text { (mm/dd//yvy) }\end{array}$ & $\begin{array}{c}\text { Blank } \\
\text { analysis } \\
\text { date } \\
\text { (mm/dd/ } \\
\text { Iyyy) }\end{array}$ & $\begin{array}{c}\text { Dichloro- } \\
\text { difluoro- } \\
\text { methane } \\
\text { (CFC-12) }\end{array}$ & $\begin{array}{c}\text { Chloro- } \\
\text { methane }\end{array}$ & $\begin{array}{c}\text { Chloro- } \\
\text { ethene } \\
\text { (vinyl } \\
\text { chloride) }\end{array}$ & $\begin{array}{c}\text { Bromo- } \\
\text { methane }\end{array}$ & $\begin{array}{c}\text { Chloro- } \\
\text { ethane }\end{array}$ & $\begin{array}{c}\text { Bromo- } \\
\text { ethene } \\
\text { (vinyl bromide) }\end{array}$ & $\begin{array}{c}\text { 1,1,2,-Trichloro- } \\
\text { 1,2-trifluoro- } \\
\text { ethane } \\
\text { (CFC-113) }\end{array}$ \\
\hline 12/26/2000 & $01 / 12 / 2001$ & E0.005 & - & - & - & - & - & - \\
$01 / 07 / 2001$ & $01 / 12 / 2001$ & NA & NA & NA & NA & NA & NA & NA \\
$01 / 19 / 2001$ & $02 / 06 / 2001$ & - & - & - & - & - & - & - \\
$01 / 31 / 2001$ & $02 / 06 / 2001$ & E0.008 & - & - & - & - & - & - \\
$02 / 12 / 2001$ & $03 / 05 / 2001$ & - & - & - & - & - & - & - \\
$02 / 24 / 2001$ & $03 / 05 / 2001$ & - & - & - & - & - & - & - \\
$03 / 08 / 2001$ & $03 / 26 / 2001$ & E0.025 & - & - & - & - & - & - \\
$03 / 20 / 2001$ & $03 / 26 / 2001$ & E0.025 & - & - & - & - & - & - \\
$04 / 01 / 2001$ & $04 / 24 / 2001$ & E0.012 & - & - & - & - & - & - \\
$04 / 13 / 2001$ & $04 / 24 / 2001$ & E0.026 & - & - & - & - & - & - \\
$04 / 25 / 2001$ & $05 / 10 / 2001$ & E0.010 & - & - & - & - & - & - \\
$05 / 07 / 2001$ & $05 / 10 / 2001$ & E0.020 & - & - & - & - & - & - \\
$05 / 19 / 2001$ & $06 / 07 / 2001$ & E0.007 & - & - & - & - & - & - \\
$05 / 31 / 2001$ & $06 / 07 / 2001$ & - & - & - & - & - & - & - \\
$06 / 12 / 2001$ & $06 / 28 / 2001$ & E0.017 & - & - & - & - & - & - \\
$06 / 24 / 2001$ & $06 / 28 / 2001$ & E0.003 & - & - & - & - & - & - \\
$07 / 05 / 2001$ & $07 / 24 / 2001$ & - & - & - & - & - & - & - \\
$07 / 16 / 2001$ & $08 / 09 / 2001$ & E0.007 & - & - & - & - & - & - \\
$08 / 08 / 2001$ & $08 / 21 / 2001$ & - & - & - & - & - & - & - \\
$08 / 23 / 2001$ & $09 / 12 / 2001$ & - & - & - & - & - & - & - \\
$09 / 16 / 2001$ & $10 / 10 / 2001$ & - & - & - & - & - & - & - \\
$09 / 28 / 2001$ & $10 / 10 / 2001$ & - & - & - & - & - & - & - \\
\hline
\end{tabular}


Table 16D. Quality-control analytical laboratory blank results for volatile organic compounds with low breakthrough volumes for the Sweetwater Reservoir air sampling site, San Diego County, California.

[The site identification number is 324141117001601 . Concentrations are given in parts per billion by volume (ppbv). Sample volume is 1.5 liters. Samples were analyzed at 20 degrees Celsius and 760 torr. Many samples were frozen at the laboratory and analyzed on the same day. Data for travel blanks and environmental samples collected on 10/27/2000 were not used because samples were contaminated during transport. mm/dd/yyyy, month/day/year; E, estimated value; NA, not analyzed; - , compound was not detected at a concentration above laboratory reporting level]

\begin{tabular}{|c|c|c|c|c|c|c|c|c|}
\hline $\begin{array}{c}\text { Sampling } \\
\text { date } \\
\text { (mm/dd/yyyy) }\end{array}$ & $\begin{array}{c}\text { Blank } \\
\text { analysis } \\
\text { date } \\
\text { (mm//dd/yyyy) }\end{array}$ & $\begin{array}{l}\text { Dichloro- } \\
\text { difluoro- } \\
\text { methane } \\
\text { (CFC-12) }\end{array}$ & $\begin{array}{l}\text { Chloro- } \\
\text { methane }\end{array}$ & $\begin{array}{l}\text { Chloroethene } \\
\text { (vinyl chloride) }\end{array}$ & $\begin{array}{l}\text { Bromo- } \\
\text { methane }\end{array}$ & $\begin{array}{l}\text { Chloro- } \\
\text { ethane }\end{array}$ & $\begin{array}{l}\text { Bromoethene } \\
\text { (vinyl bromide) }\end{array}$ & $\begin{array}{c}\text { 1,1,2,-Trichloro- } \\
\text { 1,2,2-trifluoro- } \\
\text { ethane } \\
\text { (CFC-113) }\end{array}$ \\
\hline $10 / 02 / 1999$ & 10/07/1999 & - & - & - & - & - & - & - \\
\hline 10/13/1999 & $10 / 21 / 1999$ & E0.020 & - & - & - & - & - & - \\
\hline $10 / 26 / 1999$ & $10 / 28 / 1999$ & - & - & - & - & - & - & - \\
\hline 11/07/1999 & $11 / 15 / 1999$ & E0.008 & - & - & - & - & - & - \\
\hline $11 / 19 / 1999$ & $11 / 29 / 1999$ & - & - & - & - & - & - & - \\
\hline $12 / 13 / 1999$ & $12 / 16 / 1999$ & - & - & - & - & - & - & - \\
\hline $12 / 25 / 1999$ & $01 / 13 / 2000$ & E0.010 & - & - & - & - & - & - \\
\hline $01 / 06 / 2000$ & $01 / 13 / 2000$ & - & - & - & - & - & - & - \\
\hline $01 / 18 / 2000$ & $01 / 24 / 2000$ & E0.027 & E0.055 & - & - & - & - & - \\
\hline $01 / 30 / 2000$ & $02 / 03 / 2000$ & - & - & - & - & - & - & - \\
\hline $02 / 11 / 2000$ & $02 / 17 / 2000$ & E0.009 & E0.164 & - & - & - & - & - \\
\hline $03 / 18 / 2000$ & $03 / 23 / 2000$ & - & - & - & - & - & - & - \\
\hline $04 / 11 / 2000$ & $04 / 25 / 2000$ & - & E0.016 & - & - & - & - & - \\
\hline $04 / 23 / 2000$ & $04 / 25 / 2000$ & - & - & - & - & - & - & - \\
\hline $05 / 05 / 2000$ & $05 / 18 / 2000$ & - & E0.112 & - & - & - & - & - \\
\hline $05 / 17 / 2000$ & $05 / 18 / 2000$ & - & - & - & - & - & - & - \\
\hline $05 / 29 / 2000$ & $06 / 12 / 2000$ & - & - & - & - & - & - & - \\
\hline 06/10/2000 & $06 / 12 / 2000$ & - & - & - & - & - & - & - \\
\hline $06 / 29 / 2000$ & $07 / 17 / 2000$ & E0.010 & - & - & - & - & - & - \\
\hline $07 / 11 / 2000$ & $07 / 17 / 2000$ & E0.012 & - & - & - & - & - & - \\
\hline $07 / 23 / 2000$ & 08/07/2000 & - & - & - & - & - & - & - \\
\hline 08/04/2000 & 08/07/2000 & - & - & - & - & - & - & - \\
\hline $08 / 16 / 2000$ & $09 / 19 / 2000$ & E0.058 & - & - & - & - & - & - \\
\hline $08 / 28 / 2000$ & $09 / 19 / 2000$ & E0.039 & - & - & - & - & - & - \\
\hline 09/09/2000 & $09 / 19 / 2000$ & E0.049 & - & - & - & - & - & - \\
\hline $09 / 21 / 2000$ & $10 / 12 / 2000$ & E0.002 & - & - & - & - & - & - \\
\hline $10 / 03 / 2000$ & $10 / 12 / 2000$ & E0.010 & - & - & - & - & - & - \\
\hline $11 / 08 / 2000$ & $11 / 16 / 2000$ & - & - & - & - & - & - & - \\
\hline $11 / 20 / 2000$ & $12 / 07 / 2000$ & - & - & - & - & - & - & - \\
\hline $12 / 02 / 2000$ & $12 / 07 / 2000$ & - & - & - & - & - & - & - \\
\hline $12 / 14 / 2000$ & $12 / 20 / 2000$ & - & - & - & - & - & - & - \\
\hline $12 / 26 / 2000$ & $01 / 12 / 2001$ & E0.014 & - & - & - & - & - & - \\
\hline
\end{tabular}


Table 16D. Quality-control analytical laboratory blank results for volatile organic compounds with low breakthrough volumes for the Sweetwater Reservoir air sampling site, San Diego County, California—Continued.

[The site identification number is 324141117001601 . Concentrations are given in parts per billion by volume (ppbv). Sample volume is 1.5 liters. Samples were analyzed at 20 degrees Celsius and 760 torr. Many samples were frozen at the laboratory and analyzed on the same day. Data for travel blanks and environmental samples collected on 10/27/2000 were not used because samples were contaminated during transport. mm/dd/yyyy, month/day/year; E, estimated value; NA, not analyzed; - , compound was not detected at a concentration above laboratory reporting level]

\begin{tabular}{ccccccccc}
$\begin{array}{c}\text { Sampling } \\
\text { date } \\
\text { (mm/dd/yvy) }\end{array}$ & $\begin{array}{c}\text { Blank } \\
\text { analysis } \\
\text { date } \\
\text { (mm//dd/yyyy) }\end{array}$ & $\begin{array}{c}\text { Dichloro- } \\
\text { difluoro- } \\
\text { methane } \\
\text { (CFC-12) }\end{array}$ & $\begin{array}{c}\text { Chloro- } \\
\text { methane }\end{array}$ & $\begin{array}{c}\text { Chloroethene } \\
\text { (vinyl chloride) }\end{array}$ & $\begin{array}{c}\text { Bromo- } \\
\text { methane }\end{array}$ & $\begin{array}{c}\text { Chloro- } \\
\text { ethane }\end{array}$ & $\begin{array}{c}\text { Bromoethene } \\
\text { (vinyl bromide) }\end{array}$ & $\begin{array}{c}\text { 1,2,2-Trichloro- } \\
\text { ethane } \\
\text { (CFC-113) }\end{array}$ \\
\hline $01 / 07 / 2001$ & $1 / 812 / 2001$ & NA & NA & NA & NA & NA & NA & NA \\
$01 / 19 / 2001$ & $02 / 06 / 2001$ & E0.013 & - & - & - & - & - & - \\
$01 / 31 / 2001$ & $02 / 06 / 2001$ & - & - & - & - & - & - & - \\
$02 / 12 / 2001$ & $03 / 05 / 2001$ & E0.008 & - & - & - & - & - & - \\
$02 / 24 / 2001$ & $03 / 05 / 2001$ & E0.016 & - & - & - & - & - & - \\
$03 / 08 / 2001$ & $03 / 26 / 2001$ & E0.005 & - & - & - & - & - & - \\
$03 / 20 / 2001$ & $03 / 26 / 2001$ & E0.026 & - & - & - & - & - & - \\
$04 / 01 / 2001$ & $04 / 24 / 2001$ & E0.051 & 0.603 & - & E0.240 & - & - & - \\
$04 / 13 / 2001$ & $04 / 24 / 2001$ & E0.018 & - & - & - & - & - & - \\
$04 / 25 / 2001$ & $05 / 10 / 2001$ & E0.010 & - & - & - & - & - & - \\
$05 / 07 / 2001$ & $05 / 10 / 2001$ & E0.004 & - & - & - & - & - & - \\
$05 / 19 / 2001$ & $06 / 07 / 2001$ & E0.002 & - & - & - & - & - & - \\
$05 / 31 / 2001$ & $06 / 07 / 2001$ & E0.011 & - & - & - & - & - & - \\
$06 / 12 / 2001$ & $06 / 28 / 2001$ & E0.020 & - & - & - & - & - & - \\
$06 / 24 / 2001$ & $06 / 28 / 2001$ & E0.006 & - & - & - & - & - & - \\
$07 / 05 / 2001$ & $07 / 24 / 2001$ & - & - & - & - & - & - & - \\
$07 / 16 / 2001$ & $08 / 09 / 2001$ & - & - & - & - & - & - \\
$08 / 08 / 2001$ & $08 / 21 / 2001$ & E0.015 & - & - & - & - & - & - \\
$08 / 23 / 2001$ & $09 / 12 / 2001$ & - & - & - & - & - & - & - \\
$09 / 16 / 2001$ & $10 / 10 / 2001$ & E0.005 & - & - & - & - & - & - \\
$09 / 28 / 2001$ & $10 / 10 / 2001$ & - & - & - & - & - & - & - \\
\hline
\end{tabular}


Table 17A. Quality-control analytical cartridge travel blank results for volatile organic compounds (VOC) with high breakthrough volumes for the Sweetwater Reservoir air sampling site, San Diego County, California.

[The site identification number is 324141117001601 . Concentrations are given in parts per billion by volume (ppbv). Sample volume for quality-assurance blank is 5.0 liters. Many samples were frozen at the laboratory and analyzed on the same day. Data for travel blanks and environmental samples collected on 10/27/2000 were not used because samples were contaminated during transport. mm/dd/yyyy, month/day/year; *, sampling data lost; ${ }^{\circ} \mathrm{C}$, degrees Celsius; E, estimated value; NA, not analyzed; - , compound was not detected at a concentration above laboratory reporting level]

\begin{tabular}{|c|c|c|c|c|c|c|c|c|}
\hline $\begin{array}{c}\text { Sampling } \\
\text { date } \\
\text { (mm/dd/yyyy) }\end{array}$ & $\begin{array}{c}\text { Blank analysis } \\
\text { date } \\
\text { (mm/dd/yyyy) }\end{array}$ & $\begin{array}{c}\text { Pressure } \\
\text { (torr) }\end{array}$ & $\begin{array}{c}\text { Tempera- } \\
\text { ture } \\
\left({ }^{\circ} \mathrm{C}\right)\end{array}$ & $\begin{array}{l}\text { Dibromo- } \\
\text { methane }\end{array}$ & $\begin{array}{l}\text { Bromo- } \\
\text { dichloro- } \\
\text { methane }\end{array}$ & $\begin{array}{c}\text { Carbon } \\
\text { tetra- } \\
\text { chloride }\end{array}$ & $\begin{array}{l}\text { 1,2-Di- } \\
\text { chloro- } \\
\text { ethane }\end{array}$ & $\begin{array}{c}\text { Bromoform } \\
\text { (Tribromo- } \\
\text { methane) }\end{array}$ \\
\hline 10/13/1999 & $10 / 21 / 1999$ & 751 & 16.8 & - & - & - & - & - \\
\hline $10 / 26 / 1999$ & $10 / 28 / 1999$ & 756 & 20.1 & - & - & - & - & - \\
\hline 11/19/1999 & $11 / 29 / 1999$ & 759 & 13.1 & - & - & - & - & - \\
\hline $12 / 13 / 1999$ & $12 / 16 / 1999$ & 758 & 10.4 & - & - & - & - & - \\
\hline $12 / 25 / 1999$ & $01 / 13 / 2000$ & 759 & 14.9 & - & - & - & - & - \\
\hline 01/06/2000 & $01 / 13 / 2000$ & 760 & 11.7 & - & - & - & - & - \\
\hline 01/18/2000 & $01 / 24 / 2000$ & 756 & 18.8 & - & - & - & - & - \\
\hline 03/18/2000 & $03 / 23 / 2000$ & 756 & 16.8 & - & - & - & - & - \\
\hline $04 / 11 / 2000$ & $04 / 25 / 2000$ & 755 & 16.9 & - & - & - & - & - \\
\hline $04 / 23 / 2000$ & $04 / 25 / 2000$ & 755 & 15.8 & - & - & - & - & - \\
\hline 05/05/2000 & 05/18/2000 & 752 & 17.2 & - & - & - & - & - \\
\hline $05 / 17 / 2000$ & $05 / 18 / 2000$ & 754 & 15.7 & - & - & - & - & - \\
\hline 05/29/2000 & $06 / 12 / 2000$ & 751 & 16.6 & - & - & - & - & - \\
\hline 06/10/2000 & $06 / 12 / 2000$ & 752 & 15.5 & - & - & - & - & - \\
\hline 06/29/2000 & $07 / 17 / 2000$ & 752 & 18.2 & - & - & - & - & - \\
\hline $07 / 11 / 2000$ & $07 / 17 / 2000$ & 755 & 15.8 & - & - & - & - & - \\
\hline $09 / 21 / 2000$ & $10 / 12 / 2000$ & 748 & 19.5 & - & - & - & - & - \\
\hline $10 / 03 / 2000$ & $10 / 12 / 2000$ & 752 & 19.6 & - & - & - & - & - \\
\hline $11 / 08 / 2000$ & $11 / 16 / 2000$ & 754 & 13.6 & - & - & - & - & - \\
\hline $11 / 20 / 2000$ & $12 / 07 / 2000$ & 755 & 16.2 & - & - & - & - & - \\
\hline $12 / 02 / 2000$ & $12 / 07 / 2000$ & 755 & 12.1 & - & - & - & - & - \\
\hline $12 / 14 / 2000$ & $12 / 20 / 2000$ & 761 & 11.8 & - & - & - & - & - \\
\hline $12 / 26 / 2000$ & $01 / 12 / 2001$ & 758 & 12.9 & - & - & - & - & - \\
\hline
\end{tabular}


Table 17A. Quality-control analytical cartridge travel blank results for volatile organic compounds (VOC) with high breakthrough volumes for the Sweetwater Reservoir air sampling site, San Diego County, California—Continued.

[The site identification number is 324141117001601 . Concentrations are given in parts per billion by volume (ppbv). Sample volume for quality-assurance blank is 5.0 liters. Many samples were frozen at the laboratory and analyzed on the same day. Data for travel blanks and environmental samples collected on 10/27/2000 were not used because samples were contaminated during transport. mm/dd/yyyy, month/day/year; *, sampling data lost; ${ }^{\circ} \mathrm{C}$, degrees Celsius; E, estimated value; NA, not analyzed; —, compound was not detected at a concentration above laboratory reporting level]

\begin{tabular}{|c|c|c|c|c|c|c|c|c|}
\hline $\begin{array}{c}\text { Sampling } \\
\text { date } \\
\text { (mm/dd/yyyy) }\end{array}$ & $\begin{array}{c}\text { Blank analysis } \\
\text { date } \\
\text { (mm/dd/yyyy) }\end{array}$ & $\begin{array}{l}\text { Pressure } \\
\text { (torr) }\end{array}$ & $\begin{array}{c}\text { Tempera- } \\
\text { ture } \\
\left({ }^{\circ} \mathrm{C}\right) \\
\end{array}$ & $\begin{array}{l}\text { Dibromo- } \\
\text { methane }\end{array}$ & $\begin{array}{l}\text { Bromo- } \\
\text { dichloro- } \\
\text { methane }\end{array}$ & $\begin{array}{c}\text { Carbon } \\
\text { tetra- } \\
\text { chloride }\end{array}$ & $\begin{array}{l}\text { 1,2-Di- } \\
\text { chloro- } \\
\text { ethane }\end{array}$ & $\begin{array}{c}\text { Bromoform } \\
\text { (Tribromo- } \\
\text { methane) }\end{array}$ \\
\hline 01/07/2001 & 01/12/2001 & 754 & 13.3 & - & - & - & - & - \\
\hline 01/19/2001 & 02/06/2001 & 759 & 11.0 & - & - & - & - & - \\
\hline $01 / 31 / 2001$ & $02 / 06 / 2001$ & 758 & 10.7 & NA & NA & NA & NA & NA \\
\hline $02 / 12 / 2001$ & 03/05/2001 & 752 & 12.2 & - & - & - & - & - \\
\hline $02 / 24 / 2001$ & 03/05/2001 & 755 & 9.6 & - & - & - & - & - \\
\hline 03/08/2001 & 03/26/2001 & 758 & 12.6 & NA & NA & NA & NA & NA \\
\hline 03/20/2001 & 03/26/2001 & 754 & 17.1 & NA & NA & NA & NA & NA \\
\hline 04/01/2001 & $04 / 24 / 2001$ & 752 & 14.5 & - & - & - & - & - \\
\hline $04 / 13 / 2001$ & $04 / 24 / 2001$ & 756 & 14.4 & - & - & - & - & - \\
\hline $04 / 25 / 2001$ & 05/10/2001 & 753 & 17.3 & - & - & - & - & - \\
\hline 05/07/2001 & 05/10/2001 & 754 & 18.5 & - & - & - & - & - \\
\hline 05/19/2001 & 06/07/2001 & 753 & 16.8 & - & - & - & - & - \\
\hline $05 / 31 / 2001$ & 06/07/2001 & 752 & 16.9 & - & - & - & - & - \\
\hline 06/12/2001 & $06 / 28 / 2001$ & 751 & 16.6 & - & - & - & - & - \\
\hline 06/24/2001 & $06 / 28 / 2001$ & 754 & 16.9 & - & - & - & - & - \\
\hline 07/05/2001 & $07 / 24 / 2001$ & 753 & 19.6 & - & - & - & - & - \\
\hline 07/16/2001 & 08/09/2001 & 753 & 17.5 & - & - & - & - & - \\
\hline 08/08/2001 & $08 / 21 / 2001$ & 753 & 17.7 & - & - & - & - & - \\
\hline $08 / 23 / 2001$ & $09 / 12 / 2001$ & 753 & 20.4 & - & - & - & - & - \\
\hline 09/16/2001 & $10 / 10 / 2001$ & 752 & 20.0 & - & - & - & - & - \\
\hline 09/28/2001 & $10 / 10 / 2001$ & 749 & 19.0 & - & - & - & - & - \\
\hline
\end{tabular}


Table 17A. Quality-control analytical cartridge travel blank results for volatile organic compounds (VOC) with high breakthrough volumes for the Sweetwater Reservoir air sampling site, San Diego County, California—Continued.

[The site identification number is 324141117001601 . Concentrations are given in parts per billion by volume (ppbv). Sample volume for quality-assurance blank is 5.0 liters. Many samples were frozen at the laboratory and analyzed on the same day. Data for travel blanks and environmental samples collected on 10/27/2000 were not used because samples were contaminated during transport. mm/dd/yyyy, month/day/year; *, sampling data lost; ${ }^{\circ} \mathrm{C}$, degrees Celsius; E, estimated value; NA, not analyzed; - , compound was not detected at a concentration above laboratory reporting level]

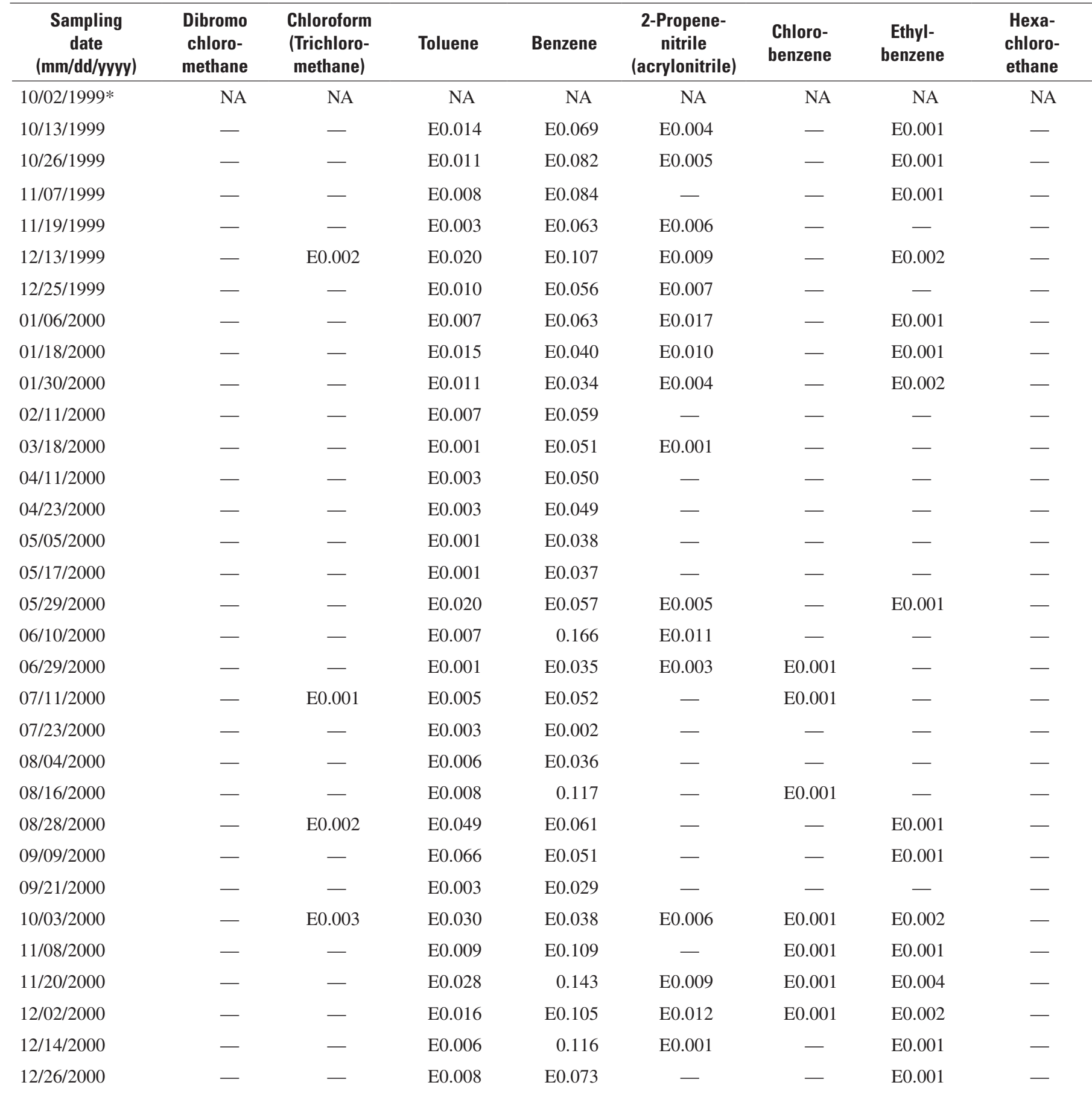


Table 17A. Quality-control analytical cartridge travel blank results for volatile organic compounds (VOC) with high breakthrough volumes for the Sweetwater Reservoir air sampling site, San Diego County, California-Continued.

[The site identification number is 324141117001601 . Concentrations are given in parts per billion by volume (ppbv). Sample volume for quality-assurance blank is 5.0 liters. Many samples were frozen at the laboratory and analyzed on the same day. Data for travel blanks and environmental samples collected on 10/27/2000 were not used because samples were contaminated during transport. mm/dd/yyyy, month/day/year; *, sampling data lost; ${ }^{\circ} \mathrm{C}$, degrees Celsius; E, estimated value; NA, not analyzed; — , compound was not detected at a concentration above laboratory reporting level]

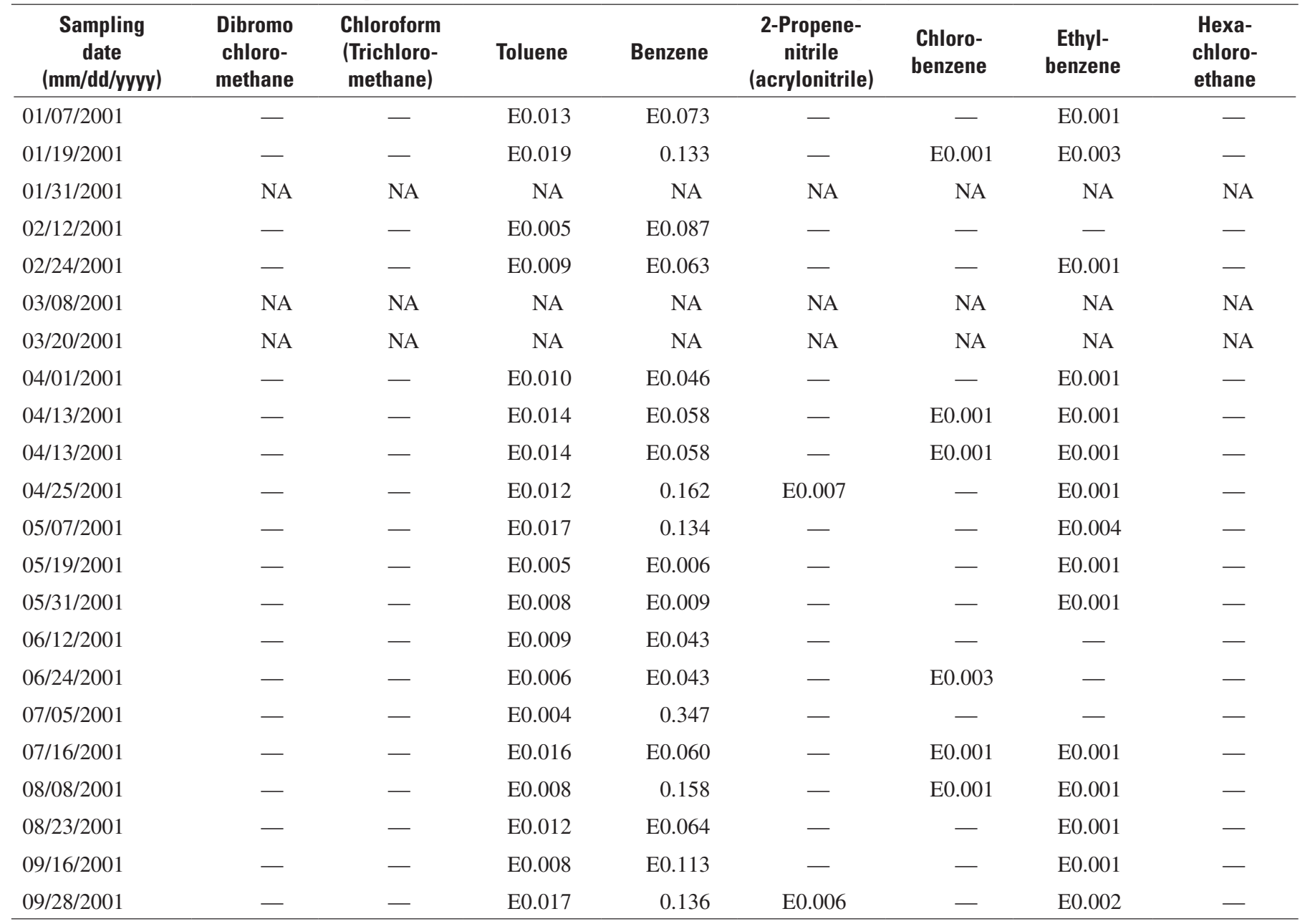


Table 17A. Quality-control analytical cartridge travel blank results for volatile organic compounds (VOC) with high breakthrough volumes for the Sweetwater Reservoir air sampling site, San Diego County, California—Continued.

[The site identification number is 324141117001601 . Concentrations are given in parts per billion by volume (ppbv). Sample volume for quality-assurance blank is 5.0 liters. Many samples were frozen at the laboratory and analyzed on the same day. Data for travel blanks and environmental samples collected on 10/27/2000 were not used because samples were contaminated during transport. mm/dd/yyyy, month/day/year; *, sampling data lost; ${ }^{\circ} \mathrm{C}$, degrees Celsius; E, estimated value; NA, not analyzed; —, compound was not detected at a concentration above laboratory reporting level]

\begin{tabular}{|c|c|c|c|c|c|c|c|c|}
\hline $\begin{array}{c}\text { Sampling } \\
\text { date } \\
\text { (mm/dd/yyy) }\end{array}$ & $\begin{array}{l}\text { Methylene } \\
\text { chloride }\end{array}$ & $\begin{array}{l}\text { Tetrachloro- } \\
\text { ethene } \\
\text { (PCE) }\end{array}$ & $\begin{array}{l}\text { 1,1-Dichloro- } \\
\text { ethane }\end{array}$ & $\begin{array}{l}\text { 1,1-Dichloro- } \\
\text { ethene }\end{array}$ & $\begin{array}{l}\text { 1,1,1-Tri- } \\
\text { chloro- } \\
\text { ethane }\end{array}$ & $\begin{array}{l}\text { 1,1,2-Tri- } \\
\text { chloro- } \\
\text { ethane }\end{array}$ & $\begin{array}{l}\text { 1,1,2,2-Tet- } \\
\text { rachloro- } \\
\text { ethane }\end{array}$ & $\begin{array}{l}\text { 1,2-Di- } \\
\text { chloro- } \\
\text { benzene }\end{array}$ \\
\hline 10/02/1999* & NA & NA & NA & NA & NA & NA & NA & NA \\
\hline 10/13/1999 & E0.008 & - & - & E0.004 & - & - & - & - \\
\hline 10/26/1999 & E0.020 & - & - & - & - & - & - & - \\
\hline 11/07/1999 & E0.004 & - & - & - & - & - & - & - \\
\hline 11/19/1999 & E0.004 & - & - & - & - & - & - & - \\
\hline $12 / 13 / 1999$ & E0.008 & E0.001 & - & E0.006 & - & - & - & - \\
\hline $12 / 25 / 1999$ & E0.009 & E0.003 & - & - & - & - & - & - \\
\hline 01/06/2000 & E0.009 & - & - & - & - & - & - & - \\
\hline 01/18/2000 & E0.044 & - & - & - & - & - & - & - \\
\hline 01/30/2000 & E0.011 & - & - & E0.003 & - & - & - & - \\
\hline $02 / 11 / 2000$ & - & - & - & - & - & - & - & - \\
\hline 03/18/2000 & - & - & - & - & - & - & - & - \\
\hline $04 / 11 / 2000$ & - & - & - & - & - & - & - & - \\
\hline $04 / 23 / 2000$ & E0.001 & - & - & - & - & - & - & - \\
\hline 05/05/2000 & - & - & - & - & - & - & - & - \\
\hline 05/17/2000 & - & E0.007 & - & - & - & - & - & - \\
\hline 05/29/2000 & E0.002 & E0.021 & - & E0.007 & - & - & - & - \\
\hline 06/10/2000 & E0.002 & E0.009 & - & E0.002 & - & - & - & - \\
\hline 06/29/2000 & - & - & - & - & - & - & - & - \\
\hline 07/11/2000 & - & - & - & - & - & - & - & - \\
\hline 07/23/2000 & - & - & - & - & - & - & - & - \\
\hline 08/04/2000 & - & - & - & - & - & - & - & - \\
\hline 08/16/2000 & - & - & - & 0.121 & - & - & - & - \\
\hline 08/28/2000 & - & - & - & E0.041 & - & - & - & - \\
\hline 09/09/2000 & - & - & - & - & - & - & - & - \\
\hline 09/21/2000 & - & - & - & - & - & - & - & - \\
\hline $10 / 03 / 2000$ & E0.005 & - & - & E0.019 & - & - & - & - \\
\hline $11 / 08 / 2000$ & E0.005 & - & - & - & - & - & - & - \\
\hline $11 / 20 / 2000$ & E0.010 & E0.001 & - & E0.003 & - & - & - & - \\
\hline $12 / 02 / 2000$ & - & - & - & - & - & - & - & - \\
\hline $12 / 14 / 2000$ & - & - & - & - & - & - & - & - \\
\hline $12 / 26 / 2000$ & - & - & - & - & - & - & - & - \\
\hline
\end{tabular}


Table 17A. Quality-control analytical cartridge travel blank results for volatile organic compounds (VOC) with high breakthrough volumes for the Sweetwater Reservoir air sampling site, San Diego County, California-Continued.

[The site identification number is 324141117001601 . Concentrations are given in parts per billion by volume (ppbv). Sample volume for quality-assurance blank is 5.0 liters. Many samples were frozen at the laboratory and analyzed on the same day. Data for travel blanks and environmental samples collected on 10/27/2000 were not used because samples were contaminated during transport. mm/dd/yyyy, month/day/year; *, sampling data lost; ${ }^{\circ} \mathrm{C}$, degrees Celsius; E, estimated value; NA, not analyzed; - - compound was not detected at a concentration above laboratory reporting level]

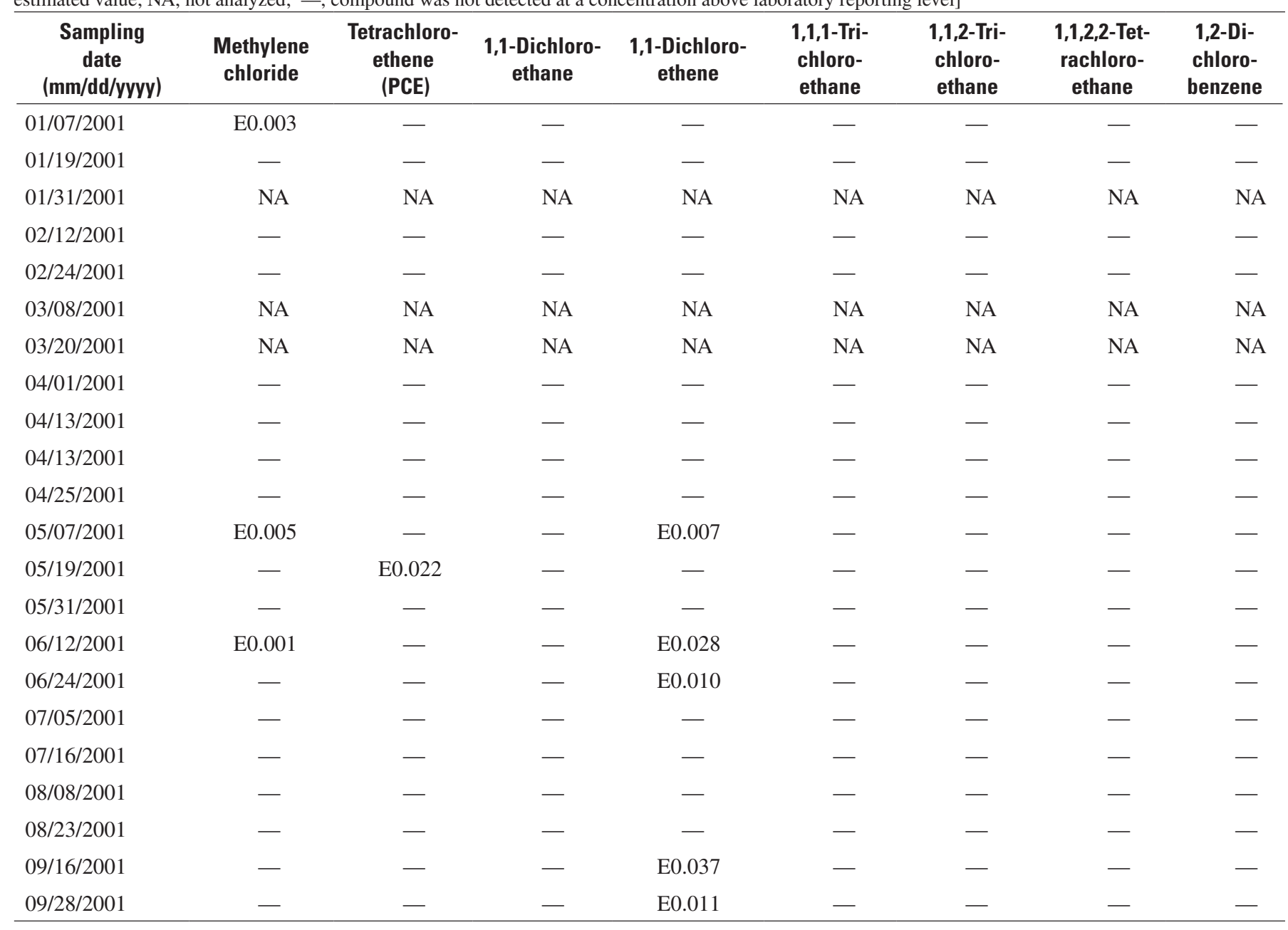


Table 17A. Quality-control analytical cartridge travel blank results for volatile organic compounds (VOC) with high breakthrough volumes for the Sweetwater Reservoir air sampling site, San Diego County, California—Continued.

[The site identification number is 324141117001601 . Concentrations are given in parts per billion by volume (ppbv). Sample volume for quality-assurance blank is 5.0 liters. Many samples were frozen at the laboratory and analyzed on the same day. Data for travel blanks and environmental samples collected on 10/27/2000 were not used because samples were contaminated during transport. mm/dd/yyyy, month/day/year; *, sampling data lost; ${ }^{\circ} \mathrm{C}$, degrees Celsius; E, estimated value; NA, not analyzed; —-, compound was not detected at a concentration above laboratory reporting level]

\begin{tabular}{|c|c|c|c|c|c|c|c|c|}
\hline $\begin{array}{c}\text { Sampling } \\
\text { date } \\
\text { (mm/dd/yyyy) }\end{array}$ & $\begin{array}{l}\text { 1,2-Dichloro- } \\
\text { propane }\end{array}$ & $\begin{array}{l}\text { trans-1,2-Di- } \\
\text { chloro- } \\
\text { ethene }\end{array}$ & $\begin{array}{c}\text { 1,2,4-Tri- } \\
\text { chloro- } \\
\text { benzene }\end{array}$ & $\begin{array}{l}\text { 1,3-Di- } \\
\text { chloro- } \\
\text { benzene }\end{array}$ & $\begin{array}{l}\text { 1,4-Di- } \\
\text { chloro- } \\
\text { benzene }\end{array}$ & $\begin{array}{l}\text { Naph- } \\
\text { thalene }\end{array}$ & $\begin{array}{l}\text { trans-1,3- } \\
\text { Dichloro- } \\
\text { propene }\end{array}$ & $\begin{array}{l}\text { cis-1,3-Di- } \\
\text { chloro- } \\
\text { propene }\end{array}$ \\
\hline $10 / 02 / 1999 *$ & NA & NA & NA & NA & NA & NA & NA & NA \\
\hline $10 / 13 / 1999$ & - & - & - & - & - & E0.001 & - & - \\
\hline $10 / 26 / 1999$ & - & - & - & - & - & E0.002 & - & - \\
\hline 11/07/1999 & - & - & - & - & - & E0.001 & - & - \\
\hline $11 / 19 / 1999$ & - & - & - & - & - & - & - & - \\
\hline $12 / 13 / 1999$ & - & - & - & - & - & - & - & - \\
\hline $12 / 25 / 1999$ & - & - & - & - & - & - & - & - \\
\hline $01 / 06 / 2000$ & - & - & - & - & - & E0.001 & - & - \\
\hline $01 / 18 / 2000$ & - & - & - & - & - & E0.002 & - & - \\
\hline $01 / 30 / 2000$ & - & - & - & - & - & E0.001 & - & - \\
\hline $02 / 11 / 2000$ & - & - & - & - & - & E0.002 & - & - \\
\hline $03 / 18 / 2000$ & - & - & - & - & - & E0.001 & - & - \\
\hline $04 / 11 / 2000$ & - & - & - & - & - & - & - & - \\
\hline $04 / 23 / 2000$ & - & - & - & - & - & - & - & - \\
\hline $05 / 05 / 2000$ & - & - & - & - & - & - & - & - \\
\hline 05/17/2000 & - & - & - & - & - & - & - & - \\
\hline $05 / 29 / 2000$ & - & - & - & - & - & E0.001 & - & - \\
\hline 06/10/2000 & - & - & - & - & - & E0.002 & - & - \\
\hline $06 / 29 / 2000$ & - & - & - & - & - & - & - & - \\
\hline $07 / 11 / 2000$ & - & - & - & - & - & - & - & - \\
\hline $07 / 23 / 2000$ & - & - & - & - & - & E0.001 & - & - \\
\hline 08/04/2000 & - & - & - & - & - & E0.001 & - & - \\
\hline $08 / 16 / 2000$ & - & - & - & - & - & E0.001 & - & - \\
\hline $08 / 28 / 2000$ & - & - & - & - & - & E0.001 & - & - \\
\hline 09/09/2000 & - & - & - & - & - & E0.001 & - & - \\
\hline $09 / 21 / 2000$ & - & - & - & - & - & E0.001 & - & - \\
\hline $10 / 03 / 2000$ & - & - & - & - & - & E0.002 & - & - \\
\hline $11 / 08 / 2000$ & - & - & - & - & - & E0.001 & - & - \\
\hline $11 / 20 / 2000$ & - & - & - & - & - & E0.006 & - & - \\
\hline $12 / 02 / 2000$ & - & - & - & - & - & E0.005 & - & - \\
\hline $12 / 14 / 2000$ & - & - & - & - & - & E0.002 & - & - \\
\hline $12 / 26 / 2000$ & - & - & - & - & - & E0.001 & - & - \\
\hline
\end{tabular}


Table 17A. Quality-control analytical cartridge travel blank results for volatile organic compounds (VOC) with high breakthrough volumes for the Sweetwater Reservoir air sampling site, San Diego County, California-Continued.

[The site identification number is 324141117001601 . Concentrations are given in parts per billion by volume (ppbv). Sample volume for quality-assurance blank is 5.0 liters. Many samples were frozen at the laboratory and analyzed on the same day. Data for travel blanks and environmental samples collected on 10/27/2000 were not used because samples were contaminated during transport. mm/dd/yyyy, month/day/year; *, sampling data lost; ${ }^{\circ} \mathrm{C}$, degrees Celsius; E, estimated value; NA, not analyzed; —, compound was not detected at a concentration above laboratory reporting level]

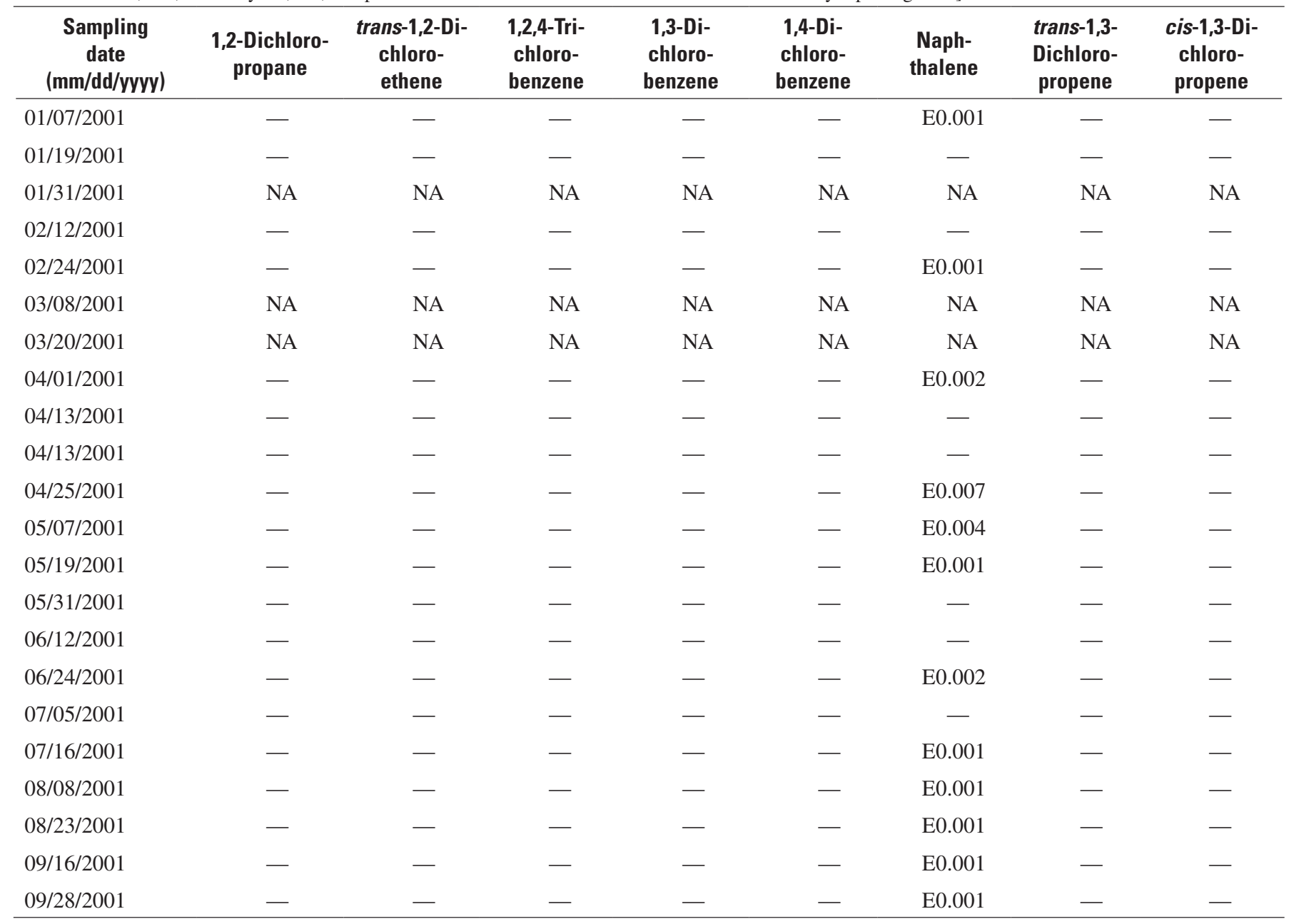


Table 17A. Quality-control analytical cartridge travel blank results for volatile organic compounds (VOC) with high breakthrough volumes for the Sweetwater Reservoir air sampling site, San Diego County, California—Continued.

[The site identification number is 324141117001601 . Concentrations are given in parts per billion by volume (ppbv). Sample volume for quality-assurance blank is 5.0 liters. Many samples were frozen at the laboratory and analyzed on the same day. Data for travel blanks and environmental samples collected on 10/27/2000 were not used because samples were contaminated during transport. mm/dd/yyyy, month/day/year; *, sampling data lost; ${ }^{\circ} \mathrm{C}$, degrees Celsius; E, estimated value; NA, not analyzed; —, compound was not detected at a concentration above laboratory reporting level]

\begin{tabular}{|c|c|c|c|c|c|c|c|c|}
\hline $\begin{array}{l}\text { Sampling } \\
\text { date } \\
\text { (mm/dd/yyyy) }\end{array}$ & $\begin{array}{l}\text { Trichloro- } \\
\text { ethene } \\
\text { (TCE) }\end{array}$ & $\begin{array}{l}\text { Hexachloro- } \\
\text { butadiene }\end{array}$ & $\begin{array}{l}\text { Methyl } \\
\text { meth- } \\
\text { acrylate }\end{array}$ & $\begin{array}{c}\text { 1,2,3,4-Tetra- } \\
\text { methyl- } \\
\text { benzene }\end{array}$ & $\begin{array}{l}\text { Ethyl tert- } \\
\text { butyl ether } \\
\text { (ETBE) }\end{array}$ & $\begin{array}{l}\text { tert-Amyl } \\
\text { methyl } \\
\text { ether } \\
\text { (TAME) }\end{array}$ & $\begin{array}{c}\text { trans-1,4- } \\
\text { Dichloro-2- } \\
\text { butene }\end{array}$ & $\begin{array}{l}\text { Ethyl } \\
\text { meth- } \\
\text { acrylate }\end{array}$ \\
\hline $10 / 02 / 1999 *$ & NA & NA & NA & NA & NA & NA & NA & NA \\
\hline 10/13/1999 & - & - & - & - & - & - & - & - \\
\hline $10 / 26 / 1999$ & - & - & - & - & - & - & - & - \\
\hline 11/07/1999 & - & - & - & - & - & - & - & - \\
\hline 11/19/1999 & - & - & - & - & - & - & - & - \\
\hline $12 / 13 / 1999$ & - & - & - & - & - & - & - & - \\
\hline $12 / 25 / 1999$ & - & - & - & - & - & - & - & - \\
\hline $01 / 06 / 2000$ & - & - & - & - & - & - & - & - \\
\hline 01/18/2000 & - & - & - & - & - & - & - & - \\
\hline 01/30/2000 & - & - & - & - & - & - & - & - \\
\hline $02 / 11 / 2000$ & - & - & - & - & - & - & - & - \\
\hline $03 / 18 / 2000$ & - & - & - & - & - & - & - & - \\
\hline $04 / 11 / 2000$ & - & - & - & - & - & - & - & - \\
\hline $04 / 23 / 2000$ & - & - & - & - & - & - & - & - \\
\hline $05 / 05 / 2000$ & - & - & - & - & - & - & - & - \\
\hline $05 / 17 / 2000$ & E0.003 & - & - & - & - & - & - & - \\
\hline $05 / 29 / 2000$ & E0.015 & - & - & - & - & - & - & - \\
\hline $06 / 10 / 2000$ & E0.005 & - & - & - & - & - & - & - \\
\hline $06 / 29 / 2000$ & - & - & - & - & - & - & - & - \\
\hline $07 / 11 / 2000$ & - & - & - & - & - & - & - & - \\
\hline $07 / 23 / 2000$ & - & - & - & - & - & - & - & - \\
\hline $08 / 04 / 2000$ & - & - & - & - & - & - & - & - \\
\hline $08 / 16 / 2000$ & - & - & - & - & - & - & - & - \\
\hline $08 / 28 / 2000$ & - & - & - & - & - & - & - & - \\
\hline 09/09/2000 & - & - & - & - & - & - & - & - \\
\hline $09 / 21 / 2000$ & - & - & - & - & - & - & - & - \\
\hline $10 / 03 / 2000$ & - & - & - & - & - & - & - & - \\
\hline $11 / 08 / 2000$ & - & - & - & - & - & - & - & - \\
\hline $11 / 20 / 2000$ & - & - & - & - & - & - & - & - \\
\hline $12 / 02 / 2000$ & - & - & - & - & - & - & - & - \\
\hline $12 / 14 / 2000$ & - & - & - & - & - & - & - & - \\
\hline $12 / 26 / 2000$ & - & - & - & - & - & - & - & - \\
\hline
\end{tabular}


Table 17A. Quality-control analytical cartridge travel blank results for volatile organic compounds (VOC) with high breakthrough volumes for the Sweetwater Reservoir air sampling site, San Diego County, California—Continued.

[The site identification number is 324141117001601 . Concentrations are given in parts per billion by volume (ppbv). Sample volume for quality-assurance blank is 5.0 liters. Many samples were frozen at the laboratory and analyzed on the same day. Data for travel blanks and environmental samples collected on 10/27/2000 were not used because samples were contaminated during transport. mm/dd/yyyy, month/day/year; *, sampling data lost; ${ }^{\circ} \mathrm{C}$, degrees Celsius; E, estimated value; NA, not analyzed; —, compound was not detected at a concentration above laboratory reporting level]

\begin{tabular}{|c|c|c|c|c|c|c|c|c|}
\hline $\begin{array}{c}\text { Sampling } \\
\text { date } \\
\text { (mm/dd/yyyy) }\end{array}$ & $\begin{array}{l}\text { Trichloro- } \\
\text { ethene } \\
\text { (TCE) }\end{array}$ & $\begin{array}{l}\text { Hexachloro- } \\
\text { butadiene }\end{array}$ & $\begin{array}{l}\text { Methyl } \\
\text { meth- } \\
\text { acrylate }\end{array}$ & $\begin{array}{c}\text { 1,2,3,4-Tetra- } \\
\text { methyl- } \\
\text { benzene }\end{array}$ & $\begin{array}{l}\text { Ethyl tert- } \\
\text { butyl ether } \\
\text { (ETBE) }\end{array}$ & $\begin{array}{l}\text { tert-Amyl } \\
\text { methyl } \\
\text { ether } \\
\text { (TAME) }\end{array}$ & $\begin{array}{l}\text { trans-1,4- } \\
\text { Dichloro-2- } \\
\text { butene }\end{array}$ & $\begin{array}{c}\text { Ethyl } \\
\text { meth- } \\
\text { acrylate }\end{array}$ \\
\hline 01/07/2001 & - & - & - & - & - & - & - & - \\
\hline 01/19/2001 & - & - & - & - & - & - & - & - \\
\hline $01 / 31 / 2001$ & NA & NA & NA & NA & NA & NA & NA & NA \\
\hline $02 / 12 / 2001$ & - & - & - & - & - & - & - & - \\
\hline $02 / 24 / 2001$ & - & - & - & - & - & - & - & - \\
\hline 03/08/2001 & NA & NA & NA & NA & NA & NA & NA & NA \\
\hline 03/20/2001 & NA & NA & NA & NA & NA & NA & NA & NA \\
\hline $04 / 01 / 2001$ & - & - & - & - & - & - & - & - \\
\hline 04/13/2001 & - & - & - & - & - & - & - & - \\
\hline $04 / 13 / 2001$ & - & - & - & - & - & - & - & - \\
\hline $04 / 25 / 2001$ & - & - & - & - & - & - & - & - \\
\hline 05/07/2001 & - & - & - & - & - & - & - & - \\
\hline 05/19/2001 & - & - & - & - & - & - & - & - \\
\hline $05 / 31 / 2001$ & - & - & - & - & - & - & - & - \\
\hline $06 / 12 / 2001$ & - & - & - & - & - & - & - & - \\
\hline $06 / 24 / 2001$ & - & - & - & - & - & - & - & - \\
\hline 07/05/2001 & - & - & - & - & - & - & - & - \\
\hline $07 / 16 / 2001$ & - & - & - & - & - & - & - & - \\
\hline 08/08/2001 & - & - & - & - & - & - & - & - \\
\hline $08 / 23 / 2001$ & - & - & - & - & - & - & - & - \\
\hline $09 / 16 / 2001$ & - & - & - & - & - & - & - & - \\
\hline 09/28/2001 & - & - & - & - & - & - & - & - \\
\hline
\end{tabular}


Table 17A. Quality-control analytical cartridge travel blank results for volatile organic compounds (VOC) with high breakthrough volumes for the Sweetwater Reservoir air sampling site, San Diego County, California—Continued.

[The site identification number is 324141117001601 . Concentrations are given in parts per billion by volume (ppbv). Sample volume for quality-assurance blank is 5.0 liters. Many samples were frozen at the laboratory and analyzed on the same day. Data for travel blanks and environmental samples collected on 10/27/2000 were not used because samples were contaminated during transport. mm/dd/yyyy, month/day/year; *, sampling data lost; ${ }^{\circ} \mathrm{C}$, degrees Celsius; E, estimated value; NA, not analyzed; —, compound was not detected at a concentration above laboratory reporting level]

\begin{tabular}{|c|c|c|c|c|c|c|c|c|}
\hline $\begin{array}{c}\text { Sampling } \\
\text { date } \\
\text { (mm/dd/yyyy) }\end{array}$ & $\begin{array}{l}\text { Carbon } \\
\text { disulfide }\end{array}$ & $\begin{array}{l}\text { cis- } \\
\text { 1,2-Di- } \\
\text { chloro- } \\
\text { ethene }\end{array}$ & $\begin{array}{l}\text { 2-Hexanone } \\
\text { (MBK) }\end{array}$ & $\begin{array}{l}\text { Ethenyl- } \\
\text { benzene } \\
\text { (Styrene) }\end{array}$ & o-Xylene & $\begin{array}{l}\text { 1,1-Di- } \\
\text { chloro- } \\
\text { propene }\end{array}$ & $\begin{array}{l}\text { 2,2-Di- } \\
\text { chloro- } \\
\text { propane }\end{array}$ & $\begin{array}{l}\text { 1,3-Di- } \\
\text { chloro- } \\
\text { propane }\end{array}$ \\
\hline 10/02/1999* & NA & NA & NA & NA & NA & NA & NA & NA \\
\hline $10 / 13 / 1999$ & - & - & - & - & E0.001 & - & - & - \\
\hline $10 / 26 / 1999$ & - & - & - & E0.001 & E0.001 & - & - & - \\
\hline 11/07/1999 & - & - & - & E0.001 & E0.001 & - & - & - \\
\hline 11/19/1999 & - & - & - & - & - & - & - & - \\
\hline $12 / 13 / 1999$ & E0.001 & - & - & E0.001 & E0.001 & - & - & - \\
\hline $12 / 25 / 1999$ & - & - & - & - & - & - & - & - \\
\hline 01/06/2000 & - & - & - & E0.001 & - & - & - & - \\
\hline $01 / 18 / 2000$ & - & - & - & E0.001 & E0.002 & - & - & - \\
\hline $01 / 30 / 2000$ & E0.001 & - & - & E0.001 & E0.001 & - & - & - \\
\hline $02 / 11 / 2000$ & E0.001 & - & - & - & E0.001 & - & - & - \\
\hline 03/18/2000 & - & - & - & - & - & - & - & - \\
\hline $04 / 11 / 2000$ & - & - & - & E0.001 & - & - & - & - \\
\hline $04 / 23 / 2000$ & E0.004 & - & - & E0.001 & - & - & - & - \\
\hline 05/05/2000 & - & - & - & - & - & - & - & - \\
\hline $05 / 17 / 2000$ & - & - & - & - & - & - & - & - \\
\hline $05 / 29 / 2000$ & E0.001 & - & - & E0.001 & - & - & - & - \\
\hline $06 / 10 / 2000$ & - & - & - & - & - & - & - & - \\
\hline $06 / 29 / 2000$ & - & - & - & E0.001 & - & - & - & - \\
\hline $07 / 11 / 2000$ & E0.006 & - & - & E0.001 & - & - & - & - \\
\hline $07 / 23 / 2000$ & E0.001 & - & - & - & - & - & - & - \\
\hline 08/04/2000 & - & - & - & - & - & - & - & - \\
\hline 08/16/2000 & E0.003 & - & - & - & - & - & - & - \\
\hline $08 / 28 / 2000$ & - & - & - & - & E0.001 & - & - & - \\
\hline 09/09/2000 & - & - & - & - & - & - & - & - \\
\hline $09 / 21 / 2000$ & - & - & - & - & - & - & - & - \\
\hline $10 / 03 / 2000$ & E0.001 & - & - & E0.001 & E0.001 & - & - & - \\
\hline $11 / 08 / 2000$ & E0.004 & - & - & E0.004 & E0.002 & - & - & - \\
\hline $11 / 20 / 2000$ & - & - & - & E0.005 & E0.004 & - & - & - \\
\hline $12 / 02 / 2000$ & E0.003 & - & - & E0.005 & E0.002 & - & - & - \\
\hline $12 / 14 / 2000$ & E0.013 & - & - & E0.001 & E0.001 & - & - & - \\
\hline $12 / 26 / 2000$ & - & - & - & E0.001 & E0.002 & - & - & - \\
\hline
\end{tabular}


Table 17A. Quality-control analytical cartridge travel blank results for volatile organic compounds (VOC) with high breakthrough volumes for the Sweetwater Reservoir air sampling site, San Diego County, California—Continued.

[The site identification number is 324141117001601 . Concentrations are given in parts per billion by volume (ppbv). Sample volume for quality-assurance blank is 5.0 liters. Many samples were frozen at the laboratory and analyzed on the same day. Data for travel blanks and environmental samples collected on 10/27/2000 were not used because samples were contaminated during transport. mm/dd/yyyy, month/day/year; *, sampling data lost; ${ }^{\circ} \mathrm{C}$, degrees Celsius; E, estimated value; NA, not analyzed; —, compound was not detected at a concentration above laboratory reporting level]

\begin{tabular}{|c|c|c|c|c|c|c|c|c|}
\hline $\begin{array}{c}\text { Sampling } \\
\text { date } \\
\text { (mm/dd/yyyy) }\end{array}$ & $\begin{array}{l}\text { Carbon } \\
\text { disulfide }\end{array}$ & $\begin{array}{l}\text { cis- } \\
\text { 1,2-Di- } \\
\text { chloro- } \\
\text { ethene }\end{array}$ & $\begin{array}{l}\text { 2-Hexanone } \\
\text { (MBK) }\end{array}$ & $\begin{array}{l}\text { Ethenyl- } \\
\text { benzene } \\
\text { (Styrene) }\end{array}$ & $o$-Xylene & $\begin{array}{l}\text { 1,1-Di- } \\
\text { chloro- } \\
\text { propene }\end{array}$ & $\begin{array}{l}\text { 2,2-Di- } \\
\text { chloro- } \\
\text { propane }\end{array}$ & $\begin{array}{l}\text { 1,3-Di- } \\
\text { chloro- } \\
\text { propane }\end{array}$ \\
\hline 01/07/2001 & E0.007 & - & - & - & E0.002 & - & - & - \\
\hline 01/19/2001 & - & - & - & - & - & - & - & - \\
\hline $01 / 31 / 2001$ & NA & NA & NA & NA & NA & NA & NA & NA \\
\hline $02 / 12 / 2001$ & - & - & - & - & - & - & - & - \\
\hline $02 / 24 / 2001$ & - & - & - & - & E0.001 & - & - & - \\
\hline 03/08/2001 & NA & NA & NA & NA & NA & NA & NA & NA \\
\hline 03/20/2001 & NA & NA & NA & NA & NA & NA & NA & NA \\
\hline $04 / 01 / 2001$ & E0.001 & - & - & E0.001 & E0.001 & - & - & - \\
\hline $04 / 13 / 2001$ & E0.001 & - & - & E0.001 & E0.001 & - & - & - \\
\hline $04 / 13 / 2001$ & E0.001 & - & - & E0.001 & E0.001 & - & - & - \\
\hline $04 / 25 / 2001$ & E0.005 & - & - & E0.002 & E0.001 & - & - & - \\
\hline 05/07/2001 & E0.008 & - & - & E0.026 & E0.029 & - & - & - \\
\hline 05/19/2001 & E0.007 & - & - & - & E0.001 & - & - & - \\
\hline $05 / 31 / 2001$ & E0.003 & - & - & - & E0.001 & - & - & - \\
\hline $06 / 12 / 2001$ & - & - & - & - & - & - & - & - \\
\hline $06 / 24 / 2001$ & E0.017 & - & - & E0.002 & E0.001 & - & - & - \\
\hline 07/05/2001 & - & - & - & - & - & - & - & - \\
\hline $07 / 16 / 2001$ & E0.002 & - & - & - & - & - & - & - \\
\hline 08/08/2001 & - & - & - & - & E0.001 & - & - & - \\
\hline $08 / 23 / 2001$ & - & - & - & - & E0.001 & - & - & - \\
\hline 09/16/2001 & E0.005 & - & - & E0.002 & E0.001 & - & - & - \\
\hline $09 / 28 / 2001$ & - & - & - & - & E0.002 & - & - & - \\
\hline
\end{tabular}


Table 17A. Quality-control analytical cartridge travel blank results for volatile organic compounds (VOC) with high breakthrough volumes for the Sweetwater Reservoir air sampling site, San Diego County, California—Continued.

[The site identification number is 324141117001601 . Concentrations are given in parts per billion by volume (ppbv). Sample volume for quality-assurance blank is 5.0 liters. Many samples were frozen at the laboratory and analyzed on the same day. Data for travel blanks and environmental samples collected on 10/27/2000 were not used because samples were contaminated during transport. mm/dd/yyyy, month/day/year; *, sampling data lost; ${ }^{\circ} \mathrm{C}$, degrees Celsius; E, estimated value; NA, not analyzed; — , compound was not detected at a concentration above laboratory reporting level]

\begin{tabular}{|c|c|c|c|c|c|c|c|c|}
\hline $\begin{array}{c}\text { Sampling } \\
\text { date } \\
\text { (mm/dd/yyyy) }\end{array}$ & $\begin{array}{l}\text { 2-Ethyl- } \\
\text { toluene }\end{array}$ & $\begin{array}{c}\text { 1,2,3-Tri- } \\
\text { methyl- } \\
\text { benzene }\end{array}$ & $\begin{array}{c}\text { 1,2,4-Tri- } \\
\text { methyl- } \\
\text { benzene }\end{array}$ & $\begin{array}{c}\text { Isopropyl- } \\
\text { benzene } \\
\text { (Cumene) }\end{array}$ & $\begin{array}{l}n \text {-Propyl- } \\
\text { benzene }\end{array}$ & $\begin{array}{l}\text { 1,3,5-Tri- } \\
\text { methyl- } \\
\text { benzene }\end{array}$ & $\begin{array}{c}\text { 1-Chloro-2- } \\
\text { methyl- } \\
\text { benzene }\end{array}$ & $\begin{array}{c}\text { 1-Chloro-4- } \\
\text { methyl- } \\
\text { benzene }\end{array}$ \\
\hline $10 / 02 / 1999 *$ & NA & NA & NA & NA & NA & NA & NA & NA \\
\hline 10/13/1999 & - & - & E0.001 & - & - & - & - & - \\
\hline $10 / 26 / 1999$ & - & - & E0.001 & - & - & - & - & - \\
\hline 11/07/1999 & - & - & E0.001 & - & - & - & - & - \\
\hline $11 / 19 / 1999$ & - & - & - & - & - & - & - & - \\
\hline $12 / 13 / 1999$ & - & - & - & - & - & - & - & - \\
\hline $12 / 25 / 1999$ & - & - & - & - & - & - & - & - \\
\hline $01 / 06 / 2000$ & - & - & - & - & - & - & - & - \\
\hline $01 / 18 / 2000$ & - & - & E0.001 & - & - & - & - & - \\
\hline 01/30/2000 & - & - & - & - & - & - & - & - \\
\hline $02 / 11 / 2000$ & - & - & - & - & - & - & - & - \\
\hline 03/18/2000 & - & - & - & - & - & - & - & - \\
\hline $04 / 11 / 2000$ & - & - & - & - & - & - & - & - \\
\hline $04 / 23 / 2000$ & - & - & - & - & - & - & - & - \\
\hline 05/05/2000 & - & - & - & - & - & - & - & - \\
\hline $05 / 17 / 2000$ & - & - & - & - & - & - & - & - \\
\hline $05 / 29 / 2000$ & - & - & - & - & - & - & - & - \\
\hline 06/10/2000 & - & - & - & - & - & - & - & - \\
\hline 06/29/2000 & - & - & - & - & - & - & - & - \\
\hline $07 / 11 / 2000$ & - & - & - & - & - & - & - & - \\
\hline $07 / 23 / 2000$ & - & - & - & - & - & - & - & - \\
\hline 08/04/2000 & - & - & - & - & - & - & - & - \\
\hline 08/16/2000 & - & - & - & - & - & - & - & - \\
\hline $08 / 28 / 2000$ & - & - & - & - & - & - & - & - \\
\hline 09/09/2000 & - & - & - & - & - & - & - & - \\
\hline $09 / 21 / 2000$ & - & - & - & - & - & - & - & - \\
\hline $10 / 03 / 2000$ & - & - & - & - & - & - & - & - \\
\hline $11 / 08 / 2000$ & - & - & - & - & - & - & - & - \\
\hline $11 / 20 / 2000$ & - & - & E0.004 & - & - & - & - & - \\
\hline $12 / 02 / 2000$ & - & - & E0.002 & - & - & - & - & - \\
\hline $12 / 14 / 2000$ & - & - & - & - & - & - & - & - \\
\hline $12 / 26 / 2000$ & - & - & - & - & - & - & - & - \\
\hline
\end{tabular}


Table 17A. Quality-control analytical cartridge travel blank results for volatile organic compounds (VOC) with high breakthrough volumes for the Sweetwater Reservoir air sampling site, San Diego County, California—Continued.

[The site identification number is 324141117001601 . Concentrations are given in parts per billion by volume (ppbv). Sample volume for quality-assurance blank is 5.0 liters. Many samples were frozen at the laboratory and analyzed on the same day. Data for travel blanks and environmental samples collected on 10/27/2000 were not used because samples were contaminated during transport. mm/dd/yyyy, month/day/year; *, sampling data lost; ${ }^{\circ} \mathrm{C}$, degrees Celsius; E, estimated value; NA, not analyzed; — , compound was not detected at a concentration above laboratory reporting level]

\begin{tabular}{|c|c|c|c|c|c|c|c|c|}
\hline $\begin{array}{c}\text { Sampling } \\
\text { date } \\
\text { (mm/dd/yyyy) }\end{array}$ & $\begin{array}{l}\text { 2-Ethyl- } \\
\text { toluene }\end{array}$ & $\begin{array}{l}\text { 1,2,3-Tri- } \\
\text { methyl- } \\
\text { benzene }\end{array}$ & $\begin{array}{l}\text { 1,2,4-Tri- } \\
\text { methyl- } \\
\text { benzene }\end{array}$ & $\begin{array}{l}\text { Isopropyl- } \\
\text { benzene } \\
\text { (Cumene) }\end{array}$ & $\begin{array}{l}n \text {-Propyl- } \\
\text { benzene }\end{array}$ & $\begin{array}{c}1,3,5-\text { Tri- } \\
\text { methyl- } \\
\text { benzene }\end{array}$ & $\begin{array}{c}\text { 1-Chloro-2- } \\
\text { methyl- } \\
\text { benzene }\end{array}$ & $\begin{array}{c}\text { 1-Chloro-4- } \\
\text { methyl- } \\
\text { benzene }\end{array}$ \\
\hline 01/07/2001 & - & - & - & - & - & - & - & - \\
\hline 01/19/2001 & - & - & - & - & - & - & - & - \\
\hline $01 / 31 / 2001$ & NA & NA & NA & NA & NA & NA & NA & NA \\
\hline $02 / 12 / 2001$ & - & - & - & - & - & - & - & - \\
\hline $02 / 24 / 2001$ & - & - & - & - & - & - & - & - \\
\hline 03/08/2001 & NA & NA & NA & NA & NA & NA & NA & NA \\
\hline $03 / 20 / 2001$ & NA & NA & NA & NA & NA & NA & NA & NA \\
\hline 04/01/2001 & - & - & - & - & - & - & - & - \\
\hline 04/13/2001 & - & - & - & - & - & - & - & - \\
\hline $04 / 13 / 2001$ & - & - & - & - & - & - & - & - \\
\hline $04 / 25 / 2001$ & - & - & - & - & - & - & - & - \\
\hline 05/07/2001 & - & - & - & - & - & - & - & - \\
\hline 05/19/2001 & - & - & - & - & - & - & - & - \\
\hline $05 / 31 / 2001$ & - & - & - & - & - & - & - & - \\
\hline $06 / 12 / 2001$ & - & - & - & - & - & - & - & - \\
\hline $06 / 24 / 2001$ & - & - & - & - & - & - & - & - \\
\hline 07/05/2001 & - & - & - & - & - & - & - & - \\
\hline 07/16/2001 & - & - & - & - & - & - & - & - \\
\hline 08/08/2001 & - & - & - & - & - & - & - & - \\
\hline $08 / 23 / 2001$ & - & - & - & - & - & - & - & - \\
\hline 09/16/2001 & - & - & - & - & - & - & - & - \\
\hline 09/28/2001 & - & - & - & - & - & - & - & - \\
\hline
\end{tabular}


Table 17A. Quality-control analytical cartridge travel blank results for volatile organic compounds (VOC) with high breakthrough volumes for the Sweetwater Reservoir air sampling site, San Diego County, California—Continued.

[The site identification number is 324141117001601 . Concentrations are given in parts per billion by volume (ppbv). Sample volume for quality-assurance blank is 5.0 liters. Many samples were frozen at the laboratory and analyzed on the same day. Data for travel blanks and environmental samples collected on 10/27/2000 were not used because samples were contaminated during transport. mm/dd/yyyy, month/day/year; *, sampling data lost; ${ }^{\circ} \mathrm{C}$, degrees Celsius; E, estimated value; NA, not analyzed; —, compound was not detected at a concentration above laboratory reporting level]

\begin{tabular}{|c|c|c|c|c|c|c|c|c|c|}
\hline $\begin{array}{c}\text { Sampling } \\
\text { date } \\
\text { (mm/dd/yyyy) }\end{array}$ & $\begin{array}{l}\text { Bromo- } \\
\text { chloro- } \\
\text { methane }\end{array}$ & $\begin{array}{l}n \text {-Butyl- } \\
\text { benzene }\end{array}$ & $\begin{array}{l}\text { sec-Butyl- } \\
\text { benzene }\end{array}$ & $\begin{array}{c}\text { tert- } \\
\text { Butyl- } \\
\text { benzene }\end{array}$ & $\begin{array}{l}\text { 1-Isopropyl- } \\
\text { 4-methyl- } \\
\text { benzene }\end{array}$ & $\begin{array}{c}\text { 1,2,3-Tri- } \\
\text { chloro- } \\
\text { propane }\end{array}$ & $\begin{array}{l}1,1,1,2- \\
\text { Tetra- } \\
\text { chloro- } \\
\text { ethane }\end{array}$ & $\begin{array}{c}1,2,3-\text { Tri- } \\
\text { chloro- } \\
\text { benzene }\end{array}$ & $\begin{array}{l}\text { 1,2-Di- } \\
\text { bromo- } \\
\text { ethane }\end{array}$ \\
\hline 10/02/1999* & NA & NA & NA & NA & NA & NA & NA & NA & NA \\
\hline $10 / 13 / 1999$ & - & - & - & - & - & - & - & - & - \\
\hline $10 / 26 / 1999$ & - & - & - & - & - & - & - & - & - \\
\hline $11 / 07 / 1999$ & - & - & - & - & - & - & - & - & - \\
\hline $11 / 19 / 1999$ & - & - & - & - & - & - & - & - & - \\
\hline $12 / 13 / 1999$ & - & - & - & - & - & - & - & - & - \\
\hline $12 / 25 / 1999$ & - & - & - & - & - & - & - & - & - \\
\hline $01 / 06 / 2000$ & - & - & - & - & - & - & - & - & - \\
\hline $01 / 18 / 2000$ & - & - & - & - & - & - & - & - & - \\
\hline $01 / 30 / 2000$ & - & - & - & - & - & - & - & - & - \\
\hline $02 / 11 / 2000$ & - & - & - & - & - & - & - & - & - \\
\hline 03/18/2000 & - & - & - & - & - & - & - & - & - \\
\hline $04 / 11 / 2000$ & - & - & - & - & - & - & - & - & - \\
\hline $04 / 23 / 2000$ & - & - & - & - & - & - & - & - & - \\
\hline 05/05/2000 & - & - & - & - & - & - & - & - & - \\
\hline $05 / 17 / 2000$ & - & - & - & - & - & - & - & - & - \\
\hline $05 / 29 / 2000$ & - & - & - & - & - & - & - & - & - \\
\hline $06 / 10 / 2000$ & - & - & - & - & - & - & - & - & - \\
\hline $06 / 29 / 2000$ & - & - & - & - & - & - & - & - & - \\
\hline $07 / 11 / 2000$ & - & - & - & - & - & - & - & - & - \\
\hline $07 / 23 / 2000$ & - & - & - & - & - & - & - & - & - \\
\hline $08 / 04 / 2000$ & - & - & - & - & - & - & - & - & - \\
\hline $08 / 16 / 2000$ & - & - & - & - & - & - & - & - & - \\
\hline $08 / 28 / 2000$ & - & - & - & - & - & - & - & - & - \\
\hline $09 / 09 / 2000$ & - & - & - & - & - & - & - & - & - \\
\hline $09 / 21 / 2000$ & - & - & - & - & - & - & - & - & - \\
\hline $10 / 03 / 2000$ & - & - & - & - & - & - & - & - & - \\
\hline $11 / 08 / 2000$ & - & - & - & - & - & - & - & - & - \\
\hline $11 / 20 / 2000$ & - & - & - & - & - & - & - & - & - \\
\hline $12 / 02 / 2000$ & - & - & - & - & - & - & - & - & - \\
\hline $12 / 14 / 2000$ & - & - & - & - & - & - & - & - & - \\
\hline $12 / 26 / 2000$ & - & - & - & - & - & - & - & - & - \\
\hline
\end{tabular}


Table 17A. Quality-control analytical cartridge travel blank results for volatile organic compounds (VOC) with high breakthrough volumes for the Sweetwater Reservoir air sampling site, San Diego County, California—Continued.

[The site identification number is 324141117001601 . Concentrations are given in parts per billion by volume (ppbv). Sample volume for quality-assurance blank is 5.0 liters. Many samples were frozen at the laboratory and analyzed on the same day. Data for travel blanks and environmental samples collected on 10/27/2000 were not used because samples were contaminated during transport. mm/dd/yyyy, month/day/year; *, sampling data lost; ${ }^{\circ} \mathrm{C}$, degrees Celsius; E, estimated value; NA, not analyzed; —, compound was not detected at a concentration above laboratory reporting level]

\begin{tabular}{|c|c|c|c|c|c|c|c|c|c|}
\hline $\begin{array}{l}\text { Sampling } \\
\text { date } \\
\text { (mm/dd/yyyy) }\end{array}$ & $\begin{array}{l}\text { Bromo- } \\
\text { chloro- } \\
\text { methane }\end{array}$ & $\begin{array}{l}n \text {-Butyl- } \\
\text { benzene }\end{array}$ & $\begin{array}{l}\text { sec-Butyl- } \\
\text { benzene }\end{array}$ & $\begin{array}{c}\text { tert- } \\
\text { Butyl- } \\
\text { benzene }\end{array}$ & $\begin{array}{l}\text { 1-Isopropyl- } \\
\text { 4-methyl- } \\
\text { benzene }\end{array}$ & $\begin{array}{l}\text { 1,2,3-Tri- } \\
\text { chloro- } \\
\text { propane }\end{array}$ & $\begin{array}{l}1,1,1,2- \\
\text { Tetra- } \\
\text { chloro- } \\
\text { ethane }\end{array}$ & $\begin{array}{c}1,2,3-\text { Tri- } \\
\text { chloro- } \\
\text { benzene }\end{array}$ & $\begin{array}{l}\text { 1,2-Di- } \\
\text { bromo- } \\
\text { ethane }\end{array}$ \\
\hline $01 / 07 / 2001$ & - & - & - & - & - & - & - & - & - \\
\hline $01 / 19 / 2001$ & - & - & - & - & - & - & - & - & - \\
\hline $01 / 31 / 2001$ & NA & NA & NA & NA & NA & NA & NA & NA & NA \\
\hline $02 / 12 / 2001$ & - & - & - & - & - & - & - & - & - \\
\hline $02 / 24 / 2001$ & - & - & - & - & - & - & - & - & - \\
\hline 03/08/2001 & NA & NA & NA & NA & NA & NA & NA & NA & NA \\
\hline 03/20/2001 & NA & NA & NA & NA & NA & NA & NA & NA & NA \\
\hline $04 / 01 / 2001$ & - & - & - & - & - & - & - & - & - \\
\hline $04 / 13 / 2001$ & - & - & - & - & - & - & - & - & - \\
\hline $04 / 13 / 2001$ & - & - & - & - & - & - & - & - & - \\
\hline $04 / 25 / 2001$ & - & - & - & - & - & - & - & - & - \\
\hline 05/07/2001 & - & - & - & - & - & - & - & - & - \\
\hline 05/19/2001 & - & - & - & - & - & - & - & - & - \\
\hline $05 / 31 / 2001$ & - & - & - & - & - & - & - & - & - \\
\hline $06 / 12 / 2001$ & - & - & - & - & - & - & - & - & - \\
\hline $06 / 24 / 2001$ & - & - & - & - & - & - & - & - & - \\
\hline $07 / 05 / 2001$ & - & - & - & - & - & - & - & - & - \\
\hline $07 / 16 / 2001$ & - & - & - & - & - & - & - & - & - \\
\hline 08/08/2001 & - & - & - & - & - & - & - & - & - \\
\hline $08 / 23 / 2001$ & - & - & - & - & - & - & - & - & - \\
\hline 09/16/2001 & - & - & - & - & - & - & - & - & - \\
\hline 09/28/2001 & - & - & - & - & - & - & - & - & - \\
\hline
\end{tabular}


Table 17A. Quality-control analytical cartridge travel blank results for volatile organic compounds (VOC) with high breakthrough volumes for the Sweetwater Reservoir air sampling site, San Diego County, California—Continued.

[The site identification number is 324141117001601 . Concentrations are given in parts per billion by volume (ppbv). Sample volume for quality-assurance blank is 5.0 liters. Many samples were frozen at the laboratory and analyzed on the same day. Data for travel blanks and environmental samples collected on 10/27/2000 were not used because samples were contaminated during transport. mm/dd/yyyy, month/day/year; *, sampling data lost; ${ }^{\circ} \mathrm{C}$, degrees Celsius; E, estimated value; NA, not analyzed; —, compound was not detected at a concentration above laboratory reporting level]

\begin{tabular}{|c|c|c|c|c|c|c|c|c|c|}
\hline $\begin{array}{c}\text { Sampling } \\
\text { date } \\
\text { (mm/dd/yyyy) }\end{array}$ & $\begin{array}{l}\text { Methyl tert- } \\
\text { butyl ether } \\
\text { (MTBE) }\end{array}$ & $\begin{array}{l}\text { 3-Chloro- } \\
\text { 1-propene }\end{array}$ & $\begin{array}{l}\text { 4-Methyl-2- } \\
\text { pentanone } \\
\text { (MIBK) }\end{array}$ & Acetone & $\begin{array}{l}\text { Bromo- } \\
\text { benzene }\end{array}$ & $\begin{array}{l}\text { Diethyl } \\
\text { ether }\end{array}$ & $\begin{array}{l}\text { Diiso- } \\
\text { propyl } \\
\text { ether } \\
\text { (DIPE) }\end{array}$ & $\begin{array}{l}\text { Methyl } \\
\text { acrylo- } \\
\text { nitrile }\end{array}$ & $\begin{array}{c}\text { 2-Butanone } \\
\text { (methyl ethyl } \\
\text { ketone) }\end{array}$ \\
\hline 10/02/1999* & NA & NA & NA & NA & NA & NA & NA & NA & NA \\
\hline 10/13/1999 & E0.027 & - & - & 0.323 & - & - & - & - & - \\
\hline 10/26/1999 & - & - & - & E0.158 & - & - & - & - & - \\
\hline 11/07/1999 & - & - & - & E0.074 & - & - & - & - & - \\
\hline 11/19/1999 & - & - & - & E0.144 & - & - & - & - & - \\
\hline 12/13/1999 & E0.080 & - & - & 0.562 & - & - & - & - & - \\
\hline $12 / 25 / 1999$ & E0.005 & - & - & 0.338 & - & - & - & - & - \\
\hline 01/06/2000 & - & - & - & 0.221 & - & - & - & - & - \\
\hline 01/18/2000 & 7.35 & - & - & 0.359 & - & - & - & - & - \\
\hline 01/30/2000 & E0.091 & - & - & 0.180 & - & - & - & - & - \\
\hline $02 / 11 / 2000$ & - & - & - & E0.062 & - & - & - & - & - \\
\hline 03/18/2000 & - & - & - & E0.048 & - & - & - & - & - \\
\hline $04 / 11 / 2000$ & - & - & - & E0.018 & - & - & - & - & - \\
\hline $04 / 23 / 2000$ & - & - & - & E0.083 & - & - & - & - & - \\
\hline 05/05/2000 & - & - & - & E0.053 & - & - & - & - & - \\
\hline 05/17/2000 & - & - & - & E0.056 & - & - & - & - & - \\
\hline 05/29/2000 & E0.005 & - & - & 0.312 & - & - & - & - & E0.005 \\
\hline 06/10/2000 & - & - & - & E0.117 & - & - & - & - & - \\
\hline 06/29/2000 & - & - & - & E0.052 & - & - & - & - & - \\
\hline 07/11/2000 & E0.009 & - & - & 0.277 & - & - & - & - & E0.005 \\
\hline 07/23/2000 & - & - & - & E0.112 & - & - & - & - & - \\
\hline 08/04/2000 & E0.017 & - & - & 0.207 & - & - & - & - & - \\
\hline 08/16/2000 & - & - & - & 0.541 & - & - & - & - & - \\
\hline 08/28/2000 & E0.032 & - & - & 1.21 & - & - & - & - & E0.066 \\
\hline 09/09/2000 & E0.006 & - & - & 0.236 & - & - & - & - & E0.016 \\
\hline 09/21/2000 & - & - & - & E0.078 & - & - & - & - & - \\
\hline $10 / 03 / 2000$ & E0.055 & - & E0.006 & 1.07 & - & - & - & - & E0.013 \\
\hline $11 / 08 / 2000$ & E0.002 & - & - & 0.229 & - & - & - & - & - \\
\hline $11 / 20 / 2000$ & - & - & - & 0.178 & - & - & - & - & E0.011 \\
\hline $12 / 02 / 2000$ & - & - & - & 0.289 & - & - & - & - & E0.009 \\
\hline $12 / 14 / 2000$ & - & - & - & E0.096 & - & - & - & - & - \\
\hline $12 / 26 / 2000$ & - & - & - & E0.055 & - & - & - & - & - \\
\hline
\end{tabular}


Table 17A. Quality-control analytical cartridge travel blank results for volatile organic compounds (VOC) with high breakthrough volumes for the Sweetwater Reservoir air sampling site, San Diego County, California—Continued.

[The site identification number is 324141117001601 . Concentrations are given in parts per billion by volume (ppbv). Sample volume for quality-assurance blank is 5.0 liters. Many samples were frozen at the laboratory and analyzed on the same day. Data for travel blanks and environmental samples collected on 10/27/2000 were not used because samples were contaminated during transport. mm/dd/yyyy, month/day/year; *, sampling data lost; ${ }^{\circ} \mathrm{C}$, degrees Celsius; E, estimated value; NA, not analyzed; — , compound was not detected at a concentration above laboratory reporting level]

\begin{tabular}{|c|c|c|c|c|c|c|c|c|c|}
\hline $\begin{array}{l}\text { Sampling } \\
\text { date } \\
\text { (mm/dd/yyyy) }\end{array}$ & $\begin{array}{l}\text { Methyl tert- } \\
\text { butyl ether } \\
\text { (MTBE) }\end{array}$ & $\begin{array}{l}\text { 3-Chloro- } \\
\text { 1-propene }\end{array}$ & $\begin{array}{l}\text { 4-Methyl-2- } \\
\text { pentanone } \\
\text { (MIBK) }\end{array}$ & Acetone & $\begin{array}{l}\text { Bromo- } \\
\text { benzene }\end{array}$ & $\begin{array}{c}\text { Diethyl } \\
\text { ether }\end{array}$ & $\begin{array}{l}\text { Diiso- } \\
\text { propyl } \\
\text { ether } \\
\text { (DIPE) }\end{array}$ & $\begin{array}{l}\text { Methyl } \\
\text { acrylo- } \\
\text { nitrile }\end{array}$ & $\begin{array}{l}\text { 2-Butanone } \\
\text { (methyl ethyl } \\
\text { ketone) }\end{array}$ \\
\hline $01 / 07 / 2001$ & - & - & - & E0.038 & - & - & - & - & - \\
\hline $01 / 19 / 2001$ & - & - & - & E0.149 & - & - & - & - & - \\
\hline $01 / 31 / 2001$ & NA & NA & NA & NA & NA & NA & NA & NA & NA \\
\hline $02 / 12 / 2001$ & - & - & - & E0.109 & - & - & - & - & - \\
\hline $02 / 24 / 2001$ & - & - & - & E0.127 & - & - & - & - & - \\
\hline 03/08/2001 & NA & NA & $\mathrm{NA}$ & NA & NA & NA & NA & NA & NA \\
\hline 03/20/2001 & NA & NA & NA & NA & NA & NA & NA & NA & NA \\
\hline 04/01/2001 & - & - & - & E0.111 & - & - & - & - & - \\
\hline $04 / 13 / 2001$ & E0.028 & - & - & 0.428 & E0.005 & - & - & - & - \\
\hline $04 / 13 / 2001$ & E0.028 & - & - & 0.428 & E0.005 & - & - & - & - \\
\hline $04 / 25 / 2001$ & - & - & - & E0.161 & - & - & - & - & - \\
\hline 05/07/2001 & - & - & - & 0.224 & - & - & - & - & - \\
\hline 05/19/2001 & - & - & - & E0.073 & - & - & - & - & - \\
\hline $05 / 31 / 2001$ & - & - & - & E0.093 & - & - & - & - & - \\
\hline $06 / 12 / 2001$ & E0.022 & - & - & 0.412 & - & - & - & - & - \\
\hline $06 / 24 / 2001$ & - & - & - & 0.178 & - & - & - & - & - \\
\hline 07/05/2001 & - & - & - & E0.035 & - & - & - & - & - \\
\hline $07 / 16 / 2001$ & - & - & - & 0.319 & - & - & - & - & - \\
\hline 08/08/2001 & - & - & - & 0.181 & - & - & - & - & - \\
\hline 08/23/2001 & - & - & - & 0.184 & - & - & - & - & - \\
\hline 09/16/2001 & - & - & - & E0.131 & - & - & - & - & - \\
\hline $09 / 28 / 2001$ & - & - & - & 0.219 & - & - & - & - & - \\
\hline
\end{tabular}


Table 17A. Quality-control analytical cartridge travel blank results for volatile organic compounds (VOC) with high breakthrough volumes for the Sweetwater Reservoir air sampling site, San Diego County, California—Continued.

[The site identification number is 324141117001601 . Concentrations are given in parts per billion by volume (ppbv). Sample volume for quality-assurance blank is 5.0 liters. Many samples were frozen at the laboratory and analyzed on the same day. Data for travel blanks and environmental samples collected on 10/27/2000 were not used because samples were contaminated during transport. mm/dd/yyyy, month/day/year; *, sampling data lost; ${ }^{\circ} \mathrm{C}$, degrees Celsius; E, estimated value; NA, not analyzed; —, compound was not detected at a concentration above laboratory reporting level]

\begin{tabular}{|c|c|c|c|c|c|c|c|c|c|}
\hline $\begin{array}{c}\text { Sampling } \\
\text { date } \\
\text { (mm/dd/yyyy) }\end{array}$ & $\begin{array}{l}\text { Methyl } \\
\text { acrylate }\end{array}$ & $\begin{array}{l}\text { Tetra- } \\
\text { hydro- } \\
\text { furan }\end{array}$ & $\begin{array}{c}\text { 1,2-Di- } \\
\text { bromo-3- } \\
\text { chloro- } \\
\text { propane } \\
\text { (DBCP) }\end{array}$ & $\begin{array}{c}\text { m- and p- } \\
\text { Xylene }\end{array}$ & $\begin{array}{c}1,2,3,5- \\
\text { Tetra } \\
\text { methyl- } \\
\text { benzene }\end{array}$ & $\begin{array}{c}\text { 1,2,4,5- } \\
\text { Tetra- } \\
\text { methyl- } \\
\text { benzene }\end{array}$ & $\begin{array}{l}\text { Methyl } \\
\text { acetate }\end{array}$ & $\begin{array}{c}\text { 2-Methyl- } \\
\text { 2-butanol } \\
\text { (tert-Amyl } \\
\text { alcohol) }\end{array}$ & $\begin{array}{c}\text { 2-Methyl- } \\
\text { 2-propanol } \\
\text { (tert-Butyl } \\
\text { alcohol) }\end{array}$ \\
\hline 10/02/1999* & NA & NA & NA & NA & NA & NA & NA & NA & NA \\
\hline 10/13/1999 & - & - & - & E0.003 & - & - & - & - & - \\
\hline $10 / 26 / 1999$ & - & - & - & E0.004 & - & - & - & - & - \\
\hline 11/07/1999 & - & - & - & E0.003 & - & - & - & - & - \\
\hline 11/19/1999 & - & - & - & - & - & - & - & - & - \\
\hline $12 / 13 / 1999$ & - & - & - & E0.004 & - & - & - & - & E0.003 \\
\hline $12 / 25 / 1999$ & - & - & - & E0.002 & - & - & - & - & - \\
\hline 01/06/2000 & - & - & - & E0.002 & - & - & - & - & - \\
\hline $01 / 18 / 2000$ & - & - & - & E0.005 & - & - & - & - & E0.004 \\
\hline $01 / 30 / 2000$ & - & - & - & E0.005 & - & - & - & - & E0.002 \\
\hline $02 / 11 / 2000$ & - & - & - & E0.002 & - & - & - & - & - \\
\hline $03 / 18 / 2000$ & - & - & - & - & - & - & - & - & - \\
\hline $04 / 11 / 2000$ & - & - & - & - & - & - & - & - & - \\
\hline $04 / 23 / 2000$ & - & - & - & - & - & - & - & - & - \\
\hline $05 / 05 / 2000$ & - & - & - & - & - & - & - & - & - \\
\hline $05 / 17 / 2000$ & - & - & - & - & - & - & - & - & - \\
\hline $05 / 29 / 2000$ & - & - & - & E0.001 & - & - & - & - & E0.003 \\
\hline $06 / 10 / 2000$ & - & - & - & - & - & - & - & - & E0.001 \\
\hline $06 / 29 / 2000$ & - & - & - & - & - & - & - & - & - \\
\hline $07 / 11 / 2000$ & - & - & - & - & - & - & - & - & - \\
\hline 07/23/2000 & - & - & - & - & - & - & - & - & - \\
\hline 08/04/2000 & - & - & - & E0.001 & - & - & - & - & - \\
\hline 08/16/2000 & - & - & - & E0.001 & - & - & - & - & - \\
\hline $08 / 28 / 2000$ & - & - & - & E0.003 & - & - & - & - & - \\
\hline 09/09/2000 & - & - & - & E0.001 & - & - & - & - & - \\
\hline $09 / 21 / 2000$ & - & - & - & - & - & - & - & - & - \\
\hline $10 / 03 / 2000$ & - & E0.003 & - & E0.004 & - & - & - & - & E0.051 \\
\hline $11 / 08 / 2000$ & - & - & - & E0.002 & - & - & - & - & - \\
\hline $11 / 20 / 2000$ & - & - & - & E0.012 & - & - & - & - & - \\
\hline $12 / 02 / 2000$ & - & E0.044 & - & E0.008 & - & - & - & - & - \\
\hline $12 / 14 / 2000$ & - & - & - & E0.003 & - & - & - & - & - \\
\hline $12 / 26 / 2000$ & - & - & - & E0.003 & - & - & - & - & - \\
\hline
\end{tabular}


Table 17A. Quality-control analytical cartridge travel blank results for volatile organic compounds (VOC) with high breakthrough volumes for the Sweetwater Reservoir air sampling site, San Diego County, California—Continued.

[The site identification number is 324141117001601 . Concentrations are given in parts per billion by volume (ppbv). Sample volume for quality-assurance blank is 5.0 liters. Many samples were frozen at the laboratory and analyzed on the same day. Data for travel blanks and environmental samples collected on 10/27/2000 were not used because samples were contaminated during transport. mm/dd/yyyy, month/day/year; *, sampling data lost; ${ }^{\circ} \mathrm{C}$, degrees Celsius; E, estimated value; NA, not analyzed; —, compound was not detected at a concentration above laboratory reporting level]

\begin{tabular}{|c|c|c|c|c|c|c|c|c|c|}
\hline $\begin{array}{l}\text { Sampling } \\
\text { date } \\
\text { (mm/dd/yyyy) }\end{array}$ & $\begin{array}{l}\text { Methyl } \\
\text { acrylate }\end{array}$ & $\begin{array}{l}\text { Tetra- } \\
\text { hydro- } \\
\text { furan }\end{array}$ & $\begin{array}{c}\text { 1,2-Di- } \\
\text { bromo-3- } \\
\text { chloro- } \\
\text { propane } \\
\text { (DBCP) }\end{array}$ & $\begin{array}{c}m \text { - and p- } \\
\text { Xylene }\end{array}$ & $\begin{array}{c}\text { 1,2,3,5- } \\
\text { Tetra } \\
\text { methyl- } \\
\text { benzene }\end{array}$ & $\begin{array}{c}1,2,4,5- \\
\text { Tetra- } \\
\text { methyl- } \\
\text { benzene }\end{array}$ & $\begin{array}{l}\text { Methyl } \\
\text { acetate }\end{array}$ & $\begin{array}{c}\text { 2-Methyl- } \\
\text { 2-butanol } \\
\text { (tert-Amyl } \\
\text { alcohol) }\end{array}$ & $\begin{array}{c}\text { 2-Methyl- } \\
\text { 2-propanol } \\
\text { (tert-Butyl } \\
\text { alcohol) }\end{array}$ \\
\hline 01/07/2001 & - & - & - & E0.005 & - & - & - & - & - \\
\hline 01/19/2001 & - & - & - & E0.006 & - & - & - & - & - \\
\hline $01 / 31 / 2001$ & NA & NA & NA & NA & NA & NA & NA & NA & NA \\
\hline $02 / 12 / 2001$ & - & - & - & E0.001 & - & - & - & - & - \\
\hline $02 / 24 / 2001$ & - & - & - & E0.003 & - & - & - & - & - \\
\hline 03/08/2001 & NA & NA & NA & NA & NA & NA & NA & NA & NA \\
\hline 03/20/2001 & NA & NA & NA & NA & NA & NA & NA & NA & NA \\
\hline $04 / 01 / 2001$ & - & - & - & E0.002 & - & - & - & - & - \\
\hline $04 / 13 / 2001$ & - & - & - & E0.003 & - & - & - & - & E0.006 \\
\hline $04 / 13 / 2001$ & - & - & - & E0.003 & - & - & - & - & E0.006 \\
\hline $04 / 25 / 2001$ & - & - & - & E0.002 & - & - & - & - & - \\
\hline 05/07/2001 & - & - & - & E0.032 & - & - & - & - & - \\
\hline 05/19/2001 & - & - & - & E0.002 & - & - & - & - & - \\
\hline $05 / 31 / 2001$ & - & - & - & E0.002 & - & - & - & - & - \\
\hline $06 / 12 / 2001$ & - & - & - & - & - & - & - & - & - \\
\hline $06 / 24 / 2001$ & - & - & - & E0.002 & - & - & - & - & - \\
\hline 07/05/2001 & - & - & - & - & - & - & - & - & - \\
\hline $07 / 16 / 2001$ & - & - & - & E0.002 & - & - & - & - & - \\
\hline 08/08/2001 & - & - & - & E0.002 & - & - & - & - & - \\
\hline $08 / 23 / 2001$ & - & - & - & E0.002 & - & - & - & - & - \\
\hline 09/16/2001 & - & - & - & E0.002 & - & - & - & - & - \\
\hline 09/28/2001 & - & - & - & E0.004 & - & - & - & - & - \\
\hline
\end{tabular}


Table 17B. Quality-control analytical results for cartridge spike for volatile organic compounds (VOC) with high breakthrough volumes for the Sweetwater Reservoir air sampling site, San Diego County, California.

[The site identification number is 324141117001601 . Values are given in percent recovery. Sample volume for quality-assurance spike is 5.0 liters. Samples processed at 20.0 degrees Celsius and 760 torr; many samples were frozen at the laboratory and analyzed on the same day. Data for travel blanks and environmental samples collected on 10/27/2000 were not used because samples were contaminated during transport. mm/dd/yyyy, month/day/year; *, sample data lost; NA, not analyzed]

\begin{tabular}{|c|c|c|c|c|c|c|c|c|}
\hline $\begin{array}{c}\text { Sampling } \\
\text { date } \\
\text { (mm/dd/yyyy) }\end{array}$ & $\begin{array}{c}\text { Spike analysis } \\
\text { date } \\
\text { (mm/dd/yyyy) }\end{array}$ & $\begin{array}{l}\text { Dibromo- } \\
\text { methane }\end{array}$ & $\begin{array}{l}\text { Bromo- } \\
\text { dichloro- } \\
\text { methane }\end{array}$ & $\begin{array}{c}\text { Carbon } \\
\text { tetra- } \\
\text { chloride }\end{array}$ & $\begin{array}{l}\text { 1,2-Di- } \\
\text { chloro- } \\
\text { ethane }\end{array}$ & $\begin{array}{c}\text { Bromform } \\
\text { (Tribromo- } \\
\text { methane) }\end{array}$ & $\begin{array}{l}\text { Dibromo- } \\
\text { chloro- } \\
\text { methane }\end{array}$ & $\begin{array}{c}\text { Chloroform } \\
\text { (Trichloro- } \\
\text { methane) }\end{array}$ \\
\hline $10 / 02 / 1999 *$ & 10/07/1999* & NA & NA & NA & NA & NA & NA & NA \\
\hline 10/13/1999 & 10/21/1999 & 88.1 & 71.6 & 115 & 94.9 & 97.3 & 80.7 & 88.4 \\
\hline 10/26/1999 & 10/28/1999 & 94.3 & 104 & 103 & 97.3 & 112 & 106 & 98.5 \\
\hline 12/13/1999 & $12 / 16 / 1999$ & 102 & 102 & 102 & 101 & 105 & 101 & 101 \\
\hline 12/25/1999, 01/06/2000 & 01/13/2000 & 97.5 & 100 & 104 & 101 & 99.7 & 101 & 98.0 \\
\hline $01 / 18 / 2000$ & $01 / 24 / 2000$ & 100 & 101 & 80 & 84.6 & 93.3 & 102 & 89.0 \\
\hline $01 / 30 / 2000$ & $02 / 03 / 2000$ & 95.1 & 103 & 108 & 103 & 108 & 126 & 99.9 \\
\hline 05/05/2000, 05/17/2000 & 05/18/2000 & 96.9 & 111 & 105 & 103 & 114 & 111 & 109 \\
\hline 05/29/2000, 06/10/2000 & $06 / 12 / 2000$ & 102 & 105 & 110 & 104 & 104 & 105 & 107 \\
\hline 06/29/2000, 07/11/2000 & $07 / 17 / 2000$ & 89.5 & 97.6 & 78.8 & 97.8 & 85.2 & 86.6 & 79.7 \\
\hline 07/23/2000, 08/04/2000 & 08/07/2000 & 99.6 & 94.9 & 89.3 & 86.8 & 95.3 & 96.8 & 93.5 \\
\hline $\begin{array}{l}\text { 08/16/2000, 08/28/2000, } \\
\text { 09/09/2000 }\end{array}$ & 09/19/2000 & 96.1 & 105 & 112 & 99.7 & 119 & 123 & 95.6 \\
\hline 09/21/2000, 10/03/2000 & $10 / 12 / 2000$ & 85.4 & 86.1 & 93.2 & 84.2 & 82.3 & 89.0 & 85.3 \\
\hline $11 / 08 / 2000$ & $11 / 16 / 2000$ & 95.0 & 100 & 103 & 93.6 & 109 & 99.8 & 92.4 \\
\hline 04/01/2001, 04/13/2001 & $04 / 24 / 2001$ & 88.6 & 98.9 & 104 & 91.5 & 113 & 102 & 87.7 \\
\hline 04/25/2001, 05/07/2001 & 05/10/2001 & 96.8 & 101 & 99.8 & 96.3 & 97.5 & 104 & 94.9 \\
\hline 05/19/2001, 05/31/2001 & 06/07/2001 & 100 & 94.3 & 96.4 & 96.0 & 93.0 & 97.2 & 103 \\
\hline 06/12/2001, 06/24/2001 & $06 / 28 / 2001$ & 85.2 & 94.0 & 92.2 & 90.6 & 91.9 & 101 & 86.6 \\
\hline 07/05/2001 & 07/24/2001 & 95.4 & 107 & 96.1 & 106 & 95.4 & 101 & 93.5 \\
\hline 07/16/2001 & 08/09/2001 & 89.0 & 98.7 & 95.6 & 94.0 & 113 & 103 & 89.4 \\
\hline 08/08/2001 & $08 / 21 / 2001$ & 89.4 & 101 & 75.5 & 85.8 & 91.6 & 104 & 71.5 \\
\hline 08/23/2001 & $09 / 12 / 2001$ & 90.5 & 96.4 & 94.0 & 99.3 & 91.7 & 115 & 76.9 \\
\hline 09/16/2001, 09/28/2001 & $10 / 10 / 2001$ & 92.2 & 99.6 & 105 & 96.7 & 98.6 & 104 & 94.5 \\
\hline
\end{tabular}


Table 17B. Quality-control analytical results for cartridge spike for volatile organic compounds (VOC) with high breakthrough volumes for the Sweetwater Reservoir air sampling site, San Diego County, California—Continued.

[The site identification number is 324141117001601 . Values are given in percent recovery. Sample volume for quality-assurance spike is 5.0 liters. Samples processed at 20.0 degrees Celsius and 760 torr; many samples were frozen at the laboratory and analyzed on the same day. Data for travel blanks and environmental samples collected on 10/27/2000 were not used because samples were contaminated during transport. mm/dd/yyyy, month/day/year; *, sample data lost; NA, not analyzed]

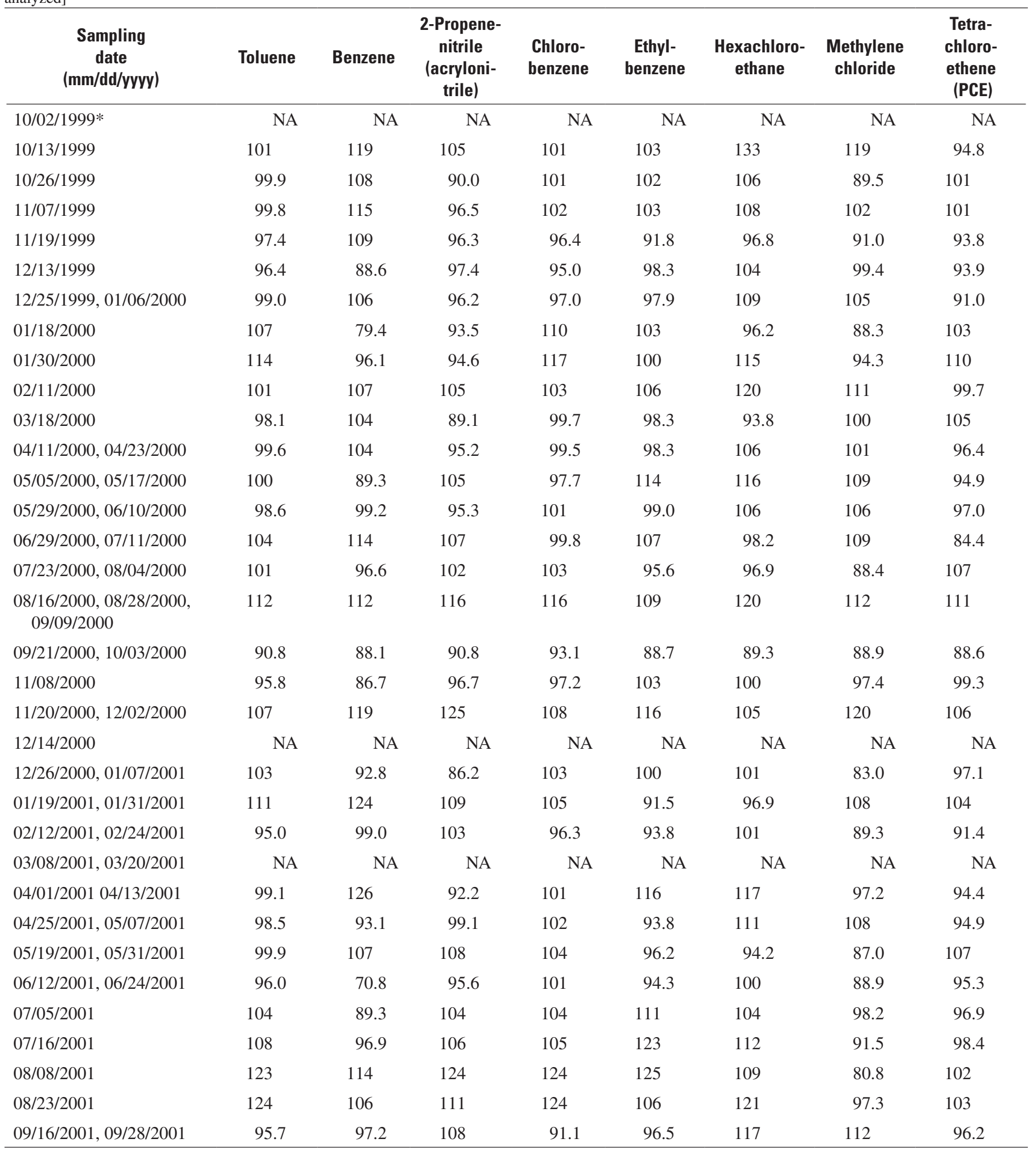


Table 17B. Quality-control analytical results for cartridge spike for volatile organic compounds (VOC) with high breakthrough volumes for the Sweetwater Reservoir air sampling site, San Diego County, California-Continued.

[The site identification number is 324141117001601 . Values are given in percent recovery. Sample volume for quality-assurance spike is 5.0 liters. Samples processed at 20.0 degrees Celsius and 760 torr; many samples were frozen at the laboratory and analyzed on the same day. Data for travel blanks and environmental samples collected on 10/27/2000 were not used because samples were contaminated during transport. mm/dd/yyyy, month/day/year; *, sample data lost; NA, not analyzed]

\begin{tabular}{|c|c|c|c|c|c|c|c|c|}
\hline $\begin{array}{c}\text { Sampling } \\
\text { date } \\
\text { (mm/dd/yyyy) }\end{array}$ & $\begin{array}{l}\text { 1,1-Di- } \\
\text { chloro- } \\
\text { ethane }\end{array}$ & $\begin{array}{l}\text { 1,1-Di- } \\
\text { chloro- } \\
\text { ethene }\end{array}$ & $\begin{array}{l}\text { 1,1,1-Tri- } \\
\text { chloro- } \\
\text { ethane }\end{array}$ & $\begin{array}{l}\text { 1,1,2-Tri- } \\
\text { chloro- } \\
\text { ethane }\end{array}$ & $\begin{array}{l}\text { 1,1,2,2-Tetra- } \\
\text { chloro- } \\
\text { ethane }\end{array}$ & $\begin{array}{c}\text { 1,2-Di- } \\
\text { chloro- } \\
\text { benzene }\end{array}$ & $\begin{array}{l}\text { 1,2-Dichloro- } \\
\text { propane }\end{array}$ & $\begin{array}{c}\text { trans-1,2- } \\
\text { Dichloro- } \\
\text { ethene }\end{array}$ \\
\hline $10 / 02 / 1999 *$ & NA & NA & NA & NA & NA & NA & NA & NA \\
\hline $10 / 13 / 1999$ & 90.7 & 105 & 111 & 85 & 92.8 & 110 & 84.5 & 116 \\
\hline $10 / 26 / 1999$ & 91.5 & 105 & 101 & 101 & 109 & 102 & 97.4 & 101 \\
\hline $12 / 13 / 1999$ & 98.8 & 98.4 & 98.3 & 97.5 & 94.1 & 94.3 & 98.5 & 98.3 \\
\hline $12 / 25 / 1999,01 / 06 / 2000$ & 99.3 & 94.7 & 98.2 & 99.1 & 82.2 & 94.8 & 100 & 99.8 \\
\hline $01 / 18 / 2000$ & 92.7 & 98.4 & 83.6 & 101 & 87.6 & 99.7 & 102 & 94.7 \\
\hline $01 / 30 / 2000$ & 96.2 & 99.7 & 106 & 102 & 106 & 101 & 92.4 & 100 \\
\hline $04 / 11 / 2000,04 / 23 / 2000$ & 82.2 & 81.0 & 104 & 100 & 105 & 96.6 & 101 & 95.8 \\
\hline 05/05/2000, 05/17/2000 & 112 & 104 & 103 & 101 & 112 & 110 & 100 & 102 \\
\hline 05/29/2000, 06/10/2000 & 101 & 109 & 103 & 102 & 108 & 96.3 & 102 & 105 \\
\hline 06/29/2000, 07/11/2000 & 91.9 & 103 & 82.5 & 95.5 & 103 & 88.7 & 121 & 101 \\
\hline 07/23/2000, 08/04/2000 & 90.5 & 94.9 & 89.0 & 98.5 & 87.4 & 97.4 & 99.4 & 93.6 \\
\hline $\begin{array}{l}\text { 08/16/2000, 08/28/2000, } \\
\text { 09/09/2000 }\end{array}$ & 97.8 & 99.5 & 102 & 112 & 97.0 & 108 & 105 & 101 \\
\hline 09/21/2000, 10/03/2000 & 91.7 & 89.4 & 87.8 & 88.7 & 79.5 & 90.3 & 90.5 & 86.9 \\
\hline $11 / 08 / 2000$ & 97.0 & 95.3 & 95.1 & 97.0 & 107 & 108 & 97.3 & 94.9 \\
\hline 04/01/2001, 04/13/2001 & 90.1 & 89.6 & 98.5 & 96.1 & 107 & 97.9 & 95.4 & 91.0 \\
\hline 04/25/2001, 05/07/2001 & 98.8 & 100 & 99.0 & 98.8 & 101 & 99.4 & 98.3 & 95.7 \\
\hline 05/19/2001, 05/31/2001 & 100 & 107 & 96.9 & 97.0 & 94.2 & 102 & 96.6 & 99.5 \\
\hline 06/12/2001, 06/24/2001 & 87.2 & 90.1 & 90.0 & 92.0 & 89.7 & 106 & 93.2 & 91.1 \\
\hline 07/05/2001 & 102 & 103 & 102 & 99.5 & 110 & 107 & 104 & 101 \\
\hline 07/16/2001 & 91.6 & 104 & 93.9 & 102 & 126 & 110 & 101 & 98.8 \\
\hline 08/08/2001 & 83.5 & 109 & 83.1 & 101 & 96.3 & 107 & 115 & 105 \\
\hline 08/23/2001 & 75.2 & 91.3 & 95.1 & 104 & 93.1 & 105 & 113 & 101 \\
\hline 09/16/2001, 09/28/2001 & 93.3 & 98.0 & 99.1 & 100 & 99.4 & 108 & 99.3 & 95.8 \\
\hline
\end{tabular}


Table 17B. Quality-control analytical results for cartridge spike for volatile organic compounds (VOC) with high breakthrough volumes for the Sweetwater Reservoir air sampling site, San Diego County, California—Continued.

[The site identification number is 324141117001601 . Values are given in percent recovery. Sample volume for quality-assurance spike is 5.0 liters. Samples processed at 20.0 degrees Celsius and 760 torr; many samples were frozen at the laboratory and analyzed on the same day. Data for travel blanks and environmental samples collected on 10/27/2000 were not used because samples were contaminated during transport. mm/dd/yyyy, month/day/year; *, sample data lost; NA, not analyzed]

\begin{tabular}{|c|c|c|c|c|c|c|c|c|}
\hline $\begin{array}{c}\text { Sampling } \\
\text { date } \\
\text { (mm/dd/yyyy) }\end{array}$ & $\begin{array}{c}\text { 1,2,4-Tri- } \\
\text { chloro- } \\
\text { benzene }\end{array}$ & $\begin{array}{c}\text { 1,3-Di- } \\
\text { chloro- } \\
\text { benzene }\end{array}$ & $\begin{array}{l}\text { 1,4-Di- } \\
\text { chloro- } \\
\text { benzene }\end{array}$ & $\begin{array}{l}\text { Naph- } \\
\text { thalene }\end{array}$ & $\begin{array}{c}\text { trans-1,3- } \\
\text { Dichloro- } \\
\text { propene }\end{array}$ & $\begin{array}{c}\text { cis-1,3-Di- } \\
\text { chloro- } \\
\text { propene }\end{array}$ & $\begin{array}{c}\text { Trichloro- } \\
\text { ethene } \\
\text { (TCE) }\end{array}$ & $\begin{array}{c}\text { Hexa- } \\
\text { chloro- } \\
\text { butadiene }\end{array}$ \\
\hline 10/13/1999 & 117 & 109 & 110 & 117 & 79.0 & 77.2 & 103 & 109 \\
\hline 10/26/1999 & 109 & 99.9 & 103 & 104 & 94.3 & 98.4 & 97.4 & 108 \\
\hline 12/13/1999 & 96.5 & 94.7 & 95.4 & 95.7 & 100 & 99.6 & 101 & 95.5 \\
\hline $12 / 25 / 1999,01 / 06 / 2000$ & 96.8 & 96.7 & 95.5 & 98.5 & 103 & 101 & 104 & 95.2 \\
\hline $01 / 18 / 2000$ & 101 & 102 & 101 & 103 & 99.2 & 99.9 & 107 & 97.7 \\
\hline $01 / 30 / 2000$ & 110 & 97.3 & 98.3 & 103 & 115 & 104 & 92.4 & 105 \\
\hline $04 / 11 / 2000,04 / 23 / 2000$ & 99.4 & 97.3 & 98.4 & 99.3 & 112 & 104 & 95.1 & 103 \\
\hline 05/05/2000, 05/17/2000 & 113 & 113 & 115 & 109 & 105 & 105 & 91.1 & 114 \\
\hline 05/29/2000, 06/10/2000 & 96.1 & 97.9 & 97.9 & 95.2 & 110 & 106 & 93.2 & 94.3 \\
\hline 06/29/2000, 07/11/2000 & 88.8 & 93.3 & 94.2 & 90.6 & 114 & 113 & 84.9 & 87.4 \\
\hline 07/23/2000, 08/04/2000 & 102 & 101 & 102 & 102 & 100 & 99.0 & 106 & 102 \\
\hline $\begin{array}{l}\text { 08/16/2000, 08/28/2000, } \\
\text { 09/09/2000 }\end{array}$ & 108 & 109 & 110 & 110 & 115 & 116 & 101 & 109 \\
\hline 09/21/2000, 10/03/2000 & 95.4 & 93.1 & 91.4 & 100 & 93.1 & 92.8 & 89.2 & 93.6 \\
\hline $11 / 08 / 2000$ & 110 & 105 & 109 & 111 & 99.3 & 98.4 & 99.3 & 101 \\
\hline 03/08/2001, 03/20/2001 & NA & NA & NA & NA & NA & NA & NA & NA \\
\hline 04/01/2001, 04/13/2001 & 107 & 99.3 & 101 & 130 & 102 & 98.7 & 94.9 & 111 \\
\hline 04/25/2001, 05/07/2001 & 109 & 91.8 & 92.3 & 101 & 104 & 102 & 93.8 & 109 \\
\hline 05/19/2001, 05/31/2001 & 125 & 104 & 104 & 128 & 94.8 & 96.5 & 102 & 105 \\
\hline 06/12/2001, 06/24/2001 & 112 & 111 & 111 & 124 & 110 & 100 & 90.0 & 103 \\
\hline 07/05/2001 & 101 & 110 & 105 & 115 & 112 & 112 & 92.8 & 98.4 \\
\hline $07 / 16 / 2001$ & 116 & 114 & 113 & 117 & 106 & 106 & 96.0 & 112 \\
\hline 08/08/2001 & 114 & 116 & 121 & 121 & 131 & 124 & 102 & 107 \\
\hline 08/23/2001 & 124 & 112 & 113 & 129 & 119 & 123 & 99.1 & 105 \\
\hline 09/16/2001, 09/28/2001 & 117 & 104 & 105 & 117 & 102 & 97.0 & 91.5 & 118 \\
\hline
\end{tabular}


Table 17B. Quality-control analytical results for cartridge spike for volatile organic compounds (VOC) with high breakthrough volumes for the Sweetwater Reservoir air sampling site, San Diego County, California—Continued.

[The site identification number is 324141117001601 . Values are given in percent recovery. Sample volume for quality-assurance spike is 5.0 liters. Samples processed at 20.0 degrees Celsius and 760 torr; many samples were frozen at the laboratory and analyzed on the same day. Data for travel blanks and environmental samples collected on 10/27/2000 were not used because samples were contaminated during transport. mm/dd/yyyy, month/day/year; *, sample data lost; NA, not analyzed]

\begin{tabular}{|c|c|c|c|c|c|c|c|c|}
\hline $\begin{array}{c}\text { Sampling } \\
\text { date } \\
\text { (mm/dd/yyyy) }\end{array}$ & $\begin{array}{l}\text { Methyl } \\
\text { meth- } \\
\text { acrylate }\end{array}$ & $\begin{array}{c}\text { 1,2,3,4- } \\
\text { Tetra- } \\
\text { methyl- } \\
\text { benzene }\end{array}$ & $\begin{array}{l}\text { Ethyl tert- } \\
\text { butyl ether } \\
\text { (ETBE) }\end{array}$ & $\begin{array}{c}\text { tert-Amyl } \\
\text { methyl ether } \\
\text { (TAME) }\end{array}$ & $\begin{array}{c}\text { trans-1,4-Di- } \\
\text { chloro-2- } \\
\text { butene }\end{array}$ & $\begin{array}{c}\text { Ethyl } \\
\text { meth- } \\
\text { acrylate }\end{array}$ & $\begin{array}{l}\text { Carbon } \\
\text { disulfide }\end{array}$ & $\begin{array}{c}\text { cis-1,2- } \\
\text { Dichloro- } \\
\text { ethene }\end{array}$ \\
\hline $10 / 02 / 1999 *$ & NA & NA & NA & NA & NA & NA & NA & NA \\
\hline 10/13/1999 & 84.7 & 116 & 84.5 & 81.7 & 90.4 & 88.8 & 102 & 110 \\
\hline 11/19/1999 & 116 & 97.2 & 99.8 & 103 & 116 & 119 & 95.4 & 97.4 \\
\hline 12/13/1999 & 89.4 & 97.2 & 93.9 & 92.4 & 104 & 87.7 & 90.2 & 98.3 \\
\hline $12 / 25 / 1999,01 / 06 / 2000$ & 65.9 & 101 & 94.1 & 92.3 & 95.4 & 71.7 & 111 & 100 \\
\hline $01 / 18 / 2000$ & 114 & 106 & 101 & 109 & 79.9 & 119 & 93.5 & 93.9 \\
\hline $04 / 11 / 2000,04 / 23 / 2000$ & 109 & 100 & 105 & 107 & 119 & 88.6 & 92.1 & 99 \\
\hline 05/05/2000, 05/17/2000 & 126 & 104 & 84.5 & 81.6 & 94.4 & 123 & 109 & 103 \\
\hline 05/29/2000, 06/10/2000 & 114 & 99.9 & 99.5 & 102 & 109 & 117 & 117 & 102 \\
\hline 06/29/2000, 07/11/2000 & 112 & 99.7 & 90.4 & 78.4 & 111 & 120 & 92.2 & 99.7 \\
\hline 07/23/2000, 08/04/2000 & 101 & 99.9 & 92.2 & 89.4 & 113 & 106 & 99.4 & 93.4 \\
\hline $\begin{array}{l}\text { 08/16/2000, 08/28/2000, } \\
\text { 09/09/2000 }\end{array}$ & 83.3 & 116 & 89.0 & 86.6 & 90.7 & 80.4 & 118 & 106 \\
\hline 09/21/2000, 10/03/2000 & 114 & 103 & 98.5 & 98.1 & 98.8 & 113 & 93.1 & 93.1 \\
\hline 03/08/2001, 03/20/2001 & NA & NA & NA & NA & NA & NA & NA & NA \\
\hline 04/01/2001, 04/13/2001 & 98.0 & 114 & 99.8 & 101 & 122 & 98.1 & 95.4 & 93.4 \\
\hline 04/25/2001, 05/07/2001 & 99.5 & 105 & 97.9 & 97.2 & 97.5 & 105 & 99.7 & 98.2 \\
\hline 05/19/2001, 05/31/2001 & 98.3 & 135 & 101 & 99.1 & 83.8 & 103 & 99.9 & 99.0 \\
\hline $06 / 12 / 2001,06 / 24 / 2001$ & 112 & 111 & 103 & 103 & 115 & 124 & 81.9 & 91.5 \\
\hline 07/05/2001 & 133 & 122 & 124 & 113 & 113 & 134 & 101 & 101 \\
\hline $07 / 16 / 2001$ & 124 & 122 & 117 & 118 & 127 & 130 & 97.9 & 101 \\
\hline 08/08/2001 & 109 & 112 & 127 & 122 & 126 & 108 & 91.2 & 102 \\
\hline 08/23/2001 & 125 & 119 & 92.9 & 87.1 & 108 & 122 & 95.4 & 97.8 \\
\hline 09/16/2001, 09/28/2001 & 101 & 118 & 93.0 & 75.8 & 108 & 101 & 108 & 97.6 \\
\hline
\end{tabular}


Table 17B. Quality-control analytical results for cartridge spike for volatile organic compounds (VOC) with high breakthrough volumes for the Sweetwater Reservoir air sampling site, San Diego County, California—Continued.

[The site identification number is 324141117001601 . Values are given in percent recovery. Sample volume for quality-assurance spike is 5.0 liters. Samples processed at 20.0 degrees Celsius and 760 torr; many samples were frozen at the laboratory and analyzed on the same day. Data for travel blanks and environmental samples collected on 10/27/2000 were not used because samples were contaminated during transport. mm/dd/yyyy, month/day/year; *, sample data lost; NA, not analyzed]

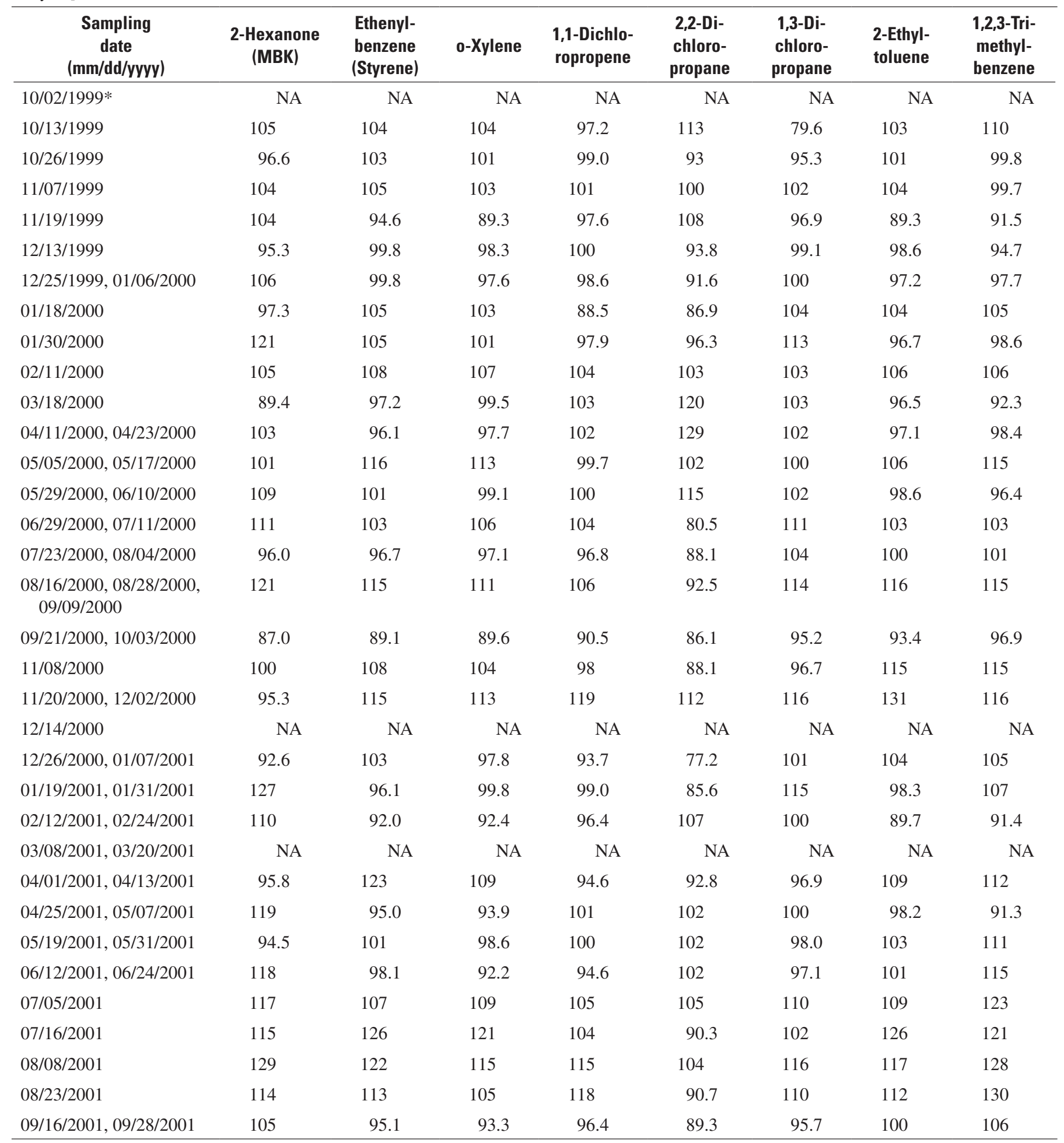


Table 17B. Quality-control analytical results for cartridge spike for volatile organic compounds (VOC) with high breakthrough volumes for the Sweetwater Reservoir air sampling site, San Diego County, California—Continued.

[The site identification number is 324141117001601 . Values are given in percent recovery. Sample volume for quality-assurance spike is 5.0 liters. Samples processed at 20.0 degrees Celsius and 760 torr; many samples were frozen at the laboratory and analyzed on the same day. Data for travel blanks and environmental samples collected on 10/27/2000 were not used because samples were contaminated during transport. mm/dd/yyyy, month/day/year; *, sample data lost; NA, not analyzed]

\begin{tabular}{|c|c|c|c|c|c|c|c|c|}
\hline $\begin{array}{c}\text { Sampling } \\
\text { date } \\
\text { (mm/dd/yyyy) }\end{array}$ & $\begin{array}{l}\text { 1,2,4-Tri- } \\
\text { methyl- } \\
\text { benzene }\end{array}$ & $\begin{array}{l}\text { Isopropyl- } \\
\text { benzene } \\
\text { (Cumene) }\end{array}$ & $\begin{array}{l}n \text {-Propyl- } \\
\text { benzene }\end{array}$ & $\begin{array}{l}\text { 1,3,5-Tri- } \\
\text { methyl- } \\
\text { benzene }\end{array}$ & $\begin{array}{l}\text { 1-Chloro- } \\
\text { 2-methyl- } \\
\text { benzene }\end{array}$ & $\begin{array}{c}\text { 1-Chloro-4- } \\
\text { methyl- } \\
\text { benzene }\end{array}$ & $\begin{array}{l}\text { Bromo- } \\
\text { chloro- } \\
\text { methane }\end{array}$ & $\begin{array}{l}n \text {-Butyl- } \\
\text { benzene }\end{array}$ \\
\hline $10 / 13 / 1999$ & 105 & 102 & 102 & 103 & 103 & 104 & 117 & 111 \\
\hline $10 / 26 / 1999$ & 104 & 103 & 103 & 103 & 101 & 102 & 86.0 & 99.3 \\
\hline $12 / 13 / 1999$ & 99.2 & 98.1 & 101 & 98.3 & 97.9 & 99.4 & 96.6 & 94.2 \\
\hline $12 / 25 / 1999,01 / 06 / 2000$ & 97.8 & 96.5 & 98.7 & 96.5 & 95.9 & 95.7 & 105 & 98.0 \\
\hline $01 / 18 / 2000$ & 105 & 103 & 105 & 105 & 102 & 107 & 104 & 104 \\
\hline $01 / 30 / 2000$ & 89.1 & 98.5 & 99.2 & 100 & 97.9 & 99.1 & 87.8 & 102 \\
\hline $04 / 11 / 2000,04 / 23 / 2000$ & 98.2 & 101 & 97.3 & 98.1 & 96.4 & 97.4 & 83.7 & 98.6 \\
\hline 05/05/2000, 05/17/2000 & 108 & 109 & 111 & 112 & 107 & 106 & 106 & 115 \\
\hline 05/29/2000, 06/10/2000 & 98.2 & 99.6 & 98.2 & 99.9 & 100 & 101 & 99.0 & 98.6 \\
\hline 06/29/2000, 07/11/2000 & 101 & 101 & 110 & 101 & 108 & 109 & 121 & 108 \\
\hline 07/23/2000, 08/04/2000 & 98.5 & 96.8 & 98.2 & 99.6 & 97.5 & 99.4 & 110 & 103 \\
\hline $\begin{array}{l}\text { 08/16/2000, 08/28/2000, } \\
\text { 09/09/2000 }\end{array}$ & 121 & 115 & 115 & 120 & 110 & 114 & 111 & 112 \\
\hline $09 / 21 / 2000,10 / 03 / 2000$ & 93.4 & 92.3 & 91.2 & 92 & 88.5 & 90.9 & 96.0 & 97.0 \\
\hline $11 / 08 / 2000$ & 116 & 107 & 115 & 116 & 104 & 110 & 98.4 & 113 \\
\hline 03/08/2001, 03/20/2001 & NA & NA & NA & NA & NA & NA & NA & NA \\
\hline 04/01/2001, 04/13/2001 & 124 & 116 & 113 & 115 & 113 & 121 & 78.1 & 115 \\
\hline 04/25/2001, 05/07/2001 & 95.8 & 98.6 & 98.1 & 96.3 & 94.7 & 95.3 & 96.9 & 100 \\
\hline 05/19/2001, 05/31/2001 & 107 & 99.9 & 102 & 103 & 100 & 101 & 125 & 122 \\
\hline $06 / 12 / 2001,06 / 24 / 2001$ & 103 & 101 & 101 & 103 & 98.9 & 101 & 92.9 & 115 \\
\hline 07/05/2001 & 111 & 108 & 110 & 108 & 108 & 108 & 105 & 124 \\
\hline $07 / 16 / 2001$ & 122 & 120 & 129 & 123 & 116 & 120 & 98.8 & 118 \\
\hline 08/08/2001 & 124 & 119 & 127 & 118 & 121 & 124 & 97.7 & 118 \\
\hline $08 / 23 / 2001$ & 119 & 108 & 109 & 117 & 110 & 112 & 90.2 & 119 \\
\hline 09/16/2001, 09/28/2001 & 99.3 & 96.7 & 97.4 & 99.2 & 98.9 & 99.6 & 97.7 & 111 \\
\hline
\end{tabular}


Table 17B. Quality-control analytical results for cartridge spike for volatile organic compounds (VOC) with high breakthrough volumes for the Sweetwater Reservoir air sampling site, San Diego County, California—Continued.

[The site identification number is 324141117001601 . Values are given in percent recovery. Sample volume for quality-assurance spike is 5.0 liters. Samples processed at 20.0 degrees Celsius and 760 torr; many samples were frozen at the laboratory and analyzed on the same day. Data for travel blanks and environmental samples collected on 10/27/2000 were not used because samples were contaminated during transport. mm/dd/yyyy, month/day/year; *, sample data lost; NA, not analyzed]

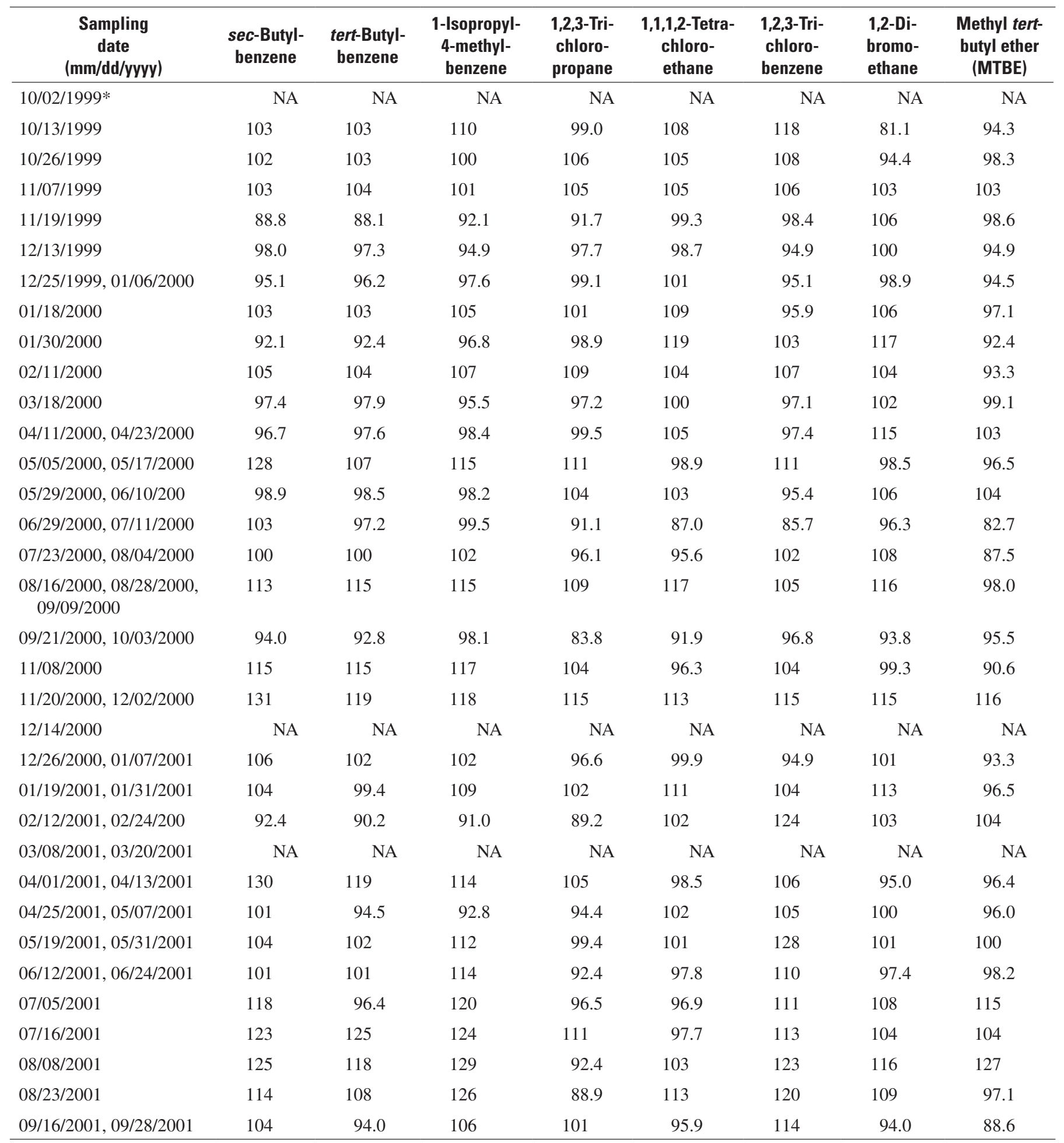


Table 17B. Quality-control analytical results for cartridge spike for volatile organic compounds (VOC) with high breakthrough volumes for the Sweetwater Reservoir air sampling site, San Diego County, California—Continued.

[The site identification number is 324141117001601 . Values are given in percent recovery. Sample volume for quality-assurance spike is 5.0 liters. Samples processed at 20.0 degrees Celsius and 760 torr; many samples were frozen at the laboratory and analyzed on the same day. Data for travel blanks and environmental samples collected on 10/27/2000 were not used because samples were contaminated during transport. mm/dd/yyyy, month/day/year; *, sample data lost; NA, not analyzed]

\begin{tabular}{|c|c|c|c|c|c|c|c|c|c|}
\hline $\begin{array}{c}\text { Sampling } \\
\text { date } \\
\text { (mm/dd/yyyy) }\end{array}$ & $\begin{array}{l}\text { 3-Chloro-1- } \\
\text { propene }\end{array}$ & $\begin{array}{c}\text { 4-Methyl-2- } \\
\text { pentanone } \\
\text { (MIBK) }\end{array}$ & Acetone & $\begin{array}{l}\text { Bromo- } \\
\text { benzene }\end{array}$ & $\begin{array}{l}\text { Diethyl } \\
\text { ether }\end{array}$ & $\begin{array}{l}\text { Diiso- } \\
\text { propyl } \\
\text { ether } \\
\text { (DIPE) }\end{array}$ & $\begin{array}{c}\text { Methyl } \\
\text { acrylo- } \\
\text { nitrile }\end{array}$ & $\begin{array}{c}\text { 2-Butanone } \\
\text { (methyl } \\
\text { ethyl } \\
\text { ketone) }\end{array}$ & $\begin{array}{l}\text { Methyl } \\
\text { acrylate }\end{array}$ \\
\hline $10 / 02 / 1999 *$ & NA & NA & NA & NA & NA & NA & NA & NA & NA \\
\hline $10 / 13 / 1999$ & 99.1 & 101 & 139 & 102 & 97.3 & 96.5 & 111 & 90.9 & 93.9 \\
\hline 10/26/1999 & 77.1 & 98.1 & 76.4 & 101 & 102 & 102 & 102 & 96.2 & 105 \\
\hline 11/19/1999 & 93.5 & 101 & 110 & 88.9 & 98.1 & 99.0 & 94.4 & 107 & 117 \\
\hline $12 / 13 / 1999$ & 88.0 & 95.8 & 98.6 & 94.6 & 102 & 97.3 & 101 & 97.0 & 92.8 \\
\hline $12 / 25 / 1999,01 / 06 / 2000$ & 94.2 & 104 & 103 & 100 & 102 & 99.8 & 104 & 102 & 68.1 \\
\hline $01 / 18 / 2000$ & 101 & 103 & 91.4 & 102 & 97.7 & 92.2 & 98.3 & 86.9 & 109 \\
\hline $04 / 11 / 2000,04 / 23 / 2000$ & 69.8 & 101 & 82.4 & 96.2 & 99.7 & 99.7 & 101 & 108 & 117 \\
\hline 05/05/2000, 05/17/2000 & 117 & 102 & 110 & 105 & 106 & 102 & 102 & 114 & 137 \\
\hline 05/29/2000, 06/10/200 & 108 & 104 & 114 & 99 & 107 & 100 & 101 & 111 & 114 \\
\hline 06/29/2000, 07/11/2000 & 118 & 113 & 123 & 118 & 110 & 119 & 118 & 101 & 104 \\
\hline $07 / 23 / 2000,08 / 04 / 2000$ & 94.9 & 96.2 & 97.0 & 96.2 & 97.5 & 94.8 & 95.3 & 101 & 102 \\
\hline $\begin{array}{l}08 / 16 / 2000,08 / 28 / 2000 \\
09 / 09 / 2000\end{array}$ & 90.6 & 117 & 89.3 & 107 & 107 & 105 & 107 & 121 & 72.2 \\
\hline 09/21/2000, 10/03/2000 & 102 & 86.4 & 89.0 & 85.4 & 92.8 & 92.1 & 90.9 & 90.0 & 106 \\
\hline 03/08/2001, 03/20/2001 & NA & NA & NA & NA & NA & NA & NA & NA & NA \\
\hline 04/01/2001, 04/13/2001 & 56.6 & 116 & 69.8 & 104 & 94.0 & 99.4 & 95.7 & 101 & 96.1 \\
\hline 04/25/2001, 05/07/2001 & 91.4 & 110 & 92.0 & 90.8 & 100 & 103 & 98.3 & 99.6 & 96.3 \\
\hline 05/19/2001, 05/31/2001 & 125 & 97.1 & 121 & 99.3 & 107 & 98.3 & 96.4 & 102 & 103 \\
\hline $06 / 12 / 2001,06 / 24 / 2001$ & 110 & 110 & 77.5 & 95.5 & 94.4 & 102 & 102 & 100 & 105 \\
\hline 07/05/2001 & 128 & 126 & 101 & 110 & 111 & 115 & 119 & 113 & 124 \\
\hline $07 / 16 / 2001$ & 104 & 116 & 107 & 116 & 102 & 110 & 110 & 107 & 107 \\
\hline 08/08/2001 & 126 & 131 & 106 & 120 & 124 & 127 & 129 & 125 & 134 \\
\hline $08 / 23 / 2001$ & 123 & 116 & 101 & 110 & 106 & 124 & 123 & 97.9 & 116 \\
\hline 09/16/2001, 09/28/2001 & 88.8 & 107 & 86.2 & 97.9 & 94.0 & 96.9 & 101 & 96.6 & 102 \\
\hline
\end{tabular}


Table 17B. Quality-control analytical results for cartridge spike for volatile organic compounds (VOC) with high breakthrough volumes for the Sweetwater Reservoir air sampling site, San Diego County, California—Continued.

[The site identification number is 324141117001601 . Values are given in percent recovery. Sample volume for quality-assurance spike is 5.0 liters. Samples processed at 20.0 degrees Celsius and 760 torr; many samples were frozen at the laboratory and analyzed on the same day. Data for travel blanks and environmental samples collected on 10/27/2000 were not used because samples were contaminated during transport. mm/dd/yyyy, month/day/year; *, sample data lost; NA, not analyzed]

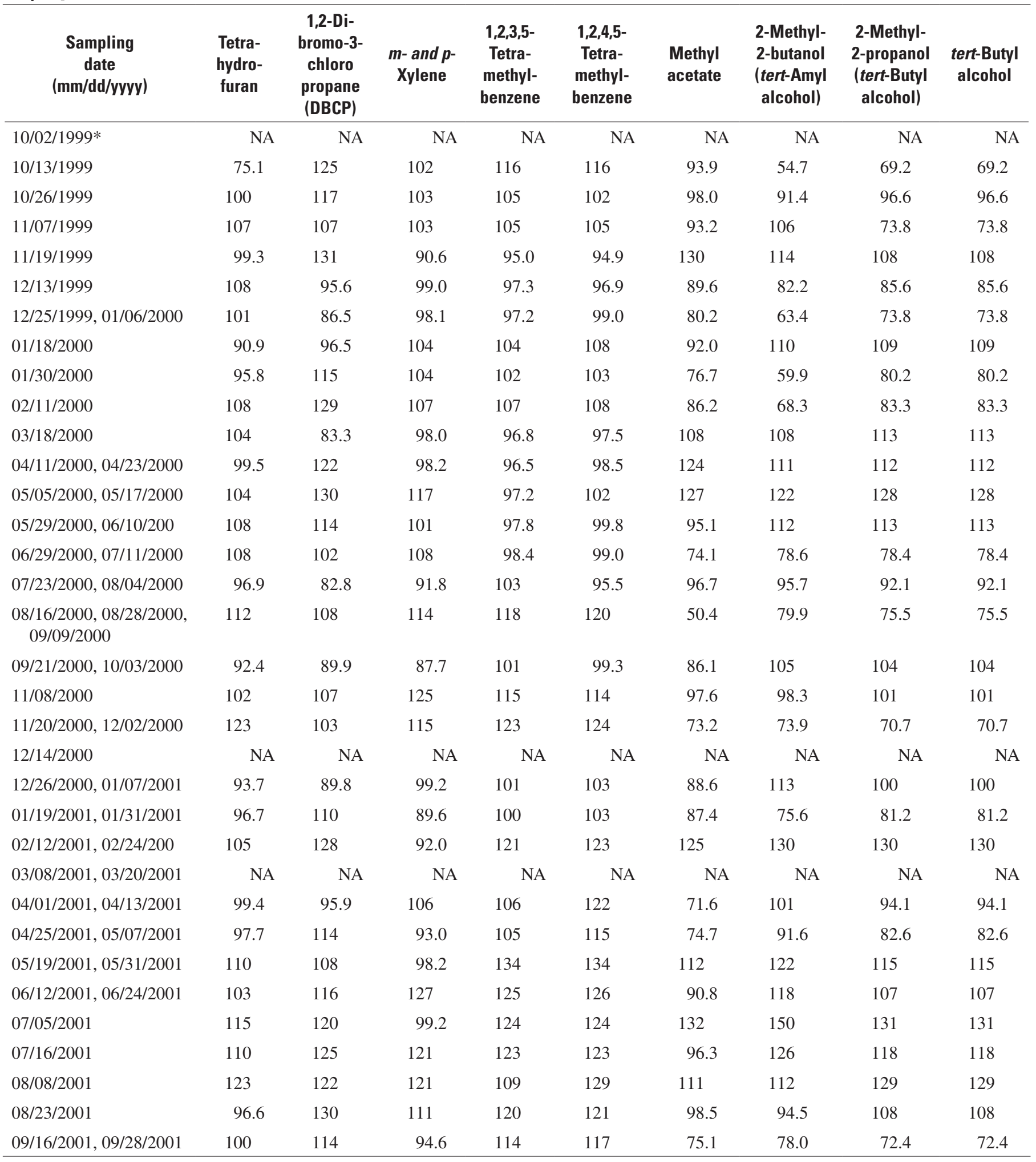


Table 17C. Quality-control analytical cartridge lot blank results for volatile organic compounds (VOC) with high breakthrough volumes for the Sweetwater Reservoir air sampling site, San Diego County, California.

[The site identification number is 324141117001601. Concentrations are given in parts per billion by volume (ppbv). Sample volume for quality-assurance blank is 5.0 liters. Samples processed at 20.0 degrees Celsius and 760 torr; many samples were frozen at the laboratory and analyzed on the same day. Data for travel blanks and environmental samples collected on 10/27/2000 were not used because samples were contaminated during transport. mm/dd/yyyy, month/day/year; E, estimated value; NA, not analyzed; - , compound was not detected at a concentration above laboratory reporting level]

\begin{tabular}{|c|c|c|c|c|c|c|c|c|}
\hline $\begin{array}{c}\text { Sampling } \\
\text { date } \\
\text { (mm/dd/yyyy) }\end{array}$ & $\begin{array}{c}\text { Blank analysis } \\
\text { date } \\
\text { (mm/dd/yyyy) }\end{array}$ & $\begin{array}{l}\text { Dibromo } \\
\text { methane }\end{array}$ & $\begin{array}{l}\text { Bromo- } \\
\text { dichloro- } \\
\text { methane }\end{array}$ & $\begin{array}{l}\text { Carbon } \\
\text { tetra- } \\
\text { chloride }\end{array}$ & $\begin{array}{l}\text { 1,2-Di- } \\
\text { chloro- } \\
\text { ethane }\end{array}$ & $\begin{array}{l}\text { Bromoform } \\
\text { (Tribromo- } \\
\text { methane) }\end{array}$ & $\begin{array}{c}\text { Dibromo } \\
\text { chloro- } \\
\text { methane }\end{array}$ & $\begin{array}{c}\text { Chloroform } \\
\text { (Trichloro- } \\
\text { methane) }\end{array}$ \\
\hline $10 / 02 / 1999$ & $10 / 07 / 1999$ & - & - & - & - & - & - & - \\
\hline $10 / 13 / 1999$ & 10/21/1999 & - & - & - & - & - & - & - \\
\hline $10 / 26 / 1999$ & $10 / 28 / 1999$ & - & - & - & - & - & - & - \\
\hline 11/07/1999 & $11 / 15 / 1999$ & - & - & - & - & - & - & - \\
\hline 11/19/1999 & $11 / 29 / 1999$ & - & - & - & - & - & - & - \\
\hline $12 / 13 / 1999$ & $12 / 16 / 1999$ & - & - & - & - & - & - & - \\
\hline $12 / 25 / 1999$ & $01 / 13 / 2000$ & - & - & - & - & - & - & - \\
\hline $01 / 06 / 2000$ & $01 / 13 / 2000$ & - & - & - & - & - & - & - \\
\hline 01/18/2000 & $01 / 24 / 2000$ & - & - & - & - & - & - & - \\
\hline 01/30/2000 & $02 / 03 / 2000$ & - & - & - & - & - & - & - \\
\hline $02 / 11 / 2000$ & $02 / 17 / 2000$ & - & - & - & - & - & - & - \\
\hline $03 / 18 / 2000$ & $03 / 23 / 2000$ & - & - & - & - & - & - & - \\
\hline $04 / 11 / 2000$ & $04 / 25 / 2000$ & - & - & - & - & - & - & - \\
\hline $04 / 23 / 2000$ & $04 / 25 / 2000$ & - & - & - & - & - & - & - \\
\hline 05/05/2000 & $05 / 18 / 2000$ & - & - & - & - & - & - & - \\
\hline $05 / 17 / 2000$ & $05 / 18 / 2000$ & - & - & - & - & - & - & - \\
\hline $05 / 29 / 2000$ & $06 / 12 / 2000$ & - & - & - & - & - & - & - \\
\hline $06 / 10 / 2000$ & $06 / 12 / 2000$ & - & - & - & - & - & - & - \\
\hline $06 / 29 / 2000$ & $07 / 17 / 2000$ & - & - & - & - & - & - & - \\
\hline $07 / 11 / 2000$ & $07 / 17 / 2000$ & - & - & - & - & - & - & - \\
\hline $07 / 23 / 2000$ & 08/07/2000 & NA & NA & NA & NA & NA & NA & NA \\
\hline 08/04/2000 & 08/07/2000 & - & - & - & - & - & - & - \\
\hline $08 / 16 / 2000$ & $09 / 19 / 2000$ & - & - & - & - & - & - & - \\
\hline $08 / 28 / 2000$ & $09 / 19 / 2000$ & - & - & - & - & - & - & - \\
\hline 09/09/2000 & 09/19/2000 & - & - & - & - & - & - & - \\
\hline $09 / 21 / 2000$ & $10 / 12 / 2000$ & - & - & - & - & - & - & - \\
\hline $10 / 03 / 2000$ & $10 / 12 / 2000$ & - & - & - & - & - & - & - \\
\hline $11 / 08 / 2000$ & $11 / 16 / 2000$ & - & - & - & - & - & - & - \\
\hline $11 / 20 / 2000$ & $12 / 07 / 2000$ & - & - & - & - & - & - & - \\
\hline $12 / 02 / 2000$ & $12 / 07 / 2000$ & - & - & - & - & - & - & - \\
\hline $12 / 14 / 2000$ & $12 / 20 / 2000$ & - & - & - & - & - & - & - \\
\hline $12 / 26 / 2000$ & $01 / 12 / 2001$ & - & - & - & - & - & - & - \\
\hline
\end{tabular}


Table 17C. Quality-control analytical cartridge lot blank results for volatile organic compounds (VOC) with high breakthrough volumes for the Sweetwater Reservoir air sampling site, San Diego County, California-Continued.

[The site identification number is 324141117001601 . Concentrations are given in parts per billion by volume (ppbv). Sample volume for quality-assurance blank is 5.0 liters. Samples processed at 20.0 degrees Celsius and 760 torr; many samples were frozen at the laboratory and analyzed on the same day. Data for travel blanks and environmental samples collected on 10/27/2000 were not used because samples were contaminated during transport. mm/dd/yyyy, month/day/year; E, estimated value; NA, not analyzed; - , compound was not detected at a concentration above laboratory reporting level]

\begin{tabular}{ccccccccc}
\hline $\begin{array}{c}\text { Sampling } \\
\text { date } \\
\text { (mm/dd/yyyy) }\end{array}$ & $\begin{array}{c}\text { Blank analysis } \\
\text { date } \\
\text { (mm/dd/yyyy) }\end{array}$ & $\begin{array}{c}\text { Dibromo } \\
\text { methane }\end{array}$ & $\begin{array}{c}\text { Bromo- } \\
\text { dichloro- } \\
\text { methane }\end{array}$ & $\begin{array}{c}\text { Carbon } \\
\text { tetra- } \\
\text { chloride }\end{array}$ & $\begin{array}{c}\mathbf{1 , 2 - D i -} \\
\text { chloro- } \\
\text { ethane }\end{array}$ & $\begin{array}{c}\text { Bromoform } \\
\text { (Tribromo- } \\
\text { methane) }\end{array}$ & $\begin{array}{c}\text { Dibromo } \\
\text { chloro- } \\
\text { methane }\end{array}$ & $\begin{array}{c}\text { Chloroform } \\
\text { (Trichloro- } \\
\text { methane) }\end{array}$ \\
\hline $01 / 07 / 2001$ & $01 / 12 / 2001$ & - & - & - & - & - & - & - \\
$01 / 19 / 2001$ & $02 / 06 / 2001$ & - & - & - & - & - & - & - \\
$01 / 31 / 2001$ & $02 / 06 / 2001$ & - & - & - & - & - & - & - \\
$02 / 12 / 2001$ & $03 / 05 / 2001$ & - & - & - & - & - & - & - \\
$02 / 24 / 2001$ & $03 / 05 / 2001$ & - & - & - & - & - & - & - \\
$03 / 08 / 2001$ & $03 / 26 / 2001$ & - & - & - & - & - & - & - \\
$03 / 20 / 2001$ & $03 / 26 / 2001$ & NA & NA & NA & NA & NA & NA & NA \\
$04 / 01 / 2001$ & $04 / 24 / 2001$ & - & - & - & - & - & - & - \\
$04 / 13 / 2001$ & $04 / 24 / 2001$ & - & - & - & - & - & - & - \\
$04 / 25 / 2001$ & $05 / 10 / 2001$ & - & - & - & - & - & - & - \\
$05 / 07 / 2001$ & $05 / 10 / 2001$ & - & - & - & - & - & - & - \\
$05 / 19 / 2001$ & $06 / 07 / 2001$ & - & - & - & - & - & - & - \\
$05 / 31 / 2001$ & $06 / 07 / 2001$ & - & - & - & - & - & - & - \\
$06 / 12 / 2001$ & $06 / 28 / 2001$ & - & - & - & - & - & - & - \\
$06 / 24 / 2001$ & $06 / 28 / 2001$ & - & - & - & - & - & - & - \\
$07 / 05 / 2001$ & $07 / 24 / 2001$ & - & - & - & - & - & - & - \\
$07 / 16 / 2001$ & $08 / 09 / 2001$ & - & - & - & - & - & - \\
$08 / 08 / 2001$ & $08 / 21 / 2001$ & - & - & - & - & - & - \\
$08 / 23 / 2001$ & $09 / 12 / 2001$ & - & - & - & - & - & - \\
$09 / 16 / 2001$ & $10 / 10 / 2001$ & - & - & - & - & - & - \\
$09 / 28 / 2001$ & $10 / 10 / 2001$ & - & - & - & - & - & - \\
\hline
\end{tabular}


Table 17C. Quality-control analytical cartridge lot blank results for volatile organic compounds (VOC) with high breakthrough volumes for the Sweetwater Reservoir air sampling site, San Diego County, California-Continued.

[The site identification number is 324141117001601 . Concentrations are given in parts per billion by volume (ppbv). Sample volume for quality-assurance blank is 5.0 liters. Samples processed at 20.0 degrees Celsius and 760 torr; many samples were frozen at the laboratory and analyzed on the same day. Data for travel blanks and environmental samples collected on 10/27/2000 were not used because samples were contaminated during transport. mm/dd/yyyy, month/day/year; E, estimated value; NA, not analyzed; - , compound was not detected at a concentration above laboratory reporting level]

\begin{tabular}{|c|c|c|c|c|c|c|c|c|}
\hline $\begin{array}{l}\text { Sampling } \\
\text { date } \\
\text { (mm/dd/yyyy) }\end{array}$ & Toluene & Benzene & $\begin{array}{l}\text { 2-Propen- } \\
\text { enitrile } \\
\text { (Acryloni- } \\
\text { trile) }\end{array}$ & $\begin{array}{l}\text { Chloro- } \\
\text { benzene }\end{array}$ & $\begin{array}{c}\text { Ethyl- } \\
\text { benzene }\end{array}$ & $\begin{array}{l}\text { Hexa- } \\
\text { chloro- } \\
\text { ethane }\end{array}$ & $\begin{array}{l}\text { Methylene } \\
\text { chloride }\end{array}$ & $\begin{array}{l}\text { Tetrachlo- } \\
\text { roethene } \\
\text { (PCE) }\end{array}$ \\
\hline $10 / 02 / 1999$ & E0.001 & E0.083 & - & - & - & - & - & - \\
\hline 10/13/1999 & E0.004 & E0.058 & E0.022 & - & E0.001 & - & - & - \\
\hline 10/26/1999 & E0.018 & E0.057 & - & - & E0.002 & - & E0.0109 & - \\
\hline 11/07/1999 & E0.007 & 0.120 & - & - & E0.001 & - & - & - \\
\hline 11/19/1999 & E0.004 & E0.052 & E0.011 & - & E0.001 & - & E0.003 & - \\
\hline $12 / 13 / 1999$ & E0.003 & 0.150 & - & - & - & - & - & - \\
\hline $12 / 25 / 1999$ & E0.005 & E0.084 & - & - & - & - & E0.004 & - \\
\hline $01 / 06 / 2000$ & E0.001 & E0.029 & E0.008 & - & - & - & - & - \\
\hline $01 / 18 / 2000$ & E0.002 & E0.066 & - & - & - & - & - & - \\
\hline $01 / 30 / 2000$ & E0.004 & E0.030 & - & - & E0.001 & - & - & - \\
\hline $02 / 11 / 2000$ & E0.004 & E0.058 & - & - & - & - & - & - \\
\hline 03/18/2000 & E0.002 & E0.056 & E0.001 & - & - & - & - & - \\
\hline $04 / 11 / 2000$ & E0.002 & E0.027 & - & - & - & - & - & - \\
\hline $04 / 23 / 2000$ & E0.001 & E0.032 & - & - & - & - & - & - \\
\hline 05/05/2000 & E0.001 & E0.024 & - & - & - & - & - & - \\
\hline 05/17/2000 & - & E0.040 & - & - & - & - & - & - \\
\hline 05/29/2000 & E0.001 & E0.051 & - & - & - & - & - & - \\
\hline $06 / 10 / 2000$ & E0.003 & E0.044 & - & - & - & - & - & - \\
\hline 06/29/2000 & - & E0.062 & - & - & - & - & - & - \\
\hline $07 / 11 / 2000$ & E0.003 & E0.066 & - & - & - & - & - & - \\
\hline $07 / 23 / 2000$ & NA & NA & NA & NA & NA & NA & NA & NA \\
\hline 08/04/2000 & E0.001 & E0.044 & - & - & - & - & - & - \\
\hline 08/16/2000 & E0.003 & E0.034 & - & - & - & - & - & - \\
\hline $08 / 28 / 2000$ & E0.002 & 0.127 & - & - & - & - & - & - \\
\hline 09/09/2000 & E0.003 & E0.034 & - & - & - & - & - & - \\
\hline 09/21/2000 & E0.002 & E0.039 & - & - & - & - & - & - \\
\hline $10 / 03 / 2000$ & E0.003 & E0.100 & - & - & - & - & - & - \\
\hline $11 / 08 / 2000$ & E0.006 & E0.068 & - & E0.001 & E0.001 & - & - & - \\
\hline $11 / 20 / 2000$ & E0.014 & 0.338 & - & E0.001 & E0.002 & - & - & - \\
\hline $12 / 02 / 2000$ & E0.002 & E0.037 & - & - & - & - & - & - \\
\hline $12 / 14 / 2000$ & E0.044 & 0.132 & E0.016 & E0.001 & E0.008 & - & - & - \\
\hline $12 / 26 / 2000$ & E0.008 & E0.099 & - & - & E0.001 & - & - & - \\
\hline
\end{tabular}


Table 17C. Quality-control analytical cartridge lot blank results for volatile organic compounds (VOC) with high breakthrough volumes for the Sweetwater Reservoir air sampling site, San Diego County, California-Continued.

[The site identification number is 324141117001601 . Concentrations are given in parts per billion by volume (ppbv). Sample volume for quality-assurance blank is 5.0 liters. Samples processed at 20.0 degrees Celsius and 760 torr; many samples were frozen at the laboratory and analyzed on the same day. Data for travel blanks and environmental samples collected on 10/27/2000 were not used because samples were contaminated during transport. mm/dd/yyyy, month/day/year; E, estimated value; NA, not analyzed; - , compound was not detected at a concentration above laboratory reporting level]

\begin{tabular}{|c|c|c|c|c|c|c|c|c|}
\hline $\begin{array}{l}\text { Sampling } \\
\text { date } \\
\text { (mm/dd/yyyy) }\end{array}$ & Toluene & Benzene & $\begin{array}{c}\text { 2-Propen- } \\
\text { enitrile } \\
\text { (Acryloni- } \\
\text { trile) }\end{array}$ & $\begin{array}{l}\text { Chloro- } \\
\text { benzene }\end{array}$ & $\begin{array}{c}\text { Ethyl- } \\
\text { benzene }\end{array}$ & $\begin{array}{l}\text { Hexa- } \\
\text { chloro- } \\
\text { ethane }\end{array}$ & $\begin{array}{c}\text { Methylene } \\
\text { chloride }\end{array}$ & $\begin{array}{l}\text { Tetrachlo- } \\
\text { roethene } \\
\text { (PCE) }\end{array}$ \\
\hline 01/07/2001 & E0.015 & 0.142 & - & E0.001 & E0.002 & - & - & - \\
\hline 01/19/2001 & E0.009 & 0.151 & - & - & E0.002 & - & - & - \\
\hline $01 / 31 / 2001$ & E0.009 & 0.144 & - & - & - & - & - & - \\
\hline $02 / 12 / 2001$ & E0.007 & E0.098 & - & - & - & - & - & - \\
\hline $02 / 24 / 2001$ & E0.006 & E0.088 & - & - & E0.001 & - & - & - \\
\hline 03/08/2001 & E0.010 & 0.114 & - & - & E0.001 & - & - & - \\
\hline 03/20/2001 & NA & NA & NA & NA & NA & NA & NA & NA \\
\hline $04 / 01 / 2001$ & E0.003 & E0.025 & - & - & - & - & - & - \\
\hline $04 / 13 / 2001$ & E0.004 & E0.080 & - & E0.001 & E0.001 & - & - & - \\
\hline $04 / 25 / 2001$ & E0.015 & 0.169 & E0.013 & - & E0.003 & - & - & - \\
\hline 05/07/2001 & E0.002 & E0.043 & - & - & - & - & - & - \\
\hline 05/19/2001 & E0.004 & E0.076 & - & - & - & - & - & E0.009 \\
\hline $05 / 31 / 2001$ & E0.003 & 0.226 & - & - & - & - & - & - \\
\hline $06 / 12 / 2001$ & E0.003 & E0.002 & - & - & - & - & - & - \\
\hline $06 / 24 / 2001$ & E0.015 & E0.058 & - & E0.006 & E0.002 & - & - & - \\
\hline $07 / 05 / 2001$ & E0.003 & 0.209 & - & - & - & - & - & - \\
\hline $07 / 16 / 2001$ & E0.002 & E0.044 & - & - & - & - & - & - \\
\hline 08/08/2001 & 0.004 & 0.098 & - & - & - & - & - & - \\
\hline $08 / 23 / 2001$ & E0.002 & E0.107 & - & - & - & - & - & - \\
\hline 09/16/2001 & E0.005 & E0.068 & - & - & E0.001 & - & - & - \\
\hline $09 / 28 / 2001$ & - & - & - & - & - & - & - & - \\
\hline
\end{tabular}


Table 17C. Quality-control analytical cartridge lot blank results for volatile organic compounds (VOC) with high breakthrough volumes for the Sweetwater Reservoir air sampling site, San Diego County, California-Continued.

[The site identification number is 324141117001601 . Concentrations are given in parts per billion by volume (ppbv). Sample volume for quality-assurance blank is 5.0 liters. Samples processed at 20.0 degrees Celsius and 760 torr; many samples were frozen at the laboratory and analyzed on the same day. Data for travel blanks and environmental samples collected on 10/27/2000 were not used because samples were contaminated during transport. mm/dd/yyyy, month/day/year; E, estimated value; NA, not analyzed; - , compound was not detected at a concentration above laboratory reporting level]

\begin{tabular}{|c|c|c|c|c|c|c|c|c|}
\hline $\begin{array}{c}\text { Sampling } \\
\text { date } \\
\text { (mm/dd/yyyy) }\end{array}$ & $\begin{array}{c}1,1- \\
\text { Dichloro- } \\
\text { ethane }\end{array}$ & $\begin{array}{c}1,1- \\
\text { Dichloro- } \\
\text { ethene }\end{array}$ & $\begin{array}{c}1,1,1- \\
\text { Trichloro- } \\
\text { ethane }\end{array}$ & $\begin{array}{c}1,1,2- \\
\text { Trichloro- } \\
\text { ethane }\end{array}$ & $\begin{array}{l}\text { 1,1,2,2- } \\
\text { Tetra- } \\
\text { chloro- } \\
\text { ethane }\end{array}$ & $\begin{array}{c}1,2- \\
\text { Dichloro- } \\
\text { benzene }\end{array}$ & $\begin{array}{c}1,2- \\
\text { Dichloro- } \\
\text { propane }\end{array}$ & $\begin{array}{c}\text { trans-1,2- } \\
\text { Dichloro- } \\
\text { ethene }\end{array}$ \\
\hline $10 / 02 / 1999$ & - & - & - & - & - & - & - & - \\
\hline $10 / 13 / 1999$ & - & - & - & - & - & - & - & - \\
\hline 10/26/1999 & - & - & - & - & - & - & - & - \\
\hline $11 / 07 / 1999$ & - & - & - & - & - & - & - & - \\
\hline 11/19/1999 & - & - & - & - & - & - & - & - \\
\hline $12 / 13 / 1999$ & - & - & - & - & - & - & - & - \\
\hline $12 / 25 / 1999$ & - & - & - & - & - & - & - & - \\
\hline $01 / 06 / 2000$ & - & - & - & - & - & - & - & - \\
\hline $01 / 18 / 2000$ & - & - & - & - & - & - & - & - \\
\hline $01 / 30 / 2000$ & - & - & - & - & - & - & - & - \\
\hline $02 / 11 / 2000$ & - & - & - & - & - & - & - & - \\
\hline 03/18/2000 & - & - & - & - & - & - & - & - \\
\hline $04 / 11 / 2000$ & - & - & - & - & - & - & - & - \\
\hline $04 / 23 / 2000$ & - & - & - & - & - & - & - & - \\
\hline 05/05/2000 & - & - & - & - & - & - & - & - \\
\hline 05/17/2000 & - & - & - & - & - & - & - & - \\
\hline 05/29/2000 & - & - & - & - & - & - & - & - \\
\hline $06 / 10 / 2000$ & - & - & - & - & - & - & - & - \\
\hline 06/29/2000 & - & - & - & - & - & - & - & - \\
\hline $07 / 11 / 2000$ & - & - & - & - & - & - & - & - \\
\hline $07 / 23 / 2000$ & NA & NA & NA & NA & NA & NA & NA & NA \\
\hline 08/04/2000 & - & - & - & - & - & - & - & - \\
\hline 08/16/2000 & - & - & - & - & - & - & - & - \\
\hline $08 / 28 / 2000$ & - & - & - & - & - & - & - & - \\
\hline 09/09/2000 & - & - & - & - & - & - & - & - \\
\hline $09 / 21 / 2000$ & - & - & - & - & - & - & - & - \\
\hline $10 / 03 / 2000$ & - & - & - & - & - & - & - & - \\
\hline $11 / 08 / 2000$ & - & - & - & - & - & - & - & - \\
\hline $11 / 20 / 2000$ & - & - & - & - & - & - & - & - \\
\hline $12 / 02 / 2000$ & - & - & - & - & - & - & - & - \\
\hline $12 / 14 / 2000$ & - & - & - & - & - & - & - & - \\
\hline $12 / 26 / 2000$ & - & - & - & - & - & - & - & - \\
\hline
\end{tabular}


Table 17C. Quality-control analytical cartridge lot blank results for volatile organic compounds (VOC) with high breakthrough volumes for the Sweetwater Reservoir air sampling site, San Diego County, California-Continued.

[The site identification number is 324141117001601 . Concentrations are given in parts per billion by volume (ppbv). Sample volume for quality-assurance blank is 5.0 liters. Samples processed at 20.0 degrees Celsius and 760 torr; many samples were frozen at the laboratory and analyzed on the same day. Data for travel blanks and environmental samples collected on 10/27/2000 were not used because samples were contaminated during transport. mm/dd/yyyy, month/day/year; E, estimated value; NA, not analyzed; - , compound was not detected at a concentration above laboratory reporting level]

\begin{tabular}{|c|c|c|c|c|c|c|c|c|}
\hline $\begin{array}{l}\text { Sampling } \\
\text { date } \\
\text { (mm/dd/yyyy) }\end{array}$ & $\begin{array}{c}1,1- \\
\text { Dichloro- } \\
\text { ethane }\end{array}$ & $\begin{array}{c}1,1- \\
\text { Dichloro- } \\
\text { ethene }\end{array}$ & $\begin{array}{c}1,1,1- \\
\text { Trichloro- } \\
\text { ethane }\end{array}$ & $\begin{array}{c}1,1,2- \\
\text { Trichloro- } \\
\text { ethane }\end{array}$ & $\begin{array}{l}1,1,2,2- \\
\text { Tetra- } \\
\text { chloro- } \\
\text { ethane }\end{array}$ & $\begin{array}{c}1,2- \\
\text { Dichloro- } \\
\text { benzene }\end{array}$ & $\begin{array}{c}1,2- \\
\text { Dichloro- } \\
\text { propane }\end{array}$ & $\begin{array}{l}\text { trans-1,2- } \\
\text { Dichloro- } \\
\text { ethene }\end{array}$ \\
\hline 01/07/2001 & - & - & - & - & - & - & - & - \\
\hline 01/19/2001 & - & - & - & - & - & - & - & - \\
\hline $01 / 31 / 2001$ & - & - & - & - & - & - & - & - \\
\hline $02 / 12 / 2001$ & - & - & - & - & - & - & - & - \\
\hline $02 / 24 / 2001$ & - & - & - & - & - & - & - & - \\
\hline 03/08/2001 & - & - & - & - & - & - & - & - \\
\hline 03/20/2001 & NA & NA & NA & NA & NA & NA & NA & NA \\
\hline $04 / 01 / 2001$ & - & - & - & - & - & - & - & - \\
\hline 04/13/2001 & - & - & - & - & - & - & - & - \\
\hline $04 / 25 / 2001$ & - & - & - & - & - & - & - & - \\
\hline 05/07/2001 & - & - & - & - & - & - & - & - \\
\hline 05/19/2001 & - & - & - & - & - & - & - & - \\
\hline 05/31/2001 & - & - & - & - & - & - & - & - \\
\hline $06 / 12 / 2001$ & - & - & - & - & - & - & - & - \\
\hline $06 / 24 / 2001$ & - & - & - & - & - & - & - & - \\
\hline 07/05/2001 & - & - & - & - & - & - & - & - \\
\hline 07/16/2001 & - & - & - & - & - & - & - & - \\
\hline 08/08/2001 & - & - & - & - & - & - & - & - \\
\hline 08/23/2001 & - & - & - & - & - & - & - & - \\
\hline 09/16/2001 & - & - & - & - & - & - & - & - \\
\hline 09/28/2001 & - & - & - & - & - & - & - & - \\
\hline
\end{tabular}


Table 17C. Quality-control analytical cartridge lot blank results for volatile organic compounds (VOC) with high breakthrough volumes for the Sweetwater Reservoir air sampling site, San Diego County, California-Continued.

[The site identification number is 324141117001601 . Concentrations are given in parts per billion by volume (ppbv). Sample volume for quality-assurance blank is 5.0 liters. Samples processed at 20.0 degrees Celsius and 760 torr; many samples were frozen at the laboratory and analyzed on the same day. Data for travel blanks and environmental samples collected on 10/27/2000 were not used because samples were contaminated during transport. mm/dd/yyyy, month/day/year; E, estimated value; NA, not analyzed; - , compound was not detected at a concentration above laboratory reporting level]

\begin{tabular}{|c|c|c|c|c|c|c|c|c|}
\hline $\begin{array}{c}\text { Sampling } \\
\text { date } \\
\text { (mm/dd/yyyy) }\end{array}$ & $\begin{array}{c}\text { 1,2,4-Tri- } \\
\text { chloro- } \\
\text { benzene }\end{array}$ & $\begin{array}{l}\text { 1,3-Di- } \\
\text { chloro- } \\
\text { benzene }\end{array}$ & $\begin{array}{l}\text { 1,4-Di- } \\
\text { chloro- } \\
\text { benzene }\end{array}$ & $\begin{array}{l}\text { Naph- } \\
\text { thalene }\end{array}$ & $\begin{array}{l}\text { trans-1,3- } \\
\text { Dichloro- } \\
\text { propene }\end{array}$ & $\begin{array}{c}\text { cis-1,3- } \\
\text { Dichloro- } \\
\text { propene }\end{array}$ & $\begin{array}{l}\text { Trichloro- } \\
\text { ethene } \\
\text { (TCE) }\end{array}$ & $\begin{array}{l}\text { Hexa- } \\
\text { chlorobuta- } \\
\text { diene }\end{array}$ \\
\hline 10/02/1999 & - & - & - & - & - & - & - & - \\
\hline 10/13/1999 & - & - & - & E0.001 & - & - & - & - \\
\hline $10 / 26 / 1999$ & - & - & - & E0.001 & - & - & - & - \\
\hline 11/07/1999 & - & - & - & E0.001 & - & - & - & - \\
\hline 11/19/1999 & - & - & - & E0.001 & - & - & - & - \\
\hline 12/13/1999 & - & - & - & - & - & - & - & - \\
\hline 12/25/1999 & - & - & - & - & - & - & - & - \\
\hline 01/06/2000 & - & - & - & - & - & - & - & - \\
\hline 01/18/2000 & - & - & - & - & - & - & - & - \\
\hline 01/30/2000 & - & - & - & - & - & - & - & - \\
\hline $02 / 11 / 2000$ & - & - & - & E0.001 & - & - & - & - \\
\hline 03/18/2000 & - & - & - & E0.001 & - & - & - & - \\
\hline $04 / 11 / 2000$ & - & - & - & - & - & - & - & - \\
\hline $04 / 23 / 2000$ & - & - & - & - & - & - & - & - \\
\hline 05/05/2000 & - & - & - & - & - & - & - & - \\
\hline 05/17/2000 & - & - & - & - & - & - & - & - \\
\hline 05/29/2000 & - & - & - & - & - & - & - & - \\
\hline 06/10/2000 & - & - & - & - & - & - & - & - \\
\hline 06/29/2000 & - & - & - & - & - & - & - & - \\
\hline 07/11/2000 & - & - & - & - & - & - & - & - \\
\hline 07/23/2000 & NA & NA & NA & NA & NA & NA & NA & NA \\
\hline 08/04/2000 & - & - & - & E0.001 & - & - & - & - \\
\hline 08/16/2000 & - & - & - & E0.001 & - & - & - & - \\
\hline 08/28/2000 & - & - & - & E0.001 & - & - & - & - \\
\hline 09/09/2000 & - & - & - & E0.001 & - & - & - & - \\
\hline 09/21/2000 & - & - & - & - & - & - & - & - \\
\hline $10 / 03 / 2000$ & - & - & - & E0.001 & - & - & - & - \\
\hline $11 / 08 / 2000$ & - & - & - & E0.003 & - & - & - & - \\
\hline $11 / 20 / 2000$ & - & - & - & E0.002 & - & - & - & - \\
\hline $12 / 02 / 2000$ & - & - & - & E0.003 & - & - & - & - \\
\hline $12 / 14 / 2000$ & - & - & - & E0.009 & - & - & - & - \\
\hline $12 / 26 / 2000$ & - & - & - & E0.002 & - & - & - & - \\
\hline
\end{tabular}


Table 17C. Quality-control analytical cartridge lot blank results for volatile organic compounds (VOC) with high breakthrough volumes for the Sweetwater Reservoir air sampling site, San Diego County, California—Continued.

[The site identification number is 324141117001601 . Concentrations are given in parts per billion by volume (ppbv). Sample volume for quality-assurance blank is 5.0 liters. Samples processed at 20.0 degrees Celsius and 760 torr; many samples were frozen at the laboratory and analyzed on the same day. Data for travel blanks and environmental samples collected on 10/27/2000 were not used because samples were contaminated during transport. mm/dd/yyyy, month/day/year; E, estimated value; NA, not analyzed; _, compound was not detected at a concentration above laboratory reporting level]

\begin{tabular}{|c|c|c|c|c|c|c|c|c|}
\hline $\begin{array}{c}\text { Sampling } \\
\text { date } \\
\text { (mm/dd/yyyy) }\end{array}$ & $\begin{array}{l}\text { 1,2,4-Tri- } \\
\text { chloro- } \\
\text { benzene }\end{array}$ & $\begin{array}{l}\text { 1,3-Di- } \\
\text { chloro- } \\
\text { benzene }\end{array}$ & $\begin{array}{l}\text { 1,4-Di- } \\
\text { chloro- } \\
\text { benzene }\end{array}$ & $\begin{array}{l}\text { Naph- } \\
\text { thalene }\end{array}$ & $\begin{array}{l}\text { trans-1,3- } \\
\text { Dichloro- } \\
\text { propene }\end{array}$ & $\begin{array}{c}\text { cis-1,3- } \\
\text { Dichloro- } \\
\text { propene }\end{array}$ & $\begin{array}{l}\text { Trichloro- } \\
\text { ethene } \\
\text { (TCE) }\end{array}$ & $\begin{array}{l}\text { Hexa- } \\
\text { chlorobuta- } \\
\text { diene }\end{array}$ \\
\hline 01/07/2001 & - & - & - & E0.001 & - & - & - & - \\
\hline 01/19/2001 & - & - & - & - & - & - & - & - \\
\hline $01 / 31 / 2001$ & - & - & - & - & - & - & - & - \\
\hline $02 / 12 / 2001$ & - & - & - & - & - & - & - & - \\
\hline $02 / 24 / 2001$ & - & - & - & E0.001 & - & - & - & - \\
\hline 03/08/2001 & - & - & - & - & - & - & - & - \\
\hline 03/20/2001 & NA & NA & NA & NA & NA & NA & NA & NA \\
\hline $04 / 01 / 2001$ & - & - & - & E0.001 & - & - & - & - \\
\hline $04 / 13 / 2001$ & - & - & - & E0.001 & - & - & - & - \\
\hline $04 / 25 / 2001$ & - & - & - & E0.004 & - & - & - & - \\
\hline 05/07/2001 & - & - & - & E0.001 & - & - & - & - \\
\hline 05/19/2001 & - & - & - & - & - & - & - & - \\
\hline $05 / 31 / 2001$ & - & - & - & - & - & - & - & - \\
\hline $06 / 12 / 2001$ & - & - & - & - & - & - & - & - \\
\hline $06 / 24 / 2001$ & - & - & - & E0.003 & - & - & - & - \\
\hline 07/05/2001 & - & - & - & E0.002 & - & - & - & - \\
\hline $07 / 16 / 2001$ & - & - & - & - & - & - & - & - \\
\hline 08/08/2001 & - & - & - & - & - & - & - & - \\
\hline $08 / 23 / 2001$ & - & - & - & - & - & - & - & - \\
\hline 09/16/2001 & - & - & - & - & - & - & - & - \\
\hline 09/28/2001 & - & - & - & - & - & - & - & - \\
\hline
\end{tabular}


Table 17C. Quality-control analytical cartridge lot blank results for volatile organic compounds (VOC) with high breakthrough volumes for the Sweetwater Reservoir air sampling site, San Diego County, California-Continued.

[The site identification number is 324141117001601 . Concentrations are given in parts per billion by volume (ppbv). Sample volume for quality-assurance blank is 5.0 liters. Samples processed at 20.0 degrees Celsius and 760 torr; many samples were frozen at the laboratory and analyzed on the same day. Data for travel blanks and environmental samples collected on 10/27/2000 were not used because samples were contaminated during transport. mm/dd/yyyy, month/day/year; E, estimated value; NA, not analyzed; - , compound was not detected at a concentration above laboratory reporting level]

\begin{tabular}{|c|c|c|c|c|c|c|c|c|}
\hline $\begin{array}{c}\text { Sampling } \\
\text { date } \\
\text { (mm/dd/yyyy) }\end{array}$ & $\begin{array}{l}\text { Methyl } \\
\text { metha- } \\
\text { crylate }\end{array}$ & $\begin{array}{c}1,2,3,4- \\
\text { Tetra- } \\
\text { methyl- } \\
\text { benzene }\end{array}$ & $\begin{array}{l}\text { Ethyl tert- } \\
\text { butyl ether } \\
\text { (ETBE) }\end{array}$ & $\begin{array}{l}\text { tert-Amyl } \\
\text { methyl } \\
\text { ether } \\
\text { (TAME) }\end{array}$ & $\begin{array}{l}\text { trans-1,4- } \\
\text { Dichloro-2- } \\
\text { butene }\end{array}$ & $\begin{array}{l}\text { Ethyl } \\
\text { metha- } \\
\text { crylate }\end{array}$ & $\begin{array}{c}\text { Carbon } \\
\text { disulfide }\end{array}$ & $\begin{array}{r}\text { cis-1,2- } \\
\text { Dichloro } \\
\text { ethene }\end{array}$ \\
\hline 10/02/1999 & - & - & - & - & - & - & - & - \\
\hline 10/13/1999 & - & - & - & - & - & - & E0.001 & - \\
\hline 10/26/1999 & - & - & - & - & - & - & - & - \\
\hline 11/07/1999 & - & - & - & - & - & - & - & - \\
\hline 11/19/1999 & - & - & - & - & - & - & - & - \\
\hline 12/13/1999 & - & - & - & - & - & - & - & - \\
\hline $12 / 25 / 1999$ & - & - & - & - & - & - & - & - \\
\hline 01/06/2000 & - & - & - & - & - & - & - & - \\
\hline $01 / 18 / 2000$ & - & - & - & - & - & - & - & - \\
\hline $01 / 30 / 2000$ & - & - & - & - & - & - & - & - \\
\hline $02 / 11 / 2000$ & - & - & - & - & - & - & E0.003 & - \\
\hline 03/18/2000 & - & - & - & - & - & - & - & - \\
\hline $04 / 11 / 2000$ & - & - & - & - & - & - & - & - \\
\hline $04 / 23 / 2000$ & - & - & - & - & - & - & - & - \\
\hline 05/05/2000 & - & - & - & - & - & - & - & - \\
\hline 05/17/2000 & - & - & - & - & - & - & - & - \\
\hline 05/29/2000 & - & - & - & - & - & - & - & - \\
\hline $06 / 10 / 2000$ & - & - & - & - & - & - & - & - \\
\hline 06/29/2000 & - & - & - & - & - & - & - & - \\
\hline $07 / 11 / 2000$ & - & - & - & - & - & - & E0.005 & - \\
\hline 07/23/2000 & NA & NA & NA & NA & NA & NA & NA & NA \\
\hline 08/04/2000 & - & - & - & - & - & - & E0.004 & - \\
\hline 08/16/2000 & - & - & - & - & - & - & - & - \\
\hline 08/28/2000 & - & - & - & - & - & - & - & - \\
\hline 09/09/2000 & - & - & - & - & - & - & - & - \\
\hline $09 / 21 / 2000$ & - & - & - & - & - & - & - & - \\
\hline $10 / 03 / 2000$ & - & - & - & - & - & - & - & - \\
\hline $11 / 08 / 2000$ & - & - & - & - & - & - & - & - \\
\hline $11 / 20 / 2000$ & - & - & - & - & - & - & - & - \\
\hline $12 / 02 / 2000$ & - & - & - & - & - & - & E0.009 & - \\
\hline $12 / 14 / 2000$ & - & - & - & - & - & - & E0.004 & - \\
\hline $12 / 26 / 2000$ & - & - & - & - & - & - & - & - \\
\hline
\end{tabular}


Table 17C. Quality-control analytical cartridge lot blank results for volatile organic compounds (VOC) with high breakthrough volumes for the Sweetwater Reservoir air sampling site, San Diego County, California-Continued.

[The site identification number is 324141117001601 . Concentrations are given in parts per billion by volume (ppbv). Sample volume for quality-assurance blank is 5.0 liters. Samples processed at 20.0 degrees Celsius and 760 torr; many samples were frozen at the laboratory and analyzed on the same day. Data for travel blanks and environmental samples collected on 10/27/2000 were not used because samples were contaminated during transport. mm/dd/yyyy, month/day/year; E, estimated value; NA, not analyzed; - , compound was not detected at a concentration above laboratory reporting level]

\begin{tabular}{|c|c|c|c|c|c|c|c|c|}
\hline $\begin{array}{l}\text { Sampling } \\
\text { date } \\
\text { (mm/dd/yyyy) }\end{array}$ & $\begin{array}{l}\text { Methyl } \\
\text { metha- } \\
\text { crylate }\end{array}$ & $\begin{array}{c}1,2,3,4- \\
\text { Tetra- } \\
\text { methyl- } \\
\text { benzene }\end{array}$ & $\begin{array}{l}\text { Ethyl tert- } \\
\text { butyl ether } \\
\text { (ETBE) }\end{array}$ & $\begin{array}{c}\text { tert-Amyl } \\
\text { methyl } \\
\text { ether } \\
\text { (TAME) }\end{array}$ & $\begin{array}{l}\text { trans-1,4- } \\
\text { Dichloro-2- } \\
\text { butene }\end{array}$ & $\begin{array}{l}\text { Ethyl } \\
\text { metha- } \\
\text { crylate }\end{array}$ & $\begin{array}{l}\text { Carbon } \\
\text { disulfide }\end{array}$ & $\begin{array}{c}\text { cis-1,2- } \\
\text { Dichloro- } \\
\text { ethene }\end{array}$ \\
\hline 01/07/2001 & - & - & - & - & - & - & E0.015 & - \\
\hline 01/19/2001 & - & - & - & - & - & - & - & - \\
\hline $01 / 31 / 2001$ & - & - & - & - & - & - & - & - \\
\hline $02 / 12 / 2001$ & - & - & - & - & - & - & - & - \\
\hline $02 / 24 / 2001$ & - & - & - & - & - & - & - & - \\
\hline 03/08/2001 & - & - & - & - & - & - & E0.002 & - \\
\hline 03/20/2001 & NA & NA & NA & NA & NA & NA & NA & NA \\
\hline 04/01/2001 & - & - & - & - & - & - & - & - \\
\hline $04 / 13 / 2001$ & - & - & - & - & - & - & E0.003 & - \\
\hline $04 / 25 / 2001$ & - & - & - & - & - & - & E0.007 & - \\
\hline 05/07/2001 & - & - & - & - & - & - & - & - \\
\hline 05/19/2001 & - & - & - & - & - & - & - & - \\
\hline $05 / 31 / 2001$ & - & - & - & - & - & - & E0.001 & - \\
\hline 06/12/2001 & - & - & - & - & - & - & - & - \\
\hline $06 / 24 / 2001$ & - & - & - & - & - & - & E0.015 & - \\
\hline 07/05/2001 & - & - & - & - & - & - & E0.005 & - \\
\hline $07 / 16 / 2001$ & - & - & - & - & - & - & E0.001 & - \\
\hline 08/08/2001 & - & - & - & - & - & - & - & - \\
\hline $08 / 23 / 2001$ & - & - & - & - & - & - & - & - \\
\hline 09/16/2001 & - & - & - & - & - & - & E0.013 & - \\
\hline 09/28/2001 & - & - & - & - & - & - & - & - \\
\hline
\end{tabular}


Table 17C. Quality-control analytical cartridge lot blank results for volatile organic compounds (VOC) with high breakthrough volumes for the Sweetwater Reservoir air sampling site, San Diego County, California-Continued.

[The site identification number is 324141117001601 . Concentrations are given in parts per billion by volume (ppbv). Sample volume for quality-assurance blank is 5.0 liters. Samples processed at 20.0 degrees Celsius and 760 torr; many samples were frozen at the laboratory and analyzed on the same day. Data for travel blanks and environmental samples collected on 10/27/2000 were not used because samples were contaminated during transport. mm/dd/yyyy, month/day/year; E, estimated value; NA, not analyzed; - , compound was not detected at a concentration above laboratory reporting level]

\begin{tabular}{|c|c|c|c|c|c|c|c|c|}
\hline $\begin{array}{l}\text { Sampling } \\
\text { date } \\
\text { (mm/dd/yyyy) }\end{array}$ & $\begin{array}{l}\text { 2-Hexanone } \\
\text { (MBK) }\end{array}$ & $\begin{array}{l}\text { Ethenyl- } \\
\text { benzene } \\
\text { (styrene) }\end{array}$ & $\begin{array}{c}o^{-} \\
\text {Xylene }\end{array}$ & $\begin{array}{l}\text { 1,1-Dichloro- } \\
\text { propene }\end{array}$ & $\begin{array}{l}\text { 2,2-Dichloro- } \\
\text { propane }\end{array}$ & $\begin{array}{l}\text { 1,3-Dichloro- } \\
\text { propane }\end{array}$ & $\begin{array}{l}\text { 2-Ethyl- } \\
\text { toluene }\end{array}$ & $\begin{array}{c}1,2,3-\text { Tri- } \\
\text { methyl- } \\
\text { benzene }\end{array}$ \\
\hline 10/02/1999 & - & - & - & - & - & - & - & - \\
\hline 10/13/1999 & - & E0.001 & E0.001 & - & - & - & - & - \\
\hline 10/26/1999 & - & E0.001 & E0.003 & - & - & - & - & - \\
\hline 11/07/1999 & - & E0.001 & E0.001 & - & - & - & - & - \\
\hline 11/19/1999 & - & E0.001 & - & - & - & - & - & - \\
\hline 12/13/1999 & - & - & - & - & - & - & - & - \\
\hline $12 / 25 / 1999$ & - & - & - & - & - & - & - & - \\
\hline 01/06/2000 & - & - & - & - & - & - & - & - \\
\hline $01 / 18 / 2000$ & - & - & - & - & - & - & - & - \\
\hline $01 / 30 / 2000$ & - & - & E0.001 & - & - & - & - & - \\
\hline $02 / 11 / 2000$ & - & - & E0.001 & - & - & - & - & - \\
\hline 03/18/2000 & - & - & - & - & - & - & - & - \\
\hline 04/11/2000 & - & - & - & - & - & - & - & - \\
\hline $04 / 23 / 2000$ & - & - & - & - & - & - & - & - \\
\hline 05/05/2000 & - & - & - & - & - & - & - & - \\
\hline 05/17/2000 & - & - & - & - & - & - & - & - \\
\hline 05/29/2000 & - & - & - & - & - & - & - & - \\
\hline $06 / 10 / 2000$ & - & - & - & - & - & - & - & - \\
\hline 06/29/2000 & - & - & - & - & - & - & - & - \\
\hline 07/11/2000 & - & - & - & - & - & - & - & - \\
\hline $07 / 23 / 2000$ & NA & NA & $\mathrm{NA}$ & NA & NA & NA & NA & NA \\
\hline 08/04/2000 & - & - & - & - & - & - & - & - \\
\hline 08/16/2000 & - & - & - & - & - & - & - & - \\
\hline 08/28/2000 & - & E0.002 & E0.001 & - & - & - & - & - \\
\hline 09/09/2000 & - & - & - & - & - & - & - & - \\
\hline 09/21/2000 & - & - & - & - & - & - & - & - \\
\hline $10 / 03 / 2000$ & - & - & - & - & - & - & - & - \\
\hline $11 / 08 / 2000$ & - & E0.001 & - & - & - & - & - & - \\
\hline $11 / 20 / 2000$ & - & - & - & - & - & - & - & - \\
\hline $12 / 02 / 2000$ & - & E0.001 & E0.002 & - & - & - & - & - \\
\hline $12 / 14 / 2000$ & - & E0.012 & E0.008 & - & - & - & - & - \\
\hline $12 / 26 / 2000$ & - & E0.001 & E0.001 & - & - & - & - & - \\
\hline
\end{tabular}


Table 17C. Quality-control analytical cartridge lot blank results for volatile organic compounds (VOC) with high breakthrough volumes for the Sweetwater Reservoir air sampling site, San Diego County, California—Continued.

[The site identification number is 324141117001601 . Concentrations are given in parts per billion by volume (ppbv). Sample volume for quality-assurance blank is 5.0 liters. Samples processed at 20.0 degrees Celsius and 760 torr; many samples were frozen at the laboratory and analyzed on the same day. Data for travel blanks and environmental samples collected on 10/27/2000 were not used because samples were contaminated during transport. mm/dd/yyyy, month/day/year; E, estimated value; NA, not analyzed; - , compound was not detected at a concentration above laboratory reporting level]

\begin{tabular}{|c|c|c|c|c|c|c|c|c|}
\hline $\begin{array}{c}\text { Sampling } \\
\text { date } \\
\text { (mm/dd/yyyy) }\end{array}$ & $\begin{array}{l}\text { 2-Hexanone } \\
\text { (MBK) }\end{array}$ & $\begin{array}{l}\text { Ethenyl- } \\
\text { benzene } \\
\text { (styrene) }\end{array}$ & $\begin{array}{c}o- \\
\text { Xylene }\end{array}$ & $\begin{array}{l}\text { 1,1-Dichloro- } \\
\text { propene }\end{array}$ & $\begin{array}{l}\text { 2,2-Dichloro- } \\
\text { propane }\end{array}$ & $\begin{array}{l}\text { 1,3-Dichloro- } \\
\text { propane }\end{array}$ & $\begin{array}{l}\text { 2-Ethyl- } \\
\text { toluene }\end{array}$ & $\begin{array}{l}\text { 1,2,3-Tri- } \\
\text { methyl- } \\
\text { benzene }\end{array}$ \\
\hline $01 / 07 / 2001$ & - & - & - & - & - & - & - & - \\
\hline 01/19/2001 & - & - & - & - & - & - & - & - \\
\hline $01 / 31 / 2001$ & - & - & - & - & - & - & - & - \\
\hline $02 / 12 / 2001$ & - & - & - & - & - & - & - & - \\
\hline $02 / 24 / 2001$ & - & - & E0.001 & - & - & - & - & - \\
\hline 03/08/2001 & - & - & E0.001 & - & - & - & - & - \\
\hline 03/20/2001 & NA & NA & NA & NA & NA & NA & NA & NA \\
\hline $04 / 01 / 2001$ & - & - & - & - & - & - & - & - \\
\hline 04/13/2001 & - & E0.001 & - & - & - & - & - & - \\
\hline $04 / 25 / 2001$ & - & E0.018 & E0.001 & - & - & - & - & - \\
\hline 05/07/2001 & - & - & - & - & - & - & - & - \\
\hline 05/19/2001 & - & - & - & - & - & - & - & - \\
\hline $05 / 31 / 2001$ & - & - & - & - & - & - & - & - \\
\hline $06 / 12 / 2001$ & - & - & - & - & - & - & - & - \\
\hline $06 / 24 / 2001$ & - & E0.013 & E0.007 & - & - & - & - & - \\
\hline 07/05/2001 & - & - & - & - & - & - & - & - \\
\hline $07 / 16 / 2001$ & - & - & - & - & - & - & - & - \\
\hline 08/08/2001 & - & - & - & - & - & - & - & - \\
\hline $08 / 23 / 2001$ & - & - & - & - & - & - & - & - \\
\hline 09/16/2001 & - & E0.002 & E0.003 & - & - & - & - & - \\
\hline 09/28/2001 & - & - & - & - & - & - & - & - \\
\hline
\end{tabular}


Table 17C. Quality-control analytical cartridge lot blank results for volatile organic compounds (VOC) with high breakthrough volumes for the Sweetwater Reservoir air sampling site, San Diego County, California-Continued.

[The site identification number is 324141117001601 . Concentrations are given in parts per billion by volume (ppbv). Sample volume for quality-assurance blank is 5.0 liters. Samples processed at 20.0 degrees Celsius and 760 torr; many samples were frozen at the laboratory and analyzed on the same day. Data for travel blanks and environmental samples collected on 10/27/2000 were not used because samples were contaminated during transport. mm/dd/yyyy, month/day/year; E, estimated value; NA, not analyzed; - , compound was not detected at a concentration above laboratory reporting level]

\begin{tabular}{|c|c|c|c|c|c|c|c|c|}
\hline $\begin{array}{c}\text { Sampling } \\
\text { date } \\
\text { (mm/dd/yyyy) }\end{array}$ & $\begin{array}{c}\text { 1,2,4-Tri- } \\
\text { methyl- } \\
\text { benzene }\end{array}$ & $\begin{array}{l}\text { Isopropyl- } \\
\text { benzene } \\
\text { (Cumene) }\end{array}$ & $\begin{array}{l}n \text {-Propyl- } \\
\text { benzene }\end{array}$ & $\begin{array}{c}\text { 1,3,5-Tri- } \\
\text { methyl- } \\
\text { benzene }\end{array}$ & $\begin{array}{c}\text { 1-Chloro-2- } \\
\text { methyl- } \\
\text { benzene }\end{array}$ & $\begin{array}{c}\text { 1-Chloro-4- } \\
\text { methyl- } \\
\text { benzene }\end{array}$ & $\begin{array}{l}\text { Bromo- } \\
\text { chloro- } \\
\text { methane }\end{array}$ & $\begin{array}{l}n \text {-Butyl- } \\
\text { benzene }\end{array}$ \\
\hline 10/02/1999 & - & - & - & - & - & - & - & - \\
\hline 10/13/1999 & - & - & - & - & - & - & - & - \\
\hline 10/26/1999 & - & - & - & - & - & - & - & - \\
\hline 11/07/1999 & - & - & - & - & - & - & - & - \\
\hline 11/19/1999 & - & - & - & - & - & - & - & - \\
\hline $12 / 13 / 1999$ & - & - & - & - & - & - & - & - \\
\hline $12 / 25 / 1999$ & - & - & - & - & - & - & - & - \\
\hline $01 / 06 / 2000$ & - & - & - & - & - & - & - & - \\
\hline $01 / 18 / 2000$ & - & - & - & - & - & - & - & - \\
\hline 01/30/2000 & - & - & - & - & - & - & - & - \\
\hline $02 / 11 / 2000$ & - & - & - & - & - & - & - & - \\
\hline 03/18/2000 & - & - & - & - & - & - & - & - \\
\hline $04 / 11 / 2000$ & - & - & - & - & - & - & - & - \\
\hline $04 / 23 / 2000$ & - & - & - & - & - & - & - & - \\
\hline 05/05/2000 & - & - & - & - & - & - & - & - \\
\hline 05/17/2000 & - & - & - & - & - & - & - & - \\
\hline 05/29/2000 & - & - & - & - & - & - & - & - \\
\hline 06/10/2000 & - & - & - & - & - & - & - & - \\
\hline 06/29/2000 & - & - & - & - & - & - & - & - \\
\hline $07 / 11 / 2000$ & - & - & - & - & - & - & - & - \\
\hline 07/23/2000 & NA & NA & $\mathrm{NA}$ & NA & NA & NA & NA & NA \\
\hline 08/04/2000 & - & - & - & - & - & - & - & - \\
\hline 08/16/2000 & - & - & - & - & - & - & - & - \\
\hline $08 / 28 / 2000$ & - & - & - & - & - & - & - & - \\
\hline 09/09/2000 & - & - & - & - & - & - & - & - \\
\hline $09 / 21 / 2000$ & - & - & - & - & - & - & - & - \\
\hline $10 / 03 / 2000$ & - & - & - & - & - & - & - & - \\
\hline $11 / 08 / 2000$ & - & - & - & - & - & - & - & - \\
\hline $11 / 20 / 2000$ & - & - & - & - & - & - & - & - \\
\hline $12 / 02 / 2000$ & - & - & - & - & - & - & - & - \\
\hline $12 / 14 / 2000$ & - & - & - & - & - & - & - & - \\
\hline $12 / 26 / 2000$ & - & - & - & - & - & - & - & - \\
\hline
\end{tabular}


Table 17C. Quality-control analytical cartridge lot blank results for volatile organic compounds (VOC) with high breakthrough volumes for the Sweetwater Reservoir air sampling site, San Diego County, California—Continued.

[The site identification number is 324141117001601 . Concentrations are given in parts per billion by volume (ppbv). Sample volume for quality-assurance blank is 5.0 liters. Samples processed at 20.0 degrees Celsius and 760 torr; many samples were frozen at the laboratory and analyzed on the same day. Data for travel blanks and environmental samples collected on 10/27/2000 were not used because samples were contaminated during transport. mm/dd/yyyy, month/day/year; E, estimated value; NA, not analyzed; - , compound was not detected at a concentration above laboratory reporting level]

\begin{tabular}{ccccccccc}
\hline $\begin{array}{c}\text { Sampling } \\
\text { date } \\
\text { (mm/dd/yyyy) }\end{array}$ & $\begin{array}{c}\text { 1,2,4-Tri- } \\
\text { methyl- } \\
\text { benzene }\end{array}$ & $\begin{array}{c}\text { Isopropyl- } \\
\text { benzene } \\
\text { (Cumene) }\end{array}$ & $\begin{array}{c}\text { n-Propyl- } \\
\text { benzene }\end{array}$ & $\begin{array}{c}\text { 1,3,5-Tri- } \\
\text { methyl- } \\
\text { benzene }\end{array}$ & $\begin{array}{c}\text { 1-Chloro-2- } \\
\text { methyl- } \\
\text { benzene }\end{array}$ & $\begin{array}{c}\text { 1-Chloro-4- } \\
\text { methyl- } \\
\text { benzene }\end{array}$ & $\begin{array}{c}\text { Bromo- } \\
\text { chloro- } \\
\text { methane }\end{array}$ & $\begin{array}{c}\text { n-Butyl- } \\
\text { benzene }\end{array}$ \\
\hline $01 / 07 / 2001$ & - & - & - & - & - & - & - & - \\
$01 / 19 / 2001$ & - & - & - & - & - & - & - & - \\
$01 / 31 / 2001$ & - & - & - & - & - & - & - & - \\
$02 / 12 / 2001$ & - & - & - & - & - & - & - & - \\
$02 / 24 / 2001$ & - & - & - & - & - & - & - & - \\
$03 / 08 / 2001$ & - & - & - & - & - & - & - & - \\
$03 / 20 / 2001$ & NA & NA & NA & NA & NA & NA & NA & NA \\
$04 / 01 / 2001$ & - & - & - & - & - & - & - & - \\
$04 / 13 / 2001$ & - & - & - & - & - & - & - & - \\
$04 / 25 / 2001$ & - & - & - & - & - & - & - & - \\
$05 / 07 / 2001$ & - & - & - & - & - & - & - & - \\
$05 / 19 / 2001$ & - & - & - & - & - & - & - & - \\
$05 / 31 / 2001$ & - & - & - & - & - & - & - & - \\
$06 / 12 / 2001$ & - & - & - & - & - & - & - & - \\
$06 / 24 / 2001$ & - & - & - & - & - & - & - & - \\
$07 / 05 / 2001$ & - & - & - & - & - & - & - & - \\
$07 / 16 / 2001$ & - & - & - & - & - & - & - & - \\
$08 / 08 / 2001$ & - & - & - & - & - & - & - & - \\
$08 / 23 / 2001$ & - & - & - & - & - & - & - & - \\
$09 / 16 / 2001$ & - & - & - & - & - & - & - & - \\
$09 / 28 / 2001$ & - & - & - & - & - & - & - & - \\
\hline
\end{tabular}


Table 17C. Quality-control analytical cartridge lot blank results for volatile organic compounds (VOC) with high breakthrough volumes for the Sweetwater Reservoir air sampling site, San Diego County, California-Continued.

[The site identification number is 324141117001601 . Concentrations are given in parts per billion by volume (ppbv). Sample volume for quality-assurance blank is 5.0 liters. Samples processed at 20.0 degrees Celsius and 760 torr; many samples were frozen at the laboratory and analyzed on the same day. Data for travel blanks and environmental samples collected on 10/27/2000 were not used because samples were contaminated during transport. mm/dd/yyyy, month/day/year; E, estimated value; NA, not analyzed; - , compound was not detected at a concentration above laboratory reporting level]

\begin{tabular}{|c|c|c|c|c|c|c|c|c|}
\hline $\begin{array}{c}\text { Sampling } \\
\text { date } \\
\text { (mm/dd/yyyy) }\end{array}$ & $\begin{array}{c}\text { sec- } \\
\text { Butyl- } \\
\text { benzene }\end{array}$ & $\begin{array}{c}\text { tert- } \\
\text { Butyl- } \\
\text { benzene }\end{array}$ & $\begin{array}{c}\text { 1-Isopropyl- } \\
\text { 4-methyl- } \\
\text { benzene }\end{array}$ & $\begin{array}{c}1,2,3-\text { Tri- } \\
\text { chloro- } \\
\text { propane }\end{array}$ & $\begin{array}{l}1,1,1,2- \\
\text { Tetra- } \\
\text { chloro- } \\
\text { ethane }\end{array}$ & $\begin{array}{c}\text { 1,2,3-Tri- } \\
\text { chloro- } \\
\text { benzene }\end{array}$ & $\begin{array}{l}\text { 1,2-Di- } \\
\text { bromo- } \\
\text { ethane }\end{array}$ & $\begin{array}{l}\text { Methyl tert- } \\
\text { butyl ether } \\
\text { (MTBE) }\end{array}$ \\
\hline $10 / 02 / 1999$ & - & - & - & - & - & - & - & - \\
\hline $10 / 13 / 1999$ & - & - & - & - & - & - & - & - \\
\hline $10 / 26 / 1999$ & - & - & - & - & - & - & - & - \\
\hline 11/07/1999 & - & - & - & - & - & - & - & - \\
\hline 11/19/1999 & - & - & - & - & - & - & - & - \\
\hline $12 / 13 / 1999$ & - & - & - & - & - & - & - & - \\
\hline $12 / 25 / 1999$ & - & - & - & - & - & - & - & - \\
\hline 01/06/2000 & - & - & - & - & - & - & - & - \\
\hline $01 / 18 / 2000$ & - & - & - & - & - & - & - & - \\
\hline $01 / 30 / 2000$ & - & - & - & - & - & - & - & - \\
\hline $02 / 11 / 2000$ & - & - & - & - & - & - & - & - \\
\hline 03/18/2000 & - & - & - & - & - & - & - & - \\
\hline $04 / 11 / 2000$ & - & - & - & - & - & - & - & - \\
\hline $04 / 23 / 2000$ & - & - & - & - & - & - & - & - \\
\hline 05/05/2000 & - & - & - & - & - & - & - & - \\
\hline $05 / 17 / 2000$ & - & - & - & - & - & - & - & - \\
\hline $05 / 29 / 2000$ & - & - & - & - & - & - & - & - \\
\hline $06 / 10 / 2000$ & - & - & - & - & - & - & - & - \\
\hline 06/29/2000 & - & - & - & - & - & - & - & - \\
\hline $07 / 11 / 2000$ & - & - & - & - & - & - & - & - \\
\hline $07 / 23 / 2000$ & NA & NA & NA & NA & NA & NA & NA & NA \\
\hline $08 / 04 / 2000$ & - & - & - & - & - & - & - & - \\
\hline $08 / 16 / 2000$ & - & - & - & - & - & - & - & - \\
\hline $08 / 28 / 2000$ & - & - & - & - & - & - & - & - \\
\hline 09/09/2000 & - & - & - & - & - & - & - & - \\
\hline 09/21/2000 & - & - & - & - & - & - & - & - \\
\hline $10 / 03 / 2000$ & - & - & - & - & - & - & - & - \\
\hline $11 / 08 / 2000$ & - & - & - & - & - & - & - & - \\
\hline $11 / 20 / 2000$ & - & - & - & - & - & - & - & - \\
\hline $12 / 02 / 2000$ & - & - & - & - & - & - & - & - \\
\hline $12 / 14 / 2000$ & - & - & - & - & - & - & - & - \\
\hline $12 / 26 / 2000$ & - & - & - & - & - & - & - & - \\
\hline
\end{tabular}


Table 17C. Quality-control analytical cartridge lot blank results for volatile organic compounds (VOC) with high breakthrough volumes for the Sweetwater Reservoir air sampling site, San Diego County, California-Continued.

[The site identification number is 324141117001601 . Concentrations are given in parts per billion by volume (ppbv). Sample volume for quality-assurance blank is 5.0 liters. Samples processed at 20.0 degrees Celsius and 760 torr; many samples were frozen at the laboratory and analyzed on the same day. Data for travel blanks and environmental samples collected on 10/27/2000 were not used because samples were contaminated during transport. mm/dd/yyyy, month/day/year; E, estimated value; NA, not analyzed; - , compound was not detected at a concentration above laboratory reporting level]

\begin{tabular}{|c|c|c|c|c|c|c|c|c|}
\hline $\begin{array}{c}\text { Sampling } \\
\text { date } \\
\text { (mm/dd/yyyy) }\end{array}$ & $\begin{array}{c}\text { sec- } \\
\text { Butyl- } \\
\text { benzene }\end{array}$ & $\begin{array}{c}\text { tert- } \\
\text { Butyl- } \\
\text { benzene }\end{array}$ & $\begin{array}{l}\text { 1-Isopropyl- } \\
\text { 4-methyl- } \\
\text { benzene }\end{array}$ & $\begin{array}{l}1,2,3-\text { Tri- } \\
\text { chloro- } \\
\text { propane }\end{array}$ & $\begin{array}{l}1,1,1,2- \\
\text { Tetra- } \\
\text { chloro- } \\
\text { ethane }\end{array}$ & $\begin{array}{c}1,2,3-\text { Tri- } \\
\text { chloro- } \\
\text { benzene }\end{array}$ & $\begin{array}{l}\text { 1,2-Di- } \\
\text { bromo- } \\
\text { ethane }\end{array}$ & $\begin{array}{l}\text { Methyl tert- } \\
\text { butyl ether } \\
\text { (MTBE) }\end{array}$ \\
\hline $01 / 07 / 2001$ & - & - & - & - & - & - & - & - \\
\hline $01 / 19 / 2001$ & - & - & - & - & - & - & - & - \\
\hline $01 / 31 / 2001$ & - & - & - & - & - & - & - & - \\
\hline $02 / 12 / 2001$ & - & - & - & - & - & - & - & - \\
\hline $02 / 24 / 2001$ & - & - & - & - & - & - & - & - \\
\hline 03/08/2001 & - & - & - & - & - & - & - & - \\
\hline 03/20/2001 & NA & NA & NA & NA & NA & NA & NA & NA \\
\hline 04/01/2001 & - & - & - & - & - & - & - & - \\
\hline $04 / 13 / 2001$ & - & - & - & - & - & - & - & - \\
\hline $04 / 25 / 2001$ & - & - & - & - & - & - & - & - \\
\hline 05/07/2001 & - & - & - & - & - & - & - & - \\
\hline 05/19/2001 & - & - & - & - & - & - & - & - \\
\hline 05/31/2001 & - & - & - & - & - & - & - & - \\
\hline $06 / 12 / 2001$ & - & - & - & - & - & - & - & - \\
\hline $06 / 24 / 2001$ & - & - & - & - & - & - & - & - \\
\hline 07/05/2001 & - & - & - & - & - & - & - & - \\
\hline 07/16/2001 & - & - & - & - & - & - & - & - \\
\hline 08/08/2001 & - & - & - & - & - & - & - & - \\
\hline $08 / 23 / 2001$ & - & - & - & - & - & - & - & - \\
\hline 09/16/2001 & - & - & - & - & - & - & - & - \\
\hline 09/28/2001 & - & - & - & - & - & - & - & - \\
\hline
\end{tabular}


Table 17C. Quality-control analytical cartridge lot blank results for volatile organic compounds (VOC) with high breakthrough volumes for the Sweetwater Reservoir air sampling site, San Diego County, California—Continued.

[The site identification number is 324141117001601 . Concentrations are given in parts per billion by volume (ppbv). Sample volume for quality-assurance blank is 5.0 liters. Samples processed at 20.0 degrees Celsius and 760 torr; many samples were frozen at the laboratory and analyzed on the same day. Data for travel blanks and environmental samples collected on 10/27/2000 were not used because samples were contaminated during transport. mm/dd/yyyy, month/day/year; E, estimated value; NA, not analyzed; - , compound was not detected at a concentration above laboratory reporting level]

\begin{tabular}{|c|c|c|c|c|c|c|c|c|c|}
\hline $\begin{array}{c}\text { Sampling } \\
\text { date } \\
\text { (mm/dd/yyyy) }\end{array}$ & $\begin{array}{l}\text { 3-Chloro-1- } \\
\text { propene }\end{array}$ & $\begin{array}{l}\text { 4-Methyl-2- } \\
\text { pentanone } \\
\text { (MIBK) }\end{array}$ & Acetone & $\begin{array}{l}\text { Bromo- } \\
\text { benzene }\end{array}$ & $\begin{array}{l}\text { Diethyl } \\
\text { ether }\end{array}$ & $\begin{array}{l}\text { Diiso- } \\
\text { propyl- } \\
\text { ether } \\
\text { (DIPE) }\end{array}$ & $\begin{array}{l}\text { Methyl } \\
\text { acrylo- } \\
\text { nitrile }\end{array}$ & $\begin{array}{c}\text { 2-Bu- } \\
\text { tanone } \\
\text { (Methyl } \\
\text { ethyl } \\
\text { ketone) }\end{array}$ & $\begin{array}{l}\text { Methyl } \\
\text { acrylate }\end{array}$ \\
\hline $10 / 02 / 1999$ & - & - & E0.008 & - & - & - & - & - & - \\
\hline 10/13/1999 & - & - & E0.083 & - & - & - & - & - & - \\
\hline $10 / 26 / 1999$ & - & - & E0.106 & - & - & - & - & - & - \\
\hline $11 / 07 / 1999$ & - & - & E0.048 & - & - & - & - & - & - \\
\hline $11 / 19 / 1999$ & - & - & E0.049 & - & - & - & - & - & - \\
\hline $12 / 13 / 1999$ & - & - & E0.035 & - & - & - & - & - & - \\
\hline $12 / 25 / 1999$ & - & - & E0.103 & - & - & - & - & - & - \\
\hline $01 / 06 / 2000$ & - & - & E0.029 & - & - & - & - & - & - \\
\hline 01/18/2000 & - & - & E0.041 & - & - & - & - & - & - \\
\hline 01/30/2000 & - & - & E0.023 & - & - & - & - & - & - \\
\hline $02 / 11 / 2000$ & - & - & E0.034 & - & - & - & - & - & - \\
\hline 03/18/2000 & - & - & E0.014 & - & - & - & - & - & - \\
\hline $04 / 11 / 2000$ & - & - & E0.037 & - & - & - & - & - & - \\
\hline $04 / 23 / 2000$ & - & - & E0.020 & - & - & - & - & - & - \\
\hline $05 / 05 / 2000$ & - & - & E0.008 & - & - & - & - & - & - \\
\hline $05 / 17 / 2000$ & - & - & E0.012 & - & - & - & - & - & - \\
\hline $05 / 29 / 2000$ & - & - & E0.056 & - & - & - & - & - & - \\
\hline $06 / 10 / 2000$ & - & - & E0.071 & - & - & - & - & - & - \\
\hline $06 / 29 / 2000$ & - & - & E0.033 & - & - & - & - & - & - \\
\hline $07 / 11 / 2000$ & - & - & E0.024 & - & - & - & - & - & - \\
\hline $07 / 23 / 2000$ & NA & NA & NA & NA & NA & NA & NA & NA & NA \\
\hline 08/04/2000 & - & - & E0.049 & - & - & - & - & - & - \\
\hline $08 / 16 / 2000$ & - & - & E0.066 & - & - & - & - & - & - \\
\hline $08 / 28 / 2000$ & - & - & E0.046 & - & - & - & - & - & - \\
\hline 09/09/2000 & - & - & E0.066 & - & - & - & - & - & - \\
\hline $09 / 21 / 2000$ & - & - & E0.104 & - & - & - & - & - & - \\
\hline $10 / 03 / 2000$ & - & - & E0.051 & - & - & - & - & - & - \\
\hline $11 / 08 / 2000$ & - & - & E0.060 & - & - & - & - & - & - \\
\hline $11 / 20 / 2000$ & - & - & E0.079 & - & - & - & - & - & - \\
\hline $12 / 02 / 2000$ & - & - & E0.093 & - & - & - & - & - & - \\
\hline $12 / 14 / 2000$ & - & - & 0.272 & - & - & - & - & - & - \\
\hline $12 / 26 / 2000$ & - & - & E0.046 & - & - & - & - & - & - \\
\hline
\end{tabular}


Table 17C. Quality-control analytical cartridge lot blank results for volatile organic compounds (VOC) with high breakthrough volumes for the Sweetwater Reservoir air sampling site, San Diego County, California-Continued.

[The site identification number is 324141117001601 . Concentrations are given in parts per billion by volume (ppbv). Sample volume for quality-assurance blank is 5.0 liters. Samples processed at 20.0 degrees Celsius and 760 torr; many samples were frozen at the laboratory and analyzed on the same day. Data for travel blanks and environmental samples collected on 10/27/2000 were not used because samples were contaminated during transport. mm/dd/yyyy, month/day/year; E, estimated value; NA, not analyzed; - , compound was not detected at a concentration above laboratory reporting level]

\begin{tabular}{|c|c|c|c|c|c|c|c|c|c|}
\hline $\begin{array}{l}\text { Sampling } \\
\text { date } \\
\text { (mm/dd/yyyy) }\end{array}$ & $\begin{array}{c}\text { 3-Chloro-1- } \\
\text { propene }\end{array}$ & $\begin{array}{l}\text { 4-Methyl-2- } \\
\text { pentanone } \\
\text { (MIBK) }\end{array}$ & Acetone & $\begin{array}{l}\text { Bromo- } \\
\text { benzene }\end{array}$ & $\begin{array}{l}\text { Diethyl } \\
\text { ether }\end{array}$ & $\begin{array}{c}\text { Diiso- } \\
\text { propyl- } \\
\text { ether } \\
\text { (DIPE) }\end{array}$ & $\begin{array}{l}\text { Methyl } \\
\text { acrylo- } \\
\text { nitrile }\end{array}$ & $\begin{array}{c}\text { 2-Bu- } \\
\text { tanone } \\
\text { (Methyl } \\
\text { ethyl } \\
\text { ketone) }\end{array}$ & $\begin{array}{l}\text { Methyl } \\
\text { acrylate }\end{array}$ \\
\hline $01 / 07 / 2001$ & - & - & E0.062 & - & - & - & - & - & - \\
\hline $01 / 19 / 2001$ & - & - & E0.037 & - & - & - & - & - & - \\
\hline $01 / 31 / 2001$ & - & - & E0.136 & - & - & - & - & - & - \\
\hline $02 / 12 / 2001$ & - & - & E0.083 & - & - & - & - & - & - \\
\hline $02 / 24 / 2001$ & - & - & E0.030 & - & - & - & - & - & - \\
\hline 03/08/2001 & - & - & E0.122 & - & - & - & - & - & - \\
\hline $03 / 20 / 2001$ & $\mathrm{NA}$ & NA & NA & NA & NA & NA & $\mathrm{NA}$ & NA & NA \\
\hline $04 / 01 / 2001$ & - & - & E0.036 & - & - & - & - & - & - \\
\hline $04 / 13 / 2001$ & - & - & E0.057 & - & - & - & - & - & - \\
\hline $04 / 25 / 2001$ & - & - & 0.252 & - & - & - & - & - & - \\
\hline $05 / 07 / 2001$ & - & - & E0.078 & - & - & - & - & - & - \\
\hline 05/19/2001 & - & - & E0.065 & - & - & - & - & - & - \\
\hline $05 / 31 / 2001$ & - & - & E0.042 & - & - & - & - & - & - \\
\hline $06 / 12 / 2001$ & - & - & E0.081 & - & - & - & - & - & - \\
\hline $06 / 24 / 2001$ & - & - & E0.089 & - & - & - & - & - & - \\
\hline $07 / 05 / 2001$ & - & - & E0.105 & - & - & - & - & - & - \\
\hline $07 / 16 / 2001$ & - & - & E0.090 & - & - & - & - & - & - \\
\hline 08/08/2001 & - & - & 0.143 & - & - & - & - & - & - \\
\hline $08 / 23 / 2001$ & - & - & E0.090 & - & - & - & - & - & - \\
\hline 09/16/2001 & - & - & E0.073 & - & - & - & - & - & - \\
\hline 09/28/2001 & - & - & - & - & - & - & - & - & - \\
\hline
\end{tabular}


Table 17C. Quality-control analytical cartridge lot blank results for volatile organic compounds (VOC) with high breakthrough volumes for the Sweetwater Reservoir air sampling site, San Diego County, California-Continued.

[The site identification number is 324141117001601 . Concentrations are given in parts per billion by volume (ppbv). Sample volume for quality-assurance blank is 5.0 liters. Samples processed at 20.0 degrees Celsius and 760 torr; many samples were frozen at the laboratory and analyzed on the same day. Data for travel blanks and environmental samples collected on 10/27/2000 were not used because samples were contaminated during transport. mm/dd/yyyy, month/day/year; E, estimated value; NA, not analyzed; - , compound was not detected at a concentration above laboratory reporting level]

\begin{tabular}{|c|c|c|c|c|c|c|c|c|}
\hline $\begin{array}{c}\text { Sampling } \\
\text { date } \\
\text { (mm/dd/yyyy) }\end{array}$ & $\begin{array}{l}\text { Tetrahy- } \\
\text { drofuran }\end{array}$ & $\begin{array}{c}\text { 1,2-Dibromo- } \\
\text { 3-chloro- } \\
\text { propane } \\
\text { (DBCP) }\end{array}$ & $\begin{array}{c}m \text { - and } p- \\
\text { Xylene }\end{array}$ & $\begin{array}{c}\text { 1,2,3,5- } \\
\text { Tetra- } \\
\text { methyl- } \\
\text { benzene }\end{array}$ & $\begin{array}{c}1,2,4,5- \\
\text { Tetra- } \\
\text { methyl- } \\
\text { benzene }\end{array}$ & $\begin{array}{l}\text { Methyl } \\
\text { acetate }\end{array}$ & $\begin{array}{c}\text { 2-Methyl- } \\
\text { 2-butanol } \\
\text { (tert-Amyl } \\
\text { alcohol) }\end{array}$ & $\begin{array}{c}\text { 2-Methyl-2-pro- } \\
\text { panol (tert-Bu- } \\
\text { tyl alcohol) }\end{array}$ \\
\hline 10/02/1999 & - & - & - & - & - & - & - & - \\
\hline 10/13/1999 & - & - & E0.001 & - & - & - & - & - \\
\hline 10/26/1999 & - & - & E0.005 & - & - & - & - & - \\
\hline 11/07/1999 & - & - & E0.003 & - & - & - & - & - \\
\hline 11/19/1999 & - & - & E0.001 & - & - & - & - & - \\
\hline $12 / 13 / 1999$ & - & - & - & - & - & - & - & - \\
\hline $12 / 25 / 1999$ & - & - & - & - & - & - & - & - \\
\hline 01/06/2000 & - & - & - & - & - & - & - & - \\
\hline 01/18/2000 & - & - & - & - & - & - & - & - \\
\hline $01 / 30 / 2000$ & - & - & - & - & - & - & - & - \\
\hline $02 / 11 / 2000$ & - & - & E0.001 & - & - & - & - & - \\
\hline 03/18/2000 & - & - & - & - & - & - & - & - \\
\hline $04 / 11 / 2000$ & - & - & - & - & - & - & - & - \\
\hline $04 / 23 / 2000$ & - & - & - & - & - & - & - & - \\
\hline 05/05/2000 & - & - & - & - & - & - & - & - \\
\hline 05/17/2000 & - & - & - & - & - & - & - & - \\
\hline 05/29/2000 & - & - & - & - & - & - & - & - \\
\hline 06/10/2000 & - & - & - & - & - & - & - & - \\
\hline $06 / 29 / 2000$ & - & - & - & - & - & - & - & - \\
\hline $07 / 11 / 2000$ & - & - & - & - & - & - & - & - \\
\hline $07 / 23 / 2000$ & NA & NA & NA & NA & NA & NA & NA & NA \\
\hline 08/04/2000 & - & - & - & - & - & - & - & - \\
\hline 08/16/2000 & - & - & - & - & - & - & - & - \\
\hline $08 / 28 / 2000$ & - & - & E0.001 & - & - & - & - & - \\
\hline 09/09/2000 & - & - & - & - & - & - & - & - \\
\hline $09 / 21 / 2000$ & - & - & - & - & - & - & - & - \\
\hline $10 / 03 / 2000$ & - & - & E0.001 & - & - & - & - & - \\
\hline $11 / 08 / 2000$ & - & - & E0.003 & - & - & - & - & - \\
\hline $11 / 20 / 2000$ & - & - & E0.003 & - & - & - & - & - \\
\hline $12 / 02 / 2000$ & - & - & E0.002 & - & - & - & - & - \\
\hline $12 / 14 / 2000$ & - & - & E0.022 & - & - & - & - & - \\
\hline $12 / 26 / 2000$ & - & - & E0.003 & - & - & - & - & - \\
\hline
\end{tabular}


Table 17C. Quality-control analytical cartridge lot blank results for volatile organic compounds (VOC) with high breakthrough volumes for the Sweetwater Reservoir air sampling site, San Diego County, California-Continued.

[The site identification number is 324141117001601 . Concentrations are given in parts per billion by volume (ppbv). Sample volume for quality-assurance blank is 5.0 liters. Samples processed at 20.0 degrees Celsius and 760 torr; many samples were frozen at the laboratory and analyzed on the same day. Data for travel blanks and environmental samples collected on 10/27/2000 were not used because samples were contaminated during transport. mm/dd/yyyy, month/day/year; E, estimated value; NA, not analyzed; - , compound was not detected at a concentration above laboratory reporting level]

\begin{tabular}{|c|c|c|c|c|c|c|c|c|}
\hline $\begin{array}{l}\text { Sampling } \\
\text { date } \\
\text { (mm/dd/yyyy) }\end{array}$ & $\begin{array}{l}\text { Tetrahy- } \\
\text { drofuran }\end{array}$ & $\begin{array}{c}\text { 1,2-Dibromo- } \\
\text { 3-chloro- } \\
\text { propane } \\
\text { (DBCP) }\end{array}$ & $\begin{array}{c}m \text { - and } p- \\
\text { Xylene }\end{array}$ & $\begin{array}{c}1,2,3,5- \\
\text { Tetra- } \\
\text { methyl- } \\
\text { benzene }\end{array}$ & $\begin{array}{c}1,2,4,5- \\
\text { Tetra- } \\
\text { methyl- } \\
\text { benzene }\end{array}$ & $\begin{array}{l}\text { Methyl } \\
\text { acetate }\end{array}$ & $\begin{array}{l}\text { 2-Methyl- } \\
\text { 2-butanol } \\
\text { (tert-Amyl } \\
\text { alcohol) }\end{array}$ & $\begin{array}{c}\text { 2-Methyl-2-pro- } \\
\text { panol (tert-Bu- } \\
\text { tyl alcohol) }\end{array}$ \\
\hline 01/07/2001 & - & - & E0.005 & - & - & - & - & - \\
\hline $01 / 19 / 2001$ & - & - & E0.005 & - & - & - & - & - \\
\hline $01 / 31 / 2001$ & - & - & - & - & - & - & - & - \\
\hline $02 / 12 / 2001$ & - & - & - & - & - & - & - & - \\
\hline $02 / 24 / 2001$ & - & - & E0.001 & - & - & - & - & - \\
\hline 03/08/2001 & - & - & E0.002 & - & - & - & - & - \\
\hline 03/20/2001 & NA & NA & NA & NA & NA & NA & NA & NA \\
\hline $04 / 01 / 2001$ & - & - & - & - & - & - & - & - \\
\hline $04 / 13 / 2001$ & - & - & E0.001 & - & - & - & - & - \\
\hline $04 / 25 / 2001$ & - & - & E0.017 & - & - & - & - & - \\
\hline 05/07/2001 & - & - & - & - & - & - & - & - \\
\hline 05/19/2001 & - & - & E0.001 & - & - & - & - & - \\
\hline $05 / 31 / 2001$ & - & - & E0.001 & - & - & - & - & - \\
\hline $06 / 12 / 2001$ & - & - & - & - & - & - & - & - \\
\hline $06 / 24 / 2001$ & - & - & E0.008 & - & - & - & - & - \\
\hline $07 / 05 / 2001$ & - & - & - & - & - & - & - & - \\
\hline 07/16/2001 & - & - & - & - & - & - & - & - \\
\hline 08/08/2001 & - & - & - & - & - & - & - & - \\
\hline $08 / 23 / 2001$ & - & - & - & - & - & - & - & - \\
\hline 09/16/2001 & - & - & E0.004 & - & - & - & - & - \\
\hline 09/28/2001 & - & - & - & - & - & - & - & - \\
\hline
\end{tabular}


Table 17D. Quality-control analytical laboratory blank results for volatile organic compounds (VOC) with high breakthrough volumes for the Sweetwater Reservoir air sampling site, San Diego County, California.

[The site identification number is 324141117001601. Concentrations are given in parts per billion by volume (ppbv). Sample volume for quality-assurance blank is 5.0 liters. Samples processed at 20.0 degrees Celsius and 760 torr; many samples were frozen at the laboratory and analyzed on the same day. Data for travel blanks and environmental samples collected on 10/27/2000 were not used because samples were contaminated during transport. mm/dd/yyyy, month/day/year; *, sample data lost; E, estimated value; NA, not analyzed; - , compound was not detected at a concentration above laboratory reporting level]

\begin{tabular}{|c|c|c|c|c|c|c|c|c|}
\hline $\begin{array}{c}\text { Sampling } \\
\text { date } \\
\text { (mm/dd/yyyy) }\end{array}$ & $\begin{array}{c}\text { Blank analysis } \\
\text { date } \\
\text { (mm/dd/yyyy) }\end{array}$ & $\begin{array}{l}\text { Dibromo- } \\
\text { methane }\end{array}$ & $\begin{array}{l}\text { Bromodi- } \\
\text { chlorometh- } \\
\text { ane }\end{array}$ & $\begin{array}{c}\text { Carbon } \\
\text { tetra- } \\
\text { chloride }\end{array}$ & $\begin{array}{l}\text { 1,2-Di- } \\
\text { chloro- } \\
\text { ethane }\end{array}$ & $\begin{array}{l}\text { Bromoform } \\
\text { (Tribromo- } \\
\text { methane) }\end{array}$ & $\begin{array}{c}\text { Dibro- } \\
\text { mochloro- } \\
\text { methane }\end{array}$ & $\begin{array}{l}\text { Chloroform } \\
\text { (Trichloro- } \\
\text { methane) }\end{array}$ \\
\hline 10/02/1999* & 10/07/1999* & NA & NA & NA & NA & NA & NA & NA \\
\hline 10/13/1999 & $10 / 21 / 1999$ & - & - & - & - & - & - & - \\
\hline 10/26/1999 & 10/28/1999 & - & - & - & - & - & - & - \\
\hline 11/07/1999 & $11 / 15 / 1999$ & - & - & - & - & - & - & - \\
\hline 11/19/1999 & $11 / 29 / 1999$ & - & - & - & - & - & - & - \\
\hline $12 / 13 / 1999$ & $12 / 16 / 1999$ & - & - & - & - & - & - & - \\
\hline $12 / 25 / 1999$ & $01 / 13 / 2000$ & - & - & - & - & - & - & - \\
\hline 01/06/2000 & $01 / 13 / 2000$ & - & - & - & - & - & - & - \\
\hline 01/18/2000 & $01 / 24 / 2000$ & - & - & - & - & - & - & - \\
\hline 01/30/2000 & $02 / 03 / 2000$ & - & - & - & - & - & - & - \\
\hline $02 / 11 / 2000$ & $02 / 17 / 2000$ & - & - & - & - & - & - & - \\
\hline 03/18/2000 & $03 / 23 / 2000$ & - & - & - & - & - & - & - \\
\hline $04 / 11 / 2000$ & $04 / 25 / 2000$ & - & - & - & - & - & - & - \\
\hline $04 / 23 / 2000$ & $04 / 25 / 2000$ & - & - & - & - & - & - & - \\
\hline 05/05/2000 & $05 / 18 / 2000$ & - & - & - & - & - & - & - \\
\hline 05/17/2000 & 05/18/2000 & - & - & - & - & - & - & - \\
\hline 05/29/2000 & $06 / 12 / 2000$ & - & - & - & - & - & - & - \\
\hline 06/10/2000 & $06 / 12 / 2000$ & - & - & - & - & - & - & - \\
\hline $06 / 29 / 2000$ & $07 / 17 / 2000$ & - & - & - & - & - & - & - \\
\hline 07/11/2000 & $07 / 17 / 2000$ & - & - & - & - & - & - & - \\
\hline 07/23/2000 & 08/07/2000 & - & - & - & - & - & - & - \\
\hline 08/04/2000 & $08 / 07 / 2000$ & - & - & - & - & - & - & - \\
\hline 08/16/2000 & 09/19/2000 & - & - & - & - & - & - & - \\
\hline 08/28/2000 & 09/19/2000 & - & - & - & - & - & - & - \\
\hline 09/09/2000 & 09/19/2000 & - & - & - & - & - & - & - \\
\hline $09 / 21 / 2000$ & $10 / 12 / 2000$ & - & - & - & - & - & - & - \\
\hline $10 / 03 / 2000$ & $10 / 12 / 2000$ & - & - & - & - & - & - & - \\
\hline $11 / 08 / 2000$ & $11 / 16 / 2000$ & - & - & - & - & - & - & - \\
\hline $11 / 20 / 2000$ & $12 / 07 / 2000$ & - & - & - & - & - & - & - \\
\hline $12 / 02 / 2000$ & $12 / 07 / 2000$ & - & - & - & - & - & - & - \\
\hline $12 / 14 / 2000$ & $12 / 20 / 2000$ & - & - & - & - & - & - & - \\
\hline $12 / 26 / 2000$ & $01 / 12 / 2001$ & - & - & - & - & - & - & - \\
\hline
\end{tabular}


Table 17D. Quality-control analytical laboratory blank results for volatile organic compounds (VOC) with high breakthrough volumes for the Sweetwater Reservoir air sampling site, San Diego County, California—Continued.

[The site identification number is 324141117001601 . Concentrations are given in parts per billion by volume (ppbv). Sample volume for quality-assurance blank is 5.0 liters. Samples processed at 20.0 degrees Celsius and 760 torr; many samples were frozen at the laboratory and analyzed on the same day. Data for travel blanks and environmental samples collected on 10/27/2000 were not used because samples were contaminated during transport. mm/dd/yyyy, month/day/year; *, sample data lost; E, estimated value; NA, not analyzed; - , compound was not detected at a concentration above laboratory reporting level]

\begin{tabular}{|c|c|c|c|c|c|c|c|c|}
\hline $\begin{array}{c}\text { Sampling } \\
\text { date } \\
\text { (mm/dd/yyyy) }\end{array}$ & $\begin{array}{c}\text { Blank analysis } \\
\text { date } \\
\text { (mm/dd/yyyy) }\end{array}$ & $\begin{array}{l}\text { Dibromo- } \\
\text { methane }\end{array}$ & $\begin{array}{l}\text { Bromodi- } \\
\text { chlorometh- } \\
\text { ane }\end{array}$ & $\begin{array}{l}\text { Carbon } \\
\text { tetra- } \\
\text { chloride }\end{array}$ & $\begin{array}{l}\text { 1,2-Di- } \\
\text { chloro- } \\
\text { ethane }\end{array}$ & $\begin{array}{l}\text { Bromoform } \\
\text { (Tribromo- } \\
\text { methane) }\end{array}$ & $\begin{array}{c}\text { Dibro- } \\
\text { mochloro- } \\
\text { methane }\end{array}$ & $\begin{array}{c}\text { Chloroform } \\
\text { (Trichloro- } \\
\text { methane) }\end{array}$ \\
\hline $01 / 07 / 2001$ & $01 / 12 / 2001$ & - & - & - & - & - & - & - \\
\hline 01/19/2001 & 02/06/2001 & - & - & - & - & - & - & - \\
\hline $01 / 31 / 2001$ & 02/06/2001 & - & - & - & - & - & - & - \\
\hline $02 / 12 / 2001$ & 03/05/2001 & - & - & - & - & - & - & - \\
\hline $02 / 24 / 2001$ & 03/05/2001 & - & - & - & - & - & - & - \\
\hline 03/08/2001 & 03/26/2001 & - & - & - & - & - & - & - \\
\hline 03/20/2001 & $03 / 26 / 2001$ & NA & NA & NA & NA & NA & NA & NA \\
\hline 04/01/2001 & $04 / 24 / 2001$ & - & - & - & - & - & - & - \\
\hline 04/13/2001 & $04 / 24 / 2001$ & - & - & - & - & - & - & - \\
\hline $04 / 25 / 2001$ & 05/10/2001 & - & - & - & - & - & - & - \\
\hline 05/07/2001 & 05/10/2001 & - & - & - & - & - & - & - \\
\hline $05 / 19 / 2001$ & 06/07/2001 & - & - & - & - & - & - & - \\
\hline $05 / 31 / 2001$ & $06 / 07 / 2001$ & - & - & - & - & - & - & - \\
\hline $06 / 12 / 2001$ & $06 / 28 / 2001$ & - & - & - & - & - & - & - \\
\hline $06 / 24 / 2001$ & $06 / 28 / 2001$ & - & - & - & - & - & - & - \\
\hline 07/05/2001 & $07 / 24 / 2001$ & - & - & - & - & - & - & - \\
\hline 07/16/2001 & 08/09/2001 & - & - & - & - & - & - & - \\
\hline 08/08/2001 & $08 / 21 / 2001$ & - & - & - & - & - & - & - \\
\hline $08 / 23 / 2001$ & $09 / 12 / 2001$ & - & - & - & - & - & - & - \\
\hline 09/16/2001 & $10 / 10 / 2001$ & - & - & - & - & - & - & - \\
\hline 09/28/2001 & $10 / 10 / 2001$ & - & - & - & - & - & - & - \\
\hline
\end{tabular}


Table 17D. Quality-control analytical laboratory blank results for volatile organic compounds (VOC) with high breakthrough volumes for the Sweetwater Reservoir air sampling site, San Diego County, California—Continued.

[The site identification number is 324141117001601 . Concentrations are given in parts per billion by volume (ppbv). Sample volume for quality-assurance blank is 5.0 liters. Samples processed at 20.0 degrees Celsius and 760 torr; many samples were frozen at the laboratory and analyzed on the same day. Data for travel blanks and environmental samples collected on 10/27/2000 were not used because samples were contaminated during transport. mm/dd/yyyy, month/day/year; *, sample data lost; E, estimated value; NA, not analyzed; - , compound was not detected at a concentration above laboratory reporting level]

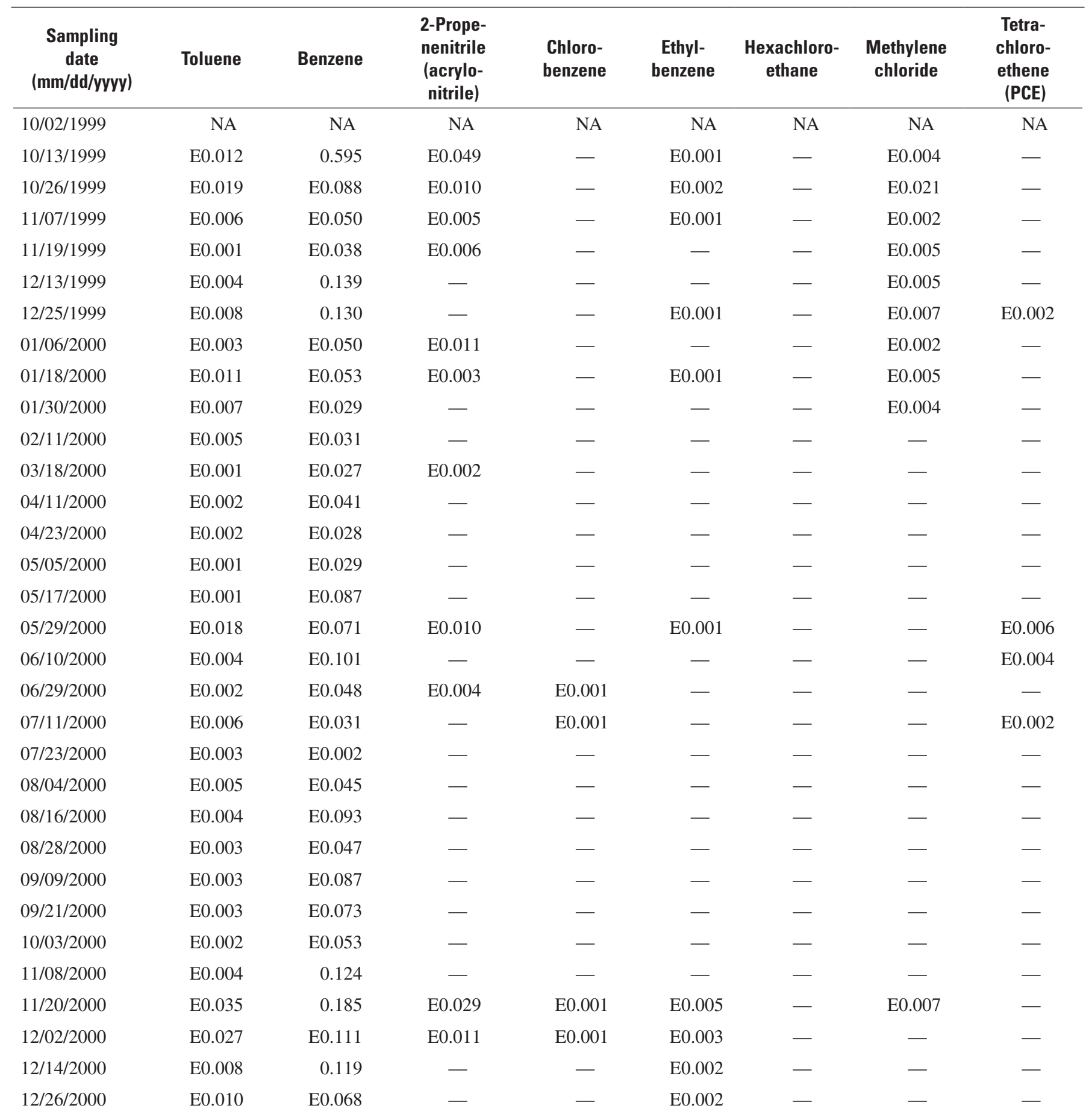


Table 17D. Quality-control analytical laboratory blank results for volatile organic compounds (VOC) with high breakthrough volumes for the Sweetwater Reservoir air sampling site, San Diego County, California—Continued.

[The site identification number is 324141117001601. Concentrations are given in parts per billion by volume (ppbv). Sample volume for quality-assurance blank is 5.0 liters. Samples processed at 20.0 degrees Celsius and 760 torr; many samples were frozen at the laboratory and analyzed on the same day. Data for travel blanks and environmental samples collected on 10/27/2000 were not used because samples were contaminated during transport. mm/dd/yyyy, month/day/year; *, sample data lost; E, estimated value; NA, not analyzed; - , compound was not detected at a concentration above laboratory reporting level]

\begin{tabular}{|c|c|c|c|c|c|c|c|c|}
\hline $\begin{array}{c}\text { Sampling } \\
\text { date } \\
\text { (mm/dd/yyyy) }\end{array}$ & Toluene & Benzene & $\begin{array}{c}\text { 2-Prope- } \\
\text { nenitrile } \\
\text { (acrylo- } \\
\text { nitrile) }\end{array}$ & $\begin{array}{l}\text { Chloro- } \\
\text { benzene }\end{array}$ & $\begin{array}{c}\text { Ethyl- } \\
\text { benzene }\end{array}$ & $\begin{array}{l}\text { Hexachloro- } \\
\text { ethane }\end{array}$ & $\begin{array}{l}\text { Methylene } \\
\text { chloride }\end{array}$ & $\begin{array}{l}\text { Tetra- } \\
\text { chloro- } \\
\text { ethene } \\
\text { (PCE) }\end{array}$ \\
\hline 01/07/2001 & E0.010 & 0.191 & - & - & E0.002 & - & - & - \\
\hline 01/19/2001 & E0.018 & 0.114 & - & - & E0.003 & - & - & - \\
\hline $01 / 31 / 2001$ & E0.033 & 0.139 & - & - & E0.006 & - & - & - \\
\hline $02 / 12 / 2001$ & E0.024 & 0.199 & - & - & E0.002 & - & - & - \\
\hline $02 / 24 / 2001$ & E0.008 & E0.109 & - & - & E0.001 & - & - & - \\
\hline 03/08/2001 & E0.009 & E0.082 & - & - & E0.001 & - & - & - \\
\hline 03/20/2001 & NA & NA & NA & NA & NA & NA & NA & NA \\
\hline 04/01/2001 & E0.007 & E0.113 & - & - & E0.001 & - & - & - \\
\hline $04 / 13 / 2001$ & E0.004 & E0.074 & - & E0.001 & E0.001 & - & - & - \\
\hline $04 / 25 / 2001$ & E0.011 & 0.191 & E0.009 & - & E0.001 & - & - & - \\
\hline 05/07/2001 & E0.010 & 0.419 & E0.011 & - & E0.001 & - & - & - \\
\hline 05/19/2001 & E0.004 & E0.013 & - & - & - & - & - & E0.007 \\
\hline $05 / 31 / 2001$ & E0.004 & E0.029 & - & - & - & - & - & - \\
\hline $06 / 12 / 2001$ & E0.003 & E0.042 & - & - & - & - & - & - \\
\hline $06 / 24 / 2001$ & E0.001 & E0.031 & - & E0.003 & - & - & - & - \\
\hline $07 / 05 / 2001$ & E0.004 & E0.084 & - & - & - & - & - & - \\
\hline 07/16/2001 & E0.003 & E0.069 & - & - & E0.001 & - & - & - \\
\hline 08/08/2001 & E0.002 & E0.013 & - & E0.001 & E0.007 & - & - & - \\
\hline 08/23/2001 & E0.009 & 0.256 & - & - & - & - & - & - \\
\hline $09 / 16 / 2001$ & E0.021 & 0.151 & - & - & E0.003 & - & - & - \\
\hline $09 / 28 / 2001$ & E0.009 & E0.124 & - & - & E0.001 & - & - & - \\
\hline
\end{tabular}


Table 17D. Quality-control analytical laboratory blank results for volatile organic compounds (VOC) with high breakthrough volumes for the Sweetwater Reservoir air sampling site, San Diego County, California-Continued.

[The site identification number is 324141117001601 . Concentrations are given in parts per billion by volume (ppbv). Sample volume for quality-assurance blank is 5.0 liters. Samples processed at 20.0 degrees Celsius and 760 torr; many samples were frozen at the laboratory and analyzed on the same day. Data for travel blanks and environmental samples collected on 10/27/2000 were not used because samples were contaminated during transport. mm/dd/yyyy, month/day/year; *, sample data lost; E, estimated value; NA, not analyzed; - , compound was not detected at a concentration above laboratory reporting level]

\begin{tabular}{|c|c|c|c|c|c|c|c|c|}
\hline $\begin{array}{l}\text { Sampling } \\
\text { date } \\
\text { (mm/dd/yyyy) }\end{array}$ & $\begin{array}{l}\text { 1,1-Di- } \\
\text { chloro- } \\
\text { ethane }\end{array}$ & $\begin{array}{l}\text { 1,1-Di- } \\
\text { chloro- } \\
\text { ethene }\end{array}$ & $\begin{array}{l}\text { 1,1,1-Tri- } \\
\text { chloro- } \\
\text { ethane }\end{array}$ & $\begin{array}{l}\text { 1,1,2-Tri- } \\
\text { chloro- } \\
\text { ethane }\end{array}$ & $\begin{array}{c}1,1,2,2- \\
\text { Tetra- } \\
\text { chloro- } \\
\text { ethane }\end{array}$ & $\begin{array}{c}\text { 1,2-Di- } \\
\text { chloro- } \\
\text { benzene }\end{array}$ & $\begin{array}{l}\text { 1,2-Di- } \\
\text { chloro- } \\
\text { propane }\end{array}$ & $\begin{array}{l}\text { trans-1,2- } \\
\text { Dichloro- } \\
\text { ethene }\end{array}$ \\
\hline 10/02/1999 & NA & NA & NA & $\mathrm{NA}$ & NA & NA & NA & NA \\
\hline 10/13/1999 & - & - & - & - & - & - & - & - \\
\hline 10/26/1999 & - & - & - & - & - & - & - & - \\
\hline 11/07/1999 & - & - & - & - & - & - & - & - \\
\hline 11/19/1999 & - & - & - & - & - & - & - & - \\
\hline 12/13/1999 & - & - & - & - & - & - & - & - \\
\hline 12/25/1999 & - & - & - & - & - & - & - & - \\
\hline 01/06/2000 & - & - & - & - & - & - & - & - \\
\hline 01/18/2000 & - & - & - & - & - & - & - & - \\
\hline 01/30/2000 & - & - & - & - & - & - & - & - \\
\hline $02 / 11 / 2000$ & - & - & - & - & - & - & - & - \\
\hline 03/18/2000 & - & - & - & - & - & - & - & - \\
\hline 04/11/2000 & - & - & - & - & - & - & - & - \\
\hline $04 / 23 / 2000$ & - & - & - & - & - & - & - & - \\
\hline 05/05/2000 & - & - & - & - & - & - & - & - \\
\hline 05/17/2000 & - & - & - & - & - & - & - & - \\
\hline 05/29/2000 & - & - & - & - & - & - & - & - \\
\hline 06/10/2000 & - & 0.214 & - & - & - & - & - & - \\
\hline 06/29/2000 & - & - & - & - & - & - & - & - \\
\hline 07/11/2000 & - & - & - & - & - & - & - & - \\
\hline 07/23/2000 & - & E0.054 & - & - & - & - & - & - \\
\hline 08/04/2000 & - & - & - & - & - & - & - & - \\
\hline 08/16/2000 & - & - & - & - & - & - & - & - \\
\hline 08/28/2000 & - & - & - & - & - & - & - & - \\
\hline 09/09/2000 & - & - & - & - & - & - & - & - \\
\hline 09/21/2000 & - & - & - & - & - & - & - & - \\
\hline $10 / 03 / 2000$ & - & - & - & - & - & - & - & - \\
\hline $11 / 08 / 2000$ & - & - & - & - & - & - & - & - \\
\hline $11 / 20 / 2000$ & - & - & - & - & - & - & - & - \\
\hline $12 / 02 / 2000$ & - & - & - & - & - & - & - & - \\
\hline $12 / 14 / 2000$ & - & - & - & - & - & - & - & - \\
\hline $12 / 26 / 2000$ & - & - & - & - & - & - & - & - \\
\hline
\end{tabular}


Table 17D. Quality-control analytical laboratory blank results for volatile organic compounds (VOC) with high breakthrough volumes for the Sweetwater Reservoir air sampling site, San Diego County, California—Continued.

[The site identification number is 324141117001601 . Concentrations are given in parts per billion by volume (ppbv). Sample volume for quality-assurance blank is 5.0 liters. Samples processed at 20.0 degrees Celsius and 760 torr; many samples were frozen at the laboratory and analyzed on the same day. Data for travel blanks and environmental samples collected on 10/27/2000 were not used because samples were contaminated during transport. mm/dd/yyyy, month/day/year; *, sample data lost; E, estimated value; NA, not analyzed; - , compound was not detected at a concentration above laboratory reporting level]

\begin{tabular}{|c|c|c|c|c|c|c|c|c|}
\hline $\begin{array}{c}\text { Sampling } \\
\text { date } \\
\text { (mm/dd/yyyy) }\end{array}$ & $\begin{array}{l}\text { 1,1-Di- } \\
\text { chloro- } \\
\text { ethane }\end{array}$ & $\begin{array}{l}\text { 1,1-Di- } \\
\text { chloro- } \\
\text { ethene }\end{array}$ & $\begin{array}{l}\text { 1,1,1-Tri- } \\
\text { chloro- } \\
\text { ethane }\end{array}$ & $\begin{array}{l}\text { 1,1,2-Tri- } \\
\text { chloro- } \\
\text { ethane }\end{array}$ & $\begin{array}{l}\text { 1,1,2,2- } \\
\text { Tetra- } \\
\text { chloro- } \\
\text { ethane }\end{array}$ & $\begin{array}{l}\text { 1,2-Di- } \\
\text { chloro- } \\
\text { benzene }\end{array}$ & $\begin{array}{l}\text { 1,2-Di- } \\
\text { chloro- } \\
\text { propane }\end{array}$ & $\begin{array}{c}\text { trans-1,2- } \\
\text { Dichloro- } \\
\text { ethene }\end{array}$ \\
\hline 01/07/2001 & - & - & - & - & - & - & - & - \\
\hline 01/19/2001 & - & - & - & - & - & - & - & - \\
\hline $01 / 31 / 2001$ & - & - & - & - & - & - & - & - \\
\hline $02 / 12 / 2001$ & - & - & - & - & - & - & - & - \\
\hline $02 / 24 / 2001$ & - & - & - & - & - & - & - & - \\
\hline 03/08/2001 & - & - & - & - & - & - & - & - \\
\hline 03/20/2001 & NA & NA & NA & NA & NA & NA & NA & NA \\
\hline $04 / 01 / 2001$ & - & - & - & - & - & - & - & - \\
\hline $04 / 13 / 2001$ & - & - & - & - & - & - & - & - \\
\hline $04 / 25 / 2001$ & - & - & - & - & - & - & - & - \\
\hline 05/07/2001 & - & - & - & - & - & - & - & - \\
\hline 05/19/2001 & - & - & - & - & - & - & - & - \\
\hline $05 / 31 / 2001$ & - & - & - & - & - & - & - & - \\
\hline $06 / 12 / 2001$ & - & - & - & - & - & - & - & - \\
\hline $06 / 24 / 2001$ & - & - & - & - & - & - & - & - \\
\hline 07/05/2001 & - & - & - & - & - & - & - & - \\
\hline $07 / 16 / 2001$ & - & - & - & - & - & - & - & - \\
\hline 08/08/2001 & - & - & - & - & - & - & - & - \\
\hline $08 / 23 / 2001$ & - & - & - & - & - & - & - & - \\
\hline 09/16/2001 & - & - & - & - & - & - & - & - \\
\hline $09 / 28 / 2001$ & - & - & - & - & - & - & - & - \\
\hline
\end{tabular}


Table 17D. Quality-control analytical laboratory blank results for volatile organic compounds (VOC) with high breakthrough volumes for the Sweetwater Reservoir air sampling site, San Diego County, California—Continued.

[The site identification number is 324141117001601 . Concentrations are given in parts per billion by volume (ppbv). Sample volume for quality-assurance blank is 5.0 liters. Samples processed at 20.0 degrees Celsius and 760 torr; many samples were frozen at the laboratory and analyzed on the same day. Data for travel blanks and environmental samples collected on 10/27/2000 were not used because samples were contaminated during transport. mm/dd/yyyy, month/day/year; *, sample data lost; E, estimated value; NA, not analyzed; - , compound was not detected at a concentration above laboratory reporting level]

\begin{tabular}{|c|c|c|c|c|c|c|c|c|}
\hline $\begin{array}{c}\text { Sampling } \\
\text { date } \\
\text { (mm/dd/yyyy) }\end{array}$ & $\begin{array}{c}\text { 1,2,4-Tri- } \\
\text { chloro- } \\
\text { benzene }\end{array}$ & $\begin{array}{l}\text { 1,3-Di- } \\
\text { chloro- } \\
\text { benzene }\end{array}$ & $\begin{array}{l}\text { 1,4-Di- } \\
\text { chloro- } \\
\text { benzene }\end{array}$ & $\begin{array}{l}\text { Naph- } \\
\text { thalene }\end{array}$ & $\begin{array}{l}\text { trans-1,3- } \\
\text { Dichloro- } \\
\text { propene }\end{array}$ & $\begin{array}{c}\text { cis-1,3-Di- } \\
\text { chloro- } \\
\text { propene }\end{array}$ & $\begin{array}{l}\text { Trichloro- } \\
\text { ethene } \\
\text { (TCE) }\end{array}$ & $\begin{array}{c}\text { Hexa- } \\
\text { chloro- } \\
\text { butadiene }\end{array}$ \\
\hline $10 / 02 / 1999$ & NA & NA & NA & $\mathrm{NA}$ & NA & NA & NA & NA \\
\hline 10/13/1999 & - & - & - & E0.001 & - & - & - & - \\
\hline $10 / 26 / 1999$ & - & - & - & E0.004 & - & - & - & - \\
\hline $11 / 07 / 1999$ & - & - & - & E0.003 & - & - & - & - \\
\hline $11 / 19 / 1999$ & - & - & - & - & - & - & - & - \\
\hline $12 / 13 / 1999$ & - & - & - & E0.001 & - & - & - & - \\
\hline $12 / 25 / 1999$ & - & - & - & - & - & - & - & - \\
\hline $01 / 06 / 2000$ & - & - & - & E0.003 & - & - & - & - \\
\hline $01 / 18 / 2000$ & - & - & - & E0.003 & - & - & - & - \\
\hline $01 / 30 / 2000$ & - & - & - & - & - & - & - & - \\
\hline $02 / 11 / 2000$ & - & - & - & E0.001 & - & - & - & - \\
\hline 03/18/2000 & - & - & - & - & - & - & - & - \\
\hline $04 / 11 / 2000$ & - & - & - & - & - & - & - & - \\
\hline $04 / 23 / 2000$ & - & - & - & - & - & - & - & - \\
\hline $05 / 05 / 2000$ & - & - & - & - & - & - & - & - \\
\hline 05/17/2000 & - & - & - & - & - & - & - & - \\
\hline $05 / 29 / 2000$ & - & - & - & E0.001 & - & - & E0.005 & - \\
\hline $06 / 10 / 2000$ & - & - & - & E0.001 & - & - & E0.002 & - \\
\hline $06 / 29 / 2000$ & - & - & - & E0.001 & - & - & - & - \\
\hline $07 / 11 / 2000$ & - & - & - & E0.001 & - & - & - & - \\
\hline $07 / 23 / 2000$ & - & - & - & - & - & - & - & - \\
\hline 08/04/2000 & - & - & - & E0.001 & - & - & - & - \\
\hline $08 / 16 / 2000$ & - & - & - & E0.001 & - & - & - & - \\
\hline $08 / 28 / 2000$ & - & - & - & E0.001 & - & - & - & - \\
\hline 09/09/2000 & - & - & - & E0.001 & - & - & - & - \\
\hline $09 / 21 / 2000$ & - & - & - & E0.001 & - & - & - & - \\
\hline $10 / 03 / 2000$ & - & - & - & E0.001 & - & - & - & - \\
\hline $11 / 08 / 2000$ & - & - & - & E0.001 & - & - & - & - \\
\hline $11 / 20 / 2000$ & - & - & - & E0.005 & - & - & - & - \\
\hline $12 / 02 / 2000$ & - & - & - & E0.009 & - & - & - & - \\
\hline $12 / 14 / 2000$ & - & - & - & E0.007 & - & - & - & - \\
\hline $12 / 26 / 2000$ & - & - & - & E0.002 & - & - & - & - \\
\hline
\end{tabular}


Table 17D. Quality-control analytical laboratory blank results for volatile organic compounds (VOC) with high breakthrough volumes for the Sweetwater Reservoir air sampling site, San Diego County, California—Continued.

[The site identification number is 324141117001601. Concentrations are given in parts per billion by volume (ppbv). Sample volume for quality-assurance blank is 5.0 liters. Samples processed at 20.0 degrees Celsius and 760 torr; many samples were frozen at the laboratory and analyzed on the same day. Data for travel blanks and environmental samples collected on 10/27/2000 were not used because samples were contaminated during transport. mm/dd/yyyy, month/day/year; *, sample data lost; E, estimated value; NA, not analyzed; - , compound was not detected at a concentration above laboratory reporting level]

\begin{tabular}{ccccccccc}
\hline $\begin{array}{c}\text { Sampling } \\
\text { date } \\
\text { (mm/dd/yyyy) }\end{array}$ & $\begin{array}{c}\mathbf{1 , 2 , 4 - T r i -} \\
\text { chloro- } \\
\text { benzene }\end{array}$ & $\begin{array}{c}\mathbf{1 , 3 - D i -} \\
\text { chloro- } \\
\text { benzen }\end{array}$ & $\begin{array}{c}\text { 1,4-Di- } \\
\text { chloro- } \\
\text { benzene }\end{array}$ & $\begin{array}{c}\text { Naph- } \\
\text { thalene }\end{array}$ & $\begin{array}{c}\text { trans-1,3- } \\
\text { Dichloro- } \\
\text { propene }\end{array}$ & $\begin{array}{c}\text { cis-1,3-Di- } \\
\text { chloro- } \\
\text { propene }\end{array}$ & $\begin{array}{c}\text { Trichloro- } \\
\text { ethene } \\
\text { (TCE) }\end{array}$ & $\begin{array}{c}\text { Hexa- } \\
\text { chloro- } \\
\text { butadiene }\end{array}$ \\
\hline $01 / 07 / 2001$ & - & - & - & E0.003 & - & - & - & - \\
$01 / 19 / 2001$ & - & - & - & E0.005 & - & - & - & - \\
$01 / 31 / 2001$ & - & - & - & E0.002 & - & - & - & - \\
$02 / 12 / 2001$ & - & - & - & - & - & - & - & - \\
$02 / 24 / 2001$ & - & - & - & E0.001 & - & - & - & - \\
$03 / 08 / 2001$ & - & - & - & E0.002 & - & - & - & - \\
$03 / 20 / 2001$ & NA & NA & NA & NA & NA & NA & NA & NA \\
$04 / 01 / 2001$ & - & - & - & E0.002 & - & - & - & - \\
$04 / 13 / 2001$ & - & - & - & E0.002 & - & - & - & - \\
$04 / 25 / 2001$ & - & - & - & E0.008 & - & - & - & - \\
$05 / 07 / 2001$ & - & - & - & E0.008 & - & - & - & - \\
$05 / 19 / 2001$ & - & - & - & E0.001 & - & - & - & - \\
$05 / 31 / 2001$ & - & - & - & - & - & - & - & - \\
$06 / 12 / 2001$ & - & - & - & E0.001 & - & - & - & - \\
$06 / 24 / 2001$ & - & - & - & - & - & - & - & - \\
$07 / 05 / 2001$ & - & - & - & E0.003 & - & - & - & - \\
$07 / 16 / 2001$ & - & - & - & E0.002 & - & - & - & - \\
$08 / 08 / 2001$ & - & - & - & E0.005 & - & - & - \\
$08 / 23 / 2001$ & - & - & - & E0.001 & - & - & - & - \\
$09 / 16 / 2001$ & - & - & - & E0.007 & - & - & - & - \\
$09 / 28 / 2001$ & - & - & - & E0.002 & - & - & - & - \\
\hline
\end{tabular}


Table 17D. Quality-control analytical laboratory blank results for volatile organic compounds (VOC) with high breakthrough volumes for the Sweetwater Reservoir air sampling site, San Diego County, California-Continued.

[The site identification number is 324141117001601 . Concentrations are given in parts per billion by volume (ppbv). Sample volume for quality-assurance blank is 5.0 liters. Samples processed at 20.0 degrees Celsius and 760 torr; many samples were frozen at the laboratory and analyzed on the same day. Data for travel blanks and environmental samples collected on 10/27/2000 were not used because samples were contaminated during transport. mm/dd/yyyy, month/day/year; *, sample data lost; E, estimated value; NA, not analyzed; - , compound was not detected at a concentration above laboratory reporting level]

\begin{tabular}{|c|c|c|c|c|c|c|c|c|}
\hline $\begin{array}{l}\text { Sampling } \\
\text { date } \\
\text { (mm/dd/yyyy) }\end{array}$ & $\begin{array}{l}\text { Methyl } \\
\text { meth- } \\
\text { acrylate }\end{array}$ & $\begin{array}{c}1,2,3,4- \\
\text { Tetra- } \\
\text { methyl- } \\
\text { benzene }\end{array}$ & $\begin{array}{l}\text { Ethyl tert- } \\
\text { butyl } \\
\text { ether } \\
\text { (ETBE) }\end{array}$ & $\begin{array}{l}\text { tert-Amyl } \\
\text { methyl ether } \\
\text { (TAME) }\end{array}$ & $\begin{array}{c}\text { trans-1,4- } \\
\text { Dichloro-2- } \\
\text { butene }\end{array}$ & $\begin{array}{c}\text { Ethyl } \\
\text { meth- } \\
\text { acrylate }\end{array}$ & $\begin{array}{l}\text { Carbon } \\
\text { disulfide }\end{array}$ & $\begin{array}{l}\text { cis-1,2-Di- } \\
\text { chloro- } \\
\text { ethene }\end{array}$ \\
\hline $10 / 02 / 1999$ & NA & NA & NA & NA & NA & NA & NA & NA \\
\hline $10 / 13 / 1999$ & - & - & - & - & - & - & E0.003 & - \\
\hline $10 / 26 / 1999$ & - & - & - & - & - & - & - & - \\
\hline 11/07/1999 & - & - & - & - & - & - & - & - \\
\hline 11/19/1999 & - & - & - & - & - & - & - & - \\
\hline $12 / 13 / 1999$ & - & - & - & - & - & - & - & - \\
\hline $12 / 25 / 1999$ & - & - & - & - & - & - & - & - \\
\hline $01 / 06 / 2000$ & - & - & - & - & - & - & - & - \\
\hline $01 / 18 / 2000$ & - & - & - & - & - & - & - & - \\
\hline $01 / 30 / 2000$ & - & - & - & - & - & - & - & - \\
\hline $02 / 11 / 2000$ & - & - & - & - & - & - & - & - \\
\hline 03/18/2000 & - & - & - & - & - & - & - & - \\
\hline $04 / 11 / 2000$ & - & - & - & - & - & - & - & - \\
\hline 04/23/2000 & - & - & - & - & - & - & - & - \\
\hline $05 / 05 / 2000$ & - & - & - & - & - & - & - & - \\
\hline 05/17/2000 & - & - & - & - & - & - & - & - \\
\hline $05 / 29 / 2000$ & - & - & - & - & - & - & E0.002 & - \\
\hline $06 / 10 / 2000$ & - & - & - & - & - & - & E0.006 & - \\
\hline $06 / 29 / 2000$ & - & - & - & - & - & - & E0.006 & - \\
\hline $07 / 11 / 2000$ & - & - & - & - & - & - & E0.009 & - \\
\hline $07 / 23 / 2000$ & - & - & - & - & - & - & E0.001 & - \\
\hline 08/04/2000 & - & - & - & - & - & - & - & - \\
\hline $08 / 16 / 2000$ & - & - & - & - & - & - & - & - \\
\hline $08 / 28 / 2000$ & - & - & - & - & - & - & - & - \\
\hline 09/09/2000 & - & - & - & - & - & - & - & - \\
\hline $09 / 21 / 2000$ & - & - & - & - & - & - & E0.005 & - \\
\hline $10 / 03 / 2000$ & - & - & - & - & - & - & E0.004 & - \\
\hline $11 / 08 / 2000$ & - & - & - & - & - & - & - & - \\
\hline $11 / 20 / 2000$ & - & - & - & - & - & - & E0.006 & - \\
\hline $12 / 02 / 2000$ & - & - & - & - & - & - & E0.002 & - \\
\hline $12 / 14 / 2000$ & - & - & - & - & - & - & E0.010 & - \\
\hline $12 / 26 / 2000$ & - & - & - & - & - & - & - & - \\
\hline
\end{tabular}


Table 17D. Quality-control analytical laboratory blank results for volatile organic compounds (VOC) with high breakthrough volumes for the Sweetwater Reservoir air sampling site, San Diego County, California—Continued.

[The site identification number is 324141117001601. Concentrations are given in parts per billion by volume (ppbv). Sample volume for quality-assurance blank is 5.0 liters. Samples processed at 20.0 degrees Celsius and 760 torr; many samples were frozen at the laboratory and analyzed on the same day. Data for travel blanks and environmental samples collected on 10/27/2000 were not used because samples were contaminated during transport. mm/dd/yyyy, month/day/year; *, sample data lost; E, estimated value; NA, not analyzed; - , compound was not detected at a concentration above laboratory reporting level]

\begin{tabular}{ccccccccc}
\hline $\begin{array}{c}\text { Sampling } \\
\text { date } \\
\text { (mm/dd/yyyy) }\end{array}$ & $\begin{array}{c}\text { Methyl } \\
\text { meth- } \\
\text { acrylate }\end{array}$ & $\begin{array}{c}\mathbf{1 , 2 , 3 , 4 -} \\
\text { Tetra- } \\
\text { methyl- } \\
\text { benzene }\end{array}$ & $\begin{array}{c}\text { Ethyl tert- } \\
\text { butyl } \\
\text { ether } \\
\text { (ETBE) }\end{array}$ & $\begin{array}{c}\text { tert-Amyl } \\
\text { methyl ether } \\
\text { (TAME) }\end{array}$ & $\begin{array}{c}\text { trans-1,4- } \\
\text { Dichloro-2- } \\
\text { butene }\end{array}$ & $\begin{array}{c}\text { Ethyl } \\
\text { meth- } \\
\text { acrylate }\end{array}$ & $\begin{array}{c}\text { Carbon } \\
\text { disulfide }\end{array}$ & $\begin{array}{c}\text { cis-1,2-Di- } \\
\text { chloro- } \\
\text { ethene }\end{array}$ \\
\hline $01 / 07 / 2001$ & - & - & - & - & - & - & E0.049 & - \\
$01 / 19 / 2001$ & - & - & - & - & - & - & - & - \\
$01 / 31 / 2001$ & - & - & - & - & - & - & - & - \\
$02 / 12 / 2001$ & - & - & - & - & - & - & E0.018 & - \\
$02 / 24 / 2001$ & - & - & - & - & - & - & - & - \\
$03 / 08 / 2001$ & - & - & - & - & - & - & E0.003 & - \\
$03 / 20 / 2001$ & NA & NA & NA & NA & NA & NA & NA & NA \\
$04 / 01 / 2001$ & - & - & - & - & - & - & E0.008 & - \\
$04 / 13 / 2001$ & - & - & - & - & - & - & E0.005 & - \\
$04 / 25 / 2001$ & - & - & - & - & - & - & E0.012 & - \\
$05 / 07 / 2001$ & - & - & - & - & - & - & E0.017 & - \\
$05 / 19 / 2001$ & - & - & - & - & - & - & E0.005 & - \\
$05 / 31 / 2001$ & - & - & - & - & - & - & E0.006 & - \\
$06 / 12 / 2001$ & - & - & - & - & - & - & - & - \\
$06 / 24 / 2001$ & - & - & - & - & - & - & - & - \\
$07 / 05 / 2001$ & - & - & - & - & - & - & - & - \\
$07 / 16 / 2001$ & - & - & - & - & - & - & E0.023 & - \\
$08 / 08 / 2001$ & - & - & - & - & - & - & - & - \\
$08 / 23 / 2001$ & - & - & - & - & - & - & - & - \\
$09 / 16 / 2001$ & - & - & - & - & - & - & E0.026 & - \\
$09 / 28 / 2001$ & - & - & - & - & - & - & - & - \\
\hline
\end{tabular}


Table 17D. Quality-control analytical laboratory blank results for volatile organic compounds (VOC) with high breakthrough volumes for the Sweetwater Reservoir air sampling site, San Diego County, California-Continued.

[The site identification number is 324141117001601 . Concentrations are given in parts per billion by volume (ppbv). Sample volume for quality-assurance blank is 5.0 liters. Samples processed at 20.0 degrees Celsius and 760 torr; many samples were frozen at the laboratory and analyzed on the same day. Data for travel blanks and environmental samples collected on 10/27/2000 were not used because samples were contaminated during transport. mm/dd/yyyy, month/day/year; , sample data lost; E, estimated value; NA, not analyzed; - , compound was not detected at a concentration above laboratory reporting level]

\begin{tabular}{|c|c|c|c|c|c|c|c|c|}
\hline $\begin{array}{c}\text { Sampling } \\
\text { date } \\
\text { (mm/dd/yyyy) }\end{array}$ & $\begin{array}{l}\text { 2-Hexanone } \\
\text { (MBK) }\end{array}$ & $\begin{array}{l}\text { Ethenyl- } \\
\text { benzene } \\
\text { (styrene) }\end{array}$ & o-Xylene & $\begin{array}{l}\text { 1,1-Di- } \\
\text { chloro- } \\
\text { propene }\end{array}$ & $\begin{array}{c}2,2-\mathrm{Di} \\
\text { chloro- } \\
\text { propane }\end{array}$ & $\begin{array}{l}\text { 1,3-Di- } \\
\text { chloro- } \\
\text { propane }\end{array}$ & $\begin{array}{l}\text { 2-Ethyl- } \\
\text { toluene }\end{array}$ & $\begin{array}{c}1,2,3-T r i- \\
\text { methyl- } \\
\text { benzene }\end{array}$ \\
\hline 10/02/1999 & NA & NA & NA & NA & NA & NA & NA & NA \\
\hline 10/13/1999 & - & E0.001 & E0.001 & - & - & - & - & - \\
\hline 10/26/1999 & - & E0.002 & E0.003 & - & - & - & - & - \\
\hline 11/07/1999 & - & E0.001 & E0.001 & - & - & - & - & - \\
\hline 11/19/1999 & - & - & - & - & - & - & - & - \\
\hline $12 / 13 / 1999$ & - & E0.001 & - & - & - & - & - & - \\
\hline $12 / 25 / 1999$ & - & - & E0.001 & - & - & - & - & - \\
\hline 01/06/2000 & - & E0.001 & - & - & - & - & - & - \\
\hline 01/18/2000 & - & E0.001 & E0.002 & - & - & - & - & - \\
\hline 01/30/2000 & - & - & E0.001 & - & - & - & - & - \\
\hline $02 / 11 / 2000$ & - & - & - & - & - & - & - & - \\
\hline 03/18/2000 & - & - & - & - & - & - & - & - \\
\hline $04 / 11 / 2000$ & - & E0.001 & - & - & - & - & - & - \\
\hline $04 / 23 / 2000$ & - & E0.001 & - & - & - & - & - & - \\
\hline 05/05/2000 & - & - & - & - & - & - & - & - \\
\hline 05/17/2000 & - & - & - & - & - & - & - & - \\
\hline 05/29/2000 & - & E0.001 & - & - & - & - & - & - \\
\hline 06/10/2000 & - & - & - & - & - & - & - & - \\
\hline 06/29/2000 & - & E0.002 & - & - & - & - & - & - \\
\hline 07/11/2000 & - & E0.001 & - & - & - & - & - & - \\
\hline 07/23/2000 & - & - & - & - & - & - & - & - \\
\hline 08/04/2000 & - & - & - & - & - & - & - & - \\
\hline 08/16/2000 & - & - & - & - & - & - & - & - \\
\hline 08/28/2000 & - & - & - & - & - & - & - & - \\
\hline 09/09/2000 & - & - & - & - & - & - & - & - \\
\hline 09/21/2000 & - & - & - & - & - & - & - & - \\
\hline $10 / 03 / 2000$ & - & - & - & - & - & - & - & - \\
\hline $11 / 08 / 2000$ & - & - & - & - & - & - & - & - \\
\hline $11 / 20 / 2000$ & - & E0.005 & E0.003 & - & - & - & - & - \\
\hline $12 / 02 / 2000$ & - & E0.002 & E0.004 & - & - & - & - & - \\
\hline $12 / 14 / 2000$ & - & E0.001 & E0.002 & - & - & - & - & - \\
\hline $12 / 26 / 2000$ & - & - & E0.002 & - & - & - & - & - \\
\hline
\end{tabular}


Table 17D. Quality-control analytical laboratory blank results for volatile organic compounds (VOC) with high breakthrough volumes for the Sweetwater Reservoir air sampling site, San Diego County, California—Continued.

[The site identification number is 324141117001601. Concentrations are given in parts per billion by volume (ppbv). Sample volume for quality-assurance blank is 5.0 liters. Samples processed at 20.0 degrees Celsius and 760 torr; many samples were frozen at the laboratory and analyzed on the same day. Data for travel blanks and environmental samples collected on 10/27/2000 were not used because samples were contaminated during transport. mm/dd/yyyy, month/day/year; *, sample data lost; E, estimated value; NA, not analyzed; - , compound was not detected at a concentration above laboratory reporting level]

\begin{tabular}{ccccccccc}
\hline $\begin{array}{c}\text { Sampling } \\
\text { date } \\
\text { (mm/dd/yyy) }\end{array}$ & $\begin{array}{c}\text { 2-Hexanone } \\
\text { (MBK) }\end{array}$ & $\begin{array}{c}\text { Ethenyl- } \\
\text { benzene } \\
\text { (styrene) }\end{array}$ & o-Xylene & $\begin{array}{c}\text { 1,1-Di- } \\
\text { chloro- } \\
\text { propene }\end{array}$ & $\begin{array}{c}\text { 2,2-Di } \\
\text { chloro- } \\
\text { propane }\end{array}$ & $\begin{array}{c}\text { 1,3-Di- } \\
\text { chloro- } \\
\text { propane }\end{array}$ & $\begin{array}{c}\text { 2-Ethyl- } \\
\text { toluene }\end{array}$ & $\begin{array}{c}\text { 1,2,3-Tri- } \\
\text { methyl- } \\
\text { benzene }\end{array}$ \\
\hline $01 / 07 / 2001$ & - & - & - & - & - & - & - & - \\
$01 / 19 / 2001$ & - & - & - & - & - & - & - & - \\
$01 / 31 / 2001$ & - & - & - & - & - & - & - & - \\
$02 / 12 / 2001$ & - & E0.005 & E0.002 & - & - & - & - & - \\
$02 / 24 / 2001$ & - & - & E0.001 & - & - & - & - & - \\
$03 / 08 / 2001$ & - & E0.001 & E0.001 & - & - & - & - & - \\
$03 / 20 / 2001$ & NA & NA & NA & NA & NA & NA & NA & NA \\
$04 / 01 / 2001$ & - & E0.002 & E0.001 & - & - & - & - & - \\
$04 / 13 / 2001$ & - & E0.001 & E0.002 & - & - & - & - & - \\
$04 / 25 / 2001$ & - & E0.002 & E0.001 & - & - & - & - & - \\
$05 / 07 / 2001$ & - & E0.003 & E0.001 & - & - & - & - & - \\
$05 / 19 / 2001$ & - & - & - & - & - & - & - & - \\
$05 / 31 / 2001$ & - & - & E0.001 & - & - & - & - & - \\
$06 / 12 / 2001$ & - & - & - & - & - & - & - & - \\
$06 / 24 / 2001$ & - & - & - & - & - & - & - & - \\
$07 / 05 / 2001$ & - & - & - & - & - & - & - & - \\
$07 / 16 / 2001$ & - & - & - & - & - & - & - & - \\
$08 / 08 / 2001$ & - & E0.012 & E0.013 & - & - & - & - & - \\
$08 / 23 / 2001$ & - & - & - & - & - & - & - & - \\
$09 / 16 / 2001$ & - & E0.004 & E0.001 & - & - & - & - & - \\
$09 / 28 / 2001$ & - & - & E0.001 & - & - & - & - & - \\
\hline
\end{tabular}


Table 17D. Quality-control analytical laboratory blank results for volatile organic compounds (VOC) with high breakthrough volumes for the Sweetwater Reservoir air sampling site, San Diego County, California-Continued.

[The site identification number is 324141117001601 . Concentrations are given in parts per billion by volume (ppbv). Sample volume for quality-assurance blank is 5.0 liters. Samples processed at 20.0 degrees Celsius and 760 torr; many samples were frozen at the laboratory and analyzed on the same day. Data for travel blanks and environmental samples collected on 10/27/2000 were not used because samples were contaminated during transport. mm/dd/yyyy, month/day/year; *, sample data lost; E, estimated value; NA, not analyzed; - , compound was not detected at a concentration above laboratory reporting level]

\begin{tabular}{|c|c|c|c|c|c|c|c|c|}
\hline $\begin{array}{c}\text { Sampling } \\
\text { date } \\
\text { (mm/dd/yyyy) }\end{array}$ & $\begin{array}{c}\text { 1,2,4-Tri- } \\
\text { methyl- } \\
\text { benzene }\end{array}$ & $\begin{array}{l}\text { Isopropyl- } \\
\text { benzene } \\
\text { (Cumene) }\end{array}$ & $\begin{array}{l}n \text {-Propyl- } \\
\text { benzene }\end{array}$ & $\begin{array}{c}\text { 1,3,5-Tri- } \\
\text { methyl- } \\
\text { benzene }\end{array}$ & $\begin{array}{c}\text { 1-Chloro-2- } \\
\text { methyl- } \\
\text { benzene }\end{array}$ & $\begin{array}{c}\text { 1-Chloro-4- } \\
\text { methyl- } \\
\text { benzene }\end{array}$ & $\begin{array}{l}\text { Bromo- } \\
\text { chloro- } \\
\text { methane }\end{array}$ & $\begin{array}{l}n \text {-Butyl- } \\
\text { benzene }\end{array}$ \\
\hline $10 / 02 / 1999$ & NA & NA & NA & NA & NA & NA & NA & NA \\
\hline 10/13/1999 & E0.001 & - & - & - & - & - & - & - \\
\hline 10/26/1999 & E0.002 & - & - & - & - & - & - & - \\
\hline 11/07/1999 & E0.001 & - & - & - & - & - & - & - \\
\hline 11/19/1999 & - & - & - & - & - & - & - & - \\
\hline 12/13/1999 & - & - & - & - & - & - & - & - \\
\hline $12 / 25 / 1999$ & - & - & - & - & - & - & - & - \\
\hline 01/06/2000 & - & - & - & - & - & - & - & - \\
\hline $01 / 18 / 2000$ & E0.001 & - & - & - & - & - & - & - \\
\hline 01/30/2000 & - & - & - & - & - & - & - & - \\
\hline 02/11/2000 & - & - & - & - & - & - & - & - \\
\hline 03/18/2000 & - & - & - & - & - & - & - & - \\
\hline $04 / 11 / 2000$ & - & - & - & - & - & - & - & - \\
\hline $04 / 23 / 2000$ & - & - & - & - & - & - & - & - \\
\hline 05/05/2000 & - & - & - & - & - & - & - & - \\
\hline 05/17/2000 & - & - & - & - & - & - & - & - \\
\hline 05/29/2000 & - & - & - & - & - & - & - & - \\
\hline 06/10/2000 & - & - & - & - & - & - & - & - \\
\hline 06/29/2000 & - & - & - & - & - & - & - & - \\
\hline $07 / 11 / 2000$ & - & - & - & - & - & - & - & - \\
\hline 07/23/2000 & - & - & - & - & - & - & - & - \\
\hline 08/04/2000 & - & - & - & - & - & - & - & - \\
\hline 08/16/2000 & - & - & - & - & - & - & - & - \\
\hline $08 / 28 / 2000$ & - & - & - & - & - & - & - & - \\
\hline 09/09/2000 & - & - & - & - & - & - & - & - \\
\hline 09/21/2000 & - & - & - & - & - & - & - & - \\
\hline $10 / 03 / 2000$ & - & - & - & - & - & - & - & - \\
\hline $11 / 08 / 2000$ & - & - & - & - & - & - & - & - \\
\hline $11 / 20 / 2000$ & - & - & - & - & - & - & - & - \\
\hline $12 / 02 / 2000$ & E0.007 & - & - & - & - & - & - & - \\
\hline $12 / 14 / 2000$ & - & - & - & - & - & - & - & - \\
\hline $12 / 26 / 2000$ & - & - & - & - & - & - & - & - \\
\hline
\end{tabular}


Table 17D. Quality-control analytical laboratory blank results for volatile organic compounds (VOC) with high breakthrough volumes for the Sweetwater Reservoir air sampling site, San Diego County, California—Continued.

[The site identification number is 324141117001601. Concentrations are given in parts per billion by volume (ppbv). Sample volume for quality-assurance blank is 5.0 liters. Samples processed at 20.0 degrees Celsius and 760 torr; many samples were frozen at the laboratory and analyzed on the same day. Data for travel blanks and environmental samples collected on 10/27/2000 were not used because samples were contaminated during transport. mm/dd/yyyy, month/day/year; *, sample data lost; E, estimated value; NA, not analyzed; - , compound was not detected at a concentration above laboratory reporting level]

\begin{tabular}{|c|c|c|c|c|c|c|c|c|}
\hline $\begin{array}{c}\text { Sampling } \\
\text { date } \\
\text { (mm/dd/yyyy) }\end{array}$ & $\begin{array}{c}\text { 1,2,4-Tri- } \\
\text { methyl- } \\
\text { benzene }\end{array}$ & $\begin{array}{l}\text { Isopropyl- } \\
\text { benzene } \\
\text { (Cumene) }\end{array}$ & $\begin{array}{l}n \text {-Propyl- } \\
\text { benzene }\end{array}$ & $\begin{array}{c}\text { 1,3,5-Tri- } \\
\text { methyl- } \\
\text { benzene }\end{array}$ & $\begin{array}{c}\text { 1-Chloro-2- } \\
\text { methyl- } \\
\text { benzene }\end{array}$ & $\begin{array}{c}\text { 1-Chloro-4- } \\
\text { methyl- } \\
\text { benzene }\end{array}$ & $\begin{array}{l}\text { Bromo- } \\
\text { chloro- } \\
\text { methane }\end{array}$ & $\begin{array}{l}n \text {-Butyl- } \\
\text { benzene }\end{array}$ \\
\hline 01/07/2001 & - & - & - & - & - & - & - & - \\
\hline 01/19/2001 & - & - & - & - & - & - & - & - \\
\hline $01 / 31 / 2001$ & - & - & - & - & - & - & - & - \\
\hline $02 / 12 / 2001$ & - & - & - & - & - & - & - & - \\
\hline $02 / 24 / 2001$ & - & - & - & - & - & - & - & - \\
\hline 03/08/2001 & - & - & - & - & - & - & - & - \\
\hline 03/20/2001 & NA & NA & NA & NA & NA & NA & NA & NA \\
\hline 04/01/2001 & - & - & - & - & - & - & - & - \\
\hline $04 / 13 / 2001$ & - & - & - & - & - & - & - & - \\
\hline $04 / 25 / 2001$ & - & - & - & - & - & - & - & - \\
\hline 05/07/2001 & - & - & - & - & - & - & - & - \\
\hline 05/19/2001 & - & - & - & - & - & - & - & - \\
\hline $05 / 31 / 2001$ & - & - & - & - & - & - & - & - \\
\hline $06 / 12 / 2001$ & - & - & - & - & - & - & - & - \\
\hline $06 / 24 / 2001$ & - & - & - & - & - & - & - & - \\
\hline 07/05/2001 & - & - & - & - & - & - & - & - \\
\hline $07 / 16 / 2001$ & - & - & - & - & - & - & - & - \\
\hline 08/08/2001 & - & - & - & - & - & - & - & - \\
\hline $08 / 23 / 2001$ & - & - & - & - & - & - & - & - \\
\hline 09/16/2001 & - & - & - & - & - & - & - & - \\
\hline $09 / 28 / 2001$ & - & - & - & - & - & - & - & - \\
\hline
\end{tabular}


Table 17D. Quality-control analytical laboratory blank results for volatile organic compounds (VOC) with high breakthrough volumes for the Sweetwater Reservoir air sampling site, San Diego County, California-Continued.

[The site identification number is 324141117001601 . Concentrations are given in parts per billion by volume (ppbv). Sample volume for quality-assurance blank is 5.0 liters. Samples processed at 20.0 degrees Celsius and 760 torr; many samples were frozen at the laboratory and analyzed on the same day. Data for travel blanks and environmental samples collected on 10/27/2000 were not used because samples were contaminated during transport. mm/dd/yyyy, month/day/year; *, sample data lost; E, estimated value; NA, not analyzed; - , compound was not detected at a concentration above laboratory reporting level]

\begin{tabular}{|c|c|c|c|c|c|c|c|c|}
\hline $\begin{array}{c}\text { Sampling } \\
\text { date } \\
\text { (mm/dd/yyyy) }\end{array}$ & $\begin{array}{c}\text { sec-Butyl- } \\
\text { benzene }\end{array}$ & $\begin{array}{c}\text { tert-Butyl- } \\
\text { benzene }\end{array}$ & $\begin{array}{l}\text { 1-Isopropyl- } \\
\text { 4-methyl- } \\
\text { benzene }\end{array}$ & $\begin{array}{l}\text { 1,2,3-Tri- } \\
\text { chloro- } \\
\text { propane }\end{array}$ & $\begin{array}{l}\text { 1,1,1,2-Tetra- } \\
\text { chloro- } \\
\text { ethane }\end{array}$ & $\begin{array}{c}\text { 1,2,3-Tri- } \\
\text { chloro- } \\
\text { benzene }\end{array}$ & $\begin{array}{l}\text { 1,2-Di- } \\
\text { bromo- } \\
\text { ethane }\end{array}$ & $\begin{array}{l}\text { Methyl tert- } \\
\text { butyl ether } \\
\text { (MTBE) }\end{array}$ \\
\hline $10 / 02 / 1999$ & NA & NA & NA & NA & NA & NA & NA & NA \\
\hline 10/13/1999 & - & - & - & - & - & - & - & - \\
\hline 10/26/1999 & - & - & - & - & - & - & - & - \\
\hline 11/07/1999 & - & - & - & - & - & - & - & - \\
\hline 11/19/1999 & - & - & - & - & - & - & - & - \\
\hline 12/13/1999 & - & - & - & - & - & - & - & - \\
\hline $12 / 25 / 1999$ & - & - & - & - & - & - & - & - \\
\hline 01/06/2000 & - & - & - & - & - & - & - & - \\
\hline $01 / 18 / 2000$ & - & - & - & - & - & - & - & - \\
\hline 01/30/2000 & - & - & - & - & - & - & - & - \\
\hline $02 / 11 / 2000$ & - & - & - & - & - & - & - & - \\
\hline 03/18/2000 & - & - & - & - & - & - & - & - \\
\hline $04 / 11 / 2000$ & - & - & - & - & - & - & - & - \\
\hline $04 / 23 / 2000$ & - & - & - & - & - & - & - & - \\
\hline 05/05/2000 & - & - & - & - & - & - & - & - \\
\hline 05/17/2000 & - & - & - & - & - & - & - & - \\
\hline 05/29/2000 & - & - & - & - & - & - & - & - \\
\hline $06 / 10 / 2000$ & - & - & - & - & - & - & - & - \\
\hline 06/29/2000 & - & - & - & - & - & - & - & - \\
\hline $07 / 11 / 2000$ & - & - & - & - & - & - & - & - \\
\hline $07 / 23 / 2000$ & - & - & - & - & - & - & - & - \\
\hline 08/04/2000 & - & - & - & - & - & - & - & - \\
\hline $08 / 16 / 2000$ & - & - & - & - & - & - & - & - \\
\hline $08 / 28 / 2000$ & - & - & - & - & - & - & - & - \\
\hline 09/09/2000 & - & - & - & - & - & - & - & - \\
\hline 09/21/2000 & - & - & - & - & - & - & - & - \\
\hline $10 / 03 / 2000$ & - & - & - & - & - & - & - & - \\
\hline $11 / 08 / 2000$ & - & - & - & - & - & - & - & - \\
\hline $11 / 20 / 2000$ & - & - & - & - & - & - & - & - \\
\hline $12 / 02 / 2000$ & - & - & - & - & - & - & - & - \\
\hline $12 / 14 / 2000$ & - & - & - & - & - & - & - & - \\
\hline $12 / 26 / 2000$ & - & - & - & - & - & - & - & - \\
\hline
\end{tabular}


Table 17D. Quality-control analytical laboratory blank results for volatile organic compounds (VOC) with high breakthrough volumes for the Sweetwater Reservoir air sampling site, San Diego County, California—Continued.

[The site identification number is 324141117001601. Concentrations are given in parts per billion by volume (ppbv). Sample volume for quality-assurance blank is 5.0 liters. Samples processed at 20.0 degrees Celsius and 760 torr; many samples were frozen at the laboratory and analyzed on the same day. Data for travel blanks and environmental samples collected on 10/27/2000 were not used because samples were contaminated during transport. mm/dd/yyyy, month/day/year; *, sample data lost; E, estimated value; NA, not analyzed; - , compound was not detected at a concentration above laboratory reporting level]

\begin{tabular}{ccccccccc}
\hline $\begin{array}{c}\text { Sampling } \\
\text { date } \\
\text { (mm/dd/yyyy) }\end{array}$ & $\begin{array}{c}\text { sec-Butyl- } \\
\text { benzene }\end{array}$ & $\begin{array}{c}\text { tert-Butyl- } \\
\text { benzene }\end{array}$ & $\begin{array}{c}\text { 1-Isopropyl- } \\
\text { 4-methyl- } \\
\text { benzen }\end{array}$ & $\begin{array}{c}\mathbf{1 , 2 , 3 - T r i -} \\
\text { chloro- } \\
\text { propane }\end{array}$ & $\begin{array}{c}\mathbf{1 , 1 , 1 , 2 - T e t r a - ~} \\
\text { chloro- } \\
\text { ethane }\end{array}$ & $\begin{array}{c}\text { 1,2,3-Tri- } \\
\text { chloro- } \\
\text { benzene }\end{array}$ & $\begin{array}{c}\text { 1,2-Di- } \\
\text { bromo- } \\
\text { ethane }\end{array}$ & $\begin{array}{c}\text { Methyl tert- } \\
\text { butyl ether } \\
\text { (MTBE) }\end{array}$ \\
\hline $01 / 07 / 2001$ & - & - & - & - & - & - & - & - \\
$01 / 19 / 2001$ & - & - & - & - & - & - & - & - \\
$01 / 31 / 2001$ & - & - & - & - & - & - & - & - \\
$02 / 12 / 2001$ & - & - & - & - & E0.009 & - & - & - \\
$02 / 24 / 2001$ & - & - & - & - & - & - & - & - \\
$03 / 08 / 2001$ & - & - & - & - & - & - & - & - \\
$03 / 20 / 2001$ & NA & NA & NA & NA & NA & NA & NA & NA \\
$04 / 01 / 2001$ & - & - & - & - & - & - & - & - \\
$04 / 13 / 2001$ & - & - & - & - & - & - & - & - \\
$04 / 25 / 2001$ & - & - & - & - & - & - & - & - \\
$05 / 07 / 2001$ & - & - & - & - & - & - & - & - \\
$05 / 19 / 2001$ & - & - & - & - & - & - & - & - \\
$05 / 31 / 2001$ & - & - & - & - & - & - & - & - \\
$06 / 12 / 2001$ & - & - & - & - & - & - & - & - \\
$06 / 24 / 2001$ & - & - & - & - & - & - & - & - \\
$07 / 05 / 2001$ & - & - & - & - & - & - & - & - \\
$07 / 16 / 2001$ & - & - & - & - & - & - & - \\
$08 / 08 / 2001$ & - & - & - & - & - & - & - \\
$08 / 23 / 2001$ & - & - & - & - & - & - & - \\
$09 / 16 / 2001$ & - & - & - & - & - & - & - \\
$09 / 28 / 2001$ & - & - & - & - & - & - & - & - \\
\hline
\end{tabular}


Table 17D. Quality-control analytical laboratory blank results for volatile organic compounds (VOC) with high breakthrough volumes for the Sweetwater Reservoir air sampling site, San Diego County, California-Continued.

[The site identification number is 324141117001601 . Concentrations are given in parts per billion by volume (ppbv). Sample volume for quality-assurance blank is 5.0 liters. Samples processed at 20.0 degrees Celsius and 760 torr; many samples were frozen at the laboratory and analyzed on the same day. Data for travel blanks and environmental samples collected on 10/27/2000 were not used because samples were contaminated during transport. mm/dd/yyyy, month/day/year; *, sample data lost; E, estimated value; NA, not analyzed; - , compound was not detected at a concentration above laboratory reporting level]

\begin{tabular}{|c|c|c|c|c|c|c|c|c|c|}
\hline $\begin{array}{c}\text { Sampling } \\
\text { date } \\
\text { (mm/dd/yyyy) }\end{array}$ & $\begin{array}{c}\text { 3-Chloro-1- } \\
\text { propene }\end{array}$ & $\begin{array}{l}\text { 4-Methyl-2- } \\
\text { pentanone } \\
\text { (MIBK) }\end{array}$ & Acetone & $\begin{array}{l}\text { Bromo- } \\
\text { benzene }\end{array}$ & $\begin{array}{l}\text { Diethyl } \\
\text { ether }\end{array}$ & $\begin{array}{l}\text { Diisopro- } \\
\text { pyl ether } \\
\text { (DIPE) }\end{array}$ & $\begin{array}{l}\text { Methyl } \\
\text { acrylo- } \\
\text { nitrile }\end{array}$ & $\begin{array}{c}\text { 2-Butanone } \\
\text { (methyl ethyl } \\
\text { ketone) }\end{array}$ & $\begin{array}{l}\text { Methyl } \\
\text { acrylate }\end{array}$ \\
\hline 10/02/1999 & NA & NA & NA & NA & NA & NA & NA & NA & NA \\
\hline $10 / 13 / 1999$ & - & - & E0.075 & - & - & - & - & - & - \\
\hline 10/26/1999 & - & - & 0.225 & - & - & - & - & - & - \\
\hline 11/07/1999 & - & - & E0.087 & - & - & - & - & - & - \\
\hline $11 / 19 / 1999$ & - & - & E0.044 & - & - & - & - & - & - \\
\hline $12 / 13 / 1999$ & - & - & E0.057 & - & - & - & - & - & - \\
\hline $12 / 25 / 1999$ & - & - & E0.055 & - & - & - & - & - & - \\
\hline 01/06/2000 & - & - & E0.046 & - & - & - & - & - & - \\
\hline $01 / 18 / 2000$ & - & - & E0.073 & - & - & - & - & - & - \\
\hline $01 / 30 / 2000$ & - & - & E0.040 & - & - & - & - & - & - \\
\hline $02 / 11 / 2000$ & - & - & E0.065 & - & - & - & - & - & - \\
\hline 03/18/2000 & - & - & 0.165 & - & - & - & - & - & - \\
\hline $04 / 11 / 2000$ & - & - & E0.017 & - & - & - & - & - & - \\
\hline $04 / 23 / 2000$ & - & - & E0.020 & - & - & - & - & - & - \\
\hline 05/05/2000 & - & - & E0.067 & - & - & - & - & - & - \\
\hline $05 / 17 / 2000$ & - & - & E0.015 & - & - & - & - & - & - \\
\hline 05/29/2000 & - & - & 0.475 & - & - & - & - & - & - \\
\hline $06 / 10 / 2000$ & - & - & 0.237 & - & - & - & - & - & - \\
\hline 06/29/2000 & - & - & E0.076 & - & - & - & - & - & - \\
\hline $07 / 11 / 2000$ & - & - & E0.076 & - & - & - & - & - & - \\
\hline 07/23/2000 & - & - & 0.202 & - & - & - & - & - & - \\
\hline 08/04/2000 & - & - & 0.463 & - & - & - & - & - & - \\
\hline 08/16/2000 & - & - & 0.163 & - & - & - & - & - & - \\
\hline $08 / 28 / 2000$ & - & - & E0.112 & - & - & - & - & - & - \\
\hline 09/09/2000 & - & - & E0.127 & - & - & - & - & - & - \\
\hline $09 / 21 / 2000$ & - & - & E0.076 & - & - & - & - & - & - \\
\hline $10 / 03 / 2000$ & - & - & E0.042 & - & - & - & - & - & - \\
\hline $11 / 08 / 2000$ & - & - & E0.046 & - & - & - & - & - & - \\
\hline $11 / 20 / 2000$ & - & - & 0.248 & - & - & - & - & E0.020 & - \\
\hline $12 / 02 / 2000$ & - & - & E0.133 & - & - & - & - & E0.002 & - \\
\hline $12 / 14 / 2000$ & - & - & E0.126 & - & - & - & - & - & - \\
\hline $12 / 26 / 2000$ & - & - & E0.032 & - & - & - & - & - & - \\
\hline
\end{tabular}


Table 17D. Quality-control analytical laboratory blank results for volatile organic compounds (VOC) with high breakthrough volumes for the Sweetwater Reservoir air sampling site, San Diego County, California—Continued.

[The site identification number is 324141117001601. Concentrations are given in parts per billion by volume (ppbv). Sample volume for quality-assurance blank is 5.0 liters. Samples processed at 20.0 degrees Celsius and 760 torr; many samples were frozen at the laboratory and analyzed on the same day. Data for travel blanks and environmental samples collected on 10/27/2000 were not used because samples were contaminated during transport. mm/dd/yyyy, month/day/year; *, sample data lost; E, estimated value; NA, not analyzed; - , compound was not detected at a concentration above laboratory reporting level]

\begin{tabular}{cccccccccc}
\hline $\begin{array}{c}\text { Sampling } \\
\text { date } \\
\text { (mm/dd/yyyy) }\end{array}$ & $\begin{array}{c}\text { 3-Chloro-1- } \\
\text { propene }\end{array}$ & $\begin{array}{c}\text { 4-Methyl-2- } \\
\text { pentanone } \\
\text { (MIBK) }\end{array}$ & Acetone & $\begin{array}{c}\text { Bromo- } \\
\text { benzene }\end{array}$ & $\begin{array}{c}\text { Diethyl } \\
\text { ether }\end{array}$ & $\begin{array}{c}\text { Diisopro- } \\
\text { pyl ether } \\
\text { (DIPE) }\end{array}$ & $\begin{array}{c}\text { Methyl } \\
\text { acrylo- } \\
\text { nitrile }\end{array}$ & $\begin{array}{c}\text { 2-Butanone } \\
\text { (methyl ethyl } \\
\text { ketone) }\end{array}$ & $\begin{array}{c}\text { Methyl } \\
\text { acrylate }\end{array}$ \\
\hline $01 / 07 / 2001$ & - & - & E0.058 & - & - & - & - & - & - \\
$01 / 19 / 2001$ & - & - & E0.144 & - & - & - & - & - & - \\
$01 / 31 / 2001$ & - & - & E0.144 & - & - & - & - & - & - \\
$02 / 12 / 2001$ & - & - & 0.245 & - & - & - & - & - & - \\
$02 / 24 / 2001$ & - & - & E0.117 & - & - & - & - & - & - \\
$03 / 08 / 2001$ & - & - & E0.123 & - & - & - & - & - & - \\
$03 / 20 / 2001$ & NA & NA & NA & NA & NA & NA & NA & NA & NA \\
$04 / 01 / 2001$ & - & - & E0.080 & - & - & - & - & - & - \\
$04 / 13 / 2001$ & - & - & E0.050 & - & - & - & - & - & - \\
$04 / 25 / 2001$ & - & - & E0.141 & - & - & - & - & - & - \\
$05 / 07 / 2001$ & - & - & 0.192 & - & - & - & - & - & - \\
$05 / 19 / 2001$ & - & - & E0.082 & - & - & - & - & - & - \\
$05 / 31 / 2001$ & - & - & E0.048 & - & - & - & - & - & - \\
$06 / 12 / 2001$ & - & - & E0.090 & - & - & - & - & - & - \\
$06 / 24 / 2001$ & - & - & 0.213 & - & - & - & - & - & - \\
$07 / 05 / 2001$ & - & - & E0.074 & - & - & - & - & - \\
$07 / 16 / 2001$ & - & - & E0.165 & - & - & - & - & - \\
$08 / 08 / 2001$ & - & - & - & - & - & - & - & - \\
$08 / 23 / 2001$ & - & - & E0.126 & - & - & - & - & - & - \\
$09 / 16 / 2001$ & - & - & 0.318 & - & - & - & - & - & - \\
$09 / 28 / 2001$ & - & - & 0.078 & - & - & - & - & - & - \\
\hline
\end{tabular}


Table 17D. Quality-control analytical laboratory blank results for volatile organic compounds (VOC) with high breakthrough volumes for the Sweetwater Reservoir air sampling site, San Diego County, California-Continued.

[The site identification number is 324141117001601 . Concentrations are given in parts per billion by volume (ppbv). Sample volume for quality-assurance blank is 5.0 liters. Samples processed at 20.0 degrees Celsius and 760 torr; many samples were frozen at the laboratory and analyzed on the same day. Data for travel blanks and environmental samples collected on 10/27/2000 were not used because samples were contaminated during transport. mm/dd/yyyy, month/day/year; *, sample data lost; E, estimated value; NA, not analyzed; - , compound was not detected at a concentration above laboratory reporting level]

\begin{tabular}{|c|c|c|c|c|c|c|c|c|}
\hline $\begin{array}{c}\text { Sampling } \\
\text { date } \\
\text { (mm/dd/yyyy) }\end{array}$ & $\begin{array}{l}\text { Tetrahy- } \\
\text { drofuran }\end{array}$ & $\begin{array}{c}\text { 1,2-Dibromo- } \\
\text { 3-chloro- } \\
\text { propane } \\
\text { (DBCP) }\end{array}$ & $\begin{array}{c}m \text { - and p- } \\
\text { Xylene }\end{array}$ & $\begin{array}{c}\text { 1,2,3,5-Tetra- } \\
\text { methyl- } \\
\text { benzene }\end{array}$ & $\begin{array}{c}1,2,4,5- \\
\text { Tetra- } \\
\text { methyl- } \\
\text { benzene }\end{array}$ & $\begin{array}{l}\text { Methyl } \\
\text { acetate }\end{array}$ & $\begin{array}{l}\text { 2-Methyl- } \\
\text { 2-butanol } \\
\text { (tert-Amyl } \\
\text { alcohol) }\end{array}$ & $\begin{array}{l}\text { 2-Methyl-2- } \\
\text { propanol (tert- } \\
\text { Butyl alcohol) }\end{array}$ \\
\hline 10/02/1999 & NA & NA & NA & NA & NA & NA & $\mathrm{NA}$ & NA \\
\hline 10/13/1999 & - & - & E0.003 & - & - & - & - & - \\
\hline 10/26/1999 & - & - & E0.007 & - & - & - & - & - \\
\hline 11/07/1999 & - & - & E0.002 & - & - & - & - & - \\
\hline 11/19/1999 & - & - & - & - & - & - & - & - \\
\hline 12/13/1999 & - & - & E0.001 & - & - & - & - & - \\
\hline 12/25/1999 & - & - & E0.002 & - & - & - & - & - \\
\hline 01/06/2000 & - & - & E0.001 & - & - & - & - & - \\
\hline 01/18/2000 & - & - & E0.004 & - & - & - & - & - \\
\hline 01/30/2000 & - & - & E0.003 & - & - & - & - & - \\
\hline $02 / 11 / 2000$ & - & - & E0.001 & - & - & - & - & - \\
\hline 03/18/2000 & - & - & - & - & - & - & - & - \\
\hline $04 / 11 / 2000$ & - & - & - & - & - & - & - & - \\
\hline $04 / 23 / 2000$ & - & - & - & - & - & - & - & - \\
\hline 05/05/2000 & - & - & - & - & - & - & - & - \\
\hline 05/17/2000 & - & - & - & - & - & - & - & - \\
\hline 05/29/2000 & - & - & E0.001 & - & - & - & - & E0.004 \\
\hline 06/10/2000 & - & - & - & - & - & - & - & - \\
\hline 06/29/2000 & - & - & E0.001 & - & - & - & - & - \\
\hline $07 / 11 / 2000$ & - & - & E0.001 & - & - & - & - & - \\
\hline 07/23/2000 & - & - & - & - & - & - & - & - \\
\hline 08/04/2000 & - & - & E0.001 & - & - & - & - & - \\
\hline 08/16/2000 & - & - & - & - & - & - & - & - \\
\hline 08/28/2000 & - & - & - & - & - & - & - & - \\
\hline 09/09/2000 & - & - & - & - & - & - & - & - \\
\hline 09/21/2000 & - & - & E0.001 & - & - & - & - & - \\
\hline $10 / 03 / 2000$ & - & - & - & - & - & - & - & - \\
\hline $11 / 08 / 2000$ & - & - & - & - & - & - & - & - \\
\hline $11 / 20 / 2000$ & - & - & E0.009 & - & - & - & - & - \\
\hline $12 / 02 / 2000$ & - & - & E0.012 & - & - & - & - & - \\
\hline $12 / 14 / 2000$ & - & - & E0.005 & - & - & - & - & - \\
\hline $12 / 26 / 2000$ & - & - & E0.003 & - & - & - & - & - \\
\hline
\end{tabular}


Table 17D. Quality-control analytical laboratory blank results for volatile organic compounds (VOC) with high breakthrough volumes for the Sweetwater Reservoir air sampling site, San Diego County, California—Continued.

[The site identification number is 324141117001601 . Concentrations are given in parts per billion by volume (ppbv). Sample volume for quality-assurance blank is 5.0 liters. Samples processed at 20.0 degrees Celsius and 760 torr; many samples were frozen at the laboratory and analyzed on the same day. Data for travel blanks and environmental samples collected on 10/27/2000 were not used because samples were contaminated during transport. mm/dd/yyyy, month/day/year; *, sample data lost; E, estimated value; NA, not analyzed; - , compound was not detected at a concentration above laboratory reporting level]

\begin{tabular}{|c|c|c|c|c|c|c|c|c|}
\hline $\begin{array}{l}\text { Sampling } \\
\text { date } \\
\text { (mm/dd/yyyy) }\end{array}$ & $\begin{array}{l}\text { Tetrahy- } \\
\text { drofuran }\end{array}$ & $\begin{array}{c}\text { 1,2-Dibromo- } \\
\text { 3-chloro- } \\
\text { propane } \\
\text { (DBCP) }\end{array}$ & $\begin{array}{c}\text { m- and p- } \\
\text { Xylene }\end{array}$ & $\begin{array}{c}\text { 1,2,3,5-Tetra- } \\
\text { methyl- } \\
\text { benzene }\end{array}$ & $\begin{array}{c}1,2,4,5- \\
\text { Tetra- } \\
\text { methyl- } \\
\text { benzene }\end{array}$ & $\begin{array}{l}\text { Methyl } \\
\text { acetate }\end{array}$ & $\begin{array}{c}\text { 2-Methyl- } \\
\text { 2-butanol } \\
\text { (tert-Amyl } \\
\text { alcohol) }\end{array}$ & $\begin{array}{l}\text { 2-Methyl-2- } \\
\text { propanol (tert- } \\
\text { Butyl alcohol) }\end{array}$ \\
\hline 01/07/2001 & - & - & E0.006 & - & - & - & - & - \\
\hline 01/19/2001 & - & - & E0.006 & - & - & - & - & - \\
\hline $01 / 31 / 2001$ & - & - & E0.014 & - & - & - & - & - \\
\hline $02 / 12 / 2001$ & - & - & E0.005 & - & - & - & - & - \\
\hline $02 / 24 / 2001$ & - & - & E0.002 & - & - & - & - & - \\
\hline 03/08/2001 & - & - & E0.003 & - & - & - & - & - \\
\hline 03/20/2001 & NA & NA & NA & NA & NA & NA & NA & NA \\
\hline $04 / 01 / 2001$ & - & - & E0.003 & - & - & - & - & - \\
\hline $04 / 13 / 2001$ & - & - & E0.002 & - & - & - & - & - \\
\hline $04 / 25 / 2001$ & - & - & E0.001 & - & - & - & - & - \\
\hline 05/07/2001 & - & - & E0.002 & - & - & - & - & - \\
\hline 05/19/2001 & - & - & - & - & - & - & - & - \\
\hline $05 / 31 / 2001$ & - & - & E0.001 & - & - & - & - & - \\
\hline $06 / 12 / 2001$ & - & - & E0.001 & - & - & - & - & - \\
\hline $06 / 24 / 2001$ & - & - & - & - & - & - & - & - \\
\hline $07 / 05 / 2001$ & - & - & - & - & - & - & - & - \\
\hline 07/16/2001 & - & - & E0.001 & - & - & - & - & - \\
\hline 08/08/2001 & - & - & E0.009 & - & - & - & - & - \\
\hline 08/23/2001 & - & - & - & - & - & - & - & - \\
\hline $09 / 16 / 2001$ & - & - & E0.006 & - & - & - & - & - \\
\hline $09 / 28 / 2001$ & - & - & E0.005 & - & - & - & - & - \\
\hline
\end{tabular}



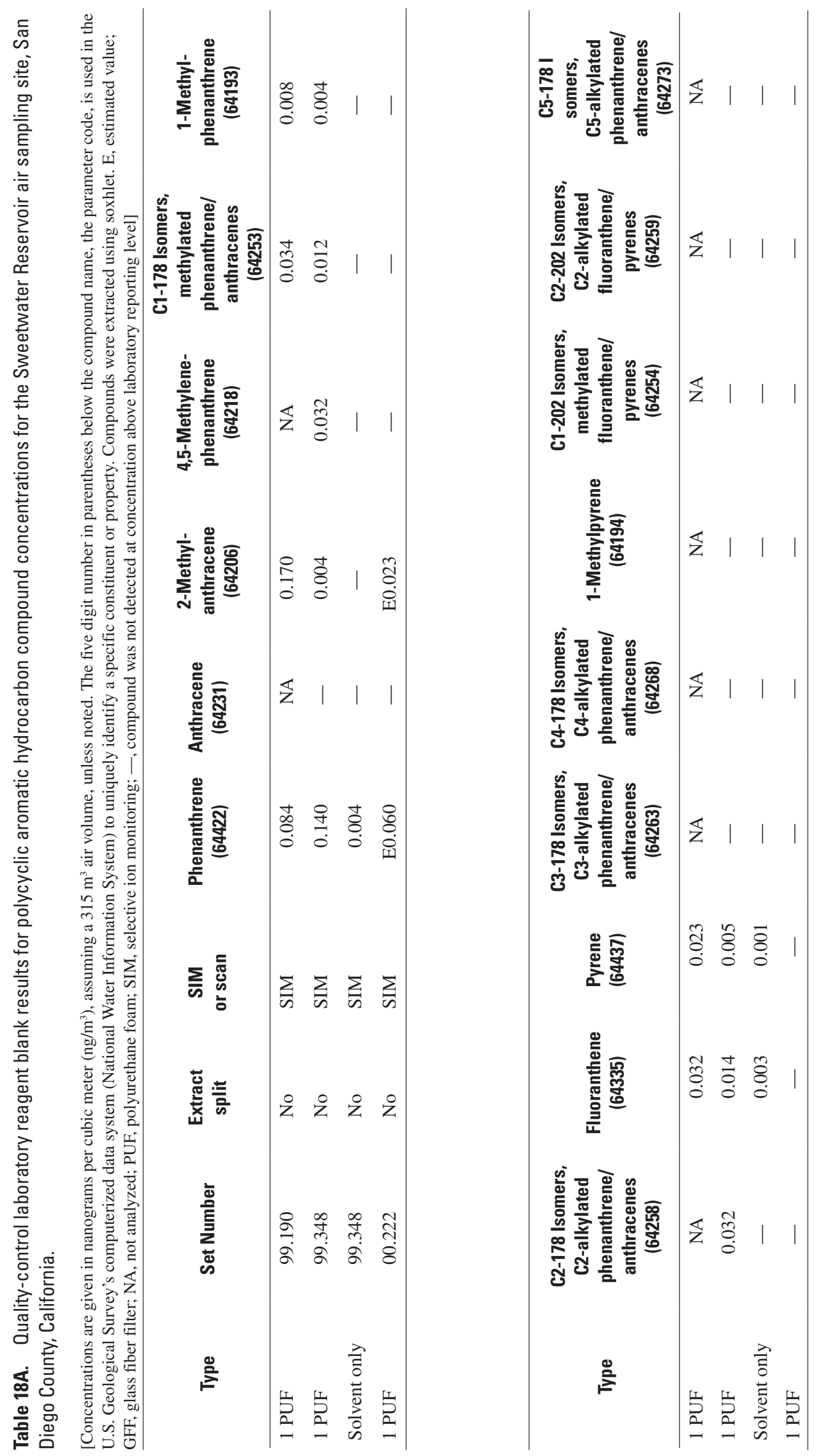

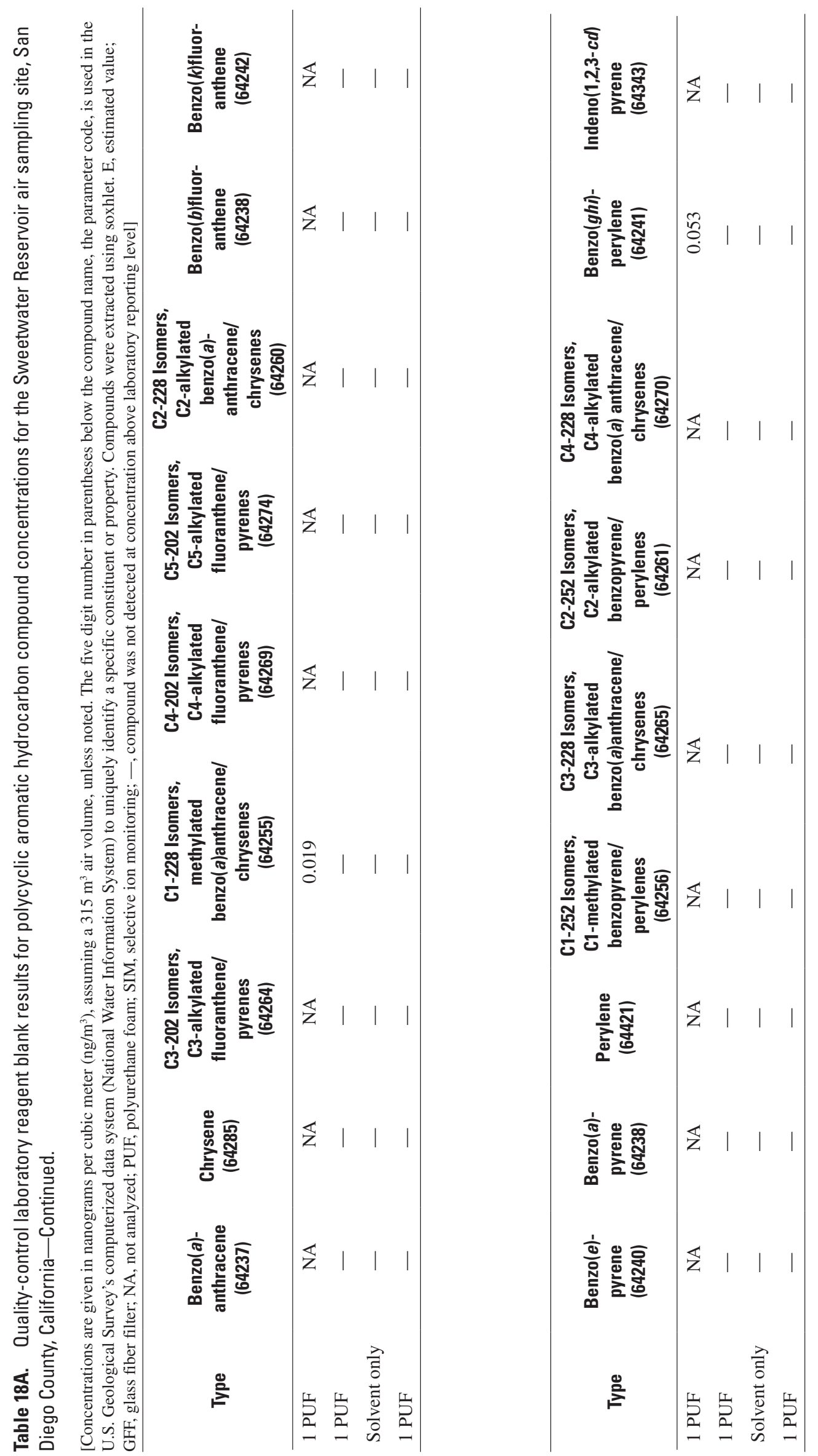


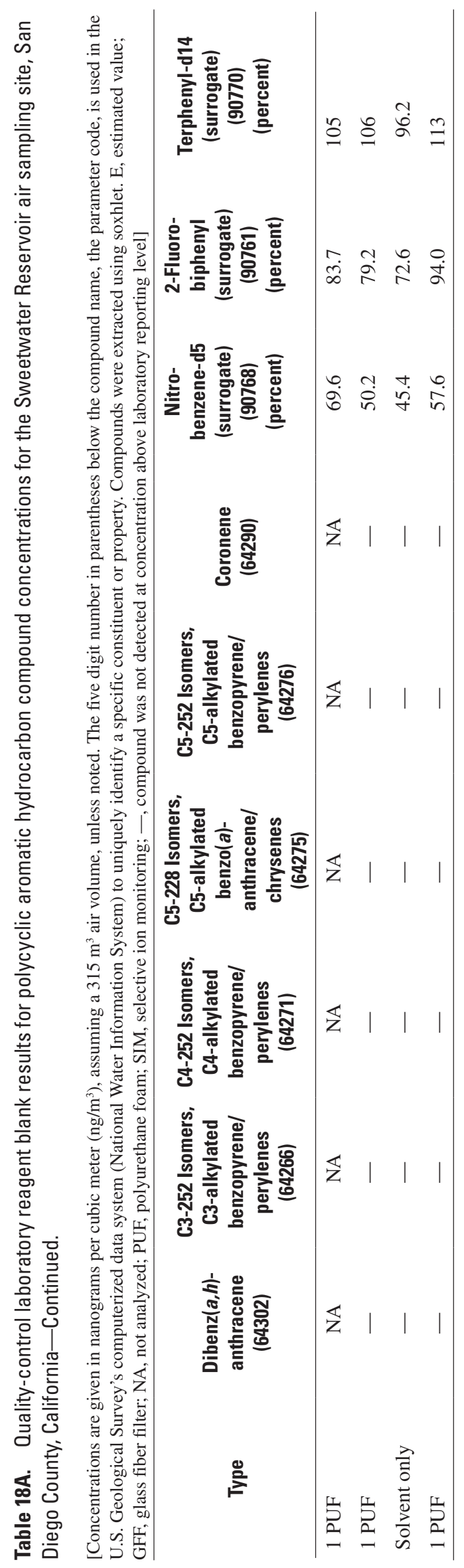


Table 18B. Quality-control laboratory reagent spike results for polycyclic aromatic hydrocarbon compound concentrations for the Sweetwater Reservoir air sampling site, San Diego County, California.

[The five digit number in parentheses below the compound name, the parameter code, is used in the U.S. Geological Survey's computerized data system (National Water Information System) to uniquely identify a specific constituent or property. The 21 Alkyl isomeric analytes on table 6 were not spiked, hence not included in this table. Compounds were extracted using soxhlet. Values are given in percent recovery. E, estimated value; NA, not analyzed]

\begin{tabular}{cccccc}
\hline Set number & $\begin{array}{c}\text { Extract } \\
\text { split }\end{array}$ & $\begin{array}{c}\text { Phenan- } \\
\text { threne } \\
\mathbf{( 6 4 4 2 2 )}\end{array}$ & $\begin{array}{c}\text { Anthracene } \\
\mathbf{( 6 4 2 3 1 )}\end{array}$ & $\begin{array}{c}\text { 2-Methyl- } \\
\text { anthracene } \\
\text { (64206) }\end{array}$ & $\begin{array}{c}\text { 4,5-Methylene- } \\
\text { phenanthrene } \\
\text { (64218) }\end{array}$ \\
\hline 99.190 & No & 96.3 & 80.0 & 87.8 & 93.3 \\
99.348 & No & 94.3 & 51.2 & 45.1 & 80.8 \\
00.222 & No & 108 & 40.5 & 35.8 & 89.2 \\
\hline
\end{tabular}

\begin{tabular}{ccccccc}
\hline Set number & $\begin{array}{c}\text { 1-Methyl- } \\
\text { phenanthrene } \\
\text { (64193) }\end{array}$ & $\begin{array}{c}\text { Fluoranthene } \\
\text { (64335) }\end{array}$ & $\begin{array}{c}\text { Pyrene } \\
\mathbf{( 6 4 4 3 7 )}\end{array}$ & $\begin{array}{c}\text { 1-Methylpyrene } \\
\text { (64194) }\end{array}$ & $\begin{array}{c}\text { Benzo(a)- } \\
\text { anthracene } \\
\text { (64237) }\end{array}$ & $\begin{array}{c}\text { Chrysene } \\
\text { (64285) }\end{array}$ \\
\hline 99.190 & 93.2 & 99.8 & 94.3 & NA & 96.3 & 89.9 \\
99.348 & 89.9 & 92.3 & 90.2 & 92.3 & 85.6 & 96.19 \\
00.222 & 101 & 92.1 & 93.1 & 93.9 & 58.6 & 101 \\
\hline
\end{tabular}

\begin{tabular}{ccccccc}
\hline $\begin{array}{c}\text { Sample } \\
\text { identification }\end{array}$ & $\begin{array}{c}\text { Benzo(b)- } \\
\text { fluor- } \\
\text { anthene } \\
(\mathbf{6 4 2 3 9 )}\end{array}$ & $\begin{array}{c}\text { Benzo(k)- } \\
\text { fluor- } \\
\text { anthene } \\
\mathbf{( 6 4 2 4 2 )}\end{array}$ & $\begin{array}{c}\text { Benzo(e)- } \\
\text { pyrene } \\
(\mathbf{6 4 2 4 0 )}\end{array}$ & $\begin{array}{c}\text { Benzo(a)- } \\
\text { pyrene } \\
(\mathbf{6 4 2 3 8}\end{array}$ & $\begin{array}{c}\text { Perylene } \\
\mathbf{( 6 4 4 2 1 )}\end{array}$ & $\begin{array}{c}\text { Benzo(ghi)- } \\
\text { perylene } \\
(\mathbf{6 4 2 4 1 )}\end{array}$ \\
\hline 99.190 & 138 & 99.5 & 116 & 93.9 & 102 & 96.4 \\
99.348 & 101 & 96.3 & 119 & 68.6 & 77.1 & 98.1 \\
00.222 & 83.0 & 57.4 & 77.1 & NA & NA & 64.6 \\
\hline
\end{tabular}

\begin{tabular}{ccccccc}
\hline $\begin{array}{c}\text { Sample } \\
\text { identification }\end{array}$ & $\begin{array}{c}\text { Indeno(1,2,3-cd)- } \\
\text { pyrene- } \\
\mathbf{( 6 4 3 4 3 )}\end{array}$ & $\begin{array}{c}\text { Debenz(a,h)- } \\
\text { anthracene } \\
\mathbf{( 6 4 3 0 1 )}\end{array}$ & $\begin{array}{c}\text { Coronene } \\
\mathbf{( 6 4 2 9 0 )}\end{array}$ & $\begin{array}{c}\text { Nirtro- } \\
\text { benzene-d5 } \\
\text { (surrogate) } \\
\text { (90768) }\end{array}$ & $\begin{array}{c}\text { 2-Fluoro- } \\
\text { biphenyl } \\
\text { (surrogate) } \\
\text { (90761) }\end{array}$ & $\begin{array}{c}\text { Terphenyl-d14 } \\
\text { (surrogate) } \\
\text { (90770) }\end{array}$ \\
\hline 99.190 & 93.8 & 97.5 & 117 & 88.3 & 90.5 & 104 \\
99.348 & 83.1 & 99.1 & 99.5 & 65.8 & 82.1 & 109 \\
00.222 & 25.4 & 63.4 & 74.4 & 60.6 & 96.2 & 116 \\
\hline
\end{tabular}



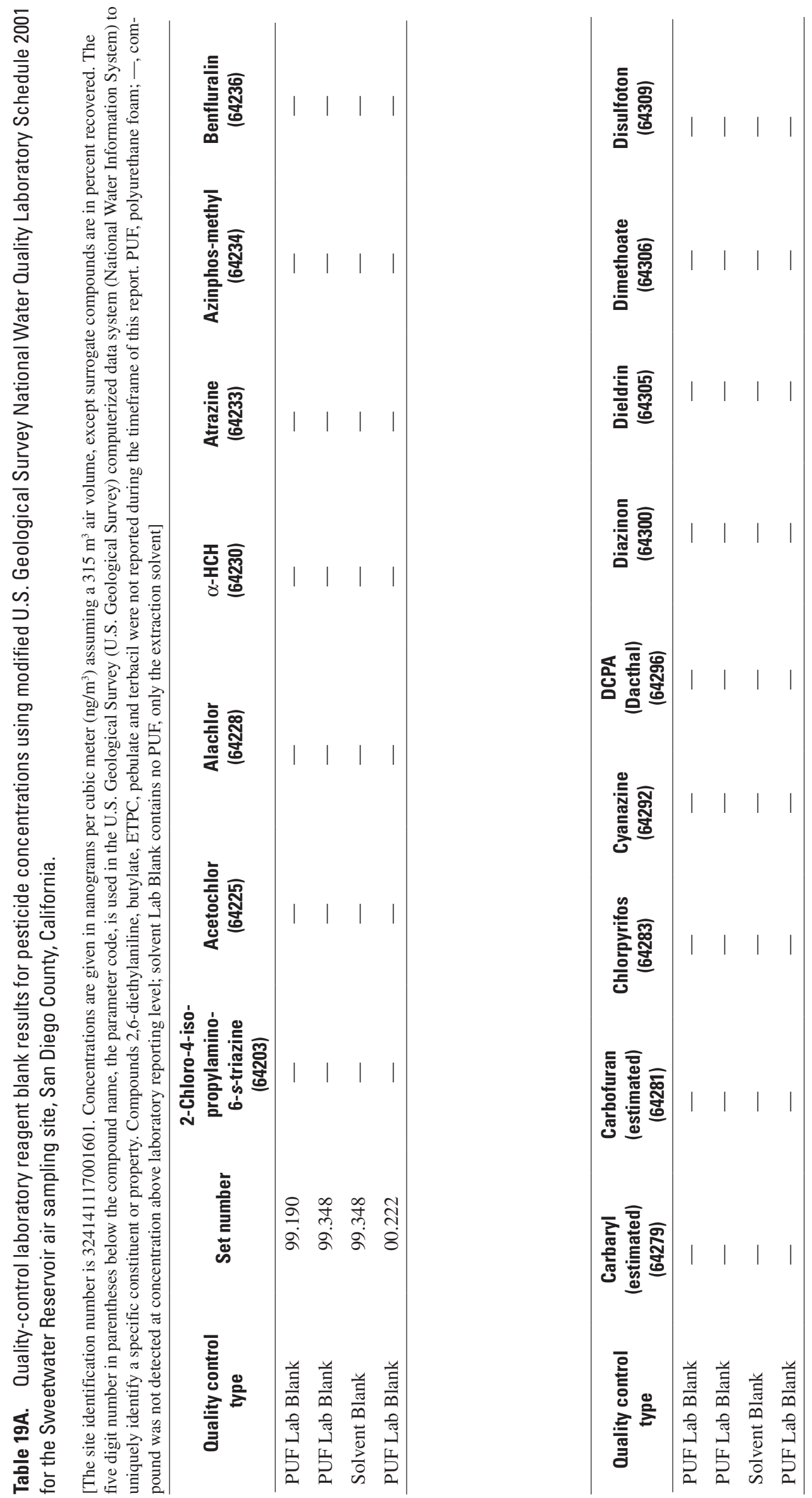

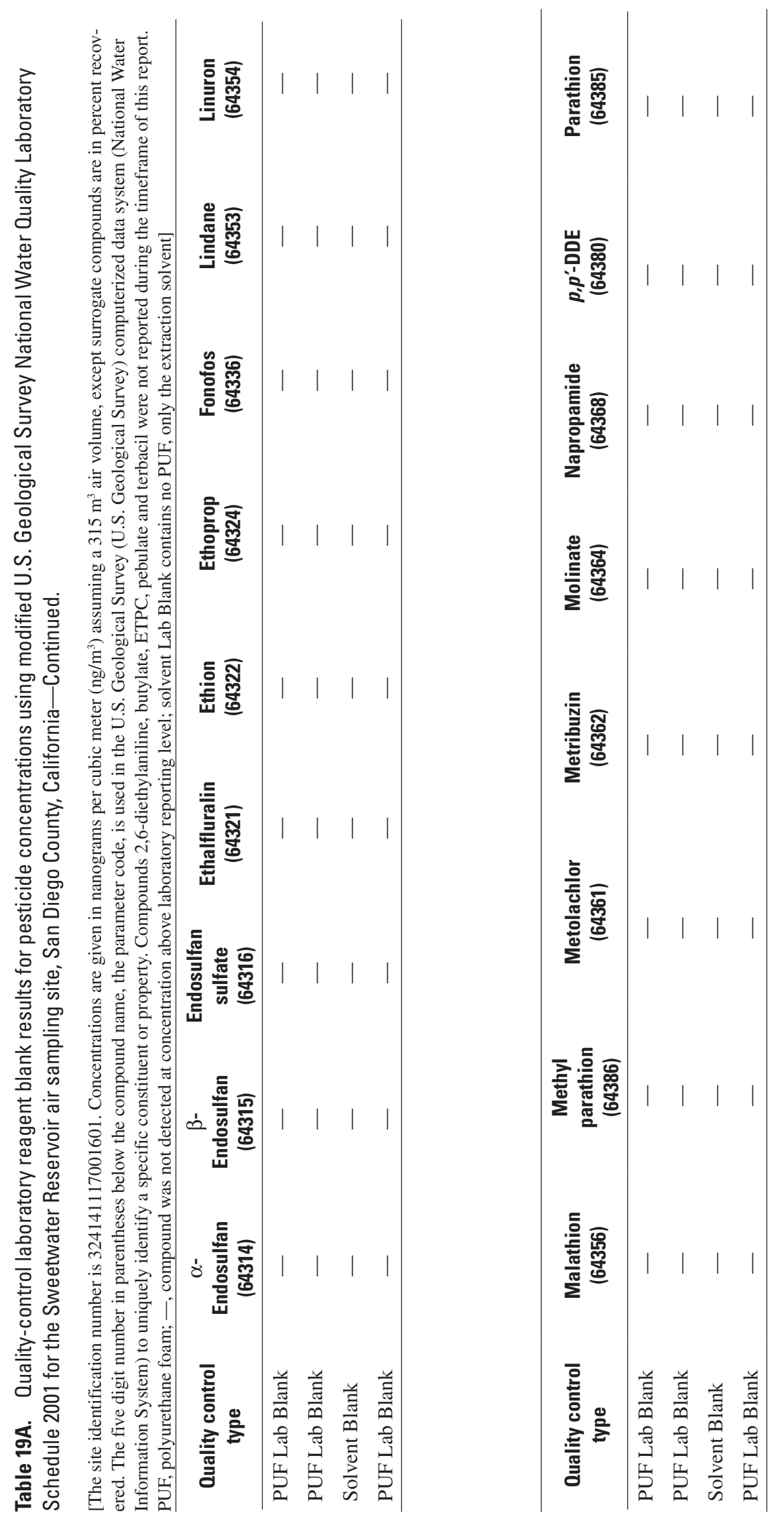

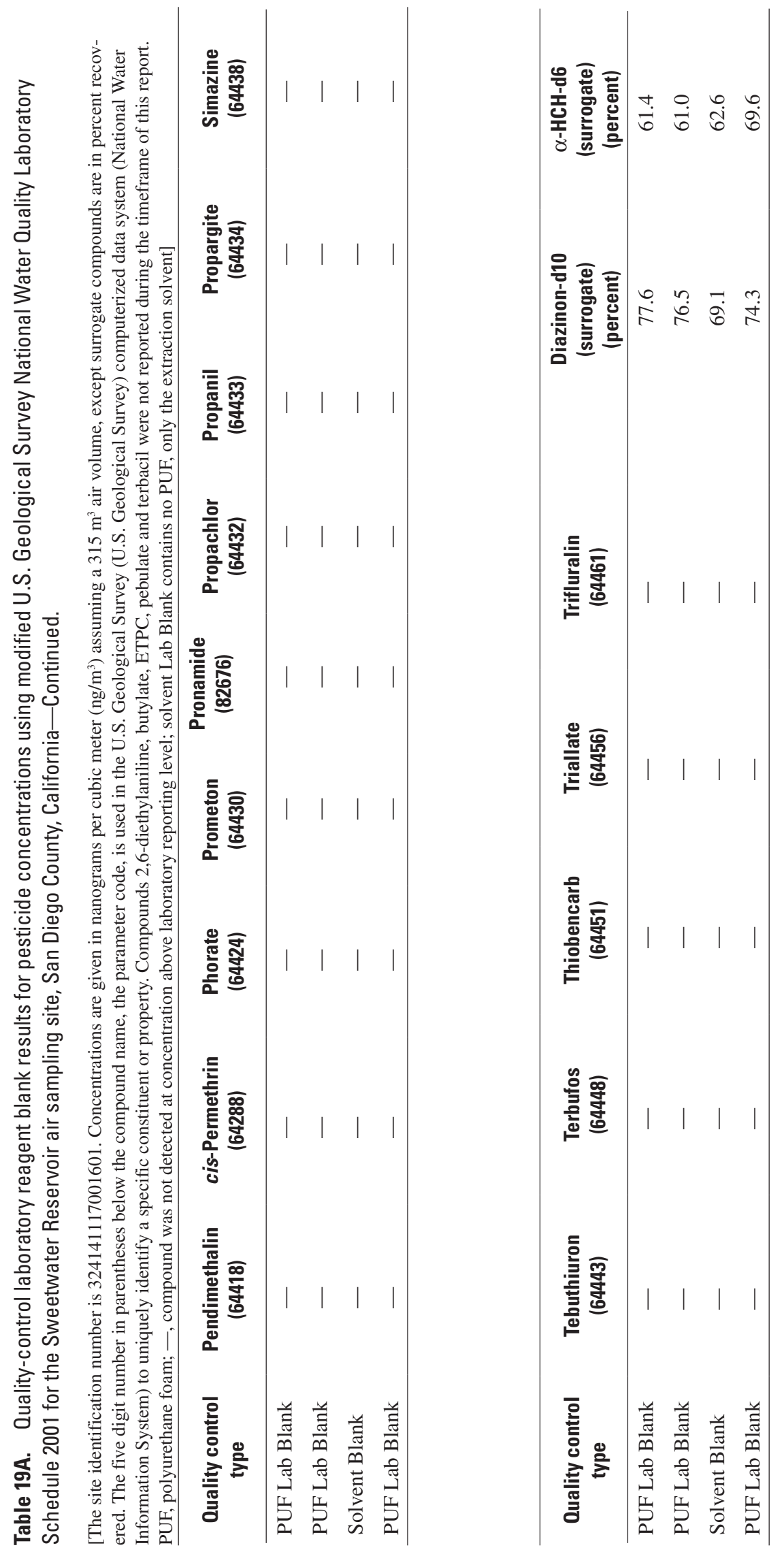

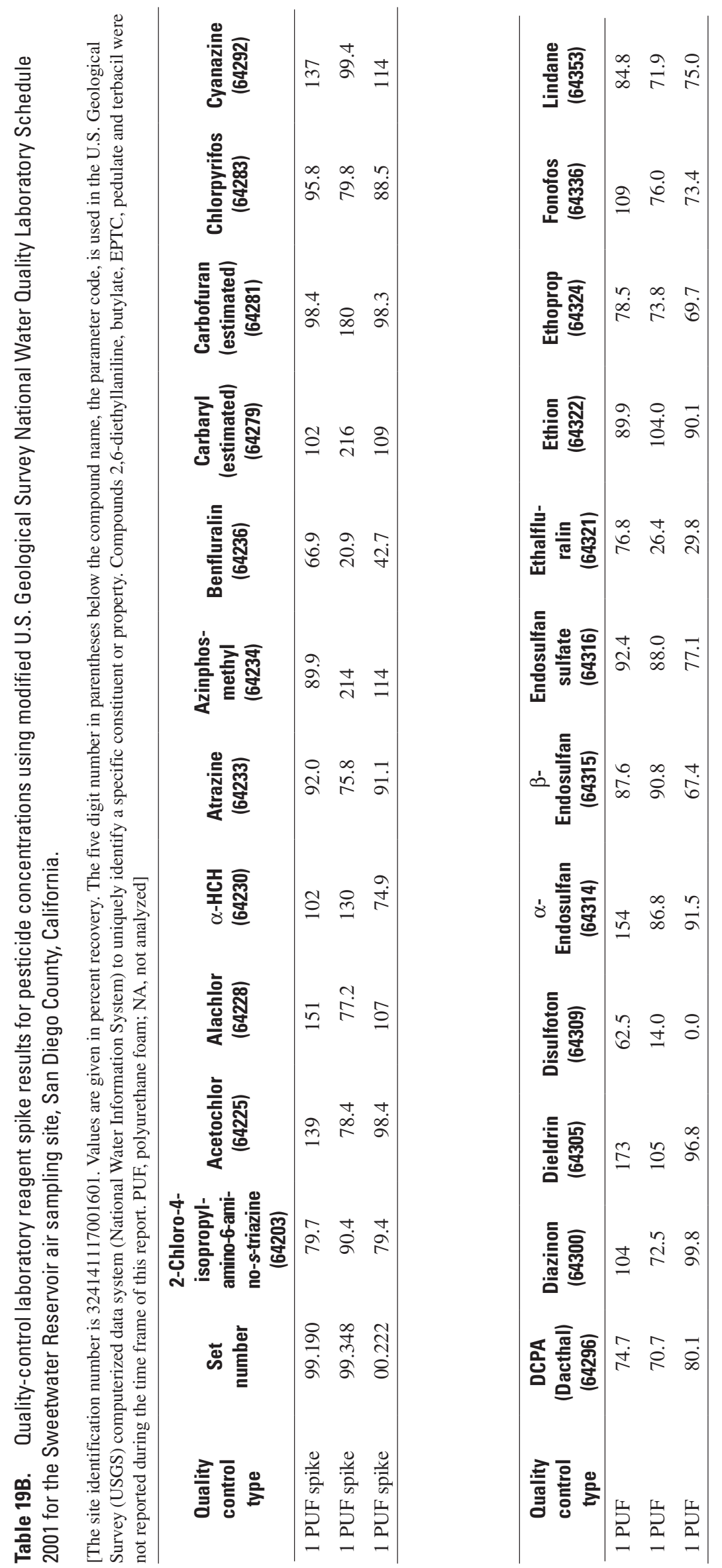


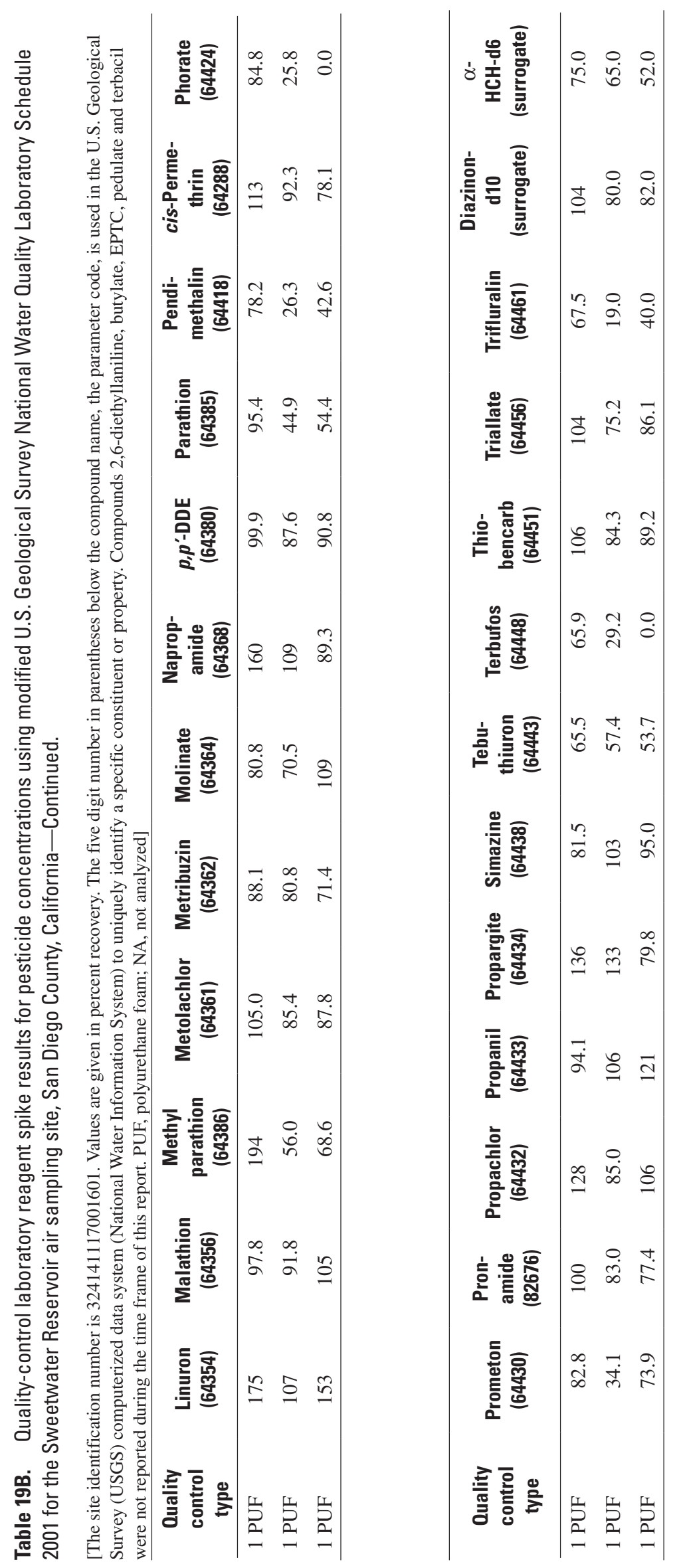



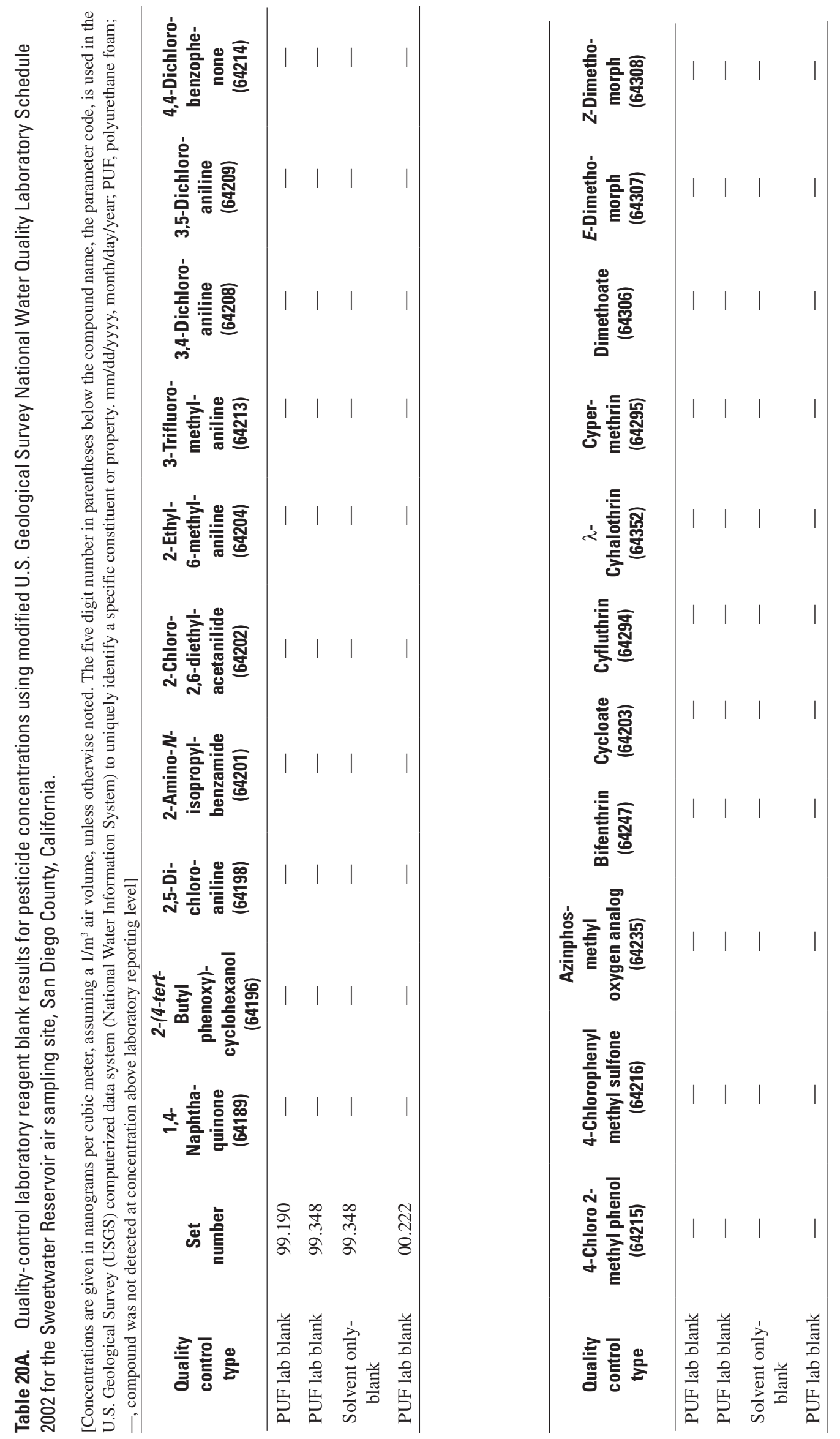

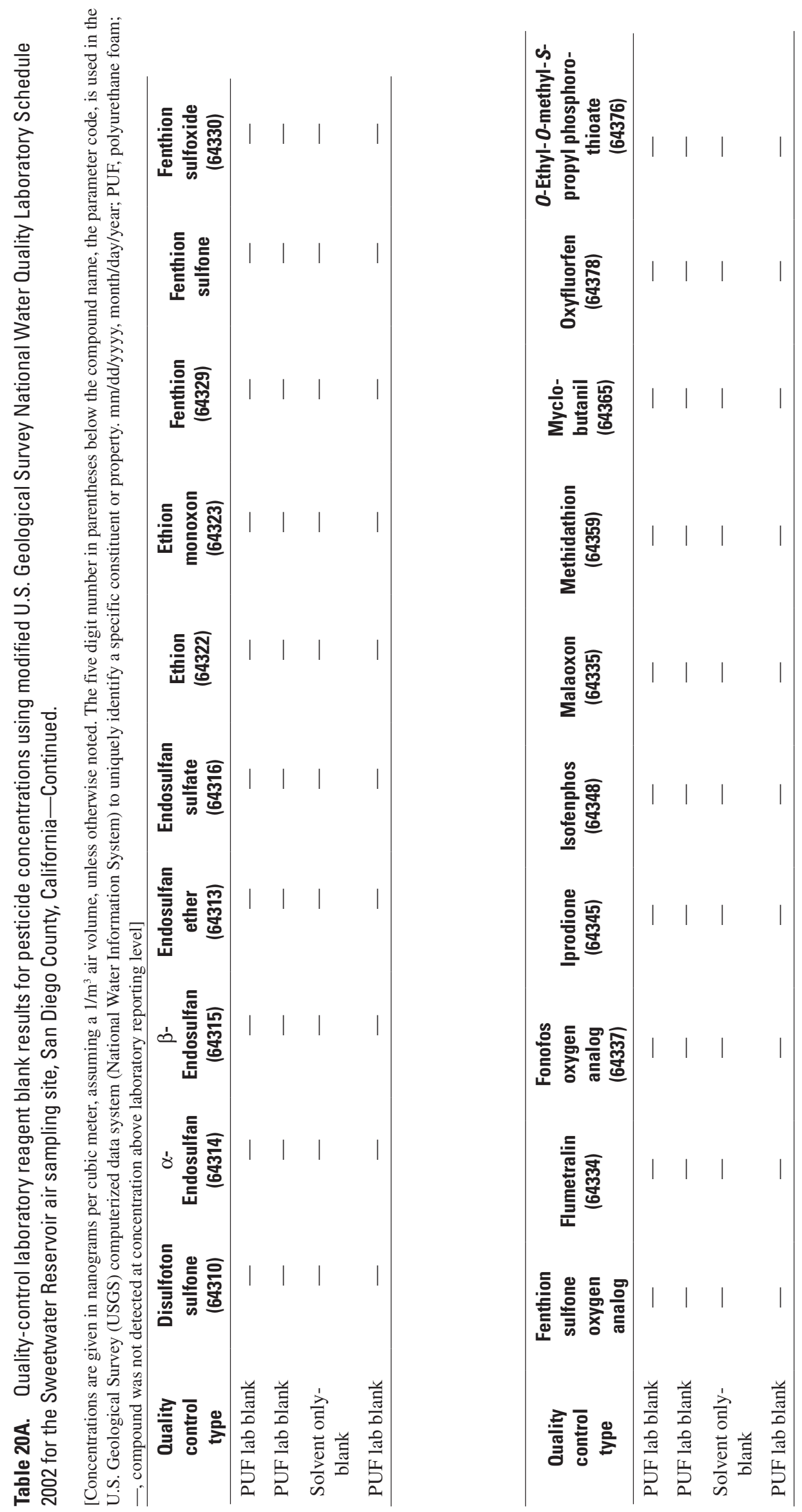

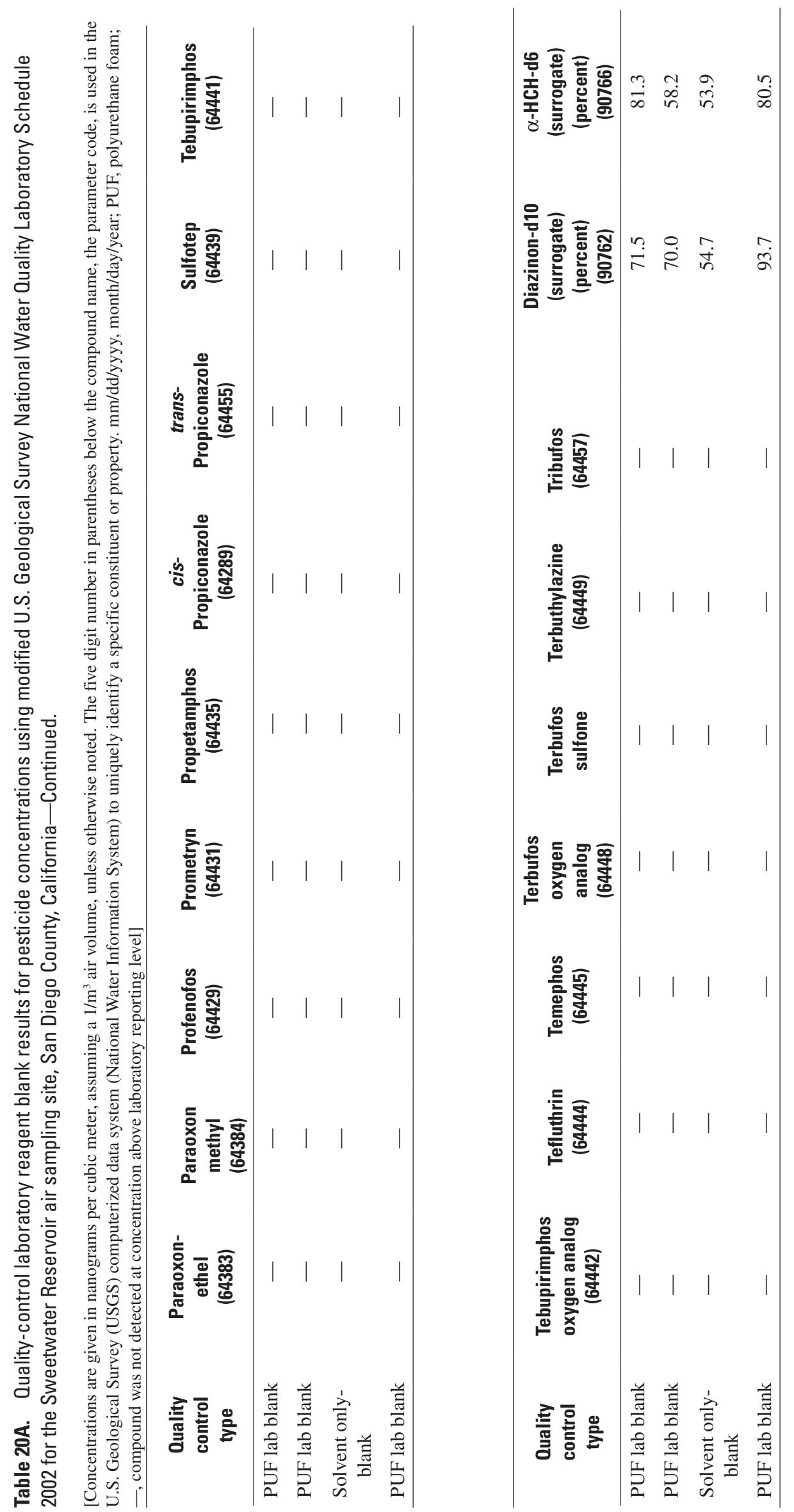

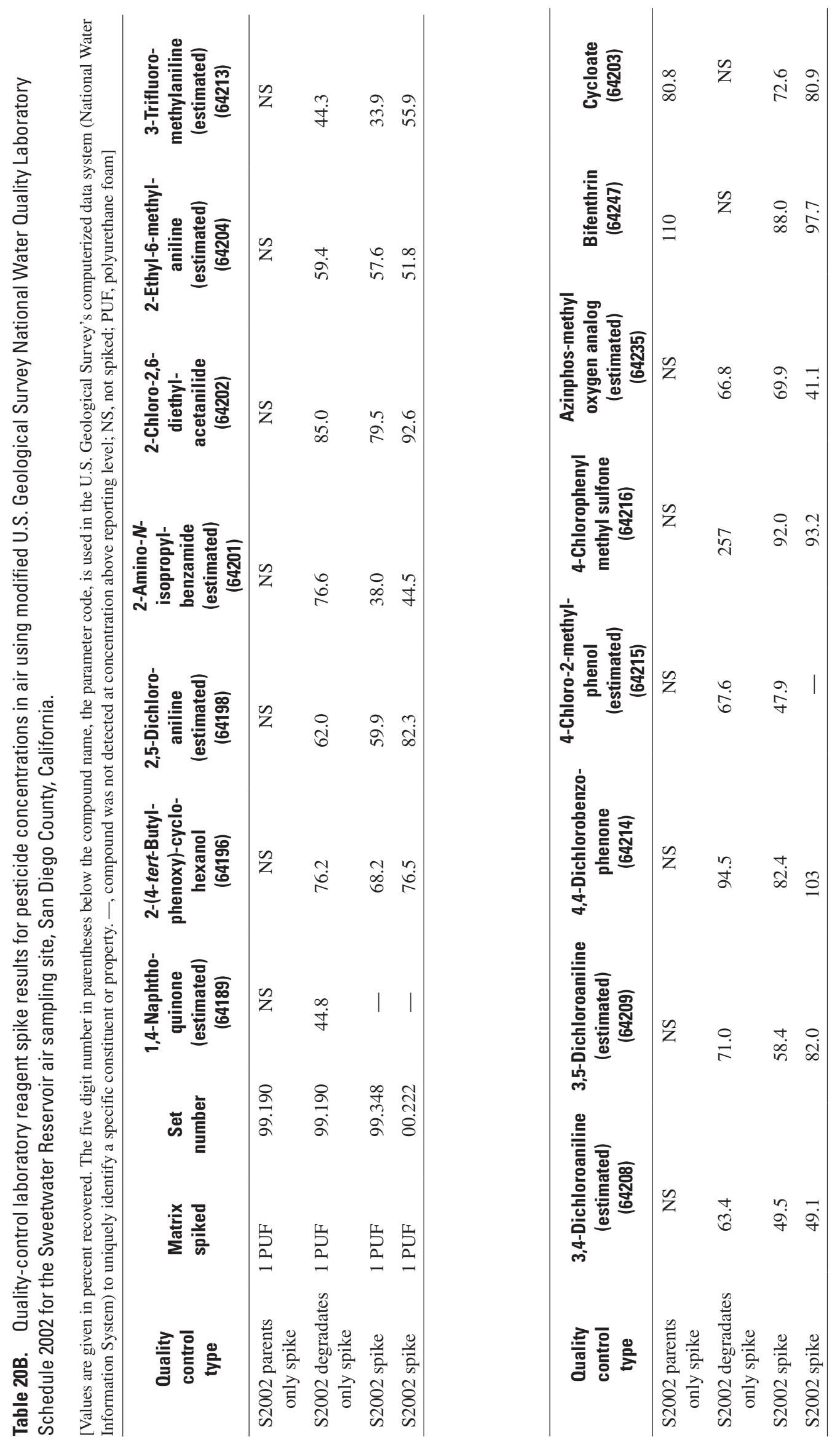


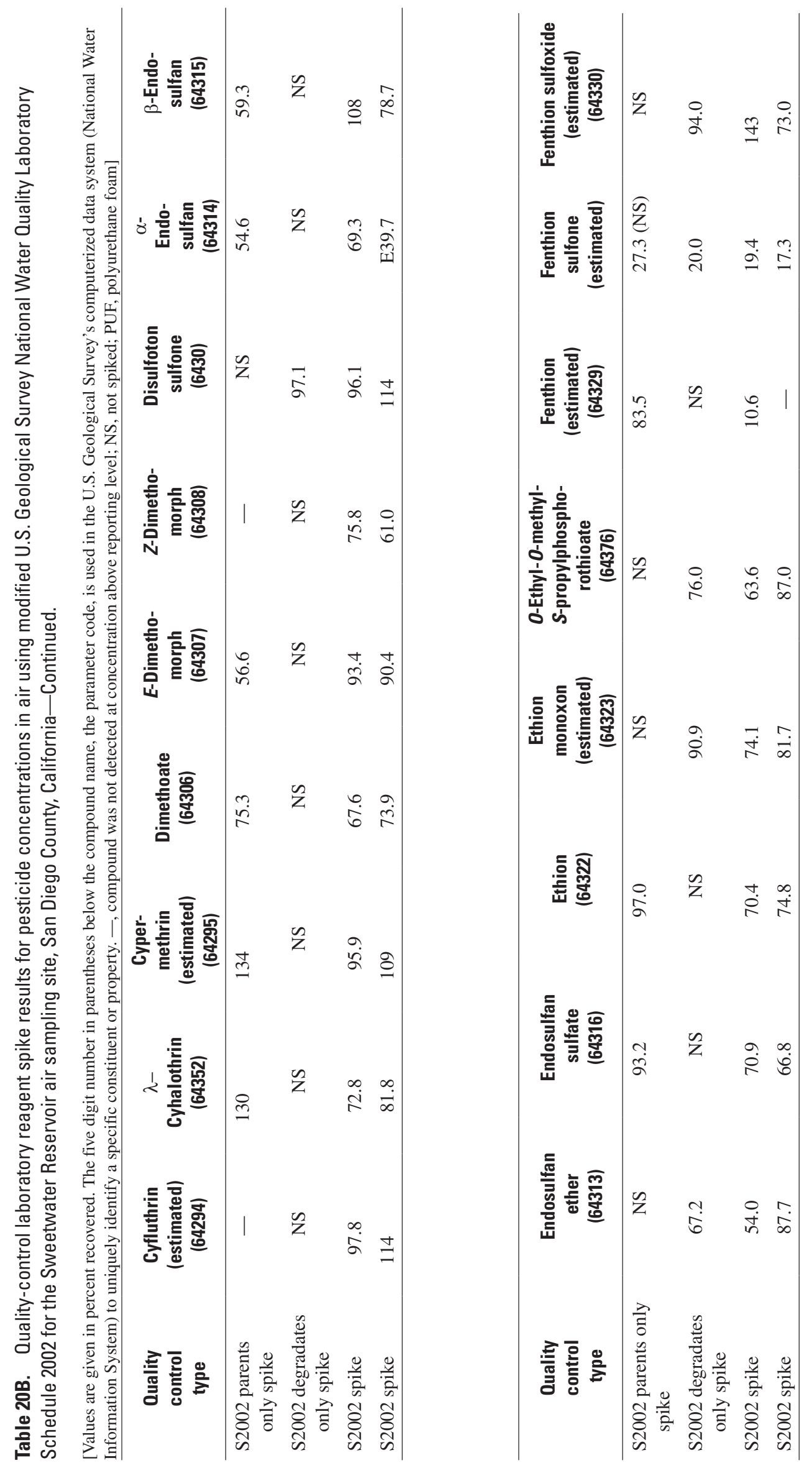




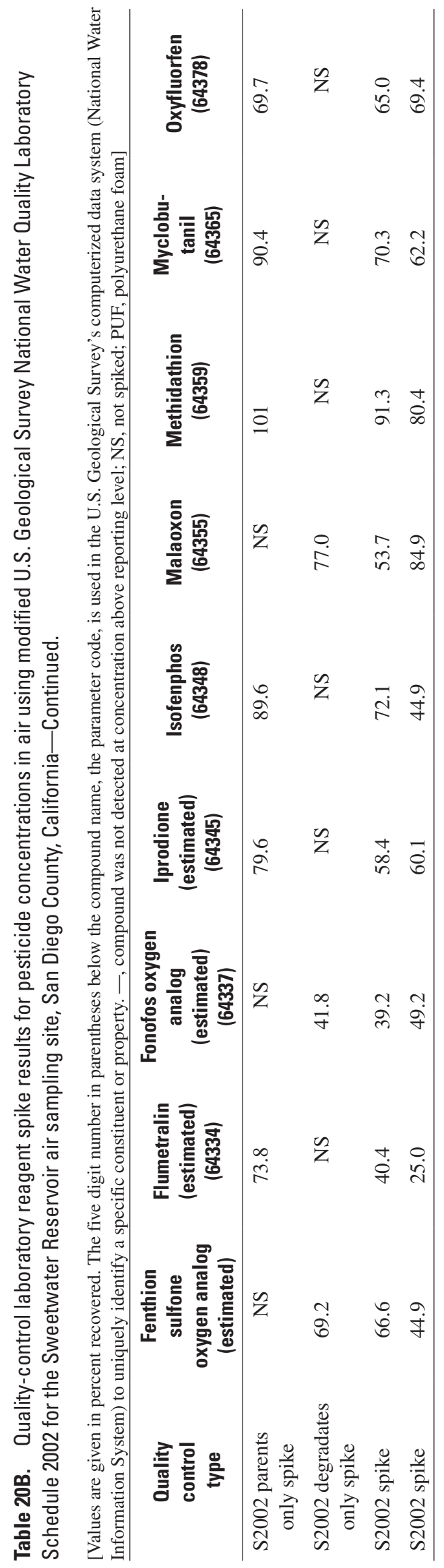

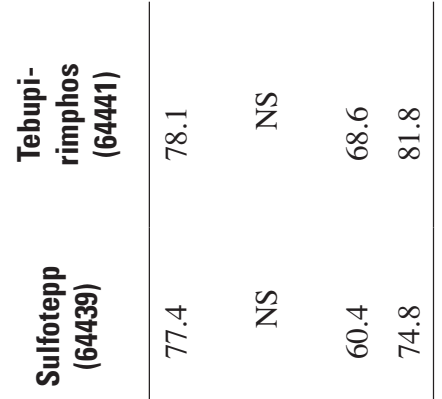

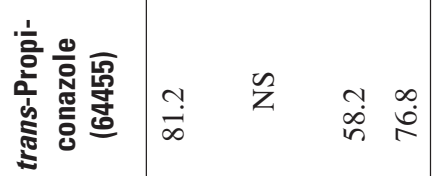

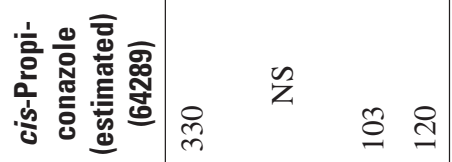

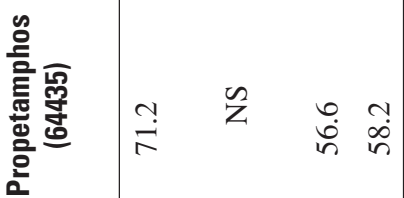

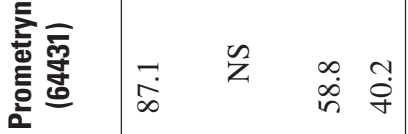

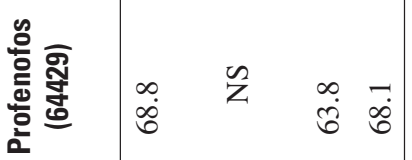

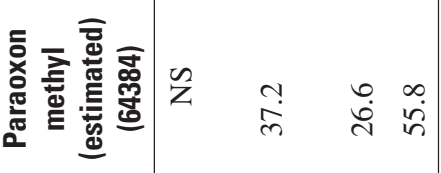

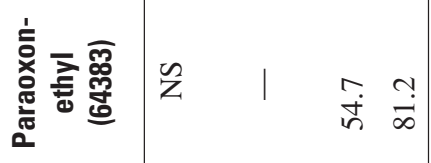

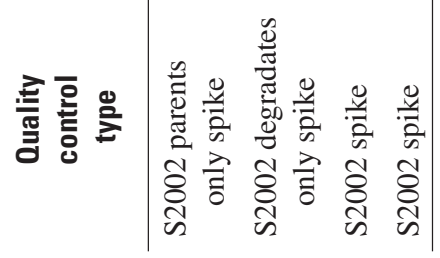




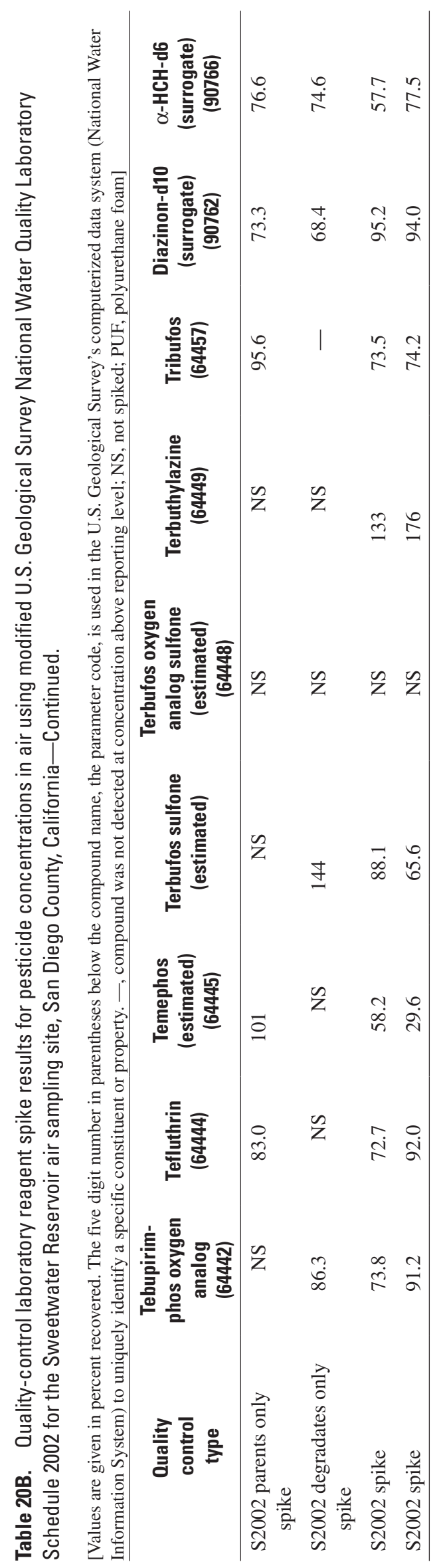


Table 21. Quality-control analytical results for surrogates added to the semipermeable membrane device in the Sweetwater Reservoir, San Diego County, California.

[Time denoted in 24-hour scale; surrogate values reported as percent recovered; mm/dd/yyyy, month/day/year; SWR, Sweetwater Reservoir]

\begin{tabular}{|c|c|c|c|c|c|c|}
\hline \multicolumn{7}{|l|}{ Pesticide surrogates } \\
\hline Station name & & & $\begin{array}{c}\text { SWR near } \\
\text { pump tower } \\
\text { (30-day SPMD } \\
\text { sample) }\end{array}$ & $\begin{array}{c}\text { SWR near } \\
\text { pump tower } \\
\text { (60-day SPMD } \\
\text { sample) }\end{array}$ & $\begin{array}{l}\text { SWR near } \\
\text { pump tower } \\
\text { (trip blank) }\end{array}$ & $\begin{array}{l}\text { SWR near } \\
\text { pump tower } \\
\text { (60-day } \\
\text { processing } \\
\text { blank) }\end{array}$ \\
\hline Begin date (mm/dd/yyyy) & & & $01 / 25 / 2001$ & $01 / 25 / 2001$ & $01 / 25 / 2001$ & $01 / 25 / 2001$ \\
\hline End date (mm/dd/yyyy) & & & $02 / 22 / 2001$ & $03 / 21 / 2001$ & $03 / 21 / 2001$ & $03 / 21 / 2001$ \\
\hline End time & & & 1000 & 1600 & 1605 & 1610 \\
\hline a-HCH-d6 & & & 90.0 & 93.0 & 84.0 & 77.0 \\
\hline $\begin{array}{l}\text { Nonachlorobiphenyl } \\
\text { (PCB-207) }\end{array}$ & & & 97.0 & 92.0 & 96.0 & 88.0 \\
\hline \multicolumn{7}{|c|}{ Polycyclic aromatic hydrocarbon surrogates } \\
\hline Station name & Lab spike & Lab blank & $\begin{array}{c}\text { SWR near } \\
\text { pump tower } \\
\text { (30-day SPMD } \\
\text { sample) }\end{array}$ & $\begin{array}{c}\text { SWR near } \\
\text { pump tower } \\
\text { (60-day SPMD } \\
\text { sample) }\end{array}$ & $\begin{array}{l}\text { SWR near } \\
\text { pump tower } \\
\text { (trip blank) }\end{array}$ & $\begin{array}{l}\text { SWR near } \\
\text { pump tower } \\
\text { (60-day } \\
\text { processing } \\
\text { blank) }\end{array}$ \\
\hline Begin date & 08/27/2001 & 08/27/2001 & $01 / 25 / 2001$ & $01 / 25 / 2001$ & $01 / 25 / 2001$ & $01 / 25 / 2001$ \\
\hline Begin time & & & 1000 & 1000 & 1005 & 1010 \\
\hline End date & & & $02 / 22 / 2001$ & 03/21/2001 & $03 / 21 / 2001$ & 03/21/2001 \\
\hline End time & & & 1000 & 1600 & 1605 & 1610 \\
\hline
\end{tabular}



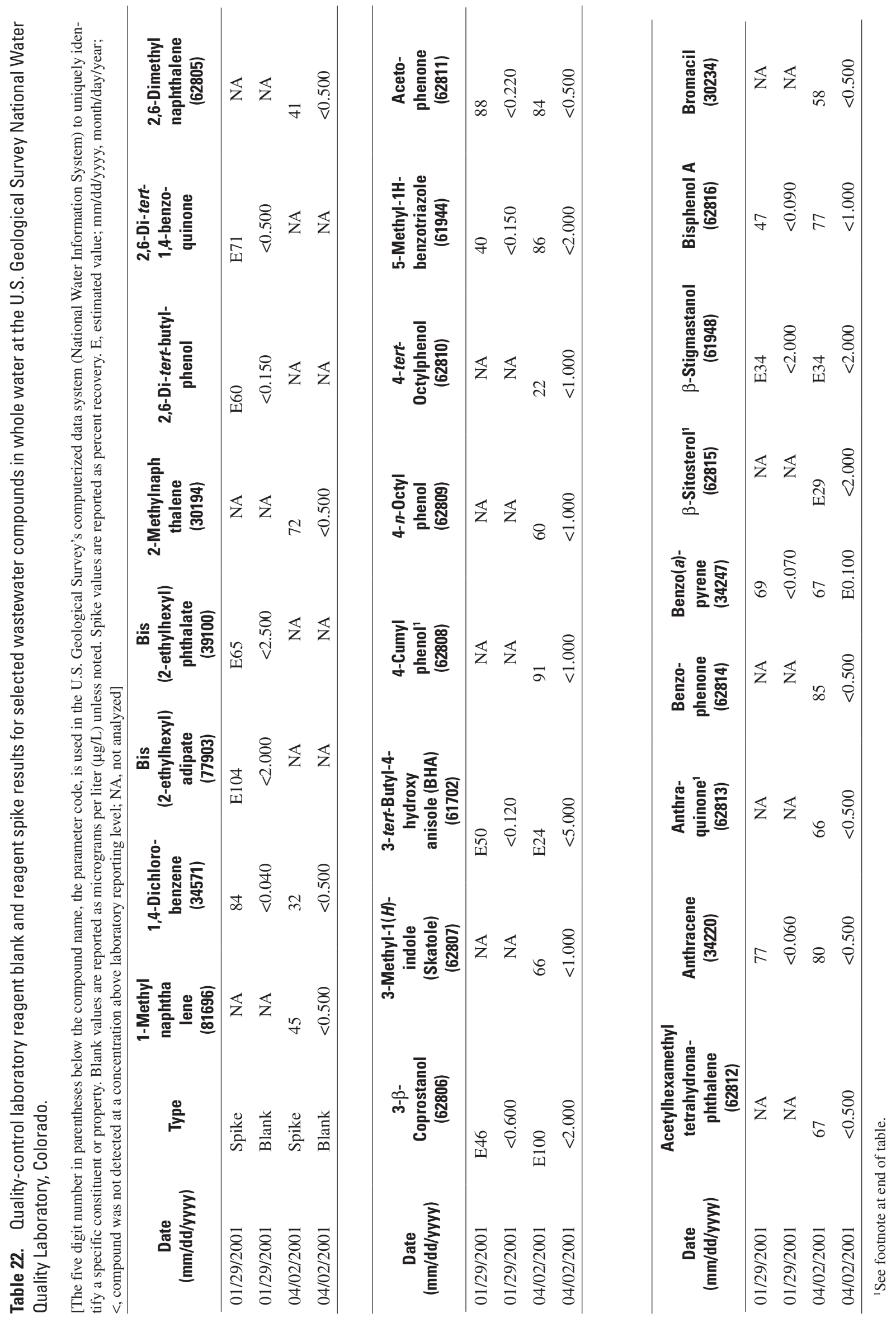


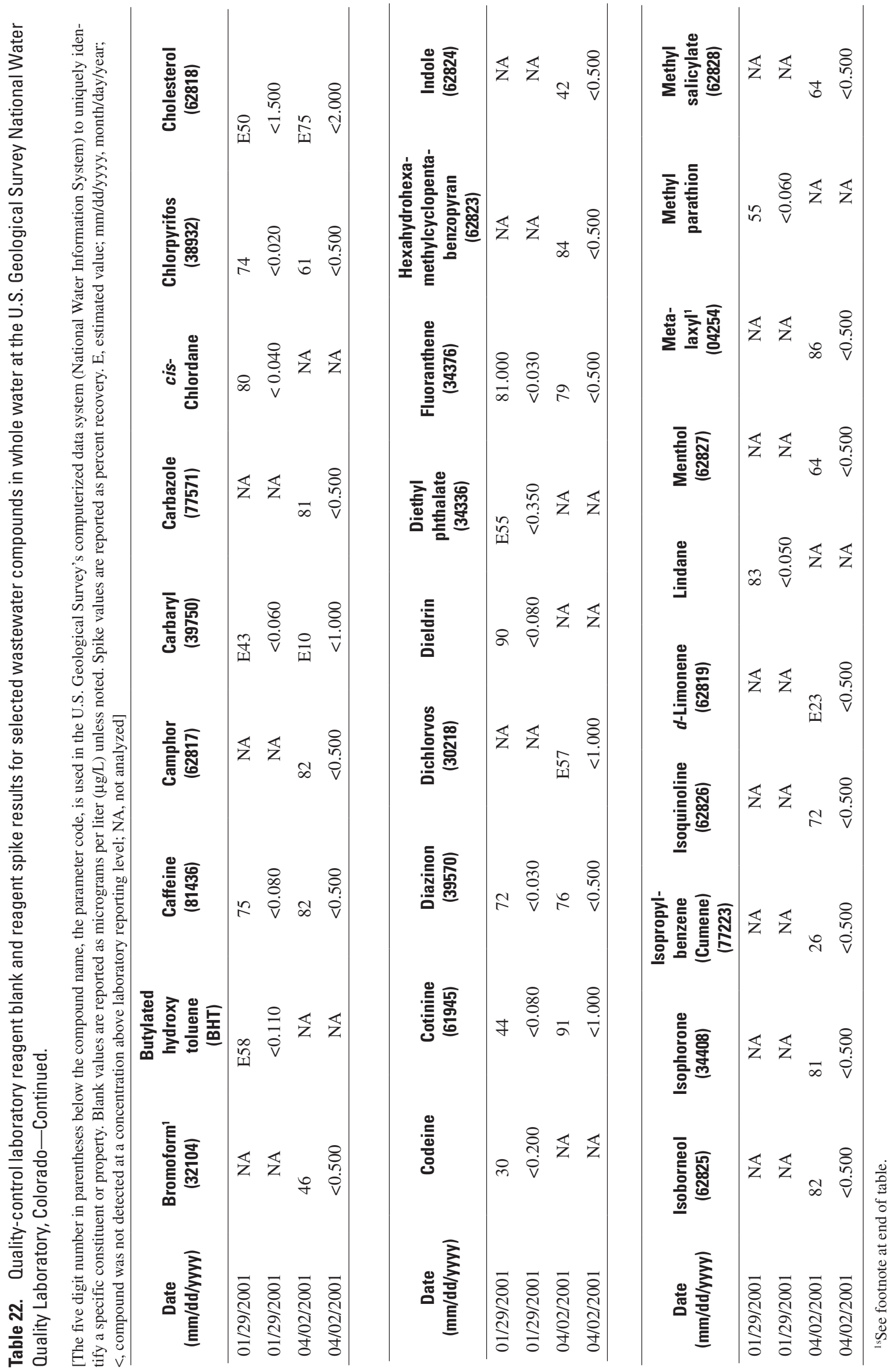




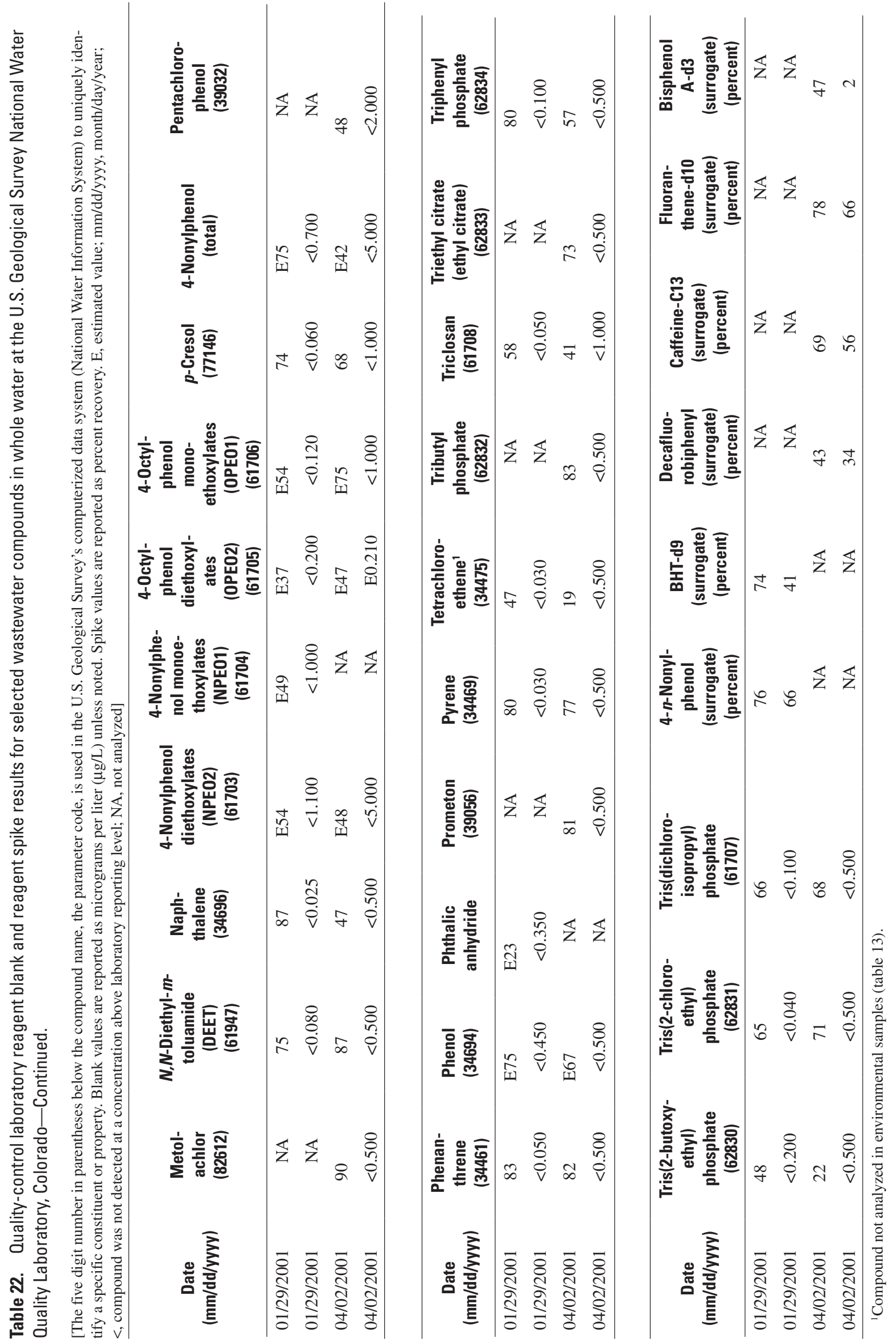

